\title{
Energy Levels, Classified Lines, and Zeeman Effect of Neutral Thorium
}

\author{
Romuald Zalubas
}

Institute for Basic Standards, National Bureau of Standards, Washington, D.C. 20234

(December 3, 1975)

\begin{abstract}
A list of about 9500 classified lines of Th I in the range 2345-29 $662 \AA$ is given. Lines in the range 2345-9239 A were observed and measured at NBS. Zeeman effect data for 2281 lines are listed. Lists of 254 even and 322 odd levels including their $g$ values are presented. Among them there are 72 new levels, which were not contained in earlier publications.
\end{abstract}

Key words: Energy levels; thorium; Th I; wavelengths; Zeeman-effect of Th I

\section{Introduction}

A review of early research on the spectra of neutral and singly ionized thorium has been given by Zalubas [1960]. ${ }^{1}$ In the meantime light sources and spectrographs have been improved and more standard wavelengths of thorium have become available. Their development is described by Giacchetti, Stanley, and Zalubas [1970]. Therefore, at NBS I undertook a new observation and description of Th I and Th II in order to expand the analyses of these spectra. A complete line list of about $35000 \mathrm{Th}$ I and Th II lines will be published separately. That list will include all available thorium standards and will be useful for users of thorium standards. Zalubas and Corliss have published a list of classified lines in Th II [1974].

In the present paper I will review only the work on finding the energy levels and $g$ factors of Th $\mathrm{I}$.

\section{Wavelengths and Classified Lines}

New observations were taken on the NBS 10.7 meter Eagle spectrograph in the range 2000-12 $000 \AA$. Electrodeless lamps and sliding sparks were used as light sources for the separation of Th I and Th II. Professor S. P. Davis supplied some spectrograms for range $2600-4500 \AA$ produced on the Czerny-Turner spectrograph at the University of California. I measured wavelengths in the 2800-12000 $\AA$ observation range. Stronger lines were measured on 3 to 6 plates, weaker lines (below intensity 5) on 2 to 3 plates. Small numbers of lines of intensity 1 are included which were measured only once. The wavelengths in the

${ }^{1}$ Dates enclosed in brackets indicate literature references at the end of this paper. range $2000-2800 \AA$ were measured only once. Internal thorium standards from Giachetti [1966], and Giacchetti, Stanley, and Zalubas [1970] were used.

All classified lines fit the energy level scheme to within $\pm 0.05 \mathrm{~cm}^{-1}$. More than 60 percent of them do not exceed $\pm 0.02 \mathrm{~cm}^{-1}$.

The consistency of wavelengths in this list can be demonstrated by the following test. The average absolute value of the difference between observed and calculated wavelengths expressed in $\mathrm{cm}^{-1}$ was 0.008 for 50 lines around each of the wavelengths 3000 , $4000,5000,6000$, and $8000 \AA$.

At the Laboratoire Aimé Cotton, Orsay, France wavelengths of Th I and Th II lines in the range 9239$29662 \AA$ were recorded by means of Fourier transform spectroscopy. The list of 3100 lines, including classified lines of Th I and Th II, was published by Giacchetti, Blaise, Corliss, and Zalubas [1974]. The average deviation between the observed and calculated wavenumbers from this list is less than $0.002 \mathrm{~cm}^{-1}$.

These two lists combined together have around 35000 lines and excel earlier observations in accuracy and extent. In order to have all classified lines of Th I at hand, the $1900 \mathrm{Th}$ I lines measured by Fourier transform spectroscopy are taken from Giacchetti et al. [1974] and incorporated in table 4.

Table 4 contains about 9500 classified lines in the wavelength range $2345-29662 \AA$. For the wavelength range 2345-9239 $\AA$ visually estimated relative intensities on an arbitrary scale from 1 to 5000 are given for electrodeless lamp and sliding spark. For the wavelength range 9239-29 $662 \AA$ only the electrodeless lamp intensity on a scale 0 to 9 is given. The letter symbols are used to describe the character of lines; 
$b$-blend (two wavelengths measured), $d$-double (one wavelength measured), $h$-hazy, $l$-shaded to longer wavelength, $r$-reversed, $s$-shaded to shorter wavelength, and $w$-wide. In column 4 the wavenumber is given in vacuum in units of $\mathrm{cm}^{-1}$. In the fifth and sixth columns the classification of a line is given by rounded-off values of the energy levels responsible for the transition, with their $J$-values as subscripts and a superscript degree symbol to indicate odd parity.

\section{Zeeman Effect and g-Factors}

B. E. Moore [1909] observed the splittings of thorium lines in the magnetic field in the range 3002-4721 $\AA$. He observed mostly Th II lines and at that time of course did not derive $J$ and $g$ values.

Lier [1939] observed the Zeeman effect of 40 lines of Th I in the range 3427-6182 $\AA$. Fifteen of these lines had resolved ZE patterns. These data were used by Schuurmans [1946], who derived $g$ factors for all levels he found. Charles [1958] furnished ZE data for 46 lines in the range 3041-6457 .

In the final list I used $g$ factors derived from my observations. I have measured ca. 2281 Zeeman patterns in a magnetic field of 2.4 teslas, produced by the electromagnet at Argonne National Laboratory. From completely resolved patterns I derived $J$ values and $g$ values, and from partly resolved patterns I obtained $\Delta g$, or $p$, or $n$, or a combination of these. $\Delta g$ is the difference between two $g$ values for that line. The distance from the no-field position to the most distant $p$ component (parallel polarization) is denoted by $p$, and $n$ is the distance from no-field position to the strongest $n$ component (normal polarization). All these measurements are given in Lorentz units. The type of Zeeman pattern is described by the following numbers: 1 indicates that the $n$ pattern shades out, 2 indicates that the $n$ pattern shades in, 3 indicates that the $n$ pattern is symmetrical, $J_{1}=J_{2}$, and the $p$ pattern shades in, and 7 indicates that the pattern is either triplet or unresolved. The values of $g$ derived from my measurements or measured $\Delta g, p$, and $n$ are given in table 3 .

Values of $g$ factors for the energy levels are derived from various numbers of patterns, from 60 for one level to only one for some levels. Therefore, the $g$-values are given with three, two, or one decimal digits. The estimated error is not greater than 2 in the last digit.

\section{Energy Levels}

In previous papers on ThI [Zalubas, 1959, 1968] I gave an account of the earlier analyses of Th I. Here I will give just the total number of levels contributed by those early investigators that have proved to be real. Schuurmans [1946] found 29 energy levels and properly identified the five lowest levels. Stukenbroeker and McNally [1953] contributed 7 levels. Charles [1958] found 2 levels. Steers [1967], using his far infrared observations, found 13 levels. Giacchetti and Blaise [1970] found 10 levels. The remaining levels were found by the present author. Now the total number of known even levels is 254, and 322 odd levels. There are 72 levels given here for the first time.

Th I has two very distinct systems of levels, which I have connected. One system starts with the ground state $6 d^{2} 7 s^{2}{ }^{3} \mathrm{~F}_{2}$. The other starts $7795 \mathrm{~cm}^{-1}$ above ground state with the $5 f 6 d 7 s^{2}{ }^{3} \mathrm{H}_{4}^{\circ}$ level.

For energy level searches I treated the two systems separately. In such searches, I used a set of programs called COMBO, written by J. Tech for the analysis of spectra. The numbers of combinations and the available ZE data were the critical factors in establishing new energy levels. I have not included levels which can be found by intercombinations between high odd levels, and high even levels, despite the fact that some chains are long. Some of them may be confirmed later by theoretical calculations and extension of the analysis. Even levels are given in table 1 and odd levels in table 2.

The uncertainty of the level values for levels below 15000 is $0.001 \mathrm{~cm}^{-1}$, and the uncertainties of most of the high levels do not exceed $0.005 \mathrm{~cm}^{-1}$. Levels given with two decimal places have uncertainties of 0.01 to $0.02 \mathrm{~cm}^{-1}$.

I have identified the lowest levels by using $g$ values, and by comparison of the levels with calculations for the $6 d^{2} 7 s^{2}$ and $6 d^{3} 7 s$ configurations by Trees [1960]. For the low odd levels I made preliminary identifications using Martin's [1963] Ce:I identifications, and also Klinkenberg's [1950] Th III identifications. Later I used Sugar's [1968] preliminary calculations for the $5 f 6 d 7 s^{2}$ and $5 f 6 d^{2} 7 s$ configurations. Giacchetti and Blaise [1970] identified the ${ }^{5} \mathrm{~F},{ }^{5} \mathrm{G},{ }^{5} \mathrm{H},{ }^{5} \mathrm{I}$ terms of the $5 f 5 d 7 s 7 p$ configuration. In addition, Brewer [1971] identified 14465 as ${ }^{5} \mathrm{G}_{2}^{\circ}$ of $6 d^{2} 7 s 7 p$, and 18431 as ${ }^{3} \mathrm{~F}_{3}$ of $5 f 7 s^{2} 7 p$. No attempt has been made to identify the terms or configurations of the high energy levels.

\section{Ionization Energy}

Ionov and Mittsev [1961] derived the ionization energy of Th $\mathrm{I}$ as $6.95 \pm 0.06 \mathrm{eV}$, and Smith and Hertel [1969] as $7.5 \pm 0.3 \mathrm{eV}$ by the surface ionization method. Avni and Klein [1973] got $7.4 \pm 0.3 \mathrm{eV}$ for the ionization energy from measurements in a d.c. arc plasma, using the Saha equations. Meggers, Corliss, and Scribner [1961] estimated this energy to be about $50000 \mathrm{~cm}^{-1}$. Rauh and Ackermann [1973] obtained an ionization potential of $6.0 \pm 0.1 \mathrm{eV}$ from an ionization efficiency curve and from the appearance potential by electron impact. The best value of 49000 $\pm 1000 \mathrm{~cm}^{-1}(6.08 \pm 0.12 \mathrm{eV})$ was obtained by Sugar [1973] utilizing interpolated spectral properties of actinide atoms. 
TABLE 1. Even energy levels of Th $\mathrm{I}$

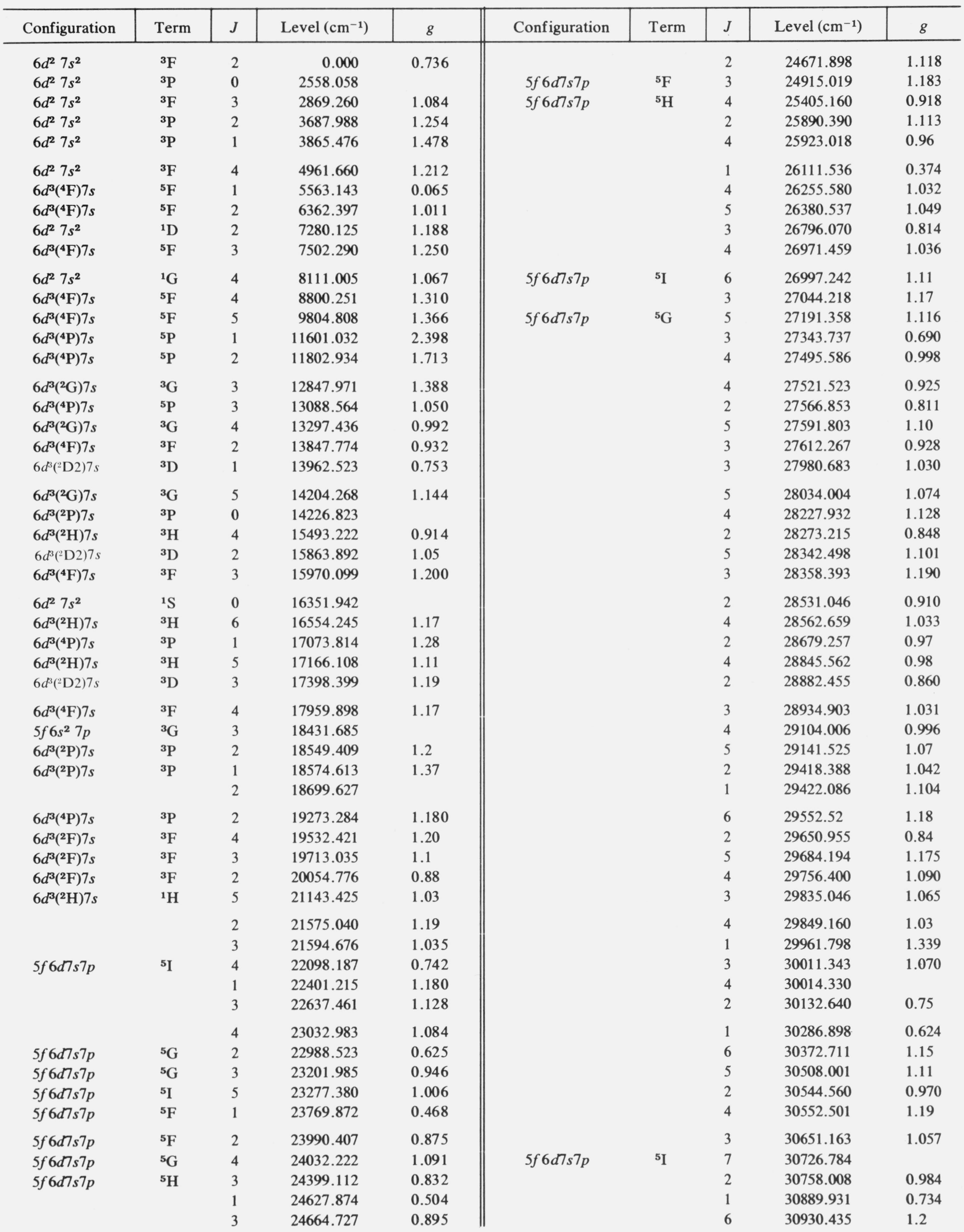


TABLE 1. Even energy levels of $\mathrm{Th} \mathrm{I}-$ continued

\begin{tabular}{|c|c|c|c|c|c|c|c|c|c|}
\hline Configuration & Term & $J$ & Level $\left(\mathrm{cm}^{-1}\right)$ & $g$ & Configuration & Term & $J$ & Level $\left(\mathrm{cm}^{-1}\right)$ & $g$ \\
\hline & & 3 & 30964.652 & 1.15 & & & 5 & 35216.987 & 1.177 \\
\hline & & 1 & 31095.84 & 1.327 & & & 5 & 35300.91 & 0.87 \\
\hline & & 3 & 31141.795 & 1.096 & & & 3 & 35405.303 & 1.067 \\
\hline & & 2 & 31245.890 & 1.336 & & & 1 & 35421.028 & 1.164 \\
\hline & & 3 & 31265.632 & 1.075 & & & 3 & 35440.891 & 1.07 \\
\hline & & 5 & 31271.000 & 1.084 & & & 3 & 35576.514 & 1.192 \\
\hline & & 4 & 31326.971 & 1.15 & & & 5 & 35582.288 & 1.15 \\
\hline & & 1 & 31429.253 & 1.294 & & & 4 & 35633.866 & 1.120 \\
\hline & & 3 & 31537.237 & 0.900 & & & 4 & 35794.375 & 1.144 \\
\hline & & 5 & 31626.821 & 1.111 & & & 6 & 35799.978 & 1.075 \\
\hline & & 3 & 31774.590 & 1.05 & & & 3 & 35831.292 & 1.190 \\
\hline & & 4 & 31793.697 & 1.090 & & & 2 & 35877.81 & 1.412 \\
\hline & & 3 & 31929.499 & 1.14 & & & 2 & 36047.30 & 0.83 \\
\hline & & 4 & 31995.652 & 0.92 & & & 4 & 36089.691 & 1.09 \\
\hline & & 4 & 32012.954 & 1.2 & & & 5 & 36133.494 & 1.041 \\
\hline & & 2 & 32041.231 & 1.08 & & & 5 & 36210.789 & 1.0 \\
\hline & & 5 & 32201.997 & 1.052 & & & 4 & 36297.296 & 1.128 \\
\hline & & 5 & 32293.919 & 1.186 & & & 3 & 36336.196 & 1.10 \\
\hline & & 4 & 32458.177 & 1.132 & & & 2 & 36485.388 & 1.275 \\
\hline & & 3 & 32551.611 & 1.25 & & & 3 & 36515.98 & 0.834 \\
\hline & & 5 & 32737.014 & 1.136 & & & 2 & 36653.567 & 1.203 \\
\hline & & 4 & 32781.597 & 1.083 & & & 6 & 36748.997 & 1.13 \\
\hline & & 3 & 32796.121 & 1.156 & & & 3 & 36775.361 & 0.97 \\
\hline & & 5 & 32963.392 & 1.20 & & & 5 & 36781.317 & 1.118 \\
\hline & & 6 & 33068.085 & 1.07 & & & 3 & 36808.30 & 1.094 \\
\hline & & 3 & 33099.412 & 1.337 & & & 2 & 36818.110 & 0.95 \\
\hline & & 5 & 33214.534 & 1.028 & & & 4 & 36885.289 & 1.048 \\
\hline & & 1 & 33273.846 & 1.380 & & & 4 & 37085.86 & 1.20 \\
\hline & & 2 & 33309.040 & 1.002 & & & 2 & 37094.849 & 1.155 \\
\hline & & 1 & 33390.746 & 0.704 & & & 3 & 37131.059 & 0.88 \\
\hline & & 3 & 33408.661 & 1.113 & & & 5 & 37211.68 & 1.03 \\
\hline & & 4 & 33418.121 & 1.121 & & & 4 & 37303.438 & 1.02 \\
\hline & & 4 & 33459.180 & 0.95 & & & 3 & 37307.14 & 0.995 \\
\hline & & 4 & 33527.562 & 1.15 & & & 6 & 37332.502 & 1.00 \\
\hline & & 3 & 33564.851 & 1.1 & & & 3 & 37404.631 & 1.3 \\
\hline & & 2 & 33606.291 & 1.417 & & & 3 & 37521.509 & 1.127 \\
\hline & & 2 & 33689.043 & 1.0 & & & 3 & 37688.21 & 1.238 \\
\hline & & 2 & 33721.308 & 1.159 & & & 6 & 37742.28 & 1.1 \\
\hline & & 1 & 33773.617 & 1.410 & & & 2 & 37748.043 & 0.99 \\
\hline & & 4 & 33831.881 & 1.15 & & & 2 & 37784.402 & 1.02 \\
\hline & & 3 & 34167.473 & 1.041 & & & 4 & 37819.119 & 1.04 \\
\hline & & 1 & 34298.062 & 0.931 & & & 5 & 37890.46 & 0.97 \\
\hline & & 4 & 34301.432 & 0.905 & & & 4 & 37892.823 & 0.86 \\
\hline & & 6 & 34407.566 & 1.19 & & & 4 & 37992.254 & 1.01 \\
\hline & & 3 & 34431.335 & 1.047 & & & 2 & 38097.10 & \\
\hline & & 2 & 34444.594 & 1.102 & & & 5 & 38106.740 & 1.16 \\
\hline & & 4 & 34447.172 & 1.11 & & & 3 & 38145.74 & 1.07 \\
\hline & & 5 & 34460.873 & 1.175 & & & 4 & 38186.12 & 0.95 \\
\hline & & 3 & 34707.730 & 1.165 & & & 4 & 38366.571 & 1.042 \\
\hline & & 5 & 34849.054 & 1.111 & & & 3 & 38382.14 & 1.0 \\
\hline & & 4 & 34873.350 & 1.11 & & & 3 & 38529.930 & 1.0 \\
\hline & & 3 & 34874.059 & 1.095 & & & 2 & 38602.98 & 1.1 \\
\hline & & 1 & 35046.030 & 0.4 & & & 4 & 38700.25 & 1.2 \\
\hline & & 3 & 35089.394 & 1.184 & & & 3 & 38734.401 & 1.10 \\
\hline & & 2 & 35149.75 & 1.09 & & & 4 & 38760.633 & 1.05 \\
\hline
\end{tabular}


TABLE 1. Even energy levels of $\mathrm{Th} \mathrm{I}$-continued

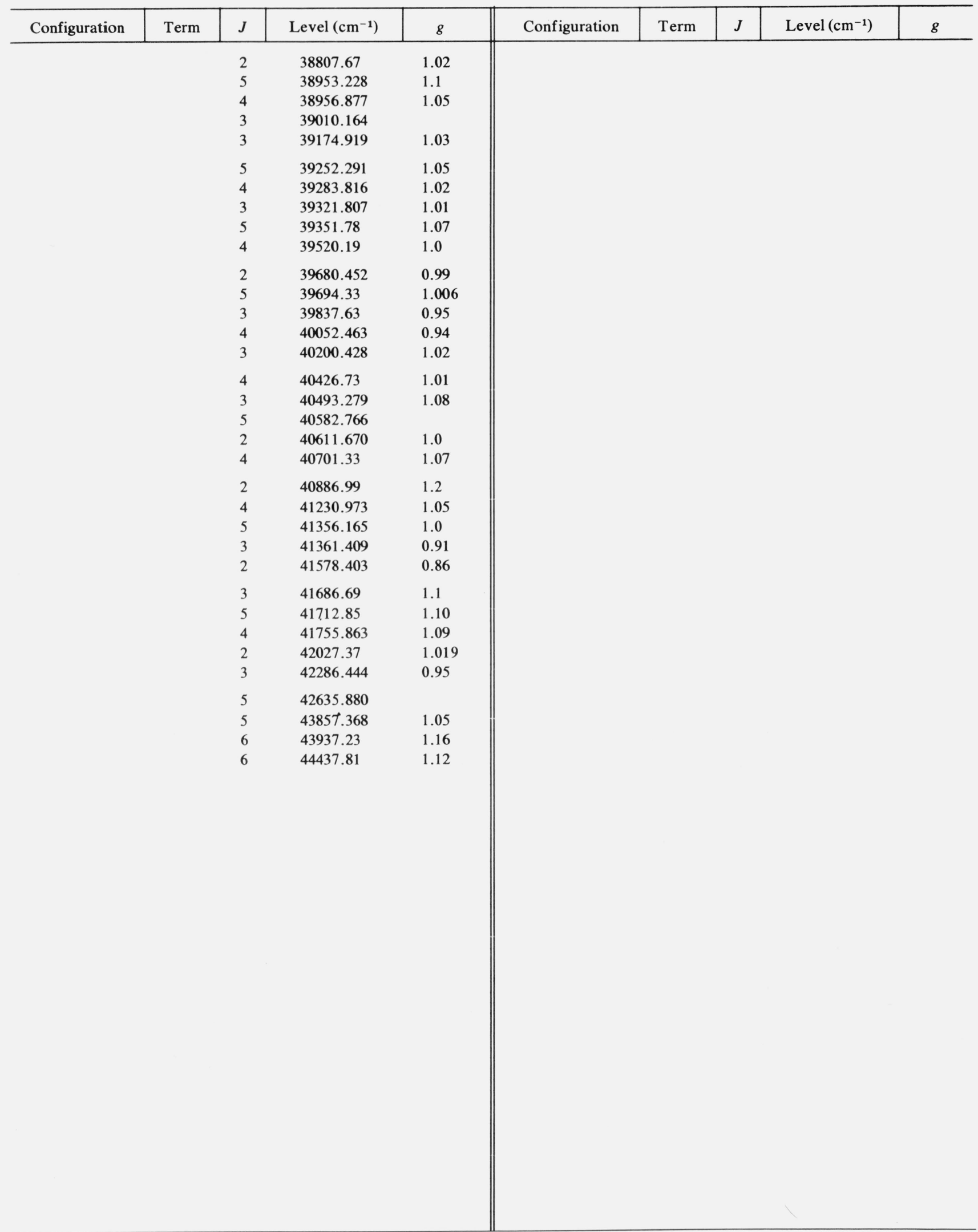


TABLE 2. Odd energy levels of Th I

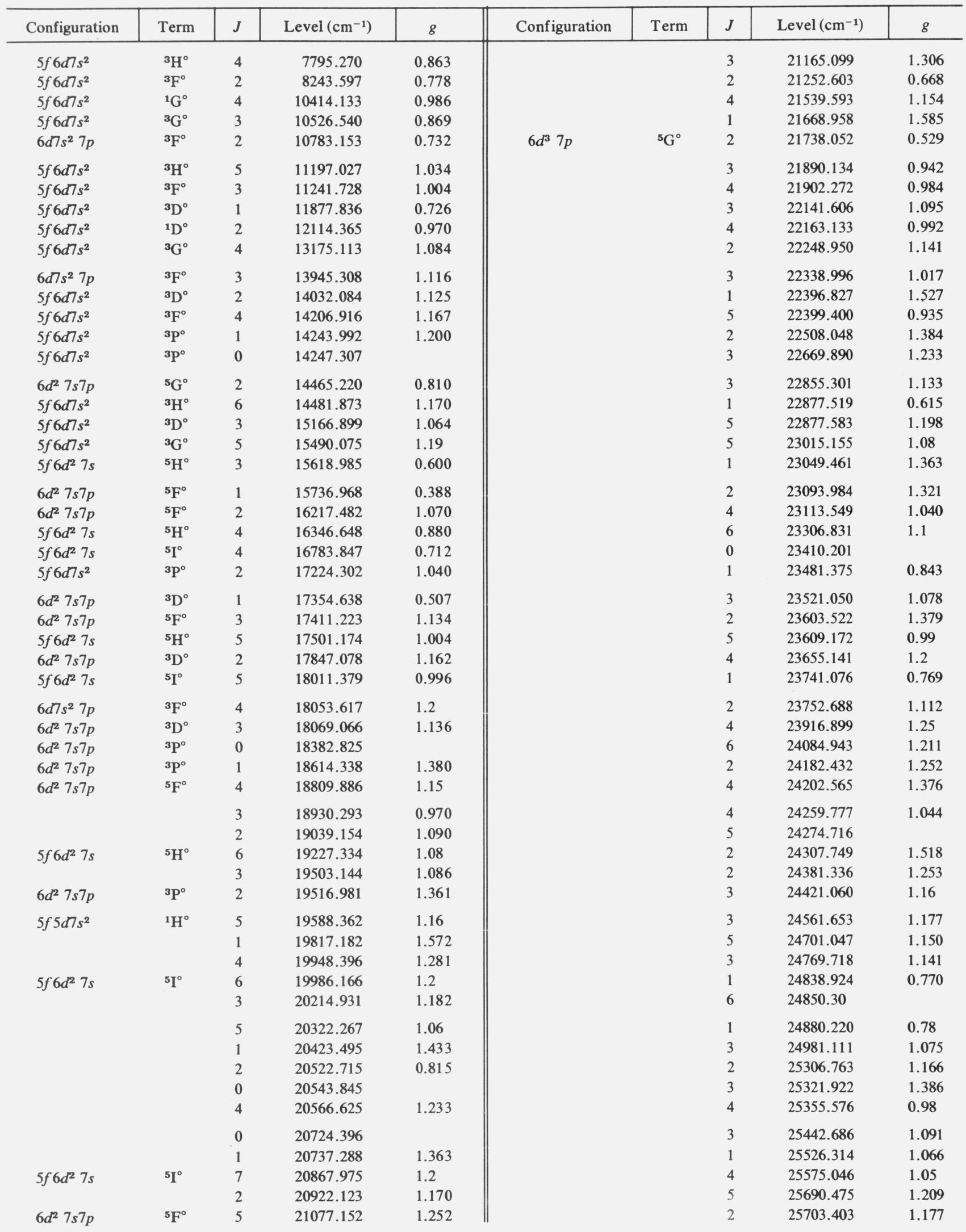


TABLE 2. Odd energy levels of $\mathrm{Th} \mathrm{I}$-continued

\begin{tabular}{|c|c|c|c|c|c|c|c|c|c|}
\hline Configuration & Term & $J$ & Level $\left(\mathrm{cm}^{-1}\right)$ & $g$ & Configuration & Term & $J$ & Level $\left(\mathrm{cm}^{-1}\right)$ & $g$ \\
\hline & & 5 & 25753.940 & 1.15 & & & 3 & 29744.522 & 1.148 \\
\hline & & 1 & 25809.306 & 1.568 & & & 2 & 29853.148 & 0.910 \\
\hline & & 4 & 25877.502 & 1.041 & & & 4 & 29881.457 & 1.165 \\
\hline & & 3 & 26036.352 & 0.954 & & & 3 & 30017.100 & 1.129 \\
\hline & & 4 & 26048.543 & 1.116 & & & 4 & 30160.006 & 1.11 \\
\hline & & 3 & 26096.986 & 1.001 & & & 2 & 30208.582 & 1.095 \\
\hline & & 2 & 26113.270 & 0.979 & & & 3 & 30255.452 & 1.082 \\
\hline & & 1 & 26287.049 & 0.710 & & & 1 & 30281.011 & 1.471 \\
\hline & & 2 & 26363.104 & 1.036 & & & 5 & 30517.108 & 1.18 \\
\hline & & 4 & 26384.927 & 1.060 & & & 4 & 30517.390 & 1.455 \\
\hline & & 3 & 26508.031 & 1.074 & & & 2 & 30553.291 & 1.042 \\
\hline & & 5 & 26645.412 & 1.1 & & & 0 & 30613.62 & \\
\hline & & 2 & 26651.831 & 1.102 & & & 1 & 30723.82 & 1.06 \\
\hline & & 4 & 26790.430 & 1.139 & & & 3 & 30761.719 & 1.192 \\
\hline & & 3 & 26878.162 & 0.903 & & & 5 & 30788.179 & 1.19 \\
\hline & & 0 & 26882.511 & & & & 2 & 30812.991 & 0.929 \\
\hline & & 3 & 26995.777 & 1.166 & & & 1 & 30928.742 & 0.976 \\
\hline & & 2 & 27061.417 & 0.987 & & & 3 & 30990.520 & 1.097 \\
\hline & & 1 & 27087.981 & 1.162 & & & 4 & 31019.110 & 1.19 \\
\hline & & 3 & 27260.171 & 1.105 & & & 2 & 31075.91 & 1.326 \\
\hline & & 4 & 27266.028 & 1.150 & & & 5 & 31141.255 & 0.947 \\
\hline & & 1 & 27297.579 & 0.6 & & & 4 & 31194.703 & 0.730 \\
\hline & & 3 & 27317.393 & 1.087 & & & 3 & 31283.117 & 1.11 \\
\hline & & 2 & 27447.746 & 1.07 & & & 3 & 31523.937 & 1.108 \\
\hline & & 3 & 27670.964 & 1.219 & & & 2 & 31599.274 & 1.216 \\
\hline & & 2 & 27674.327 & 1.090 & & & 4 & 31671.155 & 1.18 \\
\hline & & 2 & 27784.368 & 0.860 & & & 1 & 31712.73 & 1.175 \\
\hline & & 5 & 27852.743 & 1.259 & & & 5 & 31716.592 & 1.14 \\
\hline & & 4 & 27948.610 & 1.263 & & & 3 & 31780.865 & 1.175 \\
\hline & & 1 & 28024.691 & 1.030 & & & 2 & 31870.097 & 0.944 \\
\hline & & 4 & 28140.870 & 1.222 & & & 4 & 31953.464 & 1.073 \\
\hline & & 2 & 28347.551 & 1.543 & & & 5 & 32023.60 & 1.16 \\
\hline & & 1 & 28372.707 & 1.805 & & & 1 & 32080.416 & 0.795 \\
\hline & & 4 & 28480.159 & 1.02 & & & 2 & 32160.486 & 1.080 \\
\hline & & 2 & 28513.300 & 1.100 & & & 3 & 32197.119 & 1.138 \\
\hline & & 3 & 28589.314 & 1.179 & & & 3 & 32285.180 & 1.12 \\
\hline & & 1 & 28649.145 & 1.091 & & & 4 & 32294.089 & 1.16 \\
\hline & & 2 & 28673.429 & 1.130 & & & 4 & 32439.049 & 1.141 \\
\hline & & 3 & 28676.243 & 1.093 & & & 2 & 32503.62 & 1.200 \\
\hline & & 4 & 28680.738 & 1.192 & & & 2 & 32575.427 & 0.716 \\
\hline & & 3 & 28884.958 & 1.180 & & & 1 & 32650.907 & 1.14 \\
\hline & & 2 & 28917.957 & 0.945 & & & 1 & 32665.561 & 0.82 \\
\hline & & 4 & 28932.654 & 1.111 & & & 3 & 32754.504 & 1.12 \\
\hline & & 5 & 29050.740 & 1.12 & & & 4 & 32862.480 & 1.16 \\
\hline & & 1 & 29157.095 & 0.876 & & & 2 & 32954.59 & 1.114 \\
\hline & & 3 & 29157.879 & 1.210 & & & 3 & 33043.332 & 1.134 \\
\hline & & 1 & 29197.326 & 1.141 & & & 1 & 33161.77 & 1.332 \\
\hline & & 2 & 29197.906 & 1.38 & & & 4 & 33270.547 & 1.170 \\
\hline & & 2 & 29252.790 & 0.978 & & & 3 & 33294.928 & 1.10 \\
\hline & & 4 & 29310.020 & & & & 2 & 33297.133 & 1.26 \\
\hline & & 2 & 29419.232 & 1.680 & & & 2 & 33427.039 & 0.80 \\
\hline & & 1 & 29640.260 & 0.952 & & & 4 & 33560.06 & 1.18 \\
\hline & & 3 & 29686.370 & 1.260 & & & 3 & 33591.166 & 1.010 \\
\hline & & 5 & 29711.622 & 1.2 & & & 1 & 33662.23 & 1.018 \\
\hline & & 5 & 29733.325 & 1.18 & & & 2 & 33718.42 & 1.064 \\
\hline
\end{tabular}


TABLE 2. Odd energy levels of Th $\mathrm{I}^{\circ}-$ continued

\begin{tabular}{|c|c|c|c|c|c|c|c|c|c|}
\hline Configuration & Term & $J$ & Level $\left(\mathrm{cm}^{-1}\right)$ & $g$ & Configuration & Term & $J$ & Level $\left(\mathrm{cm}^{-1}\right)$ & $g$ \\
\hline & & 2 & 33734.706 & 1.302 & & & 3 & 37159.544 & 1.00 \\
\hline & & 4 & 33799.730 & 1.15 & & & 2 & 37234.01 & 1.10 \\
\hline & & 3 & 33800.621 & 1.10 & & & 5 & 37571.34 & 1.044 \\
\hline & & 5 & 33844.960 & 1.118 & & & 4 & 37605.73 & 1.13 \\
\hline & & 3 & 33967.906 & 0.794 & & & 1 & 37616.308 & \\
\hline & & 4 & 34001.333 & 1.078 & & & 3 & 37656.975 & 1.134 \\
\hline & & 1 & 34111.17 & 0.850 & & & 2 & 37736.977 & \\
\hline & & 5 & 34182.708 & 1.09 & & & 4 & 37950.044 & 1.13 \\
\hline & & 2 & 34324.08 & 1.131 & & & 3 & 37954.97 & 1.1 \\
\hline & & 4 & 34344.650 & 1.114 & & & 2 & 37970.270 & \\
\hline & & 2 & 34371.840 & 1.282 & & & 3 & 38053.028 & 1.08 \\
\hline & & 3 & 34430.22 & 1.213 & & & 2 & 38088.99 & 0.9 \\
\hline & & 4 & 34492.022 & 0.898 & & & 3 & 38216.95 & 1.13 \\
\hline & & 3 & 34583.008 & 1.172 & & & 1 & 38278.878 & 0.866 \\
\hline & & 1 & 34591.01 & 1.496 & & & 4 & 38306.376 & 1.05 \\
\hline & & 3 & 34704.406 & 1.103 & & & 2 & 38355.312 & \\
\hline & & 3 & 34725.43 & 1.090 & & & 5 & 38385.213 & 0.98 \\
\hline & & 2 & 34851.666 & 0.866 & & & 3 & 38416.222 & 1.18 \\
\hline & & 1 & 34878.665 & 1.478 & & & 4 & 38580.297 & 1.06 \\
\hline & & 5 & 34886.676 & 1.07 & & & 5 & 38665.559 & 1.078 \\
\hline & & 1 & 34989.778 & 1.619 & & & 2 & 38669.77 & 1.027 \\
\hline & & 5 & 35081.021 & 1.100 & & & 3 & 38675.356 & 0.94 \\
\hline & & 4 & 35131.22 & 1.212 & & & 3 & 38788.85 & 0.98 \\
\hline & & 3 & 35240.821 & 1.077 & & & 2 & 38814.252 & 0.77 \\
\hline & & 5 & 35273.877 & 1.16 & & & 1 & 38840.559 & 1.2 \\
\hline & & 1 & 35328.63 & 1.272 & & & 3 & 38998.975 & 1.314 \\
\hline & & 4 & 35351.439 & 1.19 & & & 5 & 39062.033 & \\
\hline & & 3 & 35462.05 & 1.250 & & & 3 & 39264.69 & 1.06 \\
\hline & & 2 & 35533.292 & 1.219 & & & 4 & 39311.261 & 1.10 \\
\hline & & 1 & 35678.85 & 1.02 & & & 1 & 39411.899 & \\
\hline & & 4 & 35733.465 & 1.1 & & & 3 & 39468.675 & \\
\hline & & 3 & 35812.518 & 1.10 & & & 4 & 39562.09 & 1.12 \\
\hline & & 2 & 35885.887 & 1.567 & & & 3 & 39611.52 & 1.26 \\
\hline & & 4 & 36062.875 & 1.100 & & & 5 & 39873.585 & 1.08 \\
\hline & & 3 & 36074.06 & 1.140 & & & 4 & 39906.930 & 1.10 \\
\hline & & 5 & 36132.494 & 1.167 & & & 3 & 39985.716 & \\
\hline & & 4 & 36178.040 & 1.04 & & & 2 & 40096.226 & 1.21 \\
\hline & & 2 & 36188.95 & 1.41 & & & 3 & 40254.944 & \\
\hline & & 5 & 36275.186 & 1.12 & & & 4 & 40270.098 & 1.10 \\
\hline & & 1 & 36361.456 & 1.109 & & & 4 & 40425.183 & 1.11 \\
\hline & & 4 & 36382.61 & 1.064 & & & 5 & 40724.599 & \\
\hline & & 3 & 36427.40 & 1.08 & & & 4 & 40776.519 & 1.16 \\
\hline & & 2 & 36488.828 & 1.0 & & & 3 & 40980.714 & \\
\hline & & 1 & 36556.77 & 1.054 & & & 4 & 41134.911 & 1.04 \\
\hline & & 5 & 36625.920 & 1.15 & & & 2 & 41200.38 & 1.35 \\
\hline & & 2 & 36668.670 & 0.92 & & & 2 & 41720.570 & 1.13 \\
\hline & & 3 & 36762.803 & 1.13 & & & 1 & 42624.127 & 1.29 \\
\hline & & 4 & 36800.658 & 0.97 & & & & & \\
\hline & & 1 & 36837.21 & 0.798 & & & & & \\
\hline & & 5 & 36837.988 & 1.168 & & & & & \\
\hline & & 2 & 36871.91 & 1.250 & & & & & \\
\hline & & 5 & 37008.760 & 1.136 & & & & & \\
\hline & & 4 & 37041.15 & 1.009 & & & & & \\
\hline & & 2 & 37149.10 & 1.060 & & & & & \\
\hline & & 1 & 37159.282 & 0.9 & & & & & \\
\hline
\end{tabular}


TABLE 3. Zeeman effect of Th I

\begin{tabular}{|c|c|c|c|c|c|c|c|c|c|c|c|}
\hline \multirow{2}{*}{$\begin{array}{l}\text { Wavelength } \\
\text { (§) }\end{array}$} & \multicolumn{2}{|c|}{ Intensity } & \multirow{2}{*}{$\begin{array}{l}\text { Wavenumber } \\
\qquad\left(\mathrm{cm}^{-1}\right)\end{array}$} & \multirow{2}{*}{ Type } & \multirow{2}{*}{$J_{1}$} & \multirow{2}{*}{$g_{1}$} & \multirow{2}{*}{$J_{2}$} & \multirow{2}{*}{$g_{2}$} & \multirow{2}{*}{$\Delta g$} & \multirow{2}{*}{$p$} & \multirow{2}{*}{$n$} \\
\hline & Lamp & Spark & & & & & & & & & \\
\hline 9094.821 & 400 & 100 & 10992.25 & 2 & 1 & 0.498 & 2 & 1.007 & & & \\
\hline 9063.948 & 200 & 10 & 11029.69 & 3 & 2 & 0.766 & 2 & 1.186 & & & \\
\hline 9048.248 & $800 \mathrm{~b}$ & 75 & 11048.83 & 2 & 2 & 1.006 & 3 & 1.136 & & & \\
\hline 9045.341 & 200 & 20 & 11052.38 & 1 & 2 & 1.710 & 3 & 1.140 & & & \\
\hline 9016.581 & 300 & 20 & 11087.63 & 7 & & & & & & & 0.918 \\
\hline 9012.517 & 150 & 8 & 11092.63 & 7 & & & & & & & 1.090 \\
\hline 8997.862 & 100 & 10 & 11110.70 & 1 & 1 & 0.727 & 2 & 0.627 & & & \\
\hline 8967.635 & 500 & 100 & 11148.15 & 3 & & & & & & 0.111 & \\
\hline 8949.114 & 100 & 20 & 11171.22 & 7 & & & & & & & 0.984 \\
\hline 8910.844 & 75 & 4 & 11219.20 & 1 & & & & & & & 0.118 \\
\hline 8892.973 & 150 & 15 & 11241.75 & 2 & 2 & 0.734 & 3 & 1.014 & & & \\
\hline 8889.187 & 100 & 10 & 11246.54 & 2 & 1 & 1.387 & 2 & 1.717 & & & \\
\hline 8875.223 & 100 & 8 & 11264.23 & 1 & 3 & 1.165 & 4 & 0.975 & & & \\
\hline 8868.822 & 200 & 50 & 11272.36 & 3 & 5 & 1.243 & 5 & 1.358 & & & \\
\hline 8854.899 & 10 & 3 & 11290.08 & 3 & & & & & & 0.288 & \\
\hline 8852.784 & 10 & 3 & 11292.78 & 1 & & & & & & & 0.628 \\
\hline 8841.170 & 150 & 20 & 11307.62 & 1 & & & & & & & 0.864 \\
\hline 8829.686 & 15 & 5 & 11322.32 & 3 & 3 & 0.895 & 3 & 1.170 & & & \\
\hline 8810.240 & 15 & & 11347.31 & 3 & & & & & & 0.359 & \\
\hline 8781.093 & 25 & 1 & 11384.98 & 7 & & & & & & & 0.892 \\
\hline 8775.575 & 200 & 40 & 11392.14 & 7 & & & & & & & 1.031 \\
\hline 8772.803 & 150 & 8 & 11395.74 & 3 & 3 & 1.001 & 3 & 1.125 & & & \\
\hline 8766.743 & 100 & 15 & 11403.61 & 2 & 4 & 0.994 & 5 & 1.150 & & & \\
\hline 8760.449 & 100 & 8 & 11411.81 & 1 & 3 & 1.392 & 4 & 1.044 & & & \\
\hline 8758.247 & 400 & 75 & 11414.67 & 2 & 3 & 1.182 & 4 & 1.310 & & & \\
\hline 8749.169 & 100 & 20 & 11426.52 & 3 & & & & & & 0.288 & \\
\hline 8748.038 & 400 & 100 & 11428.00 & 3 & 3 & 0.976 & 3 & 1.249 & & & \\
\hline 8739.779 & 75 & 10 & 11438.80 & 3 & & & & & & 0.262 & \\
\hline 8734.023 & 75 & 8 & 11446.33 & 3 & & & & & & 0.136 & \\
\hline 8732.426 & 150 & 50 & 11448.43 & 3 & 1 & 1.358 & 1 & 2.398 & & & \\
\hline 8730.818 & 15 & 2 & 11450.54 & 2 & & & & & & & 1.274 \\
\hline 8724.375 & 40 & 2 & 11458.99 & 3 & 2 & 0.928 & 2 & 1.166 & & & \\
\hline 8717.754 & 10 & 2 & 11467.70 & 1 & & & & & & & 0.965 \\
\hline 8709.233 & 200 & 50 & 11478.91 & 1 & 2 & 1.254 & 3 & 1.064 & & & \\
\hline 8701.117 & 50 & 3 & 11489.62 & 2 & & & & & 0.190 & & 1.682 \\
\hline 8691.333 & 201 & 1 & 11502.56 & 1 & & & & & & & 0.125 \\
\hline 8687.841 & 50 & 4 & 11507.18 & 1 & & & & & & & 0.698 \\
\hline 8682.216 & 10 & 1 & 11514.63 & 7 & & & & & & & 0.869 \\
\hline 8668.118 & 100 & 20 & 11533.36 & 2 & 2 & & 3 & & 0.135 & & \\
\hline 8665.491 & 400 & 100 & 11536.86 & 2 & 2 & 1.088 & 3 & 1.248 & & & \\
\hline 8655.873 & 50 & 8 & 11549.68 & 3 & & & & & & 0.075 & \\
\hline 8649.144 & 50 & 5 & 11558.66 & 2 & 4 & 0.715 & 5 & 1.115 & & & \\
\hline 8645.310 & 150 & 25 & 11563.79 & 3 & 1 & 0.759 & 1 & 1.068 & & & \\
\hline 8639.444 & 150 & 50 & 11571.64 & 1 & 3 & 0.870 & 4 & 0.746 & & & \\
\hline 8638.360 & 100 & 5 & 11573.09 & 3 & & & & & & 0.661 & \\
\hline 8631.350 & 50 & 2 & 11582.49 & 7 & & & & & & & 1.567 \\
\hline 8630.770 & $20 \mathrm{~h}$ & $2 \mathrm{~h}$ & 11583.27 & 7 & & & & & & & 1.180 \\
\hline 8621.315 & 75 & 2 & 11595.97 & 2 & 2 & 0.813 & 3 & 1.089 & & & \\
\hline 8607.724 & 8 & 2 & 11614.28 & 7 & & & & & & & 0.968 \\
\hline 8593.103 & 100 & 10 & 11634.04 & 1 & & & & & 0.150 & & \\
\hline 8577.279 & 100 & 15 & 11655.51 & 2 & 1 & 0.462 & 2 & 0.968 & & & \\
\hline 8575.327 & 75 & 5 & 11658.16 & 3 & & & & & & 0.376 & \\
\hline 8573.122 & 500 & 100 & 11661.16 & 2 & 1 & 0.055 & 2 & 1.036 & & & \\
\hline 8560.425 & 100 & 4 & 11678.46 & 2 & 1 & 0.864 & 2 & 1.706 & & & \\
\hline 8558.448 & 751 & 4 & 11681.15 & 3 & & & & & & 0.250 & \\
\hline
\end{tabular}


TABLE 3. Zeeman effect of Th $\mathrm{I}$-continued

\begin{tabular}{|c|c|c|c|c|c|c|c|c|c|c|c|}
\hline \multirow{2}{*}{$\begin{array}{l}\text { Wavelength } \\
(\AA)\end{array}$} & \multicolumn{2}{|c|}{ Intensity } & \multirow{2}{*}{$\begin{array}{l}\text { Wavenumber } \\
\qquad\left(\mathrm{cm}^{-1}\right)\end{array}$} & \multirow{2}{*}{ Type } & \multirow{2}{*}{$J_{1}$} & \multirow{2}{*}{$g_{1}$} & \multirow{2}{*}{$J_{2}$} & \multirow{2}{*}{$g_{2}$} & \multirow{2}{*}{$\Delta g$} & \multirow{2}{*}{$p$} & \multirow{2}{*}{$n$} \\
\hline & Lamp & Spark & & & & & & & & & \\
\hline 8556.324 & 100 & 20 & 11684.05 & 3 & 4 & 0.736 & 4 & 0.982 & & & \\
\hline 8554.945 & 200 & 50 & 11685.94 & 7 & & & & & & & 1.209 \\
\hline 8544.593 & 150 & 50 & 11700.09 & 2 & & & & & & & 1.455 \\
\hline 8543.721 & 150 & 40 & 11701.29 & 3 & & & & & & 0.390 & \\
\hline 8539.795 & 150 & 25 & 11706.67 & 2 & 2 & 1.004 & 3 & 1.136 & & & \\
\hline 8516.555 & 150 & 50 & 11738.61 & 7 & & & & & & & 1.144 \\
\hline 8510.622 & 150 & 25 & 11746.80 & 2 & 2 & 0.626 & 3 & 1.006 & & & \\
\hline 8501.438 & 100 & 5 & 11759.48 & 3 & & & & & & 0.065 & \\
\hline 8496.449 & $100 \mathrm{~b}$ & & 11766.39 & 3 & & & & & & 0.271 & \\
\hline 8496.340 & $75 b$ & & 11766.54 & 7 & & & & & & & 1.136 \\
\hline 8491.159 & 8 & 1 & 11773.72 & 7 & & & & & & & 1.409 \\
\hline 8483.298 & 50 & 2 & 11784.63 & 7 & & & & & & & 1.266 \\
\hline 8478.505 & 200 & & 11791.29 & 7 & & & & & & & 1.294 \\
\hline 8478.353 & 500 & & 11791.50 & 2 & 1 & 0.074 & 2 & 0.503 & & & \\
\hline 8471.823 & 200 & 50 & 11800.59 & 3 & 2 & 1.379 & 2 & 1.721 & & & \\
\hline 8465.668 & 100 & 25 & 11809.17 & 7 & & & & & & & 2.420 \\
\hline 8464.236 & 150 & 50 & 11811.17 & 3 & 2 & 0.778 & 2 & 0.878 & & & \\
\hline 8446.516 & 500 & 150 & 11835.95 & 7 & & & & & & & 0.860 \\
\hline 8445.495 & 200 & 150 & 11837.38 & 3 & 4 & 1.064 & 4 & 1.281 & & & \\
\hline 8430.586 & 15 & 1 & 11858.31 & 7 & & & & & & & 1.110 \\
\hline 8426.379 & 75 & & 11864.23 & 7 & & & & & & & 0.375 \\
\hline 8424.031 & 100 & & 11867.54 & 3 & 1 & 0.375 & 1 & 1.201 & & & \\
\hline 8421.227 & 500 & 100 & 11871.49 & 3 & 1 & 0.388 & 1 & 1.483 & & & \\
\hline 8418.004 & 200 & 75 & 11876.04 & 3 & 2 & 0.886 & 2 & 0.978 & & & \\
\hline 8416.742 & $500 w$ & 150 & 11877.82 & 7 & & & & & & & 0.745 \\
\hline 8411.913 & 100 & 5 & 11884.64 & 7 & & & & & & & 1.116 \\
\hline 8410.532 & 25 & 25 & 11886.59 & 2 & & & & & & & 1.980 \\
\hline 8407.010 & 75 & 3 & 11891.57 & 3 & & & & & & 0.364 & \\
\hline 8406.674 & 40 & 2 & 11892.04 & 3 & & & & & & 0.269 & \\
\hline 8399.253 & 100 & 8 & 11902.55 & 2 & & & & & 0.320 & & \\
\hline 8398.171 & 75 & 8 & 11904.08 & 7 & & & & & & & 0.621 \\
\hline 8388.528 & 100 & 10 & 11917.77 & 1 & 3 & 1.096 & 4 & 0.856 & & & \\
\hline 8385.726 & 100 & 10 & 11921.75 & 3 & 3 & & 3 & & & 0.737 & \\
\hline 8384.927 & 8 & 2 & 11922.89 & 7 & & & & & & & 1.101 \\
\hline 8383.073 & 8 & 2 & 11925.52 & 7 & & & & & & & 1.239 \\
\hline 8379.219 & 100 & 8 & 11931.01 & 1 & 2 & 1.264 & 3 & 0.598 & & & \\
\hline 8369.332 & 200 & 50 & 11945.10 & 7 & & & & & & & 1.084 \\
\hline 8367.390 & 150 & 20 & 11947.87 & 1 & 2 & 0.811 & 3 & 0.591 & & & \\
\hline 8366.073 & 150 & 15 & 11949.76 & 3 & 2 & 1.121 & 2 & 1.717 & & & \\
\hline 8358.728 & 300 & 200 & 11960.26 & 3 & & & & & & 0.170 & \\
\hline 8346.537 & 100 & 3 & 11977.73 & 7 & & & & & 0.130 & & \\
\hline 8345.867 & 100 & 5 & 11978.69 & 1 & 1 & 1.369 & 2 & 1.038 & & & \\
\hline 8340.933 & 20 & 3 & 11985.77 & 7 & & & & & & & 1.341 \\
\hline 8339.403 & 5 & 2 & 11987.97 & 7 & & & & & & & 1.170 \\
\hline 8335.700 & 100 & 8 & 11993.30 & 3 & & & & & & 0.969 & \\
\hline 8330.455 & 500 & 300 & 12000.85 & 3 & 3 & 1.088 & 3 & 1.240 & & & \\
\hline 8320.859 & 500 & 150 & 12014.69 & 1 & 2 & 1.353 & 3 & 1.241 & & & \\
\hline 8316.595 & 8 & 2 & 12020.85 & 1 & & & & & & & 0.226 \\
\hline 8311.621 & 100 & 8 & 12028.04 & 1 & & & & & & & 0.731 \\
\hline 8304.412 & 75 & 3 & 12038.48 & 3 & & & & & & 0.142 & \\
\hline 8297.172 & $100 \mathrm{~b}$ & 5 & 12048.99 & 2 & 1 & 0.390 & 2 & 1.255 & & & \\
\hline 8295.542 & 100 & 8 & 12051.36 & 2 & & & & & 0.170 & & \\
\hline 8292.521 & 100 & 8 & 12055.75 & 3 & 2 & 0.836 & 2 & 1.078 & & & \\
\hline 8288.404 & 100 & 5 & 12061.73 & 3 & & & & & & 1.102 & \\
\hline 8275.628 & 400 & 150 & 12080.35 & 3 & & & & & & 0.130 & \\
\hline
\end{tabular}


TABLE 3. Zeeman effect of Th $\mathrm{I}$-continued

\begin{tabular}{|c|c|c|c|c|c|c|c|c|c|c|c|}
\hline \multirow{2}{*}{$\begin{array}{l}\text { Wavelength } \\
\text { (§) }\end{array}$} & \multicolumn{2}{|c|}{ Intensity } & \multirow{2}{*}{$\begin{array}{l}\text { Wavenumber } \\
\qquad\left(\mathrm{cm}^{-1}\right)\end{array}$} & \multirow{2}{*}{ Type } & \multirow{2}{*}{$J_{1}$} & \multirow{2}{*}{$g_{1}$} & \multirow{2}{*}{$J_{2}$} & \multirow{2}{*}{$g_{2}$} & \multirow{2}{*}{$\Delta g$} & \multirow{2}{*}{$p$} & \multirow{2}{*}{$n$} \\
\hline & Lamp & Spark & & & & & & & & & \\
\hline 8263.926 & 150 & 20 & 12097.46 & 2 & 4 & 0.983 & 5 & 1.359 & & & \\
\hline 8261.010 & 100 & 25 & 12101.73 & 3 & & & & & & 0.534 & \\
\hline 8254.741 & 100 & 15 & 12110.92 & 3 & 3 & 0.870 & 3 & 1.130 & & & \\
\hline 8252.398 & 400 & 50 & 12114.36 & 2 & 2 & 0.734 & 2 & 0.966 & & & \\
\hline 8246.011 & 25 & 4 & 12123.74 & 7 & & & & & & & 1.009 \\
\hline 8237.739 & 20 & 2 & 12135.92 & 1 & & & & & & & 0.739 \\
\hline 8234.937 & 100 & 4 & 12140.05 & 3 & 1 & 0.777 & 1 & 2.399 & & & \\
\hline 8227.672 & 75 & 4 & 12150.77 & 2 & & & & & & & 1.232 \\
\hline 8227.482 & 100 & 8 & 12151.05 & 1 & & & & & 0.340 & & \\
\hline 8224.956 & 8 & 2 & 12154.78 & 7 & & & & & & & 1.151 \\
\hline 8214.147 & 100 & 10 & 12170.77 & 7 & & & & & & & 1.249 \\
\hline 8209.444 & 100 & 8 & 12177.75 & 1 & 3 & 1.200 & 4 & 0.900 & & & \\
\hline 8207.475 & 100 & 10 & 12180.67 & 2 & & & & & & & 1.440 \\
\hline 8203.199 & 100 & & 12187.02 & 2 & & & & & & & 1.246 \\
\hline 8202.148 & 100 & 10 & 12188.58 & 7 & & & & & & & 0.969 \\
\hline 8198.438 & 10 & 2 & 12194.09 & 7 & & & & & & & 1.046 \\
\hline 8195.942 & 8 & 1 & 12197.81 & 7 & & & & & & & 1.006 \\
\hline 8194.398 & 150 & 25 & 12200.11 & 2 & & & & & & & 1.863 \\
\hline 8186.915 & 500 & 100 & 12211.26 & 7 & & & & & & & 1.061 \\
\hline 8169.793 & 400 & 50 & 12236.85 & 3 & 2 & 1.194 & 2 & 1.362 & & & \\
\hline 8162.064 & 50 & 3 & 12248.44 & 2 & & & & & & & 1.277 \\
\hline 8159.737 & 400 & 100 & 12251.93 & 1 & 1 & 1.402 & 2 & 1.007 & & & \\
\hline 8157.540 & 100 & 8 & 12255.23 & 1 & & & & & & & 0.673 \\
\hline 8154.176 & 10 & & 12260.28 & 7 & & & & & & & 0.830 \\
\hline 8143.143 & 500 & 300 & 12276.89 & 1 & & & & & & & 1.063 \\
\hline 8137.940 & 100 & 4 & 12284.74 & 1 & & & & & 0.150 & & \\
\hline 8129.681 & 100 & 5 & 12297.22 & 7 & & & & & & & 1.087 \\
\hline 8122.725 & 200 & 50 & 12307.75 & 7 & & & & & & & 1.255 \\
\hline 8122.350 & 100 & 5 & 12308.32 & 2 & & & & & & & 1.460 \\
\hline 8114.539 & 75 & 4 & 12320.17 & 3 & 4 & 0.715 & 4 & 1.001 & & & \\
\hline 8096.253 & 20 & 3 & 12348.00 & 7 & & & & & & & 0.963 \\
\hline 8093.631 & 200 & 75 & 12352.00 & 1 & 1 & 1.476 & 2 & 1.066 & & & \\
\hline 8086.568 & 15 & 4 & 12362.79 & 7 & & & & & & & 1.301 \\
\hline 8075.651 & 150 & 20 & 12379.50 & 3 & 2 & 1.705 & 2 & 1.253 & & & \\
\hline 8070.094 & 25 & 1 & 12388.02 & 7 & & & & & & & 1.390 \\
\hline 8066.826 & 75 & 8 & 12393.04 & 2 & 4 & 0.999 & 5 & 1.209 & & & \\
\hline 8057.973 & 50 & 2 & 12406.66 & 7 & & & & & & & 1.141 \\
\hline 8032.433 & 200 & 50 & 12446.11 & 7 & & & & & & & 1.329 \\
\hline 8026.192 & 100 & 8 & 12455.78 & 2 & & & & & & & 1.328 \\
\hline 8025.708 & 100 & 8 & 12456.53 & 2 & & & & & & & 1.675 \\
\hline 8024.235 & 100 & 500 & 12458.82 & 2 & & & & & 0.220 & & \\
\hline 8022.310 & $100 \mathrm{~b}$ & 20 & 12461.81 & 3 & & & & & & 0.211 & \\
\hline 8022.191 & $150 \mathrm{~b}$ & 25 & 12462.00 & 2 & & & & & 0.222 & & \\
\hline 8014.499 & 50 & 3 & 12473.96 & 7 & & & & & & & 1.393 \\
\hline 8013.499 & 15 & 2 & 12475.51 & 2 & & & & & 0.170 & & \\
\hline 8010.708 & 100 & 4 & 12479.86 & 2 & & & & & 0.148 & & \\
\hline 8006.443 & 15 & & 12486.51 & 7 & & & & & & & 1.055 \\
\hline 8003.446 & 100 & 5 & 12491.18 & 2 & & & & & & & 1.038 \\
\hline 8002.179 & 50 & 5 & 12493.16 & 3 & & & & & & 0.208 & \\
\hline 8000.033 & 100 & 10 & 12496.51 & 2 & & & & & & & 1.370 \\
\hline 7996.963 & 40 & 3 & 12501.31 & 7 & & & & & & & 1.287 \\
\hline 7993.676 & 150 & 8 & 12506.45 & 2 & 3 & 0.860 & 4 & 1.084 & & & \\
\hline 7991.366 & 50 & 5 & 12510.06 & 1 & & & & & & & 0.490 \\
\hline 7978.977 & 500 & 300 & 12529.49 & 3 & 2 & 1.066 & 2 & 1.253 & & & \\
\hline 7976.175 & 8 & 2 & 12533.89 & 7 & & & & & & & 0.978 \\
\hline
\end{tabular}


TABLE 3. Zeeman effect of $\mathrm{Th} \mathrm{I}$-continued

\begin{tabular}{|c|c|c|c|c|c|c|c|c|c|c|c|}
\hline \multirow{2}{*}{$\begin{array}{l}\text { Wavelength } \\
\text { (§) }\end{array}$} & \multicolumn{2}{|c|}{ Intensity } & \multirow{2}{*}{$\begin{array}{l}\text { Wavenumber } \\
\left(\mathrm{cm}^{-1}\right)\end{array}$} & \multirow{2}{*}{ Type } & \multirow{2}{*}{$J_{1}$} & \multirow{2}{*}{$g_{1}$} & \multirow{2}{*}{$J_{2}$} & \multirow{2}{*}{$g_{2}$} & \multirow{2}{*}{$\Delta g$} & \multirow{2}{*}{$p$} & \multirow{2}{*}{$n$} \\
\hline & Lamp & Spark & & & & & & & & & \\
\hline 7972.598 & 200 & 20 & 12539.51 & 1 & 4 & 1.215 & 5 & 1.018 & & & \\
\hline 7961.994 & 100 & 8 & 12556.21 & 2 & & & & & & & 1.598 \\
\hline 7961.158 & 75 & 4 & 12557.53 & 3 & & & & & & 0.250 & \\
\hline 7954.592 & 150 & 15 & 12567.90 & 7 & & & & & & & 0.935 \\
\hline 7941.721 & 200 & 75 & 12588.27 & 1 & 3 & 1.031 & 4 & 0.886 & & & \\
\hline 7937.727 & 100 & 20 & 12594.60 & 3 & 5 & 0.935 & 5 & 1.371 & & & \\
\hline 7929.938 & 5 & & 12606.97 & 3 & & & & & & 0.236 & \\
\hline 7929.809 & $25 c$ & 2 & 12607.18 & 3 & 1 & 0.487 & 1 & 1.328 & & & \\
\hline 7927.546 & 501 & 3 & 12610.78 & 7 & & & & & & & 0.899 \\
\hline 7925.734 & 75 & 3 & 12613.66 & 3 & & & & & & 0.485 & \\
\hline 7922.235 & 15 & & 12619.23 & 3 & & & & & & 0.096 & \\
\hline 7916.790 & 40 & 5 & 12627.91 & 2 & & & & & 0.111 & & 1.460 \\
\hline 7914.493 & 8 & 2 & 12631.57 & 7 & & & & & & & 1.306 \\
\hline 7908.148 & 100 & 5 & 12641.71 & 3 & 2 & 1.081 & 2 & 1.373 & & & \\
\hline 7904.411 & 75 & 5 & 12647.69 & 3 & 4 & 0.907 & 4 & 1.231 & & & \\
\hline 7903.319 & 8 & 1 & 12649.43 & 7 & & & & & & & 1.075 \\
\hline 7900.309 & 200 & 75 & 12654.25 & 1 & 2 & 0.864 & 3 & 0.614 & & & \\
\hline 7899.614 & 100 & 50 & 12655.36 & 7 & & & & & & & 1.276 \\
\hline 7897.230 & 15 & 3 & 12659.18 & 7 & & & & & & & 1.241 \\
\hline 7893.614 & 75 & 5 & 12664.98 & 3 & & & & & & 0.452 & \\
\hline 7869.771 & 15 & 2 & 12703.35 & 7 & & & & & & & 1.338 \\
\hline 7868.924 & 40 & 10 & 12704.72 & 3 & & & & & & 0.147 & \\
\hline 7868.396 & 75 & $10 \mathrm{~h}$ & 12705.57 & 3 & & & & & & 0.159 & \\
\hline 7865.954 & 400 & 75 & 12709.52 & 7 & & & & & & & 1.362 \\
\hline 7862.336 & 15 & 3 & 12715.37 & 7 & & & & & & & 1.040 \\
\hline 7853.284 & 20 & & 12730.02 & 7 & & & & & & & 1.384 \\
\hline 7849.892 & 5 & 1 & 12735.52 & 7 & & & & & & & 1.146 \\
\hline 7848.441 & 100 & 40 & 12737.88 & 2 & & & & & & & 1.455 \\
\hline 7847.536 & $500 \mathrm{~b}$ & 150 & 12739.35 & 3 & 4 & 1.154 & 4 & 1.307 & & & \\
\hline 7842.254 & 100 & 10 & 12747.93 & 3 & & & & & 0.130 & & \\
\hline 7841.781 & 200 & 50 & 12748.70 & 2 & 2 & 0.862 & 3 & 1.002 & & & \\
\hline 7836.456 & 100 & 5 & 12757.36 & 3 & & & & & & 0.475 & \\
\hline 7823.475 & 50 & 2 & 12778.53 & 7 & & & & & & & 0.911 \\
\hline 7822.392 & 100 & 4 & 12780.30 & 2 & 1 & 2.380 & 2 & 1.245 & & & \\
\hline 7817.771 & 500 & 200 & 12787.85 & 2 & & & & & & & 1.070 \\
\hline 7809.452 & 100 & 15 & 12801.47 & 2 & & & & & & & 1.282 \\
\hline 7807.879 & 40 & & 12804.05 & 3 & & & & & & 0.327 & \\
\hline 7804.533 & 100 & 5 & 12809.54 & 3 & & & & & & 0.131 & \\
\hline 7804.146 & 75 & 4 & 12810.18 & 2 & & & & & & & 1.362 \\
\hline 7802.824 & 10 & 2 & 12812.35 & 7 & & & & & & & 1.101 \\
\hline 7798.360 & 600 & 25 & 12819.68 & 7 & & & & & & & 0.059 \\
\hline 7793.128 & 10 & 2 & 12828.29 & 7 & & & & & & & 1.255 \\
\hline 7788.932 & 500 & 75 & 12835.20 & 7 & & & & & & & 0.808 \\
\hline 7782.318 & 150 & 50 & 12846.11 & 7 & & & & & & & 1.272 \\
\hline 7778.488 & 75 & 5 & 12852.43 & 3 & & & & & & 0.481 & \\
\hline 7776.671 & 40 & 2 & 12855.43 & 2 & 2 & 1.170 & 3 & 1.394 & & & \\
\hline 7773.744 & 50 & 5 & 12860.28 & 7 & & & & & & & 1.100 \\
\hline 7761.704 & 40 & 4 & 12880.22 & 2 & & & & & & & 1.357 \\
\hline 7749.325 & 8 & 2 & 12900.80 & 7 & & & & & & & 1.099 \\
\hline 7742.552 & 100 & 2 & 12912.09 & 1 & & & & & 0.320 & & \\
\hline 7734.617 & 8 & 2 & 12925.33 & 7 & & & & & & & 1.096 \\
\hline 7728.939 & 100 & 15 & 12934.83 & 7 & & & & & & & 1.178 \\
\hline 7721.188 & 100 & 8 & 12947.81 & 3 & & & & & & 0.290 & \\
\hline 7710.258 & 100 & 15 & 12966.17 & 2 & 4 & 1.072 & 5 & 1.262 & & & \\
\hline 7703.679 & 100 & 10 & 12977.24 & 7 & & & & & & & 1.195 \\
\hline
\end{tabular}


TABLE 3. Zeeman effect of Th $\mathrm{I}$-continued

\begin{tabular}{|c|c|c|c|c|c|c|c|c|c|c|c|}
\hline \multirow{2}{*}{$\begin{array}{l}\text { Wavelength } \\
\text { (Å) }\end{array}$} & \multicolumn{2}{|c|}{ Intensity } & \multirow{2}{*}{$\begin{array}{l}\text { Wavenumber } \\
\qquad\left(\mathrm{cm}^{-1}\right)\end{array}$} & \multirow{2}{*}{ Type } & \multirow{2}{*}{$J_{1}$} & \multirow{2}{*}{$g_{1}$} & \multirow{2}{*}{$J_{2}$} & \multirow{2}{*}{$g_{2}$} & \multirow{2}{*}{$\Delta g$} & \multirow{2}{*}{$p$} & \multirow{2}{*}{$n$} \\
\hline & Lamp & Spark & & & & & & & & & \\
\hline 7699.765 & 100 & 5 & 12983.84 & 3 & & & & & & 0.191 & \\
\hline 7697.922 & 75 & 5 & 12986.94 & 3 & & & & & & 0.472 & \\
\hline 7693.797 & 100 & 5 & 12993.91 & 7 & & & & & & & 1.117 \\
\hline 7691.765 & 75 & 3 & 12997.34 & 2 & 2 & 1.286 & 3 & 1.414 & & & \\
\hline 7683.010 & 50 & 1 & 13012.15 & 7 & & & & & & & 1.239 \\
\hline 7676.212 & 100 & 15 & 13023.67 & 3 & 2 & 0.892 & 2 & 1.192 & & & \\
\hline 7668.955 & 50 & 1 & 13036.00 & 2 & 1 & 0.784 & 2 & 1.710 & & & \\
\hline 7666.566 & 100 & 2 & 13040.06 & 7 & & & & & & & 1.279 \\
\hline 7660.889 & 150 & 4 & 13049.72 & 1 & 4 & 1.207 & 5 & 1.017 & & & \\
\hline 7654.695 & 100 & 5 & 13060.28 & 1 & & & & & 0.370 & & \\
\hline 7653.827 & 200 & 10 & 13061.76 & 7 & & & & & & & 1.137 \\
\hline 7652.317 & 100 & 3 & 13064.34 & 7 & & & & & & & 1.188 \\
\hline 7647.380 & 500 & 50 & 13072.78 & 3 & 5 & 1.198 & 5 & 1.370 & & & \\
\hline 7642.873 & 15 & & 13080.48 & 3 & & & & & & 0.231 & \\
\hline 7637.385 & 75 & 2 & 13089.88 & 2 & & & & & 0.381 & & 2.444 \\
\hline 7636.173 & 100 & 4 & 13091.96 & 3 & & & & & & 0.095 & \\
\hline 7633.767 & 150 & 2 & 13096.09 & 1 & & & & & 0.270 & & \\
\hline 7630.310 & 100 & 5 & 13102.02 & 3 & 4 & 0.986 & 4 & 1.306 & & & \\
\hline 7627.175 & 150 & 5 & 13107.41 & 2 & & & & & & & 1.401 \\
\hline 7620.080 & 100 & 5 & 13119.61 & 7 & & & & & & & 1.166 \\
\hline 7616.686 & 50 & 1 & 13125.46 & 2 & 1 & 0.748 & 2 & 1.164 & & & \\
\hline 7607.519 & 40 & 1 & 13141.27 & 2 & & & & & & & 1.368 \\
\hline 7605.083 & 5 & & 13145.48 & 2 & & & & & 0.485 & & \\
\hline 7602.795 & 40 & 1 & 13149.44 & 7 & & & & & & & 1.264 \\
\hline 7598.203 & 200 & 3 & 13157.38 & 3 & & & & & & 0.580 & \\
\hline 7591.999 & 10 & & 13168.14 & 7 & & & & & & & 1.109 \\
\hline 7591.531 & 20 & & 13168.95 & 3 & & & & & & 0.157 & \\
\hline 7586.988 & 8 & & 13176.83 & 1 & & & & & 0.208 & & \\
\hline 7585.783 & 200 & 10 & 13178.93 & 7 & & & & & & & 0.386 \\
\hline 7585.688 & 200 & 15 & 13179.09 & 7 & & & & & & & 1.106 \\
\hline 7583.422 & 25 & & 13183.03 & 1 & 3 & 1.092 & 4 & 0.920 & & & \\
\hline 7580.686 & 40 & 1 & 13187.79 & 7 & & & & & & & 1.212 \\
\hline 7577.266 & 20 & & 13193.74 & 3 & & & & & & 0.182 & \\
\hline 7573.342 & 50 & 1 & 13200.58 & 1 & 3 & 1.377 & 4 & 1.111 & & & \\
\hline 7571.029 & 8 & & 13204.61 & 7 & & & & & & & 1.046 \\
\hline 7569.509 & 100 & 2 & 13207.26 & 3 & 2 & 0.728 & 2 & 0.876 & & & \\
\hline 7567.739 & 300 & 15 & 13210.35 & 3 & 5 & 1.080 & 5 & 1.372 & & & \\
\hline 7557.751 & 50 & 1 & 13227.81 & 1 & & & & & 0.166 & & \\
\hline 7555.326 & 50 & 1 & 13232.05 & 1 & 2 & 0.986 & 3 & 0.902 & & & \\
\hline 7549.316 & 200 & 8 & 13242.59 & 3 & 2 & 0.815 & 2 & 1.187 & & & \\
\hline 7537.429 & 100 & 2 & 13263.47 & 1 & & & & & 0.270 & & \\
\hline 7531.818 & 50 & 2 & 13273.35 & 3 & & & & & & 0.089 & \\
\hline 7531.142 & 75 & 2 & 13274.54 & 7 & & & & & & & 1.067 \\
\hline 7523.133 & 100 & 3 & 13288.67 & 3 & 4 & 0.998 & 4 & 1.169 & & & \\
\hline 7520.694 & 15 & & 13292.99 & 3 & & & & & & 0.190 & \\
\hline 7520.127 & 8 & & 13293.99 & 2 & 2 & 1.034 & 3 & 1.174 & & & \\
\hline 7517.963 & 100 & 2 & 13297.81 & 1 & 1 & 1.400 & 2 & 1.146 & & & \\
\hline 7516.737 & 50 & 1 & 13299.98 & 3 & & & & & & 0.420 & \\
\hline 7511.788 & 100 & 4 & 13308.74 & 1 & 4 & 1.040 & 5 & 1.384 & & & \\
\hline 7508.477 & 100 & 4 & 13314.61 & 3 & 4 & 0.938 & 4 & 1.176 & & & \\
\hline 7505.292 & 75 & & 13320.26 & 3 & & & & & & 0.135 & \\
\hline 7501.180 & 8 & & 13327.57 & 7 & & & & & & & 1.250 \\
\hline 7499.643 & 10 & 1 & 13330.30 & 3 & & & & & & 0.124 & \\
\hline 7499.000 & 100 & 2 & 13331.44 & 3 & 2 & 0.778 & 2 & 1.186 & & & \\
\hline 7495.564 & 75 & 2 & 13337.55 & 2 & 4 & 0.887 & 5 & 1.176 & & & \\
\hline
\end{tabular}


TABlE 3. Zeeman effect of Th $\mathrm{I}$-continued

\begin{tabular}{|c|c|c|c|c|c|c|c|c|c|c|c|}
\hline \multirow{2}{*}{$\begin{array}{c}\text { Wavelength } \\
\text { (Å) }\end{array}$} & \multicolumn{2}{|c|}{ Intensity } & \multirow{2}{*}{$\begin{array}{l}\text { Wavenumber } \\
\left(\mathrm{cm}^{-1}\right)\end{array}$} & \multirow{2}{*}{ Type } & \multirow{2}{*}{$J_{1}$} & \multirow{2}{*}{$g_{1}$} & \multirow{2}{*}{$J_{2}$} & \multirow{2}{*}{$g_{2}$} & \multirow{2}{*}{$\Delta g$} & \multirow{2}{*}{$p$} & \multirow{2}{*}{$n$} \\
\hline & Lamp & Spark & & & & & & & & & \\
\hline 7493.427 & 100 & 2 & 13341.36 & 2 & 3 & 1.092 & 4 & 1.304 & & & \\
\hline 7487.972 & 100 & 3 & 13351.07 & 2 & 2 & 0.780 & 3 & 1.040 & & & \\
\hline 7483.624 & 200 & 10 & 13358.83 & 1 & 1 & 1.471 & 2 & 1.040 & & & \\
\hline 7481.352 & 200 & 15 & 13362.89 & 3 & 4 & 0.996 & 4 & 1.306 & & & \\
\hline 7474.559 & 10 & 1 & 13375.03 & 3 & & & & & & 0.267 & \\
\hline 7469.054 & 40 & 1 & 13384.89 & 7 & & & & & & & 0.770 \\
\hline 7465.443 & 100 & 3 & 13391.36 & 1 & 2 & 1.439 & 3 & 1.189 & & & \\
\hline 7462.990 & 75 & 1 & 13395.77 & 7 & & & & & & & 0.961 \\
\hline 7461.290 & 8 & 1 & 13398.82 & 3 & 2 & 1.170 & 2 & 1.336 & & & \\
\hline 7458.753 & 50 & 2 & 13403.38 & 2 & 1 & 0.506 & 2 & 0.984 & & & \\
\hline 7457.648 & 25 & 1 & 13405.36 & 2 & 3 & 0.925 & 4 & 1.170 & & & \\
\hline 7456.818 & 20 & 1 & 13406.85 & 2 & & & & & & & 1.283 \\
\hline 7455.204 & 100 & 4 & 13409.76 & 3 & 4 & 0.878 & 4 & 1.090 & & & \\
\hline 7452.516 & 20 & 1 & 13414.59 & 2 & 2 & 0.944 & 3 & 1.194 & & & \\
\hline 7450.213 & $50 w$ & 5 & 13418.74 & 7 & & & & & & & 1.201 \\
\hline 7447.848 & 150 & 5 & 13423.00 & 3 & 3 & 0.890 & 3 & 1.002 & & & \\
\hline 7443.873 & 50 & 1 & 13430.17 & 1 & & & & & & & 0.840 \\
\hline 7442.040 & 15 & & 13433.48 & 3 & & & & & & 0.463 & \\
\hline 7430.255 & 500 & 15 & 13454.78 & 1 & 1 & 1.574 & 2 & 1.012 & & & \\
\hline 7428.943 & 800 & 50 & 13457.16 & 1 & 1 & 1.363 & 2 & 1.179 & & & \\
\hline 7422.013 & 100 & 2 & 13469.72 & 3 & & & & & & 0.601 & \\
\hline 7418.548 & 200 & & 13476.02 & 2 & 1 & 0.066 & 2 & 1.088 & & & \\
\hline 7417.789 & 100 & & 13477.39 & 1 & 3 & 1.075 & 4 & 0.871 & & & \\
\hline 7411.739 & 100 & 4 & 13488.39 & 2 & 3 & 1.050 & 4 & 0.872 & & & \\
\hline 7410.968 & 50 & 1 & 13489.80 & 3 & & & & & & 0.275 & \\
\hline 7409.221 & 40 & & 13492.98 & 3 & & & & & & 0.525 & \\
\hline 7404.719 & 401 & & 13501.18 & 7 & & & & & & & 1.174 \\
\hline 7403.993 & 100 & 1 & 13502.51 & 3 & & & & & & 0.603 & \\
\hline 7402.248 & 400 & 8 & 13505.69 & 2 & 3 & 0.864 & 4 & 1.094 & & & \\
\hline 7396.895 & 20 & 1 & 13515.46 & 2 & & & & & & & 1.499 \\
\hline 7392.637 & 15 & 1 & 13523.25 & 7 & & & & & & & 1.296 \\
\hline 7383.712 & 150 & 8 & 13539.59 & 7 & & & & & & & 1.203 \\
\hline 7376.879 & 200 & 8 & 13552.14 & 2 & 5 & 1.082 & 6 & 1.172 & & & \\
\hline 7376.178 & 25 & & 13553.42 & 7 & & & & & & & 1.143 \\
\hline 7370.827 & 100 & 2 & 13563.26 & 7 & & & & & & & 0.969 \\
\hline 7362.195 & 50 & 1 & 13579.16 & 3 & & & & & & 0.525 & \\
\hline 7355.179 & 10 & & 13592.12 & 3 & & & & & & 0.270 & \\
\hline 7353.644 & 15 & & 13594.96 & 7 & & & & & & & 1.084 \\
\hline 7353.007 & 15 & & 13596.13 & 7 & & & & & & & 0.962 \\
\hline 7350.454 & 75 & & 13600.85 & 2 & 3 & 1.041 & 4 & 1.233 & & & \\
\hline 7341.150 & 500 & 15 & 13618.09 & 3 & 4 & 0.986 & 4 & 1.088 & & & \\
\hline 7339.599 & 150 & 3 & 13620.97 & 1 & & & & & 0.280 & & \\
\hline 7335.568 & 100 & 15 & 13628.45 & 7 & & & & & & & 0.834 \\
\hline 7329.488 & 100 & 5 & 13639.76 & 1 & 2 & 1.713 & 3 & 1.093 & & & \\
\hline 7324.808 & 300 & 10 & 13648.47 & 3 & & & & & & 0.590 & \\
\hline 7323.207 & 100 & 3 & 13651.46 & 3 & & & & & & 0.571 & \\
\hline 7315.609 & 150 & 3 & 13665.64 & 2 & 1 & 0.734 & 2 & 1.058 & & & \\
\hline 7315.064 & 200 & 3 & 13666.65 & 2 & 1 & 0.522 & 2 & 1.262 & & & \\
\hline 7311.510 & 100 & 3 & 13673.30 & 3 & 3 & 0.994 & 3 & 1.174 & & & \\
\hline 7309.255 & 100 & 2 & 13677.52 & 1 & & & & & & & 0.938 \\
\hline 7302.172 & 50 & 2 & 13690.78 & 2 & & & & & & & 1.622 \\
\hline 7298.138 & $200 \mathrm{~b}$ & 3 & 13698.35 & 1 & 3 & 1.162 & 4 & 0.990 & & & \\
\hline 7296.265 & 100 & 3 & 13701.87 & 2 & & & & & 0.094 & & \\
\hline 7294.316 & 15 & 1 & 13705.53 & 3 & & & & & & 0.239 & \\
\hline 7284.905 & 500 & 20 & 13723.23 & 1 & 2 & 1.248 & 3 & 1.133 & & & \\
\hline & & & & & & & & & & & \\
\hline
\end{tabular}


TABLE 3. Zeeman effect of Th $\mathrm{I}$-continued

\begin{tabular}{|c|c|c|c|c|c|c|c|c|c|c|c|}
\hline \multirow{2}{*}{$\begin{array}{l}\text { Wavelength } \\
\text { (̊) }\end{array}$} & \multicolumn{2}{|c|}{ Intensity } & \multirow{2}{*}{$\begin{array}{l}\text { Wavenumber } \\
\left(\mathrm{cm}^{-1}\right)\end{array}$} & \multirow{2}{*}{ Type } & \multirow{2}{*}{$J_{1}$} & \multirow{2}{*}{$g_{1}$} & \multirow{2}{*}{$J_{2}$} & \multirow{2}{*}{$g_{2}$} & \multirow{2}{*}{$\Delta g$} & \multirow{2}{*}{$p$} & \multirow{2}{*}{$n$} \\
\hline & Lamp & Spark & & & & & & & & & \\
\hline 7282.732 & 100 & 2 & 13727.33 & 2 & & & & & & & 1.436 \\
\hline 7279.142 & 8 & & 13734.10 & 3 & & & & & & 0.579 & \\
\hline 7277.013 & 75 & 1 & 13738.12 & 3 & & & & & & 0.099 & \\
\hline 7274.294 & 15 & 1 & 13743.25 & 3 & & & & & & 0.224 & \\
\hline 7272.550 & 100 & 2 & 13746.55 & 3 & & & & & & 0.294 & \\
\hline 7270.558 & 100 & 2 & 13750.31 & 2 & 2 & 0.673 & 3 & 1.255 & & & \\
\hline 7268.834 & 100 & 2 & 13753.58 & 7 & & & & & & & 1.182 \\
\hline 7267.096 & 50 & 1 & 13756.86 & 7 & & & & & & & 1.362 \\
\hline 7264.118 & 50 & & 13762.50 & 2 & & & & & & & 1.546 \\
\hline 7262.190 & 10 & & 13766.16 & 3 & & & & & & 0.187 & \\
\hline 7258.177 & 200 & 5 & 13773.77 & 2 & 3 & 1.034 & 4 & 1.164 & & & \\
\hline 7256.981 & 150 & 4 & 13776.04 & 3 & 2 & 0.974 & 2 & 1.110 & & & \\
\hline 7255.351 & 200 & 5 & 13779.13 & 2 & 3 & 0.942 & 4 & 1.074 & & & \\
\hline 7247.988 & 25 & 2 & 13793.13 & 7 & & & & & & & 1.144 \\
\hline 7247.599 & 40 & & 13793.87 & 7 & & & & & & & 1.018 \\
\hline 7244.689 & 200 & 8 & 13799.41 & 1 & 3 & 1.030 & 4 & 0.856 & & & \\
\hline 7242.086 & 200 & 10 & 13804.37 & 3 & 5 & 0.990 & 5 & 1.370 & & & \\
\hline 7235.890 & 50 & 1 & 13816.19 & 1 & & & & & 0.160 & & \\
\hline 7233.390 & 100 & & 13820.97 & 7 & & & & & & & 1.184 \\
\hline 7230.888 & 500 & 5 & 13825.75 & 1 & & & & & & & 0.472 \\
\hline 7220.989 & 100 & 2 & 13844.70 & 2 & 1 & 0.500 & 2 & 0.720 & & & \\
\hline 7219.156 & 300 & 10 & 13848.22 & 3 & & & & & & 0.230 & \\
\hline 7208.000 & 500 & 150 & 13869.65 & 2 & 3 & 1.233 & 4 & 1.323 & & & \\
\hline 7206.476 & 400 & 8 & 13872.58 & 3 & 3 & 0.836 & 3 & 0.881 & & & \\
\hline 7201.803 & 100 & 2 & 13881.59 & 2 & 2 & 0.740 & 3 & 0.900 & & & \\
\hline 7201.042 & 50 & 1 & 13883.05 & 2 & & & & & 0.284 & & \\
\hline 7200.041 & 400 & 10 & 13884.98 & 2 & 2 & 1.188 & 3 & 1.304 & & & \\
\hline 7179.725 & 150 & 4 & 13924.27 & 3 & & & & & & 0.332 & \\
\hline 7177.284 & 75 & 2 & 13929.01 & 2 & & & & & 0.154 & & \\
\hline 7173.372 & 500 & 20 & 13936.60 & 1 & & & - & & & & 0.879 \\
\hline 7170.357 & 100 & 2 & 13942.46 & 1 & & & & & 0.260 & & \\
\hline 7168.896 & 800 & 75 & 13945.31 & 2 & 2 & 0.747 & 3 & 1.116 & & & \\
\hline 7167.202 & 150 & 4 & 13948.60 & 1 & & & & & 0.088 & & \\
\hline 7159.943 & $300 \mathrm{~b}$ & 8 & 13962.74 & 1 & 3 & 1.105 & 4 & 0.984 & & & \\
\hline 7159.877 & $100 \mathrm{~b}$ & 2 & 13962.87 & 7 & & & & & & & 1.132 \\
\hline 7156.936 & 300 & 15 & 13968.61 & 3 & 4 & 0.989 & 4 & 1.155 & & & \\
\hline 7154.952 & 200 & 8 & 13972.48 & 3 & 2 & 0.665 & 2 & 1.186 & & & \\
\hline 7153.587 & 200 & 10 & 13975.15 & 3 & & & & & & 0.829 & \\
\hline 7150.285 & 400 & 25 & 13981.60 & 1 & 1 & 1.475 & 2 & 1.167 & & & \\
\hline 7148.557 & 400 & 15 & 13984.98 & 2 & 3 & 0.826 & 4 & 0.988 & & & \\
\hline 7142.328 & 300 & 8 & 13997.18 & 2 & 1 & 0.370 & 2 & 0.970 & & & \\
\hline 7138.142 & 20 & & 14005.39 & 7 & & & & & & & 1.171 \\
\hline 7132.603 & 200 & 4 & 14016.26 & 2 & . & & & & & & 1.192 \\
\hline 7131.353 & 150 & 40 & 14018.72 & 3 & & & & & & 0.076 & 1.140 \\
\hline 7130.718 & 200 & 5 & 14019.97 & 1 & & & & & .080 & & \\
\hline 7130.179 & 100 & 4 & 14021.03 & 3 & & & & & & 0.174 & \\
\hline 7126.814 & 100 & 3 & 14027.65 & 3 & & & & & & 0.051 & \\
\hline 7125.515 & 150 & 2 & 14030.21 & 3 & 3 & 3 & & & 0.480 & & \\
\hline 7124.562 & 500 & 50 & 14032.08 & 3 & 2 & 0.737 & 2 & 1.125 & & & \\
\hline 7112.917 & 100 & 4 & 14055.06 & 2 & & & & & 0.180 & & \\
\hline 7111.045 & 75 & 2 & 14058.76 & 7 & & & & & 0.190 & & \\
\hline 7102.589 & 100 & 2 & 14075.49 & 1 & & & & & & & 0.968 \\
\hline 7088.822 & 150 & 2 & 14102.83 & 7 & & & & & & & 1.199 \\
\hline 7084.165 & 500 & 75 & 14112.10 & 2 & 4 & 1.248 & 5 & 1.363 & & & \\
\hline 7072.394 & 300 & 8 & 14135.59 & 1 & 4 & 1.172 & 5 & 1.104 & & & \\
\hline
\end{tabular}


TABLE 3. Zeeman effect of Th $\mathrm{I}$-continued

\begin{tabular}{|c|c|c|c|c|c|c|c|c|c|c|c|}
\hline \multirow{2}{*}{$\begin{array}{l}\text { Wavelength } \\
\text { (A) }\end{array}$} & \multicolumn{2}{|c|}{ Intensity } & \multirow{2}{*}{$\begin{array}{l}\text { Wa venumber } \\
\left(\mathrm{cm}^{-1}\right)\end{array}$} & \multirow{2}{*}{ Type } & \multirow{2}{*}{$J_{1}$} & \multirow{2}{*}{$g_{1}$} & \multirow{2}{*}{$J_{2}$} & \multirow{2}{*}{$g_{2}$} & \multirow{2}{*}{$\Delta g$} & \multirow{2}{*}{$p$} & \multirow{2}{*}{$n$} \\
\hline & Lamp & Spark & & & & & & & & & \\
\hline 7067.513 & 75 & & 14145.35 & 7 & & & & & 0.250 & & \\
\hline 7066.287 & 100 & 2 & 14147.80 & 3 & 3 & 1.164 & 3 & 1.385 & & & \\
\hline 7064.451 & 300 & 10 & 14151.48 & 7 & & & & & & & 1.143 \\
\hline 7062.418 & 40 & 1 & 14155.55 & 3 & & & & & & 0.125 & \\
\hline 7061.392 & 100 & 2 & 14157.61 & 1 & 1 & 1.180 & 2 & 0.770 & & & \\
\hline 7060.039 & 300 & 8 & 14160.32 & 3 & 2 & 0.820 & 2 & 1.008 & & & \\
\hline 7059.526 & 150 & 3 & 14161.35 & 2 & & & & & 0.230 & & \\
\hline 7058.488 & 150 & 5 & 14163.43 & 1 & 3 & 0.998 & 4 & 0.913 & & & \\
\hline 7054.416 & 150 & 2 & 14171.61 & 3 & & & & & & 0.189 & \\
\hline 7038.717 & 75 & 1 & 14203.22 & 7 & & & & & & & 1.194 \\
\hline 7036.277 & 400 & 8 & 14208.14 & 2 & & & & & & & 1.479 \\
\hline 7033.352 & 100 & 2 & 14214.05 & 3 & & & & & & 0.313 & \\
\hline 7028.017 & 100 & 2 & 14224.84 & 3 & 1 & 0.401 & 1 & 1.340 & & & \\
\hline 7023.523 & 100 & 3 & 14233.94 & 2 & & & & & & & 1.057 \\
\hline 7023.145 & 100 & 3 & 14234.71 & 1 & 1 & 1.380 & 2 & 1.090 & & & \\
\hline 7021.279 & 150 & 8 & 14238.49 & 2 & & & & & & & 1.389 \\
\hline 7009.047 & 50 & 1 & 14263.34 & 7 & & & & & & & 1.274 \\
\hline 7007.092 & 100 & 4 & 14267.32 & 3 & & & & & & 0.202 & \\
\hline 7002.881 & 200 & 8 & 14275.90 & 2 & & & & & & & 1.609 \\
\hline 7000.806 & 500 & 75 & 14280.13 & 1 & 5 & 1.349 & 6 & 1.211 & & & \\
\hline 6998.247 & 100 & 2 & 14285.35 & 3 & & & & & & 0.346 & \\
\hline 6995.141 & 75 & 2 & 14291.70 & 7 & & & & & & & 1.106 \\
\hline 6989.657 & 1000 & 150 & 14302.91 & 3 & 4 & 0.744 & 4 & 0.874 & & & \\
\hline 6986.031 & 150 & 4 & 14310.33 & 3 & 2 & 0.962 & 2 & 1.702 & & & \\
\hline 6981.082 & 100 & 3 & 14320.48 & 3 & & & & & & 0.336 & \\
\hline 6969.301 & 100 & 3 & 14344.68 & 3 & & & & & & 0.079 & \\
\hline 6965.946 & 5001 & 4 & 14351.59 & 2 & 1 & 0.699 & 2 & 1.097 & & & \\
\hline 6962.862 & 75 & 2 & 14357.95 & 7 & & & & & 0.397 & & \\
\hline 6954.655 & 200 & 5 & 14374.89 & 1 & 1 & 1.364 & 2 & 1.008 & & & \\
\hline 6948.205 & 300 & 20 & 14388.24 & 3 & 4 & 0.925 & 4 & 1.169 & & & \\
\hline 6945.488 & 200 & 8 & 14393.87 & 2 & & & & & 0.350 & & \\
\hline 6942.535 & 150 & 8 & 14399.99 & 1 & & & & & 0.266 & & \\
\hline 6936.651 & 150 & 4 & 14412.20 & 3 & 3 & 1.383 & 3 & 1.119 & & & \\
\hline 6920.037 & 150 & 5 & 14446.80 & 1 & & & & & & & 0.596 \\
\hline 6916.129 & 200 & 10 & 14454.97 & 2 & 4 & 1.043 & 5 & 1.376 & & & \\
\hline 6911.222 & $800 \mathrm{r}$ & 200 & 14465.22 & 3 & & & & & & 0.147 & \\
\hline 6904.578 & 100 & & 14479.15 & 3 & & & & & & 0.105 & \\
\hline 6900.762 & 100 & 5 & 14487.16 & 2 & & & & & 0.370 & & \\
\hline 6886.404 & 200 & 10 & 14517.36 & 7 & & & & & & & 1.478 \\
\hline 6883.311 & 200 & 10 & 14523.89 & 1 & 3 & 1.132 & 4 & 0.912 & & & \\
\hline 6880.769 & 15 & 1 & 14529.25 & 7 & & & & & & & 1.173 \\
\hline 6874.752 & 200 & 10 & 14541.97 & 3 & & & & & & 0.143 & \\
\hline 6870.988 & 150 & 3 & 14549.93 & 3 & 1 & 0.392 & 1 & 0.619 & & & \\
\hline 6868.455 & 100 & 3 & 14555.30 & 2 & 4 & 0.989 & 5 & 1.259 & & & \\
\hline 6866.756 & $100 \mathrm{~s}$ & 2 & 14558.90 & 7 & & & & & 0.140 & & \\
\hline 6866.368 & 150 & 3 & 14559.72 & 3 & & & & & & 0.312 & \\
\hline 6862.873 & 100 & 2 & 14567.14 & 3 & 2 & 1.099 & 2 & 1.410 & & & \\
\hline 6855.315 & 150 & 4 & 14583.20 & 2 & & & & & 0.157 & & \\
\hline 6853.511 & 100 & 4 & 14587.04 & 2 & & & & & 0.220 & & \\
\hline 6852.344 & 100 & 2 & 14589.52 & 7 & & & & & & & 1.150 \\
\hline 6850.704 & $100 \mathrm{~h}$ & 2 & 14593.01 & 7 & & & & & & & 1.197 \\
\hline 6846.469 & 100 & 2 & 14602.04 & 7 & & & & & & & 0.718 \\
\hline 6841.690 & 10 & & 14612.24 & 7 & & & & & & & 1.043 \\
\hline 6839.287 & 100 & 2 & 14617.37 & 7 & & & & & & & 0.864 \\
\hline 6834.925 & 400 & 50 & 14626.70 & 1 & & & & & & & 0.928 \\
\hline
\end{tabular}


TABLE 3. Zeeman effect of Th $\mathrm{I}$-continued

\begin{tabular}{|c|c|c|c|c|c|c|c|c|c|c|c|}
\hline \multirow{2}{*}{$\begin{array}{l}\text { Wavelength } \\
\text { (§) }\end{array}$} & \multicolumn{2}{|c|}{ Intensity } & \multirow{2}{*}{$\begin{array}{l}\text { Wavenumber } \\
\qquad\left(\mathrm{cm}^{-1}\right)\end{array}$} & \multirow{2}{*}{ Type } & \multirow{2}{*}{$J_{1}$} & \multirow{2}{*}{$g_{1}$} & \multirow{2}{*}{$J_{2}$} & \multirow{2}{*}{$g_{2}$} & \multirow{2}{*}{$\Delta g$} & \multirow{2}{*}{$p$} & \multirow{2}{*}{$n$} \\
\hline & Lamp & Spark & & & & & & & & & \\
\hline 6829.037 & 500 & 25 & 14639.31 & 3 & 3 & 1.095 & 3 & 1.249 & & & \\
\hline 6824.678 & 400 & 20 & 14648.66 & 1 & 2 & 1.101 & 3 & 1.000 & & & \\
\hline 6823.500 & 150 & 4 & 14651.19 & 3 & & & & & & 1.010 & \\
\hline 6815.604 & 100 & 2 & 14668.17 & 7 & & & & & & & 1.054 \\
\hline 6809.311 & 150 & 4 & 14681.72 & 1 & & & & & 0.160 & & \\
\hline 6805.746 & 100 & 2 & 14689.41 & 2 & & & & & & & 1.050 \\
\hline 6802.783 & 100 & 3 & 14695.81 & 2 & 2 & 0.864 & 3 & 1.049 & & & \\
\hline 6798.483 & 150 & 3 & 14705.10 & 1 & & & & & 0.630 & & \\
\hline 6795.797 & 100 & 2 & 14710.92 & 2 & & & & & 0.360 & & \\
\hline 6795.046 & 75 & 2 & 14712.54 & 7 & & & & & & & 0.952 \\
\hline 6791.232 & 400 & 25 & 14720.81 & 2 & 3 & 1.087 & 4 & 1.318 & & & \\
\hline 6788.837 & 300 & 25 & 14726.00 & 2 & 4 & 0.970 & 5 & 1.042 & & & \\
\hline 6787.734 & 300 & 25 & 14728.39 & 2 & & & & & & & 1.232 \\
\hline 6780.126 & 400 & 75 & 14744.91 & 3 & 2 & 0.622 & 2 & 0.772 & & & \\
\hline 6776.230 & 100 & 3 & 14753.40 & 1 & & & & & 0.200 & & \\
\hline 6772.176 & $400 \mathrm{~b}$ & 15 & 14762.23 & 1 & & & & & 0.165 & & \\
\hline 6765.683 & 200 & 100 & 14776.40 & 3 & & & & & & 0.720 & \\
\hline 6763.340 & 50 & 2 & 14781.51 & 7 & & & & & & & 1.126 \\
\hline 6762.500 & 40 & 1 & 14783.35 & 7 & & & & & & & 1.224 \\
\hline 6760.667 & 100 & 3 & 14787.36 & 7 & & & & & & & 1.212 \\
\hline 6758.202 & 200 & 8 & 14792.75 & 3 & & & & & & 0.837 & \\
\hline 6757.105 & 100 & 5 & 14795.75 & 1 & 3 & 1.099 & 4 & 0.891 & & & \\
\hline 6751.426 & 100 & 3 & 14807.60 & 2 & 1 & 0.378 & 2 & 0.970 & & & \\
\hline 6749.315 & 50 & 1 & 14812.23 & 3 & & & & & & 0.370 & \\
\hline 6742.881 & 200 & 8 & 14826.36 & 2 & 2 & 1.090 & 3 & 1.386 & & & \\
\hline 6738.179 & 200 & 5 & 14836.71 & 3 & 3 & 1.020 & 3 & 1.241 & & & \\
\hline 6735.128 & 100 & 2 & 14843.43 & 3 & & & & & & 0.899 & \\
\hline 6733.748 & 150 & 5 & 14846.47 & 3 & & & & & & 0.100 & \\
\hline 6732.650 & 100 & 3 & 14848.89 & 3 & & & & & & 1.191 & \\
\hline 6728.757 & 150 & 5 & 14857.48 & 3 & & & & & & 0.203 & \\
\hline 6727.459 & 500 & 75 & 14860.35 & 3 & 1 & 0.069 & 1 & 1.434 & & & \\
\hline 6719.198 & 200 & 5 & 14878.62 & 2 & & & & & 0.044 & & \\
\hline 6717.384 & 100 & 3 & 14882.64 & 7 & & & & & & & 0.982 \\
\hline 6715.515 & 100 & 2 & 14886.78 & 3 & & & & & & 0.199 & \\
\hline 6713.967 & 300 & 8 & 14890.21 & 3 & 2 & 0.665 & 2 & 1.015 & & & \\
\hline 6711.249 & 100 & 4 & 14896.24 & 3 & & & & & & 1.252 & \\
\hline 6697.708 & 200 & 5 & 14926.36 & 1 & 1 & 1.380 & 2 & 1.240 & & & \\
\hline 6696.140 & 200 & 5 & 14929.86 & 2 & & & & & & & 1.595 \\
\hline 6694.492 & 100 & 2 & 14933.53 & 2 & & & & & 0.630 & & \\
\hline 6687.521 & 150 & 3 & 14949.10 & 3 & & & & & & 0.258 & \\
\hline 6684.049 & 100 & 2 & 14956.86 & 1 & & & & & 0.294 & & \\
\hline 6683.368 & 200 & 3 & 14958.39 & 2 & 2 & 0.782 & 3 & 0.946 & & & \\
\hline 6680.087 & 100 & 1 & 14965.73 & 2 & 2 & 0.750 & 3 & 1.080 & & & \\
\hline 6678.707 & 200 & 8 & 14968.83 & 3 & & & & & & 0.083 & \\
\hline 6673.590 & 200 & 5 & 14980.30 & 3 & 4 & 0.887 & 4 & 1.157 & & & \\
\hline 6668.826 & 100 & 2 & 14991.00 & 3 & 4 & 0.922 & 4 & 0.982 & & & \\
\hline 6663.702 & 100 & 3 & 15002.53 & 3 & & & & & & 0.152 & \\
\hline 6662.270 & 800 & 200 & 15005.75 & 1 & 2 & 1.379 & 3 & 1.250 & & & \\
\hline 6658.678 & 400 & 15 & 15013.85 & 7 & & & & & & & 1.054 \\
\hline 6654.371 & 100 & 3 & 15023.57 & 2 & & & & & & & 1.536 \\
\hline 6638.912 & 150 & 20 & 15058.55 & 7 & & & & & & & 1.051 \\
\hline 6626.125 & 75 & 1 & 15087.61 & 3 & 1 & 1.018 & 1 & 1.378 & & & \\
\hline 6613.376 & 200 & 8 & 15116.70 & 1 & 1 & 1.518 & 2 & 1.182 & & & \\
\hline 6593.940 & 500 & 150 & 15161.25 & 7 & & & & & & & 0.069 \\
\hline 6593.464 & 100 & 15 & 15162.35 & 2 & & & & & & & 1.362 \\
\hline
\end{tabular}


TABLE 3. Zeeman effect of Th $\mathrm{I}$-continued

\begin{tabular}{|c|c|c|c|c|c|c|c|c|c|c|c|}
\hline \multirow{2}{*}{$\begin{array}{c}\text { Wavelength } \\
(\AA)\end{array}$} & \multicolumn{2}{|c|}{ Intensity } & \multirow{2}{*}{$\begin{array}{l}\text { Wavenumber } \\
\quad\left(\mathrm{cm}^{-1}\right)\end{array}$} & \multirow{2}{*}{ Type } & \multirow{2}{*}{$J_{1}$} & \multirow{2}{*}{$g_{1}$} & \multirow{2}{*}{$J_{2}$} & \multirow{2}{*}{$g_{2}$} & \multirow{2}{*}{$\Delta g$} & \multirow{2}{*}{$p$} & \multirow{2}{*}{$n$} \\
\hline & Lamp & Spark & & & & & & & & & \\
\hline 6591.485 & 400 & 75 & 15166.90 & 2 & 2 & 0.736 & 3 & 1.064 & & & \\
\hline 6588.540 & $500 \mathrm{r}$ & 200 & 15173.68 & 1 & 1 & 1.482 & 2 & 1.092 & & & \\
\hline 6583.906 & $400 \mathrm{r}$ & 200 & 15184.36 & 2 & & & & & & & 1.482 \\
\hline 6580.231 & 75 & 3 & 15192.84 & 1 & & & & & 0.530 & & \\
\hline 6579.482 & 20 & 2 & 15194.57 & 3 & & & & & & 0.122 & \\
\hline 6577.216 & 100 & 75 & 15199.80 & 3 & & & & & & 0.163 & \\
\hline 6564.443 & 150 & 8 & 15229.38 & 1 & 2 & 0.966 & 3 & 0.686 & & & \\
\hline 6562.105 & 50 & & 15234.80 & 3 & 1 & 0.761 & 1 & 1.141 & & & \\
\hline 6558.871 & 100 & 8 & 15242.32 & 1 & 2 & 1.246 & 3 & 0.964 & & & \\
\hline 6551.703 & 100 & 5 & 15258.99 & 1 & 2 & 1.524 & 3 & 1.038 & & & \\
\hline 6538.301 & 50 & 4 & 15290.27 & 2 & 1 & 0.762 & 2 & 0.998 & & & \\
\hline 6531.342 & $800 \mathrm{r}$ & 200 & 15306.56 & 1 & 1 & 1.582 & 2 & 1.014 & & & \\
\hline 6522.044 & 100 & 15 & 15328.38 & 2 & 1 & 0.376 & 2 & 0.723 & & & \\
\hline 6512.366 & 400 & 15 & 15351.16 & 3 & 2 & 1.093 & 2 & 1.249 & & & \\
\hline 6506.989 & 100 & 15 & 15363.85 & 2 & 2 & 1.135 & 3 & 0.866 & & & \\
\hline 6501.992 & 100 & 5 & 15375.65 & 2 & 2 & 0.532 & 2 & 1.004 & & & \\
\hline 6497.490 & 50 & 3 & 15386.31 & 3 & & & & & & 0.187 & \\
\hline 6488.882 & 100 & 4 & 15406.72 & 7 & & & & & & & 0.638 \\
\hline 6462.606 & $200 \mathrm{~b}$ & 100 & 15469.36 & 7 & & & & & & & 1.184 \\
\hline 6457.280 & $800 \mathrm{r}$ & 300 & 15482.12 & 2 & 4 & 0.866 & 5 & 1.006 & & & \\
\hline 6450.007 & 200 & 15 & 15499.58 & 1 & 2 & 1.542 & 3 & 1.386 & & & \\
\hline 6446.771 & 100 & 8 & 15507.35 & 3 & & & & & & 0.216 & \\
\hline 6438.918 & 100 & 8 & 15526.27 & 2 & 1 & 0.464 & 2 & 0.794 & & & \\
\hline 6437.769 & 200 & 20 & 15529.04 & 3 & & & & & & 0.164 & \\
\hline 6427.507 & 50 & 4 & 15553.83 & 3 & & & & & & 0.328 & \\
\hline 6422.106 & 100 & 8 & 15566.91 & 2 & & & & & & & 1.410 \\
\hline 6417.908 & 15 & 2 & 15577.09 & 3 & & & & & & 0.141 & \\
\hline 6415.536 & 75 & 5 & 15582.86 & 2 & & & & & 0.264 & & \\
\hline 6413.615 & 400 & 100 & 15587.52 & 1 & 3 & 1.200 & 4 & 1.002 & & & \\
\hline 6411.899 & 500 & 75 & 15591.69 & 1 & & & & & & & 1.095 \\
\hline 6406.446 & 100 & 5 & 15604.96 & 3 & & & & & & 0.098 & \\
\hline 6400.696 & 100 & 8 & 15618.98 & 1 & & & & & & & 0.310 \\
\hline 6396.951 & 100 & 8 & 15628.13 & 2 & & & & & & & 1.510 \\
\hline 6394.045 & 100 & 8 & 15635.23 & 3 & & & & & & 0.440 & \\
\hline 6392.371 & 75 & 8 & 15639.32 & 7 & & & & & & & 0.995 \\
\hline 6387.393 & 200 & 10 & 15651.51 & 1 & 1 & 1.466 & 2 & 1.356 & & & \\
\hline 6376.930 & 500 & 200 & 15677.19 & 7 & & & & & & & 1.138 \\
\hline 6369.141 & 200 & 25 & 15696.37 & 7 & & & & & & & 1.062 \\
\hline 6362.256 & 100 & 20 & 15713.35 & 7 & & & & & & & 1.008 \\
\hline 6359.677 & 75 & 5 & 15719.72 & 7 & & & & & & & 0.824 \\
\hline 6355.911 & 800 & 200 & 15729.04 & 2 & 3 & 0.862 & 4 & 1.032 & & & \\
\hline 6355.633 & 75 & 8 & 15729.72 & 7 & & & & & & & 1.025 \\
\hline 6348.737 & 200 & 15 & 15746.81 & 3 & & & & & & 0.171 & \\
\hline 6342.860 & 500 & 150 & 15761.40 & 2 & & & & & 0.120 & & 1.668 \\
\hline 6339.663 & 100 & 15 & 15769.35 & 1 & 1 & 1.366 & 2 & 1.179 & & & \\
\hline 6331.415 & 200 & 15 & 15789.89 & 1 & & & & & 0.220 & & \\
\hline 6327.279 & 500 & 100 & 15800.21 & 2 & & & & & & & 1.496 \\
\hline 6326.365 & 200 & 20 & 15802.50 & 3 & & & & & & 0.527 & \\
\hline 6322.866 & 20 & 2 & 15811.24 & 7 & & & & & & & 1.051 \\
\hline 6317.183 & 150 & 8 & 15825.47 & 2 & 2 & 1.135 & 3 & 1.395 & & & \\
\hline 6315.774 & 100 & 8 & 15829.00 & 3 & & & & & & 0.232 & \\
\hline 6307.868 & 25 & 3 & 15848.83 & 7 & & & & & & & 0.953 \\
\hline 6303.251 & 200 & 20 & 15860.44 & 1 & 3 & 1.218 & 4 & 0.995 & & & \\
\hline 6301.421 & 200 & 20 & 15865.05 & 7 & & & & & & & 0.832 \\
\hline 6300.917 & 150 & 10 & 15866.32 & 7 & & & & & & & 1.119 \\
\hline
\end{tabular}


TABLE 3. Zeeman effect of Th $\mathrm{I}$-continued

\begin{tabular}{|c|c|c|c|c|c|c|c|c|c|c|c|}
\hline \multirow{2}{*}{$\begin{array}{l}\text { Wavelength } \\
\text { (§) }\end{array}$} & \multicolumn{2}{|c|}{ Intensity } & \multirow{2}{*}{$\begin{array}{l}\text { Wa venumber } \\
\left(\mathrm{cm}^{-1}\right)\end{array}$} & \multirow{2}{*}{ Type } & \multirow{2}{*}{$J_{1}$} & \multirow{2}{*}{$g_{1}$} & \multirow{2}{*}{$J_{2}$} & \multirow{2}{*}{$g_{2}$} & \multirow{2}{*}{$\Delta g$} & \multirow{2}{*}{$p$} & \multirow{2}{*}{$n$} \\
\hline & Lamp & Spark & & & & & & & & & \\
\hline 6298.903 & 100 & 5 & 15871.39 & 3 & 2 & 1.099 & 2 & 1.705 & & & \\
\hline 6293.241 & 150 & 15 & 15885.67 & 3 & & & & & & 0.693 & \\
\hline 6292.891 & 100 & 8 & 15886.55 & 3 & & & & & & 0.296 & \\
\hline 6291.192 & 300 & 15 & 15890.84 & 3 & & & & & & 0.100 & \\
\hline 6271.544 & 75 & 5 & 15940.63 & 7 & & & & & & & 1.332 \\
\hline 6261.418 & 400 & 150 & 15966.41 & 7 & & & & & & & 1.148 \\
\hline 6246.173 & 150 & 8 & 16005.38 & 7 & & & & & & & 0.929 \\
\hline 6240.949 & 150 & 8 & 16018.77 & 3 & & & & & & 0.525 & \\
\hline 6234.856 & 400 & 75 & 16034.42 & 2 & 1 & 1.527 & 2 & 1.014 & & & \\
\hline 6224.527 & 400 & 40 & 16061.03 & 3 & & & & & & 0.239 & \\
\hline 6220.011 & 100 & 5 & 16072.69 & 7 & & & & & 0.325 & & \\
\hline 6208.686 & 100 & 5 & 16102.01 & 7 & & & & & 0.320 & & \\
\hline 6207.220 & 500 & 200 & 16105.82 & 3 & 1 & 0.071 & 1 & 1.589 & & & \\
\hline 6205.861 & 100 & 8 & 16109.34 & 7 & & & & & 0.340 & & \\
\hline 6203.493 & 500 & 100 & 16115.49 & 7 & & & & & & & 1.359 \\
\hline 6198.223 & 400 & 75 & 16129.19 & 1 & 1 & 1.582 & 2 & 1.262 & & & \\
\hline 6191.906 & 400 & 50 & 16145.65 & 3 & 2 & 1.009 & 2 & 1.381 & & & \\
\hline 6189.145 & 75 & 2 & 16152.85 & 7 & & & & & 0.090 & & \\
\hline 6180.703 & 200 & 8 & 16174.91 & 2 & 1 & 0.058 & 2 & 0.518 & & & \\
\hline 6178.432 & 200 & 20 & 16180.86 & 2 & 3 & 1.072 & 4 & 1.304 & & & \\
\hline 6169.822 & 500 & 200 & 16203.44 & 1 & 3 & 1.305 & 4 & 1.213 & & & \\
\hline 6164.480 & 200 & 20 & 16217.48 & 2 & 2 & 0.739 & 2 & 1.077 & & & \\
\hline 6162.968 & 40 & 3 & 16221.46 & 7 & & & & & & & 1.018 \\
\hline 6161.353 & 150 & 8 & 16225.71 & 7 & & & & & & & 1.317 \\
\hline 6157.093 & 100 & 8 & 16236.94 & 3 & & & & & & 0.916 & \\
\hline 6155.587 & 200 & 15 & 16240.91 & 1 & 2 & 1.189 & 3 & 1.077 & & & \\
\hline 6154.068 & 100 & 15 & 16244.92 & 7 & & & & & & & 1.435 \\
\hline 6151.993 & $150 \mathrm{r}$ & & 16250.40 & 2 & 2 & 1.112 & 3 & 1.250 & & & \\
\hline 6124.485 & 200 & 15 & 16323.39 & 3 & 2 & 1.189 & 2 & 1.379 & & & \\
\hline 6123.838 & 75 & 8 & 16325.11 & 1 & & & & & 0.200 & & \\
\hline 6121.413 & 150 & 50 & 16331.58 & 3 & & & & & 0.110 & 0.665 & \\
\hline 6119.704 & 75 & 8 & 16336.14 & 1 & 1 & 1.210 & 2 & 0.976 & & & \\
\hline 6116.172 & 100 & 8 & 16345.57 & 3 & & & & & & 0.130 & \\
\hline 6114.546 & 150 & 10 & 16349.92 & 7 & & & & & & & 1.399 \\
\hline 6107.539 & 400 & 15 & 16368.68 & 7 & & & & & & & 1.167 \\
\hline 6106.847 & 10 & 2 & 16370.53 & 3 & & & & & & 0.257 & \\
\hline 6104.572 & 300 & 200 & 16376.63 & 2 & & & & & 0.335 & & \\
\hline 6102.595 & 300 & 50 & 16381.93 & 2 & 3 & 0.814 & 4 & 0.982 & & & \\
\hline 6101.726 & 100 & 10 & 16384.27 & 2 & 1 & 0.507 & 2 & 0.782 & & & \\
\hline 6088.031 & 400 & 50 & 16421.13 & 2 & 2 & 0.786 & 3 & 0.894 & & & \\
\hline 6085.375 & 100 & 75 & 16428.29 & 3 & 2 & 0.782 & 2 & 1.118 & & & \\
\hline 6079.220 & 200 & 25 & 16444.93 & 2 & & & & & & & 1.546 \\
\hline 6077.870 & 150 & 25 & 16448.58 & 3 & & & & & & 0.185 & \\
\hline 6055.592 & 100 & 10 & 16509.09 & 7 & & & & & 0.080 & & \\
\hline 6053.381 & 300 & 15 & 16515.12 & 2 & 1 & 0.616 & 2 & 1.009 & & & \\
\hline 6035.194 & 100 & 5 & 16564.89 & 7 & & & & & & & 0.967 \\
\hline 6032.876 & 75 & 8 & 16571.25 & 1 & & & & & 0.300 & & \\
\hline 6030.448 & $75 \mathrm{~s}$ & 5 & 16577.93 & 3 & & & & & & 0.205 & \\
\hline 6021.036 & $200 \mathrm{~s}$ & 75 & 16603.84 & 2 & 3 & 0.834 & 4 & 0.870 & & & \\
\hline 6014.062 & 75 & 5 & 16623.09 & 7 & & & & & & & 1.194 \\
\hline 6007.073 & $300 \mathrm{~s}$ & 75 & 16642.43 & 2 & 3 & 1.095 & 4 & 1.313 & & & \\
\hline 6005.174 & 200 & 15 & 16647.70 & 1 & 2 & 1.367 & 3 & 1.087 & & & \\
\hline 6000.766 & 100 & 8 & 16659.92 & 7 & & & & & & & 1.137 \\
\hline 5996.630 & 100 & 4 & 16671.42 & 2 & & & & & 0.404 & & \\
\hline 5995.221 & 100 & 4 & 16675.33 & 3 & & & & & & 0.996 & \\
\hline
\end{tabular}


TABLE 3. Zeeman effect of $\mathrm{Th} \mathrm{I}-$ continued

\begin{tabular}{|c|c|c|c|c|c|c|c|c|c|c|c|}
\hline \multirow{2}{*}{$\begin{array}{l}\text { Wavelength } \\
(\AA)\end{array}$} & \multicolumn{2}{|c|}{ Intensity } & \multirow{2}{*}{$\begin{array}{l}\text { Wavenumber } \\
\qquad\left(\mathrm{cm}^{-1}\right)\end{array}$} & \multirow{2}{*}{ Type } & \multirow{2}{*}{$J_{1}$} & \multirow{2}{*}{$g_{1}$} & \multirow{2}{*}{$J_{2}$} & \multirow{2}{*}{$g_{2}$} & \multirow{2}{*}{$\Delta g$} & \multirow{2}{*}{$p$} & \multirow{2}{*}{$n$} \\
\hline & Lamp & Spark & & & & & & & & & \\
\hline 5994.126 & $500 \mathrm{r}$ & 100 & 16678.38 & 7 & & & & & & & 1.483 \\
\hline 5991.004 & $400 \mathrm{~s}$ & 50 & 16687.07 & 1 & 1 & 1.354 & 2 & 1.006 & & & \\
\hline 5989.742 & 50 & 5 & 16690.59 & 3 & & & & & & 0.391 & \\
\hline 5982.095 & 100 & 5 & 16711.92 & 3 & & & & & & 0.729 & \\
\hline 5975.066 & 500 & 150 & 16731.58 & 3 & 2 & 1.010 & 2 & 1.323 & & & \\
\hline 5973.665 & $500 \mathrm{r}$ & 150 & 16735.50 & 1 & 1 & 1.433 & 2 & 1.257 & & & \\
\hline 5969.742 & 150 & 10 & 16746.50 & 1 & 1 & 2.403 & 2 & 1.543 & & & \\
\hline 5959.681 & 50 & 3 & 16774.77 & 3 & & & & & & 1.043 & \\
\hline 5956.263 & 100 & 8 & 16784.40 & 3 & & & & & & 0.233 & \\
\hline 5955.566 & 100 & 5 & 16786.37 & 1 & 2 & 1.709 & 3 & 1.179 & & & \\
\hline 5953.587 & 100 & 4 & 16791.94 & 7 & & & & & 0.218 & & \\
\hline 5950.148 & 15 & 2 & 16801.65 & 7 & & & & & 0.234 & & \\
\hline 5949.373 & 50 & 5 & 16803.84 & 3 & & & & & & 0.016 & \\
\hline 5944.648 & 150 & 40 & 16817.19 & 3 & 3 & 0.696 & 3 & 0.875 & & & \\
\hline 5942.359 & 40 & 3 & 16823.67 & 7 & & & & & & & 1.105 \\
\hline 5940.567 & 50 & 3 & 16828.75 & 1 & & & & & 0.120 & & \\
\hline 5938.459 & 150 & 20 & 16834.72 & 3 & & & & & & 0.869 & \\
\hline 5937.665 & 100 & 10 & 16836.97 & 3 & & & & & & 0.178 & \\
\hline 5929.934 & 100 & 20 & 16858.92 & 7 & & & & & & & 1.481 \\
\hline 5926.232 & 150 & 40 & 16869.45 & 7 & & & & & & & 0.798 \\
\hline 5916.730 & 100 & 20 & 16896.55 & 3 & & & & & & 0.735 & \\
\hline 5914.648 & $100 \mathrm{~b}$ & 10 & 16902.49 & 1 & & & & & & & 0.068 \\
\hline 5905.571 & 150 & 20 & 16928.48 & 2 & 3 & 0.932 & 4 & 1.214 & & & \\
\hline 5902.602 & 100 & 2 & 16936.98 & 3 & & & & & & 0.989 & \\
\hline 5899.846 & 150 & 5 & 16944.90 & 2 & 1 & 0.071 & 2 & 1.387 & & & \\
\hline 5899.521 & 75 & 4 & 16945.83 & 3 & & & & & & 0.936 & \\
\hline 5894.699 & 100 & 25 & 16959.70 & 3 & & & & & & 0.796 & \\
\hline 5892.434 & 75 & 5 & 16966.21 & 3 & & & & & & 0.225 & \\
\hline 5891.451 & 200 & 25 & 16969.04 & 2 & 3 & 0.859 & 4 & 0.997 & & & \\
\hline 5886.531 & 100 & 8 & 16983.23 & 7 & & & & & & & 0.972 \\
\hline 5885.702 & 200 & 50 & 16985.62 & 2 & 4 & 1.146 & 5 & 1.379 & & & \\
\hline 5874.993 & 100 & 8 & 17016.58 & 3 & & & & & & 0.084 & \\
\hline 5874.040 & 50 & 3 & 17019.34 & 3 & & & & & & 0.134 & \\
\hline 5869.854 & 100 & 5 & 17031.48 & 2 & 2 & 0.833 & 3 & 1.011 & & & \\
\hline 5868.382 & 150 & 25 & 17035.75 & 7 & & & & & & & 1.162 \\
\hline 5866.814 & 75 & 5 & 17040.30 & 7 & & & & & & & 0.932 \\
\hline 5863.720 & 100 & 8 & 17049.29 & 2 & 1 & 1.364 & 2 & 1.252 & & & \\
\hline 5854.120 & 150 & 8 & 17077.26 & 3 & 4 & 1.041 & 4 & 1.313 & & & \\
\hline 5853.474 & 100 & 5 & 17079.14 & 2 & & & & & 0.192 & & \\
\hline 5852.682 & 200 & 25 & 17081.45 & 3 & & & & & & 0.050 & \\
\hline 5843.805 & 100 & 5 & 17107.40 & 3 & & & & & & 0.232 & \\
\hline 5842.050 & 50 & 4 & 17112.54 & 3 & & & & & & 0.236 & \\
\hline 5840.639 & 100 & 5 & 17116.67 & 3 & 3 & & & & & 0.524 & \\
\hline 5832.371 & 100 & 15 & 17140.94 & 7 & & & & & & & 1.144 \\
\hline 5830.827 & 75 & 5 & 17145.47 & 3 & & & & & & 0.264 & \\
\hline 5829.876 & 50 & 5 & 17148.27 & 3 & & & & & & 0.480 & \\
\hline 5829.110 & 50 & 3 & 17150.52 & 3 & 2 & 1.117 & 2 & 1.339 & & & \\
\hline 5822.796 & 100 & 8 & 17169.12 & 3 & & & & & & 0.772 & \\
\hline 5819.607 & 50 & 3 & 17178.53 & 7 & & & & & & & 1.132 \\
\hline 5818.453 & 75 & 5 & 17181.93 & 7 & & & & & & & 1.296 \\
\hline 5812.976 & 100 & 8 & 17198.13 & 2 & & & & & & & 1.154 \\
\hline 5807.685 & 100 & 4 & 17213.79 & 3 & & & & & & 0.433 & \\
\hline 5806.201 & 40 & 2 & 17218.19 & 7 & & & & & 0.213 & & \\
\hline 5805.702 & 100 & 3 & 17219.67 & 7 & & & & & 0.186 & & \\
\hline 5804.141 & $500 \mathrm{r}$ & 50 & 17224.30 & 3 & 2 & 0.734 & 2 & 1.045 & & & \\
\hline
\end{tabular}


TABLE 3. Zeeman effect of $\mathrm{Th} \mathrm{I}-$ continued

\begin{tabular}{|c|c|c|c|c|c|c|c|c|c|c|c|}
\hline \multirow{2}{*}{$\begin{array}{l}\text { Wavelength } \\
\text { (§) }\end{array}$} & \multicolumn{2}{|c|}{ Intensity } & \multirow{2}{*}{$\begin{array}{l}\text { Wa venumber } \\
\quad\left(\mathrm{cm}^{-1}\right)\end{array}$} & \multirow{2}{*}{ Type } & \multirow{2}{*}{$J_{1}$} & \multirow{2}{*}{$g_{1}$} & \multirow{2}{*}{$J_{2}$} & \multirow{2}{*}{$g_{2}$} & \multirow{2}{*}{$\Delta g$} & \multirow{2}{*}{$p$} & \multirow{2}{*}{$n$} \\
\hline & Lamp & Spark & & & & & & & & & \\
\hline 5800.830 & 300 & 20 & 17234.14 & 3 & 2 & 1.172 & 2 & 1.254 & & & \\
\hline 5797.319 & 75 & 3 & 17244.57 & 3 & & & & & & 0.282 & \\
\hline 5796.070 & 200 & 15 & 17248.29 & 3 & 4 & 1.120 & 4 & 1.307 & & & \\
\hline 5792.431 & 150 & 8 & 17259.12 & 7 & & & & & & & 1.573 \\
\hline 5791.704 & 50 & 2 & 17261.29 & 7 & & & & & 0.200 & & \\
\hline 5789.644 & 200 & 15 & 17267.43 & 3 & 3 & 1.139 & 3 & 1.246 & & & \\
\hline 5782.307 & $40 \mathrm{~b}$ & 4 & 17289.34 & 2 & 2 & 0.927 & 3 & 1.019 & & & \\
\hline 5779.833 & 50 & 2 & 17296.74 & 7 & & & & & 0.298 & & \\
\hline 5777.398 & 100 & 8 & 17304.03 & 3 & & & & & & 0.118 & \\
\hline 5776.164 & 25 & 2 & 17307.73 & 7 & & & & & 0.128 & & \\
\hline 5773.946 & 150 & 10 & 17314.38 & 3 & 1 & 0.070 & 1 & 0.615 & & & \\
\hline 5763.529 & 200 & 10 & 17345.67 & 3 & 3 & 1.085 & 3 & 1.180 & & & \\
\hline 5762.796 & 100 & 10 & 17347.88 & 3 & & & & & & 0.462 & \\
\hline 5760.550 & $600 \mathrm{r}$ & 150 & 17354.64 & 2 & 1 & 0.504 & 2 & 0.734 & & & \\
\hline 5753.026 & 200 & 25 & 17377.34 & 2 & 3 & 1.016 & 4 & 1.208 & & & \\
\hline 5736.031 & 150 & 8 & 17428.82 & 2 & 3 & 1.062 & 4 & 1.464 & & & \\
\hline 5729.992 & $200 \mathrm{~d}$ & 10 & 17447.19 & 3 & & & & & & 0.378 & \\
\hline 5727.711 & 40 & 3 & 17454.14 & 3 & & & & & & 0.511 & \\
\hline 5727.021 & 50 & 3 & 17456.24 & 7 & & & & & & & 1.181 \\
\hline 5725.390 & $400 \mathrm{r}$ & 75 & 17461.21 & 2 & 4 & 1.150 & 5 & 1.368 & & & \\
\hline 5724.463 & 75 & 4 & 17464.04 & 3 & & & & & & 0.051 & \\
\hline 5721.425 & 50 & 4 & 17473.32 & 3 & & & & & & 0.053 & \\
\hline 5719.625 & 200 & 50 & 17478.81 & 3 & 3 & 1.082 & 3 & 1.251 & & & \\
\hline 5711.998 & 75 & 5 & 17502.15 & 7 & & & & & 0.080 & & \\
\hline 5708.679 & 50 & 3 & 17512.33 & 7 & & & & & & & 1.142 \\
\hline 5705.637 & 50 & 4 & 17521.67 & 3 & & & & & & 0.286 & \\
\hline 5698.550 & 40 & 4 & 17543.46 & 3 & & & & & & 0.346 & \\
\hline 5698.292 & 100 & 5 & 17544.25 & 3 & 1 & 0.724 & 1 & 1.104 & & & \\
\hline 5696.391 & 100 & 8 & 17550.10 & 1 & 3 & 1.168 & 4 & 0.921 & & & \\
\hline 5685.192 & 100 & 8 & 17584.68 & 3 & 4 & 1.060 & 4 & 1.308 & & & \\
\hline 5679.004 & 75 & 5 & 17603.84 & 7 & & & & & & & 0.903 \\
\hline 5677.051 & $200 \mathrm{~b}$ & 8 & 17609.89 & 3 & & & & & & 0.203 & \\
\hline 5673.835 & 50 & 5 & 17619.87 & 2 & & & & & & & 1.436 \\
\hline 5667.129 & 150 & 15 & 17640.72 & 2 & 2 & 0.871 & 3 & 1.016 & & & \\
\hline 5665.181 & 200 & 50 & 17646.79 & 3 & 2 & 0.775 & 2 & 1.118 & & & \\
\hline 5664.621 & 100 & 15 & 17648.53 & 7 & & & & & 0.057 & & \\
\hline 5660.662 & 25 & 3 & 17660.88 & 7 & & & & & 0.590 & & \\
\hline 5657.926 & 200 & 25 & 17669.41 & 2 & & & & & & & 1.632 \\
\hline 5648.990 & 150 & 10 & 17697.37 & 2 & 3 & 1.083 & 4 & 1.233 & & & \\
\hline 5646.455 & 100 & 10 & 17705.31 & 2 & 2 & 1.041 & 3 & 1.385 & & & \\
\hline 5645.529 & 50 & 8 & 17708.22 & 7 & & & & & & & 1.235 \\
\hline 5641.735 & 75 & 8 & 17720.13 & 2 & & & & & 0.125 & & \\
\hline 5641.562 & 50 & 5 & 17720.67 & 2 & & & & & 0.102 & & \\
\hline 5635.889 & 100 & 15 & 17738.50 & 7 & & & & & & & 1.233 \\
\hline 5633.297 & 100 & 8 & 17746.67 & 7 & & & & & & & 0.907 \\
\hline 5630.297 & 100 & 10 & 17756.12 & 3 & & & & & & 0.754 & \\
\hline 5629.312 & 50 & 3 & 17759.23 & 7 & & & & & & & 0.855 \\
\hline 5624.911 & 50 & 3 & 17773.12 & 2 & & & & & 0.110 & & \\
\hline 5620.013 & $15 \mathrm{~b}$ & 5 & 17788.62 & 7 & & & & & 0.080 & & \\
\hline 5619.976 & $50 \mathrm{~b}$ & 8 & 17788.73 & 3 & & & & & & 0.962 & \\
\hline 5615.320 & $400 \mathrm{r}$ & 15 & 17803.48 & 3 & 1 & 1.493 & 1 & 1.559 & & & \\
\hline 5612.619 & 75 & 3 & 17812.05 & 7 & & & & & & & 1.126 \\
\hline 5612.068 & 150 & 20 & 17813.80 & 3 & 4 & 0.986 & 4 & 1.128 & & & \\
\hline 5610.685 & 200 & 20 & 17818.19 & 1 & 1 & 2.396 & 2 & 1.680 & & & \\
\hline 5609.585 & 200 & 10 & 17821.68 & 2 & & & & & 0.660 & & \\
\hline
\end{tabular}


TABLE 3. Zeeman effect of Th $\mathrm{I}$-continued

\begin{tabular}{|c|c|c|c|c|c|c|c|c|c|c|c|}
\hline \multirow{2}{*}{$\begin{array}{c}\text { Wavelength } \\
(\AA)\end{array}$} & \multicolumn{2}{|c|}{ Intensity } & \multirow{2}{*}{$\begin{array}{l}\text { Wavenumber } \\
\quad\left(\mathrm{cm}^{-1}\right)\end{array}$} & \multirow{2}{*}{ Type } & \multirow{2}{*}{$J_{1}$} & \multirow{2}{*}{$g_{1}$} & \multirow{2}{*}{$J_{2}$} & \multirow{2}{*}{$g_{2}$} & \multirow{2}{*}{$\Delta g$} & \multirow{2}{*}{$p$} & \multirow{2}{*}{$n$} \\
\hline & Lamp & Spark & & & & & & & & & \\
\hline 5606.390 & 150 & 10 & 17831.84 & 3 & 3 & 0.878 & 3 & 1.192 & & & \\
\hline 5605.297 & $100 \mathrm{~b}$ & 8 & 17835.32 & 3 & & & & & & 0.512 & \\
\hline 5602.866 & 50 & 5 & 17843.05 & 2 & 2 & 0.789 & 3 & 0.844 & & & \\
\hline 5601.604 & 200 & 8 & 17847.07 & 3 & 2 & 0.734 & 2 & 1.158 & & & \\
\hline 5595.064 & 300 & 25 & 17867.94 & 2 & 1 & 0.374 & 2 & 0.775 & & & \\
\hline 5587.027 & $500 \mathrm{r}$ & 50 & 17893.64 & 2 & & & & & & & 1.425 \\
\hline 5582.367 & 75 & 4 & 17908.58 & 2 & & & & & 0.546 & & \\
\hline 5580.756 & 100 & 10 & 17913.75 & 3 & & & & & & 0.587 & \\
\hline 5580.395 & 50 & 10 & 17914.90 & 3 & & & & & & 0.054 & \\
\hline 5579.359 & 300 & 40 & 17918.23 & 3 & 1 & 0.065 & 1 & 0.842 & & & \\
\hline 5576.205 & 100 & 10 & 17928.36 & 2 & 4 & 0.992 & 5 & 1.098 & & & \\
\hline 5573.355 & 400 & 20 & 17937.54 & 3 & & & & & & 0.198 & \\
\hline 5572.466 & 200 & 8 & 17940.40 & 3 & 3 & 1.094 & 3 & 1.248 & & & \\
\hline 5570.928 & 50 & 2 & 17945.35 & 3 & 2 & 1.006 & 2 & 1.516 & & & \\
\hline 5568.000 & $300 \mathrm{~b}$ & 150 & 17954.79 & 3 & & & & & & 0.081 & \\
\hline 5559.894 & 150 & 8 & 17980.96 & 1 & 1 & 1.570 & 2 & 1.252 & & & \\
\hline 5558.342 & $400 \mathrm{~b}$ & 50 & 17985.98 & 2 & & & & & & & 1.227 \\
\hline 5557.045 & 300 & 15 & 17990.18 & 3 & 4 & 1.132 & 4 & 1.306 & & & \\
\hline 5555.534 & 75 & 4 & 17995.07 & 7 & & & & & 0.128 & & \\
\hline 5554.502 & 20 & 4 & 17998.42 & 7 & & & & & & & 1.276 \\
\hline 5552.624 & 75 & 4 & 18004.50 & 1 & & & & & & & 0.791 \\
\hline 5551.193 & 40 & 2 & 18009.14 & 3 & & & & & & 0.092 & \\
\hline 5548.176 & $400 \mathrm{r}$ & $40 \mathrm{~b}$ & 18018.94 & 3 & 2 & 1.012 & 2 & 1.255 & & & \\
\hline 5546.122 & 75 & 25 & 18025.61 & 3 & & & & & & 0.131 & \\
\hline 5542.890 & 300 & 10 & 18036.12 & 2 & 3 & 0.875 & 4 & 1.040 & & & \\
\hline 5528.226 & $50 \mathrm{~b}$ & 5 & 18083.96 & 3 & 1 & 0.721 & 1 & 1.343 & & & \\
\hline 5527.295 & 200 & 8 & 18087.01 & 7 & & & & & & & 1.217 \\
\hline 5519.232 & 100 & 2 & 18113.43 & 7 & & & & & & & 1.270 \\
\hline 5518.988 & $200 \mathrm{~b}$ & 50 & 18114.23 & 2 & 4 & 0.883 & 5 & 1.175 & & & \\
\hline 5514.930 & $75 b$ & 3 & 18127.56 & 7 & & & & & & & 1.274 \\
\hline 5514.873 & $200 \mathrm{~b}$ & 10 & 18127.74 & 3 & & & & & & 0.354 & \\
\hline 5509.993 & 400 & 20 & 18143.80 & 2 & 4 & 1.263 & 5 & 1.373 & & & \\
\hline 5508.557 & 100 & 5 & 18148.53 & 3 & & & & & & 0.177 & \\
\hline 5507.542 & 1001 & 2 & 18151.88 & 3 & & & & & & 0.752 & \\
\hline 5501.281 & 100 & 10 & 18172.53 & 2 & 1 & 0.627 & 2 & 0.977 & & & \\
\hline 5496.138 & 200 & 10 & 18189.54 & 2 & 1 & 0.068 & 2 & 1.106 & & & \\
\hline 5494.330 & 75 & 10 & 18195.53 & 2 & 3 & 1.170 & 4 & 1.314 & & & \\
\hline 5492.642 & 100 & 10 & 18201.12 & 2 & 2 & 1.177 & 3 & 1.263 & & & \\
\hline 5492.416 & 50 & 10 & 18201.87 & 7 & & & & & 0.270 & & \\
\hline 5489.086 & 100 & 5 & 18212.91 & 7 & & & & & 0.544 & & \\
\hline 5482.498 & 100 & 8 & 18234.79 & 7 & & & & & & & 1.157 \\
\hline 5479.072 & 75 & 75 & 18246.20 & 1 & & & & & 0.100 & & 0.983 \\
\hline 5478.742 & 50 & 3 & 18247.29 & 7 & & & & & 0.222 & & \\
\hline 5477.550 & 40 & 2 & 18251.26 & 3 & & & & & & 0.084 & \\
\hline 5474.733 & 50 & 3 & 18260.66 & 3 & & & & & & 0.467 & \\
\hline 5470.759 & 150 & 10 & 18273.94 & 7 & & & & & & & 1.064 \\
\hline 5470.145 & 50 & 8 & 18275.97 & 3 & & & & & & 0.192 & \\
\hline 5464.205 & $200 w$ & 8 & 18295.84 & 3 & 3 & 1.082 & 3 & 1.310 & & & \\
\hline 5463.771 & 50 & 4 & 18297.29 & 7 & & & & & 0.109 & & \\
\hline 5457.289 & 75 & 3 & 18319.02 & 7 & & & & & 0.110 & & \\
\hline 5455.149 & 100 & 8 & 18326.21 & 3 & & & & & & 0.620 & \\
\hline 5452.219 & 300 & 40 & 18336.06 & 2 & 4 & 1.222 & 5 & 1.364 & & & \\
\hline 5441.213 & 100 & 8 & 18373.15 & 7 & & & & & & & 1.136 \\
\hline 5440.600 & 75 & 2 & 18375.22 & 7 & & & & & 0.212 & & \\
\hline 5439.850 & 75 & 8 & 18377.75 & 3 & & & & & & 0.522 & \\
\hline
\end{tabular}


TABLE 3. Zeeman effect of Th $\mathrm{I}$-continued

\begin{tabular}{|c|c|c|c|c|c|c|c|c|c|c|c|}
\hline \multirow{2}{*}{$\begin{array}{l}\text { Wavelength } \\
\text { (̊) }\end{array}$} & \multicolumn{2}{|c|}{ Intensity } & \multirow{2}{*}{$\begin{array}{l}\text { Wavenumber } \\
\qquad\left(\mathrm{cm}^{-1}\right)\end{array}$} & \multirow{2}{*}{ Type } & \multirow{2}{*}{$J_{1}$} & \multirow{2}{*}{$g_{1}$} & \multirow{2}{*}{$J_{2}$} & \multirow{2}{*}{$g_{2}$} & \multirow{2}{*}{$\Delta g$} & \multirow{2}{*}{$p$} & \multirow{2}{*}{$n$} \\
\hline & Lamp & Spark & & & & & & & & & \\
\hline 5431.112 & 200 & 40 & 18407.32 & 2 & 2 & 1.014 & 3 & 1.140 & & & \\
\hline 5429.105 & 100 & 5 & 18414.12 & 3 & & & & & & 0.305 & \\
\hline 5424.008 & 300 & 40 & 18431.43 & 7 & & & & & & & 0.981 \\
\hline 5419.136 & 100 & 8 & 18448.00 & 3 & & & & & & 0.371 & \\
\hline 5417.486 & $400 r$ & 40 & 18453.62 & 1 & 2 & 1.257 & 3 & 1.101 & & & \\
\hline 5415.528 & $200 \mathrm{~b}$ & 2 & 18460.29 & 3 & 4 & 0.860 & 4 & 1.030 & & & \\
\hline 5410.769 & 400 & 15 & 18476.52 & 2 & 1 & 0.770 & 2 & 1.012 & & & \\
\hline 5407.652 & $400 \mathrm{~b}$ & $40 \mathrm{~b}$ & 18487.17 & 3 & 5 & 1.035 & 5 & 1.174 & & & \\
\hline 5403.200 & 200 & 15 & 18502.41 & 2 & 4 & 0.884 & 5 & 1.109 & & & \\
\hline 5400.145 & 150 & 5 & 18512.87 & 2 & & & & & 0.035 & & \\
\hline 5399.175 & 200 & 10 & 18516.20 & 3 & & & & & & 1.208 & \\
\hline 5398.920 & 400 & 40 & 18517.08 & 2 & & & & & 0.184 & & 1.622 \\
\hline 5398.704 & 150 & 4 & 18517.82 & 2 & & & & & 0.215 & & \\
\hline 5398.205 & 150 & 4 & 18519.53 & 7 & & & & & 0.110 & & \\
\hline 5393.972 & 150 & 8 & 18534.06 & 3 & & & & & & 0.881 & \\
\hline 5388.057 & 300 & 20 & 18554.41 & 1 & & & & & & & 0.907 \\
\hline 5387.812 & 100 & 4 & 18555.25 & 7 & & & & & & & 1.195 \\
\hline 5387.152 & 25 & 2 & 18557.52 & 7 & & & & & 0.213 & & \\
\hline 5386.611 & 500 & 20 & 18559.39 & 2 & 3 & 1.082 & 4 & 1.216 & & & \\
\hline 5384.031 & 75 & 15 & 18568.28 & 1 & & & & & & & 0.452 \\
\hline 5379.112 & 300 & 20 & 18585.26 & 2 & 4 & 0.877 & 5 & 1.049 & & & \\
\hline 5378.837 & 400 & 40 & 18586.21 & 3 & & & & & & 0.505 & \\
\hline 5376.778 & 200 & 50 & 18593.33 & 3 & & & & & & 0.134 & \\
\hline 5374.823 & 150 & 15 & 18600.09 & 7 & & & & & & & 1.479 \\
\hline 5372.703 & 200 & 25 & 18607.43 & 2 & & & & & & & 1.085 \\
\hline 5369.284 & 200 & 8 & 18619.28 & 2 & 1 & 0.072 & 2 & 1.245 & & & \\
\hline 5361.156 & 100 & 3 & 18647.51 & 7 & & & & & 0.238 & & \\
\hline 5360.150 & 300 & 5 & 18651.01 & 1 & 2 & 1.249 & 3 & 1.016 & & & \\
\hline 5359.827 & 100 & 4 & 18652.13 & 7 & & & & & & & 1.032 \\
\hline 5355.638 & 100 & 3 & 18666.72 & 2 & & & & & 0.260 & & \\
\hline 5351.839 & 150 & 8 & 18679.97 & 3 & & & & & & 0.928 & \\
\hline 5351.126 & 200 & 25 & 18682.46 & 2 & 3 & 0.590 & 4 & 0.905 & & & \\
\hline 5349.003 & 100 & 3 & 18689.87 & 3 & & & & & & 0.049 & \\
\hline 5347.973 & 150 & 5 & 18693.48 & 3 & & & & & & 0.160 & \\
\hline 5343.581 & $500 \mathrm{r}$ & 40 & 18708.84 & 1 & 1 & 1.526 & 2 & 1.252 & & & \\
\hline 5340.501 & 200 & 10 & 18719.63 & 7 & & & & & & & 1.181 \\
\hline 5337.018 & $200 \mathrm{~b}$ & 10 & 18731.85 & 3 & & & & & & 0.580 & \\
\hline 5330.082 & $200 \mathrm{~b}$ & 5 & 18756.22 & 1 & 2 & 1.186 & 3 & 0.954 & & & \\
\hline 5326.976 & $500 \mathrm{r}$ & 40 & 18767.15 & 2 & 3 & 0.903 & 4 & 1.068 & & & \\
\hline 5326.278 & $200 \mathrm{~b}$ & 8 & 18769.61 & 3 & & & & & & 0.171 & \\
\hline 5320.767 & 75 & 20 & 18789.06 & 3 & & & & & & 0.161 & \\
\hline 5320.099 & 50 & 2 & 18791.42 & 7 & & & & & & & 1.111 \\
\hline 5318.110 & 100 & 3 & 18798.44 & 7 & & & & & 0.430 & & \\
\hline 5317.494 & 200 & 4 & 18800.62 & 2 & & & & & 0.400 & & \\
\hline 5315.230 & 150 & 3 & 18808.63 & 7 & & & & & 0.576 & & \\
\hline 5312.904 & 300 & 15 & 18816.86 & 1 & 2 & 1.193 & 3 & 1.006 & & & \\
\hline 5312.643 & $150 \mathrm{~b}$ & 15 & 18817.79 & 7 & & & & & 0.086 & & \\
\hline 5312.532 & 400 & 40 & 18818.18 & 2 & 1 & 0.077 & 2 & 1.257 & & & \\
\hline 5312.001 & $400 \mathrm{r}$ & 25 & 18820.06 & 3 & 2 & 1.259 & 2 & 1.386 & & & \\
\hline 5311.870 & $75 b$ & 2 & 18820.53 & 3 & & & & & & 0.670 & \\
\hline 5309.616 & 300 & 5 & 18828.52 & 7 & & & & & 0.687 & & \\
\hline 5306.987 & 100 & 4 & 18837.84 & 3 & & & & & & 0.453 & \\
\hline 5303.483 & 100 & 3 & 18850.29 & 7 & & & & & 0.180 & & \\
\hline 5303.334 & 50 & 2 & 18850.82 & 3 & & & & & & 0.140 & \\
\hline 5300.524 & 300 & 5 & 18860.81 & 2 & & & & & 0.215 & 0.429 & \\
\hline
\end{tabular}


TABLE 3. Zeeman effect of Th $\mathrm{I}$-continued

\begin{tabular}{|c|c|c|c|c|c|c|c|c|c|c|c|}
\hline \multirow{2}{*}{$\begin{array}{l}\text { Wavelength } \\
\text { (§) }\end{array}$} & \multicolumn{2}{|c|}{ Intensity } & \multirow{2}{*}{$\begin{array}{l}\text { Wavenumber } \\
\left(\mathrm{cm}^{-1}\right)\end{array}$} & \multirow{2}{*}{ Type } & \multirow{2}{*}{$J_{1}$} & \multirow{2}{*}{$g_{1}$} & \multirow{2}{*}{$J_{2}$} & \multirow{2}{*}{$g_{2}$} & \multirow{2}{*}{$\Delta g$} & \multirow{2}{*}{$p$} & \multirow{2}{*}{$n$} \\
\hline & Lamp & Spark & & & & & & & & & \\
\hline 5298.284 & 200 & 4 & 18868.79 & 2 & 2 & 0.537 & 3 & 1.089 & & & \\
\hline 5297.746 & $300 \mathrm{~b}$ & 15 & 18870.70 & & & & & & & & 1.587 \\
\hline 5296.279 & 300 & 5 & 18875.93 & 2 & 4 & 1.192 & 5 & 1.372 & & & \\
\hline 5295.674 & $50 \mathrm{~b}$ & $4 b$ & 18878.08 & 7 & & & & & & & 1.172 \\
\hline 5295.089 & 75 & 1 & 18880.17 & 7 & & & & & 0.270 & & \\
\hline 5294.397 & 200 & 3 & 18882.64 & 1 & 3 & 1.253 & 4 & 1.073 & & & \\
\hline 5291.818 & 200 & 5 & 18891.84 & 1 & 2 & 1.026 & 3 & 0.860 & & & \\
\hline 5281.070 & 300 & 2 & 18930.29 & 2 & 2 & 0.728 & 3 & 0.969 & & & \\
\hline 5280.346 & 75 & 2 & 18932.89 & 3 & & & & & & 0.634 & \\
\hline 5277.147 & 100 & 1 & 18944.36 & 3 & & & & & & 0.324 & \\
\hline 5274.391 & 100 & 3 & 18954.26 & 7 & & & & & & & 0.830 \\
\hline 5274.121 & 300 & 5 & 18955.23 & 3 & & & & & & 0.130 & \\
\hline 5273.134 & $100 \mathrm{~b}$ & 10 & 18958.78 & 7 & & & & & 0.520 & & \\
\hline 5269.794 & 100 & 4 & 18970.80 & 3 & & & & & & 0.067 & \\
\hline 5262.621 & $100 \mathrm{~b}$ & 4 & 18996.65 & 3 & & & & & & 0.620 & \\
\hline 5261.474 & $75 b$ & 3 & 19000.79 & 7 & & & & & & 0.106 & \\
\hline 5258.361 & $400 \mathrm{r}$ & 25 & 19012.41 & 3 & 1 & 0.615 & 1 & 1.481 & & & \\
\hline 5258.212 & $150 \mathrm{~b}$ & 3 & 19012.58 & 7 & & & & & & & 2.402 \\
\hline 5254.262 & 3001 & 8 & 19026.87 & 7 & & & & & 0.044 & & 0.922 \\
\hline 5238.812 & $75 \mathrm{~h}$ & 10 & 19082.98 & 3 & 2 & 1.047 & 2 & 1.193 & & & \\
\hline 5234.107 & 150 & 1 & 19100.14 & 7 & & & & & 0.074 & & \\
\hline 5228.996 & 100 & 1 & 19118.81 & 2 & & & & & & & 1.460 \\
\hline 5228.227 & 200 & 3 & 19121.62 & 3 & & & & & & 0.198 & \\
\hline 5220.928 & 100 & 1 & 19148.35 & 3 & & & & & & 0.214 & \\
\hline 5213.350 & 400 & 5 & 19176.18 & 3 & 4 & 0.863 & 4 & 1.036 & & & \\
\hline 5209.724 & 500 & 3 & 19189.53 & 2 & 1 & 0.614 & 2 & 1.250 & & & \\
\hline 5207.803 & 200 & 2 & 19196.61 & 7 & & & & & & & 1.078 \\
\hline 5206.800 & 75 & 3 & 19200.31 & 3 & & & & & & 0.158 & \\
\hline 5205.154 & 200 & 2 & 19206.38 & 7 & & & & & & & 1.029 \\
\hline 5203.850 & 500 & 25 & 19211.19 & 7 & & & & & 0.190 & & \\
\hline 5202.007 & 75 & 3 & 19218.00 & 2 & 1 & 0.724 & 2 & 1.327 & & & \\
\hline 5199.323 & $300 \mathrm{~b}$ & 3 & 19227.92 & 1 & & & & & 0.100 & & \\
\hline 5198.802 & 500 & $100 \mathrm{~d}$ & 19229.85 & 2 & 3 & 0.869 & 4 & 1.101 & & & \\
\hline 5197.236 & 200 & 5 & 19235.64 & 7 & & & & & 0.270 & & \\
\hline 5195.815 & 500 & 8 & 19240.90 & 3 & 4 & 1.212 & 4 & 1.380 & & & \\
\hline 5194.456 & 400 & 12 & 19245.93 & 3 & & & & & & 1.223 & \\
\hline 5184.452 & 100 & 3 & 19283.07 & 3 & & & & & & 0.183 & \\
\hline 5177.621 & 200 & 3 & 19308.51 & 3 & & & & & & 0.578 & \\
\hline 5176.961 & 500 & 50 & 19310.94 & 3 & & & & & & 0.354 & \\
\hline 5175.323 & 200 & 2 & 19317.08 & 3 & & & & & & 0.701 & \\
\hline 5174.367 & 200 & 3 & 19320.65 & 3 & & & & & & 0.067 & \\
\hline 5174.242 & 200 & 75 & 19321.12 & 2 & & & & & & & 1.003 \\
\hline 5173.671 & 400 & 2 & 19323.25 & 3 & & & & & & 0.044 & \\
\hline 5172.479 & 75 & 3 & 19327.71 & 3 & & & & & & 1.439 & \\
\hline 5161.540 & 400 & 8 & 19368.67 & 2 & 2 & 0.797 & 3 & 0.931 & & & \\
\hline 5160.718 & $500 \mathrm{~b}$ & 150 & 19371.75 & 3 & & & & & & 0.176 & \\
\hline 5154.243 & $500 \mathrm{r}$ & 40 & 19396.09 & 2 & 4 & 0.859 & 5 & 1.116 & & & \\
\hline 5149.210 & 300 & 3 & 19415.05 & 7 & & & & & 0.170 & & \\
\hline 5146.059 & 300 & 10 & 19426.93 & 7 & & & & & 0.310 & & \\
\hline 5143.916 & $300 \mathrm{~b}$ & 15 & 19435.02 & 3 & & & & & & 0.185 & \\
\hline 5140.771 & $200 \mathrm{~b}$ & 5 & 19446.92 & 1 & & & & & 0.550 & & \\
\hline 5130.235 & 150 & 2 & 19486.86 & 7 & & & & & 0.324 & & \\
\hline 5125.950 & 300 & 3 & 19503.14 & 2 & 2 & 0.734 & 3 & 1.085 & & & \\
\hline 5115.045 & $1000 \mathrm{r}$ & 40 & 19544.72 & 7 & & & & & & & 1.481 \\
\hline 5098.933 & 150 & 2 & 19606.48 & 7 & & & & & & & 1.093 \\
\hline
\end{tabular}


TABLE 3. Zeeman effect of Th $\mathrm{I}$-continued

\begin{tabular}{|c|c|c|c|c|c|c|c|c|c|c|c|}
\hline \multirow{2}{*}{$\begin{array}{l}\text { Wavelength } \\
(\AA)\end{array}$} & \multicolumn{2}{|c|}{ Intensity } & \multirow{2}{*}{$\begin{array}{c}\text { Wavenumber } \\
\left(\mathrm{cm}^{-1}\right)\end{array}$} & \multirow{2}{*}{ Type } & \multirow{2}{*}{$J_{1}$} & \multirow{2}{*}{$g_{1}$} & \multirow{2}{*}{$J_{2}$} & \multirow{2}{*}{$g_{2}$} & \multirow{2}{*}{$\Delta g$} & \multirow{2}{*}{$p$} & \multirow{2}{*}{$n$} \\
\hline & Lamp & Spark & & & & & & & & & \\
\hline 5096.484 & 800 & 5 & 19615.90 & 3 & 1 & 0.842 & 1 & 1.480 & & & \\
\hline 5090.052 & 400 & 5 & 19640.69 & 3 & & & & & & 0.125 & \\
\hline 5082.625 & 100 & 8 & 19669.39 & 3 & & & & & & 0.338 & \\
\hline 5067.974 & $1000 \mathrm{r}$ & 75 & 19726.25 & 3 & & & & & & 0.208 & \\
\hline 5066.134 & 300 & 10 & 19733.42 & 7 & & & & & 0.166 & & \\
\hline 5065.190 & 300 & 5 & 19737.10 & 2 & 2 & 0.767 & 3 & 1.027 & & & \\
\hline 5063.514 & 2001 & 501 & 19743.63 & 2 & & & & & 1.090 & & \\
\hline 5062.932 & 300 & 5 & 19745.90 & 1 & 3 & 1.136 & 4 & 0.985 & & & \\
\hline 5059.861 & $400 \mathrm{~b}$ & $75 \mathrm{~h}$ & 19757.88 & 3 & & & & & & 0.374 & \\
\hline 5057.982 & 300 & 2 & 19765.22 & 7 & & & & & & & 1.479 \\
\hline 5051.885 & 500 & 5 & 19789.08 & 2 & 3 & 1.182 & 4 & 1.315 & & & \\
\hline 5050.780 & 300 & 5 & 19793.40 & 2 & 1 & 0.844 & 2 & 1.256 & & & \\
\hline 5044.715 & 100 & 8 & 19817.20 & 1 & 1 & 1.561 & 2 & 0.731 & & & \\
\hline 5043.520 & 75 & 151 & 19821.89 & 3 & & & & & & 1.432 & \\
\hline 5041.126 & 200 & 5 & 19831.31 & 1 & & & & & & & 0.967 \\
\hline 5039.231 & $300 \mathrm{r}$ & 81 & 19838.77 & 2 & & & & & & & 1.536 \\
\hline 5038.306 & 75 & 3 & 19842.41 & 3 & & & & & & 0.336 & \\
\hline 5034.297 & 100 & 2 & 19858.21 & 3 & & & & & & 1.178 & \\
\hline 5029.893 & 200 & 3 & 19875.60 & 3 & 1 & 0.768 & 1 & 1.480 & & & \\
\hline 5029.013 & 75 & 2 & 19879.07 & 3 & & & & & & 0.247 & \\
\hline 5028.656 & $300 \mathrm{r}$ & 15 & 19880.48 & 3 & 4 & 1.190 & 4 & 1.310 & & & \\
\hline 5023.709 & 50 & 1 & 19900.06 & 3 & & & & & & 0.233 & \\
\hline 5023.486 & 50 & 1 & 19900.95 & 3 & & & & & & 0.324 & \\
\hline 5022.174 & 40 & 2 & 19906.14 & 3 & & & & & & 0.798 & \\
\hline 5018.059 & 150 & 5 & 19922.47 & 3 & & & & & & 0.376 & \\
\hline 5017.510 & 100 & 15 & 19924.65 & 2 & 1 & 0.708 & 2 & 1.012 & & & \\
\hline 5011.478 & 75 & 4 & 19948.63 & 7 & & & & & & & 1.126 \\
\hline 5010.417 & 50 & 4 & 19952.85 & 7 & & & & & 0.160 & & \\
\hline 5009.938 & $50 \mathrm{~b}$ & & 19954.76 & 3 & & & & & & 0.291 & \\
\hline 5004.001 & 50 & 3 & 19978.44 & 3 & & & & & & 0.233 & \\
\hline 5002.097 & $500 \mathrm{r}$ & 4 & 19986.05 & 3 & & & & & & 0.116 & \\
\hline 4992.638 & 20 & 5 & 20023.91 & 3 & & & & & & 0.178 & \\
\hline 4989.309 & 150 & 10 & 20037.26 & 1 & & & & & & & 0.890 \\
\hline 4985.948 & 50 & 3 & 20050.77 & 7 & & & & & & & 0.926 \\
\hline 4985.373 & 300 & 10 & 20053.09 & 2 & 1 & 0.770 & 2 & 1.254 & & & \\
\hline 4982.488 & 150 & $25 \mathrm{~h}$ & 20064.70 & 3 & & & & & & 0.292 & \\
\hline 4980.186 & 150 & 10 & 20073.97 & 3 & & & & & & 0.240 & \\
\hline 4972.754 & 20 & 2 & 20103.97 & 3 & & & & & & 0.179 & \\
\hline 4972.057 & 40 & 1 & 20106.79 & 7 & & & & & & & 0.771 \\
\hline 4965.731 & 200 & 10 & 20132.40 & 3 & & & & & & 0.808 & \\
\hline 4961.724 & 100 & 10 & 20148.66 & 3 & & & & & & 0.095 & \\
\hline 4961.379 & 10 & 2 & 20150.06 & 3 & & & & & & 0.162 & \\
\hline 4960.421 & $40 \mathrm{~b}$ & 10 & 20153.95 & 7 & & & & & & & 1.083 \\
\hline 4958.093 & 50 & 2 & 20163.42 & 2 & & & & & 0.360 & & \\
\hline 4957.293 & 75 & 5 & 20166.67 & 3 & & & & & & 0.890 & \\
\hline 4946.663 & 100 & 100 & 20210.02 & 7 & & & & & & & 1.297 \\
\hline 4945.458 & $200 \mathrm{r}$ & 10 & 20214.93 & 2 & 2 & 0.741 & $\dot{3}$ & 1.182 & & & \\
\hline 4943.064 & $200 \mathrm{~b}$ & 10 & 20224.72 & 1 & 2 & 1.321 & 3 & 1.084 & & & \\
\hline 4939.642 & $300 \mathrm{r}$ & 15 & 20238.73 & 2 & 4 & 0.865 & 5 & 1.074 & & & \\
\hline 4939.270 & 75 & 2 & 20240.26 & 3 & & & & & & 0.252 & \\
\hline 4938.597 & 15 & 1 & 20243.02 & 3 & & & & & & 0.129 & \\
\hline 4937.829 & 300 & 8 & 20246.16 & 3 & 1 & 0.072 & 1 & 1.570 & & & \\
\hline 4936.775 & 150 & 100 & 20250.49 & 1 & & & & & 0.188 & & \\
\hline 4933.003 & 20 & 1 & 20265.97 & 7 & & & & & 0.186 & & \\
\hline 4928.683 & 50 & 2 & 20283.73 & 7 & & & & & & & 1.150 \\
\hline
\end{tabular}


TABLE 3. Zeeman effect of Th $\mathrm{I}$-continued

\begin{tabular}{|c|c|c|c|c|c|c|c|c|c|c|c|}
\hline \multirow{2}{*}{$\begin{array}{c}\text { Wavelength } \\
(\AA)\end{array}$} & \multicolumn{2}{|c|}{ Intensity } & \multirow{2}{*}{$\begin{array}{l}\text { Wavenumber } \\
\left(\mathrm{cm}^{-1}\right)\end{array}$} & \multirow{2}{*}{ Type } & \multirow{2}{*}{$J_{1}$} & \multirow{2}{*}{$g_{1}$} & \multirow{2}{*}{$J_{2}$} & \multirow{2}{*}{$g_{2}$} & \multirow{2}{*}{$\Delta g$} & \multirow{2}{*}{$p$} & \multirow{2}{*}{$n$} \\
\hline & Lamp & Spark & & & & & & & & & \\
\hline 4927.781 & 200 & 5 & 20287.45 & 3 & 2 & 0.779 & 2 & 0.910 & & & \\
\hline 4927.299 & 50 & 1 & 20289.43 & 3 & & & & & & 0.172 & \\
\hline 4925.951 & 100 & 8 & 20294.98 & 7 & & & & & & & 1.017 \\
\hline 4924.246 & $75 b$ & 2 & 20302.01 & 3 & & & & & & 2.026 & \\
\hline 4920.053 & $75 b$ & $25 \mathrm{~s}$ & 20319.31 & 2 & & & & & 0.180 & & \\
\hline 4911.380 & 200 & 10 & 20355.19 & 2 & & & & & 0.254 & & \\
\hline 4909.844 & 50 & 3 & 20361.56 & 2 & 1 & 0.751 & 2 & 1.131 & & & \\
\hline 4907.209 & 100 & 4 & 20372.49 & 7 & & & & & & & 1.271 \\
\hline 4907.044 & 50 & 2 & 20373.18 & 7 & & & & & & & 1.330 \\
\hline 4901.987 & 50 & 2 & 20394.20 & 3 & & & & & & 0.185 & \\
\hline 4899.336 & 50 & 3 & 20405.23 & 3 & & & & & & 0.341 & \\
\hline 4899.240 & 50 & 3 & 20405.63 & 7 & & & & & & & 0.936 \\
\hline 4892.758 & 50 & 2 & 20432.67 & 3 & & & & & & 1.122 & \\
\hline 4891.036 & 10 & 1 & 20439.86 & 7 & & & & & & & 1.089 \\
\hline 4887.096 & 50 & 2 & 20456.33 & 7 & & & & & & & 1.210 \\
\hline 4886.869 & 50 & 2 & 20457.29 & 3 & & & & & & 0.354 & \\
\hline 4886.133 & 25 & 2 & 20460.37 & 7 & & & & & & & 1.007 \\
\hline 4881.204 & 75 & 2 & 20481.03 & 7 & & & & & 0.130 & & \\
\hline 4877.809 & 15 & 5 & 20495.28 & 3 & & & & & & 0.092 & \\
\hline 4877.265 & 20 & 1 & 20497.57 & 7 & & & & & & & 1.051 \\
\hline 4876.495 & 100 & 5 & 20500.81 & 7 & & & & & & & 1.178 \\
\hline 4876.244 & 50 & 2 & 20501.86 & 7 & & & & & & & 1.088 \\
\hline 4874.365 & 150 & 10 & 20509.76 & 3 & & & & & 0.270 & 1.085 & \\
\hline 4872.922 & $300 \mathrm{r}$ & $25 b$ & 20515.84 & 1 & 1 & 1.467 & 2 & 1.246 & & & \\
\hline 4872.030 & 100 & 4 & 20519.59 & 3 & & & & & & 1.298 & \\
\hline 4868.882 & 150 & 10 & 20532.86 & 3 & & & & & & 0.114 & \\
\hline 4865.974 & 501 & 81 & 20545.13 & 3 & & & & & & 0.173 & \\
\hline 4865.477 & $300 \mathrm{r}$ & $150 \mathrm{~b}$ & 20547.23 & 2 & 4 & 0.862 & 5 & 1.088 & & & \\
\hline 4861.720 & $75 b$ & $20 \mathrm{~h}$ & 20563.11 & 7 & & & & & 0.312 & & \\
\hline 4861.223 & $300 \mathrm{r}$ & 50 & 20565.21 & 7 & & & & & & & 1.045 \\
\hline 4852.868 & 200 & 50 & 20600.62 & 3 & & & & & & 0.286 & \\
\hline 4849.861 & 50 & 15 & 20613.39 & 3 & & & & & & 0.648 & \\
\hline 4849.139 & 50 & $25 b$ & 20616.46 & 2 & 4 & 0.684 & 5 & 0.848 & & & \\
\hline 4848.363 & $400 \mathrm{r}$ & 150 & 20619.76 & 3 & 2 & 1.253 & 2 & 1.512 & & & \\
\hline 4847.330 & 150 & 15 & 20624.15 & 2 & & & & & 0.386 & & \\
\hline 4843.938 & $100 \mathrm{~b}$ & $50 \mathrm{~b}$ & 20638.59 & 7 & & & & & & & 1.171 \\
\hline 4842.166 & 50 & 40 & 20646.14 & 7 & & & & & 0.256 & & \\
\hline 4840.928 & $100 \mathrm{~b}$ & 50 & 20651.43 & 3 & & & & & & 0.812 & \\
\hline 4833.674 & $20 \mathrm{~s}$ & $4 \mathrm{~s}$ & 20682.42 & 3 & & & & & & 0.289 & \\
\hline 4833.179 & $200 \mathrm{~b}$ & 50 & 20684.54 & 3 & & & & & & 0.525 & \\
\hline $4831.597^{\circ}$ & $300 \mathrm{r}$ & 150 & 20691.31 & 2 & 2 & 0.791 & 3 & 1.035 & & & \\
\hline 4826.700 & $200 \mathrm{~b}$ & $50 \mathrm{~b}$ & 20712.30 & 3 & & & & & & 0.931 & \\
\hline 4823.997 & $200 \mathrm{r}$ & $100 \mathrm{~h}$ & 20723.91 & 3 & & & & & & 0.639 & v \\
\hline 4823.604 & $300 \mathrm{r}$ & 75 & 20725.59 & 1 & & & & & & & 0.860 \\
\hline 4822.855 & $300 \mathrm{r}$ & 75 & 20728.81 & 7 & & & & & & & 1.187 \\
\hline 4821.587 & 150 & 25 & 20734.26 & 1 & 2 & 1.386 & 3 & 1.082 & & & \\
\hline 4820.463 & $150 \mathrm{~b}$ & $40 \mathrm{~h}$ & 20739.10 & 3 & & & & & & 0.630 & \\
\hline 4819.191 & 150 & 15 & 20744.57 & 1 & & & & & 0.123 & & \\
\hline 4817.017 & 75 & 8 & 20753.94 & 7 & & & & & & & 0.716 \\
\hline 4814.919 & 15 & 3 & 20762.98 & 7 & & & & & & & 1.612 \\
\hline 4813.893 & 200 & 100 & 20767.41 & 3 & & & & & & 0.670 & \\
\hline 4813.719 & 150 & 20 & 20768.15 & 7 & & & & & 0.220 & & \\
\hline 4813.004 & 75 & 15 & 20771.24 & 7 & & & & & 0.200 & & \\
\hline 4809.615 & 200 & 50 & 20785.88 & 2 & & & & & & & 1.352 \\
\hline 4808.134 & $500 \mathrm{r}$ & 150 & 20792.28 & 1 & 4 & 1.220 & 5 & 1.155 & & & \\
\hline
\end{tabular}


TABLE 3. Zeeman effect of Th $\mathrm{I}$-continued

\begin{tabular}{|c|c|c|c|c|c|c|c|c|c|c|c|}
\hline \multirow{2}{*}{$\begin{array}{c}\text { Wavelength } \\
(\AA)\end{array}$} & \multicolumn{2}{|c|}{ Intensity } & \multirow{2}{*}{$\begin{array}{l}\text { Wavenumber } \\
\qquad\left(\mathrm{cm}^{-1}\right)\end{array}$} & \multirow{2}{*}{ Type } & \multirow{2}{*}{$J_{1}$} & \multirow{2}{*}{$g_{1}$} & \multirow{2}{*}{$J_{2}$} & \multirow{2}{*}{$g_{2}$} & \multirow{2}{*}{$\Delta g$} & \multirow{2}{*}{$p$} & \multirow{2}{*}{$n$} \\
\hline & Lamp & Spark & & & & & & & & & \\
\hline 4784.038 & 150 & 20 & 20897.00 & 3 & & & & & & 0.671 & \\
\hline 4778.294 & $400 \mathrm{r}$ & 50 & 20922.12 & 3 & 2 & 0.736 & 2 & 1.169 & & & \\
\hline 4775.313 & 1001 & $5 b$ & 20935.18 & 7 & & & & & 0.370 & & \\
\hline 4766.600 & $300^{r}$ & 100 & 20973.45 & 3 & 1 & 0.760 & 1 & 1.481 & & & \\
\hline 4764.344 & 200 & 300 & 20983.38 & 3 & & & & & & 0.830 & \\
\hline 4758.145 & 100 & 75 & 21010.72 & 7 & & & & & & & 0.878 \\
\hline 4749.968 & $300 \mathrm{~b}$ & 50 & 21046.89 & 1 & 3 & 1.216 & 4 & 1.064 & & & \\
\hline 4749.200 & $400 \mathrm{r}$ & 150 & 21050.30 & 3 & 4 & 0.858 & 4 & 0.972 & & & \\
\hline 4743.693 & $150 w$ & 300 & 21074.73 & 7 & & & & & 0.250 & & \\
\hline 4741.301 & 150 & 40 & 21085.36 & 3 & & & & & & 0.112 & \\
\hline 4739.674 & 300 & 40 & 21092.60 & 1 & 1 & 1.802 & 2 & 1.188 & & & \\
\hline 4737.915 & 150 & 15. & 21100.43 & 7 & & & & & & & 1.212 \\
\hline 4734.046 & 200 & 10 & 21117.67 & 3 & & & & & & 0.100 & \\
\hline 4729.128 & $400 \mathrm{~b}$ & $100 \mathrm{~b}$ & 21139.64 & 1 & 3 & 1.028 & 4 & 0.865 & & & \\
\hline 4723.438 & $800 \mathrm{r}$ & 200 & 21165.10 & 2 & 2 & 0.728 & 3 & 1.298 & & & \\
\hline 4722.089 & 300 & 50 & 21171.15 & 2 & 2 & 1.135 & 3 & 1.251 & & & \\
\hline 4721.276 & 200 & 15 & 21174.79 & 3 & 2 & 0.779 & 2 & 1.049 & & & \\
\hline 4712.481 & $300 \mathrm{~b}$ & 100 & 21214.31 & 2 & & & & & 0.175 & & 2.053 \\
\hline 4706.575 & 100 & 5 & 21240.93 & 3 & & & & & & 0.298 & \\
\hline 4695.456 & 200 & 50 & 21291.23 & 3 & & & & & & 0.315 & \\
\hline 4695.039 & $300 \mathrm{r}$ & 100 & 21293.12 & 1 & 2 & 1.244 & 3 & 1.073 & & & \\
\hline 4691.883 & 100 & 8 & 21307.44 & 3 & & & & & & 0.171 & \\
\hline 4691.631 & $300 \mathrm{~b}$ & $50 \mathrm{~b}$ & 21308.59 & 2 & 2 & 1.015 & 3 & 1.219 & & & \\
\hline 4691.500 & 100 & 15 & 21309.18 & 7 & & & & & & & 1.174 \\
\hline 4689.252 & 2001 & $50 \mathrm{~b}$ & 21319.40 & 1 & & & & & & & 0.033 \\
\hline 4686.195 & $500 \mathrm{r}$ & 50 & 21333.30 & 2 & 3 & 1.079 & 4 & 1.373 & & & \\
\hline 4683.349 & 200 & 20 & 21346.27 & 2 & & & & & 0.210 & & \\
\hline 4682.229 & 150 & 15 & 21351.37 & 3 & & & & & & 0.292 & \\
\hline 4678.233 & 150 & $20 \mathrm{~b}$ & 21369.61 & 7 & & & & & & & 1.120 \\
\hline 4676.971 & 150 & $25 b$ & 21375.38 & 7 & & & & & & & 1.116 \\
\hline 4676.056 & $500 \mathrm{r}$ & 150 & 21379.56 & 3 & & & & & & 0.425 & \\
\hline 4673.661 & $800 \mathrm{r}$ & 150 & 21390.52 & 1 & & & & & & & 0.975 \\
\hline 4673.042 & 200 & 25 & 21393.35 & 3 & & & & & & 0.102 & \\
\hline 4669.984 & $500 \mathrm{r}$ & 100 & 21407.36 & 3 & & & & & & 0.120 & \\
\hline 4668.172 & $800 \mathrm{r}$ & 150 & 21415.67 & 2 & 2 & 0.941 & 3 & 1.241 & & & \\
\hline 4666.797 & $500 \mathrm{r}$ & 150 & 21421.97 & 2 & 2 & 0.867 & 2 & 1.004 & & & \\
\hline 4663.202 & $300 \mathrm{r}$ & 10 & 21438.49 & 1 & 2 & 1.510 & 3 & 1.080 & & & \\
\hline 4658.522 & 100 & 8 & 21460.03 & 7 & & & & & & & 1.102 \\
\hline 4658.376 & 100 & $5 b$ & 21460.70 & 7 & & & & & & & 1.002 \\
\hline 4655.210 & 200 & 20 & 21475.30 & 2 & & & & & & & 1.338 \\
\hline 4650.233 & 200 & 5 & 21498.28 & 2 & 1 & 0.064 & 2 & 0.988 & & & \\
\hline 4649.975 & 100 & 8 & 21499.47 & 3 & & & & & & 0.153 & \\
\hline 4647.249 & 200 & 20 & 21512.08 & 1 & 2 & 1.261 & 3 & 1.091 & & & \\
\hline 4643.557 & 150 & 10 & 21529.19 & 2 & & & & & & & 1.273 \\
\hline 4641.244 & $400 \mathrm{~b}$ & $150 \mathrm{~b}$ & 21539.92 & 2 & & & & & & & 1.284 \\
\hline 4639.896 & 100 & 25 & 21546.18 & 3 & & & & & & 0.118 & \\
\hline 4639.520 & 300 & 50 & 21547.92 & 3 & & & & & & 0.420 & \\
\hline 4638.686 & 150 & 10 & 21551.79 & 3 & & & & & & 0.236 & \\
\hline 4633.765 & $300 \mathrm{~b}$ & $75 b$ & 21574.68 & 7 & & & & & & & 0.981 \\
\hline 4631.644 & 200 & 50 & 21584.56 & 7 & & & & & & & 0.880 \\
\hline 4624.314 & 100 & 4 & 21618.78 & 3 & & & & & & 0.162 & \\
\hline 4621.165 & 150 & 40 & 21633.51 & 7 & & & & & & & 0.886 \\
\hline 4620.448 & 200 & 10 & 21636.86 & 3 & & & & & & 0.110 & \\
\hline 4615.334 & 200 & 15 & 21660.84 & 3 & 1 & 1.063 & 1 & 1.483 & & & \\
\hline 4615.025 & 200 & 40 & 21662.29 & 7 & & & & & & & 1.004 \\
\hline
\end{tabular}


TABLE 3. Zeeman effect of Th $\mathrm{I}$-continued

\begin{tabular}{|c|c|c|c|c|c|c|c|c|c|c|c|}
\hline \multirow{2}{*}{ 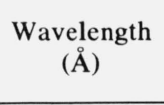 } & \multicolumn{2}{|c|}{ Intensity } & \multirow{2}{*}{$\begin{array}{l}\text { Wavenumber } \\
\qquad\left(\mathrm{cm}^{-1}\right)\end{array}$} & \multirow{2}{*}{ Type } & \multirow{2}{*}{$J_{1}$} & \multirow{2}{*}{$g_{1}$} & \multirow{2}{*}{$J_{2}$} & \multirow{2}{*}{$g_{2}$} & \multirow{2}{*}{$\Delta g$} & \multirow{2}{*}{$p$} & \multirow{2}{*}{$n$} \\
\hline & Lamp & Spark & & & & & & & & & \\
\hline 4613.605 & 200 & 15 & 21668.96 & 1 & 1 & 1.584 & 2 & 0.744 & & & \\
\hline 4610.628 & $300 \mathrm{~b}$ & 50 & 21682.95 & 3 & & & & & & 0.113 & \\
\hline 4608.620 & 150 & 8 & 21692.39 & 3 & & & & & & 0.319 & \\
\hline 4603.143 & $300 \mathrm{~b}$ & $100 \mathrm{~b}$ & 21718.20 & 1 & 1 & 1.335 & 2 & 0.774 & & & \\
\hline 4599.704 & 100 & 10 & 21734.44 & 3 & & & & & & 0.515 & \\
\hline 4595.420 & $800 r$ & 200 & 21754.70 & 1 & 2 & 1.240 & 3 & 1.084 & & & \\
\hline 4593.645 & 300 & 150 & 21763.11 & 2 & 2 & 0.863 & 3 & 1.055 & & & \\
\hline 4592.668 & $500 \mathrm{r}$ & 150 & 21767.74 & 2 & 2 & 0.782 & 3 & 1.077 & & & \\
\hline 4570.972 & $500 \mathrm{r}$ & 200 & 21871.05 & 7 & & & & & & & 1.199 \\
\hline 4567.240 & 300 & 100 & 21888.92 & 2 & & & & & 0.310 & & \\
\hline 4561.348 & $500 \mathrm{r}$ & 200 & 21917.20 & 7 & & & & & & & 1.208 \\
\hline 4558.346 & 300 & 40 & 21931.63 & 2 & & & & & 0.280 & & 1.966 \\
\hline 4551.473 & 200 & 75 & 21964.75 & 2 & & & & & 0.148 & & \\
\hline 4548.326 & $100 \mathrm{~b}$ & 10 & 21979.95 & 7 & & & & & & & 0.938 \\
\hline 4545.915 & 200 & 300 & 21991.61 & 1 & & & & & 0.090 & & 0.755 \\
\hline 4544.512 & 150 & 200 & 21998.40 & 7 & & & & & & & 0.814 \\
\hline 4540.999 & $300 \mathrm{r}$ & 75 & 22015.41 & 3 & & & & & & 0.207 & \\
\hline 4535.255 & $300 r$ & 150 & 22043.30 & 2 & 1 & 0.621 & 2 & 0.772 & & & \\
\hline 4530.319 & 200 & 100 & 22067.31 & 7 & & & & & & & 1.002 \\
\hline 4519.259 & 300 & 75 & 22121.32 & 1 & 1 & 1.560 & 2 & 1.250 & & & \\
\hline 4515.118 & $300 \mathrm{r}$ & 50 & 22141.60 & 2 & 2 & 0.729 & 3 & 1.093 & & & \\
\hline 4513.681 & $400 r$ & 150 & 22148.65 & 2 & 4 & 1.073 & 5 & 1.364 & & & \\
\hline 4505.216 & 500 & 100 & 22190.27 & 2 & 3 & 1.092 & 4 & 1.306 & & & \\
\hline 4499.984 & 500 & 200 & 22216.07 & 1 & 3 & 1.063 & 4 & 0.858 & & & \\
\hline 4498.947 & $800 \mathrm{r}$ & 200 & 22221.19 & 2 & 1 & 0.052 & 2 & 0.852 & & & \\
\hline 4493.334 & $1000 \mathrm{r}$ & 300 & 22248.95 & 3 & 2 & 0.738 & 2 & 1.141 & & & \\
\hline 4489.664 & 200 & 100 & 22267.13 & 3 & & & & & & 0.235 & \\
\hline 4488.312 & 200 & 100 & 22273.84 & 2 & & & & & & & 1.621 \\
\hline 4486.898 & $300 r$ & 100 & 22280.86 & 7 & & & & & & & 0.767 \\
\hline 4485.713 & 150 & 40 & 22286.75 & 7 & & & & & & & 0.962 \\
\hline 4482.169 & $400 r$ & 100 & 22304.37 & 3 & & & & & & 0.227 & \\
\hline 4480.823 & 200 & 200 & 22311.07 & 3 & & & & & & 0.222 & \\
\hline 4479.637 & 200 & 75 & 22316.98 & 2 & 2 & 0.989 & 3 & 1.051 & & & \\
\hline 4478.594 & 150 & 40 & 22322.17 & 7 & & & & & & & 0.793 \\
\hline 4475.221 & 200 & 40 & 22338.99 & 2 & 2 & 0.735 & 3 & 1.020 & & & \\
\hline 4470.990 & 200 & 25 & 22360.14 & 2 & 1 & 0.956 & 2 & 1.191 & & & \\
\hline 4469.526 & $400 \mathrm{r}$ & 150 & 22367.46 & 3 & & & & & & 0.395 & \\
\hline 4468.320 & 200 & 50 & 22373.49 & 3 & & & & & & 0.608 & \\
\hline 4463.834 & 200 & 100 & 22395.98 & 7 & & & & & & & 1.070 \\
\hline 4461.789 & $200 \mathrm{~b}$ & 100 & 22406.24 & 7 & & & & & & & 1.405 \\
\hline 4461.528 & 200 & 150 & 22407.56 & 2 & 2 & 0.777 & 3 & 1.057 & & & \\
\hline 4461.241 & 200 & 100 & 22409.00 & 1 & 2 & 1.244 & 3 & 0.994 & & & \\
\hline 4459.004 & 100 & 75 & 22420.24 & 3 & 1 & 0.729 & 1 & 0.931 & & & \\
\hline 4458.736 & 150 & 75 & 22421.59 & 3 & 1 & 0.723 & 1 & 1.482 & & & \\
\hline 4458.002 & $600 \mathrm{r}$ & 200 & 22425.28 & 3 & 2 & 0.980 & 2 & 1.250 & & & \\
\hline 4457.236 & 200 & 100 & 22429.14 & 7 & & & & & & & 0.942 \\
\hline 4452.565 & 200 & 100 & 22452.66 & 3 & 3 & 1.082 & 3 & 1.388 & & & \\
\hline 4445.032 & 200 & 75 & 22490.71 & 1 & & & & & 0.650 & & \\
\hline 4443.665 & 150 & 100 & 22497.63 & 1 & 1 & 1.463 & 2 & 1.018 & & & \\
\hline 4443.086 & 20 & 200 & 22500.56 & 3 & & & & & & 0.272 & \\
\hline 4438.746 & 400 & 200 & 22522.56 & 2 & 2 & 1.000 & 3 & 1.180 & & & \\
\hline 4432.252 & 400 & 150 & 22555.56 & 3 & & & & & & 0.112 & \\
\hline 4424.836 & $200 \mathrm{~b}$ & 50 & 22593.36 & 2 & 2 & 0.984 & 3 & 1.187 & & & \\
\hline 4423.719 & 400 & 150 & 22599.07 & 2 & 1 & 0.712 & 2 & 1.252 & & & \\
\hline 4422.048 & 600 & 200 & 22607.61 & 7 & & & & & & & 0.772 \\
\hline
\end{tabular}


TABLE 3. Zeeman effect of $\mathrm{Th} \mathrm{I}$-continued

\begin{tabular}{|c|c|c|c|c|c|c|c|c|c|c|c|}
\hline \multirow{2}{*}{$\begin{array}{l}\text { Wavelength } \\
\text { (̊̊) }\end{array}$} & \multicolumn{2}{|c|}{ Intensity } & \multirow{2}{*}{$\begin{array}{l}\text { Wa venumber } \\
\left(\mathrm{cm}^{-1}\right)\end{array}$} & \multirow{2}{*}{ Type } & \multirow{2}{*}{$J_{1}$} & \multirow{2}{*}{$g_{1}$} & \multirow{2}{*}{$J_{2}$} & \multirow{2}{*}{$g_{2}$} & \multirow{2}{*}{$\Delta g$} & \multirow{2}{*}{$p$} & \multirow{2}{*}{$n$} \\
\hline & Lamp & Spark & & & & & & & & & \\
\hline 4420.256 & 200 & 20 & 22616.77 & 7 & & & & & & & 1.209 \\
\hline 4416.844 & 500 & 100 & 22634.24 & 2 & 4 & 1.141 & 5 & 1.362 & & & \\
\hline 4414.486 & $500 \mathrm{~d}$ & 150 & 22646.34 & 7 & & & & & & & 0.804 \\
\hline 4408.883 & $800 \mathrm{r}$ & 200 & 22675.12 & 3 & 2 & 1.036 & 2 & 1.256 & & & \\
\hline 4402.927 & 500 & 150 & 22705.79 & 7 & & & & & & & 0.976 \\
\hline 4401.581 & 500 & 200 & 22712.73 & 2 & 4 & 0.885 & 5 & 1.126 & & & \\
\hline 4393.759 & 400 & 150 & 22753.16 & 3 & 3 & 1.084 & 3 & 1.244 & & & \\
\hline 4392.974 & 400 & 100 & 22757.23 & 3 & 4 & 0.854 & 4 & 1.194 & & & \\
\hline 4391.390 & 150 & 50 & 22765.44 & 3 & & & & & & 0.395 & \\
\hline 4388.967 & 50 & 25 & 22778.00 & 7 & & & & & & & 1.032 \\
\hline 4387.734 & 150 & 40 & 22784.40 & 2 & 1 & 0.071 & 2 & 1.541 & & & \\
\hline 4384.656 & $150 \mathrm{~b}$ & $50 \mathrm{~b}$ & 22800.40 & 7 & & & & & & & 1.106 \\
\hline 4380.289 & 200 & 25 & 22823.13 & 3 & 2 & & 2 & & & 1.396 & \\
\hline 4376.532 & 100 & 20 & 22842.73 & 7 & & & & & & & 1.287 \\
\hline 4374.124 & $600 \mathrm{r}$ & 150 & 22855.30 & 2 & 2 & 0.738 & 3 & 1.133 & & & \\
\hline 4373.041 & 200 & 50 & 22860.96 & 1 & & & & & & & 0.771 \\
\hline 4369.288 & $50 \mathrm{~b}$ & 25 & 22880.59 & 3 & & & & & & 0.134 & \\
\hline 4365.930 & 600 & 150 & 22898.19 & 2 & 2 & 0.778 & 3 & 1.093 & & & \\
\hline 4359.372 & $600 r$ & 100 & 22932.64 & 2 & & & & & & & 1.151 \\
\hline 4358.321 & 500 & 150 & 22938.17 & 3 & 2 & 0.732 & 2 & 1.159 & & & \\
\hline 4354.483 & 400 & 100 & 22958.39 & 7 & & & & & & & 0.953 \\
\hline 4353.449 & $300 \mathrm{~b}$ & $100 \mathrm{~b}$ & 22963.84 & 3 & 2 & 1.102 & 2 & 1.253 & & & \\
\hline 4351.466 & 100 & 50 & 22974.30 & 7 & & & & & & & 1.177 \\
\hline 4349.072 & 400 & 40 & 22986.95 & 3 & & & & & & 0.142 & \\
\hline 4348.599 & 400 & 75 & 22989.45 & 1 & 1 & 0.798 & 2 & 0.934 & & & \\
\hline 4346.432 & $500 \mathrm{r}$ & 200 & 23000.91 & 1 & 1 & 1.468 & 2 & 1.187 & & & \\
\hline 4345.851 & 150 & 50 & 23003.99 & 3 & & & & & & 0.582 & \\
\hline 4343.381 & 100 & 25 & 23017.07 & 7 & & & & & & & 1.430 \\
\hline 4342.445 & $500 \mathrm{r}$ & 100 & 23022.03 & 1 & 2 & 0.777 & 3 & 1.074 & & & \\
\hline 4340.896 & 200 & 40 & 23030.24 & 1 & 4 & 1.057 & 5 & 0.945 & & & \\
\hline 4338.108 & 400 & 100 & 23045.04 & 3 & & & & & & 0.130 & \\
\hline 4337.277 & $800 \mathrm{r}$ & 100 & 23049.45 & 1 & 1 & 1.356 & 2 & 0.726 & & & \\
\hline 4332.339 & 150 & 40 & 23075.73 & 2 & 1 & 1.480 & 2 & 1.710 & & & \\
\hline 4330.844 & 300 & 25 & 23083.70 & 3 & 4 & & 4 & & & 1.307 & \\
\hline 4328.915 & $300 r$ & 25 & 23093.98 & 3 & 2 & 0.733 & 2 & 1.320 & & & \\
\hline 4327.715 & 150 & 25 & 23100.39 & 1 & 2 & 1.414 & 3 & 1.048 & & & \\
\hline 4327.036 & 100 & 25 & 23104.01 & 7 & & & & & & & 1.135 \\
\hline 4325.274 & 200 & 50 & 23113.42 & 3 & & & & & & 0.639 & \\
\hline 4320.738 & 75 & 8 & 23137.69 & 7 & & & & & 0.374 & & \\
\hline 4315.254 & $500 \mathrm{r}$ & 150 & 23167.09 & 3 & 3 & 0.954 & 3 & 1.077 & & & \\
\hline 4314.319 & 300 & 50 & 23172.11 & 1 & 3 & 1.122 & 4 & 1.072 & & & \\
\hline 4312.992 & $800 \mathrm{r}$ & 200 & 23179.24 & 7 & & & & & & & 1.208 \\
\hline 4311.798 & 300 & 100 & 23185.66 & 1 & 1 & 1.294 & 2 & 0.774 & & & \\
\hline 4311.625 & $150 \mathrm{~b}$ & 25 & 23186.59 & 7 & & & & & & & 1.396 \\
\hline 4311.062 & 150 & 15 & 23189.62 & 3 & & & & & & 0.139 & \\
\hline 4308.121 & 300 & 100 & 23205.45 & 2 & & & & & & & 1.348 \\
\hline 4307.176 & $600 \mathrm{r}$ & 200 & 23210.54 & 2 & 5 & 1.041 & 6 & 1.192 & & & \\
\hline 4306.365 & $200 \mathrm{~b}$ & $200 \mathrm{~b}$ & 23214.91 & 1 & & & & & 0.285 & & \\
\hline 4304.955 & 150 & 10 & 23222.51 & 3 & 1 & 1.164 & 1 & 1.467 & & & \\
\hline 4304.417 & 100 & 25 & 23225.42 & 7 & & & & & & & 1.013 \\
\hline 4303.096 & 50 & 8 & 23232.55 & 7 & & & & & & & 0.487 \\
\hline 4299.839 & $500 \mathrm{r}$ & 100 & 23250.15 & 1 & & & & & & & 0.796 \\
\hline 4299.633 & 200 & 50 & 23251.26 & 7 & & & & & & & 1.570 \\
\hline 4297.307 & $300 \mathrm{~b}$ & 75 & 23263.85 & 3 & 5 & 1.042 & 5 & 1.180 & & & \\
\hline 4293.770 & 10 & 3 & 23283.01 & 7 & & & & & & & 1.376 \\
\hline
\end{tabular}


TABLE 3. Zeeman effect of Th $\mathrm{I}$-continued

\begin{tabular}{|c|c|c|c|c|c|c|c|c|c|c|c|}
\hline \multirow{2}{*}{$\begin{array}{c}\text { Wavelength } \\
(\AA ̊)\end{array}$} & \multicolumn{2}{|c|}{ Intensity } & \multirow{2}{*}{$\begin{array}{l}\text { Wa venumber } \\
\qquad\left(\mathrm{cm}^{-1}\right)\end{array}$} & \multirow{2}{*}{ Type } & \multirow{2}{*}{$J_{1}$} & \multirow{2}{*}{$g_{1}$} & \multirow{2}{*}{$J_{2}$} & \multirow{2}{*}{$g_{2}$} & \multirow{2}{*}{$\Delta g$} & \multirow{2}{*}{$p$} & \multirow{2}{*}{$n$} \\
\hline & Lamp & Spark & & & & & & & & & \\
\hline 4291.810 & 400 & 100 & 23293.64 & 2 & -2 & 0.790 & 3 & 0.899 & & & \\
\hline 4289.655 & 100 & 40 & 23305.34 & 2 & 3 & 0.884 & 4 & 1.152 & & & \\
\hline 4288.669 & 200 & 75 & 23310.70 & 2 & 2 & 0.927 & 3 & 1.248 & & & \\
\hline 4288.471 & $200 \mathrm{~b}$ & $50 \mathrm{~b}$ & 23311.78 & 2 & & & & & & & 1.106 \\
\hline 4287.082 & 200 & 40 & 23319.33 & 2 & & & & & & & 1.499 \\
\hline 4286.229 & $200 \mathrm{~b}$ & $75 b$ & 23323.97 & 2 & 2 & 1.020 & 3 & 1.251 & & & \\
\hline 4283.103 & $200 \mathrm{~b}$ & $50 \mathrm{~b}$ & 23340.99 & 7 & & & & & & & 1.340 \\
\hline 4280.568 & $300 r$ & 100 & 23354.81 & 2 & 1 & 0.062 & 2 & 0.943 & & & \\
\hline 4278.324 & $500 \mathrm{r}$ & 200 & 23367.07 & 3 & 5 & 1.044 & 5 & 1.144 & & & \\
\hline 4272.875 & $400 \mathrm{r}$ & 50 & 23396.86 & 2 & 3 & 1.138 & 4 & 1.309 & & & \\
\hline 4269.943 & 300 & 75 & 23412.92 & 1 & & & & & & & 1.000 \\
\hline 4262.611 & 200 & 2 & 23453.20 & 2 & & & & & 0.170 & & \\
\hline 4260.984 & 100 & 2 & 23462.15 & 2 & 2 & & 3 & & 0.220 & & \\
\hline 4260.332 & $600 \mathrm{r}$ & 5 & 23465.74 & 2 & 4 & 1.170 & 5 & 1.364 & & & \\
\hline 4259.494 & 200 & 1 & 23470.36 & 7 & & & & & 0.216 & & \\
\hline 4258.521 & 400 & 2 & 23475.73 & 2 & 4 & 0.862 & 5 & 1.087 & & & \\
\hline 4257.496 & $600 \mathrm{r}$ & 4 & 23481.37 & 1 & 1 & 0.839 & 2 & 0.728 & & & \\
\hline 4256.254 & $500 \mathrm{r}$ & 8 & 23488.23 & 3 & 3 & 1.102 & 3 & 1.248 & & & \\
\hline 4253.539 & $600 \mathrm{r}$ & 20 & 23503.22 & 3 & & & & & & 0.129 & \\
\hline 4250.315 & $800 \mathrm{r}$ & 75 & 23521.04 & 2 & 2 & 0.724 & 3 & 1.066 & & & \\
\hline 4248.391 & 200 & 3 & 23531.70 & 3 & 4 & 0.880 & 4 & 1.160 & & & \\
\hline 4235.463 & $600 \mathrm{r}$ & 5 & 23603.52 & 3 & 2 & 0.732 & 2 & 1.375 & & & \\
\hline 4230.427 & 500 & 5 & 23631.62 & 2 & & & & & 0.098 & & \\
\hline 4229.148 & $300 \mathrm{r}$ & 4 & 23638.77 & 7 & & & & & & & 1.072 \\
\hline 4228.761 & $150 \mathrm{~b}$ & 3 & 23640.93 & 3 & & & & & & 0.522 & \\
\hline 4227.387 & 400 & 8 & 23648.61 & 2 & 1 & 0.982 & 2 & 1.190 & & & \\
\hline 4226.298 & 200 & 3 & 23654.71 & 2 & & & & & 0.107 & & \\
\hline 4221.691 & 75 & 2 & 23680.52 & 2 & 2 & 0.819 & 3 & 1.076 & & & \\
\hline 4220.064 & 200 & 15 & 23689.65 & 2 & 1 & 0.064 & 2 & 0.979 & & & \\
\hline 4216.068 & $300 \mathrm{~s}$ & 5 & 23712.10 & 1 & & & & & 0.080 & & \\
\hline 4214.828 & 200 & 4 & 23719.08 & 3 & & & & & & 0.088 & \\
\hline 4210.923 & $500 \mathrm{r}$ & 10 & 23741.08 & 7 & & & & & & & 0.713 \\
\hline 4210.765 & 400 & 10 & 23741.96 & 7 & & & & & & & 0.807 \\
\hline 4210.455 & 75 & 2 & 23743.72 & 7 & & & & & & & 0.990 \\
\hline 4208.412 & 150 & 4 & 23755.24 & 2 & & & & & 0.180 & & \\
\hline 4194.936 & 200 & 5 & 23831.55 & 2 & 4 & 0.861 & 5 & 1.111 & & & \\
\hline 4187.142 & 50 & 3 & 23875.91 & 2 & 1 & 1.047 & 2 & 1.727 & & & \\
\hline 4185.143 & 50 & 2 & 23887.31 & 3 & & & & & & 0.304 & \\
\hline 4184.603 & 50 & 3 & 23890.40 & 7 & & & & & & & 0.847 \\
\hline 4179.314 & 100 & 8 & 23920.63 & 7 & & & & & 0.230 & & \\
\hline 4177.165 & 100 & 4 & 23932.94 & 3 & & & & & & 0.256 & \\
\hline 4170.533 & $500 \mathrm{~b}$ & 25 & 23970.99 & 3 & 4 & 1.111 & 4 & 1.205 & & & \\
\hline 4165.766 & $800 \mathrm{r}$ & 15 & 23998.42 & 3 & 4 & 0.869 & 4 & 1.090 & & & \\
\hline 4162.509 & 400 & 15 & 24017.20 & 1 & 3 & 1.068 & 4 & 0.998 & & & \\
\hline 4158.535 & 800 & 20 & 24040.15 & 3 & 5 & 1.119 & 5 & 1.362 & & & \\
\hline 4157.280 & 150 & 5 & 24047.41 & 7 & & & & & & & 1.064 \\
\hline 4154.719 & 200 & 5 & 24062.23 & 3 & & & & & & 0.656 & \\
\hline 4138.040 & 200 & 3 & 24159.22 & 3 & 1 & 1.030 & 1 & 1.478 & & & \\
\hline 4136.432 & $200 \mathrm{~b}$ & $5 b$ & 24168.61 & 2 & 1 & 0.707 & 2 & 0.795 & & & \\
\hline 4136.286 & 300 & 8 & 24169.46 & 2 & & & & & 0.104 & & \\
\hline 4134.062 & $200 \mathrm{~b}$ & $10 \mathrm{~b}$ & 24182.46 & 3 & 2 & 0.737 & 2 & 1.250 & & & \\
\hline 4134.039 & $100 \mathrm{~b}$ & $8 \mathrm{~b}$ & 24182.60 & 7 & & & & & & & 2.397 \\
\hline 4133.958 & 200 & 10 & 24183.07 & 3 & & & & & & 0.420 & \\
\hline 4131.207 & 50 & 2 & 24199.17 & 3 & & & & & & 0.145 & \\
\hline 4127.411 & $600 \mathrm{~b}$ & $40 \mathrm{~b}$ & 24221.43 & 7 & & & & & & & 0.876 \\
\hline
\end{tabular}


TABLE 3. Zeeman effect of Th $\mathrm{I}$-continued

\begin{tabular}{|c|c|c|c|c|c|c|c|c|c|c|c|}
\hline \multirow{2}{*}{$\begin{array}{l}\text { Wavelength } \\
\text { (A) }\end{array}$} & \multicolumn{2}{|c|}{ Intensity } & \multirow{2}{*}{$\begin{array}{l}\text { Wavenumber } \\
\left(\mathrm{cm}^{-1}\right)\end{array}$} & \multirow{2}{*}{ Type } & \multirow{2}{*}{$J_{1}$} & \multirow{2}{*}{$g_{1}$} & \multirow{2}{*}{$J_{2}$} & \multirow{2}{*}{$g_{2}$} & \multirow{2}{*}{$\Delta g$} & \multirow{2}{*}{$p$} & \multirow{2}{*}{$n$} \\
\hline & Lamp & Spark & & & & & & & & & \\
\hline 4118.490 & 200 & 4 & 24273.90 & 2 & & & & & & & 1.156 \\
\hline 4115.759 & $500 \mathrm{r}$ & 50 & 24290.00 & 2 & 1 & 0.059 & 2 & 0.900 & & & \\
\hline 4112.753 & $600 \mathrm{~b}$ & 15 & 24307.76 & 3 & 2 & 0.738 & 2 & 1.525 & & & \\
\hline 4109.323 & $400 \mathrm{r}$ & 5 & 24328.04 & 3 & & & & & & 0.302 & \\
\hline 4107.861 & 100 & 3 & 24336.70 & 2 & 1 & 1.024 & 2 & 1.250 & & & \\
\hline 4107.050 & 40 & 2 & 24341.51 & 7 & & & & & & & 1.482 \\
\hline 4102.617 & 400 & 3 & 24367.81 & 2 & 2 & 0.948 & 3 & 1.250 & & & \\
\hline 4100.341 & $1000 \mathrm{r}$ & 20 & 24381.33 & 3 & 2 & 0.734 & 2 & 1.252 & & & \\
\hline 4099.553 & 100 & 4 & 24386.02 & 3 & 2 & 1.433 & 2 & 1.713 & & & \\
\hline 4097.321 & 200 & 8 & 24399.30 & 2 & 2 & 1.014 & 3 & 1.192 & & & \\
\hline 4096.077 & 150 & 4 & 24406.71 & 2 & 4 & 0.872 & 5 & 1.052 & & & \\
\hline 4093.672 & 40 & 2 & 24421.06 & 2 & 2 & 0.741 & 3 & 1.172 & & & \\
\hline 4091.737 & 40 & 2 & 24432.60 & 7 & & & & & & & 1.191 \\
\hline 4087.285 & 200 & 5 & 24459.21 & 3 & & & & & & 0.479 & \\
\hline 4085.434 & $300 \mathrm{r}$ & 10 & 24470.30 & 3 & & & & & & 0.542 & \\
\hline 4085.257 & 200 & 8 & 24471.36 & 2 & & & & & & & 1.472 \\
\hline 4082.082 & 75 & 4 & 24490.39 & 2 & & & & & 0.085 & & 1.413 \\
\hline 4081.592 & 50 & 2 & 24493.33 & 3 & & & & & & 0.197 & \\
\hline 4081.368 & 800 & 8 & 24494.67 & 2 & & & & & 0.210 & & \\
\hline 4080.707 & 400 & 20 & 24498.64 & 2 & 4 & 0.866 & 5 & 1.186 & & & \\
\hline 4080.359 & 100 & 4 & 24500.73 & 7 & & & & & & & 1.057 \\
\hline 4078.875 & 75 & 4 & 24509.64 & 7 & & & & & & & 1.153 \\
\hline 4073.856 & 200 & 3 & 24539.84 & 2 & 4 & 1.114 & 5 & 1.376 & & & \\
\hline 4072.628 & $200 \mathrm{~s}$ & 5 & 24547.24 & 1 & & & & & & & 0.919 \\
\hline 4071.750 & $200 \mathrm{~b}$ & 15 & 24552.53 & 2 & 2 & 0.758 & 3 & 1.145 & & & \\
\hline 4070.756 & 100 & 2 & 24558.53 & 2 & 1 & 1.109 & 2 & 1.707 & & & \\
\hline 4070.238 & 100 & 3 & 24561.65 & 2 & 2 & 0.735 & 3 & 1.189 & & & \\
\hline 4067.451 & $400 \mathrm{r}$ & 5 & 24578.48 & 7 & & & & & & & 1.098 \\
\hline 4066.822 & 100 & 2 & 24582.28 & 7 & & & & & & & 1.110 \\
\hline 4065.618 & 100 & 4 & 24589.56 & 3 & & & & & & 0.550 & \\
\hline 4064.332 & 400 & 10 & 24597.34 & 1 & 4 & 1.144 & 5 & 1.034 & & & \\
\hline 4063.407 & $1500 \mathrm{r}$ & 75 & 24602.94 & 2 & & & & & & & 1.279 \\
\hline 4061.625 & 200 & 3 & 24613.73 & 7 & & & & & & & 1.210 \\
\hline 4057.940 & 200 & 2 & 24636.08 & 1 & 2 & 1.405 & 3 & 0.985 & & & \\
\hline 4057.327 & 50 & & 24639.81 & 3 & 2 & 1.050 & 2 & 1.150 & & & \\
\hline 4056.636 & 200 & 2 & 24644.01 & 3 & & & & & & 0.183 & \\
\hline 4054.302 & 200 & 8 & 24658.19 & 2 & 2 & 1.088 & 3 & 1.253 & & & \\
\hline 4053.527 & 100 & 75 & 24662.91 & 3 & 4 & 0.862 & 4 & 1.133 & & & \\
\hline 4050.888 & 800 & 25 & 24678.98 & 3 & & & & & & 0.246 & \\
\hline 4049.944 & 500 & 40 & 24684.72 & 1 & 1 & 1.802 & 2 & 1.252 & & & \\
\hline 4048.432 & 800 & 50 & 24693.94 & 2 & 2 & 0.979 & 3 & 1.089 & & & \\
\hline 4048.288 & 800 & 40 & 24694.83 & 3 & & & & & & 0.322 & \\
\hline 4047.058 & 50 & 1 & 24702.33 & 7 & & & & & & & 1.090 \\
\hline 4046.824 & 200 & 15 & 24703.75 & 3 & & & & & & 0.067 & \\
\hline 4046.252 & 200 & 8 & 24707.25 & 2 & 1 & 0.760 & 2 & 1.036 & & & \\
\hline 4045.226 & 200 & 2 & 24713.52 & 3 & & & & & & 0.634 & \\
\hline 4040.915 & 200 & 15 & 24739.88 & 2 & & & & & & & 1.067 \\
\hline 4039.865 & 500 & 20 & 24746.31 & 1 & & & & & 0.060 & & \\
\hline 4038.960 & 100 & 2 & 24751.86 & 3 & & & & & & 0.604 & \\
\hline 4038.228 & 300 & 10 & 24756.34 & 1 & & & & & 0.390 & & \\
\hline 4037.562 & 500 & 15 & 24760.43 & 3 & 1 & 1.104 & 1 & 2.400 & & & \\
\hline 4036.047 & $2000 \mathrm{r}$ & 50 & 24769.72 & 2 & 2 & 0.744 & 3 & 1.143 & & & \\
\hline 4035.352 & 150 & 5 & 24773.99 & 7 & 2 & & & 3 & 0.310 & & \\
\hline 4034.878 & 200 & 8 & 24776.90 & 3 & & & & & & 0.789 & \\
\hline 4033.907 & $600 \mathrm{r}$ & 8 & 24782.86 & 2 & & & & & & & 1.381 \\
\hline
\end{tabular}


TABLE 3. Zeeman effect of Th $\mathrm{I}$-continued

\begin{tabular}{|c|c|c|c|c|c|c|c|c|c|c|c|}
\hline \multirow{2}{*}{$\begin{array}{l}\text { Wavelength } \\
(\AA)\end{array}$} & \multicolumn{2}{|c|}{ Intensity } & \multirow{2}{*}{$\begin{array}{l}\text { Wavenumber } \\
\left(\mathrm{cm}^{-1}\right)\end{array}$} & \multirow{2}{*}{ Type } & \multirow{2}{*}{$J_{1}$} & \multirow{2}{*}{$g_{1}$} & \multirow{2}{*}{$J_{2}$} & \multirow{2}{*}{$g_{2}$} & \multirow{2}{*}{$\Delta g$} & \multirow{2}{*}{$p$} & \multirow{2}{*}{$n$} \\
\hline & Lamp & Spark & & & & & & & & & \\
\hline 4033.776 & 500 & 8 & 24783.67 & 3 & 1 & 1.082 & 1 & 1.477 & & & \\
\hline 4032.595 & $600 \mathrm{r}$ & 200 & 24790.92 & 2 & 3 & 1.013 & 4 & 1.312 & & & \\
\hline 4029.827 & $400 \mathrm{r}$ & 15 & 24807.95 & 1 & 1 & 1.481 & 2 & 1.128 & & & \\
\hline 4029.658 & 400 & 15 & 24808.99 & 3 & 3 & 1.134 & 3 & 1.385 & & & \\
\hline 4028.334 & 200 & 10 & 24817.15 & 3 & & & & & & 0.258 & \\
\hline 4027.546 & 1501 & 20 & 24822.00 & 3 & & & & & & 0.163 & \\
\hline 4027.007 & $500 \mathrm{~b}$ & 10 & 24825.32 & 3 & 2 & 1.104 & 2 & 1.248 & & & \\
\hline 4026.159 & $150 \mathrm{~b}$ & & 24830.55 & 7 & & & & & & & 1.042 \\
\hline 4022.067 & $500 \mathrm{~b}$ & & 24855.81 & 2 & 2 & 0.780 & 3 & 1.337 & & & \\
\hline 4021.149 & 300 & 10 & 24861.49 & 7 & & & & & & & 1.297 \\
\hline 4020.354 & 500 & 20 & 24866.41 & 3 & & & & & & 0.172 & \\
\hline 4019.862 & 200 & 10 & 24869.45 & 1 & 2 & 1.229 & 3 & 0.864 & & & \\
\hline 4017.062 & 400 & 5 & 24886.78 & 1 & & & & & & & 0.485 \\
\hline 4012.495 & $2000 \mathrm{r}$ & 20 & 24915.11 & 2 & 2 & 0.856 & 3 & 1.074 & & & \\
\hline 4011.739 & $500 \mathrm{r}$ & $5 b$ & 24919.80 & 3 & & & & & & 0.206 & \\
\hline 4008.210 & $1500 \mathrm{r}$ & 100 & 24941.74 & 2 & 4 & 0.860 & 5 & 1.136 & & & \\
\hline 4005.960 & 300 & 8 & 24955.75 & 3 & 1 & 1.056 & 1 & 2.391 & & & \\
\hline 4005.091 & 500 & 10 & 24961.16 & 2 & 1 & 1.096 & 2 & 1.255 & & & \\
\hline 4003.574 & 400 & 25 & 24970.62 & 7 & & & & & & & 1.116 \\
\hline 4001.894 & 300 & 2 & 24981.10 & 2 & 2 & 0.726 & 3 & 1.073 & & & \\
\hline 4001.058 & $800 \mathrm{r}$ & 40 & 24986.33 & 3 & 4 & 0.863 & 4 & 1.083 & & & \\
\hline 4000.280 & 500 & 20 & 24991.18 & 1 & & & & & & & 0.767 \\
\hline 4000.041 & $200 \mathrm{~b}$ & & 24992.69 & 2 & & & & & 0.410 & & \\
\hline 3998.953 & 600 & 20 & 24999.48 & 3 & 4 & 1.157 & 4 & 1.322 & & & \\
\hline 3998.657 & $300 \mathrm{~s}$ & 2 & 25001.33 & 2 & & & & & & & 1.273 \\
\hline 3996.670 & 500 & 15 & 25013.76 & 3 & & & & & & 0.129 & \\
\hline 3991.731 & 800 & 40 & 25044.71 & 1 & 4 & 1.308 & 5 & 1.118 & & & \\
\hline 3990.892 & 300 & 15 & 25049.97 & 3 & & & & & & 0.991 & \\
\hline 3990.809 & 300 & 25 & 25050.50 & 7 & & & & & & & 0.062 \\
\hline 3990.492 & $800 r$ & 10 & 25052.48 & 1 & 1 & 1.476 & 2 & 0.947 & & & \\
\hline 3987.219 & $100 \mathrm{~b}$ & 25 & 25073.05 & & & & & & & & 0.862 \\
\hline 3987.206 & $200 \mathrm{~b}$ & & 25073.13 & 2 & 2 & 0.709 & 3 & 1.240 & & & \\
\hline 3984.879 & 300 & & 25087.77 & 7 & & & & & & & 0.941 \\
\hline 3982.607 & 100 & 2 & 25102.08 & 1 & & & & & 0.268 & & \\
\hline 3975.831 & 150 & 5 & 25144.86 & 1 & & & & & & & 0.764 \\
\hline 3975.468 & 200 & 20 & 25147.16 & 7 & & & & & & & 0.850 \\
\hline 3974.829 & 200 & 4 & 25151.20 & 2 & & & & & 0.360 & & \\
\hline 3972.640 & 500 & 75 & 25165.06 & 2 & & & & & & & 1.772 \\
\hline 3972.153 & $1500 \mathrm{r}$ & 300 & 25168.14 & 2 & & & & & 0.350 & & \\
\hline 3971.136 & 100 & 4 & 25174.59 & 2 & & & & & & & 1.336 \\
\hline 3969.665 & 300 & 15 & 25183.92 & 7 & & & & & & & 1.025 \\
\hline 3967.797 & 100 & 3 & 25195.78 & 1 & 5 & 1.380 & 6 & 1.150 & & & \\
\hline 3967.392 & $2000 \mathrm{r}$ & 300 & 25198.35 & 3 & & & & & & 0.393 & \\
\hline 3966.334 & 200 & 4 & 25205.07 & 3 & & & & & & 0.918 & \\
\hline 3965.483 & 75 & 3 & 25210.48 & 3 & & & & & & 0.219 & \\
\hline 3964.329 & 200 & 3 & 25217.81 & 7 & & & & & & & 1.066 \\
\hline 3964.030 & 300 & 15 & 25219.72 & 3 & & & & & & 0.550 & \\
\hline 3962.420 & $400 \mathrm{r}$ & 15 & 25229.97 & 3 & 2 & 0.950 & 2 & 1.253 & & & \\
\hline 3960.879 & 300 & 10 & 25239.78 & 7 & & & & & & & 1.110 \\
\hline 3960.270 & 500 & 50 & 25243.66 & 1 & 2 & 1.275 & 3 & 1.005 & & & \\
\hline 3959.300 & $1000 \mathrm{r}$ & 100 & 25249.84 & 2 & 1 & 0.067 & 2 & 0.927 & & & \\
\hline 3958.928 & 200 & 3 & 25252.22 & 3 & & & & & & 0.349 & \\
\hline 3957.798 & 100 & 2 & 25259.42 & 3 & & & & & & 0.381 & \\
\hline 3957.595 & $20 \mathrm{~h}$ & 5 & 25260.72 & 7 & & & & & & & 2.414 \\
\hline 3957.059 & 100 & 20 & 25264.15 & 3 & & & & & & 0.185 & \\
\hline
\end{tabular}


TABLE 3. Zeeman effect of Th $\mathrm{I}$-continued

\begin{tabular}{|c|c|c|c|c|c|c|c|c|c|c|c|}
\hline \multirow{2}{*}{$\begin{array}{l}\text { Wavelength } \\
\text { (§) }\end{array}$} & \multicolumn{2}{|c|}{ Intensity } & \multirow{2}{*}{$\begin{array}{l}\text { Wavenumber } \\
\qquad\left(\mathrm{cm}^{-1}\right)\end{array}$} & \multirow{2}{*}{ Type } & \multirow{2}{*}{$J_{1}$} & \multirow{2}{*}{$g_{1}$} & \multirow{2}{*}{$J_{2}$} & \multirow{2}{*}{$g_{2}$} & \multirow{2}{*}{$\Delta g$} & \multirow{2}{*}{$p$} & \multirow{2}{*}{$n$} \\
\hline & Lamp & Spark & & & & & & & & & \\
\hline 3956.481 & 150 & 20 & 25267.83 & 7 & & & & & 0.284 & & \\
\hline 3956.005 & 400 & 10 & 25270.87 & 1 & & & & & & & 0.094 \\
\hline 3955.891 & 400 & $50 \mathrm{~b}$ & 25271.60 & 3 & & & & & & 0.570 & \\
\hline 3955.170 & $800 \mathrm{r}$ & 40 & 25276.21 & 3 & 5 & 1.095 & 5 & 1.361 & & & \\
\hline 3954.727 & 150 & 2 & 25279.04 & 3 & & & & & & 0.321 & \\
\hline 3954.130 & 100 & 20 & 25282.86 & 3 & & & & & & 0.228 & \\
\hline 3953.338 & 400 & 20 & 25287.92 & 7 & & & & & & & 0.407 \\
\hline 3952.761 & $500 \mathrm{r}$ & 10 & 25291.62 & 3 & 1 & 0.876 & 1 & 1.486 & & & \\
\hline 3950.804 & 300 & 8 & 25304.14 & 1 & & & & & 0.480 & & \\
\hline 3950.393 & $1000 \mathrm{r}$ & 200 & 25306.77 & 3 & 2 & 0.742 & 2 & 1.166 & & & \\
\hline 3949.975 & 50 & 2 & 25309.45 & 1 & & & & & & & 0.850 \\
\hline 3948.133 & 500 & 40 & 25321.26 & 7 & & & & & 0.330 & & \\
\hline 3948.029 & $600 \mathrm{r}$ & 15 & 25321.92 & 2 & 2 & 0.727 & 3 & 1.383 & & & \\
\hline 3947.329 & $1000 \mathrm{r}$ & 100 & 25326.42 & 2 & 4 & 1.213 & 5 & 1.359 & & & \\
\hline 3946.481 & 400 & 10 & 25331.86 & 3 & 1 & 1.147 & 1 & 1.485 & & & \\
\hline 3946.391 & 300 & 25 & 25332.44 & 7 & & & & & & & 1.281 \\
\hline 3945.206 & 200 & 8 & 25340.04 & 1 & 3 & 1.118 & 4 & 0.882 & & & \\
\hline 3944.253 & 500 & 20 & 25346.17 & 3 & 2 & 1.067 & 2 & 1.701 & & & \\
\hline 3943.605 & 150 & 25 & 25350.33 & 7 & & & & & 0.170 & & \\
\hline 3942.072 & $500 \mathrm{~b}$ & 50 & 25360.19 & 7 & & & & & & & 0.838 \\
\hline 3941.137 & 100 & 3 & 25366.21 & 2 & & & & & & & 1.953 \\
\hline 3940.837 & 200 & 5 & 25368.14 & 2 & & & & & & & 1.309 \\
\hline 3940.705 & 200 & 4 & 25368.99 & 3 & & & & & & 0.794 & \\
\hline 3939.538 & 75 & 2 & 25376.50 & 3 & & & & & & 0.283 & \\
\hline 3939.318 & 150 & 8 & 25377.92 & 2 & & & & & & & 1.150 \\
\hline 3938.614 & $400 \mathrm{~b}$ & 150 & 25382.46 & 7 & & & & & 0.220 & & \\
\hline 3934.274 & $400 \mathrm{~b}$ & 50 & $25 \cdot, 10.46$ & 3 & & & & & & 0.510 & \\
\hline 3934.060 & 150 & 3 & 25411.84 & 1 & 2 & 1.198 & 3 & 1.008 & & & \\
\hline 3933.970 & 150 & 5 & 25412.42 & 3 & 1 & 0.812 & 1 & 1.324 & & & \\
\hline 3933.238 & 400 & 40 & 25417.15 & 1 & & & & & 0.202 & & \\
\hline 3932.911 & $1500 \mathrm{r}$ & 200 & 25419.26 & 2 & 4 & 0.876 & 5 & 1.028 & & & \\
\hline 3931.085 & 150 & 3 & 25431.07 & 3 & & & & & & 1.241 & \\
\hline 3930.658 & 100 & 3 & 25433.83 & 7 & & & & & & & 1.061 \\
\hline 3930.331 & 150 & 20 & 25435.95 & 7 & & & & & & & 1.087 \\
\hline 3928.865 & 300 & 50 & 25445.44 & 3 & & & & & & 0.432 & \\
\hline 3928.308 & 100 & 10 & 25449.05 & 3 & & & & & & 0.476 & \\
\hline 3927.806 & 400 & 10 & 25452.30 & 2 & 2 & 1.124 & 3 & 1.268 & & & \\
\hline 3926.864 & 200 & 8 & 25458.40 & 1 & & & & & 0.350 & & \\
\hline 3926.056 & 20 & 1 & 25463.65 & 3 & & & & & & 0.243 & \\
\hline 3925.738 & 100 & 5 & 25465.70 & 3 & & & & & & 0.247 & \\
\hline 3925.219 & 600 & 50 & 25469.08 & 3 & & & & & & 1.010 & \\
\hline 3924.404 & 5001 & 10 & 25474.36 & 7 & & & & & & & 1.043 \\
\hline 3923.799 & $500 \mathrm{r}$ & 20 & 25478.29 & 1 & 2 & 1.543 & 3 & 1.083 & & & \\
\hline 3922.747 & 50 & 2 & 25485.12 & 7 & & & & & & & 1.320 \\
\hline 3922.394 & 100 & 8 & 25487.42 & 1 & 4 & 1.169 & 5 & 1.006 & & & \\
\hline 3922.218 & 50 & 50 & 25488.56 & 2 & & & & & 0.160 & & 1.504 \\
\hline 3921.453 & 300 & 15 & 25493.53 & 1 & 1 & 1.248 & 2. & 0.966 & & & \\
\hline 3920.442 & 300 & 15 & 25500.11 & 7 & & & & & & & 1.024 \\
\hline 3920.058 & 300 & 10 & 25502.61 & 1 & & & & & 0.240 & & \\
\hline 3919.980 & 300 & 10 & 25503.11 & 7 & & & & & & & 1.145 \\
\hline 3918.934 & 300 & 8 & 25509.92 & 3 & & & & & & 0.243 & \\
\hline 3918.498 & 300 & 300 & 25512.76 & 2 & 1 & 0.060 & 2 & 1.322 & & & \\
\hline 3918.070 & 300 & 40 & 25515.54 & 3 & & & & & & 0.307 & \\
\hline 3916.417 & $800 \mathrm{r}$ & 25 & 25526.31 & 1 & 1 & 1.066 & 2 & 0.738 & & & \\
\hline 3915.848 & 200 & 15 & 25530.02 & 1 & 1 & 1.410 & 2 & 0.777 & & & \\
\hline
\end{tabular}


TABLE 3. Zeeman effect of $\mathrm{Th} \mathrm{I}$-continued

\begin{tabular}{|c|c|c|c|c|c|c|c|c|c|c|c|}
\hline \multirow{2}{*}{$\begin{array}{c}\text { Wavelength } \\
(\AA)\end{array}$} & \multicolumn{2}{|c|}{ Intensity } & \multirow{2}{*}{$\begin{array}{l}\text { Wavenumber } \\
\left(\mathrm{cm}^{-1}\right)\end{array}$} & \multirow{2}{*}{ Type } & \multirow{2}{*}{$J_{1}$} & \multirow{2}{*}{$g_{1}$} & \multirow{2}{*}{$J_{2}$} & \multirow{2}{*}{$g_{2}$} & \multirow{2}{*}{$\Delta g$} & \multirow{2}{*}{$p$} & \multirow{2}{*}{$n$} \\
\hline & Lamp & Spark & & & & & & & & & \\
\hline 3915.295 & 300 & 8 & 25533.63 & 3 & & & & & & 0.106 & \\
\hline 3914.291 & 50 & 3 & 25540.18 & 7 & & & & & & & 1.537 \\
\hline 3913.865 & 100 & 10 & 25542.96 & 7 & & & & & & & 1.109 \\
\hline 3913.823 & 75 & 20 & 25543.23 & 3 & & & & & & 0.611 & \\
\hline 3913.645 & 2001 & 5 & 25544.39 & 3 & & & & & & 0.823 & \\
\hline 3913.082 & 300 & 25 & 25548.07 & 1 & 1 & 2.398 & 2 & 1.067 & & & \\
\hline 3911.950 & $300 \mathrm{~b}$ & 25 & 25555.46 & 7 & & & & & & & 1.101 \\
\hline 3911.910 & $500 \mathrm{r}$ & 40 & 25555.72 & 3 & 4 & 1.223 & 4 & 1.446 & & & \\
\hline 3910.978 & $400 \mathrm{~b}$ & 10 & 25561.81 & 1 & & & & & 0.080 & & \\
\hline 3910.773 & 300 & 8 & 25563.15 & 2 & & & & & 0.210 & & \\
\hline 3910.251 & 50 & 2 & 25566.56 & 3 & & & & & & 0.268 & \\
\hline 3909.991 & 100 & 8 & 25568.26 & 3 & & & & & & 0.623 & \\
\hline 3909.139 & 300 & 20 & 25573.84 & 2 & 2 & 0.968 & 3 & 1.238 & & & \\
\hline 3908.979 & 300 & 25 & 25574.88 & 1 & & & & & & & 0.626 \\
\hline 3908.749 & 500 & 15 & 25576.39 & 7 & & & & & & & 1.118 \\
\hline 3908.031 & 100 & 2 & 25581.09 & 7 & & & & & & & 1.002 \\
\hline 3907.543 & 50 & 5 & 25584.28 & 3 & & & & & & 0.420 & \\
\hline 3907.160 & 200 & 10 & 25586.79 & 3 & & & & & & 0.362 & \\
\hline 3906.387 & 50 & 5 & 25591.85 & 7 & & & & & & & 1.055 \\
\hline 3906.308 & 50 & 3 & 25592.37 & 3 & & & & & & 0.686 & \\
\hline 3905.298 & 100 & 2 & 25598.99 & 7 & & & & & & & 1.146 \\
\hline 3903.916 & 200 & 5 & 25608.05 & 3 & & & & & & 0.344 & \\
\hline 3903.103 & $1000 \mathrm{r}$ & 150 & 25613.39 & 1 & 3 & 1.113 & 4 & 0.856 & & & \\
\hline 3902.788 & 200 & 10 & 25615.45 & 7 & & & & & & & 1.274 \\
\hline 3902.171 & 500 & 50 & 25619.50 & 7 & & & & & & & 0.958 \\
\hline 3901.662 & 500 & 15 & 25622.85 & 3 & 4 & 0.862 & 4 & 1.121 & & & \\
\hline 3900.577 & $1000 \mathrm{r}$ & 50 & 25629.97 & 2 & & & & & & & 1.595 \\
\hline 3899.019 & 300 & 75 & 25640.21 & 3 & & & & & & 0.948 & \\
\hline 3898.795 & 300 & 100 & 25641.69 & 3 & & & & & & 0.559 & \\
\hline 3898.509 & $300 \mathrm{~b}$ & 100 & 25643.57 & 2 & & & & & & & 1.139 \\
\hline 3897.778 & 200 & 5 & 25648.37 & 3 & & & & & & 0.256 & \\
\hline 3897.612 & 100 & 3 & 25649.47 & 7 & & & & & & & 1.169 \\
\hline 3897.316 & 100 & 2 & 25651.41 & 7 & & & & & & & 0.907 \\
\hline 3896.168 & 100 & 2 & 25658.98 & 7 & & & & & & & 1.029 \\
\hline 3895.418 & $2000 \mathrm{r}$ & 200 & 25663.91 & 3 & & & & & & 0.305 & \\
\hline 3894.022 & 40 & 2 & 25673.11 & 3 & & & & & & 0.242 & \\
\hline 3893.817 & 200 & 8 & 25674.47 & 3 & & & & & & 0.163 & \\
\hline 3893.651 & 300 & 40 & 25675.56 & 3 & & & & & & 0.355 & \\
\hline 3893.344 & 300 & 25 & 25677.59 & 2 & & & & & 0.268 & & \\
\hline 3892.708 & 200 & 8 & 25681.78 & 3 & & & & & & 0.448 & \\
\hline 3891.725 & 400 & 10 & 25688.27 & 7 & & & & & & & 0.993 \\
\hline 3889.906 & 300 & 4 & 25700.28 & 3 & & & & & & 0.188 & \\
\hline 3889.542 & 200 & 8 & 25702.68 & 2 & & & & & & & 1.344 \\
\hline 3888.242 & 20 & 1 & 25711.28 & 3 & & & & & & 0.257 & \\
\hline 3888.070 & 15 & 5 & 25712.41 & 7 & & & & & & & 1.041 \\
\hline 3887.509 & 300 & 4 & 25716.12 & 7 & & & & & & & 1.208 \\
\hline 3887.019 & $800 \mathrm{r}$ & 100 & 25719.37 & 2 & & & & & & & 1.277 \\
\hline 3886.917 & $800 \mathrm{r}$ & 500 & 25720.04 & 3 & & & & & & 0.238 & \\
\hline 3886.256 & 300 & 8 & 25724.42 & 7 & & & & & & & 0.600 \\
\hline 3886.064 & 75 & 25 & 25725.69 & 7 & & & & & 0.280 & & \\
\hline 3885.412 & 25 & 4 & 25730.00 & 3 & & & & & & 0.548 & \\
\hline 3885.224 & 200 & 5 & 25731.25 & 7 & & & & & 0.433 & & \\
\hline 3883.767 & 200 & 15 & 25740.90 & 2 & & & & & & & 1.696 \\
\hline 3883.536 & 200 & 5 & 25742.44 & 3 & & & & & & 0.933 & \\
\hline 3882.853 & 25 & 1 & 25746.96 & 7 & & & & & & & 1.019 \\
\hline
\end{tabular}


TABLE 3. Zeeman effect of Th $\mathrm{I}$-continued

\begin{tabular}{|c|c|c|c|c|c|c|c|c|c|c|c|}
\hline \multirow{2}{*}{$\begin{array}{l}\text { Wavelength } \\
(\AA)\end{array}$} & \multicolumn{2}{|c|}{ Intensity } & \multirow{2}{*}{$\begin{array}{l}\text { Wavenumber } \\
\qquad\left(\mathrm{cm}^{-1}\right)\end{array}$} & \multirow{2}{*}{ Type } & \multirow{2}{*}{$J_{1}$} & \multirow{2}{*}{$g_{1}$} & \multirow{2}{*}{$J_{2}$} & \multirow{2}{*}{$g_{2}$} & \multirow{2}{*}{$\Delta g$} & \multirow{2}{*}{$p$} & \multirow{2}{*}{$n$} \\
\hline & Lamp & Spark & & & & & & & & & \\
\hline 3882.324 & 200 & 8 & 25750.47 & 2 & & & & & & & 1.310 \\
\hline 3881.792 & 100 & 2 & 25754.00 & 7 & & & & & 0.243 & & \\
\hline 3881.043 & 100 & 2 & 25758.97 & 1 & & & & & & 0.485 & 0.662 \\
\hline 3880.545 & 8 & 1 & 25762.27 & 3 & & & & & & 0.126 & \\
\hline 3880.323 & 100 & 3 & 25763.75 & 2 & & & & & 0.320 & & \\
\hline 3880.194 & 300 & 15 & 25764.61 & 7 & & & & & & & 1.116 \\
\hline 3879.644 & $1000 \mathrm{r}$ & 200 & 25768.26 & 1 & & & & & & & 0.987 \\
\hline 3879.269 & 400 & 25 & 25770.75 & 2 & & & & & 0.266 & & \\
\hline 3878.662 & 400 & 150 & 25774.78 & 3 & 1 & 0.946 & 1 & 1.484 & & & \\
\hline 3878.160 & 100 & 3 & 25778.12 & 7 & & & & & & & 1.121 \\
\hline 3877.610 & $300 \mathrm{~b}$ & 15 & 25781.77 & 3 & & & & & & 0.160 & \\
\hline 3877.462 & 500 & 20 & 25782.75 & 2 & & & & & & & 1.720 \\
\hline 3874.977 & 400 & 50 & 25799.29 & 7 & & & & & & & 1.208 \\
\hline 3873.821 & $2000 \mathrm{r}$ & 500 & 25806.99 & 7 & & & & & & & 1.085 \\
\hline 3873.473 & $500 \mathrm{r}$ & 50 & 25809.31 & 1 & 1 & 1.574 & 2 & 0.744 & & & \\
\hline 3872.288 & 20 & 1 & 25817.21 & 7 & & & & & & & 1.040 \\
\hline 3870.891 & 75 & & 25826.53 & 7 & & & & & & & 1.062 \\
\hline 3870.682 & 40 & 2 & 25827.92 & 7 & & & & & & & 1.230 \\
\hline 3870.178 & 50 & 1 & 25831.28 & 7 & & & & & & & 0.707 \\
\hline 3869.663 & $800 \mathrm{r}$ & 50 & 25834.72 & 7 & & & & & & & 1.378 \\
\hline 3868.389 & 200 & 2 & 25843.23 & 3 & & & & & & 0.264 & \\
\hline 3868.254 & 400 & 10 & 25844.13 & 2 & & & & & 0.191 & & \\
\hline 3866.907 & $500 \mathrm{~b}$ & 50 & 25853.13 & 1 & 2 & 1.151 & 3 & 1.004 & & & \\
\hline 3866.771 & 200 & 2 & 25854.04 & 1 & & & & & 0.570 & & \\
\hline 3866.375 & 100 & 2 & 25856.69 & 3 & & & & & & 0.136 & \\
\hline 3864.837 & 200 & 25 & 25866.98 & 1 & & & & & & & 0.539 \\
\hline 3862.654 & $200 \mathrm{~b}$ & 200 & 25881.28 & 7 & & & & & 0.150 & & \\
\hline 3862.419 & $500 \mathrm{~b}$ & $250 \mathrm{~b}$ & 25883.17 & 3 & & & & & & 0.559 & \\
\hline 3861.574 & $300 \mathrm{~b}$ & 40 & 25888.84 & 1 & & & & & & & 0.392 \\
\hline 3861.502 & $300 \mathrm{~b}$ & 40 & 25889.32 & 3 & & & & & & 0.371 & \\
\hline 3861.051 & 200 & 2 & 25892.34 & 3 & & & & & & 0.432 & \\
\hline 3860.657 & 200 & 10 & 25894.98 & 3 & & & & & & 0.240 & \\
\hline 3859.466 & 40 & 5 & 25902.98 & 7 & & & & & & & 0.934 \\
\hline 3858.358 & 200 & 150 & 25910.42 & 3 & 3 & 1.057 & 3 & 1.312 & & & \\
\hline 3857.450 & 75 & 8 & 25916.51 & 7 & & & & & & & 1.161 \\
\hline 3856.753 & 40 & 2 & 25921.20 & 3 & & & & & & 0.350 & \\
\hline 3856.622 & 200 & 15 & 25922.07 & 7 & & & & & & & 0.630 \\
\hline 3856.354 & 200 & 40 & 25923.88 & 2 & 2 & 0.781 & 3 & 1.050 & & & \\
\hline 3855.064 & 25 & 4 & 25932.55 & 3 & & & & & & 0.640 & \\
\hline 3854.289 & 100 & 5 & 25937.77 & 3 & & & & & & 0.208 & \\
\hline 3853.825 & $150 \mathrm{~b}$ & 15 & 25940.89 & 3 & & & & & & 1.226 & \\
\hline 3852.719 & 75 & 2 & 25948.34 & 7 & & & & & & & 1.254 \\
\hline 3852.135 & $800 \mathrm{r}$ & 50 & 25952.27 & 2 & 1 & 0.954 & 2 & 1.258 & & & \\
\hline 3851.155 & 300 & 15 & 25958.87 & 1 & & & & & 0.410 & & \\
\hline 3850.690 & 50 & 2 & 25962.01 & 1 & 3 & 1.280 & 4 & 0.960 & & & \\
\hline 3848.627 & 75 & 3 & 25975.92 & 3 & & & & & & 0.300 & \\
\hline 3847.617 & 500 & 10 & 25982.74 & 7 & & & & & & & 0.985 \\
\hline 3846.886 & $1000 \mathrm{r}$ & 100 & 25987.68 & 1 & 1 & 1.480 & 2 & 0.908 & & & \\
\hline 3846.625 & 400 & 50 & 25989.44 & 3 & & & & & & 0.116 & \\
\hline 3845.092 & 400 & 10 & 25999.81 & 2 & & & & & & & 1.244 \\
\hline 3844.933 & 400 & 15 & 26000.88 & 2 & & & & & & & 1.401 \\
\hline 3844.571 & 50 & 1 & 26003.33 & 7 & & & & & & & 1.140 \\
\hline 3843.507 & 200 & 8 & 26010.52 & 1 & & & & & 0.340 & & \\
\hline 3842.898 & $400 r$ & $75 b$ & 26014.65 & 3 & & & & & & 0.074 & \\
\hline 3840.613 & 200 & 75 & 26030.12 & 7 & & & & & 0.304 & & \\
\hline
\end{tabular}


TABLE 3. Zeeman effect of $\mathrm{Th} \mathrm{I}$-continued

\begin{tabular}{|c|c|c|c|c|c|c|c|c|c|c|c|}
\hline \multirow{2}{*}{$\begin{array}{l}\text { Wavelength } \\
(\AA)\end{array}$} & \multicolumn{2}{|c|}{ Intensity } & \multirow{2}{*}{$\begin{array}{c}\text { Wavenumber } \\
\left(\mathrm{cm}^{-1}\right)\end{array}$} & \multirow{2}{*}{ Type } & \multirow{2}{*}{$J_{1}$} & \multirow{2}{*}{$g_{1}$} & \multirow{2}{*}{$J_{2}$} & \multirow{2}{*}{$g_{2}$} & \multirow{2}{*}{$\Delta g$} & \multirow{2}{*}{$p$} & \multirow{2}{*}{$n$} \\
\hline & Lamp & Spark & & & & & & & & & \\
\hline 3840.430 & 300 & 250 & 26031.37 & 7 & & & & & & & 1.284 \\
\hline 3838.801 & $50 \mathrm{~b}$ & $4 \mathrm{~b}$ & 26042.42 & 3 & & & & & & 0.115 & \\
\hline 3837.343 & $200 \mathrm{~b}$ & 5 & 26052.31 & 7 & & & & & & & 0.979 \\
\hline 3837.024 & 200 & 3 & 26054.47 & 7 & & & & & & & 0.604 \\
\hline 3836.584 & $800 \mathrm{r}$ & 100 & 26057.46 & 7 & & & & & & & 1.211 \\
\hline 3836.542 & $1000 \mathrm{r}$ & $150 \mathrm{~b}$ & 26057.75 & 7 & & & & & & & 1.009 \\
\hline 3836.213 & 100 & 4 & 26059.98 & 3 & & & & & & 0.189 & \\
\hline 3835.414 & 200 & 10 & 26065.41 & 7 & & & & & & & 1.000 \\
\hline 3834.890 & 100 & 5 & 26068.97 & 7 & & & & & & & 0.707 \\
\hline 3834.488 & 100 & 75 & 26071.51 & 7 & & & & & & & 1.168 \\
\hline 3833.087 & 400 & 75 & 26081.24 & 3 & & & & & & 0.213 & \\
\hline 3832.583 & 200 & 3 & 26084.67 & 2 & & & & & 0.430 & & \\
\hline 3832.323 & 75 & $3 b$ & 26086.43 & 7 & & & & & 0.240 & & \\
\hline 3831.640 & 300 & 50 & 26091.08 & 7 & & & & & & & 1.111 \\
\hline 3830.774 & $800 \mathrm{r}$ & 150 & 26096.98 & 2 & 2 & 0.738 & 3 & 1.004 & & & \\
\hline 3829.040 & 200 & 4 & 26108.80 & 3 & & & & & & 0.250 & \\
\hline 3828.385 & $3000 \mathrm{r}$ & 300 & 26113.27 & 3 & 2 & 0.734 & 2 & 0.978 & & & \\
\hline 3827.502 & 100 & 2 & 26119.29 & 2 & 1 & 0.733 & 2 & 1.083 & & & \\
\hline 3826.368 & $400 \mathrm{r}$ & 75 & 26127.03 & 1 & 2 & 1.205 & 3 & 0.866 & & & \\
\hline 3825.515 & 200 & 25 & 26132.85 & 3 & & & & & & 0.127 & \\
\hline 3824.553 & $100 \mathrm{~b}$ & $10 \mathrm{~b}$ & 26139.43 & 7 & & & & & & & 1.201 \\
\hline 3824.534 & $400 \mathrm{~b}$ & $15 \mathrm{~b}$ & 26139.56 & 7 & & & & & & & 1.188 \\
\hline 3823.989 & 75 & 3 & 26143.29 & 3 & 1 & 0.696 & 1 & 1.096 & & & \\
\hline 3823.067 & $500 \mathrm{r}$ & 100 & 26149.59 & 3 & 1 & 0.062 & 1 & 1.157 & & & \\
\hline 3822.709 & 300 & 25 & 26152.04 & 7 & & & & & 0.620 & & \\
\hline 3820.792 & $600 \mathrm{r}$ & 75 & 26165.16 & 3 & 2 & 0.915 & 2 & 1.248 & & & \\
\hline 3820.327 & 200 & 2 & 26168.34 & 7 & & & & & & & 0.836 \\
\hline 3819.904 & 500 & $100 \mathrm{~b}$ & 26171.24 & 7 & & & & & & & 0.927 \\
\hline 3819.189 & 200 & 10 & 26176.14 & 7 & & & & & & & 1.060 \\
\hline 3818.685 & $400 \mathrm{r}$ & 15 & 26179.60 & 1 & 4 & 1.204 & 5 & 0.939 & & & \\
\hline 3817.478 & 400 & 40 & 26187.87 & 7 & & & & & & & 0.990 \\
\hline 3817.117 & 300 & 8 & 26190.35 & 1 & 2 & 1.579 & 3 & 1.139 & & & \\
\hline 3816.166 & 300 & 10 & 26196.87 & 1 & & & & & & & 0.696 \\
\hline 3815.566 & 500 & 50 & 26201.00 & 3 & 2 & 0.772 & 2 & 1.099 & & & \\
\hline 3813.815 & $500 \mathrm{r}$ & 50 & 26213.02 & 3 & 2 & 0.718 & 2 & 1.008 & & & \\
\hline 3812.667 & 75 & 5 & 26220.92 & 7 & & & & & & & 1.272 \\
\hline 3812.398 & 400 & 40 & 26222.76 & 3 & & & & & & 0.404 & \\
\hline 3812.044 & 40 & 2 & 26225.20 & 3 & & & & & & & 1.612 \\
\hline 3811.761 & 75 & 2 & 26227.15 & 3 & & & & & & 0.255 & \\
\hline 3810.995 & $600 \mathrm{r}$ & 150 & 26232.42 & 7 & & & & & & & 1.202 \\
\hline 3810.295 & 15 & 5 & 26237.24 & 3 & & & & & & 0.372 & \\
\hline 3808.614 & 500 & 20 & 26248.82 & 3 & & & & & & 0.267 & \\
\hline 3807.273 & 400 & 50 & 26258.07 & 2 & 4 & 1.100 & 5 & 1.369 & & & \\
\hline 3806.317 & 100 & 1 & 26264.66 & 3 & & & & & & 0.459 & \\
\hline 3805.010 & 75 & & 26273.68 & 2 & 2 & 1.052 & 3 & 1.262 & & & \\
\hline 3804.699 & 500 & 50 & 26275.83 & 1 & & & & & & & 0.821 \\
\hline 3804.127 & 400 & 20 & 26279.78 & 3 & & & & & & 0.378 & \\
\hline 3803.985 & $600 \mathrm{r}$ & 150 & 26280.76 & 1 & 4 & 1.316 & 5 & 1.106 & & & \\
\hline 3803.839 & 400 & 25 & 26281.77 & 3 & & & & & & 0.694 & \\
\hline 3801.571 & $400 \mathrm{r}$ & 100 & 26297.45 & 7 & & & & & & & 0.902 \\
\hline 3799.386 & 300 & 8 & 26312.57 & 7 & & & & & & & 1.029 \\
\hline 3798.432 & 200 & 75 & 26319.22 & 1 & & & & & & & 0.582 \\
\hline 3797.205 & 200 & 10 & 26327.68 & 3 & 5 & 1.072 & 5 & 1.372 & & & \\
\hline 3796.687 & $400 \mathrm{~b}$ & 20 & 26331.27 & 7 & & & & & & & 1.111 \\
\hline 3796.052 & 200 & 3 & 26335.68 & 3 & & & & & & 0.717 & \\
\hline
\end{tabular}


TABLE 3. Zeeman effect of Th $\mathrm{I}$-continued

\begin{tabular}{|c|c|c|c|c|c|c|c|c|c|c|c|}
\hline \multirow{2}{*}{$\begin{array}{c}\text { Wavelength } \\
\text { (̊) }\end{array}$} & \multicolumn{2}{|c|}{ Intensity } & \multirow{2}{*}{$\begin{array}{l}\text { Wavenumber } \\
\qquad\left(\mathrm{cm}^{-1}\right)\end{array}$} & \multirow{2}{*}{ Type } & \multirow{2}{*}{$J_{1}$} & \multirow{2}{*}{$g_{1}$} & \multirow{2}{*}{$J_{2}$} & \multirow{2}{*}{$g_{2}$} & \multirow{2}{*}{$\Delta g$} & \multirow{2}{*}{$p$} & \multirow{2}{*}{$n$} \\
\hline & Lamp & Spark & & & & & & & & & \\
\hline 3795.386 & 600 & 100 & 26340.30 & 7 & & & & & & & 1.087 \\
\hline 3794.981 & 200 & 3 & 26343.11 & 1 & & & & & 0.387 & & \\
\hline 3794.697 & $1500 \mathrm{r}$ & 100 & 26345.09 & 3 & & & & & & 0.341 & \\
\hline 3793.815 & 300 & 25 & 26351.21 & 7 & & & & & & & 1.078 \\
\hline 3793.504 & 200 & & 26353.55 & 7 & & & & & & & 1.424 \\
\hline 3793.097 & 300 & 10 & 26356.20 & 3 & & & & & & 0.181 & \\
\hline 3792.730 & 4001 & 75 & 26358.74 & 2 & 3 & 0.873 & 4 & 1.048 & & & \\
\hline 3792.371 & $1000 \mathrm{r}$ & 100 & 26361.24 & 7 & & & & & & & 0.986 \\
\hline 3791.517 & 300 & 10 & 26367.18 & 2 & & & & & & & 1.649 \\
\hline 3790.794 & $1500 \mathrm{r}$ & 200 & 26372.21 & 1 & 3 & 1.032 & 4 & 0.860 & & & \\
\hline 3790.356 & 300 & 40 & 26375.26 & 7 & & & & & & & 0.668 \\
\hline 3787.638 & 300 & 40 & 26394.18 & 1 & & & & & & & 0.689 \\
\hline 3787.218 & 75 & 2 & 26397.11 & 7 & & & & & 0.400 & & \\
\hline 3784.793 & 300 & 15 & 26414.02 & 1 & & & & & 0.570 & & \\
\hline 3784.574 & 400 & 20 & 26415.55 & 2 & & & & & & & 1.456 \\
\hline 3784.138 & 50 & 2 & 26418.60 & 3 & & & & & & 0.736 & \\
\hline 3782.338 & 15 & 1 & 26431.17 & 7 & & & & & & & 1.134 \\
\hline 3781.318 & 300 & 75 & 26438.30 & 3 & & & & & & 0.216 & \\
\hline 3780.966 & $300 \mathrm{r}$ & $20 \mathrm{~b}$ & 26440.76 & 7 & & & & & & & 0.960 \\
\hline 3780.147 & 200 & 8 & 26446.48 & 3 & & & & & & 0.708 & \\
\hline 3779.561 & 200 & 15 & 26450.59 & 2 & 4 & 1.023 & 5 & 1.208 & & & \\
\hline 3778.498 & 300 & 2 & 26458.03 & 1 & & & & & & & 0.270 \\
\hline 3778.045 & 300 & 15 & 26461.20 & 7 & & & & & & & 0.978 \\
\hline 3777.745 & 200 & 40 & 26463.30 & 1 & & & & & 0.282 & & \\
\hline 3777.415 & 300 & 40 & 26465.61 & 3 & 3 & 0.794 & 3 & 1.254 & & & \\
\hline 3777.184 & 400 & 8 & 26467.23 & 3 & & & & & & 0.928 & \\
\hline 3776.622 & 300 & $40 \mathrm{~b}$ & 26471.17 & 3 & & & & & & 0.242 & \\
\hline 3776.502 & 300 & 8 & 26472.01 & 7 & & & & & & & 0.722 \\
\hline 3776.271 & $600 \mathrm{r}$ & 150 & 26473.63 & 1 & & & & & 0.141 & & \\
\hline 3775.423 & 300 & 15 & 26479.58 & 2 & & & & & & & 1.278 \\
\hline 3774.732 & 300 & 10 & 26484.42 & 7 & & & & & & & 1.070 \\
\hline 3773.306 & 200 & 8 & 26494.43 & 3 & & & & & & 0.376 & \\
\hline 3772.649 & 400 & 10 & 26499.04 & 1 & 3 & 1.250 & 4 & 1.078 & & & \\
\hline 3771.370 & $1500 \mathrm{r}$ & 100 & 26508.03 & 2 & 2 & 0.734 & 3 & 1.074 & & & \\
\hline 3770.722 & 100 & 2 & 26512.59 & 3 & & & & & & 0.590 & \\
\hline 3770.056 & $1500 \mathrm{r}$ & 200 & 26517.27 & 3 & 1 & 0.053 & 1 & 0.783 & & & \\
\hline 3768.047 & 200 & 8 & 26531.41 & 1 & 1 & 1.295 & 2 & 0.769 & & & \\
\hline 3767.471 & 100 & 2 & 26535.46 & 3 & & & & & & 0.312 & \\
\hline 3766.447 & 300 & 10 & 26542.68 & 7 & & & & & & & 1.048 \\
\hline 3765.412 & $400 \mathrm{r}$ & 50 & 26549.98 & 1 & 2 & 1.686 & 3 & 1.084 & & & \\
\hline 3764.193 & 200 & 8 & 26558.57 & 2 & 1 & 0.727 & 2 & 1.101 & & & \\
\hline 3763.668 & 300 & 10 & 26562.28 & 7 & & & & & 0.104 & & \\
\hline 3762.257 & 200 & 8 & 26572.24 & 7 & & & & & & & 1.101 \\
\hline 3761.704 & 200 & 20 & 26576.14 & 7 & & & & & & & 1.421 \\
\hline 3761.470 & 200 & 25 & 26577.80 & 2 & 4 & 1.062 & 5 & 1.362 & & & \\
\hline 3760.271 & $50 \mathrm{~b}$ & $25 b$ & 26586.27 & 3 & & & & & 0.130 & & \\
\hline 3758.706 & 400 & 20 & 26597.34 & 2 & 1 & 0.062 & 2 & 1.078 & & & \\
\hline 3758.467 & $400 \mathrm{r}$ & 25 & 26599.03 & 7 & & & & & & & 0.879 \\
\hline 3757.694 & $1000 \mathrm{r}$ & 200 & 26604.51 & 7 & & & & & & & 0.871 \\
\hline 3756.451 & 200 & 8 & 26613.30 & 1 & & & & & 0.530 & & \\
\hline 3756.293 & 400 & 25 & 26614.43 & 7 & & & & & & & 1.019 \\
\hline 3755.986 & 400 & 50 & 26616.60 & 3 & & & & & & 0.296 & \\
\hline 3755.211 & $800 \mathrm{r}$ & 250 & 26622.09 & 7 & & & & & & & 1.017 \\
\hline 3754.030 & $500 \mathrm{r}$ & 50 & 26630.47 & 2 & 2 & 0.780 & 3 & 1.091 & & & \\
\hline 3753.241 & 300 & 40 & 26636.07 & 1 & 3 & 1.041 & 4 & 0.853 & & & \\
\hline
\end{tabular}


TABLE 3. Zeeman effect of Th $\mathrm{I}$-continued

\begin{tabular}{|c|c|c|c|c|c|c|c|c|c|c|c|}
\hline \multirow{2}{*}{$\begin{array}{c}\text { Wavelength } \\
(\AA)\end{array}$} & \multicolumn{2}{|c|}{ Intensity } & \multirow{2}{*}{$\begin{array}{l}\text { Wavenumber } \\
\left(\mathrm{cm}^{-1}\right)\end{array}$} & \multirow{2}{*}{ Type } & \multirow{2}{*}{$J_{1}$} & \multirow{2}{*}{$g_{1}$} & \multirow{2}{*}{$J_{2}$} & \multirow{2}{*}{$g_{2}$} & \multirow{2}{*}{$\Delta g$} & \multirow{2}{*}{$p$} & \multirow{2}{*}{$n$} \\
\hline & Lamp & Spark & & & & & & & & & \\
\hline 3752.789 & 300 & 8 & 26639.28 & 7 & & & & & & & 1.149 \\
\hline 3751.121 & 300 & 25 & 26651.12 & 7 & & & & & 0.150 & & \\
\hline 3750.493 & 400 & 25 & 26655.58 & 1 & 5 & 1.353 & 6 & 1.020 & & & \\
\hline 3749.617 & 300 & 20 & 26661.81 & 7 & & & & & & & 1.432 \\
\hline 3749.514 & 300 & 10 & 26662.55 & 2 & & & & & & 0.446 & \\
\hline 3749.083 & 200 & 25 & 26665.61 & 2 & 4 & 0.860 & 5 & 1.170 & & & \\
\hline 3748.820 & 100 & 8 & 26667.48 & 3 & & & & & & 1.030 & \\
\hline 3745.658 & $500 \mathrm{r}$ & 100 & 26689.99 & 2 & & & & & & & 1.489 \\
\hline 3745.176 & 4001 & 20 & 26693.43 & 3 & & & & & & 0.279 & \\
\hline 3744.910 & 300 & 10 & 26695.33 & 2 & 3 & 1.001 & 4 & 1.172 & & & \\
\hline 3742.923 & $1000 \mathrm{r}$ & 100 & 26709.49 & 7 & & & & & & & 1.198 \\
\hline 3742.275 & 200 & 5 & 26714.12 & 1 & & & & & 0.255 & & 0.364 \\
\hline 3742.019 & $75 b$ & 5 & 26715.95 & 3 & & & & & & 0.268 & \\
\hline 3741.884 & 200 & 8 & 26716.91 & 2 & & & & & & & 1.282 \\
\hline 3739.683 & 150 & 5 & 26732.63 & 3 & & & & & & 0.374 & \\
\hline 3738.436 & 150 & 2 & 26741.55 & 3 & & & & & & 0.283 & \\
\hline 3737.935 & 100 & 10 & 26745.14 & 1 & & & & & 0.400 & & 0.516 \\
\hline 3737.514 & $600 \mathrm{r}$ & 75 & 26748.15 & 7 & & & & & & & 1.486 \\
\hline 3736.502 & 200 & $40 b$ & 26755.39 & 7 & & & & & & & 0.922 \\
\hline 3735.363 & 100 & 3 & 26763.55 & 3 & & & & & & 0.351 & \\
\hline 3734.664 & 75 & 3 & 26768.56 & 7 & & & & & & & 1.024 \\
\hline 3733.672 & $300 \mathrm{r}$ & 25 & 26775.67 & 7 & & & & & & & 1.066 \\
\hline 3732.985 & $400 \mathrm{r}$ & 40 & 26780.60 & 3 & 3 & 0.872 & 3 & 0.995 & & & \\
\hline 3732.535 & 150 & 3 & 26783.82 & 3 & & & & & & 0.443 & \\
\hline 3729.945 & 400 & 40 & 26802.43 & 2 & 1 & 0.409 & 2 & 0.786 & & & \\
\hline 3729.836 & 400 & 25 & 26803.21 & 7 & & & & & & & 1.172 \\
\hline 3727.903 & $1000 \mathrm{r}$ & 150 & 26817.10 & 3 & & & & & & 0.518 & \\
\hline 3725.966 & 75 & 2 & 26831.05 & 2 & 1 & 0.858 & 2 & 1.188 & & & \\
\hline 3724.672 & 100 & 10 & 26840.36 & 3 & & & & & & 0.181 & \\
\hline 3724.397 & 100 & 2 & 26842.35 & 7 & & & & & 0.140 & & \\
\hline 3723.921 & $300 \mathrm{~s}$ & 8 & 26845.78 & 2 & 2 & 0.772 & 3 & 1.186 & & & \\
\hline 3722.655 & 75 & 2 & 26854.91 & 3 & & & & & & 0.094 & \\
\hline 3722.179 & $300 \mathrm{~b}$ & $25 b$ & 26858.34 & 3 & & & & & & 0.420 & \\
\hline 3721.219 & $500 \mathrm{r}$ & 50 & 26865.27 & 3 & & & & & & 0.416 & \\
\hline 3719.837 & $400 \mathrm{r}$ & 40 & 26875.26 & 3 & & & & & & 0.150 & \\
\hline 3717.895 & 50 & 2 & 26889.29 & 3 & & & & & & 0.143 & \\
\hline 3717.384 & 100 & 3 & 26892.99 & 7 & & & & & & & 0.960 \\
\hline 3716.583 & 300 & 40 & 26898.78 & 1 & & & & & & & 0.912 \\
\hline 3715.862 & 300 & 20 & 26904.00 & 3 & & & & & & 0.171 & \\
\hline 3715.561 & 400 & 50 & 26906.18 & 3 & & & & & & 0.590 & \\
\hline 3715.060 & 100 & 2 & 26909.81 & 3 & & & & & & 0.606 & \\
\hline 3714.696 & 100 & 3 & 26912.45 & 1 & 3 & 1.165 & 4 & 0.857 & & & \\
\hline 3712.373 & 100 & 3 & 26929.28 & 7 & & & & & & & 1.062 \\
\hline 3710.292 & 300 & 8 & 26944.39 & 1 & & & & & & & 0.819 \\
\hline 3709.861 & 300 & 25 & 26947.52 & 1 & 1 & 1.480 & 2 & 0.928 & & & \\
\hline 3709.404 & 75 & 3 & 26950.84 & 3 & & & & & & 0.542 & \\
\hline 3708.396 & 200 & 5 & 26958.17 & 3 & & & & & & 0.696 & \\
\hline 3706.402 & 200 & 75 & 26972.67 & 3 & & & & & & 0.429 & \\
\hline 3705.979 & $150 \mathrm{~h}$ & & 26975.74 & 1 & & & & & & & 0.706 \\
\hline 3705.665 & 100 & 40 & 26978.04 & 7 & & & & & & & 1.248 \\
\hline 3704.862 & $500 \mathrm{r}$ & 50 & 26983.88 & 2 & 2 & 0.917 & 3 & 1.086 & & & \\
\hline 3704.147 & 200 & 50 & 26989.09 & 2 & & & & & 0.075 & & 1.294 \\
\hline 3703.775 & $500 \mathrm{r}$ & 50 & 26991.80 & 3 & & & & & & 0.482 & \\
\hline 3703.230 & $400 \mathrm{r}$ & 25 & 26995.77 & 2 & & & & & 0.436 & & \\
\hline 3702.479 & 100 & 2 & 27001.25 & 3 & & & & & & 0.544 & \\
\hline
\end{tabular}


TABLE 3. Zeeman effect of Th $\mathrm{I}$-continued

\begin{tabular}{|c|c|c|c|c|c|c|c|c|c|c|c|}
\hline \multirow{2}{*}{$\begin{array}{l}\text { Wavelength } \\
(\AA)\end{array}$} & \multicolumn{2}{|c|}{ Intensity } & \multirow{2}{*}{$\begin{array}{l}\text { Wa venumber } \\
\qquad\left(\mathrm{cm}^{-1}\right)\end{array}$} & \multirow{2}{*}{ Type } & \multirow{2}{*}{$J_{1}$} & \multirow{2}{*}{$g_{1}$} & \multirow{2}{*}{$J_{2}$} & \multirow{2}{*}{$g_{2}$} & \multirow{2}{*}{$\Delta g$} & \multirow{2}{*}{$p$} & \multirow{2}{*}{$n$} \\
\hline & Lamp & Spark & & & & & & & & & \\
\hline 3700.978 & 500 & & 27012.20 & 7 & & & & & & & 1.327 \\
\hline 3699.881 & $500 \mathrm{r}$ & 50 & 27020.21 & 3 & 4 & 1.066 & 4 & 1.210 & & & \\
\hline 3699.355 & 300 & 5 & 27024.05 & 3 & & & & & & 0.440 & \\
\hline 3699.182 & 500 & 75 & 27025.31 & 2 & & & & & & 0.151 & \\
\hline 3699.048 & 300 & 5 & 27026.29 & 2 & & & & & & & 1.518 \\
\hline 3698.106 & $1000 \mathrm{r}$ & 150 & 27033.17 & 3 & 5 & 1.168 & 5 & 1.362 & & & \\
\hline 3695.288 & $500 \mathrm{r}$ & 75 & 27053.79 & 2 & 4 & 0.872 & 5 & 1.113 & & & \\
\hline 3694.177 & $400 \mathrm{r}$ & 75 & 27061.93 & 7 & & & & & & & 1.034 \\
\hline 3693.993 & $400 r$ & 200 & 27063.27 & 3 & 1 & 0.971 & 1 & 1.471 & & & \\
\hline 3693.804 & $200 \mathrm{~b}$ & 25 & 27064.66 & 3 & & & & & & 0.424 & \\
\hline 3693.523 & 3001 & $25 b$ & 27066.72 & 3 & & & & & & 0.824 & \\
\hline 3693.247 & 200 & 10 & 27068.74 & 1 & 1 & 2.399 & 2 & 1.027 & & & \\
\hline 3691.876 & $400 \mathrm{r}$ & 100 & 27078.79 & 1 & 3 & 1.099 & 4 & 0.869 & & & \\
\hline 3691.613 & $500 \mathrm{r}$ & 200 & 27080.72 & 3 & & & & & & 0.266 & \\
\hline 3691.411 & $500 \mathrm{r}$ & 50 & 27082.21 & 7 & & & & & & & 0.947 \\
\hline 3690.624 & $600 \mathrm{r}$ & 4 & 27087.98 & 1 & 1 & 1.164 & 2 & 0.734 & & & \\
\hline 3690.115 & 200 & 300 & 27091.71 & 3 & & & & & & 0.209 & \\
\hline 3689.726 & 200 & 3 & 27094.57 & 7 & & & & & & & 1.106 \\
\hline 3689.582 & 100 & 2 & 27095.63 & 1 & & & & & & & 0.916 \\
\hline 3687.983 & $400 \mathrm{r}$ & 75 & 27107.37 & 1 & 3 & 1.124 & 4 & 0.976 & & & \\
\hline 3687.492 & $150 \mathrm{~b}$ & 5 & 27110.99 & 3 & & & & & & 0.720 & \\
\hline 3687.193 & 4001 & 20 & 27113.18 & 3 & & & & & & 0.133 & \\
\hline 3685.586 & $300 \mathrm{~b}$ & 101 & 27125.00 & 3 & 2 & 0.931 & 2 & 1.252 & & & \\
\hline 3685.214 & 200 & 4 & 27127.75 & 3 & & & & & & 0.467 & \\
\hline 3684.932 & 300 & 20 & 27129.82 & 7 & & & & & & & 1.052 \\
\hline 3683.493 & 500 & 25 & 27140.42 & 7 & & & & & & & 0.999 \\
\hline 3682.179 & 300 & 50 & 27150.10 & 7 & & & & & & & 1.324 \\
\hline 3680.605 & $400 \mathrm{r}$ & 40 & 27161.71 & 2 & 2 & 0.772 & 3 & 1.072 & & & \\
\hline 3680.448 & $500 \mathrm{r}$ & 50 & 27162.87 & 2 & & & & & & & 1.539 \\
\hline 3679.544 & $400 \mathrm{r}$ & 150 & 27169.54 & 7 & & & & & & & 1.036 \\
\hline 3679.134 & 400 & 50 & 27172.57 & 7 & & & & & & & 0.967 \\
\hline 3678.478 & $400 \mathrm{r}$ & $50 \mathrm{~b}$ & 27177.42 & 1 & 1 & 1.158 & 2 & 0.773 & & & \\
\hline 3677.304 & 150 & 4 & 27186.10 & 2 & & & & & 0.256 & & \\
\hline 3675.960 & 400 & 50 & 27196.04 & 1 & 2 & 1.715 & 3 & 1.315 & & & \\
\hline 3675.790 & $400 \mathrm{r}$ & 50 & 27197.29 & 2 & & & & & 0.310 & & \\
\hline 3675.137 & $500 \mathrm{r}$ & 50 & 27202.12 & 3 & & & & & & 0.445 & \\
\hline 3674.891 & $300 \mathrm{r}$ & 50 & 27203.95 & 3 & 5 & 1.137 & 5 & 1.361 & & & \\
\hline 3674.089 & 300 & 40 & 27209.88 & 7 & & & & & & & 0.952 \\
\hline 3674.013 & $300 \mathrm{r}$ & $50 \mathrm{~b}$ & 27210.44 & 1 & 1 & & 2 & & 0.149 & & \\
\hline 3672.522 & 500 & 25 & 27221.50 & 1 & & & & & 0.130 & & \\
\hline 3672.300 & $500 \mathrm{r}$ & 50 & 27223.14 & 3 & 3 & 1.090 & 3 & 1.249 & & & \\
\hline 3670.654 & $15 b$ & & 27235.35 & 2 & & & & & & & 1.422 \\
\hline 3670.520 & 200 & 4 & 27236.34 & 7 & & & & & 0.365 & & \\
\hline 3669.640 & 400 & 50 & 27242.87 & 7 & & & & & & & 1.022 \\
\hline 3668.390 & $300 \mathrm{~b}$ & 8 & 27252.16 & 3 & & & & & & 1.176 & \\
\hline 3667.882 & 20 & 4 & 27255.93 & 3 & & & & & & 0.301 & \\
\hline 3667.621 & 500 & 40 & 27257.87 & 1 & & & & & & & 0.603 \\
\hline 3666.981 & $800 \mathrm{r}$ & 75 & 27262.63 & 3 & 4 & 1.100 & 4 & 1.308 & & & \\
\hline 3665.476 & $100 \mathrm{~b}$ & $10 \mathrm{~b}$ & 27273.82 & 2 & & & & & 0.170 & & 1.821 \\
\hline 3665.443 & $150 \mathrm{~b}$ & 500 & 27274.06 & 1 & 3 & 1.252 & 4 & 0.992 & & & \\
\hline 3664.590 & 200 & 10 & 27280.41 & 7 & & & & & & & 1.103 \\
\hline 3664.108 & 300 & 10 & 27284.00 & 1 & & & & & & & 0.875 \\
\hline 3663.201 & $800 \mathrm{r}$ & 100 & 27290.75 & 7 & & & & & & & 1.170 \\
\hline 3662.955 & 150 & 15 & 27292.59 & 2 & & & & & 0.180 & & \\
\hline 3662.750 & $300 \mathrm{r}$ & 20 & 27294.12 & 1 & 3 & 1.181 & 4 & 0.849 & & & \\
\hline
\end{tabular}


TABLE 3. Zeeman effect of Th $\mathrm{I}$-continued

\begin{tabular}{|c|c|c|c|c|c|c|c|c|c|c|c|}
\hline \multirow{2}{*}{$\begin{array}{l}\text { Wavelength } \\
\text { (A) }\end{array}$} & \multicolumn{2}{|c|}{ Intensity } & \multirow{2}{*}{$\begin{array}{l}\text { Wavenumber } \\
\qquad\left(\mathrm{cm}^{-1}\right)\end{array}$} & \multirow{2}{*}{ Type } & \multirow{2}{*}{$J_{1}$} & \multirow{2}{*}{$g_{1}$} & \multirow{2}{*}{$J_{2}$} & \multirow{2}{*}{$g_{2}$} & \multirow{2}{*}{$\Delta g$} & \multirow{2}{*}{$p$} & \multirow{2}{*}{$n$} \\
\hline & Lamp & Spark & & & & & & & & & \\
\hline 3662.283 & 100 & 3 & 27297.59 & 2 & & & & & 0.116 & & \\
\hline 3660.500 & 400 & 25 & 27310.89 & 1 & 1 & 1.496 & 2 & 1.186 & & & \\
\hline 3660.091 & $200 \mathrm{~b}$ & 4 & 27313.95 & 3 & & & & & & 0.523 & \\
\hline 3659.629 & $600 \mathrm{r}$ & 150 & 27317.39 & 2 & 2 & 0.737 & 3 & 1.087 & & & \\
\hline 3658.808 & $400 \mathrm{r}$ & 25 & 27323.52 & 2 & & & & & & & 1.468 \\
\hline 3657.641 & $200 \mathrm{~s}$ & 10 & 27332.24 & 1 & 4 & 1.306 & 5 & 1.062 & & & \\
\hline 3657.054 & 300 & 20 & 27336.63 & 2 & & & & & & & 1.206 \\
\hline 3655.125 & 300 & 50 & 27351.05 & 1 & 3 & 1.250 & 4 & 1.066 & & & \\
\hline 3654.461 & $500 \mathrm{r}$ & 50 & 27356.02 & 3 & & & & & & 0.084 & \\
\hline 3653.916 & 20 & 1 & 27360.10 & 7 & & & & & & & 0.974 \\
\hline 3653.586 & 40 & & 27362.57 & 1 & & & & & 0.340 & & \\
\hline 3653.366 & 150 & 3 & 27364.22 & 7 & & & & & 0.050 & & \\
\hline 3651.570 & 100 & & 27377.68 & 7 & & & & & & & 1.256 \\
\hline 3650.204 & 3001 & 2 & 27387.93 & 3 & & & & & & 0.136 & \\
\hline 3649.735 & $1000 \mathrm{r}$ & 100 & 27391.45 & 1 & 1 & & 2 & & 1.040 & & \\
\hline 3648.856 & 150 & 8 & 27398.04 & 3 & & & & & & 0.165 & \\
\hline 3647.932 & 300 & 50 & 27404.98 & 3 & & & & & & 0.210 & \\
\hline 3647.575 & 300 & 15 & 27407.67 & 7 & & & & & & & 1.025 \\
\hline 3646.268 & 300 & $8 \mathrm{~b}$ & 27417.49 & 1 & 2 & 1.194 & 3 & 1.000 & & & \\
\hline 3645.706 & 300 & 15 & 27421.72 & 2 & 4 & 0.877 & 5 & 1.177 & & & \\
\hline 3645.566 & 200 & 8 & 27422.77 & 7 & & & & & & & 1.177 \\
\hline 3644.018 & 100 & 2 & 27434.42 & 1 & & & & & 0.273 & & \\
\hline 3643.512 & $600 \mathrm{r}$ & 50 & 27438.23 & 7 & & & & & & & 1.276 \\
\hline 3642.573 & $500 \mathrm{r}$ & 50 & 27445.30 & 1 & & & & & & & 0.870 \\
\hline 3641.363 & $100 \mathrm{~b}$ & 4 & 27454.42 & 7 & & & & & & & 0.855 \\
\hline 3640.924 & 200 & $15 \mathrm{~h}$ & 27457.73 & 3 & 1 & 0.823 & 1 & 1.178 & & & \\
\hline 3640.390 & 100 & 3 & 27461.76 & 7 & & & & & 0.660 & & \\
\hline 3639.867 & 4001 & 40 & 27465.70 & 2 & 3 & 0.901 & 4 & 1.043 & & & \\
\hline 3638.644 & $500 \mathrm{r}$ & 50 & 27474.93 & 1 & & & & & 0.186 & & \\
\hline 3638.319 & $400 \mathrm{r}$ & 25 & 27477.39 & 3 & & & & & & 0.244 & \\
\hline 3638.146 & 200 & 4 & 27478.69 & 3 & & & & & & 0.531 & \\
\hline 3637.772 & $50 \mathrm{~b}$ & & 27481.52 & 7 & & & & & 0.080 & & \\
\hline 3636.834 & $500 \mathrm{r}$ & 25 & 27488.60 & 7 & & & & & & & 1.481 \\
\hline 3636.295 & 150 & 3 & 27492.68 & 3 & & & & & & 0.277 & \\
\hline 3635.943 & $2000 \mathrm{r}$ & 150 & 27495.34 & 7 & & & & & & & 0.614 \\
\hline 3634.902 & 200 & 4 & 27503.22 & 1 & & & & & 0.194 & & \\
\hline 3634.582 & $600 \mathrm{r}$ & $100 \mathrm{~b}$ & 27505.64 & 7 & & & & & & & 0.914 \\
\hline 3633.910 & 100 & 3 & 27510.73 & 7 & & & & & 0.180 & & \\
\hline 3632.830 & $1000 \mathrm{r}$ & 100 & 27518.90 & 2 & & & & & & & 1.207 \\
\hline 3632.124 & 100 & 5 & 27524.26 & 7 & & & & & 0.080 & & 0.836 \\
\hline 3631.863 & 150 & 8 & 27526.23 & 3 & & & & & & 0.055 & \\
\hline 3631.708 & $100 \mathrm{~b}$ & 2 & 27527.41 & 3 & & & & & & 0.372 & \\
\hline 3630.986 & 25 & 2 & 27532.88 & 7 & & & & & & & 1.003 \\
\hline 3630.851 & 25 & & 27533.90 & 7 & & & & & & & 0.963 \\
\hline 3629.851 & 400 & 20 & 27541.49 & 3 & 1 & 0.716 & 1 & 1.486 & & & \\
\hline 3629.017 & 100 & 4 & 27547.82 & 3 & & & & & & 0.668 & \\
\hline 3628.867 & 100 & 4 & 27548.95 & 3 & & & & & & 0.276 & \\
\hline 3628.129 & 75 & 4 & 27554.56 & 7 & & & & & & & 1.254 \\
\hline 3628.032 & 100 & 10 & 27555.30 & 7 & & & & & & & 1.035 \\
\hline 3627.342 & 50 & 1 & 27560.54 & 3 & & & & & & 0.227 & \\
\hline 3626.939 & 300 & 50 & 27563.60 & 7 & & & & & & & 0.942 \\
\hline 3626.471 & 200 & 10 & 27567.16 & 2 & & & & & & & 1.326 \\
\hline 3625.895 & $500 \mathrm{r}$ & $400 \mathrm{~b}$ & 27571.53 & 5 & 2 & 0.833 & 2 & 1.177 & & & \\
\hline 3625.150 & 300 & 75 & 27577.20 & 1 & & & & & 0.270 & & \\
\hline 3625.026 & $300 \mathrm{r}$ & 100 & 27578.15 & 3 & & & & & & 0.091 & \\
\hline
\end{tabular}


TABLE 3. Zeeman effect of $\mathrm{Th} \mathrm{I}$-continued

\begin{tabular}{|c|c|c|c|c|c|c|c|c|c|c|c|}
\hline \multirow{2}{*}{$\begin{array}{l}\text { Wavelength } \\
\text { (̊) }\end{array}$} & \multicolumn{2}{|c|}{ Intensity } & \multirow{2}{*}{$\begin{array}{l}\text { Wavenumber } \\
\quad\left(\mathrm{cm}^{-1}\right)\end{array}$} & \multirow{2}{*}{ Type } & \multirow{2}{*}{$J_{1}$} & \multirow{2}{*}{$g_{1}$} & \multirow{2}{*}{$J_{2}$} & \multirow{2}{*}{$g_{2}$} & \multirow{2}{*}{$\Delta g$} & \multirow{2}{*}{$p$} & \multirow{2}{*}{$n$} \\
\hline & Lamp & Spark & & & & & & & & & \\
\hline 3624.472 & 300 & 50 & 27582.36 & 3 & 4 & 1.066 & 4 & 1.305 & & & \\
\hline 3624.143 & 100 & 3 & 27584.87 & 3 & & & & & & 0.485 & \\
\hline 3623.773 & 300 & 25 & 27587.68 & 2 & 2 & 0.778 & 3 & 1.190 & & & \\
\hline 3623.297 & 15 & 1 & 27591.31 & 3 & & & & & & 0.174 & \\
\hline 3622.795 & $600 \mathrm{r}$ & 50 & 27595.13 & 1 & 2 & & 3 & & & & 0.803 \\
\hline 3622.338 & $500 \mathrm{r}$ & $20 b$ & 27598.61 & 3 & & 0.06 & & 1.33 & & & \\
\hline 3622.338 & $500 \mathrm{r}$ & $20 \mathrm{~b}$ & 27598.61 & 1 & & 1.48 & & 1.19 & & & \\
\hline 3622.290 & $500 \mathrm{r}$ & 50 & 27598.98 & 2 & 2 & & 3 & & & & 1.550 \\
\hline 3621.435 & 50 & & 27605.49 & 1 & & & & & & & 0.422 \\
\hline 3620.839 & $300 \mathrm{r}$ & 50 & 27610.04 & 1 & 3 & 1.062 & 4 & 0.858 & & & \\
\hline 3619.211 & 200 & 40 & 27622.45 & 3 & & & & & & 0.090 & \\
\hline 3617.671 & 300 & 8 & 27634.21 & 3 & 2 & 0.775 & 2 & 1.412 & & & \\
\hline 3616.177 & 200 & 8 & 27645.63 & 1 & & & & & 0.206 & & \\
\hline 3615.849 & 200 & 4 & 27648.14 & 2 & & & & & 0.372 & & \\
\hline 3614.352 & $500 \mathrm{r}$ & 75 & 27659.59 & 7 & & & & & & & 1.215 \\
\hline 3614.212 & $500 \mathrm{r}$ & 75 & 27660.66 & 7 & & & & & & & 0.978 \\
\hline 3612.864 & $600 \mathrm{r}$ & 5 & 27670.98 & 7 & & & & & 0.476 & & \\
\hline 3610.650 & 300 & 20 & 27687.95 & 2 & & & & & & & 1.485 \\
\hline 3608.377 & $800 \mathrm{r}$ & 50 & 27705.39 & 1 & 5 & 1.360 & 6 & 1.123 & & & \\
\hline 3607.819 & 75 & & 27709.67 & 1 & & & & & & & 0.749 \\
\hline 3606.793 & 200 & 4 & 27717.55 & 7 & & & & & & & 1.295 \\
\hline 3606.090 & 200 & 3 & 27722.96 & 7 & & & & & & & 1.471 \\
\hline 3605.820 & 150 & 4 & 27725.03 & 1 & & & & & 0.300 & & 0.274 \\
\hline 3605.194 & 500 & 40 & 27729.85 & 1 & & & & & & & 0.770 \\
\hline 3604.680 & $600 \mathrm{r}$ & 40 & 27733.80 & 1 & 1 & 1.468 & 2 & 1.216 & & & \\
\hline 3604.064 & $200 \mathrm{~b}$ & & 27738.54 & 3 & 3 & 1.077 & 3 & 1.243 & & & \\
\hline 3602.909 & 100 & 40 & 27747.43 & 1 & & & & & & & 0.802 \\
\hline 3602.328 & 20 & & 27751.91 & 7 & & & & & & & 1.040 \\
\hline 3601.770 & 200 & 10 & 27756.21 & 3 & & & & & & 0.313 & \\
\hline 3601.295 & 300 & 10 & 27759.87 & 7 & & & & & & & 0.954 \\
\hline 3600.431 & 300 & 50 & 27766.53 & 3 & 5 & 1.361 & 5 & 1.037 & & & \\
\hline 3599.723 & 300 & 20 & 27771.99 & 3 & & & & & & 0.095 & \\
\hline 3598.691 & 100 & 5 & 27779.96 & 3 & & & & & & 0.182 & \\
\hline 3598.523 & 150 & 10 & 27781.25 & 1 & 3 & 1.192 & 4 & 0.861 & & & \\
\hline 3597.704 & 20 & 2 & 27787.57 & 3 & & & & & & 0.281 & \\
\hline 3597.021 & 75 & 2 & 27792.86 & 7 & & & & & & & 1.395 \\
\hline 3596.536 & 100 & 5 & 27796.60 & 7 & & & & & & & 1.443 \\
\hline 3595.975 & 150 & 5 & 27800.94 & 7 & & & & & 0.244 & & \\
\hline 3595.756 & 100 & 10 & 27802.63 & 2 & & & & & 0.265 & & \\
\hline 3595.617 & $500 \mathrm{r}$ & 100 & 27803.70 & 3 & & & & & & 0.109 & \\
\hline 3594.985 & 300 & 20 & 27808.59 & 1 & 2 & 1.723 & 3 & 1.267 & & & \\
\hline 3594.721 & 300 & 10 & 27810.63 & 1 & 1 & 0.972 & 2 & 0.740 & & & \\
\hline 3592.777 & $800 \mathrm{r}$ & 100 & 27825.67 & 1 & 4 & & & & 0.160 & & \\
\hline 3592.122 & 75 & & 27830.76 & 2 & & & & & 0.160 & & 1.402 \\
\hline 3591.253 & 200 & 50 & 27837.49 & 3 & & & & & & 0.200 & \\
\hline 3590.924 & 300 & & 27840.04 & 2 & 3 & 0.878 & 4 & 1.042 & & & \\
\hline 3589.992 & 200 & 20 & 27847.27 & 3 & 1 & 1.175 & 1 & 1.483 & & & \\
\hline 3587.831 & 100 & 5 & 27864.04 & 7 & & & & & & & 1.266 \\
\hline 3587.102 & 150 & 40 & 27869.70 & 7 & & & & & & & 1.071 \\
\hline 3586.703 & 100 & & 27872.80 & 3 & & & & & & 0.406 & \\
\hline 3586.478 & 150 & 4 & 27874.55 & 1 & 1 & 0.986 & 2 & 0.666 & & & \\
\hline 3585.244 & 150 & 5 & 27884.15 & 3 & & & & & & 1.220 & \\
\hline 3585.050 & 20 & & 27885.65 & 1 & & & & & 0.080 & & 0.775 \\
\hline 3583.298 & 150 & 10 & 27899.29 & 2 & & & & & & & 1.130 \\
\hline 3583.100 & $600 \mathrm{r}$ & 25 & 27900.83 & 3 & & & & & & 0.229 & \\
\hline
\end{tabular}


TABLE 3. Zeeman effect of Th $\mathrm{I}$-continued

\begin{tabular}{|c|c|c|c|c|c|c|c|c|c|c|c|}
\hline \multirow{2}{*}{$\begin{array}{l}\text { Wavelength } \\
\text { (§) }\end{array}$} & \multicolumn{2}{|c|}{ Intensity } & \multirow{2}{*}{$\begin{array}{l}\text { Wavenumber } \\
\qquad\left(\mathrm{cm}^{-1}\right)\end{array}$} & \multirow{2}{*}{ Type } & \multirow{2}{*}{$J_{1}$} & \multirow{2}{*}{$g_{1}$} & \multirow{2}{*}{$J_{2}$} & \multirow{2}{*}{$g_{2}$} & \multirow{2}{*}{$\Delta g$} & \multirow{2}{*}{$p$} & \multirow{2}{*}{$n$} \\
\hline & Lamp & Spark & & & & & & & & & \\
\hline 3581.353 & 150 & $25 b$ & 27914.44 & 2 & & & & & 0.110 & & 1.562 \\
\hline 3581.289 & 100 & 3 & 27914.93 & 7 & & & & & & & 1.141 \\
\hline 3579.454 & 200 & & 27929.25 & 7 & & & & & 0.300 & & \\
\hline 3577.746 & 200 & 8 & 27942.58 & 1 & 2 & 1.296 & 3 & 1.064 & & & \\
\hline 3574.192 & 50 & & 27970.36 & 7 & & & & & & & 0.872 \\
\hline 3572.067 & 300 & 20 & 27987.00 & 3 & & & & & & 0.515 & \\
\hline 3571.008 & 300 & 10 & 27995.30 & 3 & & & & & & 0.201 & \\
\hline 3570.827 & 100 & & 27996.72 & 7 & & & & & & & 1.054 \\
\hline 3570.523 & 300 & 10 & 27999.11 & 3 & & & & & & 1.141 & \\
\hline 3570.357 & 500 & 10 & 28000.41 & 3 & & & & & & 1.404 & \\
\hline 3569.820 & $1000 \mathrm{r}$ & 25 & 28004.62 & 1 & 1 & 1.470 & 2 & 0.937 & & & \\
\hline 3569.207 & 75 & & 28009.43 & 3 & 2 & 1.014 & 2 & 1.282 & & & \\
\hline 3566.099 & 15 & & 28033.84 & 2 & & & & & 0.088 & & 1.226 \\
\hline 3565.822 & 300 & 15 & 28036.02 & 7 & & & & & 0.332 & & \\
\hline 3564.508 & 100 & 10 & 28046.35 & 7 & & & & & & & 1.102 \\
\hline 3564.235 & 200 & 8 & 28048.50 & 7 & & & & & & & 1.125 \\
\hline 3563.911 & 200 & & 28051.05 & 7 & & & & & & & 1.130 \\
\hline 3563.375 & 600 & $75 b$ & 28055.27 & 3 & & & & & & 0.162 & \\
\hline 3563.299 & $200 \mathrm{~b}$ & 25 & 28055.87 & 3 & & & & & & 0.117 & \\
\hline 3562.599 & 75 & 5 & 28061.38 & 7 & & & & & & & 1.155 \\
\hline 3562.253 & 100 & 8 & 28064.11 & 7 & & & & & & & 0.994 \\
\hline 3561.780 & $500 \mathrm{r}$ & 75 & 28067.83 & 2 & 2 & 1.020 & 3 & 1.213 & & & \\
\hline 3561.408 & 200 & 8 & 28070.77 & 3 & & & & & & 0.207 & \\
\hline 3560.896 & 300 & 40 & 28074.80 & 7 & & & & & & & 0.958 \\
\hline 3560.688 & 200 & 5 & 28076.44 & 1 & & & & & 0.267 & & \\
\hline 3558.638 & 100 & & 28092.61 & 2 & & & & & 0.320 & & \\
\hline 3556.973 & 75 & 10 & 28105.76 & 7 & & & & & & & 1.205 \\
\hline 3555.013 & $1000 \mathrm{r}$ & 50 & 28121.26 & 7 & & & & & & & 1.091 \\
\hline 3554.336 & $75 \mathrm{~s}$ & 2 & 28126.61 & 7 & & & & & & & 1.078 \\
\hline 3553.960 & 20 & 2 & 28129.59 & 3 & & & & & & 0.784 & \\
\hline 3553.110 & 100 & 200 & 28136.32 & 3 & & & & & & 0.163 & \\
\hline 3551.744 & 75 & 2 & 28147.14 & 7 & & & & & & & 0.948 \\
\hline 3551.401 & $1000 \mathrm{r}$ & 150 & 28149.86 & 2 & & & & & & & 1.489 \\
\hline 3550.783 & 200 & 40 & 28154.76 & 3 & & & & & & 0.163 & \\
\hline 3550.718 & $100 \mathrm{~s}$ & & 28155.28 & 1 & 1 & 0.064 & 2 & 1.064 & & & \\
\hline 3548.664 & 200 & 8 & 28171.57 & 1 & 1 & 0.062 & 2 & 1.298 & & & \\
\hline 3547.918 & 300 & 15 & 28177.49 & 3 & & & & & & 0.136 & \\
\hline 3547.338 & $200 \mathrm{~b}$ & & 28182.10 & 3 & 2 & 0.951 & 2 & 1.251 & & & \\
\hline 3546.255 & 20 & & 28190.71 & 3 & & & & & & 0.306 & \\
\hline 3545.958 & $500 \mathrm{~b}$ & & 28193.07 & 1 & & & & & & & 0.831 \\
\hline 3544.250 & 150 & 3 & 28206.65 & 1 & & & & & 0.244 & & \\
\hline 3544.018 & $800 \mathrm{r}$ & 50 & 28208.50 & 1 & 4 & 1.314 & 5 & 1.136 & & & \\
\hline 3543.208 & 150 & 3 & 28214.95 & 3 & & & & & 0.667 & 0.667 & \\
\hline 3543.023 & 200 & 4 & 28216.42 & 3 & & & & & & 0.699 & \\
\hline 3542.498 & $500 \mathrm{r}$ & 50 & 28220.60 & 2 & 2 & 1.013 & 3 & 1.171 & & & \\
\hline 3539.843 & 300 & 25 & 28241.77 & 3 & & & & & & 1.015 & \\
\hline 3536.976 & 150 & 4 & 28264.66 & 3 & & & & & & 0.149 & \\
\hline 3535.250 & $150 \mathrm{~b}$ & & 28278.46 & 7 & & & & & & & 0.967 \\
\hline 3534.675 & $150 \mathrm{~b}$ & & 28283.06 & 1 & & & & & & & 0.706 \\
\hline 3534.210 & $100 \mathrm{~b}$ & 10 & 28286.78 & 1 & 1 & 2.390 & 2 & 1.195 & & & \\
\hline 3533.600 & 40 & 3 & 28291.66 & 7 & & & & & & & 1.089 \\
\hline 3533.181 & $200 \mathrm{~b}$ & 10 & 28295.02 & 1 & 1 & 1.483 & 2 & 1.080 & & & \\
\hline 3531.450 & $1000 \mathrm{r}$ & 50 & 28308.89 & 3 & & & & & & 0.186 & \\
\hline 3531.282 & 200 & 8 & 28310.24 & 3 & 3 & 1.111 & 3 & 1.240 & & & \\
\hline 3530.514 & $800 \mathrm{r}$ & 50 & 28316.39 & 7 & & & & & & & 1.051 \\
\hline
\end{tabular}


TABLE 3. Zeeman effect of Th $\mathrm{I}$-continued

\begin{tabular}{|c|c|c|c|c|c|c|c|c|c|c|c|}
\hline \multirow{2}{*}{$\begin{array}{l}\text { Wavelength } \\
(\AA)\end{array}$} & \multicolumn{2}{|c|}{ Intensity } & \multirow{2}{*}{$\begin{array}{c}\text { Wavenumber } \\
\left(\mathrm{cm}^{-1}\right)\end{array}$} & \multirow{2}{*}{ Type } & \multirow{2}{*}{$J_{1}$} & \multirow{2}{*}{$g_{1}$} & \multirow{2}{*}{$J_{2}$} & \multirow{2}{*}{$g_{2}$} & \multirow{2}{*}{$\Delta g$} & \multirow{2}{*}{$p$} & \multirow{2}{*}{$n$} \\
\hline & Lamp & Spark & & & & & & & & & \\
\hline 3529.385 & 3001 & 40 & 28325.45 & 1 & 3 & 1.082 & 4 & 0.732 & & & \\
\hline 3528.411 & 300 & 50 & 28333.27 & 2 & & & & & & & 1.547 \\
\hline 3527.793 & 200 & 10 & 28338.23 & 2 & 4 & 0.857 & 5 & 1.041 & & & \\
\hline 3526.764 & 300 & & 28346.50 & 3 & & & & & & 0.222 & \\
\hline 3526.634 & $500 \mathrm{r}$ & 10 & 28347.54 & 3 & 2 & 0.736 & 2 & 1.544 & & & \\
\hline 3524.708 & 300 & 8 & 28363.03 & 2 & & & & & & & 1.217 \\
\hline 3524.178 & 300 & 25 & 28367.30 & 3 & & & & & & 0.660 & \\
\hline 3523.758 & 300 & 8 & 28370.69 & 7 & & & & & & & 0.996 \\
\hline 3523.505 & 300 & 501 & 28372.72 & 1 & 1 & 1.812 & 2 & 0.738 & & & \\
\hline 3523.022 & 75 & & 28376.61 & 7 & & & & & & & 0.787 \\
\hline 3521.059 & $500 \mathrm{r}$ & 25 & 2892.43 & 2 & 1 & 0.803 & 2 & 1.263 & & & \\
\hline 3520.710 & 300 & 40 & 28395.24 & 3 & & & & & & 1.151 & \\
\hline 3518.885 & 4001 & 50 & 28409.97 & 3 & 2 & 0.788 & 2 & 1.206 & & & \\
\hline 3517.961 & 2001 & 10 & 28417.43 & 3 & & & & & & 0.194 & \\
\hline 3517.257 & 40 & 3 & 28423.12 & 3 & & & & & & 0.174 & \\
\hline 3515.327 & 100 & 40 & 28438.72 & 7 & & & & & & & 1.025 \\
\hline 3514.285 & 200 & 10 & 28447.16 & 7 & & & & & & & 0.974 \\
\hline 3511.789 & 100 & $10 \mathrm{~h}$ & 28467.37 & 7 & & & & & & & 1.344 \\
\hline 3511.157 & $800 \mathrm{r}$ & 25 & 28472.50 & 3 & 2 & 1.075 & 2 & 1.249 & & & \\
\hline 3510.729 & $75 \mathrm{~b}$ & & 28475.96 & 7 & & & & & & & 1.125 \\
\hline 3509.319 & 50 & & 28487.41 & 7 & & & & & 0.060 & & \\
\hline 3509.089 & 500 & 25 & 28489.28 & 3 & 2 & 0.873 & 2 & 1.003 & & & \\
\hline 3508.360 & 300 & 3 & 28495.20 & 1 & & & & & 1.183 & & \\
\hline 3508.100 & $300 \mathrm{~b}$ & & 28497.30 & 3 & & & & & & 0.133 & \\
\hline 3507.520 & 100 & & 28502.02 & 3 & & & & & & 1.059 & \\
\hline 3507.178 & 200 & 8 & 28504.80 & 3 & & & & & & 0.133 & \\
\hline 3506.345 & 300 & 10 & 28511.57 & 7 & & & & & & & 1.080 \\
\hline 3506.132 & 300 & 3 & 28513.30 & 3 & 2 & 0.729 & 2 & 1.097 & & & \\
\hline 3505.767 & 150 & 5 & 28516.27 & 1 & 1 & 1.475 & 2 & 1.025 & & & \\
\hline 3504.992 & 300 & 8 & 28522.57 & 2 & 1 & 0.721 & 2 & 1.261 & & & \\
\hline 3504.852 & 150 & 8 & 28523.71 & 1 & & & & & & & 0.745 \\
\hline 3504.569 & 15 & & 28526.02 & 3 & & & & & & 1.254 & \\
\hline 3503.786 & $500 \mathrm{r}$ & 40 & 28532.40 & 1 & & & & & & & 0.948 \\
\hline 3503.130 & 200 & 15 & 28537.74 & 7 & & & & & & & 1.136 \\
\hline 3502.963 & 200 & 10 & 28539.10 & 7 & & & & & 0.104 & & \\
\hline 3499.821 & 200 & 5 & 28564.72 & 2 & 2 & 0.780 & 3 & 1.100 & & & \\
\hline 3498.956 & $200 \mathrm{~b}$ & $20 \mathrm{~b}$ & 28571.78 & 3 & & & & & & 0.322 & \\
\hline 3498.621 & $800 \mathrm{r}$ & 75 & 28574.51 & 3 & & & & & & 0.358 & \\
\hline 3496.811 & $500 \mathrm{r}$ & 15 & 28589.31 & 2 & 2 & 0.746 & 3 & 1.179 & & & \\
\hline 3496.060 & 200 & 10 & 28595.45 & 7 & & & & & 0.190 & & \\
\hline 3495.700 & $800 \mathrm{r}$ & 25 & 28598.39 & 7 & & & & & & & 1.197 \\
\hline 3494.801 & 100 & & 28605.75 & 3 & 2 & 1.192 & 2 & 1.567 & & & \\
\hline 3494.175 & 15 & 2 & 28610.87 & 3 & & & & & & 0.334 & \\
\hline 3492.158 & 25 & & 28627.39 & 1 & & & & & 0.615 & & \\
\hline 3491.900 & 500 & 2 & 28629.51 & 2 & 3 & 1.017 & 4 & 1.210 & & & \\
\hline 3490.848 & 50 & 1 & 28638.14 & 7 & & & & & 0.266 & & \\
\hline 3489.509 & 400 & 2 & 28649.13 & 1 & 1 & 1.076 & 2 & 0.730 & & & \\
\hline 3488.834 & 500 & 3 & 28654.67 & 7 & & & & & & & 1.087 \\
\hline 3487.991 & 1501 & 4 & 28661.60 & 3 & & & & & & 0.539 & \\
\hline 3486.551 & $1000 \mathrm{r}$ & $10 \mathrm{~b}$ & 28673.44 & 3 & 2 & 0.738 & 2 & 1.123 & & & \\
\hline 3486.269 & 50 & 1 & 28675.75 & 1 & & & & & & & 0.482 \\
\hline 3486.210 & 50 & 1 & 28676.24 & 2 & 2 & 0.744 & 3 & 1.094 & & & \\
\hline 3485.175 & $200 \mathrm{~b}$ & 2 & 28684.76 & 1 & & & & & & & 0.620 \\
\hline 3484.732 & 200 & & 28688.40 & 7 & & & & & 0.560 & & \\
\hline 3483.433 & $200 \mathrm{~b}$ & 1 & 28699.10 & 7 & & & & & & & 1.256 \\
\hline
\end{tabular}


TABLE 3. Zeeman effect of Th $\mathrm{I}$-continued

\begin{tabular}{|c|c|c|c|c|c|c|c|c|c|c|c|}
\hline \multirow{2}{*}{$\begin{array}{l}\text { Wavelength } \\
(\AA)\end{array}$} & \multicolumn{2}{|c|}{ Intensity } & \multirow{2}{*}{$\begin{array}{l}\text { Wavenumber } \\
\qquad\left(\mathrm{cm}^{-1}\right)\end{array}$} & \multirow{2}{*}{ Type } & \multirow{2}{*}{$J_{1}$} & \multirow{2}{*}{$g_{1}$} & \multirow{2}{*}{$J_{2}$} & \multirow{2}{*}{$g_{2}$} & \multirow{2}{*}{$\Delta g$} & \multirow{2}{*}{$p$} & \multirow{2}{*}{$n$} \\
\hline & Lamp & Spark & & & & & & & & & \\
\hline 3481.620 & $100 \mathrm{~h}$ & & 28714.04 & 7 & & & & & & & 1.108 \\
\hline 3480.810 & 100 & & 28720.72 & 7 & & & & & & & 0.940 \\
\hline 3480.052 & 500 & 3 & 28726.98 & 7 & & & & & & & 1.581 \\
\hline 3479.684 & 300 & 2 & 28730.02 & 1 & 2 & 1.195 & 3 & 1.072 & & & \\
\hline 3478.612 & 150 & 1 & 28738.87 & 3 & & & & & & 0.652 & \\
\hline 3476.385 & 200 & 3 & 28757.28 & 2 & 3 & 0.860 & 4 & 1.010 & & & \\
\hline 3474.530 & 200 & 3 & 28772.63 & 3 & & & & & & 0.510 & \\
\hline 3473.545 & 400 & 8 & 28780.79 & 3 & & & & & & 1.515 & \\
\hline 3472.691 & 150 & 4 & 28787.87 & 7 & & & & & & & 1.021 \\
\hline 3471.959 & $500 \mathrm{r}$ & 501 & 28793.94 & 7 & & & & & & & 1.090 \\
\hline 3471.001 & 300 & 15 & 28801.89 & 2 & & & & & & & 1.442 \\
\hline 3470.568 & 300 & 20 & 28805.48 & 3 & 4 & 1.120 & 4 & 1.308 & & & \\
\hline 3469.345 & $1000 \mathrm{r}$ & 150 & 28815.63 & 7 & & & & & & & 1.237 \\
\hline 3465.061 & $200 \mathrm{~b}$ & 25 & 28851.25 & 3 & 2 & 0.776 & 2 & 1.159 & & & \\
\hline 3464.882 & 75 & & 28852.74 & 3 & & & & & & 0.184 & \\
\hline 3462.981 & 100 & 2 & 28868.58 & 7 & & & & & & & 1.118 \\
\hline 3461.574 & 200 & 3 & 28880.32 & 1 & 3 & 1.240 & 4 & 1.065 & & & \\
\hline 3459.907 & 20 & 2 & 28894.24 & 3 & & & & & & 0.237 & \\
\hline 3458.752 & 15 & & 28903.88 & 3 & & & & & & 1.247 & \\
\hline 3458.142 & 200 & 8 & 28908.98 & 7 & & & & & & & 1.194 \\
\hline 3457.829 & 75 & & 28911.60 & 3 & & & & & & 0.248 & \\
\hline 3457.434 & 150 & 5 & 28914.90 & 3 & & & & & & 0.158 & \\
\hline 3457.069 & $400 \mathrm{r}$ & 25 & 28917.95 & 3 & 2 & 0.734 & 2 & 0.949 & & & \\
\hline 3456.695 & $200 \mathrm{~b}$ & 8 & 28921.08 & 7 & & & & & & & 1.133 \\
\hline 3455.612 & $200 \mathrm{~b}$ & 10 & 28930.14 & 3 & & & & & & 0.269 & \\
\hline 3455.273 & 20 & 5 & 28932.98 & 3 & & & & & & 0.193 & \\
\hline 3454.206 & 200 & 15 & 28941.92 & 3 & & & & & & 0.448 & \\
\hline 3452.335 & 25 & & 28957.61 & 7 & & & & & & & 0.970 \\
\hline 3452.207 & 75 & 1 & 28958.68 & 7 & & & & & & & 1.014 \\
\hline 3451.308 & 100 & & 28966.22 & 1 & 1 & 1.276 & 2 & 1.016 & & & \\
\hline 3450.948 & 100 & 15 & 28969.25 & 3 & & & & & & 0.158 & \\
\hline 3450.268 & 150 & 8 & 28974.95 & 3 & 2 & 1.267 & 2 & 1.707 & & & \\
\hline 3448.949 & 300 & 10 & 28986.03 & 2 & 4 & 0.864 & 5 & 1.118 & & & \\
\hline 3446.546 & 300 & 8 & 29006.24 & 2 & 3 & 0.793 & 4 & 1.213 & & & \\
\hline 3444.795 & 100 & 3 & 29020.98 & 3 & 2 & 0.720 & 2 & 0.984 & & & \\
\hline 3443.980 & 100 & 8 & 29027.86 & 3 & & & & & & 1.435 & \\
\hline 3443.497 & 1501 & 2 & 29031.93 & 7 & & & & & & & 0.784 \\
\hline 3443.409 & $200 \mathrm{~s}$ & 3 & 29032.67 & 7 & & & & & & 0.474 & \\
\hline 3442.305 & 25 & 1 & 29041.98 & 3 & & & & & & 0.167 & \\
\hline 3441.036 & 100 & 20 & 29052.69 & 3 & & & & & & 0.488 & \\
\hline 3440.070 & 25 & 3 & 29060.84 & 7 & & & & & & & 0.916 \\
\hline 3435.487 & 251 & & 29099.61 & 7 & & & & & 0.231 & & \\
\hline 3434.727 & $200 \mathrm{~b}$ & 2 & 29106.05 & 7 & & & & & & & 0.997 \\
\hline 3433.238 & 50 & & 29118.68 & 7 & & & & & & & 0.822 \\
\hline 3432.860 & 20 & 1 & 29121.88 & 7 & & & & & & & 0.590 \\
\hline 3431.265 & 200 & 5 & 29135.42 & 7 & & & & & & & 0.816 \\
\hline 3429.871 & $100 \mathrm{~b}$ & & 29147.26 & 7 & & & & & & & 0.876 \\
\hline 3429.574 & 200 & 10 & 29149.78 & 3 & & & & & & 0.726 & \\
\hline 3427.089 & $200 \mathrm{~s}$ & & 29170.92 & 3 & 2 & 1.020 & 2 & 1.216 & & & \\
\hline 3425.613 & 100 & 2 & 29183.49 & 3 & & & & & & 0.966 & \\
\hline 3425.435 & 150 & 15 & 29185.01 & 7 & & & & & & & 0.978 \\
\hline 3425.140 & $200 \mathrm{~b}$ & 2 & 29187.52 & 3 & & & & & & 0.302 & \\
\hline 3421.678 & 200 & 4 & 29217.04 & 7 & & & & & & & 0.833 \\
\hline 3420.372 & 200 & & 29228.20 & 3 & & & & & & 0.821 & \\
\hline 3420.196 & 100 & 3 & 29229.70 & 7 & & & & & & & 1.080 \\
\hline
\end{tabular}


TABLE 3. Zeeman effect of Th $\mathrm{I}$-continued

\begin{tabular}{|c|c|c|c|c|c|c|c|c|c|c|c|}
\hline \multirow{2}{*}{$\begin{array}{l}\text { Wavelength } \\
(\AA)\end{array}$} & \multicolumn{2}{|c|}{ Intensity } & \multirow{2}{*}{$\begin{array}{l}\text { Wavenumber } \\
\qquad\left(\mathrm{cm}^{-1}\right)\end{array}$} & \multirow{2}{*}{ Type } & \multirow{2}{*}{$J_{1}$} & \multirow{2}{*}{$g_{1}$} & \multirow{2}{*}{$J_{2}$} & \multirow{2}{*}{$g_{2}$} & \multirow{2}{*}{$\Delta g$} & \multirow{2}{*}{$p$} & \multirow{2}{*}{$n$} \\
\hline & Lamp & Spark & & & & & & & & & \\
\hline 3418.032 & 15 & & 29248.21 & 3 & & & & & & 0.462 & \\
\hline 3417.498 & $400 \mathrm{r}$ & 10 & 29252.78 & 3 & 2 & 0.733 & 2 & 0.978 & & & \\
\hline 3416.595 & 150 & 3 & 29260.51 & 3 & & & & & & 0.350 & \\
\hline 3416.386 & $100 \mathrm{~b}$ & & 29262.31 & 7 & & & & & & & 1.140 \\
\hline 3415.885 & $500 \mathrm{r}$ & 75 & 29266.60 & 3 & & & & & & 0.282 & \\
\hline 3414.299 & 75 & & 29280.19 & 7 & & & & & & & 1.074 \\
\hline 3412.181 & 200 & & 29298.36 & 1 & & & & & 0.280 & & \\
\hline 3410.699 & 25 & & 29311.09 & 3 & & & & & & 0.236 & \\
\hline 3410.184 & 150 & 3 & 29315.52 & 3 & 1 & 0.076 & 1 & 1.476 & & & \\
\hline 3407.832 & $400 \mathrm{~b}$ & 10 & 29335.75 & 7 & & & & & & & 0.853 \\
\hline 3406.978 & 10 & & 29343.10 & 3 & & & & & & 0.268 & \\
\hline 3406.841 & 50 & & 29344.29 & 7 & & & & & & & 1.066 \\
\hline 3404.313 & 50 & & 29366.08 & 3 & & & & & & 0.263 & \\
\hline 3403.903 & 200 & 8 & 29369.61 & 7 & & & & & & & 1.252 \\
\hline 3400.680 & 25 & & 29397.44 & 3 & & & & & & 0.724 & \\
\hline 3400.630 & 75 & 3 & 29397.88 & 3 & 1 & 1.109 & 1 & 2.408 & & & \\
\hline 3399.041 & 100 & 4 & 29411.62 & 7 & & & & & & & 1.538 \\
\hline 3398.545 & $1500 \mathrm{r}$ & 50 & 29415.92 & 7 & & & & & & & 1.102 \\
\hline 3395.986 & $200 \mathrm{~b}$ & 1 & 29438.08 & 7 & & & & & & & 0.959 \\
\hline 3394.583 & 15 & 3 & 29450.24 & 3 & & & & & & 0.332 & \\
\hline 3393.993 & 200 & 2 & 29455.37 & 3 & & & & & & 0.079 & \\
\hline 3393.421 & 150 & 10 & 29460.33 & 7 & & & & & & & 1.036 \\
\hline 3392.473 & 100 & 2 & 29468.57 & 7 & & & & & & & 1.168 \\
\hline 3390.849 & 200 & 8 & 29482.67 & 7 & & & & & & & 1.043 \\
\hline 3390.368 & $600 \mathrm{r}$ & 75 & 29486.85 & 7 & & & & & & & 1.468 \\
\hline 3389.038 & 300 & 8 & 29498.43 & 7 & & & & & & & 0.859 \\
\hline 3388.348 & 50 & & 29504.44 & 3 & & & & & & 0.414 & \\
\hline 3388.154 & $100 \mathrm{~b}$ & 5 & 29506.13 & 3 & & & & & & 1.052 & \\
\hline 3387.119 & 15 & & 29515.14 & 7 & & & & & & & 0.949 \\
\hline 3384.177 & 15 & & 29540.80 & 3 & & & & & & 0.495 & \\
\hline 3383.586 & 200 & 8 & 29545.96 & 7 & & & & & & & 0.938 \\
\hline 3382.829 & 15 & & 29552.57 & 7 & & & & & & & 1.108 \\
\hline 3381.907 & 25 & & 29560.63 & 3 & & & & & & 0.522 & \\
\hline 3377.483 & 50 & 40 & 29599.35 & 1 & 1 & 2.407 & 2 & 1.351 & & & \\
\hline 3375.587 & $200 \mathrm{~b}$ & & 29615.97 & 2 & & & & & & & 1.623 \\
\hline 3374.975 & $1500 \mathrm{r}$ & 75 & 29621.34 & 7 & & & & & & & 1.283 \\
\hline 3372.822 & $400 \mathrm{r}$ & 100 & 29640.25 & 1 & & & & & & & 0.547 \\
\hline 3370.887 & 100 & 5 & 29657.26 & 3 & & & & & & 0.744 & \\
\hline 3370.738 & 100 & 4 & 29658.58 & 7 & & & & & & & 0.482 \\
\hline 3369.358 & 20 & & 29670.73 & 7 & & & & & & & 1.192 \\
\hline 3369.000 & 150 & 4 & 29673.87 & 3 & & & & & & 0.427 & \\
\hline 3368.318 & 10 & 3 & 29679.88 & 3 & & & & & & 0.404 & \\
\hline 3367.582 & $200 \mathrm{r}$ & 10 & 29686.37 & 2 & 2 & 0.742 & 3 & 1.270 & & & \\
\hline 3365.979 & 150 & $5 b$ & 29700.50 & 7 & & & & & & & 0.977 \\
\hline 3365.337 & $400 \mathrm{r}$ & 50 & 29706.17 & 2 & 2 & 0.724 & 3 & 1.084 & & & \\
\hline 3365.137 & 300 & 15 & 29707.93 & 7 & & & & & & & 0.993 \\
\hline 3364.154 & $20 \mathrm{~b}$ & 2 & 29716.62 & 2 & & & & & & & 1.294 \\
\hline 3360.997 & $400 \mathrm{r}$ & 150 & 29744.53 & 2 & 2 & 0.748 & 3 & 1.148 & & & \\
\hline 3350.352 & 200 & 5 & 29839.04 & 3 & & & & & & 0.193 & \\
\hline 3348.288 & 40 & 1 & 29857.43 & 3 & 1 & 1.427 & 1 & 2.444 & & & \\
\hline 3347.991 & 200 & 5 & 29860.08 & 7 & & & & & & & 1.018 \\
\hline 3347.403 & 150 & & 29865.32 & 1 & 4 & 1.303 & 5 & 1.078 & & & \\
\hline 3345.170 & 150 & 3 & 29885.25 & 3 & & & & & & 0.099 & \\
\hline 3343.813 & $75 \mathrm{~s}$ & 3 & 29897.38 & 7 & & & & & & & 0.978 \\
\hline 3343.499 & 150 & 5 & 29900.19 & 2 & & & & & & & 1.408 \\
\hline
\end{tabular}


TABLE 3. Zeeman effect of Th $\mathrm{I}-$ continued

\begin{tabular}{|c|c|c|c|c|c|c|c|c|c|c|c|}
\hline \multirow{2}{*}{ 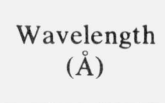 } & \multicolumn{2}{|c|}{ Intensity } & \multirow{2}{*}{$\begin{array}{c}\text { Wavenumber } \\
\left(\mathrm{cm}^{-1}\right)\end{array}$} & \multirow{2}{*}{ Type } & \multirow{2}{*}{$J_{1}$} & \multirow{2}{*}{$g_{1}$} & \multirow{2}{*}{$J_{2}$} & \multirow{2}{*}{$g_{2}$} & \multirow{2}{*}{$\Delta g$} & \multirow{2}{*}{$p$} & \multirow{2}{*}{$n$} \\
\hline & Lamp & Spark & & & & & & & & & \\
\hline 3343.344 & 200 & 10 & 29901.57 & 3 & & & & & & 0.383 & \\
\hline 3343.164 & 300 & 5 & 29903.19 & 2 & 2 & 1.244 & 3 & 1.004 & & & \\
\hline 3342.898 & 20 & & 29905.56 & 3 & & & & & & 0.483 & \\
\hline 3341.550 & 10 & 2 & 29917.63 & 3 & & & & & & 1.118 & \\
\hline 3341.205 & 50 & 2 & 29920.72 & 3 & & & & & & 0.505 & \\
\hline 3340.337 & $100 \mathrm{~b}$ & 1 & 29928.49 & 7 & & & & & & & 1.464 \\
\hline 3340.021 & 15 & & 29931.33 & 7 & & & & & & & 1.123 \\
\hline 3339.257 & 15 & & 29938.18 & 7 & & & & & & & 1.454 \\
\hline 3337.275 & 200 & 5 & 29955.95 & 3 & & & & & & 0.259 & \\
\hline 3336.074 & 150 & & 29966.74 & 3 & & & & & & 0.607 & \\
\hline 3335.694 & 150 & 4 & 29970.15 & 2 & 1 & 0.050 & 2 & 1.220 & & & \\
\hline 3335.238 & 300 & 5 & 29974.24 & 2 & & & & & 0.210 & & \\
\hline 3332.479 & $100 \mathrm{~b}$ & 1 & 29999.06 & 7 & & & & & & & 0.952 \\
\hline 3331.727 & 50 & 2 & 30005.83 & 7 & & & & & & & 1.074 \\
\hline 3330.976 & 150 & 4 & 30012.60 & 7 & & & & & & & 0.998 \\
\hline 3330.477 & $1500 \mathrm{r}$ & 40 & 30017.10 & 2 & 2 & 0.733 & 3 & 1.129 & & & \\
\hline 3329.728 & $300 \mathrm{r}$ & 75 & 30023.85 & 3 & & & & & & 0.705 & \\
\hline 3327.527 & 50 & 2 & 30043.70 & 3 & & & & & & 0.482 & \\
\hline 3327.193 & $800 \mathrm{r}$ & 25 & 30046.73 & 7 & & & & & & & 1.275 \\
\hline 3322.093 & $400 \mathrm{r}$ & 10 & 30092.85 & 7 & & & & & & & 1.139 \\
\hline 3321.574 & $300 \mathrm{r}$ & 40 & 30097.55 & 7 & & & & & & & 0.862 \\
\hline 3320.879 & 20 & & 30103.85 & 3 & & & & & & 1.020 & \\
\hline 3320.476 & $400 \mathrm{r}$ & 25 & 30107.50 & 1 & & & & & & & 0.817 \\
\hline 3320.301 & 600 & 500 & 30109.09 & 7 & & & & & & & 0.995 \\
\hline 3319.572 & 50 & & 30115.70 & 3 & & & & & & 0.941 & \\
\hline 3318.390 & 200 & 5 & 30126.43 & 7 & & & & & & & 0.993 \\
\hline 3317.576 & 25 & & 30133.82 & 7 & & & & & & & 1.051 \\
\hline 3315.848 & 200 & 2 & 30149.52 & 2 & 1 & 0.733 & 2 & 1.027 & & & \\
\hline 3314.793 & $400 \mathrm{r}$ & & 30159.12 & 7 & & & & & & & 1.044 \\
\hline 3313.647 & $300 \mathrm{r}$ & & 30169.55 & 3 & & & & & & 0.075 & 1.215 \\
\hline 3313.437 & 50 & 4 & 30171.46 & 3 & & & & & & 0.512 & \\
\hline 3313.387 & $75 \mathrm{~b}$ & & 30171.91 & 7 & & & & & & & 0.992 \\
\hline 3313.073 & 2001 & 40 & 30174.77 & 2 & & & & & & & 1.575 \\
\hline 3310.637 & $150 \mathrm{~d}$ & $15 \mathrm{~b}$ & 30196.98 & 3 & & & & & & 0.587 & \\
\hline 3310.446 & 100 & $10 \mathrm{~b}$ & 30198.72 & 7 & & & & & & & 1.298 \\
\hline 3309.365 & $1000 \mathrm{r}$ & 40 & 30208.58 & 3 & 2 & 0.744 & 2 & 1.095 & & & \\
\hline 3305.303 & $400 \mathrm{r}$ & 40 & 30245.71 & 3 & 1 & 0.846 & 1 & 1.481 & & & \\
\hline 3304.238 & $3000 \mathrm{r}$ & 200 & 30255.45 & 2 & 2 & 0.732 & 3 & 1.082 & & & \\
\hline 3303.281 & 100 & 5 & 30264.22 & 7 & & & & & & & 1.048 \\
\hline 3302.192 & $400 \mathrm{r}$ & 5 & 30274.19 & 1 & & & & & & & 0.710 \\
\hline 3301.651 & $1500 \mathrm{r}$ & 150 & 30279.16 & 2 & & & & & & & 1.556 \\
\hline 3300.775 & 75 & 4 & 30287.20 & 3 & & & & & & 0.277 & \\
\hline 3298.811 & 200 & 8 & 30305.22 & 1 & & & & & & & 0.809 \\
\hline 3298.697 & 75 & 4 & 30306.27 & 3 & & & & & & 0.178 & \\
\hline 3296.372 & 150 & 8 & 30327.64 & 1 & & & & & 0.570 & & \\
\hline 3292.926 & 200 & 4 & 30359.39 & 3 & & & & & & 0.635 & \\
\hline 3291.410 & 50 & & 30373.37 & 7 & & & & & 0.150 & & 0.566 \\
\hline 3291.037 & 401 & 1 & 30376.81 & 7 & & & & & & & 1.087 \\
\hline 3289.517 & 100 & 10 & 30390.85 & 3 & & & & & & 0.332 & \\
\hline 3283.671 & 100 & 4 & 30444.95 & 3 & & & & & & 0.314 & \\
\hline 3282.837 & 40 & 2 & 30452.68 & 3 & & & & & & 0.398 & \\
\hline 3281.049 & $400 \mathrm{r}$ & 25 & 30469.28 & 7 & & & & & & & 1.060 \\
\hline 3280.764 & $150 \mathrm{~b}$ & 20 & 30471.92 & 7 & & & & & & & 0.719 \\
\hline 3279.025 & 75 & 4 & 30488.09 & 7 & & & & & & & 0.974 \\
\hline 3278.733 & 100 & 25 & 30490.80 & 2 & & & & & & & 1.753 \\
\hline
\end{tabular}


TABLE 3. Zeeman effect of Th $\mathrm{I}$-continued

\begin{tabular}{|c|c|c|c|c|c|c|c|c|c|c|c|}
\hline \multirow{2}{*}{$\begin{array}{l}\text { Wavelength } \\
\text { (A) }\end{array}$} & \multicolumn{2}{|c|}{ Intensity } & \multirow{2}{*}{$\begin{array}{l}\text { Wavenumber } \\
\qquad\left(\mathrm{cm}^{-1}\right)\end{array}$} & \multirow{2}{*}{ Type } & \multirow{2}{*}{$J_{1}$} & \multirow{2}{*}{$g_{1}$} & \multirow{2}{*}{$J_{2}$} & \multirow{2}{*}{$g_{2}$} & \multirow{2}{*}{$\Delta g$} & \multirow{2}{*}{$p$} & \multirow{2}{*}{$n$} \\
\hline & Lamp & Spark & & & & & & & & & \\
\hline 3277.702 & 50 & 3 & 30500.39 & 7 & & & & & & & 1.145 \\
\hline 3276.721 & 300 & 5 & 30509.52 & 3 & & & & & & 0.480 & \\
\hline 3276.225 & 150 & 8 & 30514.14 & 7 & & & & & & & 1.328 \\
\hline 3276.045 & 50 & 5 & 30515.82 & 3 & & & & & & 0.287 & \\
\hline 3272.027 & $800 \mathrm{r}$ & 75 & 30553.29 & 3 & 2 & 0.730 & 2 & 1.044 & & & \\
\hline 3271.892 & $300 \mathrm{r}$ & 100 & 30554.55 & 7 & & & & & & & 1.080 \\
\hline 3271.546 & 50 & 3 & 30557.78 & 2 & & & & & 0.280 & & \\
\hline 3270.874 & 150 & 10 & 30564.06 & 3 & & & & & & 0.496 & \\
\hline 3270.100 & 100 & 5 & 30571.20 & 3 & & & & & & 0.665 & \\
\hline 3265.604 & 20 & & 30613.38 & 3 & & & & & & 0.401 & \\
\hline 3263.183 & $400 \mathrm{r}$ & 20 & 30636.09 & 3 & & & & & & 0.262 & \\
\hline 3262.316 & 75 & 4 & 30644.24 & 7 & & & & & & & 1.256 \\
\hline 3261.497 & 20 & & 30651.93 & 3 & & & & & & 0.301 & \\
\hline 3259.061 & $600 \mathrm{r}$ & 50 & 30674.84 & 7 & & & & & & & 0.961 \\
\hline 3258.103 & $200 \mathrm{r}$ & & 30683.84 & 7 & & & & & & & 1.275 \\
\hline 3256.503 & 20 & 3 & 30698.93 & 7 & & & & & & & 0.942 \\
\hline 3255.920 & 150 & 8 & 30704.43 & 2 & & & & & 0.176 & & \\
\hline 3251.916 & $2000 \mathrm{r}$ & 200 & 30742.23 & 7 & & & & & & & 1.156 \\
\hline 3249.855 & $800 \mathrm{r}$ & 25 & 30761.73 & 2 & 2 & 0.741 & 3 & 1.193 & & & \\
\hline 3248.697 & $75 b$ & & 30772.69 & 3 & & & & & & 0.283 & \\
\hline 3247.331 & 15 & & 30785.64 & 7 & & & & & & & 0.964 \\
\hline 3247.218 & 75 & 4 & 30786.71 & 3 & & & & & & 0.072 & \\
\hline 3245.994 & 200 & 8 & 30798.32 & 3 & & & & & & 1.043 & \\
\hline 3244.043 & 150 & 10 & 30816.84 & 3 & & & & & & 0.255 & \\
\hline 3243.916 & 50 & 5 & 30818.05 & 3 & & & & & & 0.290 & \\
\hline 3242.145 & 50 & $15 b$ & 30834.88 & 7 & & & & & & & 0.896 \\
\hline 3240.644 & $400 \mathrm{r}$ & $40 \mathrm{~b}$ & 30849.17 & 7 & & & & & & & 1.121 \\
\hline 3238.934 & $400 \mathrm{r}$ & 20 & 30865.45 & 1 & 2 & 1.307 & 3 & 1.095 & & & \\
\hline 3236.573 & 100 & $8 \mathrm{~b}$ & 30887.96 & 1 & & & & & 0.240 & & \\
\hline 3234.996 & $400 \mathrm{r}$ & 20 & 30903.02 & 1 & & & & & & & 1.030 \\
\hline 3231.461 & 75 & & 30936.83 & 7 & & & & & & & 1.049 \\
\hline 3225.007 & 100 & 5 & 30998.74 & 2 & 1 & 0.866 & 2 & 1.194 & & & \\
\hline 3222.807 & 200 & 15 & 31019.89 & 7 & & & & & & & 1.215 \\
\hline 3221.291 & $400 \mathrm{~b}$ & & 31034.49 & 7 & & & & & & & 1.128 \\
\hline 3220.986 & $75 b$ & & 31037.43 & 2 & & & & & & & 0.796 \\
\hline 3219.490 & $20 \mathrm{~h}$ & 4 & 31051.85 & 7 & & & & & & & 0.859 \\
\hline 3216.998 & 75 & 3 & 31075.91 & 3 & & & & & & 1.185 & \\
\hline 3215.778 & 100 & 100 & 31087.69 & 7 & & & & & & & 0.915 \\
\hline 3214.648 & 150 & 5 & 31098.62 & 3 & & & & & & 0.871 & \\
\hline 3214.381 & $400 \mathrm{r}$ & 25 & 31101.20 & 3 & & & & & & 0.400 & \\
\hline 3214.076 & $400 \mathrm{r}$ & 50 & 31104.15 & 7 & & & & & & & 1.010 \\
\hline 3211.997 & $100 \mathrm{r}$ & 5 & 31124.29 & 3 & & & & & & 0.131 & \\
\hline 3210.779 & 200 & 5 & 31136.09 & 7 & & & & & & & 1.172 \\
\hline 3207.938 & $50 \mathrm{r}$ & 5 & 31163.67 & 3 & 2 & 0.878 & 2 & 1.262 & & & \\
\hline 3202.521 & $200 \mathrm{r}$ & 8 & 31216.38 & 3 & & & & & & 0.653 & \\
\hline 3198.969 & $75 \mathrm{~s}$ & & 31251.04 & 3 & & & & & & 0.214 & \\
\hline 3198.694 & $50 \mathrm{~b}$ & & 31253.73 & 7 & & & & & & & 0.806 \\
\hline 3195.688 & $400 \mathrm{r}$ & 50 & 31283.12 & 2 & 2 & 0.750 & 3 & 1.120 & & & \\
\hline 3193.781 & $200 r$ & 5 & 31301.80 & 1 & 1 & 1.619 & 2 & 1.259 & & & \\
\hline 3192.586 & $400 \mathrm{r}$ & 20 & 31313.52 & 7 & & & & & & & 0.920 \\
\hline 3189.711 & 200 & 8 & 31341.74 & 3 & & & & & & 0.416 & \\
\hline 3189.562 & 200 & 3 & 31343.20 & 7 & & & & & & & 1.109 \\
\hline 3185.628 & 200 & 3 & 31381.91 & 3 & & & & & & 3.269 & \\
\hline 3184.843 & 300 & 8 & 31389.64 & 3 & & & & & & 0.320 & \\
\hline 3184.277 & 300 & $15 b$ & 31395.22 & 1 & & & & & 0.243 & & \\
\hline
\end{tabular}


TABLE 3. Zeeman effect of Th $\mathrm{I}$-continued

\begin{tabular}{|c|c|c|c|c|c|c|c|c|c|c|c|}
\hline \multirow{2}{*}{$\begin{array}{l}\text { Wavelength } \\
\text { (A) }\end{array}$} & \multicolumn{2}{|c|}{ Intensity } & \multirow{2}{*}{$\begin{array}{l}\text { Wavenumber } \\
\qquad\left(\mathrm{cm}^{-1}\right)\end{array}$} & \multirow{2}{*}{ Type } & \multirow{2}{*}{$J_{1}$} & \multirow{2}{*}{$g_{1}$} & \multirow{2}{*}{$J_{2}$} & \multirow{2}{*}{$g_{2}$} & \multirow{2}{*}{$\Delta g$} & \multirow{2}{*}{$p$} & \multirow{2}{*}{$n$} \\
\hline & Lamp & Spark & & & & & & & & & \\
\hline 3183.384 & 100 & 2 & 31404.03 & 7 & & & & & & & 1.056 \\
\hline 3181.670 & $500 \mathrm{r}$ & 20 & 31420.95 & 3 & & & & & & 0.546 & \\
\hline 3180.194 & $1000 \mathrm{r}$ & 1500 & 31435.53 & 7 & & & & & & & 1.077 \\
\hline 3178.622 & 200 & 4 & 31451.07 & 3 & & & & & & 0.241 & \\
\hline 3178.244 & $600 \mathrm{r}$ & 100 & 31454.82 & 7 & & & & & & & 1.028 \\
\hline 3177.402 & 100 & 2 & 31463.15 & 3 & 1 & 1.268 & 1 & 1.462 & & & \\
\hline 3176.167 & $200 \mathrm{r}$ & 5 & 31475.38 & 7 & & & & & & & 1.130 \\
\hline 3174.840 & 200 & 4 & 31488.54 & 3 & & & & & & 0.660 & \\
\hline 3174.020 & 300 & 8 & 31496.68 & 3 & & & & & & 0.142 & \\
\hline 3173.427 & $500 \mathrm{r}$ & 50 & 31502.56 & 7 & & & & & 0.220 & & \\
\hline 3171.276 & $500 \mathrm{r}$ & 75 & 31523.93 & 2 & 2 & 0.743 & 3 & 1.108 & & & \\
\hline 3168.342 & 100 & 2 & 31553.11 & 7 & & & & & & & 0.852 \\
\hline 3165.048 & 150 & 3 & 31585.95 & 2 & 1 & 0.076 & 2 & 1.073 & & & \\
\hline 3161.364 & $200 d$ & 5 & 31622.76 & 1 & 3 & 1.078 & 4 & 0.898 & & & \\
\hline 3161.148 & 100 & 2 & 31624.93 & 3 & & & & & & 0.795 & \\
\hline 3158.394 & 100 & & 31652.50 & 3 & & & & & 0.456 & & \\
\hline 3157.221 & $300 \mathrm{r}$ & 8 & 31664.25 & 7 & & & & & & & 0.952 \\
\hline 3156.866 & 300 & & 31667.81 & 1 & 1 & 1.466 & 2 & 1.221 & & & \\
\hline 3152.395 & 100 & 3 & 31712.73 & 1 & 1 & 1.175 & 2 & 0.745 & & & \\
\hline 3152.295 & 100 & 3 & 31713.74 & 3 & & & & & & 0.235 & \\
\hline 3151.184 & 300 & 5 & 31724.92 & 3 & & & & & & 0.459 & \\
\hline 3151.017 & 200 & & 31726.60 & 3 & & & & & & 0.203 & \\
\hline 3150.536 & 100 & 8 & 31731.44 & 7 & & & & & & & 1.157 \\
\hline 3147.449 & 200 & 25 & 31762.56 & 7 & & & & & & & 1.149 \\
\hline 3146.310 & $200 \mathrm{r}$ & 8 & 31774.07 & 7 & & & & & & & 1.256 \\
\hline 3145.637 & $400 \mathrm{r}$ & 25 & 31780.86 & 2 & 2 & 0.738 & 3 & 1.175 & & & \\
\hline 3144.145 & 100 & 3 & 31795.94 & 3 & & & & & & 0.102 & \\
\hline 3142.422 & 100 & 5 & 31813.37 & 3 & & & & & & 0.482 & \\
\hline 3139.892 & 150 & 100 & 31839.00 & 3 & 4 & 0.968 & 4 & 1.220 & & & \\
\hline 3138.200 & 100 & 10 & 31856.17 & 7 & & & & & & & 1.088 \\
\hline 3136.829 & $500 \mathrm{r}$ & 8 & 31870.09 & 3 & 2 & 0.730 & 2 & 0.941 & & & \\
\hline 3136.216 & $1000 \mathrm{r}$ & 100 & 31876.32 & 7 & & & & & & & 1.088 \\
\hline 3134.203 & 400 & 5 & 31896.79 & 2 & & & & & & & 1.160 \\
\hline 3132.102 & 300 & 5 & 31918.19 & 7 & & & & & & & 1.477 \\
\hline 3126.414 & 200 & 2 & 31976.26 & 3 & & & & & & 0.485 & \\
\hline 3124.986 & 75 & 2 & 31990.87 & 7 & & & & & 0.180 & & \\
\hline 3120.881 & $400 \mathrm{r}$ & 25 & 32032.94 & 7 & & & & & & & 1.498 \\
\hline 3116.352 & $300 \mathrm{r}$ & 10 & 32079.50 & 3 & 4 & 1.008 & 4 & 1.213 & & & \\
\hline 3116.263 & $500 \mathrm{r}$ & & 32080.42 & 7 & & & & & & & 0.674 \\
\hline 3111.280 & 300 & 8 & 32131.79 & 7 & & & & & & & 1.202 \\
\hline 3108.636 & 200 & 3 & 32159.12 & 3 & & & & & & 0.139 & \\
\hline 3108.504 & $300 \mathrm{r}$ & 10 & 32160.48 & 3 & & & & & 0.340 & & \\
\hline 3104.966 & $100 \mathrm{r}$ & 3 & 32197.13 & 2 & 2 & 0.761 & 3 & 1.155 & & & \\
\hline 3102.125 & 200 & 20 & 32226.61 & 7 & & & & & & 0.383 & \\
\hline 3099.184 & 100 & 2 & 32257.20 & 3 & & & & & & 0.322 & \\
\hline 3098.726 & $300 \mathrm{r}$ & 5 & 32261.96 & 2 & 3 & 1.070 & 4 & 1.200 & & & \\
\hline 3098.119 & 100 & & 32268.28 & 3 & & & & & & 0.600 & \\
\hline 3093.719 & 200 & 3 & 32314.17 & 3 & & & & & & 0.159 & \\
\hline 3092.828 & $300 \mathrm{r}$ & 8 & 32323.48 & 2 & & & & & & & 1.382 \\
\hline 3082.507 & $100 \mathrm{~b}$ & & 32431.71 & 7 & & & & & & & 1.624 \\
\hline 3079.574 & 150 & 2 & 32462.60 & 3 & & & & & & 0.825 & \\
\hline 3078.625 & $150 \mathrm{~b}$ & 2 & 32472.61 & 7 & & & & & & & 1.017 \\
\hline 3077.718 & $300 \mathrm{r}$ & 5 & 32482.17 & 2 & & & & & & & 1.483 \\
\hline 3076.410 & 150 & 30 & 32495.98 & 3 & 1 & 1.116 & 1 & 1.493 & & & \\
\hline 3075.687 & 100 & 3 & 32503.61 & 3 & & & & & & 0.956 & \\
\hline
\end{tabular}


TABLE 3. Zeeman effect of Th $\mathrm{I}-$ continued

\begin{tabular}{|c|c|c|c|c|c|c|c|c|c|c|c|}
\hline \multirow{2}{*}{$\begin{array}{l}\text { Wavelength } \\
(\AA)\end{array}$} & \multicolumn{2}{|c|}{ Intensity } & \multirow{2}{*}{$\begin{array}{c}\text { Wa venumber } \\
\left(\mathrm{cm}^{-1}\right)\end{array}$} & \multirow{2}{*}{ Type } & \multirow{2}{*}{$J_{1}$} & \multirow{2}{*}{$g_{1}$} & \multirow{2}{*}{$J_{2}$} & \multirow{2}{*}{$g_{2}$} & \multirow{2}{*}{$\Delta g$} & \multirow{2}{*}{$p$} & \multirow{2}{*}{$n$} \\
\hline & Lamp & Spark & & & & & & & & & \\
\hline 3071.632 & 50 & & 32546.53 & 7 & & & & & & & 1.225 \\
\hline 3065.683 & $200 \mathrm{r}$ & 2 & 32609.68 & 1 & & & & & 0.168 & & \\
\hline 3065.038 & 50 & 10 & 32616.54 & 7 & & & & & & & 1.164 \\
\hline 3062.454 & $200 \mathrm{r}$ & 2 & 32644.07 & 3 & & & & & & 0.325 & \\
\hline 3061.812 & $400 \mathrm{r}$ & 3 & 32650.91 & 1 & & & & & 0.400 & & \\
\hline 3058.029 & $200 \mathrm{r}$ & 2 & 32691.29 & 3 & & & & & & 0.427 & \\
\hline 3047.816 & $500 \mathrm{r}$ & 2 & 32800.84 & 3 & & & & & & 0.485 & \\
\hline 3043.248 & 100 & 5 & 32850.07 & 7 & & & & & & & 1.928 \\
\hline 3041.941 & 100 & & 32864.19 & 7 & & & & & & & 1.121 \\
\hline 3034.639 & $400 \mathrm{r}$ & 2 & 32943.26 & 7 & & & & & & & 1.097 \\
\hline 3032.016 & $100 \mathrm{r}$ & 2 & 32971.76 & 3 & 1 & & 1 & & & 0.700 & \\
\hline 3031.195 & $100 \mathrm{r}$ & 2 & 32980.68 & 3 & & & & & & 0.654 & \\
\hline 3030.487 & $400 \mathrm{r}$ & 8 & 32988.39 & 3 & & & & & & 0.218 & \\
\hline 3029.477 & 100 & & 32999.39 & 7 & & ' & & & & & 0.956 \\
\hline 3027.896 & 150 & 3 & 33016.62 & 1 & & & & & 0.480 & & \\
\hline 3025.448 & $200 \mathrm{r}$ & 2 & 33043.33 & 2 & 2 & 0.742 & 3 & 1.132 & & & \\
\hline 3021.056 & 300 & 1 & 33091.37 & 7 & & & & & & & 1.401 \\
\hline 3019.889 & 300 & & 33104.15 & 7 & & & & & & & 1.028 \\
\hline 3016.096 & 50 & & 33145.79 & 7 & & & & & & & 1.297 \\
\hline 3014.641 & $150 \mathrm{r}$ & 1 & 33161.79 & 1 & & & & & 0.572 & & \\
\hline 3011.745 & $100 \mathrm{~s}$ & 1 & 33193.67 & 7 & & & & & & & 1.274 \\
\hline 3008.924 & 200 & 2 & 33224.79 & 7 & & & & & & & 1.224 \\
\hline 3006.634 & 200 & 2 & 33250.10 & 7 & & & & & & & 1.101 \\
\hline 3006.164 & $300 \mathrm{r}$ & 2 & 33255.29 & 7 & & & & & & & 1.455 \\
\hline 3004.248 & $150 \mathrm{r}$ & 1 & 33276.50 & 7 & & & & & & & 1.488 \\
\hline 3003.210 & 200 & 1 & 33288.00 & 3 & & & & & & 0.434 & \\
\hline 3002.687 & $200 \mathrm{r}$ & 2 & 33293.80 & 3 & & & & & & 0.587 & \\
\hline 3002.390 & $100 \mathrm{r}$ & & 33297.13 & 3 & & & & & & 0.897 & \\
\hline 2999.272 & 75 & 10 & 33331.71 & 3 & & & & & & 0.329 & \\
\hline 2998.147 & $200 \mathrm{~b}$ & 1 & 33344.21 & 3 & & & & & & 0.507 & \\
\hline 2997.196 & 100 & 8 & 33354.79 & 3 & & & & & & 0.261 & \\
\hline 2995.962 & 100 & 1 & 33368.53 & 7 & & & & & & & 0.803 \\
\hline 2995.166 & 100 & 1 & 33377.40 & 7 & & & & & & & 0.862 \\
\hline 2993.079 & 100 & & 33400.67 & 7 & & & & & & & 0.966 \\
\hline 2987.672 & $300 \mathrm{r}$ & 2 & 33461.12 & 3 & & & & & & 0.464 & \\
\hline 2981.843 & 100 & 15 & 33526.52 & 7 & & & & & & & 0.788 \\
\hline 2980.335 & 150 & 25 & 33543.48 & 7 & & & & & & & 1.399 \\
\hline 2980.112 & $200 \mathrm{r}$ & 2 & 33546.04 & 3 & & & & & & 0.261 & \\
\hline 2979.043 & $100 \mathrm{~b}$ & & 33558.04 & 7 & & & & & & & 1.064 \\
\hline 2973.675 & 100 & 1 & 33618.61 & 3 & & & & & & 0.488 & \\
\hline 2970.566 & 100 & 2 & 33653.79 & 7 & & & & & & & 1.150 \\
\hline 2969.378 & 100 & 8 & 33667.26 & 3 & & & & & & 0.618 & ror \\
\hline 2963.608 & 75 & 8 & 33732.80 & 7 & & & & & & & 1.178 \\
\hline 2963.442 & 75 & & 33734.69 & 3 & & & & & & 0.844 & \\
\hline 2959.854 & 300 & $25 \mathrm{~b}$ & 33775.58 & 7 & & & & & & & 0.881 \\
\hline 2959.137 & 200 & 2 & 33783.77 & 3 & & & & & & 0.466 & \\
\hline 2958.367 & $100 \mathrm{~b}$ & 2 & 33792.57 & 7 & & & & & & & 1.374 \\
\hline 2957.332 & 150 & 10 & 33804.39 & 7 & & & & & & & 1.194 \\
\hline 2943.729 & 400 & 3 & 33960.59 & 3 & & & & & & 0.837 & \\
\hline 2931.783 & 400 & 4 & 34098.96 & 7 & & & & & & & 0.685 \\
\hline 2925.526 & $500 \mathrm{r}$ & 5 & 34171.89 & 7 & & & & & & & 0.843 \\
\hline 2918.922 & 200 & $20 \mathrm{~b}$ & 34249.20 & 7 & & & & & & & 1.167 \\
\hline 2916.369 & $500 \mathrm{r}$ & 10 & 34279.18 & 7 & & & & & & & 0.766 \\
\hline 2909.103 & $300 \mathrm{~d}$ & $8 \mathrm{~d}$ & 34364.79 & 7 & & & & & & & 0.990 \\
\hline 2907.451 & 100 & & 34384.32 & 7 & & & & & & & 1.194 \\
\hline
\end{tabular}


TABLE 3. Zeeman effect of Th $\mathrm{I}$-continued

\begin{tabular}{|c|c|c|c|c|c|c|c|c|c|c|c|}
\hline \multirow{2}{*}{$\begin{array}{c}\text { Wavelength } \\
\text { (§) }\end{array}$} & \multicolumn{2}{|c|}{ Intensity } & \multirow{2}{*}{$\begin{array}{l}\text { Wavenumber } \\
\left(\mathrm{cm}^{-1}\right)\end{array}$} & \multirow{2}{*}{ Type } & \multirow{2}{*}{$J_{1}$} & \multirow{2}{*}{$g_{1}$} & \multirow{2}{*}{$J_{2}$} & \multirow{2}{*}{$g_{2}$} & \multirow{2}{*}{$\Delta g$} & \multirow{2}{*}{$p$} & \multirow{2}{*}{$n$} \\
\hline & Lamp & Spark & & & & & & & & & \\
\hline 2905.097 & $100 \mathrm{~b}$ & 2 & 34412.18 & 7 & & & & & & & 1.238 \\
\hline 2890.078 & $150 \mathrm{r}$ & 5 & 34591.00 & 1 & & & & & 0.703 & & \\
\hline 2889.488 & 50 & 2 & 34598.07 & 7 & & & & & & & 1.506 \\
\hline 2888.785 & 200 & 2 & 34606.48 & 7 & & & & & & & 1.396 \\
\hline 2888.552 & 200 & 3 & 34609.28 & 7 & & & & & & & 1.307 \\
\hline 2886.983 & $150 \mathrm{~b}$ & & 34628.09 & 3 & & & & & & 0.318 & \\
\hline 2883.266 & 400 & 3 & 34672.72 & 7 & & & & & & & 1.329 \\
\hline 2881.577 & 200 & 10 & 34693.04 & 7 & & & & & & & 0.912 \\
\hline 2876.768 & 300 & 3 & 34751.04 & 7 & & & & & & & 1.073 \\
\hline 2862.610 & $300 \mathrm{r}$ & 20 & 34922.90 & 7 & & & & & & & 1.143 \\
\hline 2860.776 & 100 & 2 & 34945.29 & 3 & & & & & & 0.356 & \\
\hline 2860.491 & 150 & 3 & 34948.77 & 7 & & & & & 0.697 & & \\
\hline 2850.597 & $300 \mathrm{~b}$ & 2 & 35070.06 & 3 & & & & & & 0.820 & \\
\hline 2848.084 & $300 \mathrm{r}$ & 2 & 35101.01 & 7 & & & & & & & 1.426 \\
\hline 2846.036 & 150 & 2 & 35126.26 & 3 & & & & & & 0.980 & \\
\hline 2843.906 & $300 r$ & 2 & 35152.58 & 7 & & & & & & & 1.234 \\
\hline 2841.383 & $150 \mathrm{~h}$ & 2 & 35183.79 & 7 & & & & & & & 1.087 \\
\hline 2832.314 & $1000 \mathrm{r}$ & 400 & 35296.44 & 7 & & & & & & & 0.962 \\
\hline 2829.346 & 150 & 10 & 35333.46 & 7 & & & & & & & 1.196 \\
\hline 2828.502 & 2001 & 4 & 35344.00 & 7 & & & & & & & 1.079 \\
\hline 2825.909 & $100 \mathrm{~b}$ & & 35376.44 & 7 & & & & & & & 1.011 \\
\hline 2808.323 & 200 & 2 & 35597.96 & 7 & & & & & & & 1.191 \\
\hline 2804.912 & 200 & 2 & 35641.24 & 3 & & & & & & 0.684 & \\
\hline 2799.432 & 100 & 1 & 35711.01 & 7 & & & & & & & 0.990 \\
\hline 2762.458 & $50 \mathrm{r}$ & & 36188.96 & 3 & & & & & & 1.301 & \\
\hline 2747.139 & $150 \mathrm{~b}$ & & 36390.74 & 7 & & & & & & & 0.665 \\
\hline
\end{tabular}


TABLE 4. Classified lines of Th I

\begin{tabular}{|c|c|c|c|c|c|c|c|c|c|}
\hline \multirow{2}{*}{$\begin{array}{l}\text { Wavelength } \\
\text { (Å) }\end{array}$} & \multicolumn{2}{|c|}{ Intensity } & \multirow{2}{*}{$\begin{array}{l}\text { Wavenumber } \\
\left(\mathrm{cm}^{-1}\right)\end{array}$} & \multirow{2}{*}{ Classification } & \multirow{2}{*}{$\begin{array}{c}\text { Wavelength } \\
(\AA)\end{array}$} & \multicolumn{2}{|c|}{ Inten sity } & \multirow{2}{*}{$\begin{array}{l}\text { Wave number } \\
\qquad\left(\mathrm{cm}^{-1}\right)\end{array}$} & \multirow{2}{*}{ Classification } \\
\hline & Lamp & Spark & & & & Lamp & Spark & & \\
\hline 29662.822 & 0 & & 3370.304 & $98045-13175^{\circ}$ & 23217.661 & 1 & & 4305.891 & $18549_{2}-22855^{\circ}$ \\
\hline 28574.421 & 0 & & 3498.679 & $12847_{3}-16346_{4}^{\circ}$ & 23175.469 & 3 & & 4313.730 & $165546-20867^{\circ}$ \\
\hline 26734.663 & 0 & & 3739.442 & $75023-11241_{3}^{\circ}$ & 23153.597 & 4 & & 4317.805 & $15736 i-20054_{2}$ \\
\hline 26377.115 & 2 & & 3790.131 & $10414_{4}^{\circ}-142045$ & 23127.608 & 1 & & 4322.657 & $13088_{3}-17411_{3}^{\circ}$ \\
\hline 25984.374 & 1 & & 3847.417 & $18549_{2}-22396_{1}^{\circ}$ & 23121.211 & 0 & & 4323.853 & $25690{ }^{\circ}-30014_{4}$ \\
\hline 25971.332 & 0 & & 3849.349 & $142045-180533^{\circ}$ & 23085.081 & 0 & & 4330.620 & $14243 i^{\circ}-185741$ \\
\hline 25913.673 & 0 & & 3857.914 & $23113_{4}^{\circ}-26971_{4}$ & 23007.044 & 1 & & 4345.309 & $27948_{4}^{\circ}-322935$ \\
\hline 25790.101 & 1 & & 3876.399 & $20522_{2}^{\circ}-24399_{3}$ & 22978.346 & 0 & & 4350.736 & $17224_{2}^{\circ}-21575_{2}$ \\
\hline 25735.979 & 1 & & 3884.551 & $13962_{1}-17847_{2}^{\circ}$ & 22964.759 & 1 & & 4353.310 & $21902_{4}^{\circ}-26255_{4}$ \\
\hline 25561.646 & 1 & & 3911.044 & $171665-21077^{\circ}$ & 22900.906 & 2 & & 4365.448 & $21890_{3}^{\circ}-26255_{4}$ \\
\hline 25269.196 & 1 & & 3956.308 & $23015 \circ-269714$ & 22900.565 & 0 & & 4365.513 & $15166_{3}^{\circ}-195324$ \\
\hline 25111.575 & 1 & & 3981.141 & $22399^{\circ}-263805$ & 22875.089 & 0 & & 4370.375 & $17224_{2}^{\circ}-21594_{3}$ \\
\hline 25080.636 & 2 & & 3986.052 & $11877_{1}^{\circ}-15863_{2}$ & 22864.133 & 2 & & 4372.469 & $25180_{7}^{\circ}-295526$ \\
\hline 25049.573 & 2 & & 3990.995 & $13175^{\circ}-171665$ & 22860.040 & 0 & & 4373.252 & $24561 \stackrel{\circ}{3}-289343$ \\
\hline 25032.462 & 0 & & 3993.723 & $25690_{0}^{\circ}-296845$ & 22855.180 & 2 & & 4374.182 & $18614_{1}^{\circ}-229882$ \\
\hline 24997.523 & 1 & & 3999.305 & $13847_{2}-17847_{2}^{\circ}$ & 22851.632 & 3 & & 4374.861 & $8800_{4}-13175_{4}^{\circ}$ \\
\hline 24973.732 & 1 & & 4003.115 & $267236^{\circ}-307267$ & 22843.962 & 1 & & 4376.330 & $12847_{3}-17224_{2}^{\circ}$ \\
\hline 24931.344 & 1 & & 4009.921 & $154934-19503 \stackrel{8}{3}$ & 22834.596 & 2 & & 4378.125 & $3865_{1}-8243_{2}^{\circ}$ \\
\hline 24902.355 & 1 & & 4014.589 & $13945^{\circ}-17959_{4}$ & 22829.522 & 2 & & 4379.098 & $17959_{4}-22338_{3}^{\circ}$ \\
\hline 24789.343 & 1 & & 4032.891 & $21890^{\circ}-259234$ & 22806.940 & 0 & & 4383.434 & $21539^{\circ}-259234$ \\
\hline 24684.317 & 0 & & 4050.050 & $19227^{\circ}-23277_{5}$ & 22785.721 & 1 & & 4387.516 & $142260-18614^{\circ}$ \\
\hline 24516.271 & 1 & & 4077.811 & $23113_{4}^{\circ}-271915$ & 22760.862 & 0 & & 4392.308 & $20522_{2}^{\circ}-24915_{3}$ \\
\hline 24486.763 & 0 & & 4082.725 & $24259_{4}^{\circ}-283425$ & 22750.249 & 1 & & 4394.357 & $18699_{2}-23093_{2}^{\circ}$ \\
\hline 24470.262 & 0 & & 4085.478 & $23481_{1}^{\circ}-27566_{2}$ & 22723.153 & 0 & & 4399.597 & $14032_{2}^{\circ}-18431_{3}$ \\
\hline 24428.586 & 1 & & 4092.448 & $22163^{\circ}-26255_{4}$ & 22710.196 & 3 & & 4402.107 & $9804_{5}-14206_{4}^{\circ}$ \\
\hline 24420.077 & 1 & & 4093.874 & $22877^{\circ}-26971_{4}$ & 22646.195 & 3 & & 4414.548 & $118022-16217_{2}^{\circ}$ \\
\hline 24329.294 & 0 & & 4109.150 & $25575^{\circ}-296845$ & 22616.700 & 1 & & 4420.305 & $139621-183820^{\circ}$ \\
\hline 24301.853 & 1 & & 4113.790 & $132974-17411^{\circ}$ & 22614.383 & 4 & & 4420.758 & $6362_{2}-10783_{2}^{\circ}$ \\
\hline 24262.390 & 1 & & 4120.481 & $18549_{2}-22669_{3}^{\circ}$ & 22544.420 & 1 & & 4434.477 & $25321_{3}^{\circ}-29756_{4}$ \\
\hline 24216.068 & 0 & & 4128.363 & $21575_{2}-25703_{2}^{\circ}$ & 22537.763 & 3 & & 4435.787 & $156183^{\circ}-200542$ \\
\hline 24172.879 & 2 & & 4135.739 & $13088_{3}-17224_{2}^{\circ}$ & 22518.913 & 0 & & 4439.500 & $17959_{4}-22399_{0}^{\circ}$ \\
\hline 24171.733 & 0 & & 4135.935 & $11601_{1}-15736_{1}^{\circ}$ & 22513.933 & 1 & & 4440.482 & $24701 \stackrel{\circ}{\circ}-291415$ \\
\hline 24138.665 & 0 & & 4141.601 & $24421_{3}^{\circ}-285624$ & 22496.819 & 2 & & 4443.860 & $19588 \stackrel{\circ}{-240324}$ \\
\hline 24136.264 & 3 & & 4142.013 & $20522_{2}^{\circ}-24664_{3}$ & 22446.273 & 0 & & 4453.867 & $215943-26048^{\circ}$ \\
\hline 24120.715 & 1 & & 4144.683 & $175010^{\circ}-216454$ & 22439.688 & 0 & & 4455.174 & $15493_{4}-19948_{4}^{\circ}$ \\
\hline 24007.982 & 4 & & 4164.145 & $6362_{2}-10526_{3}^{\circ}$ & 22437.386 & 1 & & 4455.631 & $14243_{1}^{\circ}-18699_{2}$ \\
\hline 23940.033 & 0 & & 4175.964 & $24182_{2}^{\circ}-283583$ & 22377.781 & 1 & & 4467.499 & $18809_{4}^{\circ}-23277_{5}$ \\
\hline 23907.149 & 1 & & 4181.708 & $17959_{4}-22141_{3}^{\circ}$ & 22350.905 & 0 & & 4472.871 & $23093_{2}^{\circ}-27566_{2}$ \\
\hline 23865.994 & 0 & & 4188.919 & $22855_{3}^{\circ}-27044_{3}$ & 22344.755 & 2 & & 4474.102 & $11877^{\circ}-163510$ \\
\hline 23781.839 & 4 & & 4203.742 & $13297_{4}-175018$ & 22323.993 & 0 & & 4478.263 & $21902 \stackrel{\circ}{4}-263805$ \\
\hline 23712.604 & 0 & & 4216.016 & $25336_{6}^{\circ}-295526$ & 22318.327 & 1 & & 4479.400 & $19273_{2}-23752_{2}^{\circ}$ \\
\hline 23704.794 & 1 & & 4217.405 & $22163_{4}^{\circ}-263805$ & 22283.623 & 0 & & 4486.376 & $13945_{3}^{\circ}-18431_{3}$ \\
\hline 23672.844 & 1 & & 4223.097 & $18809_{4}^{\circ}-230324$ & 22215.926 & 1 & & 4500.047 & $18549_{2}-23049_{i}^{\circ}$ \\
\hline 23671.785 & 1 & & 4223.286 & $13175_{4}^{\circ}-173983$ & 22115.259 & 0 & & 4520.531 & $23752_{2}^{\circ}-28273_{2}$ \\
\hline 23663.487 & 1 & & 4224.767 & $14206_{4}^{\circ}-18431_{3}$ & 22103.641 & 4 & & 4522.907 & $165546-21077^{\circ}$ \\
\hline 23608.338 & 0 & & 4234.636 & $17411_{3}^{\circ}-216454$ & 22082.481 & 1 & & 4527.241 & $25321 \stackrel{\circ}{3}-29849_{4}$ \\
\hline 23588.463 & 1 & & 4238.204 & $18431_{3}-22669_{3}^{\circ}$ & 22073.514 & 1 & & 4529.080 & $19503_{3}^{\circ}-24032_{4}$ \\
\hline 23551.636 & 1 & & 4244.831 & $15970_{3}-20214_{3}^{\circ}$ & 22029.034 & 0 & & 4538.225 & $21575_{2}-26113_{2}^{\circ}$ \\
\hline 23514.732 & 5 & & 4251.493 & $11241_{3}^{\circ}-154934$ & 21990.719 & 2 & & 4546.132 & $15166^{\circ}-19713_{3}$ \\
\hline 23477.705 & 0 & & 4258.198 & $24421_{3}^{\circ}-28679_{2}$ & 21987.769 & 0 & & 4546.742 & $19713_{3}-24259_{4}^{\circ}$ \\
\hline 23403.546 & 0 & & 4271.691 & $18930_{3}^{\circ}-23201_{3}$ & 21987.769 & 0 & & 4546.742 & $26882{ }^{\circ}-31429_{1}$ \\
\hline 23330.692 & 2 & & 4285.030 & $26645_{0}^{\circ}-30930_{6}$ & 21986.275 & 0 & & 4547.051 & $211435-25690_{0}^{\circ}$ \\
\hline 23314.380 & 1 & & 4288.028 & $22508_{2}^{\circ}-267963$ & 21959.399 & 1 & & 4552.616 & $159703-20522^{\circ}$ \\
\hline 23297.038 & 0 & & 4291.220 & $248506-291415$ & 21952.080 & 1 & & 4554.134 & $17847_{2}^{\circ}-22401_{1}$ \\
\hline 23270.076 & 2 & & 4296.192 & $11197^{\circ}-15493_{4}$ & 21944.958 & 4 & & 4555.612 & $3687_{2}-8243_{2}^{\circ}$ \\
\hline
\end{tabular}


TABle 4. Classified lines of Th $\mathrm{I}$-continued

\begin{tabular}{|c|c|c|c|c|c|c|c|c|c|}
\hline \multirow{2}{*}{$\begin{array}{c}\text { Wavelength } \\
\text { (§) }\end{array}$} & \multicolumn{2}{|c|}{ Intensity } & \multirow{2}{*}{$\begin{array}{l}\text { Wavenumber } \\
\left(\mathrm{cm}^{-1}\right)\end{array}$} & \multirow{2}{*}{ Classification } & \multirow{2}{*}{$\begin{array}{c}\text { Wavelength } \\
(\AA)\end{array}$} & \multicolumn{2}{|c|}{ Inten sity } & \multirow{2}{*}{$\begin{array}{l}\text { Wave number } \\
\left(\mathrm{cm}^{-1}\right)\end{array}$} & \multirow{2}{*}{ Classification } \\
\hline & Lamp & Spark & & & & Lamp & Spark & & \\
\hline 21925.759 & 2 & & 4559.601 & $15863_{2}-20423^{\circ}$ & 20879.577 & 1 & & 4788.062 & $215752-263632^{\circ}$ \\
\hline 21883.548 & 0 & & 4568.396 & $18069_{3}^{\circ}-22637_{3}$ & 20869.452 & 4 & & 4790.385 & $17847_{2}^{\circ}-22637_{3}$ \\
\hline 21862.515 & 1 & & 4572.791 & $23655_{4}^{\circ}-28227_{4}$ & 20863.115 & 0 & & 4791.840 & $23481_{1}^{\circ}-28273_{2}$ \\
\hline 21809.793 & 0 & & 4583.845 & $18053^{\circ}-22637_{3}$ & 20862.579 & 2 & & 4791.963 & $223998-271915$ \\
\hline 21756.165 & 1 & & 4595.144 & $17073_{1}-21668_{i}^{\circ}$ & 20833.885 & 1 & & 4798.563 & $25753 \AA-305524$ \\
\hline 21749.619 & 1 & & 4596.527 & $15970_{3}-20566_{4}^{\circ}$ & 20792.737 & 1 & & 4808.059 & $14465_{2}^{\circ}-19273_{2}$ \\
\hline 21747.329 & 1 & & 4597.011 & $17501_{0}^{\circ}-22098_{4}$ & 20735.100 & 0 & & 4821.424 & $18699_{2}-23521_{3}^{\circ}$ \\
\hline 21743.999 & 5 & & 4597.715 & $7280_{2}-11877_{1}^{\circ}$ & 20717.817 & 0 & & 4825.446 & $20054_{2}-24880_{1}^{\circ}$ \\
\hline 21712.571 & 4 & & 4604.370 & $8243_{2}^{\circ}-128473$ & 20716.744 & 2 & & 4825.696 & $22669_{3}^{\circ}-27495_{4}$ \\
\hline 21706.674 & 1 & & 4605.621 & $142045-18809_{4}^{\circ}$ & 20715.963 & 0 & & 4825.878 & $25306_{2}^{\circ}-30132_{2}$ \\
\hline 21706.264 & 1 & & 4605.708 & $23752_{2}^{\circ}-283583$ & 20702.372 & 0 & & 4829.046 & $15493_{4}-203220^{\circ}$ \\
\hline 21683.632 & 0 & & 4610.515 & $211435-25753{ }^{\circ}$ & 20681.631 & 0 & & 4833.889 & $24850_{6}^{\circ}-29684_{5}$ \\
\hline 21676.298 & 0 & & 4612.075 & $75023-12114_{2}^{\circ}$ & 20680.125 & 4 & & 4834.241 & $7280_{2}-12114_{2}^{\circ}$ \\
\hline 21655.755 & 2 & & 4616.450 & $11601_{1}-16217_{2}^{\circ}$ & 20661.764 & 1 & & 4838.537 & $20566_{4}^{\circ}-25405_{4}$ \\
\hline 21628.998 & 4 & & 4622.161 & $11241_{3}^{\circ}-158632$ & 20651.478 & 0 & & 4840.947 & $215399_{4}^{\circ}-26380_{5}$ \\
\hline 21605.654 & 1 & & 4627.155 & $24307_{2}^{\circ}-289343$ & 20634.364 & 6 & & 4844.962 & $8243 \stackrel{\circ}{2}-13088_{3}$ \\
\hline 21599.082 & 1 & & 4628.563 & $22637_{3}-27266_{4}^{\circ}$ & 20618.818 & 0 & & 4848.615 & $197133-24561_{3}^{\circ}$ \\
\hline 21580.898 & 0 & & 4632.463 & $22338^{\circ}-269714$ & 20610.588 & 1 & & 4850.551 & $17398_{3}-22248_{2}^{\circ}$ \\
\hline 21556.115 & 0 & & 4637.789 & $21252_{2}^{\circ}-25890_{2}$ & 20605.987 & 2 & & 4851.634 & $22669_{3}^{\circ}-27521_{4}$ \\
\hline 21527.563 & 0 & & 4643.940 & $22877^{\circ}-27521_{4}$ & 20584.349 & 0 & & 4856.734 & $24561 \stackrel{\circ}{3}-29418_{2}$ \\
\hline 21519.111 & 0 & & 4645.764 & $23916_{4}^{\circ}-285624$ & 20575.042 & 0 & & 4858.931 & $21252_{2}^{\circ}-26111_{1}$ \\
\hline 21472.877 & 1 & & 4655.767 & $25355_{4}^{\circ}-30011_{3}$ & 20562.003 & 0 & & 4862.012 & $16783_{4}^{\circ}-21645_{4}$ \\
\hline 21442.806 & 1 & & 4662.296 & $18431_{3}-230932$ & 20561.348 & 2 & & 4862.167 & $21645_{4}-26508^{\circ}$ \\
\hline 21424.751 & 0 & & 4666.225 & $22855_{3}^{\circ}-27521_{4}$ & 20541.715 & 1 & & 4866.814 & $24274{ }^{\circ}-291415$ \\
\hline 21418.725 & 0 & & 4667.538 & $14032_{2}^{\circ}-186992$ & 20513.976 & 0 & & 4873.395 & $15863_{2}-20737_{1}^{\circ}$ \\
\hline 21415.242 & 2 & & 4668.297 & $19713_{3}-24381_{2}^{\circ}$ & 20489.032 & 1 & & 4879.328 & $6362_{2}-11241_{3}^{\circ}$ \\
\hline 21406.777 & 1 & & 4670.143 & $19532_{4}-24202^{\circ}$ & 20478.858 & 1 & & 4881.752 & $24259_{4}^{\circ}-29141_{5}$ \\
\hline 21375.118 & 3 & & 4677.060 & $98045-14481 \stackrel{\circ}{6}$ & 20453.217 & 0 & & 4887.872 & $15166_{3}^{\circ}-20054_{2}$ \\
\hline 21353.185 & 3 & & 4681.864 & $184313-23113 \stackrel{\circ}{\circ}$ & 20450.008 & 1 & & 4888.639 & $195324-24421_{3}^{\circ}$ \\
\hline 21328.149 & 0 & & 4687.360 & $23655_{4}^{\circ}-283425$ & 20428.437 & 1 & & 4893.801 & $21902_{4}^{\circ}-267963$ \\
\hline 21319.166 & 1 & & 4689.335 & $22877_{i}^{\circ}-27566_{2}$ & 20421.761 & 3 & & 4895.401 & $17959_{4}-22855_{3}^{\circ}$ \\
\hline 21260.824 & 0 & & 4702.203 & $24850 \circ-295526$ & 20419.379 & 0 & & 4895.972 & $19503 \stackrel{\circ}{3}-24399_{3}$ \\
\hline 21256.077 & 0 & & 4703.253 & $23655_{4}^{\circ}-283583$ & 20392.064 & 0 & & 4902.530 & $26363 \stackrel{\circ}{2}-31265_{3}$ \\
\hline 21250.077 & 0 & & 4704.581 & $25306_{2}^{\circ}-300113$ & 20391.719 & 0 & & 4902.613 & $221411_{3}^{\circ}-270443$ \\
\hline 21247.182 & 0 & & 4705.222 & $22338^{\circ}-270443$ & 20381.309 & 3 & & 4905.117 & $211435-260488^{\circ}$ \\
\hline 21239.694 & 1 & & 4706.881 & $23521_{3}^{\circ}-28227_{4}$ & 20377.894 & 1 & & 4905.939 & $21890_{3}^{\circ}-267963$ \\
\hline 21203.367 & 0 & & 4714.945 & $20054_{2}-24769_{3}^{\circ}$ & 20374.476 & 1 & & 4906.762 & $18574_{1}-23481_{i}$ \\
\hline 21198.664 & 0 & & 4715.991 & $21539_{4}^{\circ}-26255_{4}$ & 20321.907 & 0 & & 4919.455 & $18069_{3}^{\circ}-22988_{2}$ \\
\hline 21173.002 & 1 & & 4721.707 & $154934-20214_{3}^{\circ}$ & 20317.777 & 4 & & 4920.455 & $23113 \stackrel{\circ}{4}-280345$ \\
\hline 21148.510 & 3 & & 4727.175 & $23306_{6}^{\circ}-280345$ & 20263.141 & 0 & & 4933.722 & $26995_{3}^{\circ}-31929_{3}$ \\
\hline 21143.170 & 4 & & 4728.369 & $11241_{3}^{\circ}-15970_{3}$ & 20234.945 & 0 & & 4940.597 & $173983-22338^{\circ}$ \\
\hline 21132.654 & 2 & & 4730.722 & $19039_{2}^{\circ}-23769_{1}$ & 20205.662 & 0 & & 4947.757 & $25703_{2}^{\circ}-30651_{3}$ \\
\hline 21117.677 & 0 & & 4734.077 & $211435-25877^{\circ}$ & 20191.383 & 3 & & 4951.256 & $19039_{2}^{\circ}-23990_{2}$ \\
\hline 21108.367 & 1 & & 4736.165 & $171665-21902 \stackrel{\circ}{4}$ & 20183.242 & 0 & & 4953.253 & $27087_{1}^{\circ}-32041_{2}$ \\
\hline 21095.446 & 0 & & 4739.066 & $154934-20232_{4}^{\circ}$ & 20158.039 & 2 & & 4959.446 & $12114_{2}^{\circ}-17073_{1}$ \\
\hline 21095.446 & 0 & & 4739.066 & $21645_{4}-26384_{4}^{\circ}$ & 20139.866 & 1 & & 4963.921 & $18069_{3}^{\circ}-23032_{4}$ \\
\hline 21019.525 & 2 & & 4756.183 & $13297_{4}-18053_{4}^{\circ}$ & 20128.687 & 3 & & 4966.678 & $10526^{\circ}-15493_{4}$ \\
\hline 21011.808 & 2 & & 4757.930 & $21165_{3}^{\circ}-259234$ & 20122.241 & 0 & & 4968.269 & $20922_{2}^{\circ}-25890_{2}$ \\
\hline 21009.229 & 1 & & 4758.514 & $13088_{3}-17847_{2}^{\circ}$ & 20108.593 & 2 & & 4971.641 & $18549_{2}-23521_{3}^{\circ}$ \\
\hline 20981.790 & 0 & & 4764.737 & $173983-22163_{4}^{\circ}$ & 20077.401 & 2 & & 4979.365 & $18053 \stackrel{\circ}{4}-230324$ \\
\hline 20973.743 & 1 & & 4766.565 & $13847_{2}-18614_{i}^{\circ}$ & 20047.945 & 2 & & 4986.681 & $24769_{3}^{\circ}-297564$ \\
\hline 20965.562 & 1 & & 4768.425 & $215943-26363_{2}^{\circ}$ & 19998.121 & 1 & & 4999.105 & $12847_{3}-17847_{2}^{\circ}$ \\
\hline 20951.480 & 1 & & 4771.630 & $13297_{4}-18069_{3}^{\circ}$ & 19935.599 & 0 & & 5014.783 & $27948 \stackrel{\circ}{4}-329635$ \\
\hline 20920.513 & 1 & & 4778.693 & $26363_{2}^{\circ}-311413$ & 19922.803 & 1 & & 5018.004 & $23916 \stackrel{8}{-289343}$ \\
\hline 20893.881 & 0 & & 4784.784 & $13175_{4}^{\circ}-179594$ & 19919.437 & 0 & & 5018.852 & $23015 \circ-280345$ \\
\hline
\end{tabular}


TABLE 4. Classified lines of Th $\mathrm{I}$-continued

\begin{tabular}{|c|c|c|c|c|c|c|c|c|c|}
\hline \multirow{2}{*}{$\begin{array}{l}\text { Wavelength } \\
\text { (§) }\end{array}$} & \multicolumn{2}{|c|}{ Intensity } & \multirow{2}{*}{$\begin{array}{l}\text { Wavenumber } \\
\left(\mathrm{cm}^{-1}\right)\end{array}$} & \multirow{2}{*}{ Classification } & \multirow{2}{*}{$\begin{array}{l}\text { Wavelength } \\
\text { ( })\end{array}$} & \multicolumn{2}{|c|}{ Intensity } & \multirow{2}{*}{$\begin{array}{l}\text { Wavenumber } \\
\left(\mathrm{cm}^{-1}\right)\end{array}$} & \multirow{2}{*}{ Classification } \\
\hline & Lamp & Spark & & & & Lamp & Spark & & \\
\hline 19908.520 & 0 & & 5021.604 & $18011^{\circ}-230324$ & 19290.328 & 0 & & 5182.530 & $22338_{3}^{\circ}-27521_{4}$ \\
\hline 19902.706 & 4 & & 5023.071 & $14204_{5}-19227_{6}^{\circ}$ & 19273.916 & 2 & & 5186.943 & $10783_{2}^{\circ}-15970_{3}$ \\
\hline 19882.301 & 2 & & 5028.226 & $22163_{4}^{\circ}-271915$ & 19264.749 & 1 & & 5189.411 & $20922_{2}^{\circ}-26111_{1}$ \\
\hline 19862.586 & 0 & & 5033.217 & $24981_{3}^{\circ}-300144$ & 19261.710 & 0 & & 5190.230 & $20214^{\circ}-25405_{4}$ \\
\hline 19857.666 & 2 & & 5034.464 & $19273_{2}-24307_{2}^{\circ}$ & 19261.001 & 0 & & 5190.421 & $23655_{4}^{\circ}-28845_{4}$ \\
\hline 19852.906 & 2 & & 5035.671 & $23306_{6}^{\circ}-283425$ & 19257.450 & 0 & & 5191.378 & $13847_{2}-19039_{2}^{\circ}$ \\
\hline 19850.778 & 1 & & 5036.211 & $25336_{6}^{\circ}-303726$ & 19256.382 & 1 & & 5191.666 & $18549_{2}-23741_{i}^{\circ}$ \\
\hline 19847.455 & 0 & & 5037.054 & $24381_{2}^{\circ}-294182$ & 19244.968 & 0 & & 5194.745 & $24561_{3}^{\circ}-297564$ \\
\hline 19829.520 & 1 & & 5041.610 & $23521_{3}^{\circ}-285624$ & 19244.035 & 1 & & 5194.997 & $15970_{3}-21165_{3}^{\circ}$ \\
\hline 19797.857 & 0 & & 5049.673 & $23481_{1}^{\circ}-28531_{2}$ & 19241.246 & 0 & & 5195.750 & $21594_{3}-26790_{4}^{\circ}$ \\
\hline 19786.004 & 5 & & 5052.698 & $7795_{4}^{\circ}-128473$ & 19240.420 & 0 & & 5195.973 & $11877^{\circ}-17073_{1}$ \\
\hline 19784.595 & 1 & & 5053.058 & $18699_{2}-23752_{2}^{\circ}$ & 19204.660 & 1 & & 5205.648 & $12847_{3}-18053^{\circ}$ \\
\hline 19770.799 & 1 & & 5056.584 & $24084^{\circ}-291415$ & 19190.670 & 2 & & 5209.443 & $27084_{6}^{\circ}-322935$ \\
\hline 19765.178 & 3 & & 5058.022 & $21738_{2}^{\circ}-267963$ & 19178.389 & 2 & & 5212.779 & $230150^{\circ}-28227_{4}$ \\
\hline 19764.361 & 0 & & 5058.231 & $15863_{2}-20922_{2}^{\circ}$ & 19162.549 & 1 & & 5217.088 & $26048_{4}^{\circ}-31265_{3}$ \\
\hline 19762.115 & 0 & & 5058.806 & $22508_{2}^{\circ}-27566_{2}$ & 19151.815 & 4 & & 5220.012 & $5563_{1}-10783_{2}^{\circ}$ \\
\hline 19757.006 & 0 & & 5060.114 & $18930_{3}^{\circ}-23990_{2}$ & 19147.846 & 3 & & 5221.094 & $12847_{3}-18069_{3}^{\circ}$ \\
\hline 19741.428 & 5 & & 5064.107 & $8111_{4}-13175^{\circ}$ & 19143.281 & 1 & & 5222.339 & $18809_{4}^{\circ}-24032_{4}$ \\
\hline 19736.666 & 0 & & 5065.329 & $24769_{3}^{\circ}-29835_{3}$ & 19138.077 & 1 & & 5223.759 & $18053_{4}^{\circ}-23277_{5}$ \\
\hline 19733.467 & 1 & & 5066.150 & $26363_{2}^{\circ}-314291$ & 19138.077 & 1 & & 5223.759 & $23049_{1}^{\circ}-28273_{2}$ \\
\hline 19721.637 & 0 & & 5069.189 & $21902^{\circ}-26971_{4}$ & 19113.116 & 2 & & 5230.581 & $25321_{3}^{\circ}-305524$ \\
\hline 19705.256 & 0 & & 5073.403 & $154934-20566_{4}^{\circ}$ & 19091.898 & 0 & & 5236.394 & $23609^{\circ}-28845_{4}$ \\
\hline 19696.195 & 1 & & 5075.737 & $23603_{2}^{\circ}-28679_{2}$ & 19088.610 & 3 & & 5237.296 & $195324-24769_{3}^{\circ}$ \\
\hline 19692.738 & 1 & & 5076.628 & $13962_{1}-19039_{2}^{\circ}$ & 19080.012 & 1 & & 5239.656 & $24182_{2}^{\circ}-294221$ \\
\hline 19683.215 & 4 & & 5079.084 & $10414_{4}^{\circ}-154934$ & 19078.887 & 1 & & 5239.965 & $25690 \circ-309306$ \\
\hline 19676.823 & 1 & & 5080.734 & $10783_{2}^{\circ}-15863_{2}$ & 19074.406 & 3 & & 5241.196 & $14032_{2}^{\circ}-19273_{2}$ \\
\hline 19674.535 & 3 & & 5081.325 & $21890^{\circ}-26971_{4}$ & 19073.289 & 2 & & 5241.503 & $211435-26384_{4}^{\circ}$ \\
\hline 19669.905 & 2 & & 5082.521 & $13847_{2}-18930_{3}^{\circ}$ & 19050.366 & 0 & & 5247.810 & $14465_{2}^{\circ}-197133$ \\
\hline 19668.461 & 4 & & 5082.894 & $20322 \circ-254054$ & 19035.208 & 0 & & 5251.989 & $20054_{2}-25306_{2}^{\circ}$ \\
\hline 19643.457 & 1 & & 5089.364 & $18431_{3}-23521_{3}^{\circ}$ & 19033.646 & 1 & & 5252.420 & $24880_{1}^{\circ}-30132_{2}$ \\
\hline 19639.120 & 1 & & 5090.488 & $21165_{3}^{\circ}-26255_{4}$ & 19018.615 & 2 & & 5256.571 & $13175_{4}^{\circ}-184313$ \\
\hline 19622.548 & 0 & & 5094.787 & $22248^{\circ}-273433$ & 18984.562 & 0 & & 5266.000 & $18011^{\circ}-232775$ \\
\hline 19617.154 & 2 & & 5096.188 & $22399_{0}^{\circ}-274954$ & 18964.790 & 1 & & 5271.490 & $17398_{3}-22669_{3}^{\circ}$ \\
\hline 19595.083 & 3 & & 5101.928 & $18930^{\circ}-240324$ & 18958.367 & 1 & & 5273.276 & $22338_{3}^{\circ}-276123$ \\
\hline 19586.250 & 0 & & 5104.229 & $22508_{2}^{\circ}-276123$ & 18957.939 & 0 & & 5273.395 & $24561_{3}^{\circ}-29835_{3}$ \\
\hline 19571.602 & 2 & & 5108.049 & $19273_{2}-24381_{2}^{\circ}$ & 18942.594 & 0 & & 5277.667 & $26651_{2}^{\circ}-319293$ \\
\hline 19561.688 & 2 & & 5110.638 & $24307_{2}^{\circ}-29418_{2}$ & 18942.109 & 1 & & 5277.802 & $24274 \stackrel{\circ}{-}-295526$ \\
\hline 19535.499 & 0 & & 5117.489 & $26878 \stackrel{\circ}{-}-31995_{4}$ & 18938.047 & 1 & & 5278.934 & $23603_{2}^{\circ}-28882_{2}$ \\
\hline 19519.076 & 0 & & 5121.795 & $27674_{2}^{\circ}-327963$ & 18925.238 & 0 & & 5282.507 & $15970_{3}-21252_{2}^{\circ}$ \\
\hline 19505.411 & 0 & & 5125.383 & $22855^{\circ}-27980_{3}$ & 18919.776 & 2 & & 5284.032 & $1214_{2}^{\circ}-173983$ \\
\hline 19476.782 & 1 & & 5132.917 & $18069_{3}^{\circ}-23201_{3}$ & 18907.334 & 2 & & 5287.509 & $24561_{3}^{\circ}-29849_{4}$ \\
\hline 19444.483 & 1 & & 5141.443 & $17847_{2}^{\circ}-22988_{2}$ & 18904.263 & 0 & & 5288.368 & $19273_{2}-24561{ }^{\circ}$ \\
\hline 19430.829 & 2 & & 5145.056 & $8800_{4}-13945_{3}^{\circ}$ & 18893.288 & 0 & & 5291.440 & $27260_{3}^{\circ}-32551_{3}$ \\
\hline 19420.664 & 1 & & 5147.749 & $19516_{2}^{\circ}-24664_{3}$ & 18886.685 & 6 & & 5293.290 & $7795_{4}^{\circ}-13088_{3}$ \\
\hline 19418.336 & 1 & & 5148.366 & $18053_{4}^{\circ}-23201_{3}$ & 18885.154 & 1 & & 5293.719 & $24838_{1}^{\circ}-30132_{2}$ \\
\hline 19400.479 & 3 & & 5153.105 & $20737_{1}^{\circ}-25890_{2}$ & 18865.586 & 3 & & 5299.210 & $16346_{4}^{\circ}-21645_{4}$ \\
\hline 19396.794 & 2 & & 5154.084 & $21890 \stackrel{3}{-}-27044_{3}$ & 18858.490 & 1 & & 5301.204 & $15863_{2}-21165_{3}^{\circ}$ \\
\hline 19391.319 & 1 & & 5155.539 & $18614^{\circ}-237691$ & 18850.731 & 2 & & 5303.386 & $21077^{\circ}-26380_{5}$ \\
\hline 19388.002 & 1 & & 5156.421 & $22877{ }^{\circ}-280345$ & 18840.854 & 0 & & 5306.166 & $21738_{2}^{\circ}-27044_{3}$ \\
\hline 19345.173 & 0 & & 5167.837 & $220984-27266_{4}^{\circ}$ & 18824.439 & 0 & & 5310.793 & $22669_{3}^{\circ}-27980_{3}$ \\
\hline 19342.209 & 2 & & 5168.629 & $195324-247018$ & 18811.879 & 7 & & 5314.339 & $167833^{\circ}-220984$ \\
\hline 19336.983 & 0 & & 5170.026 & $22396 i-27566_{2}$ & 18802.414 & 3 & & 5317.014 & $16351_{0}-21668^{\circ}$ \\
\hline 19321.626 & 0 & & 5174.135 & $26363_{2}^{\circ}-315373$ & 18799.282 & 0 & & 5317.900 & $22248_{2}^{\circ}-27566_{2}$ \\
\hline 19317.885 & 3 & & 5175.137 & $17073_{1}-22248_{2}^{\circ}$ & 18788.340 & 0 & & 5320.997 & $18431_{3}-23752_{2}^{\circ}$ \\
\hline 19291.493 & 1 & & 5182.217 & $23752_{2}^{\circ}-28934_{3}$ & 18781.224 & 1 & & 5323.013 & $17073_{1}-22396_{1}^{\circ}$ \\
\hline
\end{tabular}


TABLE 4. Classified lines of Th $\mathrm{I}$-continued

\begin{tabular}{|c|c|c|c|c|c|c|c|c|c|}
\hline \multirow{2}{*}{$\begin{array}{c}\text { Wavelength } \\
(\AA)\end{array}$} & \multicolumn{2}{|c|}{ Intensity } & \multirow{2}{*}{$\begin{array}{l}\text { Wavenumber } \\
\left(\mathrm{cm}^{-1}\right)\end{array}$} & \multirow{2}{*}{ Classification } & \multirow{2}{*}{$\begin{array}{c}\text { Wavelength } \\
\text { (§) }\end{array}$} & \multicolumn{2}{|c|}{ Intensity } & \multirow{2}{*}{$\begin{array}{l}\text { Wavenumber } \\
\left(\mathrm{cm}^{-1}\right)\end{array}$} & \multirow{2}{*}{ Classification } \\
\hline & Lamp & Spark & & & & Lamp & Spark & & \\
\hline 18772.446 & 3 & & 5325.502 & $14206_{4}^{\circ}-195324$ & 17987.035 & 1 & & 5558.042 & $22669_{3}^{\circ}-28227_{4}$ \\
\hline 18763.740 & 0 & & 5327.973 & $13945_{3}^{\circ}-19273_{2}$ & 17976.979 & 4 & & 5561.151 & $179594-23521^{\circ}$ \\
\hline 18737.838 & 0 & & 5335.338 & $24421_{3}^{\circ}-297564$ & 17969.554 & 0 & & 5563.449 & $24981_{3}^{\circ}-305442$ \\
\hline 18730.785 & 2 & & 5337.347 & $10526_{3}^{\circ}-15863_{2}$ & 17964.920 & 6 & & 5564.884 & $4961_{4}-10526_{3}^{\circ}$ \\
\hline 18689.399 & 0 & & 5349.166 & $165546-21903^{\circ}$ & 17962.477 & 0 & & 5565.641 & $19273_{2}-24838_{1}^{\circ}$ \\
\hline 18685.648 & 0 & & 5350.240 & $266450-319954$ & 17960.037 & 0 & & 5566.397 & $26363_{2}^{\circ}-31929_{3}$ \\
\hline 18685.274 & 1 & & 5350.347 & $22877^{\circ}-28227_{4}$ & 17955.860 & 2 & & 5567.692 & $20543{ }^{\circ}-26111_{1}$ \\
\hline 18664.162 & 2 & & 5356.399 & $20566^{\circ}-259234$ & 17893.451 & 0 & & 5587.111 & $13945^{\circ}-195324$ \\
\hline 18657.231 & 2 & & 5358.389 & $221634-275214$ & 17887.982 & 3 & & 5588.819 & $20522_{2}^{\circ}-26111_{1}$ \\
\hline 18651.748 & 0 & & 5359.964 & $19039_{2}^{\circ}-24399_{3}$ & 17886.677 & 2 & & 5589.227 & $18809_{4}^{\circ}-24399_{3}$ \\
\hline 18625.408 & 0 & & 5367.544 & $266450-320124$ & 17886.181 & 0 & & 5589.382 & $24259_{4}^{\circ}-29849_{4}$ \\
\hline 18595.874 & 3 & & 5376.069 & $18614_{1}^{\circ}-23990_{2}$ & 17883.051 & 2 & & 5590.360 & $142260-19817_{1}^{\circ}$ \\
\hline 18557.981 & 3 & & 5387.046 & $18382{ }^{\circ}-23769_{1}$ & 17873.738 & 2 & & 5593.273 & $24421_{3}^{\circ}-30014_{4}$ \\
\hline 18546.830 & 1 & & 5390.285 & $253366^{\circ}-307267$ & 17873.620 & 1 & & 5593.310 & $21902_{4}^{\circ}-27495_{4}$ \\
\hline 18520.188 & 0 & & 5398.039 & $19516_{2}^{\circ}-24915_{3}$ & 17872.284 & 1 & & 5593.728 & $19713_{3}-25306_{2}^{\circ}$ \\
\hline 18509.757 & 1 & & 5401.081 & $23481_{1}^{\circ}-288822$ & 17854.649 & 2 & & 5599.253 & $24084{ }_{6}^{\circ}-296845$ \\
\hline 18490.644 & 4 & & 5406.664 & $8800_{4}-14206_{4}^{\circ}$ & 17849.848 & 1 & & 5600.759 & 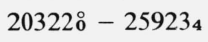 \\
\hline 18468.454 & 3 & & 5413.160 & $17224_{2}^{\circ}-22637_{3}$ & 17838.977 & 1 & & 5604.172 & $8243 \stackrel{\circ}{2}-138472$ \\
\hline 18452.242 & 0 & & 5417.916 & $22855^{\circ}-282732$ & 17834.907 & 1 & & 5605.451 & $21890_{3}^{\circ}-27495_{4}$ \\
\hline 18440.493 & 4 & & 5421.368 & $11802_{2}-17224_{2}^{\circ}$ & 17827.385 & 1 & & 5607.816 & $18574_{1}-24182_{2}^{\circ}$ \\
\hline 18427.858 & 0 & & 5425.085 & $27674_{2}^{\circ}-33099_{3}$ & 17826.416 & 0 & & 5608.121 & $18699_{2}-24307_{2}^{\circ}$ \\
\hline 18396.840 & 0 & & 5434.232 & $17073_{1}-22508_{2}^{\circ}$ & 17825.875 & 5 & & 5608.291 & $11802_{2}-17411_{3}^{\circ}$ \\
\hline 18348.035 & 1 & & 5448.687 & $195324-24981_{3}^{\circ}$ & 17823.987 & 1 & & 5608.885 & $197133-25321_{3}^{\circ}$ \\
\hline 18339.435 & 0 & & 5451.242 & $25306_{2}^{\circ}-30758_{2}$ & 17788.214 & 2 & & 5620.165 & $21645_{4}-27266_{4}^{\circ}$ \\
\hline 18335.284 & 2 & & 5452.476 & $49614-10414_{4}^{\circ}$ & 17783.170 & 4 & & 5621.759 & $17411^{\circ}-230324$ \\
\hline 18334.595 & 0 & & 5452.681 & $24561_{3}^{\circ}-30014_{4}$ & 17778.391 & 1 & & 5623.270 & $11601_{1}-17224^{\circ}$ \\
\hline 18331.495 & 1 & & 5453.603 & $21890_{3}^{\circ}-273433$ & 17763.368 & 0 & & 5628.026 & $26384 \stackrel{\circ}{4}-320124$ \\
\hline 18331.136 & 0 & & 5453.710 & $24381_{2}^{\circ}-298353$ & 17757.108 & 2 & & 5630.010 & $24381_{2}^{\circ}-30011_{3}$ \\
\hline 18320.410 & 0 & & 5456.903 & $173983-22855_{3}^{\circ}$ & 17752.753 & 1 & & 5631.391 & $21890_{3}^{\circ}-27521_{4}$ \\
\hline 18301.976 & 1 & & 5462.399 & $26995_{3}^{\circ}-32458_{4}$ & 17744.898 & 5 & & 5633.884 & $17354_{1}^{\circ}-22988_{2}$ \\
\hline 18293.550 & 1 & & 5464.915 & $22877^{\circ}-283425$ & 17742.618 & 0 & & 5634.608 & $22399 \stackrel{\circ}{-280345}$ \\
\hline 18286.901 & 1 & & 5466.902 & $20423^{\circ}-25890_{2}$ & 17720.358 & 2 & & 5641.686 & $22338_{3}^{\circ}-27980_{3}$ \\
\hline 18281.471 & 1 & & 5468.526 & $24182_{2}^{\circ}-29650_{2}$ & 17717.673 & 0 & & 5642.541 & $19713_{3}-25355_{4}^{\circ}$ \\
\hline 18280.488 & 0 & & 5468.820 & $18930_{3}^{\circ}-24399_{3}$ & 17703.673 & 3 & & 5647.003 & $211435-26790_{4}^{\circ}$ \\
\hline 18274.319 & 0 & & 5470.666 & $22141^{\circ}-276123$ & 17688.744 & 2 & & 5651.769 & $215394-271915$ \\
\hline 18225.855 & 1 & & 5485.213 & $18431_{3}-23916_{4}^{\circ}$ & 17683.810 & 3 & & 5653.346 & 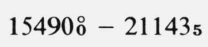 \\
\hline 18221.955 & 1 & & 5486.387 & $23655^{\circ}-291415$ & 17683.237 & 0 & & 5653.529 & $22877_{1}^{\circ}-28531_{2}$ \\
\hline 18209.323 & 0 & & 5490.193 & $27061_{2}^{\circ}-325513$ & 17677.481 & 3 & & 5655.370 & $13847_{2}-19503^{\circ}$ \\
\hline 18173.926 & 1 & & 5500.886 & $26036^{\circ}-315373$ & 17670.229 & 1 & & 5657.691 & $24850 \stackrel{6}{6}-305085$ \\
\hline 18170.303 & 1 & & 5501.983 & $211435-26645 \circ$ & 17669.614 & 3 & & 5657.888 & $25306_{2}^{\circ}-30964_{3}$ \\
\hline 18169.715 & 1 & & 5502.161 & $7795_{4}^{\circ}-13297_{4}$ & 17663.853 & 0 & & 5659.733 & $25877_{4}^{\circ}-31537_{3}$ \\
\hline 18166.638 & 1 & & 5503.093 & $22855^{\circ}-283583$ & 17658.896 & 0 & & 5661.322 & $26113_{2}^{\circ}-317743$ \\
\hline 18161.579 & 2 & & 5504.626 & $21539_{4}^{\circ}-27044_{3}$ & 17649.490 & 2 & & 5664.339 & $24880_{1}-30544_{2}$ \\
\hline 18156.664 & 0 & & 5506.116 & $14206_{4}^{\circ}-197133$ & 17634.332 & 4 & & 5669.208 & $13847_{2}-19516_{2}^{\circ}$ \\
\hline 18135.791 & 1 & & 5512.453 & $13297_{4}-18809^{\circ}$ & 17633.725 & 0 & & 5669.403 & $23752_{2}^{\circ}-294221$ \\
\hline 18125.969 & 5 & & 5515.440 & $6362_{2}-11877_{i}^{\circ}$ & 17627.118 & 0 & & 5671.528 & $21645_{4}-27317^{\circ}$ \\
\hline 18103.118 & 2 & & 5522.402 & $24850 \circ-303726$ & 17626.046 & 3 & & 5671.873 & $15493_{4}-21165_{3}^{\circ}$ \\
\hline 18087.083 & 2 & & 5527.298 & $24307_{2}^{\circ}-29835_{3}$ & 17623.094 & 6 & & 5672.823 & $7502_{3}-13175^{\circ}$ \\
\hline 18072.340 & 4 & & 5531.807 & $17501{ }^{\circ}-230324$ & 17614.900 & 1 & & 5675.462 & $20214_{3}^{\circ}-25890_{2}$ \\
\hline 18070.540 & 1 & & 5532.358 & $236090-291415$ & 17614.015 & 1 & & 5675.747 & $22855_{3}^{\circ}-28531_{2}$ \\
\hline 18023.692 & 1 & & 5546.738 & $25180_{7}^{\circ}-307267$ & 17597.889 & 1 & & 5680.948 & $14032_{2}^{\circ}-197133$ \\
\hline 18007.579 & 2 & & 5551.701 & $11802_{2}-17354_{1}^{\circ}$ & 17595.542 & 1 & & 5681.706 & $18699_{2}-24381_{2}^{\circ}$ \\
\hline 18000.660 & 1 & & 5553.835 & $24202_{4}^{\circ}-297564$ & 17584.517 & 7 & & 5685.268 & $98045-15490 \circ$ \\
\hline 17993.769 & 3 & & 5555.962 & $10414_{4}^{\circ}-15970_{3}$ & 17575.945 & 1 & & 5688.041 & $20423 i-26111_{1}$ \\
\hline 17991.742 & 0 & & 5556.588 & $26645 \circ-322025$ & 17573.115 & 3 & & 5688.957 & $20566_{4}^{\circ}-26255_{4}$ \\
\hline
\end{tabular}


TABLE 4. Classified lines of Th $\mathrm{I}$-continued

\begin{tabular}{|c|c|c|c|c|c|c|c|c|c|}
\hline \multirow{2}{*}{$\begin{array}{l}\text { Wavelength } \\
\text { (A) }\end{array}$} & \multicolumn{2}{|c|}{ Intensity } & \multirow{2}{*}{$\begin{array}{l}\text { Wavenumber } \\
\qquad\left(\mathrm{cm}^{-1}\right)\end{array}$} & \multirow{2}{*}{ Classification } & \multirow{2}{*}{$\begin{array}{c}\text { Wavelength } \\
\text { (§) }\end{array}$} & \multicolumn{2}{|c|}{ Inten sity } & \multirow{2}{*}{$\begin{array}{l}\text { Wavenumber } \\
\qquad\left(\mathrm{cm}^{-1}\right)\end{array}$} & \multirow{2}{*}{ Classification } \\
\hline & Lamp & Spark & & & & Lamp & Spark & & \\
\hline 17553.728 & 1 & & 5695.240 & $17959_{4}-23655_{4}^{\circ}$ & 17121.318 & 3 & & 5839.077 & $22141_{3}^{\circ}-27980_{3}$ \\
\hline 17552.671 & 4 & & 5695.583 & $173983-23093_{2}^{\circ}$ & 17120.840 & 0 & & 5839.240 & $27260_{3}^{\circ}-33099_{3}$ \\
\hline 17551.531 & 1 & & 5695.953 & $25575_{4}^{\circ}-31271_{5}$ & 17120.083 & 1 & & 5839.498 & $23916^{\circ}-297564$ \\
\hline 17549.205 & 0 & & 5696.708 & $26096^{\circ}-317934$ & 17113.542 & 2 & & 5841.730 & $13088_{3}-18930_{3}^{\circ}$ \\
\hline 17547.178 & 0 & & 5697.366 & $27266_{4}^{\circ}-329635$ & 17103.520 & 1 & & 5845.153 & $165546-22399^{\circ}$ \\
\hline 17521.738 & 2 & & 5705.638 & $24838^{\circ}-30544_{2}$ & 17088.335 & 2 & & 5850.347 & $22508_{2}^{\circ}-28358_{3}$ \\
\hline 17516.448 & 0 & & 5707.361 & $22855^{\circ}-28562_{4}$ & 17085.097 & 0 & & 5851.456 & $24701{ }^{\circ}-305524$ \\
\hline 17508.350 & 2 & & 5710.001 & $21902^{\circ}-276123$ & 17078.798 & 3 & & 5853.614 & $16783_{4}^{\circ}-226373$ \\
\hline 17503.828 & 1 & & 5711.476 & $171665-22877^{\circ}$ & 17075.750 & 3 & & 5854.659 & $13962_{1}-19817^{\circ}$ \\
\hline 17492.573 & 2 & & 5715.151 & $173983-23113{ }^{\circ}$ & 17054.299 & 0 & & 5862.023 & $18699_{2}-24561_{3}^{\circ}$ \\
\hline 17481.041 & 7 & & 5718.921 & $8243_{2}^{\circ}-13962_{1}$ & 17028.599 & 1 & & 5870.870 & $22163_{4}^{\circ}-280345$ \\
\hline 17473.699 & 2 & & 5721.324 & $13088_{3}-18809_{4}^{\circ}$ & 17026.334 & 1 & & 5871.651 & $18549_{2}-24421_{3}^{\circ}$ \\
\hline 17441.114 & 0 & & 5732.013 & $23113 \stackrel{\circ}{4}-288454$ & 17019.670 & 0 & & 5873.950 & $20922_{2}^{\circ}-267963$ \\
\hline 17437.704 & 1 & & 5733.134 & $18574_{1}-24307_{2}^{\circ}$ & 17018.885 & 2 & & 5874.221 & $21738_{2}^{\circ}-27612_{3}$ \\
\hline 17404.332 & 4 & & 5744.127 & $142045-19948{ }^{\circ}$ & 17014.114 & 1 & & 5875.868 & $19039_{2}^{\circ}-24915_{3}$ \\
\hline 17401.230 & 1 & & 5745.151 & $26048_{4}^{\circ}-31793_{4}$ & 17013.556 & 0 & & 5876.061 & $18431_{3}-24307_{2}^{\circ}$ \\
\hline 17388.612 & 0 & & 5749.320 & $25877_{4}^{\circ}-316265$ & 17012.360 & 0 & & 5876.474 & $24850_{6}^{\circ}-30726_{7}$ \\
\hline 17385.370 & 4 & & 5750.392 & $25180_{7}^{\circ}-309306$ & 17008.562 & 2 & & 5877.786 & $24880_{1}^{\circ}-30758_{2}$ \\
\hline 17381.909 & 7 & & 5751.537 & $16346_{4}^{\circ}-22098_{4}$ & 17005.290 & 2 & & 5878.917 & $27084_{6}^{\circ}-329635$ \\
\hline 17380.610 & 5 & & 5751.967 & $6362_{2}-12114_{2}^{\circ}$ & 17004.697 & 3 & & 5879.122 & $21165_{3}^{\circ}-27044_{3}$ \\
\hline 17375.665 & 1 & & 5753.604 & $11601_{1}-17354_{i}^{\circ}$ & 16997.972 & 0 & & 5881.448 & $24769_{3}^{\circ}-30651_{3}$ \\
\hline 17372.868 & 1 & & 5754.530 & $20054_{2}-25809_{1}^{\circ}$ & 16989.777 & 1 & & 5884.285 & $25442_{3}^{\circ}-313264$ \\
\hline 17334.404 & 1 & & 5767.299 & $23916^{\circ}-296845$ & 16976.362 & 0 & & 5888.935 & $22338_{3}^{\circ}-28227_{4}$ \\
\hline 17333.121 & 1 & & 5767.726 & $13945^{\circ}-19713_{3}$ & 16964.222 & 0 & & 5893.149 & $26036_{3}^{\circ}-319293$ \\
\hline 17323.648 & 0 & & 5770.880 & $18431_{3}-24202^{\circ}$ & 16960.892 & 1 & & 5894.306 & $21077_{0}^{\circ}-26971_{4}$ \\
\hline 17311.753 & 1 & & 5774.845 & $24769_{3}^{\circ}-30544_{2}$ & 16952.178 & 0 & & 5897.336 & $23521_{3}^{\circ}-29418_{2}$ \\
\hline 17307.662 & 7 & & 5776.210 & $17501 \stackrel{\circ}{-23277_{5}}$ & 16949.485 & 0 & & 5898.273 & $23752_{2}^{\circ}-29650_{2}$ \\
\hline 17290.627 & 1 & & 5781.901 & $142045-19986_{6}^{\circ}$ & 16945.150 & 0 & & 5899.782 & $26651_{2}^{\circ}-32551_{3}$ \\
\hline 17277.735 & 0 & & 5786.215 & $25355_{4}^{\circ}-31141_{3}$ & 16938.730 & 3 & & 5902.018 & $19503_{3}^{\circ}-25405_{4}$ \\
\hline 17271.005 & 0 & & 5788.470 & $230932-28882_{2}$ & 16918.744 & 0 & & 5908.990 & $26384_{4}^{\circ}-322935$ \\
\hline 17267.932 & 3 & & 5789.500 & $195324-253211_{3}^{\circ}$ & 16915.692 & 0 & & 5910.056 & $25355_{4}^{\circ}-31265_{3}$ \\
\hline 17264.172 & 3 & & 5790.761 & $17411^{\circ}-232013$ & 16915.097 & 1 & & 5910.264 & $19532_{4}-25442_{3}^{\circ}$ \\
\hline 17261.632 & 0 & & 5791.613 & $21252_{2}^{\circ}-27044_{3}$ & 16900.345 & 1 & & 5915.423 & $25355_{4}^{\circ}-312715$ \\
\hline 17227.152 & 0 & & 5803.205 & $25442 \stackrel{\circ}{3}-31245_{2}$ & 16887.173 & 1 & & 5920.037 & $15970_{3}-21890_{3}^{\circ}$ \\
\hline 17225.670 & 3 & & 5803.704 & $17073_{1}-22877_{1}^{\circ}$ & 16883.454 & 0 & & 5921.341 & $18069_{3}^{\circ}-23990_{2}$ \\
\hline 17221.632 & 1 & & 5805.065 & $15863_{2}-21668_{1}^{\circ}$ & 16879.312 & 2 & & 5922.794 & $17847_{2}^{\circ}-23769_{1}$ \\
\hline 17217.782 & 2 & & 5806.363 & $21165_{3}^{\circ}-26971_{4}$ & 16875.970 & 0 & & 5923.967 & $25321_{3}^{\circ}-31245_{2}$ \\
\hline 17216.726 & 0 & & 5806.719 & $185741_{1}-24381_{2}^{\circ}$ & 16852.625 & 2 & & 5932.173 & $159703-21902_{4}^{\circ}$ \\
\hline 17198.807 & 0 & & 5812.769 & $266458-324584$ & 16849.382 & 0 & & 5933.315 & $20322^{\circ}-26255_{4}$ \\
\hline 17195.420 & 0 & & 5813.914 & $20566_{4}^{\circ}-26380_{5}$ & 16846.815 & 0 & & 5934.219 & $22338^{\circ}-282732$ \\
\hline 17192.605 & 1 & & 5814.866 & $236032-29418_{2}$ & 16846.017 & 0 & & 5934.500 & $253366^{\circ}-312715$ \\
\hline 17186.894 & 2 & & 5816.798 & $19588 \AA-25405_{4}$ & 16840.767 & 0 & & 5936.350 & $25690^{\circ}-316265$ \\
\hline 17173.440 & 0 & & 5821.355 & $231134^{\circ}-289343$ & 16832.896 & 0 & & 5939.126 & $25306_{2}^{\circ}-31245_{2}$ \\
\hline 17168.126 & 0 & & 5823.157 & $195324-25355_{4}^{\circ}$ & 16820.947 & 1 & & 5943.345 & $23609^{\circ}-295526$ \\
\hline 17165.765 & 2 & & 5823.958 & $22855^{\circ}-28679_{2}$ & 16811.787 & 0 & & 5946.583 & $26790_{4}^{\circ}-32737_{5}$ \\
\hline 17163.009 & 1 & & 5824.893 & $24307_{2}^{\circ}-301322$ & 16801.531 & 0 & & 5950.213 & $24182_{2}^{\circ}-30132_{2}$ \\
\hline 17153.591 & 2 & & 5828.091 & $18431_{3}-24259_{4}^{\circ}$ & 16800.478 & 1 & & 5950.586 & $13088_{3}-19039_{2}^{\circ}$ \\
\hline 17152.288 & 1 & & 5828.534 & $223998-28227_{4}$ & 16785.229 & 2 & & 5955.992 & $21539_{4}^{\circ}-27495_{4}$ \\
\hline 17151.508 & 1 & & 5828.799 & $21738_{2}^{\circ}-27566_{2}$ & 16785.046 & 2 & & 5956.057 & $15618_{3}^{\circ}-21575_{2}$ \\
\hline 17151.178 & 0 & & 5828.911 & $24182 \stackrel{\circ}{2}-300113$ & 16782.389 & 1 & & 5957.000 & $17959_{4}-23916_{4}^{\circ}$ \\
\hline 17146.766 & 0 & & 5830.411 & $23015 \circ-288454$ & 16768.550 & 6 & & 5961.916 & $12847_{3}-18809_{4}^{\circ}$ \\
\hline 17135.330 & 4 & & 5834.302 & $8111_{4}-13945^{\circ}$ & 16761.533 & 0 & & 5964.412 & $26048_{4}^{\circ}-32012_{4}$ \\
\hline 17134.167 & 4 & & 5834.698 & $233066-291415$ & 16748.433 & 5 & & 5969.077 & $11197^{\circ}-17166_{5}$ \\
\hline 17133.189 & 1 & & 5835.031 & $25306_{2}^{\circ}-31141_{3}$ & 16747.499 & 2 & & 5969.410 & $13847_{2}-19817_{1}^{\circ}$ \\
\hline 17124.268 & 1 & & 5838.071 & $15736_{1}^{\circ}-21575_{2}$ & 16741.938 & 0 & & 5971.393 & $25355_{4}^{\circ}-31326_{4}$ \\
\hline
\end{tabular}


TABLE 4. Classified lines of Th $\mathrm{I}$-continued

\begin{tabular}{|c|c|c|c|c|c|c|c|c|c|}
\hline \multirow{2}{*}{$\begin{array}{l}\text { Wavelength } \\
(\AA)\end{array}$} & \multicolumn{2}{|c|}{ Intensity } & \multirow{2}{*}{$\begin{array}{l}\text { Wavenumber } \\
\quad\left(\mathrm{cm}^{-1}\right)\end{array}$} & \multirow{2}{*}{ Classification } & \multirow{2}{*}{$\begin{array}{c}\text { Wavelength } \\
\text { (§) }\end{array}$} & \multicolumn{2}{|c|}{ Inten sity } & \multirow{2}{*}{$\begin{array}{l}\text { Wavenumber } \\
\left(\mathrm{cm}^{-1}\right)\end{array}$} & \multirow{2}{*}{ Classification } \\
\hline & Lamp & Spark & & & & Lamp & Spark & & \\
\hline 16730.028 & 3 & & 5975.644 & $17073_{1}-230491$ & 16211.744 & 0 & & 6166.683 & $26384_{4}^{\circ}-32551_{3}$ \\
\hline 16724.318 & 1 & & 5977.684 & $17224_{2}^{\circ}-23201_{3}$ & 16204.604 & 1 & & 6169.400 & $19273_{2}-25442_{3}^{\circ}$ \\
\hline 16720.218 & 0 & & 5979.150 & $24307_{2}^{\circ}-30286_{1}$ & 16195.248 & 1 & & 6172.964 & $26790 \stackrel{\circ}{-}-329635$ \\
\hline 16712.444 & 3 & & 5981.931 & $21539_{4}^{\circ}-27521_{4}$ & 16188.147 & 0 & & 6175.672 & $226693-28845_{4}^{\circ}$ \\
\hline 16707.945 & 0 & & 5983.542 & $24981_{3}^{\circ}-309643$ & 16180.368 & 0 & & 6178.641 & $21165^{\circ}-273433$ \\
\hline 16704.639 & 3 & & 5984.726 & $18930_{3}^{\circ}-24915_{3}$ & 16178.472 & 2 & & 6179.365 & $22163 \stackrel{\circ}{4}-283425$ \\
\hline 16694.697 & 0 & & 5988.290 & $24769_{3}^{\circ}-30758_{2}$ & 16172.466 & 1 & & 6181.660 & $25355_{4}^{\circ}-31537_{3}$ \\
\hline 16691.676 & 1 & & 5989.374 & $18431_{3}-24421_{3}^{\circ}$ & 16147.600 & 3 & & 6191.179 & $12847_{3}-19039_{2}^{\circ}$ \\
\hline 16688.906 & 1 & & 5990.368 & $197133-25703_{2}^{\circ}$ & 16145.326 & 1 & & 6192.051 & $22338{ }_{3}^{\circ}-28531_{2}$ \\
\hline 16687.560 & 0 & & 5990.851 & $24561_{3}^{\circ}-305524$ & 16142.758 & 2 & & 6193.036 & $15970_{3}-22163_{4}^{\circ}$ \\
\hline 16686.678 & 1 & & 5991.168 & $26790_{4}^{\circ}-32781_{4}$ & 16140.194 & 0 & & 6194.020 & $23655_{4}^{\circ}-29849_{4}$ \\
\hline 16624.607 & 2 & & 6013.537 & $18614_{1}^{\circ}-24627_{1}$ & 16137.810 & 0 & & 6194.935 & $24769_{3}^{\circ}-30964_{3}$ \\
\hline 16606.290 & 1 & & 6020.170 & $17073_{1}-23093_{2}^{\circ}$ & 16125.805 & 0 & & 6199.547 & $25575_{4}^{\circ}-31774_{3}$ \\
\hline 16604.436 & 1 & & 6020.842 & $18011{ }^{\circ}-240324$ & 16109.798 & 5 & & 6205.707 & $132974-19503_{3}^{\circ}$ \\
\hline 16599.350 & 3 & & 6022.687 & $14032_{2}^{\circ}-200542$ & 16100.958 & 0 & & 6209.114 & $237522-29961_{1}$ \\
\hline 16594.999 & 0 & & 6024.266 & $22248_{2}^{\circ}-28273_{2}$ & 16084.899 & 0 & & 6215.313 & $253211_{3}^{\circ}-315373$ \\
\hline 16589.555 & 1 & & 6026.243 & $15863_{2}-21890_{3}^{\circ}$ & 16081.086 & 1 & & 6216.787 & $22141^{\circ}-283583$ \\
\hline 16587.815 & 3 & & 6026.875 & $15618_{3}^{\circ}-21645_{4}$ & 16071.988 & 1 & & 6220.306 & $18549_{2}-24769_{3}^{\circ}$ \\
\hline 16587.044 & 0 & & 6027.155 & $22855_{3}^{\circ}-28882_{2}$ & 16070.913 & 0 & & 6220.722 & $23741_{1}^{\circ}-29961_{1}$ \\
\hline 16569.667 & 0 & & 6033.476 & $19273_{2}-25306_{2}^{\circ}$ & 16068.852 & 1 & & 6221.520 & $195324-25753{ }^{\circ}$ \\
\hline 16540.424 & 5 & & 6044.143 & $11802_{2}-17847_{2}^{\circ}$ & 16056.201 & 0 & & 6226.422 & $228777^{\circ}-29104_{4}$ \\
\hline 16538.399 & 3 & & 6044.883 & $16351_{0}-22396^{\circ}$ & 16048.543 & 1 & & 6229.393 & $24701{ }^{\circ}-30930_{6}$ \\
\hline 16534.332 & 2 & & 6046.370 & $15493_{4}-21539_{4}^{\circ}$ & 16045.762 & 0 & & 6230.473 & $25306_{2}^{\circ}-31537_{3}$ \\
\hline 16531.417 & 1 & & 6047.436 & $23603_{2}^{\circ}-29650_{2}$ & 16038.518 & 3 & & 6233.287 & $24274 \stackrel{\circ}{-}-305085$ \\
\hline 16521.658 & 1 & & 6051.008 & $24838_{1}^{\circ}-30889_{1}$ & 16033.162 & 2 & & 6235.369 & $49614-111970$ \\
\hline 16503.791 & 4 & & 6057.559 & $18614_{1}^{\circ}-24671_{2}$ & 16014.426 & 2 & & 6242.664 & $17959_{4}-24202_{4}^{\circ}$ \\
\hline 16501.244 & 0 & & 6058.494 & $20054_{2}-26113_{2}^{\circ}$ & 16008.311 & 3 & & 6245.049 & $18382{ }^{\circ}-24627_{1}$ \\
\hline 16461.254 & 1 & & 6073.212 & $19817_{1}^{\circ}-25890_{2}$ & 16006.680 & 0 & & 6245.685 & $233066-295526$ \\
\hline 16452.932 & 0 & & 6076.284 & $21594_{3}-27670_{3}^{\circ}$ & 16005.763 & 4 & & 6246.043 & $11601_{1}-17847_{2}^{\circ}$ \\
\hline 16447.180 & 0 & & 6078.409 & $21902_{4}^{\circ}-27980_{3}$ & 15997.841 & 2 & & 6249.136 & $16783 \stackrel{\circ}{4}-230324$ \\
\hline 16414.399 & 1 & & 6090.548 & $21890_{3}^{\circ}-27980_{3}$ & 15978.393 & 2 & & 6256.742 & $173983-23655^{\circ}$ \\
\hline 16412.823 & 3 & & 6091.133 & $21252_{2}^{\circ}-273433$ & 15973.502 & 1 & & 6258.658 & $23752 \stackrel{2}{2}-30011_{3}$ \\
\hline 16403.906 & 0 & & 6094.444 & $23916_{4}^{\circ}-30011_{3}$ & 15972.593 & 0 & & 6259.014 & $25753{ }^{\circ}-32012_{4}$ \\
\hline 16394.343 & 1 & & 6097.999 & $24274^{\circ}-303726$ & 15960.024 & 2 & & 6263.943 & $22877{ }^{\circ}-291415$ \\
\hline 16390.870 & 0 & & 6099.291 & $21575_{2}-27674_{2}^{\circ}$ & 15954.449 & 4 & & 6266.132 & $11802_{2}-18069_{3}^{\circ}$ \\
\hline 16375.188 & 1 & & 6105.132 & $18809_{4}^{\circ}-24915_{3}$ & 15941.439 & 0 & & 6271.246 & $25355_{4}^{\circ}-31626_{5}$ \\
\hline 16363.631 & 2 & & 6109.444 & $22248_{2}^{\circ}-28358_{3}$ & 15936.069 & 3 & & 6273.359 & $205222_{2}^{\circ}-26796_{3}$ \\
\hline 16340.749 & 2 & & 6117.999 & $142045-20322{ }^{\circ}$ & 15925.011 & 1 & & 6277.715 & $15863_{2}-22141_{3}^{\circ}$ \\
\hline 16329.816 & 2 & & 6122.095 & $20922_{2}^{\circ}-27044_{3}$ & 15924.829 & 0 & & 6277.787 & $24274 \stackrel{\circ}{-}-305524$ \\
\hline 16326.067 & 2 & & 6123.501 & $24421_{3}^{\circ}-305442$ & 15922.130 & 4 & & 6278.851 & $15970_{3}-22248_{2}^{\circ}$ \\
\hline 16310.703 & 3 & & 6129.269 & $20867_{7}^{\circ}-26997_{6}$ & 15919.042 & 1 & & 6280.069 & $4961_{4}-11241_{3}^{\circ}$ \\
\hline 16308.851 & 2 & & 6129.965 & $18431_{3}-24561_{3}^{\circ}$ & 15915.448 & 1 & & 6281.487 & $18699_{2}-24981_{3}^{\circ}$ \\
\hline 16304.920 & 0 & & 6131.443 & $24421_{3}^{\circ}-305524$ & 15913.906 & 0 & & 6282.096 & $22248_{2}^{\circ}-28531_{2}$ \\
\hline 16304.149 & 3 & & 6131.733 & $21902^{\circ}-280345$ & 15899.553 & 3 & & 6287.767 & $240846-303726$ \\
\hline 16297.533 & 0 & & 6134.222 & $22396_{1}^{\circ}-28531_{2}$ & 15895.139 & 1 & & 6289.513 & $18549_{2}-24838^{\circ}$ \\
\hline 16284.058 & 1 & & 6139.298 & $18699_{2}-24838_{i}$ & 15893.090 & 1 & & 6290.324 & $253366_{6}^{\circ}-316265$ \\
\hline 16280.282 & 3 & & 6140.722 & $171665-233066^{\circ}$ & 15891.854 & 3 & & 6290.813 & $16346_{4}^{\circ}-22637_{3}$ \\
\hline 16273.373 & 0 & & 6143.329 & $17847_{2}^{\circ}-23990_{2}$ & 15891.564 & 4 & & 6290.928 & $13297_{4}-19588_{0}^{\circ}$ \\
\hline 16270.823 & 0 & & 6144.292 & $26651_{2}^{\circ}-32796_{3}$ & 15887.023 & 1 & & 6292.726 & $24259_{4}^{\circ}-30552_{4}$ \\
\hline 16263.051 & 1 & & 6147.228 & $236090^{\circ}-297564$ & 15868.987 & 2 & & 6299.878 & $17959_{4}-24259_{4}^{\circ}$ \\
\hline 16240.450 & 2 & & 6155.783 & $15490_{0}^{\circ}-21645_{4}$ & 15861.758 & 1 & & 6302.749 & $21645_{4}-27948_{4}^{\circ}$ \\
\hline 16238.115 & 3 & & 6156.668 & $11241_{3}^{\circ}-173983$ & 15854.989 & 1 & & 6305.440 & $24202 \stackrel{\circ}{4}-305085$ \\
\hline 16234.453 & 0 & & 6158.057 & $195324-25690_{0}^{\circ}$ & 15847.733 & 3 & & 6308.327 & $20054_{2}-26363^{\circ}$ \\
\hline 16220.740 & 3 & & 6163.263 & $22399^{\circ}-285624$ & 15833.507 & 0 & & 6313.995 & $23521_{3}^{\circ}-29835_{3}$ \\
\hline 16217.564 & 0 & & 6164.470 & $19713_{3}-25877_{4}^{\circ}$ & 15832.882 & 0 & & 6314.244 & $212522-27566_{2}$ \\
\hline
\end{tabular}


TABLE 4. Classified lines of $\mathrm{Th} \mathrm{I}$-continued

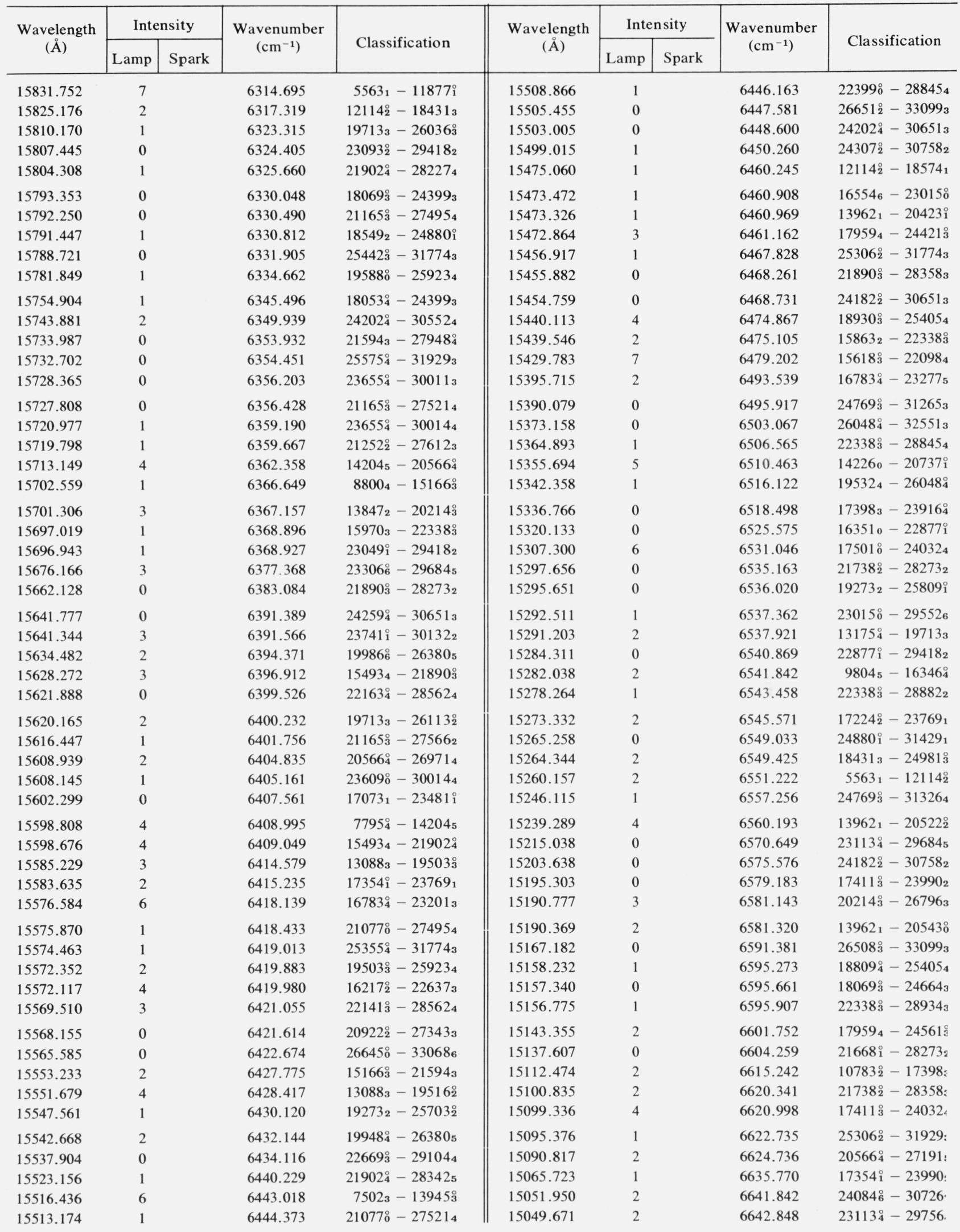


TABLE 4. Classified lines of Th I-continued

\begin{tabular}{|c|c|c|c|c|c|c|c|c|c|}
\hline \multirow{2}{*}{$\begin{array}{l}\text { Wavelength } \\
\text { (A) }\end{array}$} & \multicolumn{2}{|c|}{ Intensity } & \multirow{2}{*}{$\begin{array}{l}\text { Wavenumber } \\
\quad\left(\mathrm{cm}^{-1}\right)\end{array}$} & \multirow{2}{*}{ Classification } & \multirow{2}{*}{$\begin{array}{c}\text { Wavelength } \\
(\AA)\end{array}$} & \multicolumn{2}{|c|}{ Inten sity } & \multirow{2}{*}{$\begin{array}{l}\text { Wavenumber } \\
\qquad\left(\mathrm{cm}^{-1}\right)\end{array}$} & \multirow{2}{*}{ Classification } \\
\hline & Lamp & Spark & & & & Lamp & Spark & & \\
\hline 15045.413 & 3 & & 6644.728 & $20922_{2}^{\circ}-27566_{2}$ & 14695.579 & 3 & & 6802.908 & $21539_{4}^{\circ}-283425$ \\
\hline 15037.137 & 0 & & 6648.385 & $154934-22141_{3}^{\circ}$ & 14690.666 & 0 & & 6805.183 & $211435-27948^{\circ}$ \\
\hline 15035.312 & 0 & & 6649.192 & $20322 \circ-26971_{4}$ & 14689.932 & 0 & & 6805.523 & $23481 i-302861$ \\
\hline 15031.317 & 1 & & 6650.959 & $132974-19948{ }^{\circ}$ & 14688.909 & 0 & & 6805.997 & $15863_{2}-22669_{3}^{\circ}$ \\
\hline 15021.804 & 3 & & 6655.171 & $128473-195033^{\circ}$ & 14687.576 & 0 & & 6806.615 & $22877^{\circ}-29684_{5}$ \\
\hline 15017.893 & 0 & & 6656.904 & $24307_{2}^{\circ}-309643$ & 14680.667 & 0 & & 6809.818 & $17959_{4}-24769_{3}^{\circ}$ \\
\hline 15010.031 & 3 & & 6660.391 & $21902_{4}^{\circ}-28562_{4}$ & 14677.249 & 5 & & 6811.404 & $11802_{2}-18614_{1}^{\circ}$ \\
\hline 15007.406 & 0 & & 6661.556 & $14481 \stackrel{\circ}{6}-211435$ & 14661.334 & 0 & & 6818.798 & $21539_{4}^{\circ}-28358_{3}$ \\
\hline 14994.654 & 3 & & 6667.221 & $195888^{\circ}-26255_{4}$ & 14654.914 & 7 & & 6821.785 & $11877^{\circ}-18699_{2}$ \\
\hline 14994.569 & 2 & & 6667.259 & $17073_{1}-23741_{i}^{\circ}$ & 14651.482 & 3 & & 6823.383 & $20054_{2}-26878_{3}^{\circ}$ \\
\hline 14990.632 & 1 & & 6669.010 & $12847_{3}-19516_{2}^{\circ}$ & 14644.393 & 1 & & 6826.686 & $18699_{2}-25526_{1}^{\circ}$ \\
\hline 14988.603 & 4 & & 6669.913 & $154934-22163_{4}^{\circ}$ & 14638.811 & 0 & & 6829.289 & $20214_{3}^{\circ}-270443$ \\
\hline 14984.882 & 4 & & 6671.569 & $11877_{1}^{\circ}-18549_{2}$ & 14638.217 & 1 & & 6829.566 & $20737_{1}^{\circ}-27566_{2}$ \\
\hline 14982.726 & 1 & & 6672.529 & $21890_{3}^{\circ}-285624$ & 14628.703 & 2 & & 6834.008 & $23015_{0}^{\circ}-29849_{4}$ \\
\hline 14980.034 & 0 & & 6673.728 & $25321_{3}^{\circ}-319954$ & 14618.976 & 7 & & 6838.555 & $3687_{2}-10526_{3}^{\circ}$ \\
\hline 14977.332 & 3 & & 6674.932 & $22877^{\circ}-295526$ & 14615.924 & 1 & & 6839.983 & $19273_{2}-26113_{2}^{\circ}$ \\
\hline 14968.497 & 2 & & 6678.872 & $17073_{1}-23752_{2}^{\circ}$ & 14604.155 & 2 & & 6845.495 & $240846^{\circ}-309306$ \\
\hline 14960.529 & 0 & & 6682.429 & $22163_{4}^{\circ}-28845_{4}$ & 14603.558 & 3 & & 6845.775 & $15493_{4}-22338_{3}^{\circ}$ \\
\hline 14956.476 & 2 & & 6684.240 & $24561_{3}^{\circ}-31245_{2}$ & 14603.174 & 0 & & 6845.955 & $18069_{3}^{\circ}-24915_{3}$ \\
\hline 14955.641 & 1 & & 6684.613 & $26096_{3}^{\circ}-32781_{4}$ & 14599.499 & 1 & & 6847.678 & $19948_{4}^{\circ}-267963$ \\
\hline 14951.792 & 1 & & 6686.334 & $16346_{4}^{\circ}-230324$ & 14591.907 & 1 & & 6851.241 & $19039_{2}^{\circ}-25890_{2}$ \\
\hline 14947.310 & 2 & & 6688.339 & $21539_{4}^{\circ}-28227_{4}$ & 14573.635 & 2 & & 6859.831 & $13088_{3}-19948^{\circ}$ \\
\hline 14947.021 & 1 & & 6688.468 & $26048_{4}^{\circ}-32737_{5}$ & $14570.349^{\circ}$ & 3 & & 6861.378 & $17398_{3}-24259_{4}^{\circ}$ \\
\hline 14943.987 & 6 & & 6689.826 & $8800_{4}-15490 \AA$ & 14563.603 & 0 & & 6864.556 & $24381_{2}^{\circ}-31245_{2}$ \\
\hline 14928.485 & 4 & & 6696.773 & $11877_{i}^{\circ}-18574_{1}$ & 14553.980 & 1 & & 6869.095 & $20322{ }^{\circ}-271915$ \\
\hline 14921.758 & 0 & & 6699.792 & $15970_{3}-22669_{3}^{\circ}$ & 14548.134 & 2 & & 6871.855 & $10526_{3}^{\circ}-17398_{3}$ \\
\hline 14911.028 & 2 & & 6704.613 & $22399^{\circ}-291044$ & 14545.954 & 2 & & 6872.885 & $14204_{5}-21077^{\circ}$ \\
\hline 14880.943 & 0 & & 6718.168 & $11241_{3}^{\circ}-17959_{4}$ & 14541.314 & 1 & & 6875.078 & $18431_{3}-25306_{2}^{\circ}$ \\
\hline 14875.259 & 1 & & 6720.735 & $24421_{3}^{\circ}-31141_{3}$ & 14533.419 & 4 & & 6878.813 & $22877_{0}^{\circ}-29756_{4}$ \\
\hline 14873.575 & 1 & & 6721.496 & $23113{ }^{\circ}-298353$ & 14519.935 & 2 & & 6885.201 & $15970_{3}-22855^{\circ}$ \\
\hline 14850.034 & 0 & & 6732.151 & $18574_{1}-25306_{2}^{\circ}$ & 14510.851 & 0 & & 6889.511 & $13847_{2}-20737_{1}^{\circ}$ \\
\hline 14842.404 & 0 & & 6735.612 & $23113_{4}^{\circ}-29849_{4}$ & 14502.932 & 1 & & 6893.273 & $18549_{2}-25442_{3}^{\circ}$ \\
\hline 14830.215 & 3 & & 6741.148 & $179594-24701^{\circ}$ & 14493.424 & 2 & & 6897.795 & $23113_{4}^{\circ}-300113$ \\
\hline 14830.001 & 1 & & 6741.245 & $23015 \circ-297564$ & 14491.248 & 1 & & 6898.831 & $23609_{0}^{\circ}-305085$ \\
\hline 14809.031 & 2 & & 6750.791 & $171665-23916_{4}^{\circ}$ & 14476.391 & 0 & & 6905.911 & $24421_{3}^{\circ}-313264$ \\
\hline 14806.456 & 5 & & 6751.965 & $7280_{2}-14032_{2}^{\circ}$ & 14475.836 & 4 & & 6906.176 & $15493_{4}-22399_{0}^{\circ}$ \\
\hline 14805.415 & 3 & & 6752.440 & $19503_{3}^{\circ}-26255_{4}$ & 14469.188 & 2 & & 6909.349 & $17398_{3}-24307^{\circ}$ \\
\hline 14805.099 & 1 & & 6752.584 & $165546-23306_{6}^{\circ}$ & 14467.109 & 0 & & 6910.342 & $22508_{2}^{\circ}-29418_{2}$ \\
\hline 14796.452 & 0 & & 6756.530 & $20214_{3}^{\circ}-26971_{4}$ & 14452.427 & 0 & & 6917.362 & $23093_{2}^{\circ}-300113$ \\
\hline 14789.356 & 1 & & 6759.772 & $26036^{\circ}-327963$ & 14452.147 & 2 & & 6917.496 & $13297_{4}-20214^{\circ}$ \\
\hline 14784.758 & 1 & & 6761.874 & $139621-20724{ }^{\circ}$ & 14451.758 & 4 & & 6917.682 & $3865_{1}-10783_{2}^{\circ}$ \\
\hline 14781.114 & 0 & & 6763.541 & $23609 \circ-303726$ & 14449.799 & 1 & & 6918.620 & $25877_{4}^{\circ}-32796_{3}$ \\
\hline 14777.907 & 1 & & 6765.009 & $22338_{3}^{\circ}-29104_{4}$ & 14449.350 & 2 & & 6918.835 & $171665-240846$ \\
\hline 14777.230 & 1 & & 6765.319 & $24561_{3}^{\circ}-313264$ & 14438.799 & 3 & & 6923.891 & $18431_{3}-25355_{4}^{\circ}$ \\
\hline 14772.419 & 0 & & 6767.522 & $24769_{3}^{\circ}-31537_{3}$ & 14424.537 & 7 & & 6930.737 & $16346_{4}^{\circ}-23277_{5}$ \\
\hline 14772.031 & 3 & & 6767.700 & $25690^{\circ}-324584$ & 14423.392 & 2 & & 6931.287 & $15166_{3}^{\circ}-22098_{4}$ \\
\hline 14764.746 & 2 & & 6771.039 & $16217_{2}^{\circ}-22988_{2}$ & 14412.536 & 4 & & 6936.508 & $14206_{4}^{\circ}-211435$ \\
\hline 14756.630 & 3 & & 6774.763 & $139621-20737_{1}^{\circ}$ & 14407.785 & 1 & & 6938.795 & $19713_{3}-26651_{2}^{\circ}$ \\
\hline 14751.515 & 0 & & 6777.112 & $20566_{4}^{\circ}-27343_{3}$ & 14403.474 & 1 & & 6940.872 & $22163_{4}^{\circ}-29104_{4}$ \\
\hline 14741.329 & 5 & & 6781.795 & $116011-18382^{\circ}$ & 14403.132 & 0 & & 6941.037 & $23603_{2}^{\circ}-30544_{2}$ \\
\hline 14740.403 & 0 & & 6782.221 & $24182_{2}^{\circ}-30964_{3}$ & 14398.454 & 2 & & 6943.292 & $21902_{4}^{\circ}-28845_{4}$ \\
\hline 14736.470 & 0 & & 6784.031 & $173983-24182_{2}^{\circ}$ & 14374.424 & 2 & & 6954.899 & $20566_{4}^{\circ}-27521_{4}$ \\
\hline 14719.449 & 1 & & 6791.876 & $23752_{2}^{\circ}-30544_{2}$ & 14373.329 & 1 & & 6955.429 & $21890_{3}^{\circ}-28845_{4}$ \\
\hline 14718.799 & 5 & & 6792.176 & $19588^{\circ}-26380_{5}$ & 14370.393 & 0 & & 6956.850 & $21077^{\circ}-280345$ \\
\hline 14716.370 & 2 & & 6793.297 & $22141_{3}^{\circ}-289343$ & 14364.711 & 2 & & 6959.602 & $139621-20922_{2}^{\circ}$ \\
\hline
\end{tabular}


TABLE 4. Classified lines of Th I-continued

\begin{tabular}{|c|c|c|c|c|c|c|c|c|c|}
\hline \multirow{2}{*}{$\begin{array}{l}\text { Wavelength } \\
\text { (Å) }\end{array}$} & \multicolumn{2}{|c|}{ Intensity } & \multirow{2}{*}{$\begin{array}{l}\text { Wavenumber } \\
\left(\mathrm{cm}^{-1}\right)\end{array}$} & \multirow{2}{*}{ Classification } & \multirow{2}{*}{$\begin{array}{c}\text { Wavelength } \\
(\AA)\end{array}$} & \multicolumn{2}{|c|}{ Inten sity } & \multirow{2}{*}{$\begin{array}{c}\text { Wavenumber } \\
\left(\mathrm{cm}^{-1}\right)\end{array}$} & \multirow{2}{*}{ Classification } \\
\hline & Lamp & Spark & & & & Lamp & Spark & & \\
\hline 14363.687 & 2 & & 6960.098 & $18930_{3}^{\circ}-25890_{2}$ & 14028.560 & 4 & & 7126.367 & $13088_{3}-20214_{3}^{\circ}$ \\
\hline 14358.938 & 2 & & 6962.400 & $22141_{3}^{\circ}-291044$ & 14026.605 & 5 & & 7127.360 & $11802_{2}-18930_{3}^{\circ}$ \\
\hline 14357.839 & 3 & & 6962.933 & $75023-14465_{2}^{\circ}$ & 14008.158 & 0 & & 7136.746 & $22877^{\circ}-30014_{4}$ \\
\hline 14355.909 & 0 & & 6963.869 & $7280_{2}-14243^{\circ}$ & 14007.173 & 0 & & 7137.248 & $23752_{2}^{\circ}-30889_{1}$ \\
\hline 14340.036 & 0 & & 6971.577 & $22877_{0}^{\circ}-29849_{4}$ & 13995.011 & 0 & & 7143.450 & $15970_{3}-23113{ }^{\circ}$ \\
\hline 14326.032 & 1 & & 6978.392 & $22163_{4}^{\circ}-291415$ & 13993.145 & 1 & & 7144.403 & $21738_{2}^{\circ}-28882_{2}$ \\
\hline 14324.702 & 0 & & 6979.040 & $98045-16783 \stackrel{\circ}{4}$ & 13976.090 & 2 & & 7153.121 & $22399 \circ-295526$ \\
\hline 14323.255 & 1 & & 6979.745 & $22855_{3}^{\circ}-29835_{3}$ & 13975.932 & 4 & & 7153.202 & $19227_{6}^{\circ}-263805$ \\
\hline 14313.992 & 4 & & 6984.262 & $10414_{4}^{\circ}-173983$ & 13974.383 & 1 & & 7153.995 & $18549_{2}-25703_{2}^{\circ}$ \\
\hline 14306.560 & 2 & & 6987.890 & $17411_{3}^{\circ}-24399_{3}$ & 13970.381 & 2 & & 7156.044 & $22855_{3}^{\circ}-30011_{3}$ \\
\hline 14297.496 & 0 & & 6992.320 & $21890_{3}^{\circ}-28882_{2}$ & 13964.777 & 2 & & 7158.916 & $12114_{2}^{\circ}-19273_{2}$ \\
\hline 14296.656 & 2 & & 6992.731 & $18930_{3}^{\circ}-259234$ & 13936.736 & 5 & & 7173.320 & $20322^{\circ}-27495_{4}$ \\
\hline 14294.346 & 0 & & 6993.861 & $22855^{\circ}-29849_{4}$ & 13930.234 & 3 & & 7176.668 & $154934-22669_{3}^{\circ}$ \\
\hline 14283.487 & 1 & & 6999.178 & $230150-300144$ & 13904.491 & 6 & & 7189.955 & $11241 \stackrel{\circ}{3}-184313$ \\
\hline 14271.876 & 1 & & 7004.872 & $24769_{3}^{\circ}-31774_{3}$ & 13898.031 & 1 & & 7193.297 & $21165_{3}^{\circ}-283583$ \\
\hline 14270.962 & 1 & & 7005.321 & $23752_{2}^{\circ}-30758_{2}$ & 13886.527 & 4 & & 7199.256 & $20322{ }^{\circ}-27521_{4}$ \\
\hline 14259.243 & 5 & & 7011.078 & $19986_{6}^{\circ}-269976$ & 13882.834 & 1 & & 7201.171 & $23306_{6}^{\circ}-30508_{5}$ \\
\hline 14254.713 & 5 & & 7013.306 & $11601_{1}-18614_{i}^{\circ}$ & 13872.468 & 0 & & 7206.552 & $25575_{4}^{\circ}-32781_{4}$ \\
\hline 14254.065 & 0 & & 7013.625 & $15863_{2}-22877^{\circ}$ & 13862.050 & 0 & & 7211.968 & $23752_{2}^{\circ}-309643$ \\
\hline 14253.783 & 1 & & 7013.764 & $19273_{2}-26287_{1}^{\circ}$ & 13859.106 & 1 & & 7213.500 & $21668_{1}^{\circ}-28882_{2}$ \\
\hline 14244.211 & 2 & & 7018.477 & $15618^{\circ}-22637_{3}$ & 13844.566 & 0 & & 7221.076 & $25575_{4}^{\circ}-327963$ \\
\hline 14239.881 & 1 & & 7020.611 & $21252_{2}^{\circ}-28273_{2}$ & 13818.508 & 1 & & 7234.693 & $18574_{1}-25809_{1}^{\circ}$ \\
\hline 14238.660 & 2 & & 7021.213 & $17959_{4}-24981_{3}^{\circ}$ & 13815.598 & 1 & & 7236.217 & $11802_{2}-19039_{2}^{\circ}$ \\
\hline 14237.955 & 1 & & 7021.561 & $22396_{1}^{\circ}-29418_{2}$ & 13828.453 & 1 & & 7229.490 & $24307_{2}^{\circ}-31537_{3}$ \\
\hline 14235.729 & 2 & & 7022.659 & $17398_{3}-24421_{3}^{\circ}$ & 13814.181 & 0 & & 7236.959 & $23521 \stackrel{\circ}{3}-307582$ \\
\hline 14234.902 & 2 & & 7023.067 & $19948_{4}^{\circ}-26971_{4}$ & 13813.265 & 0 & & 7237.439 & $23049_{1}^{\circ}-30286_{1}$ \\
\hline 14234.902 & 2 & & 7023.067 & $21539_{4}^{\circ}-285624$ & 13809.796 & 1 & & 7239.257 & $21902_{4}^{\circ}-29141_{5}$ \\
\hline 14233.051 & 0 & & 7023.980 & $24769^{\circ}-317934$ & 13799.139 & 0 & & 7244.848 & $25306_{2}^{\circ}-32551_{3}$ \\
\hline 14231.327 & 4 & & 7024.831 & $13297_{4}-20322{ }^{\circ}$ & 13795.378 & 1 & & 7246.823 & $24182_{2}^{\circ}-31429_{1}$ \\
\hline 14230.620 & 0 & & 7025.180 & $20566_{4}^{\circ}-27591_{5}$ & 13792.422 & 5 & & 7248.376 & $16783^{\circ}-240324$ \\
\hline 14230.460 & 0 & & 7025.259 & $22396^{\circ}-29422_{1}$ & 13786.380 & 1 & & 7251.553 & $15736_{1}^{\circ}-22988_{2}$ \\
\hline 14217.138 & 1 & & 7031.842 & $24981^{\circ}-32012_{4}$ & 13779.592 & 1 & & 7255.125 & $22877_{1}^{\circ}-30132_{2}$ \\
\hline 14214.389 & 0 & & 7033.202 & $20054_{2}-27087^{\circ}$ & 13774.118 & 1 & & 7258.008 & $19532_{4}-26790_{4}^{\circ}$ \\
\hline 14192.323 & 4 & & 7044.137 & $20522_{2}^{\circ}-27566_{2}$ & 13760.203 & 2 & & 7265.348 & $210777_{0}^{\circ}-283425$ \\
\hline 14191.050 & 2 & & 7044.769 & $21890_{3}^{\circ}-289343$ & 13752.928 & 2 & & 7269.191 & $13297_{4}-20566_{4}^{\circ}$ \\
\hline 14189.283 & 0 & & 7045.646 & $20566_{4}^{\circ}-276123$ & 13752.277 & 0 & & 7269.535 & $20322{ }^{\circ}-275915$ \\
\hline 14187.489 & 2 & & 7046.537 & $25690_{0}^{\circ}-327375$ & 13748.535 & 0 & & 7271.514 & $24769_{3}^{\circ}-32041_{2}$ \\
\hline 14170.625 & 0 & & 7054.923 & $165546-23609^{\circ}$ & 13748.149 & 2 & & 7271.718 & $18431_{3}-25703_{2}^{\circ}$ \\
\hline 14168.671 & 7 & & 7055.896 & $8111_{4}-15166_{3}^{\circ}$ & 13745.894 & 0 & & 7272.911 & $25690 \AA-329635$ \\
\hline 14160.194 & 1 & & 7060.120 & $24981_{3}^{\circ}-32041_{2}$ & 13739.956 & 5 & & 7276.054 & $18614_{1}^{\circ}-25890_{2}$ \\
\hline 14154.750 & 0 & & 7062.835 & $21165_{3}^{\circ}-28227_{4}$ & 13738.583 & 3 & & 7276.781 & $221411_{3}^{\circ}-29418_{2}$ \\
\hline 14148.646 & 2 & & 7065.882 & $23306^{\circ}-303726$ & 13735.446 & 2 & & 7278.443 & $21252_{2}^{\circ}-28531_{2}$ \\
\hline 14107.467 & 1 & & 7086.507 & $22669_{3}^{\circ}-29756_{4}$ & 13734.218 & 2 & & 7279.094 & $19516_{2}^{\circ}-26796_{3}$ \\
\hline 14101.396 & 3 & & 7089.558 & $20522_{2}^{\circ}-27612_{3}$ & 13731.271 & 2 & & 7280.656 & $20214_{3}^{\circ}-274954$ \\
\hline 14100.879 & 2 & & 7089.818 & $19273_{2}-26363_{2}^{\circ}$ & 13727.340 & 1 & & 7282.741 & $19713_{3}-26995^{\circ}$ \\
\hline 14095.249 & 1 & & 7092.650 & $24701^{\circ}-31793_{4}$ & 13723.460 & 0 & & 7284.800 & $223990_{0}^{\circ}-296845$ \\
\hline 14090.246 & 7 & & 7095.168 & $3687_{2}-10783_{2}^{\circ}$ & 13680.790 & 0 & & 7307.521 & $170731-24381_{2}^{\circ}$ \\
\hline 14079.818 & 5 & & 7100.423 & $12847_{3}-19948_{4}^{\circ}$ & 13680.494 & 1 & & 7307.679 & $11241 \stackrel{\circ}{3}-18549_{2}$ \\
\hline 14063.614 & 2 & & 7108.604 & $171665-24274^{\circ}$ & 13677.064 & 2 & & 7309.512 & $23655_{4}^{\circ}-30964_{3}$ \\
\hline 14063.614 & 2 & & 7108.604 & $17073_{1}-24182_{2}^{\circ}$ & 13672.580 & 0 & & 7311.909 & 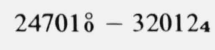 \\
\hline 14061.486 & 0 & & 7109.680 & $18699_{2}-25809_{1}^{\circ}$ & 13672.490 & 0 & & 7311.957 & $22338^{\circ}-29650_{2}$ \\
\hline 14061.215 & 1 & & 7109.817 & $14465_{2}^{\circ}-21575_{2}$ & 13668.402 & 0 & & 7314.144 & $257530^{\circ}-330686$ \\
\hline 14054.652 & 1 & & 7113.137 & $18809_{4}^{\circ}-25923_{4}$ & 13662.574 & 2 & & 7317.264 & $17354_{1}^{\circ}-24671_{2}$ \\
\hline 14038.143 & 0 & & 7121.502 & $24307_{2}^{\circ}-31429_{1}$ & 13662.466 & 1 & & 7317.322 & $13847_{2}-21165_{3}^{\circ}$ \\
\hline 14032.419 & 1 & & 7124.407 & $24202^{\circ}-313264$ & 13647.606 & 5 & & 7325.289 & $18930_{3}^{\circ}-26255_{4}$ \\
\hline
\end{tabular}


TABle 4. Classified lines of Th $\mathrm{I}$-continued

\begin{tabular}{|c|c|c|c|c|c|c|c|c|c|}
\hline \multirow{2}{*}{ 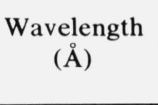 } & \multicolumn{2}{|c|}{ Intensity } & \multirow{2}{*}{$\begin{array}{l}\text { Wavenumber } \\
\left(\mathrm{cm}^{-1}\right)\end{array}$} & \multirow{2}{*}{ Classification } & \multirow{2}{*}{$\begin{array}{c}\text { Wavelength } \\
(\AA)\end{array}$} & \multicolumn{2}{|c|}{ Inten sity } & \multirow{2}{*}{$\begin{array}{l}\text { Wavenumber } \\
\left(\mathrm{cm}^{-1}\right)\end{array}$} & \multirow{2}{*}{ Classification } \\
\hline & Lamp & Spark & & & & Lamp & Spark & & \\
\hline 13628.932 & 1 & & 7335.326 & $14204_{5}-21539_{4}^{\circ}$ & 13348.625 & 1 & & 7489.360 & $25306_{2}^{\circ}-327963$ \\
\hline 13612.012 & 0 & & 7344.444 & $22669_{3}^{\circ}-30014_{4}$ & 13342.411 & 1 & & 7492.848 & $23015^{\circ}-305085$ \\
\hline 13609.614 & 2 & & 7345.738 & $19532_{4}-26878_{3}^{\circ}$ & 13336.712 & 3 & & 7496.050 & $22338_{3}^{\circ}-29835_{3}$ \\
\hline 13599.702 & 3 & & 7351.092 & $20922_{2}^{\circ}-28273_{2}$ & 13334.671 & 1 & & 7497.197 & $18614_{i}^{\circ}-2611_{1}$ \\
\hline 13598.599 & 3 & & 7351.688 & $24850 \stackrel{\circ}{-}-322025$ & 13322.946 & 0 & & 7503.795 & $17411_{3}^{\circ}-24915_{3}$ \\
\hline 13598.167 & 2 & & 7351.922 & $20214_{3}^{\circ}-27566_{2}$ & 13315.518 & 1 & & 7507.981 & $8111_{4}-15618_{3}^{\circ}$ \\
\hline 13597.830 & 1 & & 7352.104 & $24274{ }^{\circ}-31626_{5}$ & 13314.704 & 1 & & 7508.440 & $24421_{3}^{\circ}-319293$ \\
\hline 13595.363 & 0 & & 7353.438 & $25442{ }_{3}^{\circ}-32796_{3}$ & 13303.409 & 1 & & 7514.815 & $24259_{4}^{\circ}-317743$ \\
\hline 13592.829 & 0 & & 7354.809 & $24182_{2}^{\circ}-31537_{3}$ & 13296.036 & 1 & & 7518.982 & 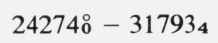 \\
\hline 13588.783 & 4 & & 7356.999 & $22399^{\circ}-297564$ & 13292.355 & 0 & & 7521.064 & $22163^{\circ}-296845$ \\
\hline 13587.748 & 0 & & 7357.559 & $23015^{\circ}-303726$ & 13290.823 & 1 & & 7521.931 & $154934-230158$ \\
\hline 13579.410 & 4 & & 7362.077 & $15493_{4}-22855^{\circ}$ & 13286.526 & 0 & & 7524.364 & $25575_{4}^{\circ}-33099_{3}$ \\
\hline 13572.266 & 0 & & 7365.952 & $21165_{3}^{\circ}-28531_{2}$ & 13281.453 & 4 & & 7527.238 & $19516_{2}^{\circ}-27044_{3}$ \\
\hline 13570.411 & 3 & & 7366.959 & $12847_{3}-20214_{3}^{\circ}$ & 13279.662 & 0 & & 7528.253 & $21890_{3}^{\circ}-29418_{2}$ \\
\hline 13565.665 & 7 & & 7369.536 & $15618_{3}^{\circ}-22988_{2}$ & 13275.351 & 5 & & 7530.698 & $165546-240846$ \\
\hline 13562.388 & 2 & & 7371.317 & $17398_{3}-24769^{\circ}$ & 13269.677 & 1 & & 7533.918 & $24259_{4}^{\circ}-317934$ \\
\hline 13549.102 & 2 & & 7378.545 & $19273_{2}-26651_{2}^{\circ}$ & 13267.880 & 2 & & 7534.938 & $17166_{5}-24701{ }^{\circ}$ \\
\hline 13540.748 & 3 & & 7383.097 & $195888^{\circ}-26971_{4}$ & 13263.633 & 2 & & 7537.351 & $23015 \circ-305524$ \\
\hline 13538.429 & 2 & & 7384.362 & $154934-22877^{\circ}$ & 13261.338 & 0 & & 7538.655 & $18574_{1}-26113_{2}^{\circ}$ \\
\hline 13532.204 & 2 & & 7387.759 & $14206_{4}^{\circ}-21594_{3}$ & 13257.081 & 3 & & 7541.076 & $195033-270443$ \\
\hline 13529.689 & 1 & & 7389.132 & $163510-23741^{\circ}$ & 13253.863 & 5 & & 7542.907 & $15490_{0}^{\circ}-230324$ \\
\hline 13518.143 & 6 & & 7395.443 & $11877_{1}^{\circ}-19273_{2}$ & 13250.406 & 1 & & 7544.875 & $2869_{3}-10414_{4}^{\circ}$ \\
\hline 13517.712 & 2 & & 7395.679 & $17959_{4}-25355_{4}^{\circ}$ & 13248.848 & 2 & & 7545.762 & $10414_{4}^{\circ}-17959_{4}$ \\
\hline 13514.671 & 1 & & 7397.343 & $20214_{3}^{\circ}-276123$ & 13247.730 & 4 & & 7546.399 & $8800_{4}-16346_{4}^{\circ}$ \\
\hline 13508.886 & 0 & & 7400.511 & $25336^{\circ}-327375$ & 13246.336 & 0 & & 7547.193 & $19948_{4}^{\circ}-27495_{4}$ \\
\hline 13501.001 & 4 & & 7404.833 & $13847_{2}-21252_{2}^{\circ}$ & 13245.664 & 2 & & 7547.576 & $18549_{2}-26096_{3}^{\circ}$ \\
\hline 13491.454 & 1 & & 7410.073 & $23916_{4}^{\circ}-313264$ & 13239.744 & 4 & & 7550.951 & $15970_{3}-23521_{3}^{\circ}$ \\
\hline 13484.963 & 1 & & 7413.640 & $18699_{2}-26113_{2}^{\circ}$ & 13236.164 & 3 & & 7552.993 & $19713_{3}-27266_{4}^{\circ}$ \\
\hline 13484.304 & 3 & & 7414.002 & $15618_{3}^{\circ}-230324$ & 13234.852 & 2 & & 7553.742 & $3687_{2}-11241_{3}^{\circ}$ \\
\hline 13478.127 & 0 & & 7417.400 & $22338^{\circ}-297564$ & 13219.366 & 0 & & 7562.591 & $14032_{2}^{\circ}-215943$ \\
\hline 13465.671 & 0 & & 7424.261 & $24202_{4}^{\circ}-316265$ & 13217.149 & 0 & & 7563.859 & $18549_{2}-26113_{2}^{\circ}$ \\
\hline 13449.197 & 4 & & 7433.355 & $10526_{3}^{\circ}-17959_{4}$ & 13216.178 & 1 & & 7564.415 & $21539_{4}^{\circ}-29104_{4}$ \\
\hline 13448.036 & 1 & & 7433.997 & $24561_{3}^{\circ}-319954$ & 13215.207 & 0 & & 7564.971 & $22396_{1}^{\circ}-29961_{1}$ \\
\hline 13447.755 & 4 & & 7434.152 & $13088_{3}-20522_{2}^{\circ}$ & 13205.290 & 6 & & 7570.652 & $18809_{4}^{\circ}-263805$ \\
\hline 13443.925 & 4 & & 7436.270 & $20922_{2}^{\circ}-283583$ & 13202.889 & 1 & & 7572.029 & $24202_{4}^{\circ}-317743$ \\
\hline 13440.583 & 4 & & 7438.119 & $11601_{1}-19039_{2}^{\circ}$ & 13200.969 & 1 & & 7573.130 & $19948 \stackrel{\circ}{4}-27521_{4}$ \\
\hline 13439.085 & 1 & & 7438.948 & $14206_{4}^{\circ}-216454$ & 13184.286 & 2 & & 7582.713 & $17398_{3}-24981_{3}^{\circ}$ \\
\hline 13436.414 & 0 & & 7440.427 & $17224_{2}^{\circ}-246643$ & 13183.941 & 3 & & 7582.911 & $6362_{2}-13945_{3}^{\circ}$ \\
\hline 13433.332 & 3 & & 7442.134 & $142260-21668^{\circ}$ & 13183.783 & 3 & & 7583.002 & $15618_{3}^{\circ}-23201_{3}$ \\
\hline 13426.907 & 3 & & 7445.695 & $18809_{4}^{\circ}-26255_{4}$ & 13176.103 & 2 & & 7587.422 & $18699_{2}-26287_{i}^{\circ}$ \\
\hline 13426.686 & 2 & & 7445.818 & $18431_{3}-25877_{4}^{\circ}$ & 13169.665 & 0 & & 7591.131 & $24202_{4}^{\circ}-31793_{4}$ \\
\hline 13423.480 & 2 & & 7447.596 & $17224_{2}^{\circ}-24671_{2}$ & 13168.345 & 0 & & 7591.892 & $24421_{3}^{\circ}-32012_{4}$ \\
\hline 13419.576 & 4 & & 7449.763 & $22399 \circ-298494$ & 13167.879 & 0 & & 7592.161 & $24182_{2}^{\circ}-317743$ \\
\hline 13418.102 & 0 & & 7450.581 & $23093_{2}^{\circ}-305442$ & 13156.601 & 1 & & 7598.669 & $12114_{2}^{\circ}-19713_{3}$ \\
\hline 13404.943 & 4 & & 7457.895 & $11241_{3}^{\circ}-18699_{2}$ & 13150.947 & 1 & & 7601.936 & $21539_{4}^{\circ}-291415$ \\
\hline 13395.136 & 1 & & 7463.355 & $195324-269953$ & 13146.765 & 1 & & 7604.354 & $19713_{3}-27317^{\circ}$ \\
\hline 13387.914 & 0 & & 7467.381 & $20566_{4}^{\circ}-280345$ & 13146.229 & 1 & & 7604.664 & $18431_{3}-26036_{3}^{\circ}$ \\
\hline 13386.236 & 2 & & 7468.317 & $19503_{3}^{\circ}-26971_{4}$ & 13144.547 & 1 & & 7605.637 & $19986_{6}^{\circ}-27591_{5}$ \\
\hline 13382.214 & 4 & & 7470.562 & $15166_{3}^{\circ}-22637_{3}$ & 13136.162 & 0 & & 7610.492 & $23655_{4}^{\circ}-31265_{3}$ \\
\hline 13370.573 & 1 & & 7477.066 & $24981_{3}^{\circ}-324584$ & 13128.501 & 2 & & 7614.933 & 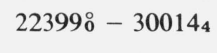 \\
\hline 13368.792 & 6 & & 7478.062 & $130883-20566_{4}^{\circ}$ & 13127.923 & 5 & & 7615.268 & $16783_{4}^{\circ}-24399_{3}$ \\
\hline 13360.349 & 0 & & 7482.788 & $17959_{4}-25442_{3}^{\circ}$ & 13126.337 & 0 & & 7616.188 & $20054_{2}-27670_{3}^{\circ}$ \\
\hline 13355.490 & 0 & & 7485.510 & $21077_{0}^{\circ}-285624$ & 13124.108 & 1 & & 7617.482 & $15863_{2}-23481_{i}^{\circ}$ \\
\hline 13353.446 & 0 & & 7486.656 & $23655^{\circ}-311413$ & 13120.544 & 1 & & 7619.551 & $20054_{2}-27674_{2}^{\circ}$ \\
\hline 13352.939 & 0 & & 7486.940 & $18549_{2}-26036_{3}^{\circ}$ & 13119.476 & 1 & & 7620.171 & $24421_{3}^{\circ}-32041_{2}$ \\
\hline
\end{tabular}


TABLE 4. Classified lines of $\mathrm{Th} \mathrm{I}-$ continued

\begin{tabular}{|c|c|c|c|c|c|c|c|c|c|}
\hline \multirow{2}{*}{$\begin{array}{l}\text { Wavelength } \\
\text { (A) }\end{array}$} & \multicolumn{2}{|c|}{ Intensity } & \multirow{2}{*}{$\begin{array}{l}\text { Wavenumber } \\
\quad\left(\mathrm{cm}^{-1}\right)\end{array}$} & \multirow{2}{*}{ Classification } & \multirow{2}{*}{$\begin{array}{c}\text { Wavelength } \\
\text { (A) }\end{array}$} & \multicolumn{2}{|c|}{ Inten sity } & \multirow{2}{*}{$\begin{array}{c}\text { Wavenumber } \\
\left(\mathrm{cm}^{-1}\right)\end{array}$} & \multirow{2}{*}{ Classification } \\
\hline & Lamp & Spark & & & & Lamp & Spark & & \\
\hline 13116.755 & 1 & & 7621.752 & $24307_{2}^{\circ}-319293$ & 12866.644 & 7 & & 7769.909 & $19227_{6}^{\circ}-269976$ \\
\hline 13111.862 & 0 & & 7624.596 & $22508_{2}^{\circ}-30132_{2}$ & 12861.651 & 4 & & 7772.925 & $16217_{2}^{\circ}-23990_{2}$ \\
\hline 13107.912 & 1 & & 7626.894 & $25336_{6}^{\circ}-329635$ & 12854.102 & 1 & & 7777.490 & $25321_{3}^{\circ}-33099_{3}$ \\
\hline 13103.036 & 0 & & 7629.732 & $13945^{\circ}-21575_{2}$ & 12846.823 & 1 & & 7781.897 & $24769_{3}^{\circ}-325513$ \\
\hline 13102.832 & 1 & & 7629.851 & $21252_{2}^{\circ}-288822$ & 12845.690 & 1 & & 7782.583 & $15970_{3}-23752_{2}^{\circ}$ \\
\hline 13101.858 & 1 & & 7630.418 & $22877_{0}^{\circ}-305085$ & 12841.750 & 2 & & 7784.971 & $195324-27317^{\circ}$ \\
\hline 13079.586 & 1 & & 7643.411 & $19948^{\circ}-275915$ & 12837.894 & 2 & & 7787.309 & $15490 \AA-23277_{5}$ \\
\hline 13070.834 & 6 & & 7648.529 & $10783^{\circ}-184313$ & 12836.536 & 1 & & 7788.133 & $19273_{2}-27061_{2}^{\circ}$ \\
\hline 13069.399 & 3 & & 7649.369 & $13945_{3}^{\circ}-215943$ & 12831.063 & 0 & & 7791.455 & $10783_{2}^{\circ}-18574_{1}$ \\
\hline 13056.097 & 2 & & 7657.162 & $15863_{2}-23521_{3}^{\circ}$ & 12830.547 & 3 & & 7791.768 & $20566_{4}^{\circ}-283583$ \\
\hline 13055.889 & 6 & & 7657.284 & $2869_{3}-10526_{3}^{\circ}$ & 12828.379 & 1 & & 7793.085 & $24202^{\circ}-31995_{4}$ \\
\hline 13049.034 & 2 & & 7661.307 & $20566_{4}^{\circ}-28227_{4}$ & 12827.456 & 0 & & 7793.646 & $22338^{\circ}-301322$ \\
\hline 13048.139 & 1 & & 7661.832 & $23609_{0}^{\circ}-312715$ & 12826.806 & 3 & & 7794.041 & $179594-257530^{\circ}$ \\
\hline 13045.340 & 1 & & 7663.476 & $18699_{2}-26363_{2}^{\circ}$ & 12799.956 & 2 & & 7810.390 & $24202_{4}^{\circ}-32012_{4}$ \\
\hline 13043.407 & 4 & & 7664.612 & $75023-15166_{3}^{\circ}$ & 12782.294 & 1 & & 7821.182 & $13847_{2}-21668_{1}^{\circ}$ \\
\hline 13034.773 & 2 & & 7669.689 & $63622-14032_{2}^{\circ}$ & 12782.059 & 2 & & 7821.326 & $18069_{3}^{\circ}-25890_{2}$ \\
\hline 13031.133 & 3 & & 7671.831 & $23655^{\circ}-313264$ & 12781.578 & 4 & & 7821.620 & $15166_{3}^{\circ}-22988_{2}$ \\
\hline 13030.257 & 0 & & 7672.347 & $223388^{\circ}-300113$ & 12773.189 & 1 & & 7826.757 & $19516_{2}^{\circ}-273433$ \\
\hline 13026.187 & 6 & & 7674.744 & $12847_{3}-20522_{2}^{\circ}$ & 12762.096 & 6 & & 7833.560 & $13088_{3}-20922_{2}^{\circ}$ \\
\hline 13023.092 & 1 & & 7676.568 & $23752_{2}^{\circ}-314291$ & 12750.649 & 0 & & 7840.593 & $195033^{\circ}-273433$ \\
\hline 13010.439 & 1 & & 7684.034 & $21738_{2}^{\circ}-294221$ & 12738.275 & 0 & & 7848.209 & $22163_{4}^{\circ}-30011_{3}$ \\
\hline 13010.154 & 3 & & 7684.202 & $171665-24850_{6}^{\circ}$ & 12733.582 & 3 & & 7851.102 & $23113_{4}^{\circ}-309643$ \\
\hline 13008.742 & 0 & & 7685.036 & $15970_{3}-23655_{4}^{\circ}$ & 12709.820 & 4 & & 7865.780 & $18930_{3}^{\circ}-267963$ \\
\hline 13007.832 & 5 & & 7685.574 & $16346^{\circ}-240324$ & 12709.330 & 5 & & 7866.083 & $15166^{\circ}-23032_{4}$ \\
\hline 12994.532 & 3 & & 7693.440 & $22141 \stackrel{\circ}{-}-298353$ & 12703.962 & 0 & & 7869.407 & $180534^{\circ}-259234$ \\
\hline 12989.585 & 1 & & 7696.370 & $98045-175010^{\circ}$ & 12703.428 & 1 & & 7869.738 & $22141 \stackrel{\circ}{3}-30011_{3}$ \\
\hline 12988.177 & 1 & & 7697.204 & $22855_{3}^{\circ}-305524$ & 12691.421 & 4 & & 7877.183 & $15863_{2}-23741_{i}^{\circ}$ \\
\hline 12986.920 & 4 & & 7697.949 & $7795_{4}^{\circ}-154934$ & 12685.466 & 1 & & 7880.881 & $16783_{4}^{\circ}-246643$ \\
\hline 12983.105 & 0 & & 7700.211 & $11802_{2}-19503_{3}^{\circ}$ & 12684.317 & 0 & & 7881.595 & $63622-14243 i$ \\
\hline 12982.529 & 1 & & 7700.553 & $13945^{\circ}-216454$ & 12683.507 & 1 & & 7882.098 & $23655_{4}^{\circ}-31537_{3}$ \\
\hline 12970.735 & 2 & & 7707.555 & $22141_{3}^{\circ}-29849_{4}$ & 12682.679 & 0 & & 7882.613 & $22669_{3}^{\circ}-305524$ \\
\hline 12966.746 & 0 & & 7709.926 & $23916_{4}^{\circ}-316265$ & 12680.944 & 1 & & 7883.691 & $22248_{2}^{\circ}-30132_{2}$ \\
\hline 12963.697 & 2 & & 7711.739 & $20322{ }^{\circ}-280345$ & 12673.959 & 2 & & 7888.036 & $25180_{7}^{\circ}-330686$ \\
\hline 12959.819 & 7 & & 7714.047 & $11802_{2}-19516_{2}^{\circ}$ & 12670.355 & 1 & & 7890.280 & $13847_{2}-21738_{2}^{\circ}$ \\
\hline 12952.082 & 5 & & 7718.655 & $12847_{3}-20566_{4}^{\circ}$ & 12668.767 & 2 & & 7891.269 & $14206_{4}^{\circ}-220984$ \\
\hline 12949.037 & 1 & & 7720.470 & $165546-24274{ }^{\circ}$ & 12648.388 & 1 & & 7903.983 & $17501 \stackrel{\circ}{\circ}-25405_{4}$ \\
\hline 12948.255 & 0 & & 7720.936 & $24274 \circ-319954$ & 12646.536 & 8 & & 7905.141 & $10526_{3}^{\circ}-18431_{3}$ \\
\hline 12938.933 & 2 & & 7726.499 & $82432-159703$ & 12645.697 & 4 & & 7905.665 & $203220^{\circ}-28227_{4}$ \\
\hline 12936.840 & 1 & & 7727.749 & $195324-27260_{3}^{\circ}$ & 12641.383 & 3 & & 7908.363 & $17398_{3}-25306_{2}^{\circ}$ \\
\hline 12935.233 & 3 & & 7728.709 & $18382 \circ-261111$ & 12636.142 & 5 & & 7911.643 & $180110^{\circ}-259234$ \\
\hline 12933.760 & 1 & & 7729.589 & $20054_{2}-27784_{2}^{\circ}$ & 12632.545 & 3 & & 7913.896 & $2869_{3}-10783_{2}^{\circ}$ \\
\hline 12932.107 & 2 & & 7730.577 & $17959_{4}-25690_{0}^{\circ}$ & 12629.269 & 0 & & 7915.949 & $11601_{1}-19516_{2}^{\circ}$ \\
\hline 12930.425 & 1 & & 7731.583 & $253366^{\circ}-330686$ & 12617.200 & 1 & & 7923.521 & $173983-25321_{3}^{\circ}$ \\
\hline 12925.197 & 1 & & 7734.710 & $19713_{3}-27447_{2}^{\circ}$ & 12611.214 & 0 & & 7927.282 & $242740^{\circ}-322025$ \\
\hline 12923.256 & 0 & & 7735.872 & $24259^{\circ}-31995_{4}$ & 12604.640 & 2 & & 7931.416 & $18431_{3}-263632$ \\
\hline 12920.304 & 0 & & 7737.639 & $18549_{2}-26287_{1}^{\circ}$ & 12587.889 & 4 & & 7941.971 & $20737_{1}^{\circ}-28679_{2}$ \\
\hline 12919.299 & 3 & & 7738.241 & $24274{ }^{\circ}-320124$ & 12583.229 & 1 & & 7944.912 & $21890_{3}^{\circ}-298353$ \\
\hline 12916.981 & 4 & & 7739.630 & $15863_{2}-236032$ & 12580.239 & 1 & & 7946.800 & $15970_{3}-23916_{4}^{\circ}$ \\
\hline 12900.246 & 2 & & 7749.670 & $19817_{1}^{\circ}-27566_{2}$ & 12580.100 & 1 & & 7946.888 & $21902_{4}^{\circ}-29849_{4}$ \\
\hline 12898.865 & 4 & & 7750.500 & $205222-282732$ & 12571.690 & 2 & & 7952.204 & $18699_{2}-26651_{2}^{\circ}$ \\
\hline 12888.182 & 2 & & 7756.924 & $19039_{2}^{\circ}-267963$ & 12563.833 & 0 & & 7957.177 & $17398_{3}-25355_{4}^{\circ}$ \\
\hline 12881.709 & 0 & & 7760.822 & $21890_{3}^{\circ}-29650_{2}$ & 12562.651 & 2 & & 7957.926 & $197133-27670_{3}^{\circ}$ \\
\hline 12874.595 & 1 & & 7765.110 & $17073_{1}-24838_{1}^{\circ}$ & 12561.555 & 3 & & 7958.620 & $18549_{2}-26508_{3}^{\circ}$ \\
\hline 12873.533 & 1 & & 7765.751 & $202143 \stackrel{\circ}{-279803}$ & 12561.165 & 1 & & 7958.867 & $142045-22163^{\circ}$ \\
\hline 12872.702 & 3 & & 7766.252 & $10783_{2}^{\circ}-18549_{2}$ & 12558.855 & 1 & & 7960.331 & $20922_{2}^{\circ}-28882_{2}$ \\
\hline
\end{tabular}


TABLE 4. Classified lines of Th $\mathrm{I}$-continued

\begin{tabular}{|c|c|c|c|c|c|c|c|c|c|}
\hline \multirow{2}{*}{$\begin{array}{c}\text { Wavelength } \\
(\AA)\end{array}$} & \multicolumn{2}{|c|}{ Intensity } & \multirow{2}{*}{$\begin{array}{l}\text { Wavenumber } \\
\qquad\left(\mathrm{cm}^{-1}\right)\end{array}$} & \multirow{2}{*}{ Classification } & \multirow{2}{*}{$\begin{array}{c}\text { Wavelength } \\
\text { (§) }\end{array}$} & \multicolumn{2}{|c|}{ Inten sity } & \multirow{2}{*}{$\begin{array}{l}\text { Wavenumber } \\
\qquad\left(\mathrm{cm}^{-1}\right)\end{array}$} & \multirow{2}{*}{ Classification } \\
\hline & Lamp & Spark & & & & Lamp & Spark & & \\
\hline 12553.032 & 5 & & 7964.024 & $19227_{6}^{\circ}-271915$ & 12312.736 & 2 & & 8119.450 & $23655_{4}^{\circ}-317743$ \\
\hline 12552.801 & 4 & & 7964.170 & $233066^{\circ}-312715$ & 12310.069 & 3 & & 8121.209 & $21890 \stackrel{3}{3}-30011_{3}$ \\
\hline 12540.971 & 2 & & 7971.683 & $23655_{4}^{\circ}-31626_{5}$ & 12295.924 & 0 & & 8130.551 & $24421_{3}^{\circ}-32551_{3}$ \\
\hline 12522.256 & 1 & & 7983.597 & $8800_{4}-16783_{4}^{\circ}$ & 12294.985 & 1 & & 8131.172 & $16783_{4}^{\circ}-24915_{3}$ \\
\hline 12518.195 & 3 & & 7986.187 & $18809_{4}^{\circ}-267963$ & 12283.851 & 1 & & 8138.542 & $19532_{4}-27670_{3}^{\circ}$ \\
\hline 12508.396 & 2 & & 7992.443 & $19503_{3}^{\circ}-27495_{4}$ & 12283.851 & 1 & & 8138.542 & $23655_{4}^{\circ}-317934$ \\
\hline 12502.774 & 2 & & 7996.037 & $20566_{4}^{\circ}-285624$ & 12276.430 & 0 & & 8143.462 & $20214_{3}^{\circ}-283583$ \\
\hline 12488.669 & 1 & & 8005.068 & $19039_{2}^{\circ}-27044_{3}$ & 12273.859 & 2 & & 8145.168 & $20737_{1}^{\circ}-28882_{2}$ \\
\hline 12477.297 & 7 & & 8012.364 & $3865_{1}-11877^{\circ}$ & 12271.398 & 2 & & 8146.801 & $165546-24701{ }^{\circ}$ \\
\hline 12476.305 & 3 & & 8013.001 & $20214_{3}^{\circ}-28227_{4}$ & 12263.449 & 1 & & 8152.082 & $23113 \stackrel{\circ}{4}-31265_{3}$ \\
\hline 12474.364 & 2 & & 8014.248 & $11802_{2}-19817_{1}^{\circ}$ & 12262.250 & 1 & & 8152.879 & $13945_{3}^{\circ}-22098_{4}$ \\
\hline 12471.346 & 1 & & 8016.187 & $23521^{\circ}-31537_{3}$ & 12261.910 & 2 & & 8153.105 & $22399^{\circ}-305524$ \\
\hline 12469.228 & 4 & & 8017.549 & $10414_{4}^{\circ}-184313$ & 12256.743 & 2 & & 8156.542 & $20522_{2}^{\circ}-28679_{2}$ \\
\hline 12466.651 & 0 & & 8019.206 & $24274{ }^{\circ}-322935$ & 12249.188 & 4 & & 8161.573 & $18809_{4}^{\circ}-26971_{4}$ \\
\hline 12465.053 & 3 & & 8020.234 & $20322{ }^{\circ}-283425$ & 12245.483 & 3 & & 8164.042 & $130883-21252_{2}^{\circ}$ \\
\hline 12460.967 & 4 & & 8022.864 & $10526_{3}^{\circ}-18549_{2}$ & 12242.874 & 0 & & 8165.782 & $21252_{2}^{\circ}-29418_{2}$ \\
\hline 12455.465 & 0 & & 8026.408 & $24769_{3}^{\circ}-327963$ & 12237.332 & 1 & & 8169.480 & $21252_{2}^{\circ}-29422_{1}$ \\
\hline 12453.262 & 3 & & 8027.828 & $15493_{4}-23521_{3}^{\circ}$ & 12236.549 & 2 & & 8170.003 & $142260-22396^{\circ}$ \\
\hline 12447.488 & 5 & & 8031.552 & $11241_{3}^{\circ}-19273_{2}$ & 12234.949 & 1 & & 8171.071 & $23603_{2}^{\circ}-317743$ \\
\hline 12446.347 & 1 & & 8032.288 & $19948_{4}^{\circ}-27980_{3}$ & 12233.199 & 5 & & 8172.240 & $14465_{2}^{\circ}-226373$ \\
\hline 12445.393 & 3 & & 8032.904 & $15736_{1}^{\circ}-23769_{1}$ & 12231.942 & 8 & & 8173.080 & $10526_{3}^{\circ}-18699_{2}$ \\
\hline 12442.013 & 5 & & 8035.086 & $15166_{3}^{\circ}-23201_{3}$ & 12229.332 & 1 & & 8174.824 & $7795_{4}^{\circ}-15970_{3}$ \\
\hline 12440.650 & 2 & & 8035.966 & $24701{ }^{\circ}-327375$ & 12226.604 & 1 & & 8176.648 & $17398_{3}-25575_{4}^{\circ}$ \\
\hline 12437.583 & 0 & & 8037.948 & $22248_{2}^{\circ}-30286_{1}$ & 12226.177 & 4 & & 8176.934 & $11877_{1}^{\circ}-20054_{2}$ \\
\hline 12430.756 & 1 & & 8042.362 & $13847_{2}-21890_{3}^{\circ}$ & 12223.788 & 2 & & 8178.532 & $18699_{2}-26878_{3}^{\circ}$ \\
\hline 12429.285 & 1 & & 8043.314 & $17847_{2}^{\circ}-25890_{2}$ & 12219.156 & 0 & & 8181.632 & $16217_{2}^{\circ}-24399_{3}$ \\
\hline 12422.300 & 4 & & 8047.837 & $19986^{\circ}-280345$ & 12216.424 & 1 & & 8183.462 & $24274 \stackrel{\circ}{-}-324584$ \\
\hline 12419.161 & 2 & & 8049.871 & $19516_{2}^{\circ}-27566_{2}$ & 12211.865 & 2 & & 8186.517 & $18069_{3}^{\circ}-26255_{4}$ \\
\hline 12415.160 & 5 & & 8052.465 & $16346_{4}^{\circ}-24399_{3}$ & 12206.894 & 7 & & 8189.851 & $3687_{2}-11877_{1}^{\circ}$ \\
\hline 12406.195 & 1 & & 8058.284 & $20214_{3}^{\circ}-282732$ & 12188.866 & 1 & & 8201.964 & $18053_{4}^{\circ}-26255_{4}$ \\
\hline 12397.849 & 4 & & 8063.709 & $19503_{3}^{\circ}-27566_{2}$ & 12182.019 & 3 & & 8206.574 & $98045-18011^{\circ}$ \\
\hline 12396.828 & 0 & & 8064.373 & $21077 \circ-291415$ & 12178.456 & 2 & & 8208.975 & $24084_{6}^{\circ}-322935$ \\
\hline 12381.813 & 3 & & 8074.152 & $12847_{3}-20922_{2}^{\circ}$ & 12171.815 & 4 & & 8213.454 & $4961_{4}-13175_{4}^{\circ}$ \\
\hline 12378.451 & 1 & & 8076.345 & $18431_{3}-26508_{3}^{\circ}$ & 12167.821 & 3 & & 8216.150 & $11601_{1}-19817_{1}^{\circ}$ \\
\hline 12378.290 & 2 & & 8076.450 & $17959_{4}-26036_{3}^{\circ}$ & 12166.851 & 0 & & 8216.805 & $21539 \stackrel{\circ}{-}-297564$ \\
\hline 12377.115 & 0 & & 8077.217 & $18574_{1}-26651_{2}^{\circ}$ & 12161.908 & 0 & & 8220.144 & $18431_{3}-26651_{2}^{\circ}$ \\
\hline 12372.007 & 2 & & 8080.552 & $24701{ }^{\circ}-32781_{4}$ & 12143.705 & 0 & & 8232.466 & $15970_{3}-24202_{4}^{\circ}$ \\
\hline 12364.267 & 1 & & 8085.610 & $19948{ }^{\circ}-280345$ & 12140.955 & 3 & & 8234.331 & $18809_{4}^{\circ}-27044_{3}$ \\
\hline 12359.625 & 2 & & 8088.647 & $17959_{4}-26048_{4}^{\circ}$ & 12140.751 & 1 & & 8234.469 & $24561_{3}^{\circ}-327963$ \\
\hline 12355.491 & 0 & & 8091.353 & $24202{ }^{\circ}-322935$ & 12139.017 & 1 & & 8235.645 & $8111_{4}-16346_{4}^{\circ}$ \\
\hline 12349.478 & 2 & & 8095.293 & $19516_{2}^{\circ}-276123$ & 12132.020 & 3 & & 8240.395 & $20322{ }^{\circ}-285624$ \\
\hline 12348.314 & 0 & & 8096.056 & $23916_{4}^{\circ}-320124$ & 12129.427 & 5 & & 8242.157 & $13297_{4}-21539_{4}^{\circ}$ \\
\hline 12346.884 & 0 & & 8096.994 & $21738_{2}^{\circ}-29835_{3}$ & 12128.913 & 1 & & 8242.506 & $21890_{3}^{\circ}-30132_{2}$ \\
\hline 12338.612 & 0 & & 8102.422 & $18549_{2}-26651_{2}^{\circ}$ & 12127.302 & 8 & & 8243.601 & $0_{2}-82432$ \\
\hline 12337.998 & 7 & & 8102.825 & $63622-14465_{2}^{\circ}$ & 12126.918 & 1 & & 8243.862 & $24307_{2}^{\circ}-32551_{3}$ \\
\hline 12330.800 & 1 & & 8107.555 & $20423 i-28531_{2}$ & 12126.419 & 5 & & 8244.201 & $18011{ }^{\circ}-26255_{4}$ \\
\hline 12329.207 & 2 & & 8108.603 & $22399^{\circ}-305085$ & 12119.644 & 3 & & 8248.810 & $98045-18053{ }^{\circ}$ \\
\hline 12328.492 & 1 & & 8109.073 & $21902^{\circ}-30011_{3}$ & 12117.950 & 0 & & 8249.963 & $22508_{2}^{\circ}-30758_{2}$ \\
\hline 12328.408 & 0 & & 8109.128 & $195033-276123$ & 12113.062 & 0 & & 8253.292 & $21165_{3}^{\circ}-29418_{2}$ \\
\hline 12323.962 & 0 & & 8112.054 & $219024-30014_{4}$ & 12109.655 & 0 & & 8255.614 & $24202_{4}^{\circ}-324584$ \\
\hline 12322.400 & 1 & & 8113.082 & $24850 \%-329635$ & 12109.434 & 3 & & 8255.765 & $20423 i-28679_{2}$ \\
\hline 12321.120 & 3 & & 8113.925 & $18930_{3}^{\circ}-270443$ & 12109.313 & 1 & & 8255.847 & $23015 \circ-312715$ \\
\hline 12318.050 & 3 & & 8115.947 & $15493_{4}-23609_{0}^{\circ}$ & 12099.785 & 2 & & 8262.348 & $24701 \stackrel{\circ}{-}-329635$ \\
\hline 12316.913 & 2 & & 8116.696 & $75023-15618_{3}^{\circ}$ & 12083.777 & 0 & & 8273.294 & $21738_{2}^{\circ}-30011_{3}$ \\
\hline 12316.372 & 1 & & 8117.053 & $24084_{6}^{\circ}-322025$ & 12082.221 & 2 & & 8274.359 & $23655_{4}^{\circ}-31929_{3}$ \\
\hline
\end{tabular}


TABLE 4. Classified lines of $\mathrm{Th} \mathrm{I}-$ continued

\begin{tabular}{|c|c|c|c|c|c|c|c|c|c|}
\hline \multirow{2}{*}{$\begin{array}{l}\text { Wavelength } \\
\text { (§) }\end{array}$} & \multicolumn{2}{|c|}{ Intensity } & \multirow{2}{*}{$\begin{array}{l}\text { Wavenumber } \\
\left(\mathrm{cm}^{-1}\right)\end{array}$} & \multirow{2}{*}{ Classification } & \multirow{2}{*}{ 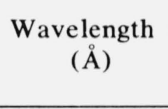 } & \multicolumn{2}{|c|}{ Intensity } & \multirow{2}{*}{$\begin{array}{c}\text { Wavenumber } \\
\left(\mathrm{cm}^{-1}\right)\end{array}$} & \multirow{2}{*}{ Classification } \\
\hline & Lamp & Spark & & & & Lamp & Spark & & \\
\hline 12075.537 & 1 & & 8278.939 & $20566_{4}^{\circ}-28845_{4}$ & 11829.730 & 4 & & 8450.965 & $15970_{3}-24421_{3}^{\circ}$ \\
\hline 12074.667 & 2 & & 8279.536 & $19948_{4}^{\circ}-28227_{4}$ & 11829.640 & 5 & & 8451.029 & $13088_{3}-21539_{4}^{\circ}$ \\
\hline 12066.559 & 0 & & 8285.099 & $23916_{4}^{\circ}-322025$ & 11827.583 & 4 & & 8452.499 & $17073_{1}-25526^{\circ}$ \\
\hline 12059.895 & 0 & & 8289.677 & $15970_{3}-24259_{4}^{\circ}$ & 11824.901 & 1 & & 8454.416 & $16217_{2}^{\circ}-24671_{2}$ \\
\hline 12058.422 & 0 & & 8290.690 & $11241_{3}^{\circ}-195324$ & 11822.640 & 4 & & 8456.033 & $19817^{\circ}-28273_{2}$ \\
\hline 12055.291 & 0 & & 8292.843 & $21668_{1}^{\circ}-29961_{1}$ & 11821.839 & 1 & & 8456.606 & $22508_{2}^{\circ}-30964_{3}$ \\
\hline 12051.267 & 1 & & 8295.612 & $22248^{\circ}-305442$ & 11821.500 & 0 & & 8456.848 & $7280_{2}-15736 i$ \\
\hline 12050.486 & 0 & & 8296.150 & $18699_{2}-26995_{3}^{\circ}$ & 11818.546 & 1 & & 8458.962 & $20423_{i}-28882_{2}$ \\
\hline 12038.242 & 1 & & 8304.588 & $19039_{2}^{\circ}-273433$ & 11811.926 & 2 & & 8463.703 & $19516_{2}^{\circ}-27980_{3}$ \\
\hline 12037.639 & 4 & & 8305.004 & $173983-25703^{\circ}$ & 11811.055 & 0 & & 8464.327 & $20214_{3}^{\circ}-28679_{2}$ \\
\hline 12020.095 & 1 & & 8317.125 & $128473-21165_{3}^{\circ}$ & 11804.617 & 6 & & 8468.943 & $5563_{1}-14032_{2}^{\circ}$ \\
\hline 12018.718 & 7 & & 8318.078 & $16346_{4}^{\circ}-246643$ & 11804.116 & 3 & & 8469.303 & $21903 \stackrel{\circ}{7}-303726$ \\
\hline 12018.054 & 4 & & 8318.538 & $15863_{2}-24182_{2}^{\circ}$ & 11802.102 & 1 & & 8470.748 & $13175_{4}^{\circ}-21645_{4}$ \\
\hline 12015.950 & 4 & & 8319.994 & $23306_{6}^{\circ}-31626_{5}$ & 11801.327 & 0 & & 8471.304 & $11241_{3}^{\circ}-197133$ \\
\hline 12015.478 & 1 & & 8320.321 & $195324-27852^{\circ}$ & 11793.069 & 1 & & 8477.236 & $24259_{4}^{\circ}-32737_{5}$ \\
\hline 12007.313 & 0 & & 8325.979 & $23603_{2}^{\circ}-31929_{3}$ & 11792.645 & 1 & & 8477.541 & $19503_{3}^{\circ}-27980_{3}$ \\
\hline 12005.953 & 1 & & 8326.922 & $18053_{4}^{\circ}-263805$ & 11790.382 & 1 & & 8479.168 & $17411_{3}^{\circ}-25890_{2}$ \\
\hline 11986.392 & 0 & & 8340.511 & $23655_{4}^{\circ}-31995_{4}$ & 11779.528 & 2 & & 8486.981 & $163510-24838^{\circ}$ \\
\hline 11980.135 & 1 & & 8344.867 & $22163_{4}^{\circ}-305085$ & 11775.163 & 4 & & 8490.127 & $10783_{2}^{\circ}-19273_{2}$ \\
\hline 11976.025 & 1 & & 8347.731 & $20214^{\circ}-285624$ & 11772.698 & 1 & & 8491.905 & $23521 \stackrel{\circ}{3}-320124$ \\
\hline 11974.134 & 1 & & 8349.049 & $24202_{4}^{\circ}-325513$ & 11761.537 & 1 & & 8499.963 & $20922_{2}^{\circ}-29422_{1}$ \\
\hline 11961.578 & 1 & & 8357.813 & $23655_{4}^{\circ}-320124$ & 11746.174 & 1 & & 8511.080 & $19273_{2}-27784_{2}^{\circ}$ \\
\hline 11956.759 & 0 & & 8361.182 & $22396^{\circ}-30758_{2}$ & 11729.339 & 1 & & 8523.296 & $14465_{2}^{\circ}-22988_{2}$ \\
\hline 11955.885 & 0 & & 8361.793 & $18699_{2}-27061_{2}^{\circ}$ & 11729.339 & 1 & & 8523.296 & $20322{ }^{\circ}-28845_{4}$ \\
\hline 11952.063 & 4 & & 8364.467 & $19227^{\circ}-275915$ & 11723.279 & 2 & & 8527.702 & $19039_{2}^{\circ}-27566_{2}$ \\
\hline 11946.618 & 1 & & 8368.279 & $20566_{4}^{\circ}-289343$ & 11722.487 & 1 & & 8528.278 & $16351_{0}-24880^{\circ}$ \\
\hline 11945.362 & 6 & & 8369.159 & $18011{ }^{\circ}-26380_{5}$ & 11712.220 & 3 & & 8535.754 & $17354_{1}^{\circ}-25890_{2}$ \\
\hline 11942.130 & 5 & & 8371.424 & $15618^{\circ}-23990_{2}$ & 11709.471 & 0 & & 8537.758 & $24561_{3}^{\circ}-33099_{3}$ \\
\hline 11940.638 & 7 & & 8372.470 & $2869_{3}-11241_{3}^{\circ}$ & 11704.645 & 1 & & 8541.278 & $23916_{4}^{\circ}-32458_{4}$ \\
\hline 11927.809 & 0 & & 8381.475 & $18809_{4}^{\circ}-271915$ & 11703.457 & 7 & & 8542.145 & $15490 \AA-240324$ \\
\hline 11916.586 & 3 & & 8389.369 & $22163_{4}^{\circ}-305524$ & 11698.831 & 3 & & 8545.523 & $13962_{1}-22508_{2}^{\circ}$ \\
\hline 11910.840 & 0 & & 8393.416 & $22877 \circ-312715$ & 11695.263 & 2 & & 8548.130 & $17959_{4}-26508_{3}^{\circ}$ \\
\hline 11909.862 & 1 & & 8394.105 & $19948{ }^{\circ}-283425$ & 11694.285 & 3 & & 8548.845 & $21738_{2}^{\circ}-30286_{1}$ \\
\hline 11904.795 & 2 & & 8397.678 & $19273_{2}-27670_{3}^{\circ}$ & 11694.000 & 2 & & 8549.053 & $13847_{2}-22396_{1}^{\circ}$ \\
\hline 11899.837 & 4 & & 8401.177 & $13847_{2}-22248_{2}^{\circ}$ & 11690.334 & 0 & & 8551.734 & $22877_{i}^{\circ}-314291$ \\
\hline 11894.942 & 3 & & 8404.634 & $12847_{3}-21252_{2}^{\circ}$ & 11682.909 & 1 & & 8557.169 & $158632-24421^{\circ}$ \\
\hline 11887.357 & 1 & & 8409.997 & $19948_{4}^{\circ}-283583$ & 11678.305 & 1 & & 8560.543 & $18699_{2}-27260_{3}^{\circ}$ \\
\hline 11886.085 & 2 & & 8410.897 & $22141_{3}^{\circ}-305524$ & 11673.465 & 1 & & 8564.092 & $18431_{3}-26995^{\circ}$ \\
\hline 11884.530 & 5 & & 8411.997 & $11802_{2}-20214_{3}^{\circ}$ & 11667.638 & 5 & & 8568.369 & $16346_{4}^{\circ}-24915_{3}$ \\
\hline 11882.776 & 2 & & 8413.239 & $15618^{\circ}-240324$ & 11661.167 & 3 & & 8573.124 & $19039_{2}^{\circ}-27612_{3}$ \\
\hline 11878.611 & 2 & & 8416.189 & $195324-27948 \stackrel{\circ}{4}$ & 11657.259 & 1 & & 8575.998 & $22669_{3}^{\circ}-31245_{2}$ \\
\hline 11874.629 & 2 & & 8419.011 & $22338^{\circ}-30758_{2}$ & 11653.134 & 1 & & 8579.034 & $24202_{4}^{\circ}-32781_{4}$ \\
\hline 11873.851 & 2 & & 8419.563 & $13175_{4}^{\circ}-215943$ & 11648.509 & 1 & & 8582.440 & $21252_{2}^{\circ}-29835_{3}$ \\
\hline 11870.629 & 1 & & 8421.848 & $17501 \stackrel{\circ}{-}-259234$ & 11641.195 & 1 & & 8587.832 & $171665-257530^{\circ}$ \\
\hline 11868.045 & 0 & & 8423.682 & $154934-23916_{4}^{\circ}$ & 11636.590 & 2 & & 8591.231 & $18930_{3}^{\circ}-27521_{4}$ \\
\hline 11864.247 & 7 & & 8426.378 & $3687_{2}-12114_{2}^{\circ}$ & 11636.496 & 1 & & 8591.300 & $21165_{3}^{\circ}-29756_{4}$ \\
\hline 11858.382 & 4 & & 8430.546 & $14206^{\circ}-22637_{3}$ & 11636.155 & 3 & & 8591.552 & $159703-24561_{3}^{\circ}$ \\
\hline 11853.098 & 1 & & 8434.304 & $13962_{1}-22396 i$ & 11634.601 & 4 & & 8592.699 & $13297_{4}-21890^{\circ}$ \\
\hline 11848.313 & 1 & & 8437.710 & $236032-320412$ & 11630.479 & 0 & & 8595.745 & $22669_{3}^{\circ}-31265_{3}$ \\
\hline 11840.529 & 0 & & 8443.257 & $23093_{2}^{\circ}-31537_{3}$ & 11618.190 & 3 & & 8604.837 & $132974-21902^{\circ}$ \\
\hline 11839.689 & 1 & & 8443.856 & $15863_{2}-24307_{2}^{\circ}$ & 11617.462 & 5 & & 8605.376 & $14032_{2}^{\circ}-22637_{3}$ \\
\hline 11837.186 & 2 & & 8445.642 & $19588 \circ-280345$ & 11615.211 & 1 & & 8607.044 & $210777^{\circ}-29684_{5}$ \\
\hline 11836.168 & 0 & & 8446.368 & $18549_{2}-26995_{3}^{\circ}$ & 11609.914 & 2 & & 8610.971 & $8800_{4}-17411_{3}^{\circ}$ \\
\hline 11834.938 & 1 & & 8447.246 & $16217_{2}^{\circ}-24664_{3}$ & 11606.246 & 1 & & 8613.692 & $24182_{2}^{\circ}-327963$ \\
\hline 11831.941 & 0 & & 8449.386 & $22877^{\circ}-313264$ & 11605.473 & 3 & & 8614.266 & $19948^{\circ}-28562_{4}$ \\
\hline
\end{tabular}


TABLE 4. Classified lines of $\mathrm{Th} \mathrm{I}$-continued

\begin{tabular}{|c|c|c|c|c|c|c|c|c|c|}
\hline \multirow{2}{*}{$\begin{array}{l}\text { Wavelength } \\
\text { (A) }\end{array}$} & \multicolumn{2}{|c|}{ Intensity } & \multirow{2}{*}{$\begin{array}{l}\text { Wavenumber } \\
\left(\mathrm{cm}^{-1}\right)\end{array}$} & \multirow{2}{*}{ Classification } & \multirow{2}{*}{$\begin{array}{c}\text { Wavelength } \\
\text { (A) }\end{array}$} & \multicolumn{2}{|c|}{ Inten sity } & \multirow{2}{*}{$\begin{array}{l}\text { Wavenumber } \\
\left(\mathrm{cm}^{-1}\right)\end{array}$} & \multirow{2}{*}{ Classification } \\
\hline & Lamp & Spark & & & & Lamp & Spark & & \\
\hline 11602.596 & 0 & & 8616.402 & $22141_{3}^{\circ}-307582$ & 11411.321 & 1 & & 8760.829 & $24202 \stackrel{\circ}{4}-329635$ \\
\hline 11600.761 & 0 & & 8617.765 & $18699_{2}-27317_{3}^{\circ}$ & 11402.009 & 0 & & 8767.984 & $18549_{2}-27317_{3}^{\circ}$ \\
\hline 11600.523 & 0 & & 8617.942 & $21668^{\circ}-30286_{1}$ & 11386.235 & 5 & & 8780.130 & $15618^{\circ}-24399_{3}$ \\
\hline 11597.002 & 1 & & 8620.558 & $118022-20423^{\circ}$ & 11384.471 & 3 & & 8781.491 & $154934-242740$ \\
\hline 11595.985 & 6 & & 8621.314 & $16783_{4}^{\circ}-254054$ & 11383.920 & 3 & & 8781.916 & $18809_{4}^{\circ}-27591_{5}$ \\
\hline 11589.953 & 4 & & 8625.801 & $165546-25180_{7}^{\circ}$ & 11371.299 & 1 & & 8791.663 & $24307_{2}^{\circ}-33099_{3}$ \\
\hline 11584.866 & 0 & & 8629.589 & $17073_{1}-25703_{2}^{\circ}$ & 11369.091 & 2 & & 8793.370 & $24274^{\circ}-330686$ \\
\hline 11584.675 & 1 & & 8629.731 & $18431_{3}-27061_{2}^{\circ}$ & 11366.315 & 2 & & 8795.518 & $14481{ }^{\circ}-232775$ \\
\hline 11583.465 & 0 & & 8630.633 & $20214_{3}^{\circ}-288454$ & 11358.564 & 1 & & 8801.520 & $22163_{4}^{\circ}-309643$ \\
\hline 11577.991 & 1 & & 8634.713 & $23916_{4}^{\circ}-325513$ & 11358.499 & 3 & & 8801.570 & $13088_{3}-21890_{3}^{\circ}$ \\
\hline 11575.518 & 4 & & 8636.558 & $18930_{3}^{\circ}-27566_{2}$ & 11357.450 & 0 & & 8802.383 & $18809_{4}^{\circ}-27612_{3}$ \\
\hline 11573.652 & 1 & & 8637.950 & $17398_{3}-26036_{3}^{\circ}$ & 11356.910 & 0 & & 8802.802 & $22338_{3}^{\circ}-31141_{3}$ \\
\hline 11571.485 & 5 & & 8639.568 & $19588^{\circ}-282274$ & 11356.608 & 1 & & 8803.036 & $23655_{4}^{\circ}-324584$ \\
\hline 11558.214 & 3 & & 8649.488 & $13088_{3}-21738_{2}^{\circ}$ & 11354.715 & 8 & & 8804.503 & $6362_{2}-15166_{3}^{\circ}$ \\
\hline 11557.337 & 3 & & 8650.144 & $17398_{3}-26048{ }^{\circ}$ & 11352.128 & 2 & & 8806.510 & $21738_{2}^{\circ}-30544_{2}$ \\
\hline 11557.225 & 0 & & 8650.228 & $21902_{4}^{\circ}-305524$ & 11351.924 & 6 & & 8806.668 & $19227^{\circ}-280345$ \\
\hline 11554.767 & 0 & & 8652.068 & $24084_{6}^{\circ}-327375$ & 11346.494 & 1 & & 8810.883 & $142045-23015^{\circ}$ \\
\hline 11551.616 & 0 & & 8654.428 & $21890 \stackrel{\circ}{-}-30544_{2}$ & 11343.709 & 4 & & 8813.046 & $11241_{3}^{\circ}-20054_{2}$ \\
\hline 11543.824 & 2 & & 8660.270 & $13847_{2}-22508_{2}^{\circ}$ & 11342.857 & 3 & & 8813.708 & $13088_{3}-21902_{4}^{\circ}$ \\
\hline 11541.028 & 0 & & 8662.368 & $21890_{3}^{\circ}-305524$ & 11335.713 & 0 & & 8819.262 & $20322 \circ-291415$ \\
\hline 11536.071 & 2 & & 8666.090 & $17224_{2}^{\circ}-25890_{2}$ & 11332.045 & 4 & & 8822.117 & $13847_{2}-22669_{3}^{\circ}$ \\
\hline 11530.938 & 3 & & 8669.948 & $21165_{3}^{\circ}-29835_{3}$ & 11331.384 & 1 & & 8822.632 & $142260-23049^{\circ}$ \\
\hline 11526.460 & 4 & & 8673.316 & $14204_{5}-22877_{0}^{\circ}$ & 11326.973 & 3 & & 8826.067 & $14206_{4}^{\circ}-230324$ \\
\hline 11517.393 & 1 & & 8680.144 & $23113^{\circ}-317934$ & 11323.876 & 0 & & 8828.481 & $18431_{3}-27260_{3}^{\circ}$ \\
\hline 11516.456 & 5 & & 8680.850 & $55631-142431$ & 11321.659 & 2 & & 8830.210 & $8243_{2}^{\circ}-170731$ \\
\hline 11514.959 & 4 & & 8681.979 & $18930_{3}^{\circ}-276123$ & 11321.249 & 2 & & 8830.530 & $17959_{4}-26790_{4}^{\circ}$ \\
\hline 11512.063 & 3 & & 8684.163 & $5563_{1}-14247^{\circ}$ & 11307.314 & 3 & & 8841.412 & $19516_{2}^{\circ}-28358_{3}$ \\
\hline 11511.566 & 6 & & 8684.538 & $20867_{7}^{\circ}-295526$ & 11303.787 & 5 & & 8844.171 & $13297_{4}-22141_{3}^{\circ}$ \\
\hline 11511.286 & 1 & & 8684.749 & $23609^{\circ}-322935$ & 11303.544 & 4 & & 8844.361 & $7502_{3}-16346_{4}^{\circ}$ \\
\hline 11510.028 & 3 & & 8685.698 & $18809_{4}^{\circ}-27495_{4}$ & 11303.544 & 4 & & 8844.361 & $17411_{3}^{\circ}-26255_{4}$ \\
\hline 11506.079 & 1 & & 8688.679 & $24274{ }^{\circ}-329635$ & 11301.136 & 1 & & 8846.246 & $21165_{3}^{\circ}-30011_{3}$ \\
\hline 11502.185 & 3 & & 8691.621 & $12847_{3}-21539_{4}^{\circ}$ & 11297.608 & 1 & & 8849.008 & $23609_{0}^{\circ}-32458_{4}$ \\
\hline 11501.478 & 4 & & 8692.155 & $13945_{3}^{\circ}-22637_{3}$ & 11289.645 & 2 & & 8855.250 & $19503_{3}^{\circ}-283583$ \\
\hline 11494.068 & 2 & & 8697.759 & $15863_{2}-24561_{3}^{\circ}$ & 11277.612 & 0 & & 8864.698 & $23916^{\circ}-32781_{4}$ \\
\hline 11489.885 & 5 & & 8700.925 & $88004-17501{ }^{\circ}$ & 11276.818 & 4 & & 8865.322 & $15166_{3}^{\circ}-240324$ \\
\hline 11478.781 & 1 & & 8709.342 & $154934-24202_{4}^{\circ}$ & 11276.338 & 2 & & 8865.700 & $132974-22163 \stackrel{\circ}{\circ}$ \\
\hline 11476.910 & 0 & & 8710.762 & $18549_{2}-27260_{3}^{\circ}$ & 11273.574 & 4 & & 8867.873 & $21890_{3}^{\circ}-30758_{2}$ \\
\hline 11476.074 & 1 & & 8711.396 & $171665-25877^{\circ}$ & 11260.143 & 1 & & 8878.451 & $240846-329635$ \\
\hline 11475.757 & 3 & & 8711.637 & $18809_{4}^{\circ}-27521_{4}$ & 11258.986 & 4 & & 8879.363 & $17501 \stackrel{\circ}{\circ}-263805$ \\
\hline 11472.823 & 2 & & 8713.865 & $19817_{1}^{\circ}-28531_{2}$ & 11258.130 & 3 & & 8880.038 & $21252_{2}^{\circ}-301322$ \\
\hline 11471.073 & 0 & & 8715.194 & $75023-16217_{2}^{\circ}$ & 11255.513 & 0 & & 8882.103 & $23113_{4}^{\circ}-319954$ \\
\hline 11465.039 & 5 & & 8719.781 & $118022-20522_{2}^{\circ}$ & 11255.092 & 3 & & 8882.435 & $17166_{5}-26048{ }^{\circ}$ \\
\hline 11464.786 & 2 & & 8719.973 & $20214_{3}^{\circ}-28934_{3}$ & 11249.018 & 0 & & 8887.231 & $17224_{2}^{\circ}-26111_{1}$ \\
\hline 11458.461 & 1 & & 8724.787 & $19503_{3}^{\circ}-282274$ & 11246.685 & 1 & & 8889.075 & $20214_{3}^{\circ}-29104_{4}$ \\
\hline 11455.545 & $1 d$ & & 8727.008 & $18069_{3}^{\circ}-26796_{3}$ & 11245.412 & 2 & & 8890.081 & $128473-21738_{2}^{\circ}$ \\
\hline 11444.419 & 2 & & 8735.492 & $17073_{1}-25809_{1}^{\circ}$ & 11238.982 & 2 & & 8895.167 & $23306_{6}^{\circ}-322025$ \\
\hline 11442.751 & 1 & & 8736.765 & $14465_{2}^{\circ}-23201_{3}$ & 11238.344 & 0 & & 8895.672 & $20522_{2}^{\circ}-29418_{2}$ \\
\hline 11432.593 & 4 & & 8744.528 & $14243^{\circ}-22988_{2}$ & 11236.858 & 1 & & 8896.849 & $24202_{4}^{\circ}-33099_{3}$ \\
\hline 11429.704 & 6 & & 8746.738 & $10526_{3}^{\circ}-19273_{2}$ & 11233.671 & 2 & & 8899.373 & $20522_{2}^{\circ}-294221$ \\
\hline 11427.901 & 1 & & 8748.118 & $18699_{2}-27447_{2}^{\circ}$ & 11230.255 & 9 & & 8902.080 & $5563_{1}-14465_{2}^{\circ}$ \\
\hline 11426.433 & 0 & & 8749.242 & $22877^{\circ}-316265$ & 11225.532 & 3 & & 8905.825 & $15863_{2}-24769_{3}^{\circ}$ \\
\hline 11420.044 & 1 & & 8754.137 & $19588 \AA-283425$ & 11221.176 & 1 & & 8909.282 & $142045-23113{ }^{\circ}$ \\
\hline 11419.693 & 1 & & 8754.406 & $175018-26255_{4}$ & 11215.651 & 2 & & 8913.671 & $20737_{1}^{\circ}-29650_{2}$ \\
\hline 11417.309 & 3 & & 8756.234 & $19516_{2}^{\circ}-28273_{2}$ & 11212.583 & 1 & & 8916.110 & $22877 \stackrel{\circ}{-317934}$ \\
\hline 11415.542 & 1 & & 8757.589 & $22508_{2}^{\circ}-31265_{3}$ & 11211.484 & 0 & & 8916.984 & $24182_{2}^{\circ}-33099_{3}$ \\
\hline
\end{tabular}


TABLE 4. Classified lines of $\mathrm{Th} \mathrm{I}$-continued

\begin{tabular}{|c|c|c|c|c|c|c|c|c|c|}
\hline \multirow{2}{*}{ 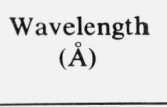 } & \multicolumn{2}{|c|}{ Intensity } & \multirow{2}{*}{$\begin{array}{l}\text { Wavenumber } \\
\qquad\left(\mathrm{cm}^{-1}\right)\end{array}$} & \multirow{2}{*}{ Classification } & \multirow{2}{*}{$\begin{array}{c}\text { Wavelength } \\
\text { (§) }\end{array}$} & \multicolumn{2}{|c|}{ Inten sity } & \multirow{2}{*}{$\begin{array}{l}\text { Wavenumber } \\
\qquad\left(\mathrm{cm}^{-1}\right)\end{array}$} & \multirow{2}{*}{ Classification } \\
\hline & Lamp & Spark & & & & Lamp & Spark & & \\
\hline 11205.579 & 4 & & 8921.683 & $11601_{1}-20522_{2}^{\circ}$ & 10956.004 & 1 & & 9124.916 & $18549_{2}-27674_{2}^{\circ}$ \\
\hline 11203.831 & 1 & & 8923.075 & $13175_{4}^{\circ}-220984$ & 10948.154 & 4 & & 9131.459 & $13962_{1}-23093_{2}^{\circ}$ \\
\hline 11195.293 & 1 & & 8929.880 & $10783_{2}^{\circ}-197133$ & 10942.432 & 3 & & 9136.234 & $165546-25690 \circ$ \\
\hline 11189.689 & 2 & & 8934.352 & $11802_{2}-20737_{1}^{\circ}$ & 10942.432 & 3 & & 9136.234 & $11601_{1}-20737^{\circ}$ \\
\hline 11185.925 & 5 & & 8937.359 & $7280_{2}-16217_{2}^{\circ}$ & 10938.909 & 1 & & 9139.176 & $16783 \stackrel{\circ}{4}-259234$ \\
\hline 11184.628 & 0 & & 8938.395 & $22855_{3}^{\circ}-31793_{4}$ & 10923.725 & 2 & & 9151.880 & $21738_{2}^{\circ}-30889_{1}$ \\
\hline 11179.105 & 3 & & 8942.811 & $11601_{1}-20543 \circ$ & 10913.581 & 3 & & 9160.386 & $130883-22248^{\circ}$ \\
\hline 11171.377 & 1 & & 8948.997 & $17847_{2}^{\circ}-267963$ & 10911.329 & 2 & & 9162.277 & $19516_{2}^{\circ}-28679_{2}$ \\
\hline 11162.099 & 3 & & 8956.436 & $14032_{2}^{\circ}-22988_{2}$ & 10901.193 & 4 & & 9170.796 & $18809_{4}^{\circ}-27980_{3}$ \\
\hline 11157.560 & 1 & & 8960.079 & $18011^{\circ}-26971_{4}$ & 10894.875 & 1 & & 9176.114 & $195033-28679_{2}$ \\
\hline 11151.802 & 0 & & 8964.706 & $173983-26363_{2}^{\circ}$ & 10890.287 & 4 & & 9179.980 & $18011{ }^{\circ}-271915$ \\
\hline 11148.272 & 0 & & 8967.544 & $21165^{\circ}-301322$ & 10887.285 & 0 & & 9182.511 & $23916_{4}^{\circ}-33099_{3}$ \\
\hline 11147.197 & 0 & & 8968.409 & $21539_{4}^{\circ}-305085$ & 10883.902 & 1 & & 9185.365 & $221411^{\circ}-313264$ \\
\hline 11139.881 & 3 & & 8974.299 & $19588^{\circ}-285624$ & 10883.233 & 0 & & 9185.930 & $22855_{3}^{\circ}-32041_{2}$ \\
\hline 11138.971 & 1 & & 8975.032 & $15863_{2}-24838_{1}^{\circ}$ & 10882.569 & 1 & & 9186.490 & $10526^{\circ}-197133$ \\
\hline 11138.821 & 4 & & 8975.153 & $18069_{3}^{\circ}-270443$ & 10882.153 & 0 & & 9186.842 & $230150^{\circ}-322025$ \\
\hline 11134.469 & 1 & & 8978.661 & $22163^{\circ}-311413$ & 10869.967 & 4 & & 9197.141 & $17847_{2}^{\circ}-27044_{3}$ \\
\hline 11128.287 & 5 & & 8983.649 & $4961_{4}-139453$ & 10866.949 & 1 & & 9199.695 & $165546-25753{ }^{\circ}$ \\
\hline 11125.541 & 4 & & 8985.866 & $18011^{\circ}-269976$ & 10864.601 & 2 & & 9201.683 & $13847_{2}-23049_{1}^{\circ}$ \\
\hline 11124.745 & 2 & & 8986.509 & $19948{ }^{\circ}-289343$ & 10862.508 & 4 & & 9203.456 & $20214_{3}^{\circ}-29418_{2}$ \\
\hline 11124.026 & 3 & & 8987.090 & $233066^{\circ}-322935$ & 10857.355 & 1 & & 9207.824 & $154934-247018$ \\
\hline 11114.372 & 0 & & 8994.896 & $20423_{1}^{\circ}-29418_{2}$ & 10853.458 & 3 & & 9211.130 & $8800_{4}-18011^{\circ}$ \\
\hline 11114.157 & 3 & & 8995.070 & $14206_{4}^{\circ}-23201_{3}$ & 10844.405 & 4 & & 9218.820 & $171665-26384_{4}^{\circ}$ \\
\hline 11101.804 & 5 & & 9005.079 & $98045-18809_{4}^{\circ}$ & 10837.713 & 0 & & 9224.512 & $20737_{1}^{\circ}-29961_{1}$ \\
\hline 11098.788 & 2 & & 9007.526 & $13847_{2}-22855_{3}^{\circ}$ & 10826.499 & 0 & & 9234.067 & $19039_{2}^{\circ}-28273_{2}$ \\
\hline 11094.494 & 2 & & 9011.012 & $15970{ }_{3}-24981_{3}^{\circ}$ & 10813.573 & 4 & & 9245.105 & $2869_{3}-12114_{2}^{\circ}$ \\
\hline 11092.159 & 1 & & 9012.909 & $21539_{4}^{\circ}-305524$ & 10813.395 & 6 & & 9245.257 & $4961_{4}-14206^{\circ}$ \\
\hline 11090.735 & 3 & & 9014.066 & $19516_{2}^{\circ}-28531_{2}$ & 10812.281 & 5 & & 9246.209 & $13847_{2}-230932^{\circ}$ \\
\hline 11073.735 & 3 & & 9027.904 & $19503_{3}^{\circ}-28531_{2}$ & 10807.344 & 3 & & 9250.433 & $13088_{3}-22338_{3}^{\circ}$ \\
\hline 11071.478 & 1 & & 9029.745 & $13847_{2}-22877_{1}^{\circ}$ & 10803.918 & 4 & & 9253.367 & $88004-18053_{4}^{\circ}$ \\
\hline 11057.009 & 4 & & 9041.561 & $132974-22338^{\circ}$ & 10802.535 & 1 & & 9254.551 & $142260-23481_{1}^{\circ}$ \\
\hline 11054.989 & 4 & & 9043.213 & $13945_{3}^{\circ}-22988_{2}$ & 10800.157 & 4 & & 9256.589 & $63622-156183$ \\
\hline 11051.898 & 7 & & 9045.742 & $15618_{3}^{\circ}-24664_{3}$ & 10800.053 & 5 & & 9256.678 & $13945_{3}^{\circ}-232013$ \\
\hline 11050.978 & 1 & & 9046.495 & $23916^{\circ}-329635$ & 10788.554 & 1 & & 9266.544 & $22508_{2}^{\circ}-31774_{3}$ \\
\hline 11046.224 & 4 & & 9050.389 & $18930_{3}^{\circ}-27980_{3}$ & 10786.371 & 3 & & 9268.420 & $20566_{4}^{\circ}-29835_{3}$ \\
\hline 11043.146 & 3 & & 9052.911 & $15618_{3}^{\circ}-24671_{2}$ & 10785.912 & 4 & & 9268.814 & $88004-18069_{3}^{\circ}$ \\
\hline 11042.986 & 2 & & 9053.042 & $130883-22141_{3}^{\circ}$ & 10779.100 & 2 & & 9274.672 & $180693-273433$ \\
\hline 11036.319 & 4 & & 9058.511 & $16346_{4}^{\circ}-25405_{4}$ & 10778.635 & 1 & & 9275.072 & $23521_{3}^{\circ}-327963$ \\
\hline 11035.091 & 0 & & 9059.519 & $19503_{3}^{\circ}-285624$ & 10776.984 & 4 & & 9276.493 & $154934-24769_{3}^{\circ}$ \\
\hline 11031.608 & 1 & & 9062.380 & $21902^{\circ}-309643$ & 10774.344 & 1 & & 9278.766 & $23015 \circ-322935$ \\
\hline 11028.089 & 1 & & 9065.271 & $19817_{1}^{\circ}-28882_{2}$ & 10759.049 & 1 & & 9291.956 & $212522-305442$ \\
\hline 11021.768 & 3 & & 9070.470 & $14206^{\circ}-23277_{5}$ & 10754.878 & 0 & & 9295.560 & $21077 \stackrel{\circ}{-303726}$ \\
\hline 11016.787 & 4 & & 9074.571 & $13088_{3}-22163_{4}^{\circ}$ & 10754.328 & 4 & & 9296.035 & $15618_{3}^{\circ}-24915_{3}$ \\
\hline 11001.800 & 3 & & 9086.933 & $139621-23049_{1}^{\circ}$ & 10752.475 & 2 & & 9297.637 & $18930_{3}^{\circ}-28227_{4}$ \\
\hline 11000.900 & 1 & & 9087.676 & $13945^{\circ}-230324$ & 10749.434 & $3 d$ & & 9300.268 & $17959_{4}-27260_{3}^{\circ}$ \\
\hline 10999.236 & 0 & & 9089.051 & $21668_{1}^{\circ}-30758_{2}$ & 10744.370 & 2 & & 9304.651 & $14465_{2}^{\circ}-23769_{1}$ \\
\hline 10983.633 & 5 & & 9101.963 & $132974-22399_{0}^{\circ}$ & 10732.245 & 5 & & 9315.163 & $128473-22163^{\circ}$ \\
\hline 10982.982 & 3 & & 9102.502 & $22163_{4}^{\circ}-31265_{3}$ & 10726.926 & 8 & & 9319.782 & $25580-11877^{\circ}$ \\
\hline 10982.910 & 4 & & 9102.562 & $142045-23306_{6}^{\circ}$ & 10725.418 & 6 & & 9321.092 & $11601_{1}-20922_{2}^{\circ}$ \\
\hline 10976.518 & 0 & & 9107.863 & $22163^{\circ}-312715$ & 10720.051 & 0 & & 9325.759 & $22669 \stackrel{\circ}{3}-319954$ \\
\hline 10974.389 & 1 & & 9109.630 & $17398_{3}-26508_{3}^{\circ}$ & 10707.531 & 1 & & 9336.663 & $159703-25306_{2}^{\circ}$ \\
\hline 10965.252 & 1 & & 9117.220 & $158632-24981_{3}^{\circ}$ & 10700.359 & 1 & & 9342.921 & $18930_{3}^{\circ}-28273_{2}$ \\
\hline 10964.829 & 1 & & 9117.572 & $20566_{4}^{\circ}-296845$ & 10700.194 & 0 & & 9343.065 & $22669_{3}^{\circ}-320124$ \\
\hline 10963.975 & 1 & & 9118.282 & $10414_{4}^{\circ}-19532_{4}$ & 10690.175 & 1 & & 9351.822 & $15970_{3}-25321^{\circ}$ \\
\hline 10962.885 & 5 & & 9119.189 & $11802_{2}-20922_{2}^{\circ}$ & 10678.631 & 1 & & 9361.931 & $20322{ }^{\circ}-296845$ \\
\hline
\end{tabular}


TABLE 4. Classified lines of Th $\mathrm{I}-$ continued

\begin{tabular}{|c|c|c|c|c|c|c|c|c|c|}
\hline \multirow{2}{*}{$\begin{array}{l}\text { Wavelength } \\
\text { (Å) }\end{array}$} & \multicolumn{2}{|c|}{ Intensity } & \multirow{2}{*}{$\begin{array}{l}\text { Wavenumber } \\
\left(\mathrm{cm}^{-1}\right)\end{array}$} & \multirow{2}{*}{ Classification } & \multirow{2}{*}{$\begin{array}{c}\text { Wavelength } \\
(\AA)\end{array}$} & \multicolumn{2}{|c|}{ Intensity } & \multirow{2}{*}{$\begin{array}{l}\text { Wavenumber } \\
\left(\mathrm{cm}^{-1}\right)\end{array}$} & \multirow{2}{*}{ Classification } \\
\hline & Lamp & Spark & & & & Lamp & Spark & & \\
\hline 10678.369 & 3 & & 9362.161 & $11802_{2}-21165_{3}^{\circ}$ & 10469.974 & 1 & & 9548.506 & $18809_{4}^{\circ}-28358_{3}$ \\
\hline 10668.486 & 2 & & 9370.834 & $7795_{4}^{\circ}-171665$ & 10477.700 & 1 & & 9541.465 & $20214_{3}^{\circ}-29756_{4}$ \\
\hline 10666.640 & 0 & & 9372.456 & $13297_{4}-22669_{3}^{\circ}$ & 10481.173 & 0 & & 9538.303 & $20423_{1}^{\circ}-29961_{1}$ \\
\hline 10664.767 & 0 & & 9374.102 & $22163_{4}^{\circ}-31537_{3}$ & 10450.448 & 3 & & 9566.346 & $199866^{\circ}-295526$ \\
\hline 10664.234 & 5 & & 9374.570 & $6362_{2}-15736_{1}^{\circ}$ & 10439.505 & 3 & & 9576.374 & $16346_{4}^{\circ}-259234$ \\
\hline 10663.178 & 1 & & 9375.499 & $21890_{3}^{\circ}-31265_{3}$ & 10436.870 & 2 & & 9578.792 & $15863_{2}-25442^{\circ}$ \\
\hline 10651.842 & 0 & & 9385.476 & $15970_{3}-25355_{4}^{\circ}$ & 10435.094 & 3 & & 9580.422 & $180110^{\circ}-275915$ \\
\hline 10649.651 & 1 & & 9387.407 & $21165_{3}^{\circ}-30552_{4}$ & 10434.109 & 1 & & 9581.326 & $13088_{3}-226699_{3}^{\circ}$ \\
\hline 10646.516 & 2 & & 9390.171 & $8111_{4}-17501_{0}^{\circ}$ & 10424.126 & 1 & & 9590.502 & $22338_{3}^{\circ}-319293$ \\
\hline 10640.329 & 1 & & 9395.631 & $22141_{3}^{\circ}-315373$ & 10421.511 & 0 & & 9592.909 & $21165_{3}^{\circ}-307582$ \\
\hline 10629.842 & 2 & & 9404.901 & $142045-23609_{0}^{\circ}$ & 10417.881 & 1 & & 9596.251 & $22399^{\circ}-319954$ \\
\hline 10615.008 & 4 & & 9418.044 & $18809_{4}^{\circ}-28227_{4}$ & 10412.996 & 4 & & 9600.753 & $18930_{3}^{\circ}-28531_{2}$ \\
\hline 10613.388 & 4 & & 9419.481 & $13088_{3}-22508_{2}^{\circ}$ & 10412.507 & 0 & & 9601.204 & $19817_{1}^{\circ}-29418_{2}$ \\
\hline 10609.955 & 1 & & 9422.529 & $9804_{5}-19227_{6}^{\circ}$ & 10410.424 & 2 & & 9603.125 & $7795_{4}^{\circ}-17398_{3}$ \\
\hline 10607.108 & 1 & & 9425.058 & $21539_{4}^{\circ}-309643$ & 10408.497 & 1 & & 9604.903 & $19817_{i}^{\circ}-29422_{1}$ \\
\hline 10605.464 & 4 & & 9426.519 & $18069_{3}^{\circ}-27495_{4}$ & 10403.056 & 1 & & 9609.927 & $20522_{2}^{\circ}-301322$ \\
\hline 10601.345 & 2 & & 9430.182 & $23306_{6}^{\circ}-32737_{5}$ & 10401.401 & 1 & & 9611.456 & $22163_{4}^{\circ}-317743$ \\
\hline 10600.597 & 1 & & 9430.847 & $21077 \circ-305085$ & 10392.041 & 0 & & 9620.113 & $20214_{3}^{\circ}-298353$ \\
\hline 10596.909 & 1 & & 9434.129 & $20322 \circ-297564$ & 10387.498 & 1 & & 9624.320 & $17166_{5}-26790_{4}^{\circ}$ \\
\hline 10595.264 & 1 & & 9435.594 & $22338_{3}^{\circ}-317743$ & 10378.821 & 1 & & 9632.366 & $18930_{3}^{\circ}-285624$ \\
\hline 10594.780 & 1 & & 9436.025 & $20214_{3}^{\circ}-29650_{2}$ & 10378.146 & 1 & & 9632.993 & $17411_{3}^{\circ}-270443$ \\
\hline 10592.495 & 0 & & 9438.061 & $23113_{4}^{\circ}-32551_{3}$ & 10377.490 & 2 & & 9633.601 & $13847_{2}-23481_{i}^{\circ}$ \\
\hline 10591.350 & 2 & & 9439.081 & $205222_{2}^{\circ}-29961_{1}$ & 10376.812 & 0 & & 9634.231 & $20214_{3}^{\circ}-29849_{4}$ \\
\hline 10587.100 & 3 & & 9442.870 & $15863_{2}-25306_{2}^{\circ}$ & 10376.020 & 1 & & 9634.966 & $21902_{4}^{\circ}-315373$ \\
\hline 10586.930 & 1 & & 9443.022 & $230158-324584$ & 10369.528 & 4 & & 9640.998 & $13962_{1}-23603_{2}^{\circ}$ \\
\hline 10585.524 & 0 & & 9444.276 & $23655_{4}^{\circ}-33099_{3}$ & 10362.966 & 1 & & 9647.103 & $21890_{3}^{\circ}-315373$ \\
\hline 10585.028 & 2 & & 9444.718 & $20566_{4}^{\circ}-30011_{3}$ & 10358.167 & 3 & & 9651.573 & $11601_{1}-21252_{2}^{\circ}$ \\
\hline 10581.711 & 1 & & 9447.679 & $139621-23410_{0}^{\circ}$ & 10357.615 & 0 & & 9652.087 & $22141_{3}^{\circ}-317934$ \\
\hline 10579.481 & 1 & & 9449.670 & $11802_{2}-21252_{2}^{\circ}$ & 10350.334 & 1 & & 9658.877 & $18614_{1}^{\circ}-28273_{2}$ \\
\hline 10578.136 & 1 & & 9450.872 & $142045-23655_{4}^{\circ}$ & 10349.051 & 5 & & 9660.074 & $12847_{3}-22508_{2}^{\circ}$ \\
\hline 10576.362 & 2 & & 9452.457 & $18069_{3}^{\circ}-27521_{4}$ & 10346.540 & 3 & & 9662.419 & $15863_{2}-25526_{1}^{\circ}$ \\
\hline 10570.129 & 0 & & 9458.031 & $15863_{2}-25321_{3}^{\circ}$ & 10345.899 & 0 & & 9663.017 & $17398_{3}-27061_{2}^{\circ}$ \\
\hline 10569.146 & 0 & & 9458.911 & $23609^{\circ}-330686$ & 10334.926 & 3 & & 9673.277 & $13847_{2}-23521_{3}^{\circ}$ \\
\hline 10567.177 & 1 & & 9460.673 & $12114_{2}^{\circ}-21575_{2}$ & 10316.894 & 4 & & 9690.184 & $17501 \stackrel{\circ}{0}-271915$ \\
\hline 10565.306 & 6 & & 9462.349 & $13175_{4}^{\circ}-226373$ & 10314.894 & 2 & & 9692.063 & $20322{ }^{\circ}-30014_{4}$ \\
\hline 10559.107 & 0 & & 9467.904 & $18053_{4}^{\circ}-27521_{4}$ & 10308.549 & 5 & & 9698.028 & $19986_{6}^{\circ}-296845$ \\
\hline 10556.454 & 7 & & 9470.283 & $17501{ }^{\circ}-26971_{4}$ & 10304.082 & 2 & & 9702.233 & $22338 \stackrel{\circ}{3}-32041_{2}$ \\
\hline 10554.837 & 4 & & 9471.734 & $16783^{\circ}-26255_{4}$ & 10294.714 & 0 & & 9711.062 & $17959_{4}-27670_{3}^{\circ}$ \\
\hline 10553.890 & 1 & & 9472.584 & $159703-25442 \stackrel{\circ}{3}$ & 10293.052 & 4 & & 9712.630 & $14204_{5}-23916^{\circ}$ \\
\hline 10545.902 & 1 & & 9479.759 & $173983-26878_{3}^{\circ}$ & 10285.485 & 0 & & 9719.775 & $17847_{2}^{\circ}-27566_{2}$ \\
\hline 10545.290 & 2 & & 9480.309 & $12114_{2}^{\circ}-21594_{3}$ & 10283.118 & 4 & & 9722.012 & $7502_{3}-17224_{2}^{\circ}$ \\
\hline 10540.958 & 5 & & 9484.205 & $18011^{\circ}-274954$ & 10278.860 & 1 & & 9726.040 & $21539_{4}^{\circ}-31265_{3}$ \\
\hline 10536.866 & 2 & & 9487.888 & $154934-24981_{3}^{\circ}$ & 10275.694 & 0 & & 9729.036 & $20922_{2}^{\circ}-30651_{3}$ \\
\hline 10533.385 & 3 & & 9491.024 & $12847_{3}-22338_{3}^{\circ}$ & 10271.191 & 2 & & 9733.302 & $159703-25703^{\circ}$ \\
\hline 10527.789 & 5 & & 9496.069 & $17501{ }^{\circ}-269976$ & 10268.554 & 1 & & 9735.801 & $19948 \stackrel{\circ}{4}-296845$ \\
\hline 10527.134 & 1 & & 9496.660 & $17847_{2}^{\circ}-27343_{3}$ & 10257.374 & 5 & & 9746.413 & $14243 i-23990_{2}$ \\
\hline 10518.190 & 2 & & 9504.735 & $20867^{\circ}-303726$ & 10255.580 & 2 & & 9748.118 & $15166_{3}^{\circ}-24915_{3}$ \\
\hline 10512.209 & 5 & & 9510.143 & $18011^{\circ}-27521_{4}$ & 10250.684 & 4 & & 9752.773 & $18809_{4}^{\circ}-28562_{4}$ \\
\hline 10502.592 & 4 & & 9518.851 & $139621-23481 i$ & 10247.561 & 4 & & 9755.746 & $13847_{2}-23603_{2}^{\circ}$ \\
\hline 10498.496 & 5 & & 9522.565 & $14247{ }^{\circ}-23769_{1}$ & 10241.779 & 2 & & 9761.253 & $233066-330686$ \\
\hline 10495.610 & 1 & & 9525.183 & $14465_{2}^{\circ}-23990_{2}$ & 10236.031 & 4 & & 9766.735 & $13088_{3}-22855_{3}^{\circ}$ \\
\hline 10494.843 & 6 & & 9525.879 & $14243 i-237691$ & 10223.662 & 3 & & 9778.551 & $139621-237411$ \\
\hline 10492.255 & 6 & & 9528.229 & $10526_{3}^{\circ}-20054_{2}$ & 10218.434 & 5 & & 9783.554 & $98045-19588 \circ$ \\
\hline 10487.427 & 1 & & 9532.615 & $18809_{4}^{\circ}-28342_{5}$ & 10214.442 & 1 & & 9787.377 & $21539_{4}^{\circ}-313264$ \\
\hline 10459.723 & 4 & & 9557.864 & $13297_{4}-22855_{3}^{\circ}$ & 10211.538 & 2 & & 9790.161 & $13962_{1}-23752_{2}^{\circ}$ \\
\hline
\end{tabular}


TABLE 4. Classified lines of Th $\mathrm{I}$-continued

\begin{tabular}{|c|c|c|c|c|c|c|c|c|c|}
\hline \multirow{2}{*}{$\begin{array}{l}\text { Wavelength } \\
(\AA)\end{array}$} & \multicolumn{2}{|c|}{ Intensity } & \multirow{2}{*}{$\begin{array}{l}\text { Wavenumber } \\
\left(\mathrm{cm}^{-1}\right)\end{array}$} & \multirow{2}{*}{ Classification } & \multirow{2}{*}{$\begin{array}{c}\text { Wavelength } \\
\text { (A) }\end{array}$} & \multicolumn{2}{|c|}{ Intensity } & \multirow{2}{*}{$\begin{array}{l}\text { Wavenumber } \\
\qquad\left(\mathrm{cm}^{-1}\right)\end{array}$} & \multirow{2}{*}{ Classification } \\
\hline & Lamp & Spark & & & & Lamp & Spark & & \\
\hline 10205.020 & 1 & & 9796.414 & $202143^{\circ}-300113$ & 9970.467 & 4 & & 10026.872 & $13175_{4}^{\circ}-23201_{3}$ \\
\hline 10202.133 & 1 & & 9799.186 & $21738_{2}^{\circ}-31537_{3}$ & 9963.495 & 3 & & 10033.888 & $16346_{4}^{\circ}-263805$ \\
\hline 10193.720 & 0 & & 9807.273 & $20737_{1}^{\circ}-30544_{2}$ & 9958.059 & 1 & & 10039.365 & $21890_{3}^{\circ}-31929_{3}$ \\
\hline 10192.965 & 0 & & 9808.000 & $19948{ }^{\circ}-297564$ & 9952.375 & 3 & & 10045.099 & $13945_{3}^{\circ}-23990_{2}$ \\
\hline 10184.539 & 2 & & 9816.114 & $132974-23113_{4}^{\circ}$ & 9948.171 & 1 & & 10049.344 & $173983-27447_{2}^{\circ}$ \\
\hline 10180.596 & 2 & & 9819.916 & $17224_{2}^{\circ}-27044_{3}$ & 9947.082 & 1 & & 10050.444 & $20322{ }^{\circ}-303726$ \\
\hline 10178.522 & 5 & & 9821.917 & $12847_{3}-22669_{3}^{\circ}$ & 9938.839 & 1 & & 10058.779 & $223990-324584$ \\
\hline 10175.012 & 4 & & 9825.305 & $14206_{4}^{\circ}-240324$ & 9935.203 & 2 & & 10062.461 & $20867^{\circ}-309306$ \\
\hline 10160.324 & 0 & & 9839.509 & $15863_{2}-25703_{2}^{\circ}$ & 9934.723 & 1 & & 10062.947 & $19948{ }^{\circ}-30011_{3}$ \\
\hline 10156.406 & 0 & & 9843.305 & $19039_{2}^{\circ}-28882_{2}$ & 9932.777 & 0 & & 10064.918 & $18614_{i}^{\circ}-28679_{2}$ \\
\hline 10144.266 & 4 & & 9855.084 & $6362_{2}-16217_{2}^{\circ}$ & 9929.814 & 3 & & 10067.922 & $11601_{1}-21668^{\circ}$ \\
\hline 10141.399 & 6 & & 9857.870 & $13175_{4}^{\circ}-230324$ & 9927.326 & 2 & & 10070.445 & $142045-242740$ \\
\hline 10140.434 & 4 & & 9858.808 & $20867 \stackrel{\circ}{-307267}$ & 9923.310 & 1 & & 10074.520 & $18574_{1}-28649_{i}^{\circ}$ \\
\hline 10137.390 & 2 & & 9861.769 & $17398_{3}-27260_{3}^{\circ}$ & 9919.448 & 0 & & 10078.443 & $15970_{3}-26048_{4}^{\circ}$ \\
\hline 10136.787 & 0 & & 9862.355 & $154934-25355_{4}^{\circ}$ & 9916.122 & 1 & & 10081.823 & $154934-25575_{4}^{\circ}$ \\
\hline 10131.371 & 2 & & 9867.628 & $173983-27266_{4}^{\circ}$ & 9913.628 & 1 & & 10084.360 & $17411_{3}^{\circ}-274954$ \\
\hline 10127.552 & 0 & & 9871.349 & $22141^{\circ}-320124$ & 9912.205 & 2 & & 10085.807 & $22877 \circ-329635$ \\
\hline 10126.557 & 1 & & 9872.318 & $21902_{4}^{\circ}-317743$ & 9911.116 & 4 & & 10086.915 & $13945_{3}^{\circ}-240324$ \\
\hline 10117.993 & 2 & & 9880.675 & $142045-24084_{6}^{\circ}$ & 9910.837 & 2 & & 10087.199 & $11802_{2}-21890_{3}^{\circ}$ \\
\hline 10111.877 & 1 & & 9886.651 & $19948{ }^{\circ}-298353$ & 9907.464 & $1 q$ & & 10090.634 & $175018-275915$ \\
\hline 10107.000 & 0 & & 9891.421 & $21902_{4}^{\circ}-317934$ & 9906.948 & 1 & & 10091.159 & $165546-266450$ \\
\hline 10105.549 & 0 & & 9892.842 & $17959_{4}-27852{ }^{\circ}$ & 9904.771 & 1 & & 10093.377 & $21902^{\circ}-31995_{4}$ \\
\hline 10105.080 & 2 & & 9893.301 & $138472-23741_{1}^{\circ}$ & 9902.362 & 4 & & 10095.833 & $19588 \stackrel{\circ}{-296845}$ \\
\hline 10104.307 & 1 & & 9894.058 & $16217_{2}^{\circ}-26111_{1}$ & 9898.356 & 2 & & 10099.918 & $17166_{5}-27266_{4}^{\circ}$ \\
\hline 10102.579 & 2 & & 9895.750 & $19039_{2}^{\circ}-28934_{3}$ & 9896.050 & 3 & & 10102.272 & $13175_{4}^{\circ}-23277_{5}$ \\
\hline 10098.624 & 1 & & 9899.625 & $22141_{3}^{\circ}-32041_{2}$ & 9873.821 & 3 & & 10125.015 & $18809_{4}^{\circ}-28934_{3}$ \\
\hline 10097.463 & 1 & & 9900.764 & $19948{ }_{4}^{\circ}-29849_{4}$ & 9872.635 & 1 & & 10126.231 & $22669{ }_{3}^{\circ}-32796_{3}$ \\
\hline 10089.136 & 7 & & 9908.935 & $75023-17411_{3}^{\circ}$ & 9871.998 & 2 & & 10126.885 & $159703-26096_{3}^{\circ}$ \\
\hline 10089.136 & 7 & & 9908.935 & $16346_{4}^{\circ}-26255_{4}$ & 9868.922 & 2 & & 10130.041 & $8800_{4}-18930_{3}^{\circ}$ \\
\hline 10086.406 & 2 & & 9911.617 & $18069_{3}^{\circ}-27980_{3}$ & 9867.890 & 2 & & 10131.101 & $7280_{2}-17411^{\circ}$ \\
\hline 10083.788 & 5 & & 9914.190 & $19227^{\circ}-291415$ & 9865.451 & 1 & & 10133.605 & $17847_{2}^{\circ}-27980_{3}$ \\
\hline 10082.880 & 4 & & 9915.083 & $15490_{0}^{\circ}-25405_{4}$ & 9862.127 & 0 & & 10137.021 & $11601_{1}-21738_{2}^{\circ}$ \\
\hline 10082.719 & 1 & & 9915.242 & $19503_{3}^{\circ}-29418_{2}$ & 9855.745 & 3 & & 10143.585 & $9804_{5}-19948_{4}^{\circ}$ \\
\hline 10082.719 & 1 & & 9915.242 & $18930_{3}^{\circ}-28845_{4}$ & 9840.923 & 1 & & 10158.863 & $18069_{3}^{\circ}-28227_{4}$ \\
\hline 10081.227 & 1 & & 9916.709 & $18614_{1}^{\circ}-28531_{2}$ & 9838.008 & 1 & & 10161.873 & $21165_{3}^{\circ}-313264$ \\
\hline 10078.906 & 1 & & 9918.993 & $17398_{3}-27317_{3}^{\circ}$ & 9837.258 & 0 & & 10162.648 & $14465_{2}^{\circ}-24627_{1}$ \\
\hline 10065.187 & 1 & & 9932.512 & $17411_{3}^{\circ}-27343_{3}$ & 9833.424 & 8 & & 10166.610 & $38651-14032 \stackrel{\circ}{2}$ \\
\hline 10056.214 & 2 & & 9941.375 & $20566_{4}^{\circ}-305085$ & 9826.452 & 7 & & 10173.823 & $5563_{1}-15736_{1}^{\circ}$ \\
\hline 10054.960 & 1 & & 9942.615 & $8111_{4}-18053 \stackrel{\circ}{4}$ & 9819.178 & 1 & & 10181.360 & $9804_{5}-19986_{6}^{\circ}$ \\
\hline 10053.375 & 0 & & 9944.182 & $7280_{2}-17224_{2}^{\circ}$ & 9814.962 & 1 & & 10185.733 & $20322 \AA-305085$ \\
\hline 10048.041 & 3 & & 9949.461 & $15493_{4}-25442_{3}^{\circ}$ & 9813.153 & 0 & & 10187.611 & $16783_{4}^{\circ}-26971_{4}$ \\
\hline 10045.316 & 2 & & 9952.160 & $18930_{3}^{\circ}-288822$ & 9812.698 & 7 & & 10188.083 & $8243 \stackrel{2}{-}-184313$ \\
\hline 10039.364 & 7 & & 9958.060 & $8111_{4}-18069_{3}^{\circ}$ & 9801.710 & 0 & & 10199.505 & $14465_{2}^{\circ}-246643$ \\
\hline 10039.101 & 2 & & 9958.321 & $14032_{2}^{\circ}-23990_{2}$ & 9797.252 & 1 & & 10204.145 & $18069_{3}^{\circ}-28273_{2}$ \\
\hline 10033.226 & 2 & & 9964.152 & $19588^{\circ}-295526$ & 9796.200 & 5 & & 10205.241 & $49614-15166^{\circ}$ \\
\hline 10029.545 & 1 & & 9967.809 & $20922_{2}^{\circ}-30889_{1}$ & 9789.508 & 2 & & 10212.218 & $17354_{1}^{\circ}-27566_{2}$ \\
\hline 10011.398 & 3 & & 9985.877 & $20566_{4}^{\circ}-305524$ & 9785.356 & 0 & & 10216.551 & $18011^{\circ}-28227_{4}$ \\
\hline 9999.614 & 0 & & 9997.645 & $21539_{4}^{\circ}-31537_{3}$ & 9782.141 & 0 & & 10219.908 & $13962_{1}-24182_{2}^{\circ}$ \\
\hline 9998.963 & 2 & & 9998.296 & $142045-24202_{4}^{\circ}$ & 9769.538 & 2 & & 10233.092 & $15863_{2}-26096^{\circ}$ \\
\hline 9992.656 & 3 & & 10004.607 & $18930_{3}^{\circ}-289343$ & 9764.606 & 0 & & 10238.261 & $15166_{3}^{\circ}-25405_{4}$ \\
\hline 9991.846 & 0 & & 10005.418 & $130883-23093_{2}^{\circ}$ & 9757.222 & 1 & & 10246.009 & $128477_{3}-23093_{2}^{\circ}$ \\
\hline 9989.939 & 2 & & 10007.327 & $128473-22855_{3}^{\circ}$ & 9754.019 & 1 & & 10249.374 & $15863_{2}-26113_{2}^{\circ}$ \\
\hline 9987.636 & 6 & & 10009.635 & $8800_{4}-18809_{4}^{\circ}$ & 9753.597 & 2 & & 10249.817 & $21077^{\circ}-313264$ \\
\hline 9985.052 & 5 & & 10012.225 & $16783^{\circ}-267963$ & 9746.464 & 7 & & 10257.318 & $3687_{2}-13945^{\circ}$ \\
\hline 9974.693 & 3 & & 10022.623 & $180110^{\circ}-280345$ & 9743.565 & 4 & & 10260.370 & $16783_{4}^{\circ}-27044_{3}$ \\
\hline
\end{tabular}


TABLE 4. Classified lines of Th $\mathrm{I}$-continued

\begin{tabular}{|c|c|c|c|c|c|c|c|c|c|}
\hline \multirow{2}{*}{$\begin{array}{l}\text { Wavelength } \\
(\AA)\end{array}$} & \multicolumn{2}{|c|}{ Intensity } & \multirow{2}{*}{$\begin{array}{l}\text { Wavenumber } \\
\left(\mathrm{cm}^{-1}\right)\end{array}$} & \multirow{2}{*}{ Classification } & \multirow{2}{*}{$\begin{array}{c}\text { Wavelength } \\
\text { (§) }\end{array}$} & \multicolumn{2}{|c|}{ Inten sity } & \multirow{2}{*}{$\begin{array}{l}\text { Wavenumber } \\
\left(\mathrm{cm}^{-1}\right)\end{array}$} & \multirow{2}{*}{ Classification } \\
\hline & Lamp & Spark & & & & Lamp & Spark & & \\
\hline 9738.624 & 1 & & 10265.576 & $12847_{3}-23113_{4}^{\circ}$ & 9436.815 & 2 & & 10593.889 & $11802_{2}-22396_{1}^{\circ}$ \\
\hline 9736.216 & 1 & & 10268.115 & $18614_{1}^{\circ}-28882_{2}$ & 9431.600 & 5 & & 10599.747 & $3865_{1}-14465_{2}^{\circ}$ \\
\hline 9716.146 & 2 & & 10289.325 & $18069_{3}^{\circ}-28358_{3}$ & 9430.921 & 0 & & 10600.510 & $17073_{1}-27674_{2}^{\circ}$ \\
\hline 9702.272 & 3 & & 10304.038 & $15618^{\circ}-25923_{4}$ & 9422.317 & 0 & & 10610.190 & $18069_{3}^{\circ}-28679_{2}$ \\
\hline 9701.580 & 1 & & 10304.773 & $180533_{4}^{\circ}-283583$ & 9420.621 & $2 u$ & & 10612.100 & $14226 o-24838^{\circ}$ \\
\hline 9700.564 & 7 & & 10305.852 & $2869_{3}-13175_{4}^{\circ}$ & 9417.463 & 0 & & 10615.659 & $19516_{2}^{\circ}-301322$ \\
\hline 9695.033 & 2 & & 10311.732 & $13297_{4}-23609_{0}^{\circ}$ & 9414.090 & 2 & & 10619.462 & $132974-23916^{\circ}$ \\
\hline 9678.235 & 0 & & 10329.629 & $20214_{3}^{\circ}-30544_{2}$ & 9409.353 & 4 & & 10624.808 & $16346_{4}^{\circ}-26971_{4}$ \\
\hline 9676.940 & 0 & & 10331.012 & $8243_{2}^{\circ}-185741$ & 9399.091 & 7 & & 10636.408 & $77955_{4}^{\circ}-184313$ \\
\hline 9676.840 & 3 & & 10331.118 & $18011{ }^{\circ}-283425$ & 9392.268 & 0 & & 10644.135 & $15863_{2}-26508 \stackrel{\circ}{\circ}$ \\
\hline 9676.107 & 2 & & 10331.901 & $19503_{3}^{\circ}-29835_{3}$ & 9390.588 & 2 & & 10646.040 & $142045-24850_{6}^{\circ}$ \\
\hline 9674.790 & 2 & & 10333.308 & $11241_{3}^{\circ}-21575_{2}$ & 9388.933 & 4 & & 10647.916 & $11601_{1}-22248_{2}^{\circ}$ \\
\hline 9664.700 & 6 & & 10344.096 & $3687_{2}-14032_{2}^{\circ}$ & 9388.933 & 4 & & 10647.916 & $18549_{2}-29197_{1}^{\circ}$ \\
\hline 9663.647 & 1 & & 10345.223 & $139621-2 \div 307_{2}^{\circ}$ & 9384.103 & 0 & & 10653.397 & $142260-24880_{1}^{\circ}$ \\
\hline 9656.439 & 1 & & 10352.945 & $11241_{3}^{\circ}-215943$ & 9383.275 & 5 & & 10654.337 & $55631-16217_{2}^{\circ}$ \\
\hline 9652.004 & 0 & & 10357.702 & $13297_{4}-23655_{4}^{\circ}$ & 9380.642 & 0 & & 10657.327 & $4961_{4}-15618_{3}^{\circ}$ \\
\hline 9643.322 & 1 & & 10367.027 & $14032_{2}^{\circ}-24399_{3}$ & 9366.797 & Ou & & 10673.079 & $128473-23521_{3}^{\circ}$ \\
\hline 9643.147 & 0 & & 10367.215 & $20522_{2}^{\circ}-30889_{1}$ & 9357.253 & 0 & & 10683.966 & $17847_{2}^{\circ}-28531_{2}$ \\
\hline 9636.906 & 1 & & 10373.929 & $17073_{1}-27447_{2}^{\circ}$ & 9344.207 & $1 \mathrm{u}$ & & 10698.882 & $8111_{4}-18809_{4}^{\circ}$ \\
\hline 9632.647 & 6 & & 10378.516 & $38651-14243 i$ & 9344.096 & $1 d$ & & 10699.009 & $20566^{\circ}-31265_{3}$ \\
\hline 9630.745 & 1 & & 10380.565 & $14247^{\circ}-24627_{1}$ & 9340.708 & 4 & & 10702.890 & $8800_{4}-19503{ }_{3}^{\circ}$ \\
\hline 9629.572 & 6 & & 10381.830 & $38651-14247^{\circ}$ & 9336.161 & $1 q$ & & 10708.102 & $14206_{4}^{\circ}-24915_{3}$ \\
\hline 9629.231 & 0 & & 10382.198 & $22399^{\circ}-32781_{4}$ & 9320.071 & 0 & & 10726.589 & $13945_{3}^{\circ}-24671_{2}$ \\
\hline 9627.672 & 1 & & 10383.879 & $14243^{\circ}-24627_{1}$ & 9317.727 & 3 & & 10729.287 & $10414 \stackrel{\circ}{4}-211435$ \\
\hline 9625.204 & 3 & & 10386.541 & $199866^{\circ}-303726$ & 9310.448 & 2 & & 10737.675 & $16783_{4}^{\circ}-27521_{4}$ \\
\hline 9623.414 & 0 & & 10388.473 & $22163{ }_{4}^{\circ}-32551_{3}$ & 9307.899 & 4 & & 10740.616 & $19986_{6}^{\circ}-307267$ \\
\hline 9619.222 & 1 & & 10393.001 & $159700_{3}-26363_{2}^{\circ}$ & 9300.018 & 1 & & 10749.718 & $20214_{3}^{\circ}-309643$ \\
\hline 9608.933 & 1 & & 10404.129 & $112411_{3}^{\circ}-216454$ & 9294.976 & 1 & & 10755.549 & $128473-23603^{\circ}$ \\
\hline 9605.809 & 0 & & 10407.513 & $16783_{4}^{\circ}-271915$ & 9289.564 & 5 & & 10761.815 & $98045-20566_{4}^{\circ}$ \\
\hline 9595.395 & 4 & & 10418.808 & $13962_{1}-24381_{2}^{\circ}$ & 9276.273 & 5 & & 10777.234 & $3687_{2}-14465_{2}^{\circ}$ \\
\hline 9588.809 & 0 & & 10425.964 & $195880-30014_{4}$ & 9271.181 & 1 & & 10783.153 & $0_{2}-10783_{2}^{\circ}$ \\
\hline 9587.027 & 1 & & 10427.902 & $14243 i-24671_{2}$ & 9270.155 & 2 & & 10784.347 & $19588 \circ-303726$ \\
\hline 9582.816 & 4 & & 10432.484 & $130888_{3}-23521{ }^{\circ}$ & 9266.920 & 4 & & 10788.111 & $8800_{4}-19588^{\circ}$ \\
\hline 9571.505 & 1 & & 10444.813 & $19516_{2}^{\circ}-29961_{1}$ & 9266.208 & 6 & & 10788.941 & $7280_{2}-18069_{3}^{\circ}$ \\
\hline 9570.405 & 1 & & 10446.013 & $118022_{2}-22248_{2}^{\circ}$ & 9263.682 & 0 & & 10791.882 & $10783_{2}^{\circ}-21575_{2}$ \\
\hline 9567.826 & 2 & & 10448.829 & $111977^{\circ}-21645_{4}$ & 9260.327 & 2 & & 10795.792 & $11601_{1}-22396^{\circ}$ \\
\hline 9567.283 & 1 & & 10449.422 & $16346_{4}^{\circ}-267963$ & 9250.579 & 0 & & 10807.168 & $12847_{3}-23655_{4}^{\circ}$ \\
\hline 9561.245 & 5 & & 10456.021 & $8243_{2}^{\circ}-18699_{2}$ & 9245.257 & 1 & & 10813.389 & $18069_{3}^{\circ}-28882_{2}$ \\
\hline 9510.950 & 0 & & 10511.313 & $17847_{2}^{\circ}-283583$ & 9239.328 & 1 & & 10820.329 & $15970_{3}-26790_{4}^{\circ}$ \\
\hline 9507.656 & 2 & & 10514.955 & $130883-23603_{2}^{\circ}$ & 9237.550 & 2 & & 10822.411 & $20423 i-312452$ \\
\hline 9505.395 & 5 & & 10517.456 & $98045-20322 \circ$ & 9234.396 & 3 & & 10826.108 & $18930_{3}^{\circ}-297564$ \\
\hline 9501.443 & 0 & & 10521.831 & 199866 - 305085 & 9233.858 & 50 & 5 & 10826.738 & $16217 \AA-27044_{3}$ \\
\hline 9500.302 & 2 & & 10523.095 & $1214_{2}^{\circ}-22637_{3}$ & 9232.493 & 50 & 4 & 10828.339 & $130883-23916_{4}^{\circ}$ \\
\hline 9497.191 & 7 & & 10526.541 & $0_{2}-10526_{3}^{\circ}$ & 9230.616 & 1 & & 10830.541 & $21165_{3}^{\circ}-31995_{4}$ \\
\hline 9495.500 & 7 & & 10528.416 & $4961_{4}-154900$ & 9229.218 & 1 & & 10832.181 & $17847_{2}^{\circ}-28679_{2}$ \\
\hline 9486.929 & 3 & & 10537.928 & $159700_{3}-26508_{3}^{\circ}$ & 9227.870 & 1 & & 10833.764 & $22855_{3}^{\circ}-33689_{2}$ \\
\hline 9474.882 & 7 & & 10551.327 & $75023-18053_{4}^{\circ}$ & 9227.517 & 300 & 50 & 10834.178 & $18011^{\circ}-28845_{4}$ \\
\hline 9470.684 & 6 & & 10556.003 & $3687_{2}-14243 i$ & 9227.057 & 2 & & 10834.718 & $21902_{4}^{\circ}-327375$ \\
\hline 9467.200 & 5 & & 10559.888 & $16783_{4}^{\circ}-273433$ & 9222.345 & 20 & 5 & 10840.254 & $197133-30553^{\circ}$ \\
\hline 9461.208 & 3 & & 10566.576 & $13088_{3}-23655_{4}^{\circ}$ & 9221.439 & 40 & 5 & 10841.319 & 17501 ○ -283425 \\
\hline 9461.031 & 6 & & 10566.774 & $7502{ }_{3}-18069_{3}^{\circ}$ & 9218.851 & 2 & & 10844.363 & $21594_{3}-32439_{4}^{\circ}$ \\
\hline 9460.871 & 2 & & 10566.952 & $7280_{2}-17847_{2}^{\circ}$ & 9218.670 & 1 & & 10844.576 & $18574_{1}-29419_{2}^{\circ}$ \\
\hline 9458.629 & 0 & & 10569.457 & $17411_{3}^{\circ}-27980_{3}$ & 9218.612 & 1 & & 10844.644 & $18574_{1}-29419_{2}^{\circ}$ \\
\hline 9455.205 & $2 q$ & & 10573.284 & $138472-24421_{3}^{\circ}$ & 9218.612 & 1 & & 10844.644 & $18574_{1}-29419_{2}^{\circ}$ \\
\hline 9450.464 & 1 & & 10578.589 & $16217_{2}^{\circ}-267963$ & 9208.593 & 50 & & 10856.443 & $112411_{3}^{\circ}-220984$ \\
\hline
\end{tabular}


TABle 4. Classified lines of $\mathrm{Th} \mathrm{I}$-continued

\begin{tabular}{|c|c|c|c|c|c|c|c|c|c|}
\hline \multirow{2}{*}{$\begin{array}{l}\text { Wavelength } \\
(\AA)\end{array}$} & \multicolumn{2}{|c|}{ Intensity } & \multirow{2}{*}{$\begin{array}{l}\text { Wavenumber } \\
\left(\mathrm{cm}^{-1}\right)\end{array}$} & \multirow{2}{*}{ Classification } & \multirow{2}{*}{ 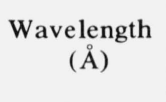 } & \multicolumn{2}{|c|}{ Intensity } & \multirow{2}{*}{$\begin{array}{l}\text { Wavenumber } \\
\left(\mathrm{cm}^{-1}\right)\end{array}$} & \multirow{2}{*}{ Classification } \\
\hline & Lamp & Spark & & & & Lamp & Spark & & \\
\hline 9208.035 & 8 & 2 & 10857.101 & $13175_{4}^{\circ}-24032_{4}$ & 9061.672 & 2 & & 11032.463 & $13847_{2}-24880_{1}^{\circ}$ \\
\hline 9203.988 & 500 & 75 & 10861.874 & $6362_{2}-17224_{2}^{\circ}$ & 9059.264 & 3 & & 11035.395 & $17847_{2}^{\circ}-28882_{2}$ \\
\hline 9200.638 & $1 \mathrm{~h}$ & & 10865.829 & $18069^{\circ}-289343$ & 9056.071 & 15 & 2 & 11039.286 & $18382^{\circ}-294221$ \\
\hline 9199.676 & 8 & & 10866.966 & $11802_{2}-22669_{3}^{\circ}$ & 9054.306 & 2 & & 11041.438 & $195033^{\circ}-30544_{2}$ \\
\hline 9197.246 & 3 & & 10869.837 & $18549_{2}-29419_{2}^{\circ}$ & 9048.503 & 50 & & 11048.519 & $10526_{3}^{\circ}-21575_{2}$ \\
\hline 9191.681 & 2 & & 10876.418 & $13962_{1}-24838_{1}^{\circ}$ & 9048.248 & $800 \mathrm{~b}$ & 75 & 11048.830 & $63622-17411_{3}^{\circ}$ \\
\hline 9188.503 & 2 & & 10880.179 & $211435-32023{ }^{\circ}$ & 9046.960 & 3 & & 11050.403 & $18053_{4}^{\circ}-29104_{4}$ \\
\hline 9186.364 & 1 & & 10882.713 & $22508_{2}^{\circ}-33390_{1}$ & 9046.137 & 4 & & 11051.409 & $22163^{\circ}-332145$ \\
\hline 9179.820 & 2 & & 10890.471 & $15490 \AA-263805$ & 9046.137 & 4 & & 11051.409 & $22669_{3}^{\circ}-33721_{2}$ \\
\hline 9178.777 & 200 & 15 & 10891.708 & $154934-26384_{4}^{\circ}$ & 9045.341 & 200 & 20 & 11052.381 & $11802_{2}-22855_{3}^{\circ}$ \\
\hline 9176.967 & 2 & & 10893.856 & $21902_{4}^{\circ}-32796_{3}$ & 9041.717 & 2 & & 11056.811 & $22508_{2}^{\circ}-33564_{3}$ \\
\hline 9175.085 & 2 & & 10896.091 & $22877_{1}^{\circ}-33773_{1}$ & 9039.277 & 2 & & 11059.796 & $22399_{0}^{\circ}-33459_{4}$ \\
\hline 9171.262 & 2 & & 10900.633 & $22508_{2}^{\circ}-33408_{3}$ & 9038.935 & 2 & & 11060.214 & $20566_{4}^{\circ}-316265$ \\
\hline 9170.810 & 100 & 4 & 10901.170 & $111977^{\circ}-220984$ & 9037.880 & 200 & 10 & 11061.505 & $17501 \stackrel{\circ}{\circ}-285624$ \\
\hline 9167.793 & 20 & 2 & 10904.758 & $18930_{3}^{\circ}-29835_{3}$ & 9034.490 & 2 & & 11065.656 & $185741-29640^{\circ}$ \\
\hline 9167.469 & 1 & & 10905.143 & $13297_{4}-24202_{4}^{\circ}$ & 9032.457 & 3 & & 11068.146 & 105263 \\
\hline 9166.738 & 1 & & 10906.013 & $21890_{3}-327963$ & 9031.811 & 150 & 10 & 11068.938 & $12847_{3}-23916_{4}^{\circ}$ \\
\hline 9165.890 & 400 & 25 & 10907.022 & $11601_{1}-22508_{2}^{\circ}$ & 9026.157 & 2 & & 11075.872 & $21575_{2}-32650_{1}^{\circ}$ \\
\hline 9165.008 & 5 & 1 & 10908.071 & $15970_{3}-26878_{3}^{\circ}$ & 9026.004 & 2 & & 11076.059 & $2869_{3}-13945_{3}^{\circ}$ \\
\hline 9164.261 & 4 & & 10908.961 & $215943-32503_{2}^{\circ}$ & 9016.581 & 300 & 20 & 11087.634 & $12114_{2}^{\circ}-232013$ \\
\hline 9156.909 & 4 & & 10917.719 & $139621-24880_{i}^{\circ}$ & 9016.382 & 3 & & 11087.879 & $18053{ }_{4}^{\circ}-291415$ \\
\hline 9155.929 & 4 & 1 & 10918.888 & $18930_{3}^{\circ}-29849_{4}$ & 9013.959 & 5 & 1 & 11090.860 & $17959_{4}-29050^{\circ}$ \\
\hline 9153.352 & 8 & 2 & 10921.962 & $13847_{2}-24769^{\circ}$ & 9013.959 & 5 & 1 & 11090.860 & $18549_{2}-29640_{1}^{\circ}$ \\
\hline 9152.771 & 3 & & 10922.655 & $19039_{2}^{\circ}-29961_{1}$ & 9013.581 & 1 & & 11091.325 & $159703-27061_{2}^{\circ}$ \\
\hline 9147.790 & 3 & 1 & 10928.602 & $21575_{2}-32503_{2}^{\circ}$ & 9012.517 & 150 & 8 & 11092.634 & $180110^{\circ}-291044$ \\
\hline 9141.782 & 8 & 2 & 10935.785 & $21077^{\circ}-320124$ & 9011.500 & 4 & 1 & 11093.886 & $13088_{3}-24182_{2}^{\circ}$ \\
\hline 9141.337 & $2 \mathrm{~h}$ & & 10936.317 & $22163_{4}^{\circ}-33099_{3}$ & 8997.862 & 100 & 10 & 11110.701 & $11877_{1}^{\circ}-22988_{2}$ \\
\hline 9141.245 & 1 & & 10936.427 & $22669_{3}^{\circ}-33606_{2}$ & 8995.178 & 100 & 10 & 11114.016 & $13088_{3}-24202_{4}^{\circ}$ \\
\hline 9137.725 & 8 & 2 & 10940.640 & $18699_{2}-29640_{1}^{\circ}$ & 8994.447 & 2 & & 11114.919 & $173983-28513_{2}^{\circ}$ \\
\hline 9134.679 & 150 & 10 & 10944.288 & $199866^{\circ}-309306$ & 8991.072 & 1 & & 11119.092 & $20922_{2}^{\circ}-32041_{2}$ \\
\hline 9132.261 & 40 & 8 & 10947.186 & $17411_{3}^{\circ}-283583$ & 8990.879 & 10 & 2 & 11119.330 & $10526_{3}^{\circ}-21645_{4}$ \\
\hline 9129.173 & 20 & 5 & 10950.889 & $17073_{1}-28024^{\circ}$ & 8987.396 & 75 & 10 & 11123.640 & $132974-24421_{3}^{\circ}$ \\
\hline 9126.321 & 20 & 4 & 10954.311 & $22877^{\circ}-33831_{4}$ & 8985.270 & 15 & 4 & 11126.271 & $16217_{2}^{\circ}-273433$ \\
\hline 9123.393 & 1 & & 10957.827 & $22141_{3}^{\circ}-33099_{3}$ & 8983.722 & 2 & & 11128.189 & $22399 \circ-33527_{4}$ \\
\hline 9118.125 & 50 & 3 & 10964.158 & $195888^{\circ}-305524$ & 8982.127 & 2 & & 11130.165 & $180110^{\circ}-291415$ \\
\hline 9113.239 & 1 & & 10970.036 & $22338^{\circ}-33309_{2}$ & 8980.723 & 10 & 3 & 11131.905 & $15863_{2}-26995_{3}^{\circ}$ \\
\hline 9110.964 & 2 & & 10972.775 & $179594-28932_{4}^{\circ}$ & 8978.886 & 3 & & 11134.182 & $19516_{2}^{\circ}-30651_{3}$ \\
\hline 9107.788 & 2 & & 10976.601 & $22855_{3}^{\circ}-33831_{4}$ & 8976.639 & 3 & 1 & 11136.969 & $18549_{2}-29686_{3}^{\circ}$ \\
\hline 9107.216 & 100 & & 10977.291 & $13297_{4}-24274{ }^{\circ}$ & 8969.853 & 100 & 15 & 11145.395 & $19227 \%-303726$ \\
\hline 9103.165 & 2 & & 10982.176 & $19273_{2}-30255_{3}^{\circ}$ & 8967.635 & 500 & 100 & 11148.151 & $8800_{4}-19948 \stackrel{\circ}{\circ}$ \\
\hline 9101.064 & 20 & 3 & 10984.711 & $195324-30517^{\circ}$ & 8965.074 & 2 & & 11151.336 & $142045-25355_{4}^{\circ}$ \\
\hline 9099.364 & 2 & & 10986.763 & $18699_{2}-29686_{3}^{\circ}$ & 8956.495 & 2 & & 11162.017 & $226699_{3}^{\circ}-33831_{4}$ \\
\hline 9095.713 & 4 & & 10991.173 & $13847_{2}-24838^{\circ}$ & 8955.834 & 200 & 15 & 11162.841 & $2869_{3}-14032_{2}^{\circ}$ \\
\hline 9094.821 & 400 & 100 & 10992.251 & $63622-17354^{\circ}$ & 8952.133 & 2 & & 11167.456 & $22141_{3}^{\circ}-33309_{2}$ \\
\hline 9090.809 & 200 & 10 & 10997.103 & $16346_{4}^{\circ}-273433$ & 8949.114 & 100 & 20 & 11171.223 & $13088_{3}-24259_{4}^{\circ}$ \\
\hline 9084.511 & 3 & & 11004.726 & $20322{ }^{\circ}-313264$ & 8944.447 & 1 & & 11177.052 & $15618_{3}^{\circ}-26796_{3}$ \\
\hline 9082.305 & 3 & 1 & 11007.399 & $20922_{2}^{\circ}-319293$ & 8942.502 & 2 & & 11179.483 & $21575_{2}-32754_{3}^{\circ}$ \\
\hline 9076.185 & 8 & 1 & 11014.821 & $154934-265088^{\circ}$ & 8941.641 & 8 & 2 & 11180.560 & $10414_{4}^{\circ}-215943$ \\
\hline 9074.980 & 1 & & 11016.284 & $19948{ }^{\circ}-309643$ & 8935.225 & 1 & & 11188.588 & $22338_{3}^{\circ}-33527_{4}$ \\
\hline 9072.954 & 1 & & 11018.744 & $22399 \circ-33418_{4}^{\circ}$ & 8933.357 & 1 & & 11190.928 & $17398_{3}-28589_{3}^{\circ}$ \\
\hline 9067.890 & 1 & & 11024.897 & $22248_{2}^{\circ}-33273_{1}$ & 8930.006 & 2 & & 11195.127 & $18549_{2}-29744^{\circ}$ \\
\hline 9067.657 & 2 & & 11025.181 & $18809_{4}^{\circ}-29835_{3}$ & 8928.384 & 2 & & 11197.161 & $21902_{4}^{\circ}-33099_{3}$ \\
\hline 9067.234 & 5 & 1 & 11025.695 & $15970_{3}-26995_{3}^{\circ}$ & 8928.078 & 25 & 5 & 11197.545 & $15863_{2}-27061_{2}^{\circ}$ \\
\hline 9063.948 & 200 & 10 & 11029.692 & $8243_{2}^{\circ}-19273_{2}$ & 8927.723 & 15 & 5 & 11197.990 & $17959_{4}-29157^{\circ}$ \\
\hline 9062.879 & 2 & & 11030.993 & $20214^{\circ}-31245_{2}$ & 8924.217 & $3 w$ & & 11202.389 & $18930_{3}^{\circ}-30132$ \\
\hline
\end{tabular}


TABLE 4. Classified lines of $\mathrm{Th} \mathrm{I}$-continued

\begin{tabular}{|c|c|c|c|c|c|c|c|c|c|}
\hline \multirow{2}{*}{$\begin{array}{l}\text { Wavelength } \\
\text { (Å) }\end{array}$} & \multicolumn{2}{|c|}{ Intensity } & \multirow{2}{*}{$\begin{array}{c}\text { Wavenumber } \\
\left(\mathrm{cm}^{-1}\right)\end{array}$} & \multirow{2}{*}{ Classification } & \multirow{2}{*}{$\begin{array}{c}\text { Wavelength } \\
(\AA)\end{array}$} & \multicolumn{2}{|c|}{ Inten sity } & \multirow{2}{*}{$\begin{array}{c}\text { Wavenumber } \\
\left(\mathrm{cm}^{-1}\right)\end{array}$} & \multirow{2}{*}{ Classification } \\
\hline & Lamp & Spark & & & & Lamp & Spark & & \\
\hline 8922.566 & 2 & & 11204.462 & $18809_{4}^{\circ}-30014_{4}$ & 8786.029 & 2 & & 11378.581 & $19948_{4}^{\circ}-31326_{4}$ \\
\hline 8918.698 & 1 & & 11209.321 & $21890_{3}^{\circ}-33099_{3}$ & 8784.139 & 3 & 1 & 11381.029 & $21077_{0}^{\circ}-32458_{4}$ \\
\hline 8918.584 & 1 & & 11209.465 & $22396_{1}^{\circ}-336062$ & 8781.093 & 25 & 1 & 11384.977 & $49614-16346^{\circ}$ \\
\hline 8912.867 & 2 & & 11216.655 & $21645_{4}-32862 \stackrel{\circ}{4}$ & 8780.337 & 1 & & 11385.958 & $22141 \stackrel{\circ}{3}-335274$ \\
\hline 8910.844 & 75 & 4 & 11219.201 & $13088_{3}-24307_{2}^{\circ}$ & 8779.898 & 1 & & 11386.527 & $21165_{3}^{\circ}-325513$ \\
\hline 8907.016 & 40 & 2 & 11224.023 & $13175_{4}^{\circ}-24399_{3}$ & 8775.575 & 200 & 40 & 11392.136 & $8111_{4}-19503 \stackrel{\circ}{3}$ \\
\hline 8905.566 & 2 & & 11225.850 & $22338^{\circ}-335643$ & 8773.524 & 5 & 1 & 11394.799 & $16217_{2}^{\circ}-27612_{3}$ \\
\hline 8902.815 & 2. & & 11229.319 & $195324-30761_{3}^{\circ}$ & 8772.803 & 150 & 8 & 11395.736 & $11241_{3}^{\circ}-226373$ \\
\hline 8900.891 & 2 & & 11231.746 & $10414_{4}^{\circ}-21645_{4}$ & 8772.384 & 8 & 1 & 11396.280 & $15863_{2}-27260_{3}^{\circ}$ \\
\hline 8893.533 & 15 & 5 & 11241.039 & $19516_{2}^{\circ}-30758_{2}$ & 8771.465 & 1 & & 11397.474 & $216454-33043$ \\
\hline 8892.973 & 150 & 15 & 11241.747 & $0_{2}-11241_{3}^{\circ}$ & 8768.195 & 5 & 1 & 11401.725 & $22163_{4}^{\circ}-335643$ \\
\hline 8892.778 & 2 & & 11241.993 & $215399_{4}^{\circ}-32781_{4}$ & 8766.743 & 100 & 15 & 11403.613 & $132974-24701^{\circ}$ \\
\hline 8889.970 & 2 & & 11245.544 & $221634^{\circ}-334083$ & 8760.449 & 100 & 8 & 11411.806 & $12847_{3}-24259_{4}^{\circ}$ \\
\hline 8889.187 & 100 & 10 & 11246.535 & $11802_{2}-23049^{\circ}$ & 8758.247 & 400 & 75 & 11414.675 & $8800_{4}-20214_{3}^{\circ}$ \\
\hline 8882.601 & 1 & & 11254.873 & $195033^{\circ}-307582$ & 8755.001 & 3 & 1 & 11418.907 & $21890_{3}^{\circ}-33309_{2}$ \\
\hline 8877.739 & 1 & & 11261.037 & $22401_{1}-33662^{\circ}$ & 8753.738 & $2 \mathrm{~h}$ & & 11420.555 & $22877^{\circ}-342981$ \\
\hline 8875.223 & 100 & 8 & 11264.229 & $13297_{4}-24561_{3}^{\circ}$ & 8751.202 & 10 & 2 & 11423.864 & $22877^{\circ}-34301_{4}$ \\
\hline 8874.156 & 2 & & 11265.584 & $22508_{2}^{\circ}-33773_{1}$ & 8750.176 & 1 & & 11425.204 & $14465_{2}^{\circ}-25890_{2}$ \\
\hline 8872.787 & 1 & & 11267.322 & $22338_{3}^{\circ}-33606_{2}$ & 8748.038 & 400 & 100 & 11427.996 & $75023-18930_{3}^{\circ}$ \\
\hline 8872.409 & 1 & & 11267.802 & $21594_{3}-32862{ }^{\circ}$ & 8747.498 & 2 & & 11428.701 & $19817^{\circ}-31245_{2}$ \\
\hline 8868.822 & 200 & 50 & 11272.359 & $98045-21077_{0}^{\circ}$ & 8747.258 & 2 & & 11429.015 & $20566_{4}^{\circ}-31995_{4}$ \\
\hline 8867.740 & 1 & & 11273.735 & $17073_{1}-28347_{2}^{\circ}$ & 8744.965 & 2 & 1 & 11432.012 & $23015^{\circ}-344474$ \\
\hline 8865.566 & 100 & 8 & 11276.499 & $11601_{1}-22877^{\circ}$ & 8744.598 & 5 & 1 & 11432.492 & $223998-338314$ \\
\hline 8862.284 & 2 & & 11280.675 & $19227 \mathbf{6}_{6}^{-305085}$ & 8743.269 & 15 & 2 & 11434.229 & $23655_{4}^{\circ}-35089_{3}$ \\
\hline 8860.973 & 4 & 1 & 11282.344 & $17398_{3}-28680_{4}^{\circ}$ & 8739.251 & 1 & & 11439.486 & $17073_{1}-28513^{\circ}$ \\
\hline 8857.869 & 4 & 1 & 11286.298 & $23015^{\circ}-343014$ & 8735.735 & 2 & & 11444.091 & $16783_{4}^{\circ}-28227_{4}$ \\
\hline 8854.899 & 10 & 3 & 11290.083 & $15970_{3}-27260_{3}^{\circ}$ & 8734.482 & 5 & 1 & 11445.732 & $230150^{\circ}-344605$ \\
\hline 8854.136 & 1 & & 11291.056 & $11802_{2}-23093_{2}^{\circ}$ & 8734.023 & 75 & 8 & 11446.334 & $20566_{4}^{\circ}-32012_{4}$ \\
\hline 8852.784 & 10 & 3 & 11292.781 & $13088_{3}-24381_{2}^{\circ}$ & 8732.992 & 2 & & 11447.685 & $19516_{2}^{\circ}-30964_{3}$ \\
\hline 8852.552 & 1 & & 11293.077 & $21165_{3}^{\circ}-32458_{4}$ & 8732.426 & 150 & 50 & 11448.427 & $11601_{1}-23049^{\circ}$ \\
\hline 8850.543 & 2 & & 11295.640 & $211435-32439_{4}^{\circ}$ & 8730.818 & 15 & 2 & 11450.536 & $19273_{2}-30723_{1}^{\circ}$ \\
\hline 8848.294 & 8 & 3 & 11298.511 & $165546-27852^{\circ}$ & 8728.550 & 2 & & 11453.511 & $15863_{2}-27317^{\circ}$ \\
\hline 8847.513 & 2 & & 11299.508 & $142260-25526^{\circ}$ & 8727.446 & 4 & & 11454.960 & $17224_{2}^{\circ}-28679_{2}$ \\
\hline 8843.549 & 2 & & 11304.573 & $20322^{\circ}-316265$ & 8724.375 & 40 & 2 & 11458.992 & $13847_{2}-25306_{2}^{\circ}$ \\
\hline 8842.359 & 1 & & 11306.095 & $197133-31019 \stackrel{\circ}{\circ}$ & 8723.718 & 15 & 2 & 11459.855 & $13945^{\circ}-25405_{4}$ \\
\hline 8841.841 & 4 & & 11306.757 & $17224^{\circ}-285312$ & 8722.459 & 15 & 2 & 11461.509 & $19503 \stackrel{\circ}{3}-309643$ \\
\hline 8841.170 & 150 & 20 & 11307.615 & $7502_{3}-18809_{4}^{\circ}$ & 8717.754 & 10 & 2 & 11467.695 & $18549_{2}-30017^{\circ}$ \\
\hline 8836.139 & 3 & & 11314.053 & $171665-28480_{4}^{\circ}$ & 8716.427 & 2 & & 11469.441 & $8243 \stackrel{\circ}{2}-19713_{3}$ \\
\hline 8833.471 & 2 & & 11317.471 & $18699_{2}-30017_{3}^{\circ}$ & 8715.065 & 1 & & 11471.233 & $17411_{3}^{\circ}-28882_{2}$ \\
\hline 8829.686 & 15 & 5 & 11322.322 & $20214^{\circ}-31537_{3}$ & 8714.917 & 1 & & 11471.428 & $20322{ }^{\circ}-31793_{4}$ \\
\hline 8829.470 & 2 & & 11322.599 & $199489-312715$ & 8714.257 & 8 & 1 & 11472.297 & $132974-24769^{\circ}$ \\
\hline 8827.897 & 2 & & 11324.616 & $17354_{1}^{\circ}-28679_{2}$ & 8713.654 & 20 & 2 & 11473.090 & $13088_{3}-24561 \stackrel{\circ}{3}$ \\
\hline 8821.739 & 4 & 1 & 11332.521 & $13088_{3}-24421_{3}^{\circ}$ & 8712.838 & 10 & 2 & 11474.165 & $13847_{2}-25321^{\circ}$ \\
\hline 8820.409 & $50 \mathrm{~b}$ & 10 & 11334.230 & $7280_{2}-18614_{1}^{\circ}$ & 8710.410 & 50 & 2 & 11477.363 & $8111_{4}-195880$ \\
\hline 8820.214 & 20 & 3 & 11334.481 & $128473-24182_{2}^{\circ}$ & 8709.233 & 200 & 50 & 11478.914 & $3687_{2}-15166^{\circ}$ \\
\hline 8812.507 & 4 & & 11344.393 & $175010^{\circ}-28845_{4}$ & 8707.356 & 75 & 3 & 11481.389 & $15490 \circ-26971_{4}$ \\
\hline 8810.240 & 15 & & 11347.312 & $15970_{3}-27317^{\circ}$ & 8704.855 & 25 & 3 & 11484.688 & $63622-17847^{\circ}$ \\
\hline 8808.667 & 2 & & 11349.339 & $18069_{3}^{\circ}-29418_{2}$ & 8703.697 & 25 & 2 & 11486.216 & $142045-25690^{\circ}$ \\
\hline 8806.220 & 2 & & 11352.492 & $15618_{3}^{\circ}-26971_{4}$ & 8703.340 & 3 & & 11486.687 & $19532_{4}-31019_{4}^{\circ}$ \\
\hline 8804.580 & 75 & 3 & 11354.607 & $12847_{3}-24202^{\circ}$ & 8702.021 & 8 & 1 & 11488.428 & $19273_{2}-307613$ \\
\hline 8800.465 & 3 & & 11359.916 & $215943-32954^{\circ}$ & 8701.117 & 50 & 3 & 11489.622 & $13175_{4}^{\circ}-246643$ \\
\hline 8799.353 & 1 & & 11361.352 & $21738_{2}^{\circ}-33099_{3}$ & 8693.669 & 2 & & 11499.465 & $19227^{\circ}-307267$ \\
\hline 8798.171 & 10 & 3 & 11362.878 & $19713_{3}-31075_{2}^{\circ}$ & 8691.333 & 201 & 1 & 11502.556 & $154934-269953$ \\
\hline 8792.058 & 100 & 5 & 11370.779 & $142045-25575^{\circ}$ & 8689.176 & 8 & 3 & 11505.411 & $19039_{2}^{\circ}-30544_{2}$ \\
\hline 8790.372 & 10 & 2 & 11372.960 & $19516_{2}^{\circ}-30889_{1}$ & 8688.429 & 2 & & 11506.400 & $21902_{4}^{\circ}-334083$ \\
\hline
\end{tabular}


TABLE 4. Classified lines of $\mathrm{Th} \mathrm{I}$-continued

\begin{tabular}{|c|c|c|c|c|c|c|c|c|c|}
\hline \multirow{2}{*}{$\begin{array}{c}\text { Wavelength } \\
\text { (§) }\end{array}$} & \multicolumn{2}{|c|}{ Intensity } & \multirow{2}{*}{$\begin{array}{l}\text { Wavenumber } \\
\quad\left(\mathrm{cm}^{-1}\right)\end{array}$} & \multirow{2}{*}{ Classification } & \multirow{2}{*}{$\begin{array}{c}\text { Wavelength } \\
\text { ( })\end{array}$} & \multicolumn{2}{|c|}{ Intensity } & \multirow{2}{*}{$\begin{array}{l}\text { Wavenumber } \\
\qquad\left(\mathrm{cm}^{-1}\right)\end{array}$} & \multirow{2}{*}{ Classification } \\
\hline & Lamp & Spark & & & & Lamp & Spark & & \\
\hline 8687.841 & 50 & 4 & 11507.179 & $15490 \AA-269976$ & 8605.146 & 2 & & 11617.762 & $20423 i-32041_{2}$ \\
\hline 8682.216 & 10 & 1 & 11514.634 & $17166_{5}-28680_{4}^{\circ}$ & 8604.922 & 4 & & 11618.064 & $10783_{2}^{\circ}-22401_{1}$ \\
\hline 8681.305 & 2 & & 11515.842 & $21902^{\circ}-334184$ & 8601.844 & 1 & & 11622.221 & $18930_{3}^{\circ}-305524$ \\
\hline 8679.439 & 15 & 1 & 11518.318 & $18614_{1}^{\circ}-30132_{2}$ & 8600.016 & 3 & & 11624.692 & $21645_{4}-33270_{4}^{\circ}$ \\
\hline 8679.303 & 1 & & 11518.499 & $20522_{2}^{\circ}-32041_{2}$ & 8596.686 & 5 & & 11629.195 & $15166_{3}^{\circ}-26796_{3}$ \\
\hline 8679.303 & 1 & & 11518.499 & $21890_{3}^{\circ}-334083$ & 8596.466 & 8 & 1 & 11629.492 & $20922_{2}^{\circ}-32551_{3}$ \\
\hline 8678.490 & 10 & 1 & 11519.578 & $173983-28917_{2}^{\circ}$ & 8595.655 & 1 & & 11630.590 & $18053_{4}^{\circ}-296845$ \\
\hline 8676.629 & $2 \mathrm{~s}$ & & 11522.048 & $8800_{4}-20322 \circ$ & 8595.359 & 1 & & 11630.990 & $21165_{3}^{\circ}-32796_{3}$ \\
\hline 8675.392 & 15 & 2 & 11523.691 & $17411_{3}^{\circ}-289343$ & 8595.307 & 1 & & 11631.060 & $21165_{3}^{\circ}-32796_{3}$ \\
\hline 8674.634 & 1 & & 11524.698 & $22248^{\circ}-337731$ & 8594.965 & 1 & & 11631.523 & $22669_{3}^{\circ}-34301_{4}$ \\
\hline 8672.281 & 1 & & 11527.825 & $17354_{1}^{\circ}-288822$ & 8593.103 & 100 & 10 & 11634.044 & $16346_{4}^{\circ}-27980_{3}$ \\
\hline 8672.159 & 3 & & 11527.987 & $21890_{3}^{\circ}-33418_{4}$ & 8589.687 & 3 & & 11638.670 & $19503 \stackrel{\circ}{3}-31141_{3}$ \\
\hline 8668.118 & 100 & 20 & 11533.362 & $128473-24381_{2}^{\circ}$ & 8588.435 & 50 & 2 & 11640.367 & $17501 \stackrel{\circ}{-291415}$ \\
\hline 8667.444 & 15 & 2 & 11534.258 & $173983-28932^{\circ}$ & 8588.221 & 8 & & 11640.657 & $19986_{6}^{\circ}-316265$ \\
\hline 8665.491 & 400 & 100 & 11536.858 & $75023-19039_{2}^{\circ}$ & 8583.974 & 2 & & 11646.416 & $14243_{1}^{\circ}-25890_{2}$ \\
\hline 8663.348 & 4 & & 11539.712 & $19273_{2}-30812_{2}^{\circ}$ & 8582.016 & 4 & & 11649.074 & $21645_{4}-33294_{3}^{\circ}$ \\
\hline 8662.270 & 2 & & 11541.148 & $18011 \stackrel{\circ}{-295526}$ & 8579.355 & 3 & & 11652.687 & $21738_{2}^{\circ}-33390_{1}$ \\
\hline 8660.494 & 1 & & 11543.515 & $21252_{2}^{\circ}-327963$ & 8577.279 & 100 & 15 & 11655.507 & $1214_{2}^{\circ}-23769_{1}$ \\
\hline 8659.754 & 2 & & 11544.501 & $20054_{2}-31599_{2}^{\circ}$ & 8575.327 & 75 & 5 & 11658.160 & $17224_{2}^{\circ}-288822$ \\
\hline 8657.541 & 3 & & 11547.452 & $22141_{3}^{\circ}-33689_{2}$ & 8574.577 & 2 & & 11659.180 & $185499_{2}-30208_{2}^{\circ}$ \\
\hline 8655.873 & 50 & 8 & 11549.677 & $142045-257530^{\circ}$ & 8574.081 & 50 & 10 & 11659.854 & $21077^{\circ}-32737_{5}$ \\
\hline 8651.256 & 3 & & 11555.841 & $18699_{2}-30255_{3}^{\circ}$ & 8573.122 & 500 & 100 & 11661.159 & $5563_{1}-17224_{2}^{\circ}$ \\
\hline 8650.455 & 2 & & 11556.911 & $21902^{\circ}-33459_{4}$ & 8572.075 & 4 & 1 & 11662.583 & $21902_{4}^{\circ}-335643$ \\
\hline 8649.144 & 50 & 5 & 11558.663 & $16783^{\circ}-283425$ & 8564.748 & 50 & 4 & 11672.560 & $18614_{1}^{\circ}-302861$ \\
\hline 8648.386 & 40 & 4 & 11559.676 & $20214_{3}^{\circ}-31774_{3}$ & 8564.570 & 5 & & 11672.803 & $180110^{\circ}-296845$ \\
\hline 8648.282 & 5 & & 11559.815 & $21539_{4}^{\circ}-33099_{3}$ & 8564.141 & 25 & 3 & 11673.387 & $20322{ }^{\circ}-31995_{4}$ \\
\hline 8645.310 & 150 & 25 & 11563.789 & $13962_{1}-25526^{\circ}$ & 8563.151 & 15 & 2 & 11674.737 & $21890_{3}^{\circ}-33564_{3}$ \\
\hline 8642.849 & 1 & & 11567.081 & $22877_{1}^{\circ}-34444_{2}$ & 8563.003 & 3 & & 11674.939 & $21539_{4}^{\circ}-33214_{5}$ \\
\hline 8641.383 & 3 & 1 & 11569.044 & $21890_{3}^{\circ}-33459_{4}$ & 8562.314 & 5 & 1 & 11675.878 & $21594_{3}-33270_{4}^{\circ}$ \\
\hline 8640.972 & 4 & & 11569.594 & $22877_{1}^{\circ}-34447_{4}$ & 8560.425 & 100 & 4 & 11678.454 & $118022-23481_{i}^{\circ}$ \\
\hline 8639.655 & 8 & & 11571.358 & $17847_{2}^{\circ}-29418_{2}$ & 8558.448 & 751 & 4 & 11681.152 & $13088_{3}-24769_{3}^{\circ}$ \\
\hline 8639.444 & 150 & 5 & 11571.640 & $10526_{3}^{\circ}-22098_{4}$ & 8556.589 & $10 \mathrm{~b}$ & & 11683.690 & $13297_{4}-24981_{3}^{\circ}$ \\
\hline 8638.360 & 100 & 5 & 11573.092 & $128473-24421_{3}^{\circ}$ & 8556.324 & 100 & 20 & 11684.052 & $10414_{4}^{\circ}-220984$ \\
\hline 8637.271 & 5 & 1 & 11574.551 & $16783_{4}^{\circ}-283583$ & 8554.945 & 200 & 50 & 11685.935 & $25580-14243 i$ \\
\hline 8636.928 & 2 & & 11575.011 & $17847_{2}^{\circ}-294221$ & 8553.895 & 1 & & 11687.370 & $16346_{4}^{\circ}-280345$ \\
\hline 8636.680 & 2 & & 11575.343 & $17073_{1}-28649_{1}^{\circ}$ & 8551.763 & 8 & 1 & 11690.284 & $22141_{3}^{\circ}-338314$ \\
\hline 8634.114 & 3 & & 11578.784 & $20214_{3}^{\circ}-31793_{4}$ & 8551.460 & 2 & & 11690.698 & $20322{ }^{\circ}-320124$ \\
\hline 8634.022 & $5 \mathrm{~h}$ & 1 & 11578.907 & $19516_{2}^{\circ}-31095_{1}$ & 8549.932 & 1 & & 11692.787 & $17411^{\circ}-291044$ \\
\hline 8633.966 & 3 & & 11578.982 & $18382{ }^{\circ}-29961_{1}$ & 8543.721 & 150 & 40 & 11701.287 & $15490 \AA-271915$ \\
\hline 8633.455 & 3 & 1 & 11579.667 & $22141_{3}^{\circ}-33721_{2}$ & 8542.618 & $3 \mathrm{~h}$ & & 11702.798 & $18053^{\circ}-297564$ \\
\hline 8632.169 & 3 & 1 & 11581.393 & $18699_{2}-30281_{i}$ & 8540.232 & 4 & 1 & 11706.068 & $18549_{2}-30255_{3}^{\circ}$ \\
\hline 8631.788 & 2 & & 11581.904 & $18069_{3}^{\circ}-29650_{2}$ & 8539.795 & 150 & 25 & 11706.667 & $63622_{2}-18069_{3}^{\circ}$ \\
\hline 8631.350 & 50 & 2 & 11582.491 & $142260-25809^{\circ}$ & 8537.379 & 3 & & 11709.979 & $22401_{1}-34111^{\circ}$ \\
\hline 8630.770 & $20 \mathrm{~h}$ & $2 \mathrm{~h}$ & 11583.270 & $22877^{\circ}-344605$ & 8534.674 & 50 & 2 & 11713.691 & $12847_{3}-24561_{3}^{\circ}$ \\
\hline 8630.330 & 5 & 1 & 11583.860 & $15863_{2}-27447_{2}^{\circ}$ & 8532.907 & 10 & 1 & 11716.117 & $14206_{4}^{\circ}-259234$ \\
\hline 8626.611 & 1 & & 11588.854 & $19948_{4}^{\circ}-31537_{3}$ & 8532.096 & 8 & 1 & 11717.230 & $19273_{2}-30990_{3}^{\circ}$ \\
\hline 8626.254 & 1 & & 11589.334 & $22855_{3}^{\circ}-34444_{2}$ & 8531.445 & 50 & 2 & 11718.124 & $11802_{2}-23521_{3}^{\circ}$ \\
\hline 8622.093 & 5 & 1 & 11594.927 & $13847_{2}-25442_{3}^{\circ}$ & 8530.911 & 40 & 3 & 11718.858 & $19039_{2}^{\circ}-30758_{2}$ \\
\hline 8621.315 & 75 & 2 & 11595.973 & $2869_{3}-14465_{2}^{\circ}$ & 8530.159 & 2 & & 11719.891 & $21575_{2}-332943$ \\
\hline 8618.598 & 5 & 1 & 11599.629 & $17073_{1}-28673_{2}^{\circ}$ & 8528.762 & 3 & & 11721.811 & $21668_{1}^{\circ}-33390_{1}$ \\
\hline 8616.218 & 100 & 10 & 11602.833 & $175010^{\circ}-29104_{4}$ & 8528.548 & 2 & & 11722.105 & $215752-33297_{2}^{\circ}$ \\
\hline 8614.687 & 5 & 1 & 11604.895 & $21668_{1}^{\circ}-33273_{1}$ & 8526.603 & 3 & & 11724.779 & $15618^{\circ}-273433$ \\
\hline 8611.754 & 3 & & 11608.847 & $195324-31141{ }^{\circ}$ & 8525.651 & 4 & & 11726.088 & $20054_{2}-31780^{\circ}$ \\
\hline 8609.370 & 4 & 1 & 11612.062 & $19817^{\circ}-314291$ & 8525.386 & 3 & & 11726.452 & $17959_{4}-29686_{3}^{\circ}$ \\
\hline 8607.724 & 8 & 2 & 11614.282 & $18930_{3}^{\circ}-30544_{2}$ & 8524.785 & 2 & & 11727.279 & $20566_{4}^{\circ}-322935$ \\
\hline
\end{tabular}


TABLE 4. Classified lines of Th $\mathrm{I}$-continued

\begin{tabular}{|c|c|c|c|c|c|c|c|c|c|}
\hline \multirow{2}{*}{$\begin{array}{c}\text { Wavelength } \\
(\AA)\end{array}$} & \multicolumn{2}{|c|}{ Intensity } & \multirow{2}{*}{$\begin{array}{l}\text { Wavenumber } \\
\quad\left(\mathrm{cm}^{-1}\right)\end{array}$} & \multirow{2}{*}{ Classification } & \multirow{2}{*}{$\begin{array}{c}\text { Wavelength } \\
\text { (̊̊) }\end{array}$} & \multicolumn{2}{|c|}{ Intensity } & \multirow{2}{*}{$\begin{array}{l}\text { Wavenumber } \\
\qquad\left(\mathrm{cm}^{-1}\right)\end{array}$} & \multirow{2}{*}{ Classification } \\
\hline & Lamp & Spark & & & & Lamp & Spark & & \\
\hline 8521.620 & 4 & & 11731.635 & $185492-30281 i$ & 8434.780 & 2 & & 11852.417 & $22855_{3}^{\circ}-34707_{3}$ \\
\hline 8519.327 & 50 & 3 & 11734.792 & $98045-215399$ & 8433.885 & 8 & 1 & 11853.674 & $18699_{2}-30553^{\circ}$ \\
\hline 8516.555 & 150 & 50 & 11738.612 & $195888^{\circ}-313264$ & 8432.485 & 10 & 2 & 11855.643 & $138472-25703_{2}^{\circ}$ \\
\hline 8515.615 & 2 & & 11739.907 & $13175_{4}^{\circ}-24915_{3}$ & 8430.586 & 15 & 1 & 11858.313 & $14032_{2}^{\circ}-25890_{2}$ \\
\hline 8514.901 & 8 & 1 & 11740.892 & $139621-25703_{2}^{\circ}$ & 8426.379 & 75 & & 11864.233 & $14247_{0}^{\circ}-26111_{1}$ \\
\hline 8513.624 & 2 & & 11742.653 & $18809_{4}^{\circ}-30552_{4}$ & 8424.031 & 100 & & 11867.540 & $14243 i-26111_{1}$ \\
\hline 8511.906 & 50 & 3 & 11745.023 & $18011 \stackrel{\circ}{-}-297564$ & 8423.529 & 3 & & 11868.247 & $21738_{2}^{\circ}-33606_{2}$ \\
\hline 8510.622 & 150 & 25 & 11746.795 & $11241_{3}^{\circ}-22988_{2}$ & 8422.942 & $2 \mathrm{~h}$ & & 11869.075 & $21539_{4}^{\circ}-33408_{3}$ \\
\hline 8509.273 & 2 & & 11748.657 & $19516_{2}^{\circ}-31265_{3}$ & 8422.464 & 1 & & 11869.748 & $22098_{4}-33967^{\circ}$ \\
\hline 8508.186 & 5 & 1 & 11750.158 & $23655_{4}^{\circ}-35405_{3}$ & 8421.227 & 500 & 100 & 11871.492 & $3865_{1}-15736^{\circ}$ \\
\hline 8501.756 & 8 & & 11759.045 & $7280_{2}-19039_{2}^{\circ}$ & 8419.431 & 2 & & 11874.024 & $20922_{2}^{\circ}-327963$ \\
\hline 8501.438 & 50 & 5 & 11759.485 & $173983-29157_{3}^{\circ}$ & 8418.004 & 200 & 75 & 11876.037 & $12114_{2}^{\circ}-23990_{2}$ \\
\hline 8500.011 & 10 & 2 & 11761.459 & $22669_{3}^{\circ}-34431_{3}$ & 8417.605 & 8 & 2 & 11876.600 & $15618^{\circ}-27495_{4}$ \\
\hline 8498.731 & 2 & & 11763.230 & $16217_{2}^{\circ}-27980_{3}$ & 8417.071 & 75 & 3 & 11877.353 & $15166_{3}^{\circ}-270443$ \\
\hline 8496.449 & $50 \mathrm{~b}$ & & 11766.390 & $88004-20566_{4}^{\circ}$ & 8416.742 & $500 w$ & 150 & 11877.818 & $0_{2}-11877^{\circ}$ \\
\hline 8496.340 & $25 b$ & & 11766.541 & $17166_{5}-28932_{4}^{\circ}$ & 8415.375 & 75 & 4 & 11879.747 & $20322{ }^{\circ}-322025$ \\
\hline 8496.044 & 4 & & 11766.951 & $15493_{4}-27260_{3}^{\circ}$ & 8414.938 & 50 & 1 & 11880.364 & $11601_{1}-23481^{\circ}$ \\
\hline 8491.811 & 10 & & 11772.816 & $154934-27266_{4}^{\circ}$ & 8414.273 & 4 & & 11881.303 & $16346_{4}^{\circ}-28227_{4}$ \\
\hline 8491.368 & 2 & & 11773.430 & $179594-297338$ & .8414 .005 & 2 & & 11881.681 & $21645_{4}^{\circ}-33527_{4}$ \\
\hline 8488.564 & 1 & & 11777.320 & $22669_{3}^{\circ}-34447_{4}$ & 8411.913 & 100 & 5 & 11884.636 & $171665-29050 \AA$ \\
\hline 8487.478 & 15 & 1 & 11778.826 & $16783_{4}^{\circ}-28562_{4}$ & 8410.763 & 5 & & 11886.261 & $19713_{3}-31599_{2}^{\circ}$ \\
\hline 8486.561 & 50 & 2 & 11780.099 & $18069_{3}^{\circ}-29849_{4}$ & 8407.010 & 75 & 3 & 11891.567 & $20566_{4}^{\circ}-32458_{4}$ \\
\hline 8486.108 & 2 & & 11780.728 & $20214_{3}^{\circ}-319954$ & 8406.674 & 40 & 2 & 11892.043 & $11877_{1}^{\circ}-23769_{1}$ \\
\hline 8485.600 & 3 & & 11781.433 & $18053^{\circ}-298353$ & 8406.314 & 20 & 1 & 11892.552 & $13088_{3}-24981_{3}^{\circ}$ \\
\hline 8483.298 & 50 & 2 & 11784.630 & $17959_{4}-29744_{3}^{\circ}$ & 8401.986 & 100 & 8 & 11898.678 & $14481 \stackrel{\circ}{6}-263805$ \\
\hline 8482.478 & 1 & & 11785.769 & $23655_{4}^{\circ}-354403$ & 8400.154 & 2 & & 11901.273 & $22396_{1}^{\circ}-34298_{1}$ \\
\hline 8478.505 & 200 & & 11791.292 & $11241^{\circ}-230324$ & 8399.253 & 100 & 8 & 11902.550 & $15618_{3}^{\circ}-27521_{4}$ \\
\hline 8478.353 & 500 & & 11791.504 & $5563_{1}-17354_{i}^{\circ}$ & 8398.171 & 100 & 8 & 11904.083 & $18382 \circ-302861$ \\
\hline 8473.657 & 1 & & 11798.038 & $20214_{3}^{\circ}-320124$ & 8392.847 & 50 & 3 & 11911.634 & $17398_{3}-29310_{4}^{\circ}$ \\
\hline 8472.593 & 40 & 3 & 11799.520 & $17398_{3}-29197_{2}^{\circ}$ & 8392.397 & 40 & & 11912.273 & $19516_{2}^{\circ}-314291$ \\
\hline 8471.823 & 200 & 50 & 11800.592 & $11802_{2}-236032_{2}^{\circ}$ & 8388.528 & 100 & 10 & 11917.767 & $7795^{\circ}-197133$ \\
\hline 8470.358 & 5 & & 11802.633 & $19273_{2}-31075_{2}^{\circ}$ & 8385.726 & 100 & 10 & 11921.750 & $128473-24769_{3}^{\circ}$ \\
\hline 8469.451 & 3 & & 11803.897 & $17847_{2}^{\circ}-29650_{2}$ & 8384.927 & 8 & 2 & 11922.886 & $22401_{1}-34324_{2}^{\circ}$ \\
\hline 8468.973 & 8 & 2 & 11804.563 & $15166_{3}^{\circ}-26971_{4}$ & 8384.639 & 1 & & 11923.295 & $22508_{2}^{\circ}-34431_{3}$ \\
\hline 8467.164 & 5 & 1 & 11807.086 & $15863_{2}-27670_{3}^{\circ}$ & 8383.073 & 8 & 2 & 11925.522 & $19039_{2}^{\circ}-309643$ \\
\hline 8465.668 & 100 & 25 & 11809.172 & $116011-234108$ & 8379.754 & 75 & 4 & 11930.246 & $18614_{1}^{\circ}-30544_{2}$ \\
\hline 8464.753 & 50 & 3 & 11810.448 & $15863_{2}-27674_{2}^{\circ}$ & 8379.219 & 100 & 8 & 11931.008 & $3687_{2}-156183$ \\
\hline 8464.236 & 150 & 50 & 11811.170 & $8243_{2}^{\circ}-200542$ & 8376.896 & 3 & & 11934.316 & $21165_{3}^{\circ}-33099_{3}$ \\
\hline 8462.015 & 4 & & 11814.270 & $159703-27784_{2}^{\circ}$ & 8375.333 & 1 & & 11936.543 & $22508_{2}^{\circ}-34444_{2}$ \\
\hline 8456.341 & 50 & 2 & 11822.197 & $4961_{4}-16783 \stackrel{\circ}{4}$ & 8374.766 & 50 & 2 & 11937.351 & $21668_{1}^{\circ}-33606_{2}$ \\
\hline 8455.209 & 2 & & 11823.780 & $195033-313264$ & 8374.202 & 50 & 3 & 11938.155 & $11802_{2}-23741^{\circ}$ \\
\hline 8454.924 & 50 & 4 & 11824.178 & $154934-27317_{3}^{\circ}$ & 8371.658 & 2 & & 11941.783 & $21890_{3}^{\circ}-33831_{4}$ \\
\hline 8453.419 & 5 & 1 & 11826.283 & $20214^{\circ}-32041_{2}$ & 8371.303 & 1 & & 11942.290 & $18069_{3}^{\circ}-30011_{3}$ \\
\hline 8451.839 & 2 & & 11828.494 & $22338_{3}^{\circ}-341673$ & 8369.332 & 200 & 50 & 11945.102 & $13945_{3}^{\circ}-25890_{2}$ \\
\hline 8450.836 & $15 \mathrm{~b}$ & & 11829.898 & $15736_{1}^{\circ}-27566_{2}$ & 8367.390 & 150 & 20 & 11947.874 & $15618_{3}^{\circ}-27566_{2}$ \\
\hline 8449.925 & 2 & & 11831.173 & $21890_{3}^{\circ}-33721_{2}$ & 8366.073 & 150 & 15 & 11949.755 & $11802_{2}-23752_{2}^{\circ}$ \\
\hline 8447.968 & 4 & 1 & 11833.914 & $23015 \circ-348495$ & 8365.198 & 2 & & 11951.005 & $21738_{2}^{\circ}-33689_{2}$ \\
\hline 8446.516 & 500 & 150 & 11835.948 & $11197^{\circ}-230324$ & 8360.487 & 75 & 5 & 11957.739 & $18053 \stackrel{\circ}{4}-300113$ \\
\hline 8445.495 & 200 & 150 & 11837.379 & $8111_{4}-19948 \stackrel{\circ}{\circ}$ & 8358.728 & 150 & 200 & 11960.256 & $11241 \stackrel{\circ}{3}-23201_{3}$ \\
\hline 8445.214 & $100 \mathrm{~b}$ & 8 & 11837.773 & $18011 \stackrel{\circ}{-}-298494$ & 8357.829 & 50 & 3 & 11961.542 & $13847_{2}-25809^{\circ}$ \\
\hline 8440.671 & 25 & & 11844.145 & $17073_{1}-28917_{2}^{\circ}$ & 8357.192 & 1 & & 11962.454 & $22338_{3}^{\circ}-34301_{4}$ \\
\hline 8440.660 & $50 w$ & 4 & 11844.160 & $17073_{1}-28917_{2}^{\circ}$ & 8355.142 & 50 & 4 & 11965.389 & $21594_{3}-33560_{4}^{\circ}$ \\
\hline 8440.578 & 25 & & 11844.275 & $142045-26048 \%$ & 8350.865 & 2 & & 11971.517 & $22877_{1}^{\circ}-34849_{5}$ \\
\hline 8439.849 & 1 & & 11845.298 & $19948_{4}^{\circ}-317934$ & 8346.537 & 50 & 3 & 11977.725 & $13945_{3}^{\circ}-259234$ \\
\hline 8438.789 & 8 & & 11846.786 & $13962_{1}-25809_{1}^{\circ}$ & 8345.867 & 50 & 5 & 11978.686 & $18574_{1}-30553_{2}^{\circ}$ \\
\hline
\end{tabular}


TABle 4. Classified lines of Th $\mathrm{I}$-continued

\begin{tabular}{|c|c|c|c|c|c|c|c|c|c|}
\hline \multirow{2}{*}{$\begin{array}{l}\text { Wavelength } \\
\text { (§) }\end{array}$} & \multicolumn{2}{|c|}{ Intensity } & \multirow{2}{*}{$\begin{array}{l}\text { Wavenumber } \\
\left(\mathrm{cm}^{-1}\right)\end{array}$} & \multirow{2}{*}{ Classification } & \multirow{2}{*}{$\begin{array}{c}\text { Wavelength } \\
(\AA)\end{array}$} & \multicolumn{2}{|c|}{ Intensity } & \multirow{2}{*}{$\begin{array}{l}\text { Wavenumber } \\
\left(\mathrm{cm}^{-1}\right)\end{array}$} & \multirow{2}{*}{ Classification } \\
\hline & Lamp & Spark & & & & Lamp & Spark & & \\
\hline 8344.174 & 8 & 2 & 11981.117 & $19948{ }^{\circ}-31929_{3}$ & 8252.398 & 400 & 50 & 12114.360 & $0_{2}-12114_{2}^{\circ}$ \\
\hline 8341.475 & 50 & 8 & 11984.993 & $20566_{4}^{\circ}-32551_{3}$ & 8246.170 & $2 \mathrm{~h}$ & & 12123.509 & $17073_{1}-29197_{1}^{\circ}$ \\
\hline 8339.403 & 5 & 2 & 11987.971 & $17847_{2}^{\circ}-29835_{3}$ & 8246.011 & 25 & 4 & 12123.743 & $21594_{3}-33718_{2}^{\circ}$ \\
\hline 8337.330 & $2 \mathrm{~h}$ & 1 & 11990.952 & $21077 \circ-330686$ & 8245.781 & 2 & & 12124.081 & $17073_{1}-29197_{2}^{\circ}$ \\
\hline 8336.934 & 20 & 2 & 11991.521 & $195324-31523^{\circ}$ & 8243.712 & 2 & & 12127.124 & $211435-33270_{4}^{\circ}$ \\
\hline 8335.700 & 50 & 8 & 11993.297 & $15618^{\circ}-276123$ & 8239.619 & 5 & 1 & 12133.148 & $128473-24981_{3}^{\circ}$ \\
\hline 8333.441 & 50 & 5 & 11996.548 & $215943-335911_{3}^{\circ}$ & 8237.739 & 20 & 2 & 12135.917 & $20322{ }^{\circ}-324584$ \\
\hline 8330.455 & 500 & 300 & 12000.848 & $75023-195033^{\circ}$ & 8236.739 & 4 & & 12137.390 & $21077^{\circ}-332145$ \\
\hline 8329.299 & 2 & & 12002.513 & $11601_{1}-236032$ & 8235.819 & 4 & 1 & 12138.746 & $195324-31671_{4}^{\circ}$ \\
\hline 8328.990 & 15 & 2 & 12002.959 & $18011^{\circ}-30014_{4}$ & 8235.484 & 1 & & 12139.240 & $23655_{4}^{\circ}-35794_{4}$ \\
\hline 8328.338 & 15 & 2 & 12003.898 & $18549_{2}-30553_{2}^{\circ}$ & 8234.937 & 50 & 4 & 12140.046 & $11601_{1}-23741_{1}^{\circ}$ \\
\hline 8327.213 & 15 & 2 & 12005.520 & $15490 \circ-27495_{4}$ & 8234.937 & 50 & 4 & 12140.046 & $215943-33734_{2}^{\circ}$ \\
\hline 8326.068 & 5 & 1 & 12007.171 & $17411^{\circ}-29418_{2}$ & 8234.346 & 25 & 3 & 12140.918 & $16217_{2}^{\circ}-28358_{3}$ \\
\hline 8322.891 & 5 & 1 & 12011.754 & $16346_{4}^{\circ}-283583$ & 8233.377 & 2 & & 12142.347 & $20054_{2}-32197^{\circ}$ \\
\hline 8320.859 & 500 & 150 & 12014.688 & $75023-19516_{2}^{\circ}$ & 8232.673 & 1 & & 12143.385 & $21575_{2}-33718_{2}^{\circ}$ \\
\hline 8317.106 & 2 & & 12020.109 & $21668^{\circ}-33689_{2}$ & 8232.451 & 3 & & 12143.712 & $18614_{1}^{\circ}-30758_{2}$ \\
\hline 8316.595 & 8 & 2 & 12020.848 & $17398_{3}-29419_{2}^{\circ}$ & 8232.310 & 2 & & 12143.920 & $21165_{3}^{\circ}-33309_{2}$ \\
\hline 8314.281 & 4 & 1 & 12024.193 & $18699_{2}-30723^{\circ}$ & 8231.410 & 100 & 15 & 12145.248 & $13297_{4}-25442^{\circ}$ \\
\hline 8314.067 & 5 & 1 & 12024.503 & $13297_{4}-25321_{3}^{\circ}$ & 8228.725 & 1 & & 12149.211 & $18574_{1}-30723^{\circ}$ \\
\hline 8313.281 & 2 & & 12025.640 & $20054_{2}-32080_{1}^{\circ}$ & 8227.672 & 50 & 4 & 12150.766 & $139621-261132$ \\
\hline 8311.019 & 2 & & 12028.913 & $20522_{2}^{\circ}-32551_{3}$ & 8227.482 & 50 & 8 & 12151.046 & $16783 \stackrel{9}{4}-289343$ \\
\hline 8309.257 & 15 & & 12031.463 & $15490_{0}^{\circ}-27521_{4}$ & 8227.096 & 3 & & 12151.617 & $11601_{1}-23752_{2}^{\circ}$ \\
\hline 8307.245 & 1 & & 12034.377 & $18930_{3}^{\circ}-30964_{3}$ & 8224.956 & 8 & 2 & 12154.778 & $21645_{4}-33800_{3}^{\circ}$ \\
\hline 8306.411 & 1 & & 12035.586 & $21738_{2}^{\circ}-33773_{1}$ & 8223.407 & 8 & & 12157.068 & $19713_{3}-31870_{2}^{\circ}$ \\
\hline 8304.844 & 5 & 1 & 12037.857 & $22669_{3}^{\circ}-347073$ & 8221.666 & 1 & & 12159.642 & $21575_{2}-33734_{2}^{\circ}$ \\
\hline 8304.412 & 50 & 3 & 12038.483 & $19588^{\circ}-316265$ & 8220.872 & 3 & & 12160.817 & $15863_{2}-28024_{1}^{\circ}$ \\
\hline 8304.030 & 8 & 1 & 12039.037 & $185741-30613{ }^{\circ}$ & 8214.147 & 100 & 10 & 12170.773 & $15970_{3}-28140_{4}^{\circ}$ \\
\hline 8298.353 & 10 & 2 & 12047.273 & $19948{ }_{4}^{\circ}-31995_{4}$ & 8212.219 & 5 & 1 & 12173.630 & $14206_{4}^{\circ}-263805$ \\
\hline 8297.984 & 3 & & 12047.808 & $22399^{\circ}-34447_{4}$ & 8210.525 & 2 & & 12176.142 & $23655_{4}^{\circ}-358313$ \\
\hline 8297.377 & 25 & 2 & 12048.690 & $14206^{\circ}-26255_{4}$ & 8210.047 & 4 & & 12176.851 & $15166^{\circ}-273433$ \\
\hline 8297.172 & $50 \mathrm{~b}$ & 5 & 12048.987 & $3687_{2}-15736_{1}^{\circ}$ & 8209.444 & 100 & 8 & 12177.745 & $15493_{4}-27670_{3}^{\circ}$ \\
\hline 8295.542 & 100 & 8 & 12051.355 & $17501 \stackrel{\circ}{-}-295526$ & 8208.596 & 2 & & 12179.003 & $17073_{1}-29252_{2}^{\circ}$ \\
\hline 8294.880 & $8 \mathrm{c}$ & & 12052.317 & $216681-33721_{2}$ & 8208.596 & 2 & & 12179.003 & $17073_{1}-29252_{2}^{\circ}$ \\
\hline 8292.521 & 100 & 8 & 12055.745 & $16217_{2}^{\circ}-28273_{2}$ & 8207.475 & 100 & 10 & 12180.666 & $142045-26384_{4}^{\circ}$ \\
\hline 8292.039 & 4 & & 12056.446 & $21252_{2}^{\circ}-33309_{2}$ & 8205.892 & 8 & & 12183.016 & $17501{ }^{\circ}-296845$ \\
\hline 8291.869 & 1 & & 12056.693 & $19039_{2}^{\circ}-31095_{1}$ & 8205.103 & 20 & 2 & 12184.188 & $195324-31716^{\circ}$ \\
\hline 8291.523 & 2 & & 12057.196 & $17959_{4}-30017_{3}^{\circ}$ & 8202.148 & 100 & 10 & 12188.577 & $13847_{2}-26036^{\circ}$ \\
\hline 8290.864 & 2 & & 12058.155 & $13297_{4}-25355_{4}^{\circ}$ & 8201.354 & 1 & & 12189.757 & $22401_{1}-34591^{\circ}$ \\
\hline 8289.447 & 2 & & 12060.216 & $142260-26287_{i}^{\circ}$ & 8198.438 & 10 & 2 & 12194.093 & $17224_{2}^{\circ}-29418_{2}$ \\
\hline 8288.404 & 50 & 5 & 12061.733 & $16783^{\circ}-288454$ & 8195.942 & 8 & 1 & 12197.806 & $17224_{2}^{\circ}-294221$ \\
\hline 8287.110 & 25 & 3 & 12063.617 & $18069_{3}^{\circ}-301322$ & 8194.698 & 3 & & 12199.658 & $22508_{2}^{\circ}-34707_{3}$ \\
\hline 8287.023 & 8 & & 12063.744 & $17354_{1}^{\circ}-29418_{2}$ & 8194.398 & 100 & 25 & 12200.105 & $17959_{4}-30160_{4}^{\circ}$ \\
\hline 8284.481 & 3 & & 12067.445 & $17354^{\circ}-294221$ & 8194.398 & 100 & 25 & 12200.105 & $20867_{7}^{\circ}-330686$ \\
\hline 8276.232 & 20 & 2 & 12079.473 & $14032_{2}^{\circ}-26111_{1}$ & 8193.239 & 5 & 1 & 12201.831 & $230158-352165$ \\
\hline 8275.628 & 400 & 150 & 12080.354 & $11197^{\circ}-23277_{5}$ & 8192.136 & 3 & & 12203.473 & $22669 \stackrel{\circ}{3}-348734$ \\
\hline 8273.609 & 8 & 1 & 12083.302 & $17073_{1}-29157_{1}^{\circ}$ & 8191.069 & 10 & 1 & 12205.063 & $21594_{3}-33799_{4}^{\circ}$ \\
\hline 8270.940 & 3 & 1 & 12087.202 & $21575_{2}-33662_{1}^{\circ}$ & 8190.889 & 50 & 8 & 12205.331 & $19588 \circ-31793_{4}$ \\
\hline 8267.412 & 2 & & 12092.360 & $22338_{3}^{\circ}-344313$ & 8190.479 & 2 & & 12205.942 & $215943-338003$ \\
\hline 8263.926 & 150 & 20 & 12097.460 & $98045-21902{ }^{\circ}$ & 8189.938 & 4 & & 12206.749 & $19039_{2}^{\circ}-31245_{2}$ \\
\hline 8261.010 & 100 & 25 & 12101.731 & $15490_{0}^{\circ}-275915$ & 8186.915 & 500 & 100 & 12211.256 & $81114-203220$ \\
\hline 8260.391 & 1 & & 12102.638 & $19039_{2}^{\circ}-31141_{3}$ & 8186.217 & 8 & & 12212.297 & $18549_{2}-30761_{3}^{\circ}$ \\
\hline 8259.505 & 100 & 8 & 12103.936 & $8111_{4}-20214_{3}^{\circ}$ & 8183.838 & 10 & 2 & 12215.847 & $19986_{6}^{\circ}-322025$ \\
\hline 8258.339 & 8 & 2 & 12105.645 & $22338_{3}^{\circ}-34444_{2}$ & 8183.723 & 2 & & 12216.019 & $16346 \stackrel{\circ}{4}-285624$ \\
\hline 8254.741 & 100 & 15 & 12110.921 & $10526_{3}^{\circ}-22637_{3}$ & 8179.030 & 8 & 1 & 12223.028 & $7280_{2}-195033$ \\
\hline 8253.613 & 75 & 8 & 12112.576 & $11877_{1}^{\circ}-23990_{2}$ & 8178.829 & 2 & & 12223.329 & $10414_{4}^{\circ}-226373$ \\
\hline
\end{tabular}


TABLE 4. Classified lines of Th $\mathrm{I}$-continued

\begin{tabular}{|c|c|c|c|c|c|c|c|c|c|}
\hline \multirow{2}{*}{$\begin{array}{l}\text { Wavelength } \\
\text { (Å) }\end{array}$} & \multicolumn{2}{|c|}{ Intensity } & \multirow{2}{*}{$\begin{array}{l}\text { Wavenumber } \\
\left(\mathrm{cm}^{-1}\right)\end{array}$} & \multirow{2}{*}{ Classification } & \multirow{2}{*}{$\begin{array}{c}\text { Wavelength } \\
\text { (̊̊) }\end{array}$} & \multicolumn{2}{|c|}{ Inten sity } & \multirow{2}{*}{$\begin{array}{c}\text { Wavenumber } \\
\left(\mathrm{cm}^{-1}\right)\end{array}$} & \multirow{2}{*}{ Classification } \\
\hline & Lamp & Spark & & & & Lamp & Spark & & \\
\hline 8178.318 & 2 & & 12224.092 & $19817_{1}^{\circ}-32041_{2}$ & 8092.238 & 75 & 5 & 12354.124 & $18574_{1}-30928_{1}^{\circ}$ \\
\hline 8174.704 & 2 & & 12229.496 & $20566_{4}^{\circ}-32796_{3}$ & 8091.356 & 2 & & 12355.470 & $216454-34001_{4}^{\circ}$ \\
\hline 8174.328 & 2 & & 12230.059 & $13175_{4}^{\circ}-25405_{4}$ & 8089.896 & 4 & & 12357.700 & $16783{ }^{\circ}-291415$ \\
\hline 8174.086 & 4 & & 12230.421 & $20054_{2}-32285_{3}^{\circ}$ & 8089.481 & 75 & 8 & 12358.334 & $98045-22163_{4}^{\circ}$ \\
\hline 8172.122 & 50 & 3 & 12233.360 & $13088_{3}-25321{ }_{3}^{\circ}$ & 8088.701 & 4 & & 12359.526 & $154934-27852^{\circ}$ \\
\hline 8171.652 & 2 & & 12234.064 & $22855_{3}^{\circ}-35089_{3}$ & 8087.495 & 5 & 2 & 12361.369 & $18011^{\circ}-303726$ \\
\hline 8169.793 & 400 & 50 & 12236.848 & $7280_{2}-19516_{2}^{\circ}$ & 8087.263 & 2 & & 12361.723 & $15618_{3}^{\circ}-27980_{3}$ \\
\hline 8168.775 & 5 & & 12238.373 & $18574_{1}-30812_{2}^{\circ}$ & 8085.228 & 200 & 50 & 12364.835 & $8800_{4}-21165_{3}^{\circ}$ \\
\hline 8167.865 & 3 & & 12239.736 & $17411_{3}^{\circ}-29650_{2}$ & 8084.451 & 1 & & 12366.023 & $22508_{2}^{\circ}-34874_{3}$ \\
\hline 8163.355 & 1 & & 12246.498 & $220984-34344_{4}^{\circ}$ & 8076.980 & 5 & 1 & 12377.461 & $15970_{3}-28347_{2}^{\circ}$ \\
\hline 8162.064 & 50 & 3 & 12248.435 & $19532_{4}-31780_{3}^{\circ}$ & 8075.651 & 150 & 20 & 12379.498 & $11802_{2}-24182_{2}^{\circ}$ \\
\hline 8161.552 & 2 & & 12249.204 & $13847_{2}-26096_{3}^{\circ}$ & 8074.472 & 3 & & 12381.306 & $18431_{3}-30812_{2}^{\circ}$ \\
\hline 8159.737 & 400 & 100 & 12251.928 & $6362_{2}-18614_{1}^{\circ}$ & 8070.785 & 2 & & 12386.962 & $20922_{2}^{\circ}-333092$ \\
\hline 8159.016 & 2 & & 12253.011 & $21165_{3}^{\circ}-33418_{4}$ & 8068.729 & 5 & 1 & 12390.118 & $19039_{2}^{\circ}-31429_{1}$ \\
\hline 8157.540 & 50 & 8 & 12255.228 & $175010-297564$ & 8066.826 & 75 & 8 & 12393.041 & $13297_{4}-25690^{\circ}$ \\
\hline 8155.949 & 5 & 1 & 12257.619 & $19516_{2}^{\circ}-31774_{3}$ & 8064.380 & 2 & & 12396.800 & $20566_{4}^{\circ}-329635$ \\
\hline 8151.973 & 2 & & 12263.597 & $18549_{2}-30812_{2}^{\circ}$ & 8062.640 & 200 & 50 & 12399.475 & $19227_{6}^{\circ}-316265$ \\
\hline 8150.878 & 1 & & 12265.245 & $21902_{4}^{\circ}-34167_{3}$ & 8062.345 & 4 & & 12399.929 & $15166_{3}^{\circ}-27566_{2}$ \\
\hline 8150.703 & 25 & & 12265.508 & $13847_{2}-26113_{2}^{\circ}$ & 8061.918 & 3 & & 12400.586 & $13962_{1}-26363_{2}^{\circ}$ \\
\hline 8149.704 & 50 & 4 & 12267.011 & $13088_{3}-25355_{4}^{\circ}$ & 8057.973 & 50 & 2 & 12406.657 & $215943-34001_{4}^{\circ}$ \\
\hline 8148.901 & 3 & & 12268.220 & $22163_{4}^{\circ}-344313$ & 8054.929 & 2 & & 12411.345 & $21890 \stackrel{3}{-3} 34301_{4}$ \\
\hline 8146.748 & 5 & & 12271.462 & $19503_{3}^{\circ}-317743$ & 8052.715 & 5 & & 12414.758 & $203220^{\circ}-327375$ \\
\hline 8146.237 & 5 & & 12272.232 & $22877_{1}^{\circ}-35149_{2}$ & 8051.486 & 1 & & 12416.653 & $211435-33560_{4}^{\circ}$ \\
\hline 8144.017 & 4 & & 12275.577 & $18614_{1}^{\circ}-30889_{1}$ & 8050.067 & 5 & 1 & 12418.841 & $10783_{2}^{\circ}-23201_{3}$ \\
\hline 8143.143 & 500 & 300 & 12276.895 & $8800_{4}-21077_{0}^{\circ}$ & 8048.611 & 2 & & 12421.088 & $19532_{4}-31953^{\circ}$ \\
\hline 8142.679 & 20 & 3 & 12277.595 & $13297_{4}-25575_{4}^{\circ}$ & 8046.838 & 5 & & 12423.825 & $17411_{3}^{\circ}-29835_{3}$ \\
\hline 8138.474 & 200 & 100 & 12283.938 & $5563_{1}-17847_{2}^{\circ}$ & 8046.331 & 8 & 3 & 12424.608 & $19588^{\circ}-320124$ \\
\hline 8137.940 & 50 & 4 & 12284.744 & $12114_{2}^{\circ}-24399_{3}$ & 8045.182 & 5 & 1 & 12426.382 & $19503_{3}^{\circ}-31929_{3}$ \\
\hline 8137.367 & 4 & & 12285.609 & $17847_{2}^{\circ}-301322$ & 8044.989 & $4 \mathrm{~b}$ & & 12426.680 & $17224_{2}^{\circ}-29650_{2}$ \\
\hline 8137.256 & 2 & & 12285.777 & $230150^{\circ}-353005$ & 8043.239 & 1 & & 12429.384 & $21738_{2}^{\circ}-34167_{3}$ \\
\hline 8135.798 & 2 & & 12287.979 & $17398_{3}-29686_{3}^{\circ}$ & 8038.691 & 2 & & 12436.416 & $21252_{2}^{\circ}-33689_{2}$ \\
\hline 8134.633 & 5 & & 12289.738 & $22141_{3}^{\circ}-344313$ & 8037.699 & 3 & & 12437.951 & $17411_{3}^{\circ}-29849_{4}$ \\
\hline 8134.085 & 2 & & 12290.566 & $19503_{3}^{\circ}-31793_{4}$ & 8035.618 & 50 & & 12441.172 & $21165_{3}^{\circ}-33606_{2}$ \\
\hline 8133.855 & 1 & & 12290.914 & $18699_{2}-30990_{3}^{\circ}$ & 8032.883 & 10 & & 12445.408 & $15166_{3}^{\circ}-276123$ \\
\hline 8132.964 & 50 & 1 & 12292.260 & $21539^{\circ}-338314$ & 8032.433 & 200 & 50 & 12446.105 & $7502_{3}-19948^{\circ}$ \\
\hline 8131.517 & 5 & 1 & 12294.448 & $22855_{3}^{\circ}-35149_{2}$ & 8031.574 & 1 & & 12447.436 & $19713_{3}-32160_{2}^{\circ}$ \\
\hline 8129.681 & 50 & 5 & 12297.224 & $16351_{0}-28649_{1}^{\circ}$ & 8030.188 & 50 & 4 & 12449.585 & $4961_{4}-17411_{3}^{\circ}$ \\
\hline 8129.405 & 150 & 25 & 12297.642 & $2869_{3}-15166_{3}^{\circ}$ & 8027.103 & 3 & & 12454.369 & $18053_{4}^{\circ}-305085$ \\
\hline 8125.877 & 4 & & 12302.981 & $22141_{3}^{\circ}-344442$ & 8026.438 & 10 & 1 & 12455.401 & $15493_{4}-27948_{4}^{\circ}$ \\
\hline 8122.725 & 200 & 50 & 12307.755 & $19986_{6}^{\circ}-322935$ & 8026.192 & 50 & 8 & 12455.783 & $18809_{4}^{\circ}-31265_{3}$ \\
\hline 8121.058 & 15 & 1 & 12310.282 & $13945_{3}^{\circ}-26255_{4}$ & 8025.708 & 50 & 8 & 12456.534 & $13297_{4}-25753^{\circ}$ \\
\hline 8117.557 & 5 & & 12315.591 & $18930_{3}^{\circ}-31245_{2}$ & 8024.235 & 100 & 100 & 12458.821 & $12847_{3}-25306_{2}^{\circ}$ \\
\hline 8114.539 & 50 & 4 & 12320.171 & $16783_{4}^{\circ}-291044$ & 8023.886 & 2 & & 12459.363 & $20322{ }^{\circ}-32781_{4}$ \\
\hline 8108.929 & 4 & 1 & 12328.695 & $15166_{3}^{\circ}-27495_{4}$ & 8022.733 & 3 & & 12461.153 & $18809_{4}^{\circ}-31271_{5}$ \\
\hline 8107.508 & 3 & & 12330.855 & $14465_{2}^{\circ}-267963$ & 8022.191 & $75 b$ & 25 & 12461.995 & $10526^{\circ}-22988_{2}$ \\
\hline 8106.805 & 2 & & 12331.925 & $18809_{4}^{\circ}-31141_{3}$ & 8014.499 & 50 & 3 & 12473.955 & $128473-25321_{3}^{\circ}$ \\
\hline 8104.536 & 2 & & 12335.377 & $18930_{3}^{\circ}-31265_{3}$ & 8013.499 & 15 & 2 & 12475.512 & $18069_{3}^{\circ}-30544_{2}$ \\
\hline 8103.669 & 3 & 1 & 12336.697 & $20214_{3}^{\circ}-32551_{3}$ & 8010.708 & 50 & 4 & 12479.859 & $22669_{3}^{\circ}-35149_{2}$ \\
\hline 8101.873 & 2 & & 12339.432 & $22877_{1}^{\circ}-352165$ & 8008.645 & 2 & & 12483.073 & $173983-29881_{4}^{\circ}$ \\
\hline 8100.839 & 1 & & 12341.007 & $210770^{\circ}-33418_{4}$ & 8008.397 & 5 & & 12483.460 & $18069_{3}^{\circ}-30552_{4}$ \\
\hline 8097.857 & 4 & & 12345.551 & $19948_{4}^{\circ}-32293_{5}$ & 8008.272 & 2 & & 12483.655 & $15863_{2}-28347_{2}^{\circ}$ \\
\hline 8097.483 & 4 & & 12346.122 & $173983-29744_{3}^{\circ}$ & 8007.988 & 1 & & 12484.098 & $197133-32197^{\circ}$ \\
\hline 8096.253 & 20 & 3 & 12347.997 & $17501_{0}^{\circ}-29849_{4}$ & 8006.477 & 25 & & 12486.454 & $13088_{3}-25575_{4}^{\circ}$ \\
\hline 8093.631 & 200 & 75 & 12351.997 & $3865_{1}-16217_{2}^{\circ}$ & 8006.443 & 15 & & 12486.507 & $20922_{2}^{\circ}-33408_{3}$ \\
\hline 8092.238 & 75 & 5 & 12354.124 & $13088_{3}-25442_{3}^{\circ}$ & 8003.446 & 50 & 5 & 12491.182 & $195324-320230^{\circ}$ \\
\hline
\end{tabular}


TABLE 4. Classified lines of Th I-continued

\begin{tabular}{|c|c|c|c|c|c|c|c|c|c|}
\hline \multirow{2}{*}{$\begin{array}{l}\text { Wavelength } \\
\text { (Å) }\end{array}$} & \multicolumn{2}{|c|}{ Intensity } & \multirow{2}{*}{$\begin{array}{l}\text { Wavenumber } \\
\quad\left(\mathrm{cm}^{-1}\right)\end{array}$} & \multirow{2}{*}{ Classification } & \multirow{2}{*}{$\begin{array}{c}\text { Wavelength } \\
\text { (§) }\end{array}$} & \multicolumn{2}{|c|}{ Intensity } & \multirow{2}{*}{$\begin{array}{l}\text { Wavenumber } \\
\left(\mathrm{cm}^{-1}\right)\end{array}$} & \multirow{2}{*}{ Classification } \\
\hline & Lamp & Spark & & & & Lamp & Spark & & \\
\hline 8002.589 & 4 & & 12492.520 & $19503^{\circ}-31995_{4}$ & 7941.164 & 2 & & 12589.149 & $14206_{4}^{\circ}-26796_{3}$ \\
\hline 8000.033 & 100 & 10 & 12496.511 & $165546-29050_{0}^{\circ}$ & 7937.727 & 100 & 20 & 12594.600 & $98045-22399_{0}^{\circ}$ \\
\hline 7999.045 & 2 & & 12498.055 & $19039_{2}^{\circ}-31537_{3}$ & 7936.762 & 4 & & 12596.132 & $20054_{2}-32650_{i}^{\circ}$ \\
\hline 7999.009 & 4 & & 12498.111 & $19039_{2}^{\circ}-31537_{3}$ & 7932.280 & 3 & 1 & 12603.249 & $19948{ }^{\circ}-32551_{3}$ \\
\hline 7998.488 & 2 & & 12498.925 & $16346_{4}^{\circ}-28845_{4}$ & 7929.938 & 5 & & 12606.971 & $18930 \stackrel{3}{-}-31537_{3}$ \\
\hline 7996.963 & 40 & 3 & 12501.309 & $18574_{1}-31075_{2}^{\circ}$ & 7929.809 & $25 \mathrm{c}$ & 2 & 12607.176 & $17354^{\circ}-29961_{1}$ \\
\hline 7994.709 & 3 & & 12504.833 & $11802_{2}-24307_{2}^{\circ}$ & 7928.680 & 3 & & 12608.971 & $156183-28227_{4}$ \\
\hline 7993.676 & 150 & 8 & 12506.449 & $10526_{3}^{\circ}-230324$ & 7927.546 & 25 & 3 & 12610.775 & $20054_{2}-32665^{\circ}$ \\
\hline 7993.253 & 15 & 1 & 12507.111 & $18382^{\circ}-30889_{1}$ & 7925.734 & 50 & 3 & 12613.658 & $195888^{\circ}-322025$ \\
\hline 7992.936 & 10 & 2 & 12507.607 & $128473-25355_{4}^{\circ}$ & 7924.977 & 50 & 4 & 12614.863 & $130883-25703^{\circ}$ \\
\hline 7992.936 & 10 & 2 & 12507.607 & $19273_{2}-31780_{3}^{\circ}$ & 7922.912 & 8 & 2 & 12618.151 & $11802_{2}-24421_{3}^{\circ}$ \\
\hline 7992.156 & 50 & 3 & 12508.828 & $15863_{2}-28372^{\circ}$ & 7922.549 & 50 & 5 & 12618.729 & $173983-30017^{\circ}$ \\
\hline 7991.515 & $4 \mathrm{~h}$ & & 12509.831 & $195033^{\circ}-320124$ & 7922.235 & 15 & & 12619.229 & $15970_{3}-28589_{3}^{\circ}$ \\
\hline 7991.515 & $4 \mathrm{~h}$ & & 12509.831 & $19948 \stackrel{\circ}{-}-324584$ & 7917.236 & 15 & 1 & 12627.197 & $22098_{4}-34725^{\circ}$ \\
\hline 7991.366 & 50 & 5 & 12510.064 & $15970_{3}-28480_{4}^{\circ}$ & 7916.790 & 40 & 5 & 12627.908 & $21539_{4}^{\circ}-34167_{3}$ \\
\hline 7989.163 & 8 & 1 & 12513.514 & $12114_{2}^{\circ}-24627_{1}$ & 7914.493 & 8 & 2 & 12631.573 & $18614_{1}^{\circ}-31245_{2}$ \\
\hline 7987.974 & 200 & 50 & 12515.377 & $14481 \stackrel{\circ}{-}-269976$ & 7908.484 & 8 & 2 & 12641.171 & $20322 \circ-329635$ \\
\hline 7986.904 & 1 & & 12517.053 & $18809_{4}^{\circ}-313264$ & 7908.148 & 50 & 5 & 12641.708 & $22508_{2}^{\circ}-35149_{2}$ \\
\hline 7984.603 & 1 & & 12520.660 & $20054_{2}-32575_{2}^{\circ}$ & 7907.856 & 4 & & 12642.175 & $23655_{4}^{\circ}-36297_{4}$ \\
\hline 7984.032 & 1 & & 12521.556 & $21645_{4}^{\circ}-341673$ & 7904.411 & 50 & 5 & 12647.685 & $154934-28140_{4}^{\circ}$ \\
\hline 7982.495 & 10 & 2 & 12523.967 & $21165_{3}^{\circ}-33689_{2}$ & 7904.294 & 4 & & 12647.872 & $20566_{4}^{\circ}-332145$ \\
\hline 7982.289 & 8 & 2 & 12524.290 & $19516_{2}^{\circ}-32041_{2}$ & 7903.319 & 8 & 1 & 12649.432 & $15863_{2}-28513^{\circ}$ \\
\hline 7980.878 & 2 & & 12526.504 & $18549_{2}-31075_{2}^{\circ}$ & 7900.778 & 5 & & 12653.500 & $20737_{i}^{\circ}-33390_{1}$ \\
\hline 7978.977 & 500 & 300 & 12529.489 & $3687_{2}-16217_{2}^{\circ}$ & 7900.309 & 200 & 75 & 12654.251 & $15618^{\circ}-28273_{2}$ \\
\hline 7976.870 & 3 & & 12532.798 & $20566_{4}^{\circ}-33099_{3}$ & 7899.018 & 8 & 1 & 12656.320 & $211435-33799_{4}^{\circ}$ \\
\hline 7975.874 & 2 & & 12534.363 & $22338_{3}^{\circ}-34873_{4}$ & 7896.541 & 5 & & 12660.290 & $13847_{2}-265083$ \\
\hline 7974.743 & 3 & & 12536.141 & $21575_{2}-34111^{\circ}$ & 7893.614 & 50 & 5 & 12664.984 & $16217_{2}^{\circ}-28882_{2}$ \\
\hline 7974.459 & 4 & & 12536.587 & $20737_{1}^{\circ}-33273_{1}$ & 7892.770 & 8 & & 12666.338 & $20724 \stackrel{\circ}{-}-333901$ \\
\hline 7974.164 & 100 & 8 & 12537.051 & $7280_{2}-19817_{i}^{\circ}$ & 7886.273 & 4001 & 50 & 12676.773 & $63622-19039_{2}^{\circ}$ \\
\hline 7972.598 & 200 & 20 & 12539.514 & $49614-175018$ & 7883.595 & 4 & & 12681.080 & $23655_{4}^{\circ}-363363$ \\
\hline 7970.254 & 50 & 5 & 12543.201 & $15970_{3}-28513_{2}^{\circ}$ & 7881.667 & 2 & & 12684.182 & $20922_{2}^{\circ}-336062$ \\
\hline 7969.785 & 10 & 1 & 12543.940 & $15490 \AA-280345$ & 7880.577 & 5 & & 12685.936 & $22163_{4}^{\circ}-348495$ \\
\hline 7969.359 & 4 & & 12544.610 & $22163_{4}^{\circ}-347073$ & 7878.483 & 5 & & 12689.308 & $139622_{1}-26651_{2}^{\circ}$ \\
\hline 7968.784 & 2 & & 12545.515 & $171665-29711^{\circ}$ & 7869.771 & 15 & 2 & 12703.355 & $159700_{3}-28673_{2}^{\circ}$ \\
\hline 7966.264 & 2 & & 12549.484 & $207240^{\circ}-332731$ & 7868.924 & 40 & 10 & 12704.722 & $22877_{1}^{\circ}-355825$ \\
\hline 7965.711 & 8 & 1 & 12550.355 & $12114_{2}^{\circ}-24664_{3}$ & 7868.396 & 75 & $10 \mathrm{~h}$ & 12705.575 & $19588^{\circ}-322935$ \\
\hline 7961.994 & 100 & 8 & 12556.214 & $21165_{3}^{\circ}-33721_{2}$ & 7867.683 & 401 & 3 & 12706.726 & $11601_{1}-24307_{2}^{\circ}$ \\
\hline 7961.343 & 50 & 8 & 12557.241 & $179594-30517^{\circ}$ & 7865.954 & 400 & 75 & 12709.519 & $14481 \stackrel{\circ}{6}-271915$ \\
\hline 7961.158 & 50 & 4 & 12557.533 & $12114_{2}^{\circ}-24671_{2}$ & 7865.248 & 10 & 2 & 12710.660 & $15970_{3}-28680_{4}^{\circ}$ \\
\hline 7960.474 & 1 & & 12558.612 & $21902^{\circ}-344605$ & 7865.077 & 8 & & 12710.936 & $22163_{4}^{\circ}-348743$ \\
\hline 7955.709 & 4 & 1 & 12566.133 & $22141_{3}^{\circ}-347073$ & 7864.009 & 100 & 8 & 12712.663 & $7502_{3}-20214_{3}^{\circ}$ \\
\hline 7955.491 & 2 & & 12566.478 & $17073_{1}-29640 i$ & 7862.336 & 15 & 3 & 12715.368 & $171665-29881_{4}^{\circ}$ \\
\hline 7955.066 & 75 & 4 & 12567.149 & $23015 \AA-355825$ & 7858.703 & 2 & & 12721.246 & $22855_{3}^{\circ}-35576_{3}$ \\
\hline 7954.592 & 150 & 15 & 12567.898 & $63622-18930 \stackrel{\circ}{2}$ & 7858.583 & 2 & & 12721.440 & $17411_{3}^{\circ}-301322$ \\
\hline 7952.148 & 3 & & 12571.760 & $20737_{1}^{\circ}-333092$ & 7855.074 & 3 & & 12727.123 & $12847_{3}-25575_{4}^{\circ}$ \\
\hline 7951.885 & 8 & 1 & 12572.176 & $197133-32285_{3}^{\circ}$ & 7854.919 & 8 & & 12727.374 & $18809_{4}^{\circ}-31537_{3}$ \\
\hline 7949.019 & 3 & 1 & 12576.709 & $20522_{2}^{\circ}-33099_{3}$ & 7853.645 & 2 & & 12729.439 & $21594_{3}-34324_{2}^{\circ}$ \\
\hline 7947.930 & 4 & & 12578.432 & $11802_{2}-24381_{2}^{\circ}$ & 7853.284 & 20 & & 12730.024 & $20543 \stackrel{\circ}{0}-33273_{1}$ \\
\hline 7946.113 & 100 & 8 & 12581.309 & $22508_{2}^{\circ}-35089_{3}$ & 7852.227 & 1 & & 12731.738 & $22141 \stackrel{\circ}{3}-348734$ \\
\hline 7946.074 & 2 & & 12581.370 & $11601_{1}-24182_{2}^{\circ}$ & 7851.006 & 3 & & 12733.718 & $18549_{2}-31283^{\circ}$ \\
\hline 7945.611 & 3 & & 12582.103 & $18069_{3}^{\circ}-30651_{3}$ & 7848.645 & $5 \mathrm{~h}$ & & 12737.548 & $17224_{2}^{\circ}-29961_{1}$ \\
\hline 7944.708 & 2 & & 12583.534 & $18699_{2}-31283^{\circ}$ & 7848.441 & 100 & 40 & 12737.879 & $15490 \circ-28227_{4}$ \\
\hline 7943.393 & 2 & & 12585.617 & $22855_{3}^{\circ}-354403$ & 7847.784 & $100 \mathrm{~b}$ & 40 & 12738.946 & $132974-26036_{3}^{\circ}$ \\
\hline 7943.050 & 20 & 2 & 12586.160 & $142045-267904$ & 7847.536 & $500 \mathrm{~b}$ & 150 & 12739.348 & $8800_{4}-21539_{4}^{\circ}$ \\
\hline 7941.721 & 200 & 75 & 12588.266 & $16346_{4}^{\circ}-289343$ & 7843.545 & 4 & 1 & 12745.830 & $20322{ }^{\circ}-330686$ \\
\hline
\end{tabular}


TABle 4. Classified lines of Th $\mathrm{I}$-continued

\begin{tabular}{|c|c|c|c|c|c|c|c|c|c|}
\hline \multirow{2}{*}{$\begin{array}{l}\text { Wavelength } \\
(\AA)\end{array}$} & \multicolumn{2}{|c|}{ Intensity } & \multirow{2}{*}{$\begin{array}{l}\text { Wavenumber } \\
\qquad\left(\mathrm{cm}^{-1}\right)\end{array}$} & \multirow{2}{*}{ Classification } & \multirow{2}{*}{$\begin{array}{l}\text { Wavelength } \\
\text { (A) }\end{array}$} & \multicolumn{2}{|c|}{ Intensity } & \multirow{2}{*}{$\begin{array}{l}\text { Wavenumber } \\
\qquad\left(\mathrm{cm}^{-1}\right)\end{array}$} & \multirow{2}{*}{ Classification } \\
\hline & Lamp & Spark & & & & Lamp & Spark & & \\
\hline 7842.254 & 100 & 10 & 12747.928 & $13175_{4}^{\circ}-25923_{4}$ & 7776.671 & 40 & 2 & 12855.435 & $12847_{3}-25703_{2}^{\circ}$ \\
\hline 7841.781 & 200 & 50 & 12748.697 & $11241_{3}^{\circ}-23990_{2}$ & 7775.688 & 2 & & 12857.060 & $17398_{3}-30255_{3}^{\circ}$ \\
\hline 7841.596 & 5 & & 12748.998 & $21575_{2}-34324_{2}^{\circ}$ & 7773.389 & 3 & & 12860.863 & $23655_{4}^{\circ}-36515_{3}$ \\
\hline 7841.134 & 8 & & 12749.749 & $2869_{3}-15618_{3}^{\circ}$ & 7772.457 & 2 & & 12862.405 & $19713_{3}-32575_{2}^{\circ}$ \\
\hline 7840.992 & 8 & & 12749.980 & $215943-34344_{4}^{\circ}$ & 7771.942 & 100 & 50 & 12863.257 & $10414_{4}^{\circ}-23277_{5}$ \\
\hline 7840.445 & 100 & 75 & 12750.870 & $19986_{6}^{\circ}-327375$ & 7769.052 & 2 & & 12868.042 & $20522_{2}^{\circ}-33390_{1}$ \\
\hline 7840.290 & 100 & 50 & 12751.122 & $13297_{4}-26048_{4}^{\circ}$ & 7768.470 & 2 & & 12869.006 & $20737_{1}^{\circ}-33606_{2}$ \\
\hline 7839.276 & 10 & 2 & 12752.771 & $195324-32285_{3}^{\circ}$ & 7767.963 & 3 & & 12869.846 & $19588^{\circ}-324584$ \\
\hline 7838.062 & 2 & & 12754.746 & $21077 \circ-33831_{4}$ & 7766.941 & 4 & & 12871.540 & $17501 \stackrel{\circ}{-303726}$ \\
\hline 7836.456 & 100 & 5 & 12757.360 & $16346_{4}^{\circ}-29104_{4}$ & 7762.722 & 50 & 3 & 12878.535 & $14465_{2}^{\circ}-273433$ \\
\hline 7835.620 & 100 & & 12758.721 & $11802_{2}-24561_{3}^{\circ}$ & 7759.127 & 2 & & 12884.502 & $20214_{3}^{\circ}-33099_{3}$ \\
\hline 7828.073 & 3 & & 12771.022 & $22669_{3}^{\circ}-35440_{3}$ & 7758.486 & 3 & & 12885.566 & $20423^{\circ}-33309_{2}$ \\
\hline 7825.245 & 2 & & 12775.637 & $21668_{1}^{\circ}-34444_{2}$ & 7758.251 & $3 \mathrm{~h}$ & & 12885.957 & $20522_{2}^{\circ}-334083$ \\
\hline 7824.291 & 1 & & 12777.195 & $21594_{3}-34371_{2}^{\circ}$ & 7755.600 & 4 & & 12890.362 & $19039_{2}^{\circ}-31929_{3}$ \\
\hline 7823.787 & 2 & & 12778.018 & $17354 i-301322$ & 7754.748 & 1 & & 12891.778 & $21539_{4}^{\circ}-34431_{3}$ \\
\hline 7823.475 & 50 & 2 & 12778.528 & $22855_{3}^{\circ}-35633_{4}$ & 7754.433 & 1 & & 12892.301 & $20322{ }^{\circ}-332145$ \\
\hline 7822.392 & 100 & 4 & 12780.297 & $11601_{1}-24381_{2}^{\circ}$ & 7754.271 & 2 & & 12892.571 & $20566_{4}^{\circ}-33459_{4}$ \\
\hline 7819.888 & 2 & & 12784.389 & $21645_{4}-34430_{3}^{\circ}$ & 7752.440 & 3 & & 12895.616 & $180693-309643$ \\
\hline 7819.350 & 3 & & 12785.269 & $15863_{2}-28649_{1}^{\circ}$ & 7751.435 & 5 & & 12897.288 & $22508_{2}^{\circ}-35405_{3}$ \\
\hline 7818.694 & 2 & & 12786.342 & $20522^{\circ}-33309_{2}$ & 7749.578 & 5 & & 12900.378 & $16783_{4}^{\circ}-296845$ \\
\hline 7817.771 & 500 & 200 & 12787.851 & $10414_{4}^{\circ}-232013$ & 7749.325 & 8 & 2 & 12900.799 & $22248{ }^{\circ}-35149_{2}$ \\
\hline 7817.130 & 4 & & 12788.900 & $13088_{3}-25877_{4}^{\circ}$ & 7748.880 & 3 & & 12901.540 & $22399^{\circ}-353005$ \\
\hline 7816.135 & 50 & 4 & 12790.528 & $11241_{3}^{\circ}-240324$ & 7745.813 & 15 & 3 & 12906.649 & $19532_{4}-32439_{4}^{\circ}$ \\
\hline 7813.970 & 50 & 5 & 12794.072 & $11877_{1}^{\circ}-24671_{2}$ & 7745.235 & 3 & & 12907.612 & $21539_{4}^{\circ}-34447_{4}$ \\
\hline 7813.970 & 50 & 5 & 12794.072 & $15736_{1}^{\circ}-28531_{2}$ & 7744.786 & 4 & & 12908.360 & $17224_{2}^{\circ}-30132_{2}$ \\
\hline 7813.481 & 50 & 8 & 12794.872 & $16346_{4}^{\circ}-29141_{5}$ & 7742.552 & 100 & 2 & 12912.085 & $15618 \stackrel{\circ}{3}-28531_{2}$ \\
\hline 7810.627 & 100 & 10 & 12799.547 & $13297_{4}-26096_{3}^{\circ}$ & 7742.019 & 2 & & 12912.974 & $22508_{2}^{\circ}-35421_{1}$ \\
\hline 7809.950 & 2 & & 12800.657 & $12114_{2}^{\circ}-24915_{3}$ & 7740.867 & 15 & 1 & 12914.895 & $21252_{2}^{\circ}-34167_{3}$ \\
\hline 7809.245 & $25 b$ & 2 & 12801.813 & $17959_{4}-30761_{3}^{\circ}$ & 7739.704 & 2 & & 12916.836 & $22877_{1}^{\circ}-35794_{4}$ \\
\hline 7807.879 & 40 & & 12804.052 & $13847_{2}-26651_{2}^{\circ}$ & 7738.356 & 5 & 1 & 12919.086 & $18011^{\circ}-309306$ \\
\hline 7804.533 & 50 & 5 & 12809.542 & $15863_{2}-28673_{2}^{\circ}$ & 7737.779 & 4 & & 12920.049 & $13962_{1}-26882^{\circ}$ \\
\hline 7804.146 & 50 & 4 & 12810.177 & $17398_{3}-30208_{2}^{\circ}$ & 7736.364 & 2 & & 12922.412 & $22877_{1}^{\circ}-35799_{6}$ \\
\hline 7803.811 & 3 & 1 & 12810.727 & $22338_{3}^{\circ}-35149_{2}$ & 7735.479 & 2 & & 12923.891 & $19273_{2}-32197_{3}^{\circ}$ \\
\hline 7802.824 & 10 & 2 & 12812.347 & $15863_{2}-28676_{3}^{\circ}$ & 7733.362 & 2 & & 12927.429 & $22401_{1}-35328^{\circ}$ \\
\hline 7801.945 & 2 & & 12813.791 & $15166_{3}^{\circ}-27980_{3}$ & 7730.107 & 5 & 1 & 12932.872 & $22508_{2}^{\circ}-35440_{3}$ \\
\hline 7800.029 & 3 & & 12816.938 & $18809_{4}^{\circ}-31626_{5}$ & 7728.939 & 100 & 15 & 12934.827 & $7280_{2}-20214_{3}^{\circ}$ \\
\hline 7799.629 & 8 & 1 & 12817.596 & $21890_{3}^{\circ}-34707_{3}$ & 7727.552 & 2 & & 12937.148 & $21645_{4}-34583_{3}^{\circ}$ \\
\hline 7799.629 & 8 & 1 & 12817.596 & $22399^{\circ}-352165$ & 7726.384 & 2 & & 12939.104 & $22855_{3}^{\circ}-35794_{4}$ \\
\hline 7798.360 & 300 & 25 & 12819.681 & $5563_{1}-18382^{\circ}$ & 7723.641 & 3 & & 12943.699 & $15618_{3}^{\circ}-28562_{4}$ \\
\hline 7795.533 & 1 & & 12824.330 & $18699_{2}-31523 \stackrel{\circ}{3}$ & 7721.780 & 2 & & 12946.819 & $21902_{4}^{\circ}-348495$ \\
\hline 7793.128 & 10 & 2 & 12828.288 & $17959_{4}-30788^{\circ}$ & 7721.188 & 100 & 8 & 12947.811 & $22141_{3}^{\circ}-35089_{3}$ \\
\hline 7790.132 & 10 & & 12833.221 & $199484^{\circ}-32781_{4}$ & 7718.812 & 3 & & 12951.797 & $20737_{1}^{\circ}-33689_{2}$ \\
\hline 7788.932 & 500 & 75 & 12835.199 & $11197{ }^{\circ}-240324$ & 7713.337 & 4 & & 12960.990 & $20566_{4}^{\circ}-33527_{4}$ \\
\hline 7787.659 & 25 & & 12837.297 & $14206_{4}^{\circ}-270443$ & 7712.394 & 50 & 4 & 12962.575 & $159703-28932{ }^{\circ}$ \\
\hline 7785.771 & $2 \mathrm{~h}$ & & 12840.410 & $22248_{2}^{\circ}-35089_{3}$ & 7711.113 & 40 & 3 & 12964.728 & $18809_{4}^{\circ}-31774_{3}$ \\
\hline 7784.779 & 3 & & 12842.046 & $20566^{\circ}-334083$ & 7710.258 & 100 & 15 & 12966.166 & $8111_{4}-210770$ \\
\hline 7783.417 & 5 & 1 & 12844.293 & $18930_{3}^{\circ}-317743$ & 7709.882 & 50 & & 12966.798 & $11802_{2}-24769_{3}^{\circ}$ \\
\hline 7782.742 & 4 & 1 & 12845.407 & $163510-29197^{\circ}$ & 7708.149 & 2 & & 12969.714 & $21738_{2}^{\circ}-34707_{3}$ \\
\hline 7782.318 & 150 & 50 & 12846.107 & $216454-34492 \stackrel{\circ}{\circ}$ & 7706.909 & 1 & & 12971.800 & $21902 \AA-348743$ \\
\hline 7781.843 & 100 & 4 & 12846.891 & $20543 \circ-33390_{1}$ & 7706.448 & 3 & & 12972.576 & $16783 \stackrel{9}{4}-297564$ \\
\hline 7781.329 & 2 & & 12847.740 & $19948_{4}^{\circ}-327963$ & 7705.268 & 2 & & 12974.563 & $18549_{2}-315233_{3}^{\circ}$ \\
\hline 7779.741 & 8 & 2 & 12850.362 & $20423 i-332731$ & 7703.679 & 100 & 10 & 12977.239 & $199866^{\circ}-329635$ \\
\hline 7779.472 & 2 & & 12850.806 & $13945_{3}^{\circ}-267963$ & 7699.765 & 100 & 5 & 12983.836 & $18809_{4}^{\circ}-317934$ \\
\hline 7778.488 & 50 & 5 & 12852.432 & $15490 \stackrel{\circ}{-283425}$ & 7699.660 & 10 & 1 & 12984.013 & $20737_{1}^{\circ}-33721_{2}$ \\
\hline 7776.804 & 4 & & 12855.215 & $21575_{2}-34430_{3}^{\circ}$ & 7699.399 & 75 & 1 & 12984.453 & $14206_{4}^{\circ}-271915$ \\
\hline
\end{tabular}


TABLE 4. Classified lines of Th $\mathrm{I}$-continued

\begin{tabular}{|c|c|c|c|c|c|c|c|c|c|}
\hline \multirow{2}{*}{$\begin{array}{c}\text { Wavelength } \\
\text { (A) }\end{array}$} & \multicolumn{2}{|c|}{ Intensity } & \multirow{2}{*}{$\begin{array}{l}\text { Wavenumber } \\
\left(\mathrm{cm}^{-1}\right)\end{array}$} & \multirow{2}{*}{ Classification } & \multirow{2}{*}{$\begin{array}{c}\text { Wavelength } \\
\text { (̊̊) }\end{array}$} & \multicolumn{2}{|c|}{ Intensity } & \multirow{2}{*}{$\begin{array}{l}\text { Wavenumber } \\
\left(\mathrm{cm}^{-1}\right)\end{array}$} & \multirow{2}{*}{ Classification } \\
\hline & Lamp & Spark & & & & Lamp & Spark & & \\
\hline 7698.035 & $20 \mathrm{~b}$ & & 12986.754 & $10783_{2}^{\circ}-23769_{1}$ & 7634.919 & 3 & & 13094.111 & $20214_{3}^{\circ}-33309_{2}$ \\
\hline 7697.922 & 75 & & 12986.944 & $154934-28480_{4}^{\circ}$ & 7633.767 & 75 & 2 & 13096.087 & $154934-28589^{\circ}$ \\
\hline 7697.077 & 2 & & 12988.370 & $215943-34583 \stackrel{\circ}{3}$ & 7632.129 & 50 & 2 & 13098.898 & $139621-27061_{2}^{\circ}$ \\
\hline 7693.797 & 100 & 5 & 12993.907 & $171665-30160_{4}^{\circ}$ & 7632.129 & 50 & 2 & 13098.898 & $13945_{3}^{\circ}-27044_{3}$ \\
\hline 7690.658 & 5 & & 12999.211 & $18930_{3}^{\circ}-319293$ & 7630.536 & 50 & 2 & 13101.633 & $14465_{2}^{\circ}-27566_{2}$ \\
\hline 7689.993 & 1 & & 13000.335 & $22877_{1}^{\circ}-35877_{2}$ & 7630.310 & 100 & 5 & 13102.021 & $8800_{4}-21902_{4}^{\circ}$ \\
\hline 7688.949 & 3 & & 13002.100 & $19039_{2}^{\circ}-32041_{2}$ & 7627.428 & 3 & & 13106.971 & $20054_{2}-33161_{1}^{\circ}$ \\
\hline 7686.149 & 50 & 1 & 13006.836 & $17501 \stackrel{\circ}{-}-305085$ & 7627.428 & 3 & & 13106.971 & $20054_{2}-33161^{\circ}$ \\
\hline 7683.150 & $3 b$ & & 13011.913 & $19273_{2}-32285_{3}^{\circ}$ & 7627.175 & 150 & 5 & 13107.406 & $4961_{4}-18069 \stackrel{\circ}{3}$ \\
\hline 7683.010 & 50 & 1 & 13012.150 & $14032_{2}^{\circ}-270443$ & 7625.705 & 150 & 5 & 13109.933 & $14481{ }^{\circ}-275915$ \\
\hline 7682.438 & 3 & & 13013.119 & $18699_{2}-31712^{\circ}$ & 7625.120 & 50 & 2 & 13110.938 & $18930_{3}^{\circ}-32041_{2}$ \\
\hline 7681.318 & 3 & & 13015.017 & $19948{ }^{\circ}-329635$ & 7620.815 & 5 & & 13118.345 & $23015 \circ-361335$ \\
\hline 7678.123 & 150 & 5 & 13020.432 & $75023-20522_{2}^{\circ}$ & 7620.443 & 3 & & 13118.985 & $173988_{3}-30517_{4}^{\circ}$ \\
\hline 7676.888 & 2 & & 13022.527 & $22855^{\circ}-35877_{2}$ & 7620.080 & 100 & 5 & 13119.610 & $18809_{4}^{\circ}-31929_{3}$ \\
\hline 7675.925 & 2 & & 13024.161 & $22396^{\circ}-35421_{1}$ & 7617.255 & 2 & & 13124.476 & $22669_{3}^{\circ}-357944$ \\
\hline 7675.621 & 3 & & 13024.677 & $130883-26113_{2}^{\circ}$ & 7616.686 & 50 & 1 & 13125.456 & $139621-27087_{1}^{\circ}$ \\
\hline 7675.621 & 3 & & 13024.677 & $18574_{1}-31599_{2}^{\circ}$ & 7614.421 & 1 & & 13129.360 & $21575_{2}-34704^{\circ}$ \\
\hline 7672.749 & 2 & & 13029.552 & $128473-25877_{4}^{\circ}$ & 7612.122 & 4 & 1 & 13133.326 & $17411_{3}^{\circ}-30544_{2}$ \\
\hline 7672.252 & 50 & 2 & 13030.396 & $13847_{2}-26878_{3}^{\circ}$ & 7611.293 & 8 & & 13134.756 & $17073_{1}-30208_{2}^{\circ}$ \\
\hline 7672.123 & 1 & & 13030.615 & $17959_{4}-30990_{3}^{\circ}$ & 7610.378 & 2 & & 13136.335 & $21165_{3}^{\circ}-343014$ \\
\hline 7669.750 & 8 & & 13034.647 & $19516_{2}^{\circ}-32551_{3}$ & 7610.101 & 5 & & 13136.813 & $14206 \stackrel{9}{4}-273433$ \\
\hline 7668.955 & 50 & 1 & 13035.998 & $11802_{2}-24838_{1}^{\circ}$ & 7609.546 & 2 & & 13137.772 & $22163_{4}^{\circ}-353005$ \\
\hline 7667.018 & 2 & & 13039.291 & $211435-34182{ }^{\circ}$ & 7609.359 & 3 & & 13138.094 & $185741-31712^{\circ}$ \\
\hline 7665.734 & 8 & & 13041.475 & $197133-32754^{\circ}$ & 7607.824 & 100 & 4 & 13140.745 & $63622-19503 \stackrel{\circ}{\circ}$ \\
\hline 7665.344 & 2 & & 13042.139 & $20522_{2}^{\circ}-335643$ & 7607.519 & 40 & 1 & 13141.272 & $17411_{3}^{\circ}-305524$ \\
\hline 7661.621 & 8 & 1 & 13048.476 & $195033^{\circ}-325513$ & 7606.314 & 5 & & 13143.354 & $7280_{2}-20423_{i}^{\circ}$ \\
\hline 7661.162 & 2 & & 13049.258 & $20724 \stackrel{\circ}{-}-337731$ & 7605.083 & 5 & & 13145.481 & $15736_{1}^{\circ}-28882_{2}$ \\
\hline 7660.889 & 150 & 4 & 13049.723 & $49614-18011{ }^{\circ}$ & 7604.177 & 3 & & 13147.048 & $14465_{2}^{\circ}-27612_{3}$ \\
\hline 7660.029 & 75 & 1 & 13051.188 & $5563_{1}-18614_{i}^{\circ}$ & 7603.623 & 40 & 1 & 13148.005 & $13847_{2}-26995 \stackrel{\circ}{3}$ \\
\hline 7660.029 & 75 & 1 & 13051.188 & $16783^{\circ}-29835_{3}$ & 7603.249 & 40 & 1 & 13148.652 & $195888^{\circ}-327375$ \\
\hline 7658.323 & 150 & 5 & 13054.096 & $8111_{4}-21165_{3}^{\circ}$ & 7602.795 & 40 & 1 & 13149.437 & $19713_{3}-32862_{4}^{\circ}$ \\
\hline 7655.702 & 8 & & 13058.565 & $216454-347043$ & 7602.247 & 2 & & 13150.385 & $21575_{2}-34725_{3}^{\circ}$ \\
\hline 7655.307 & 2 & & 13059.239 & $17959_{4}-31019 \%$ & 7601.880 & 5 & & 13151.020 & $19948_{4}^{\circ}-33099_{3}$ \\
\hline 7654.695 & 100 & 5 & 13060.283 & $15618_{3}^{\circ}-28679_{2}$ & 7600.637 & 2 & & 13153.171 & $23655_{4}^{\circ}-368083$ \\
\hline 7653.827 & 200 & 10 & 13061.764 & $14204_{5}-27266^{\circ}$ & 7599.815 & 2 & & 13154.593 & $6362_{2}-19516_{2}^{\circ}$ \\
\hline 7653.327 & 1 & & 13062.617 & $17224_{2}^{\circ}-302861$ & 7599.638 & 5 & & 13154.900 & $173983-30553_{2}^{\circ}$ \\
\hline 7652.317 & 100 & 3 & 13064.341 & $75023-20566_{4}^{\circ}$ & 7598.203 & 200 & 3 & 13157.384 & $165546-29711^{\circ}$ \\
\hline 7651.739 & 100 & 2 & 13065.328 & $16783_{4}^{\circ}-298494$ & 7598.203 & 200 & 3 & 13157.384 & $11241_{3}^{\circ}-24399_{3}$ \\
\hline 7651.143 & 1 & & 13066.346 & $22338_{3}^{\circ}-35405_{3}$ & 7591.999 & 10 & & 13168.136 & $21539_{4}^{\circ}-34707_{3}$ \\
\hline 7650.996 & 75 & 1 & 13066.597 & $19227 \%-322935$ & 7586.988 & 8 & & 13176.833 & $18069_{3}^{\circ}-31245_{2}$ \\
\hline 7649.891 & 2 & & 13068.484 & $22508_{2}^{\circ}-35576 \mathbf{3}$ & 7586.207 & 20 & & 13178.190 & $11802_{2}-24981_{3}^{\circ}$ \\
\hline 7648.545 & 4 & & 13070.784 & $142260-27297^{\circ}$ & 7585.783 & 200 & 10 & 13178.926 & $25580-15736^{\circ}$ \\
\hline 7647.380 & 500 & 50 & 13072.775 & $98045-22877^{\circ}$ & 7585.688 & 200 & 15 & 13179.091 & $165546-29733{ }^{\circ}$ \\
\hline 7647.380 & 500 & 50 & 13072.775 & $18069_{3}^{\circ}-31141_{3}$ & 7583.574 & 2 & & 13182.765 & $20423_{1}^{\circ}-336062$ \\
\hline 7646.337 & 2 & & 13074.558 & $23015_{0}^{\circ}-36089_{4}$ & 7583.534 & 2 & & 13182.835 & $20423 i-336062$ \\
\hline 7644.737 & 25 & 1 & 13077.295 & $11802_{2}-24880_{i}^{\circ}$ & 7583.422 & 25 & & 13183.029 & $15493_{4}-28676_{3}^{\circ}$ \\
\hline 7643.413 & 1 & & 13079.560 & $21645_{4}-34725_{3}^{\circ}$ & 7581.856 & 4 & & 13185.752 & $18809_{4}^{\circ}-319954$ \\
\hline 7642.873 & 15 & & 13080.484 & $13175_{4}^{\circ}-26255_{4}$ & 7581.088 & 2 & & 13187.088 & $21902_{4}^{\circ}-35089_{3}$ \\
\hline 7642.425 & 2 & & 13081.251 & $18699_{2}-31780_{3}^{\circ}$ & 7580.866 & 4 & & 13187.474 & $15493_{4}-28680_{4}^{\circ}$ \\
\hline 7642.028 & 10 & 1 & 13081.931 & $19986^{\circ}-330686$ & 7580.829 & 5 & & 13187.539 & $154934-28680_{4}^{\circ}$ \\
\hline 7641.605 & 2 & & 13082.655 & $18930_{3}^{\circ}-320124$ & 7580.686 & 40 & 1 & 13187.787 & $15970_{3}-29157_{3}^{\circ}$ \\
\hline 7641.066 & 1 & & 13083.578 & $20522_{2}^{\circ}-336062$ & 7580.340 & 50 & 1 & 13188.389 & $12847_{3}-26036^{\circ}$ \\
\hline 7638.777 & 100 & 3 & 13087.498 & $13297_{4}-26384_{4}^{\circ}$ & 7579.456 & 3 & & 13189.928 & $17354_{1}^{\circ}-30544_{2}$ \\
\hline 7637.385 & 75 & 2 & 13089.883 & $8800_{4}-21890_{3}^{\circ}$ & 7578.274 & 2 & & 13191.985 & $21252_{2}^{\circ}-34444_{2}$ \\
\hline 7636.173 & 100 & 4 & 13091.961 & $49614-18053 \stackrel{\circ}{4}$ & 7578.274 & 2 & & 13191.985 & $22248_{2}^{\circ}-35440_{3}$ \\
\hline
\end{tabular}


TABLE 4. Classified lines of Th $\mathrm{I}$-continued

\begin{tabular}{|c|c|c|c|c|c|c|c|c|c|}
\hline \multirow{2}{*}{$\begin{array}{c}\text { Wavelength } \\
\text { (̊̊) }\end{array}$} & \multicolumn{2}{|c|}{ Intensity } & \multirow{2}{*}{$\begin{array}{l}\text { Wavenumber } \\
\quad\left(\mathrm{cm}^{-1}\right)\end{array}$} & \multirow{2}{*}{ Classification } & \multirow{2}{*}{$\begin{array}{c}\text { Wavelength } \\
\text { (§) }\end{array}$} & \multicolumn{2}{|c|}{ Inten sity } & \multirow{2}{*}{$\begin{array}{l}\text { Wavenumber } \\
\qquad\left(\mathrm{cm}^{-1}\right)\end{array}$} & \multirow{2}{*}{ Classification } \\
\hline & Lamp & Spark & & & & Lamp & Spark & & \\
\hline 7578.274 & 2 & & 13191.985 & $22855_{3}^{\circ}-36047_{2}$ & 7517.963 & 50 & 2 & 13297.814 & $204231-33721_{2}$ \\
\hline 7577.550 & 25 & & 13193.245 & $19588^{\circ}-32781_{4}$ & 7515.504 & 1 & & 13302.164 & $19273_{2}-32575_{2}^{\circ}$ \\
\hline 7577.266 & 20 & & 13193.740 & $20214_{3}^{\circ}-334083$ & 7514.681 & 2 & & 13303.621 & $21575_{2}-34878_{i}^{\circ}$ \\
\hline 7574.484 & 5 & & 13198.585 & $20522_{2}^{\circ}-33721_{2}$ & 7512.237 & 3 & & 13307.949 & $21738_{2}^{\circ}-35046_{1}$ \\
\hline 7574.116 & 3 & & 13199.227 & $21890_{3}^{\circ}-35089_{3}$ & 7511.788 & 100 & 4 & 13308.745 & $98045-23113 \stackrel{\circ}{9}$ \\
\hline 7573.342 & 50 & 1 & 13200.576 & $12847_{3}-26048_{4}^{\circ}$ & 7510.144 & 1 & & 13311.658 & $14032_{2}^{\circ}-273433$ \\
\hline 7573.155 & 3 & & 13200.902 & $16217_{2}^{\circ}-29418_{2}$ & 7509.595 & 2 & & 13312.631 & $20214_{3}^{\circ}-33527_{4}$ \\
\hline 7572.973 & 4 & & 13201.219 & $211435-34344_{4}^{\circ}$ & 7508.477 & 100 & 4 & 13314.614 & $14206^{\circ}-27521_{4}$ \\
\hline 7571.931 & 3 & & 13203.036 & $18809_{4}^{\circ}-32012_{4}$ & 7507.923 & 75 & & 13315.596 & $18011^{\circ}-313264$ \\
\hline 7571.029 & 8 & & 13204.609 & $16217_{2}^{\circ}-29422_{1}$ & 7507.737 & 50 & & 13315.926 & $15618_{3}^{\circ}-289343$ \\
\hline 7570.636 & 2 & & 13205.294 & $20322{ }^{\circ}-33527_{4}$ & 7505.292 & 50 & & 13320.264 & $17224_{2}^{\circ}-30544_{2}$ \\
\hline 7569.509 & 50 & 2 & 13207.260 & $10783_{2}^{\circ}-23990_{2}$ & 7505.052 & 1 & & 13320.690 & $18549_{2}-31870_{2}^{\circ}$ \\
\hline 7567.739 & 300 & 15 & 13210.349 & $98045-23015 \circ$ & 7503.829 & 50 & 8 & 13322.861 & $14243 i-27566_{2}$ \\
\hline 7567.596 & 50 & 3 & 13210.599 & $13297_{4}-26508_{3}^{\circ}$ & 7503.626 & 2 & & 13323.221 & $17959_{4}-31283_{3}^{\circ}$ \\
\hline 7566.732 & 3 & & 13212.107 & $22877_{0}^{\circ}-36089_{4}$ & 7501.180 & 8 & & 13327.566 & $22248_{2}^{\circ}-35576_{3}$ \\
\hline 7565.850 & 50 & 2 & 13213.647 & $13847_{2}-27061_{2}^{\circ}$ & 7499.786 & 1 & & 13330.043 & $195324-32862_{4}^{\circ}$ \\
\hline 7563.711 & 5 & & 13217.384 & $18053^{\circ}-312715$ & 7499.579 & 8 & 1 & 13330.411 & $21077^{\circ}-344076$ \\
\hline 7557.751 & 50 & 1 & 13227.807 & $15970_{3}-29197_{2}^{\circ}$ & 7499.000 & 75 & 2 & 13331.440 & $8243 \stackrel{\circ}{-}-21575_{2}$ \\
\hline 7557.438 & 2 & & 13228.355 & $19986^{\circ}-332145$ & 7497.552 & 10 & 1 & 13334.015 & $15863_{2}-29197_{2}^{\circ}$ \\
\hline 7556.646 & 1 & & 13229.742 & $20543 \stackrel{\circ}{ }-337731$ & 7497.305 & 3 & & 13334.454 & $215399_{4}^{\circ}-348743$ \\
\hline 7556.309 & 2 & & 13230.332 & $19273_{2}-32503^{\circ}$ & 7496.988 & 15 & 1 & 13335.018 & $13962_{1}-27297_{1}^{\circ}$ \\
\hline 7556.225 & 5 & & 13230.479 & $16783_{4}^{\circ}-30014_{4}$ & 7495.564 & 50 & 2 & 13337.551 & $16346_{4}^{\circ}-296845$ \\
\hline 7553.974 & $10 w$ & 2 & 13234.421 & $22399^{\circ}-356334$ & 7494.237 & 2 & & 13339.913 & $15970_{3}-29310_{4}^{\circ}$ \\
\hline 7553.974 & $10 w$ & 2 & 13234.421 & $22855_{3}^{\circ}-36089_{4}$ & 7493.427 & 75 & 2 & 13341.355 & $8800_{4}-22141_{3}^{\circ}$ \\
\hline 7553.753 & 2 & & 13234.808 & $17959_{4}-31194_{4}^{\circ}$ & 7490.379 & 5 & & 13346.784 & $17411_{3}^{\circ}-307582$ \\
\hline 7551.997 & 4 & & 13237.886 & $11601_{1}-24838^{\circ}$ & 7489.709 & 50 & 2 & 13347.978 & $13297_{4}-26645^{\circ}$ \\
\hline 7550.831 & 1 & & 13239.930 & $17411_{3}^{\circ}-306513$ & 7489.612 & 50 & 2 & 13348.151 & $7795_{4}^{\circ}-211435$ \\
\hline 7550.677 & 5 & & 13240.200 & $13847_{2}-27087_{1}^{\circ}$ & 7489.361 & 1 & & 13348.598 & $211435-34492^{\circ}$ \\
\hline 7549.923 & 2 & & 13241.522 & $19713_{3}-32954_{2}^{\circ}$ & 7487.972 & 100 & 3 & 13351.074 & $8243_{2}^{\circ}-215943$ \\
\hline 7549.532 & 4 & & 13242.208 & $22163{ }^{\circ}-354053$ & 7487.869 & 3 & & 13351.258 & $17166_{5}-30517_{4}^{\circ}$ \\
\hline 7549.439 & 1 & & 13242.371 & $200542-33297_{2}^{\circ}$ & 7485.500 & 5 & & 13355.483 & $15490 \AA-288454$ \\
\hline 7549.316 & 200 & 8 & 13242.587 & $7280_{2}-20522_{2}^{\circ}$ & 7483.624 & 200 & 10 & 13358.831 & $3865_{1}-17224_{2}^{\circ}$ \\
\hline 7547.742 & 3 & & 13245.348 & $20922_{2}^{\circ}-34167_{3}$ & 7481.352 & 200 & 15 & 13362.888 & $8800_{4}-22163 \stackrel{\circ}{\circ}$ \\
\hline 7545.657 & 5 & & 13249.008 & $128473-26096_{3}^{\circ}$ & 7481.110 & 50 & 1 & 13363.320 & $173983-30761_{3}^{\circ}$ \\
\hline 7544.592 & 2 & & 13250.879 & $20522_{2}^{\circ}-33773_{1}$ & 7480.802 & 2 & & 13363.870 & $220984-35462_{3}^{\circ}$ \\
\hline 7540.596 & 15 & & 13257.901 & $18069_{3}^{\circ}-313264$ & 7477.364 & 8 & & 13370.015 & $21077_{0}^{\circ}-34447_{4}$ \\
\hline 7539.622 & 4 & & 13259.613 & $18011{ }^{\circ}-312715$ & 7474.559 & 10 & 1 & 13375.032 & $195888^{\circ}-329635$ \\
\hline 7537.429 & 50 & 2 & 13263.471 & $15618_{3}^{\circ}-28882_{2}$ & 7474.043 & 2 & & 13375.956 & $20922_{2}^{\circ}-34298_{1}$ \\
\hline 7536.407 & 50 & 10 & 13265.270 & $20566_{4}^{\circ}-33831_{4}$ & 7473.440 & 20 & 1 & 13377.035 & $21668_{i}^{\circ}-350461$ \\
\hline 7536.244 & 1 & & 13265.557 & $20423_{1}^{\circ}-33689_{2}$ & 7471.346 & 5 & & 13380.784 & $18699_{2}-32080_{1}^{\circ}$ \\
\hline 7535.850 & 5 & & 13266.250 & $211655^{\circ}-344313$ & 7469.705 & 4 & & 13383.724 & $210777^{\circ}-344605$ \\
\hline 7531.818 & 50 & 2 & 13273.352 & $18053{ }^{\circ}-313264$ & 7469.054 & 40 & 1 & 13384.890 & $14206_{4}^{\circ}-275915$ \\
\hline 7531.142 & 75 & 2 & 13274.543 & $13088_{3}-26363_{2}^{\circ}$ & 7466.819 & 5 & & 13388.897 & $15863_{2}-29252_{2}^{\circ}$ \\
\hline 7529.408 & 2 & & 13277.600 & $22401_{1}-35678 i$ & 7465.443 & 100 & 3 & 13391.364 & $20214_{3}^{\circ}-336062$ \\
\hline 7529.318 & 3 & & 13277.759 & $22163_{4}^{\circ}-354403$ & 7465.236 & 50 & 1 & 13391.736 & $154934-288843$ \\
\hline 7528.919 & 5 & & 13278.463 & $195033^{\circ}-32781_{4}$ & 7465.025 & 15 & 1 & 13392.114 & $18809_{4}^{\circ}-32202_{5}$ \\
\hline 7528.490 & 40 & 8 & 13279.220 & $11601_{1}-24880_{1}^{\circ}$ & 7463.427 & 1 & & 13394.981 & $22399^{\circ}-35794_{4}$ \\
\hline 7526.832 & 2 & & 13282.145 & $23015 \circ-362974$ & 7462.990 & 75 & 1 & 13395.766 & $15166_{3}^{\circ}-28562_{4}$ \\
\hline 7523.335 & 8 & & 13288.318 & $163510-29640_{1}^{\circ}$ & 7461.502 & 5 & & 13398.437 & $13945_{3}^{\circ}-27343_{3}$ \\
\hline 7523.133 & 100 & 3 & 13288.675 & $14206^{\circ}-27495_{4}$ & 7461.385 & 3 & & 13398.647 & $21902 \stackrel{\circ}{4}-353005$ \\
\hline 7520.694 & 15 & & 13292.985 & $195033^{\circ}-327963$ & 7461.290 & 8 & 1 & 13398.818 & $17847_{2}^{\circ}-31245_{2}$ \\
\hline 7520.567 & 2 & & 13293.209 & $15863_{2}-29157_{1}^{\circ}$ & 7460.310 & 10 & 1 & 13400.578 & $22399 \AA-357996$ \\
\hline 7520.127 & 8 & & 13293.987 & $15863_{2}-29157_{3}^{\circ}$ & 7458.753 & 50 & 2 & 13403.375 & $17354_{1}^{\circ}-30758_{2}$ \\
\hline 7519.279 & 1 & & 13295.486 & $18574_{1}-31870_{2}^{\circ}$ & 7457.648 & 25 & 1 & 13405.361 & $14206_{4}^{\circ}-276123$ \\
\hline 7518.780 & 50 & 1 & 13296.369 & $13088_{3}-26384_{4}^{\circ}$ & 7455.204 & 100 & 4 & 13409.756 & $16346 \stackrel{8}{4}-297564$ \\
\hline
\end{tabular}


TABLE 4. Classified lines of Th $\mathrm{I}$-continued

\begin{tabular}{|c|c|c|c|c|c|c|c|c|c|}
\hline \multirow{2}{*}{$\begin{array}{l}\text { Wavelength } \\
\text { (A) }\end{array}$} & \multicolumn{2}{|c|}{ Intensity } & \multirow{2}{*}{$\begin{array}{l}\text { Wavenumber } \\
\qquad\left(\mathrm{cm}^{-1}\right)\end{array}$} & \multirow{2}{*}{ Classification } & \multirow{2}{*}{$\begin{array}{c}\text { Wavelength } \\
\text { (§) }\end{array}$} & \multicolumn{2}{|c|}{ Intensity } & \multirow{2}{*}{$\begin{array}{l}\text { Wavenumber } \\
\qquad\left(\mathrm{cm}^{-1}\right)\end{array}$} & \multirow{2}{*}{ Classification } \\
\hline & Lamp & Spark & & & & Lamp & Spark & & \\
\hline 7453.730 & 20 & 1 & 13412.408 & $13847_{2}-27260_{3}^{\circ}$ & 7398.583 & 50 & 1 & 13512.380 & $15166_{3}^{\circ}-28679_{2}$ \\
\hline 7453.188 & 5 & & 13413.383 & $22163^{\circ}-355763$ & 7398.540 & 8 & & 13512.458 & $19039_{2}^{\circ}-32551_{3}$ \\
\hline 7452.516 & 20 & 1 & 13414.593 & $17398_{3}-30812_{2}^{\circ}$ & 7397.074 & 4 & & 13515.136 & $128473-26363_{2}^{\circ}$ \\
\hline 7452.432 & 2 & & 13414.744 & $21575_{2}-34989^{\circ}$ & 7396.895 & 20 & 1 & 13515.463 & $14465_{2}^{\circ}-27980_{3}$ \\
\hline 7450.306 & 8 & & 13418.572 & $17847_{2}^{\circ}-31265_{3}$ & 7394.970 & 1 & & 13518.981 & $11802_{2}-25321_{3}^{\circ}$ \\
\hline 7449.979 & 3 & & 13419.161 & $22163_{4}^{\circ}-355825$ & 7390.097 & 50 & & 13527.896 & $18930_{3}^{\circ}-324584$ \\
\hline 7449.808 & 75 & 2 & 13419.469 & $13088_{3}-26508_{3}^{\circ}$ & 7388.403 & 5 & & 13530.997 & $18549_{2}-32080_{1}^{\circ}$ \\
\hline 7449.605 & 75 & 3 & 13419.834 & $75023-20922_{2}^{\circ}$ & 7386.924 & 50 & 2 & 13533.706 & $17224_{2}^{\circ}-30758_{2}$ \\
\hline 7447.848 & 150 & 5 & 13423.000 & $11241_{3}^{\circ}-246643$ & 7386.347 & 50 & 10 & 13534.764 & $14032_{2}^{\circ}-275662$ \\
\hline 7445.697 & 3 & & 13426.878 & $17224_{2}^{\circ}-30651_{3}$ & 7386.055 & 8 & & 13535.299 & $17354_{1}^{\circ}-308891$ \\
\hline 7445.697 & 3 & & 13426.878 & $18614_{1}^{\circ}-32041_{2}$ & 7385.498 & 500 & 15 & 13536.320 & $3687_{2}-17224_{2}^{\circ}$ \\
\hline 7444.751 & 200 & 8 & 13428.584 & $8111_{4}-21539^{\circ}$ & 7385.391 & $8 b$ & & 13536.516 & $21594_{3}-35131_{4}^{\circ}$ \\
\hline 7444.373 & 8 & 1 & 13429.266 & $17501 \stackrel{\circ}{-309306}$ & 7385.154 & 20 & & 13536.950 & $128473-26384_{4}^{\circ}$ \\
\hline 7443.873 & 50 & 1 & 13430.168 & $11241_{3}^{\circ}-24671_{2}$ & 7384.174 & 200 & 5 & 13538.747 & $8800_{4}-223388^{\circ}$ \\
\hline 7443.573 & 1 & & 13430.709 & $23655_{4}^{\circ}-37085_{4}$ & 7383.712 & 150 & 8 & 13539.594 & $20867_{7}^{\circ}-344076$ \\
\hline 7442.040 & 15 & & 13433.476 & $16217_{2}^{\circ}-29650_{2}$ & 7383.596 & 50 & 2 & 13539.806 & $170731-306130^{\circ}$ \\
\hline 7441.125 & 25 & 1 & 13435.128 & $216454-35081{ }^{\circ}$ & 7378.165 & 2 & & 13549.773 & $21539_{4}^{\circ}-35089_{3}$ \\
\hline 7439.314 & 3 & & 13438.398 & $18431_{3}-31870_{2}^{\circ}$ & 7377.891 & 40 & 1 & 13550.276 & $13945_{3}^{\circ}-27495_{4}$ \\
\hline 7437.331 & 1 & & 13441.981 & $22855_{3}^{\circ}-36297_{4}$ & 7377.626 & 2 & & 13550.763 & $21890_{3}^{\circ}-35440_{3}$ \\
\hline 7433.375 & 8 & & 13449.135 & $15970_{3}-29419_{2}^{\circ}$ & 7376.879 & 200 & 8 & 13552.135 & $14481^{\circ}-280345$ \\
\hline 7433.005 & 5 & & 13449.804 & $13847_{2}-27297_{1}^{\circ}$ & 7376.178 & 25 & & 13553.423 & $17411_{3}^{\circ}-309643$ \\
\hline 7430.255 & 500 & 15 & 13454.782 & $63622-19817_{1}^{\circ}$ & 7375.142 & 1 & & 13555.327 & $15863_{2}-29419_{2}^{\circ}$ \\
\hline 7429.212 & 3 & & 13456.671 & $19817^{\circ}-33273_{1}$ & 7374.483 & 5 & & 13556.538 & $23655_{4}^{\circ}-372115$ \\
\hline 7428.943 & 800 & 50 & 13457.159 & $7280_{2}-20737_{1}^{\circ}$ & 7373.960 & 1 & & 13557.500 & $154934-29050 \circ$ \\
\hline 7427.224 & 2 & & 13460.273 & $19948_{4}^{\circ}-334083$ & 7373.960 & 1 & & 13557.500 & $19713_{3}-33270_{4}^{\circ}$ \\
\hline 7426.906 & 4 & & 13460.849 & $18699_{2}-32160_{2}^{\circ}$ & 7370.827 & 50 & 2 & 13563.262 & $130883-26651_{2}^{\circ}$ \\
\hline 7422.013 & 75 & 2 & 13469.724 & $19948^{\circ}-334184$ & 7370.409 & 4 & & 13564.031 & $17959_{4}-315233^{\circ}$ \\
\hline 7421.465 & 5 & & 13470.718 & $22163_{4}^{\circ}-35633_{4}$ & 7365.427 & 50 & 20 & 13573.206 & $18053_{4}^{\circ}-316265$ \\
\hline 7419.593 & 10 & & 13474.117 & $20214_{3}^{\circ}-33689_{2}$ & 7365.233 & 50 & 20 & 13573.564 & $19817_{1}^{\circ}-33390_{1}$ \\
\hline 7418.548 & 200 & & 13476.015 & $55631-190392$ & 7365.166 & $3 \mathrm{~h}$ & & 13573.687 & 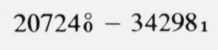 \\
\hline 7417.789 & 100 & & 13477.394 & $2869_{3}-16346_{4}^{\circ}$ & 7363.794 & 10 & 1 & 13576.216 & $139453_{3}^{\circ}-27521_{4}$ \\
\hline 7416.503 & 3 & & 13479.731 & $19588 \stackrel{\circ}{-3} 330686$ & 7362.195 & 50 & 1 & 13579.165 & $19948_{4}^{\circ}-33527_{4}$ \\
\hline 7415.923 & 10 & & 13480.785 & $21668_{1}^{\circ}-35149_{2}$ & 7361.348 & 150 & 5 & 13580.727 & $13297_{4}-26878_{3}^{\circ}$ \\
\hline 7415.817 & 5 & & 13480.978 & $22396_{1}^{\circ}-35877_{2}$ & 7360.715 & 2 & & 13581.895 & $197133-33294^{\circ}$ \\
\hline 7415.679 & 3 & & 13481.228 & $19273_{2}-32754_{3}^{\circ}$ & 7360.553 & 2 & & 13582.194 & $17847_{2}^{\circ}-31429_{1}$ \\
\hline 7414.361 & 10 & & 13483.625 & $180533_{4}^{\circ}-315373$ & 7360.428 & 3 & & 13582.425 & $19516_{2}^{\circ}-33099_{3}$ \\
\hline 7414.139 & 3 & & 13484.029 & $18809_{4}^{\circ}-322935$ & 7359.530 & 2 & & 13584.082 & $19713_{3}-33297_{2}^{\circ}$ \\
\hline 7413.592 & 25 & & 13485.024 & $156188^{\circ}-29104_{4}$ & 7358.738 & 2 & & 13585.544 & $18699_{2}-32285_{3}^{\circ}$ \\
\hline 7413.484 & 3 & & 13485.220 & $139621-27447_{2}^{\circ}$ & 7358.568 & 8 & & 13585.858 & $18574_{1}-32160_{2}^{\circ}$ \\
\hline 7413.410 & 2 & & 13485.355 & $216454-351311^{\circ}$ & 7355.179 & 10 & & 13592.118 & $173983-30990_{3}^{\circ}$ \\
\hline 7411.739 & 100 & 4 & 13488.395 & $16346_{4}^{\circ}-29835_{3}$ & 7353.644 & 15 & & 13594.955 & $216454-35240^{\circ}$ \\
\hline 7411.322 & 10 & & 13489.154 & $38651-17354^{\circ}$ & 7352.937 & 8 & & 13596.262 & $19503_{3}^{\circ}-33099_{3}$ \\
\hline 7409.836 & 3 & & 13491.859 & $19817_{1}^{\circ}-33309_{2}$ & 7351.371 & 1 & & 13599.158 & $8800_{4}-22399_{0}^{\circ}$ \\
\hline 7409.618 & 1 & & 13492.256 & $22141_{3}^{\circ}-35633_{4}$ & 7350.934 & 5 & & 13599.967 & $13847_{2}-27447_{2}^{\circ}$ \\
\hline 7409.618 & 1 & & 13492.256 & $223388^{\circ}-358313$ & 7350.454 & 50 & & 13600.855 & $20566_{4}^{\circ}-34167_{3}$ \\
\hline 7409.221 & 40 & & 13492.979 & $13297_{4}-26790_{4}^{\circ}$ & 7346.898 & 2 & & 13607.438 & $20054_{2}-33662_{1}^{\circ}$ \\
\hline 7404.262 & 50 & & 13502.016 & $98045-233066$ & 7346.671 & 2 & & 13607.858 & $22877_{1}^{\circ}-36485_{2}$ \\
\hline 7403.993 & 50 & 1 & 13502.506 & $16346_{4}^{\circ}-29849_{4}$ & 7344.939 & 10 & & 13611.067 & $18549_{2}-32160_{2}^{\circ}$ \\
\hline 7403.698 & 2 & & 13503.044 & $21902_{4}^{\circ}-35405_{3}$ & 7343.396 & $5 \mathrm{~h}$ & & 13613.927 & $15490_{0}^{\circ}-29104_{4}$ \\
\hline 7403.273 & 3 & & 13503.819 & $11802_{2}-25306_{2}^{\circ}$ & 7342.573 & 150 & 5 & 13615.453 & 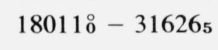 \\
\hline 7402.248 & 400 & 8 & 13505.689 & $10526^{\circ}-240324$ & 7342.300 & 75 & 2 & 13615.959 & $10783_{2}^{\circ}-24399_{3}$ \\
\hline 7401.872 & 50 & & 13506.375 & $20214_{3}^{\circ}-33721_{2}$ & 7342.035 & 75 & 2 & 13616.451 & $19948{ }_{4}^{\circ}-33564_{3}$ \\
\hline 7400.309 & 2 & & 13509.228 & $20922_{2}^{\circ}-34431_{3}$ & 7341.769 & 5 & & 13616.944 & $20214_{3}^{\circ}-33831_{4}$ \\
\hline 7400.071 & 40 & 2 & 13509.663 & $19227^{\circ}-327375$ & 7341.433 & 8 & & 13617.567 & $16217_{2}^{\circ}-29835_{3}$ \\
\hline 7399.391 & 8 & & 13510.904 & $195324-33043_{3}^{\circ}$ & 7341.150 & 500 & 15 & 13618.092 & $10414_{4}^{\circ}-240324$ \\
\hline
\end{tabular}


TABLE 4. Classified lines of Th $\mathrm{I}$-continued

\begin{tabular}{|c|c|c|c|c|c|c|c|c|c|}
\hline \multirow{2}{*}{$\begin{array}{l}\text { Wavelength } \\
\text { (A) }\end{array}$} & \multicolumn{2}{|c|}{ Intensity } & \multirow{2}{*}{$\begin{array}{l}\text { Wavenumber } \\
\left(\mathrm{cm}^{-1}\right)\end{array}$} & \multirow{2}{*}{ Classification } & \multirow{2}{*}{$\begin{array}{c}\text { Wavelength } \\
\text { (§) }\end{array}$} & \multicolumn{2}{|c|}{ Inten sity } & \multirow{2}{*}{$\begin{array}{l}\text { Wavenumber } \\
\qquad\left(\mathrm{cm}^{-1}\right)\end{array}$} & \multirow{2}{*}{ Classification } \\
\hline & Lamp & Spark & & & & Lamp & Spark & & \\
\hline 7339.729 & 3 & & 13620.729 & $173983-31019_{4}^{\circ}$ & 7284.905 & 500 & 20 & 13723.233 & $3687_{2}-17411_{3}^{\circ}$ \\
\hline 7339.599 & 150 & 3 & 13620.970 & $13175_{4}^{\circ}-267963$ & 7284.419 & 40 & 1 & 13724.149 & $16783_{4}^{\circ}-305085$ \\
\hline 7339.409 & 50 & 1 & 13621.322 & $18930_{3}^{\circ}-32551_{3}$ & 7284.166 & 8 & 1 & 13724.626 & $18069_{3}^{\circ}-31793_{4}$ \\
\hline 7339.330 & 2 & & 13621.469 & $21252_{2}^{\circ}-34874_{3}$ & 7281.962 & 2 & & 13728.780 & $18431_{3}-32160_{2}^{\circ}$ \\
\hline 7339.005 & 8 & & 13622.072 & $171665-30788^{\circ}$ & 7280.478 & 20 & 1 & 13731.578 & $21902_{4}^{\circ}-35633_{4}$ \\
\hline 7336.798 & 4 & & 13626.170 & $195888^{\circ}-332145$ & 7279.142 & 8 & & 13734.098 & $22399^{\circ}-361335$ \\
\hline 7336.134 & 2 & & 13627.403 & $22669_{3}^{\circ}-36297_{4}$ & 7278.767 & 1 & & 13734.806 & $20566_{4}^{\circ}-34301_{4}$ \\
\hline 7335.802 & 5 & & 13628.020 & 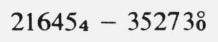 & 7278.272 & 1 & & 13735.740 & $18549_{2}-32285^{\circ}$ \\
\hline 7335.357 & 1 & & 13628.847 & $22248_{2}^{\circ}-35877_{2}$ & 7278.101 & 3 & & 13736.063 & $19227^{\circ}-329635$ \\
\hline 7334.067 & 4 & & 13631.244 & $22163{ }^{\circ}-357944$ & 7278.032 & 4 & & 13736.193 & $221411_{3}^{\circ}-35877_{2}$ \\
\hline 7331.906 & 2 & & 13635.262 & $22098_{4}-35733_{4}^{\circ}$ & 7277.013 & 75 & 1 & 13738.116 & $19532_{4}-33270_{4}^{\circ}$ \\
\hline 7329.488 & 100 & 5 & 13639.760 & $11802_{2}-25442_{3}^{\circ}$ & 7276.453 & 10 & 1 & 13739.174 & $17073_{1}-30812_{2}^{\circ}$ \\
\hline 7328.283 & $300 \mathrm{~s}$ & $50 \mathrm{~d}$ & 13642.003 & $7280_{2}-20922_{2}^{\circ}$ & 7275.972 & 2 & & 13740.082 & $18053_{4}^{\circ}-31793_{4}$ \\
\hline 7326.801 & 5 & & 13644.762 & $20522_{2}^{\circ}-34167_{3}$ & 7275.833 & 15 & 1 & 13740.344 & $17224_{2}^{\circ}-30964_{3}$ \\
\hline 7326.058 & 3 & & 13646.146 & $21594_{3}-35240_{3}^{\circ}$ & 7274.294 & 15 & 1 & 13743.251 & $211435-34886^{\circ}$ \\
\hline 7325.219 & 8 & & 13647.709 & $18549_{2}-32197_{3}^{\circ}$ & 7274.048 & 3 & & 13743.716 & $21890_{3}^{\circ}-35633_{4}$ \\
\hline 7324.894 & $20 \mathrm{~b}$ & & 13648.314 & $23655_{4}^{\circ}-37303_{4}$ & 7273.732 & 40 & 1 & 13744.313 & $16217_{2}^{\circ}-29961_{1}$ \\
\hline 7324.808 & 300 & 10 & 13648.475 & $142045-27852{ }^{\circ}$ & 7270.993 & 8 & & 13749.491 & $23655_{4}^{\circ}-37404_{3}$ \\
\hline 7323.988 & 10 & & 13650.003 & $170731-30723 i$ & 7270.558 & 100 & 2 & 13750.313 & $75023-21252_{2}^{\circ}$ \\
\hline 7323.740 & 2 & & 13650.465 & $22396_{1}^{\circ}-360472$ & 7268.834 & 100 & 2 & 13753.575 & $21575_{2}-35328_{1}^{\circ}$ \\
\hline 7323.207 & 100 & 3 & 13651.458 & $15490 \AA-291415$ & 7268.490 & 5 & 1 & 13754.226 & $20543 \AA-34298_{1}$ \\
\hline 7318.595 & 8 & & 13660.061 & $12847_{3}-26508^{\circ}$ & 7267.096 & 50 & 1 & 13756.864 & $19516_{2}^{\circ}-33273_{1}$ \\
\hline 7318.272 & $2 \mathrm{~h}$ & & 13660.664 & $22855_{3}^{\circ}-36515_{3}$ & 7264.747 & 2 & & 13761.312 & $21539_{4}^{\circ}-35300_{5}$ \\
\hline 7317.122 & 3 & & 13662.811 & $75023-21165_{3}^{\circ}$ & 7264.118 & 50 & & 13762.504 & $19532_{4}-33294_{3}^{\circ}$ \\
\hline 7316.676 & 2 & & 13663.644 & $20054_{2}-33718_{2}^{\circ}$ & 7262.578 & 2 & & 13765.422 & $18431_{3}-32197_{3}^{\circ}$ \\
\hline 7316.124 & 100 & 1 & 13664.675 & $15493_{4}-29157_{3}^{\circ}$ & 7262.190 & 10 & & 13766.157 & $23015{ }^{\circ}-367815$ \\
\hline 7315.609 & 150 & & 13665.637 & $17224_{2}^{\circ}-30889_{1}$ & 7261.217 & 5 & & 13768.002 & $15166_{3}^{\circ}-28934_{3}$ \\
\hline 7315.064 & 200 & 3 & 13666.655 & $3687_{2}-17354_{1}^{\circ}$ & 7260.874 & 5 & & 13768.652 & $16783_{4}^{\circ}-30552_{4}$ \\
\hline 7314.743 & 2 & & 13667.255 & $21738_{2}^{\circ}-35405_{3}$ & 7260.258 & 8 & & 13769.821 & $17501^{\circ}-312715$ \\
\hline 7311.510 & 100 & 3 & 13673.298 & $11241_{3}^{\circ}-24915_{3}$ & 7258.177 & 200 & 5 & 13773.769 & $14206_{4}^{\circ}-27980_{3}$ \\
\hline 7311.003 & 10 & 2 & 13674.246 & $21902_{4}^{\circ}-35576_{3}$ & 7257.334 & 2 & & 13775.369 & $20522_{2}^{\circ}-34298_{1}$ \\
\hline 7309.311 & $2 b$ & & 13677.412 & $21539^{\circ}-352165$ & 7256.981 & 150 & 2 & 13776.039 & $12114_{2}^{\circ}-25890_{2}$ \\
\hline 7309.255 & 100 & 2 & 13677.516 & $173983-31075_{2}^{\circ}$ & 7256.807 & 75 & 2 & 13776.369 & $15863_{2}-29640^{\circ}$ \\
\hline 7308.640 & 150 & 3 & 13678.667 & $15166^{\circ}-28845_{4}$ & 7255.351 & 200 & 5 & 13779.133 & $8111_{4}-21890_{3}^{\circ}$ \\
\hline 7307.933 & 4 & & 13679.991 & $21902_{4}^{\circ}-355825$ & 7253.672 & 100 & 3 & 13782.323 & $18011^{\circ}-317934$ \\
\hline 7307.233 & 4 & & 13681.301 & & 7251.943 & 2 & & 13785.609 & $20922_{2}^{\circ}-34707_{3}$ \\
\hline 7307.176 & 5 & & 13681.408 & $\begin{array}{r}192732-329542 \\
15736 i-294182\end{array}$ & 7250.100 & 15 & & 13789.113 & $19817_{1}^{\circ}-336062$ \\
\hline 7205.102 & & & & $15736_{1}^{\circ}-294182$ & 7249.842 & 75 & 1 & 13789.604 & $13088_{3}-26878_{3}^{\circ}$ \\
\hline 7305.193 & 50 & 1 & 13685.122 & $157361-294221$ & 7248.964 & 75 & 1 & 13791.274 & $8111_{4}-21902_{4}^{\circ}$ \\
\hline 7304.521 & 4 & & 13686.381 & $21890_{3}^{\circ}-35576_{3}$ & 7248.547 & 2 & & 13792.067 & $19516_{2}^{\circ}-33309_{2}$ \\
\hline 7302.496 & $3 b$ & & 13690.176 & $17847_{2}^{\circ}-315373$ & 7247.843 & 2 & & 13793.407 & $21252_{2}^{\circ}-350461$ \\
\hline 7302.428 & 75 & 2 & 13690.303 & $22399^{\circ}-360894$ & 7247.599 & 40 & & 13793.872 & $16217_{2}^{\circ}-30011_{3}$ \\
\hline 7298.138 & $200 \mathrm{~b}$ & 3 & 13698.351 & $13297_{4}-26995_{3}^{\circ}$ & 7246.370 & 3 & & 13796.211 & $21077^{\circ}-34873_{4}$ \\
\hline 7296.265 & 100 & 3 & 13701.867 & $13088_{3}-26790_{4}^{\circ}$ & 7246.318 & $5 \mathrm{~h}$ & & 13796.310 & $17398_{3}-31194_{4}^{\circ}$ \\
\hline 7294.316 & 15 & 1 & 13705.528 & $18069_{3}^{\circ}-317743$ & 7245.500 & 8 & & 13797.868 & $142260-28024^{\circ}$ \\
\hline 7294.217 & 5 & & 13705.714 & $11601_{1}-25306_{2}^{\circ}$ & 7245.292 & 2 & & 13798.264 & $22855_{3}^{\circ}-366532$ \\
\hline 7293.374 & 15 & 1 & 13707.298 & $20737_{1}^{\circ}-344442$ & 7244.689 & 200 & 8 & 13799.412 & $7795_{4}^{\circ}-215943$ \\
\hline 7292.864 & 2 & & 13708.257 & $21165_{3}^{\circ}-34873_{4}$ & 7244.689 & 200 & 8 & 13799.412 & $15618_{3}^{\circ}-29418_{2}$ \\
\hline 7292.864 & 2 & & 13708.257 & $22338_{3}^{\circ}-36047_{2}$ & 7242.345 & 150 & 2 & 13803.878 & $128473-26651_{2}^{\circ}$ \\
\hline 7292.494 & 3 & & 13708.952 & $21165^{\circ}-348743$ & 7242.282 & $20 \mathrm{~b}$ & & 13803.998 & $18699_{2}-32503_{2}^{\circ}$ \\
\hline 7291.276 & 8 & 2 & 13711.242 & $17959_{4}-31671_{4}^{\circ}$ & 7242.086 & 200 & 10 & 13804.372 & $98045-236098$ \\
\hline 7289.812 & 4 & & 13713.996 & & 7241.280 & 2 & & 13805.908 & $19503_{3}^{\circ}-33309_{2}$ \\
\hline 7289.812 & 2 & & $13 / 13.990$ & $197133-334272$ & 7240.181 & 100 & 2 & 13808.004 & $14465_{2}^{\circ}-28273_{2}$ \\
\hline 7289.659 & 2 & & 13714.284 & $220984-35812_{3}^{\circ}$ & 7238.404 & 8 & & 13811.394 & $22399_{0}^{\circ}-36210_{5}$ \\
\hline 7288.990 & 10 & 1 & 13715.543 & $15166_{3}^{\circ}-28882_{2}$ & 7236.257 & 20 & & 13815.492 & $22669_{3}^{\circ}-36485_{2}$ \\
\hline 7288.610 & 50 & 1 & 13716.258 & $15970_{3}-29686_{3}^{\circ}$ & 7235.899 & 50 & 1 & 13816.175 & $21645_{4}-35462^{\circ}$ \\
\hline 7286.110 & 3 & & 13720.964 & $18053^{\circ}-317743$ & 7235.551 & 20 & & 13816.840 & $15493_{4}-29310_{4}^{\circ}$ \\
\hline
\end{tabular}


TABLE 4. Classified lines of Th I-continued

\begin{tabular}{|c|c|c|c|c|c|c|c|c|c|}
\hline \multirow{2}{*}{$\begin{array}{l}\text { Wavelength } \\
(\AA)\end{array}$} & \multicolumn{2}{|c|}{ Intensity } & \multirow{2}{*}{$\begin{array}{l}\text { Wavenumber } \\
\left(\mathrm{cm}^{-1}\right)\end{array}$} & \multirow{2}{*}{ Classification } & \multirow{2}{*}{$\begin{array}{c}\text { Wavelength } \\
\text { (§) }\end{array}$} & \multicolumn{2}{|c|}{ Intensity } & \multirow{2}{*}{$\begin{array}{l}\text { Wavenumber } \\
\left(\mathrm{cm}^{-1}\right)\end{array}$} & \multirow{2}{*}{ Classification } \\
\hline & Lamp & Spark & & & & Lamp & Spark & & \\
\hline 7233.390 & 100 & & 13820.967 & $17959_{4}-31780_{3}^{\circ}$ & 7180.959 & 8 & & 13921.879 & $20522_{2}^{\circ}-344442$ \\
\hline 7232.946 & 10 & & 13821.816 & $13962_{1}-27784_{2}^{\circ}$ & 7179.725 & 150 & 4 & 13924.272 & $21165_{3}^{\circ}-35089_{3}$ \\
\hline 7226.246 & 15 & & 13834.631 & $17411^{\circ}-31245_{2}$ & 7179.189 & 1 & & 13925.312 & $11601_{1}-25526^{\circ}$ \\
\hline 7225.125 & 20 & 5 & 13836.778 & $21252_{2}^{\circ}-35089_{3}$ & 7178.539 & 10 & 1 & 13926.572 & $18069_{3}^{\circ}-31995_{4}$ \\
\hline 7220.989 & 100 & 2 & 13844.703 & $10783_{2}^{\circ}-24627_{1}$ & 7178.252 & 8 & & 13927.129 & $18809_{4}^{\circ}-32737_{5}$ \\
\hline 7219.782 & 25 & 1 & 13847.017 & $19713_{3}-33550_{4}^{\circ}$ & 7177.284 & 50 & 2 & 13929.008 & $185741-32503_{2}^{\circ}$ \\
\hline 7219.156 & 300 & 10 & 13848.218 & $4961_{4}-18809_{4}^{\circ}$ & 7173.372 & 500 & 20 & 13936.604 & $13847_{2}-27784_{2}^{\circ}$ \\
\hline 7218.056 & 500 & 75 & 13850.328 & $98045-23655_{4}^{\circ}$ & 7173.372 & 500 & 20 & 13936.604 & $142045-28140_{4}^{\circ}$ \\
\hline 7216.911 & 8 & & 13852.526 & $6362_{2}-20214_{3}^{\circ}$ & 7173.117 & 8 & & 13937.099 & $15166_{3}^{\circ}-291044$ \\
\hline 7216.666 & 40 & 2 & 13852.996 & $17166_{5}-31019_{4}^{\circ}$ & 7172.867 & 8 & & 13937.585 & $211435-35081{ }^{\circ}$ \\
\hline 7215.933 & 8 & & 13854.403 & $17411_{3}^{\circ}-31265_{3}$ & 7170.575 & 2 & & 13942.040 & $180534^{\circ}-319954$ \\
\hline 7215.667 & 10 & & 13854.914 & $17073_{1}-30928_{1}^{\circ}$ & 7170.357 & 100 & 2 & 13942.464 & $128473-26790_{4}^{\circ}$ \\
\hline 7212.687 & 500 & 50 & 13860.638 & $14481 \stackrel{\circ}{6}-283425$ & 7169.621 & 4 & & 13943.895 & $18069_{3}^{\circ}-320124$ \\
\hline 7211.769 & 2 & & 13862.403 & $18431_{3}-32294_{4}^{\circ}$ & 7168.896 & 800 & 75 & 13945.305 & $0_{2}-13945_{3}^{\circ}$ \\
\hline 7210.562 & 2 & & 13864.723 & $20566_{4}^{\circ}-34431_{3}$ & 7167.202 & 150 & 4 & 13948.601 & $14032_{2}^{\circ}-27980_{3}$ \\
\hline 7210.036 & 5 & & 13865.735 & $21539_{4}^{\circ}-35405_{3}$ & 7165.492 & 8 & 1 & 13951.930 & $20922_{2}^{\circ}-34874_{3}$ \\
\hline 7209.981 & 5 & & 13865.840 & $18930_{3}^{\circ}-32796_{3}$ & 7165.176 & 25 & 1 & 13952.545 & $20214_{3}^{\circ}-34167_{3}$ \\
\hline 7209.177 & 5 & & 13867.387 & $21594_{3}-35462_{3}^{\circ}$ & 7164.508 & 2 & & 13953.846 & $5563_{1}-19516_{2}^{\circ}$ \\
\hline 7208.275 & $100 \mathrm{~b}$ & & 13869.122 & $13175_{4}^{\circ}-27044_{3}$ & 7164.323 & 8 & & 13954.206 & $18549_{2}-32503_{2}^{\circ}$ \\
\hline 7208.000 & 500 & 150 & 13869.651 & $8800_{4}-22669_{3}^{\circ}$ & 7163.374 & 2 & & 13956.055 & $195033^{\circ}-33459_{4}$ \\
\hline 7207.756 & $50 \mathrm{~b}$ & 2 & 13870.121 & $23015 \circ-36885_{4}$ & 7163.180 & 5 & & 13956.433 & $19817^{\circ}-33773_{1}$ \\
\hline 7207.392 & 5 & & 13870.821 & $195888^{\circ}-33459_{4}$ & 7162.250 & 8 & 1 & 13958.245 & $21575_{2}-35533^{\circ}$ \\
\hline 7207.016 & 50 & 2 & 13871.545 & $17224_{2}^{\circ}-31095_{1}$ & 7161.689 & 2 & & 13959.339 & $18053^{\circ}-320124$ \\
\hline 7206.476 & 400 & 8 & 13872.584 & $105263_{3}^{\circ}-24399_{3}$ & $7159: 943$ & $300 \mathrm{~b}$ & 8 & 13962.743 & $13297_{4}-27260_{3}^{\circ}$ \\
\hline 7205.435 & 2 & & 13874.589 & $20423 i-342981$ & 7159.877 & $100 \mathrm{~b}$ & 2 & 13962.871 & $165546-30517{ }^{\circ}$ \\
\hline 7204.761 & $3 \mathrm{~h}$ & & 13875.887 & $18053_{4}^{\circ}-31929_{3}$ & 7156.936 & 300 & 15 & 13968.609 & $4961_{4}-18930_{3}^{\circ}$ \\
\hline 7203.593 & 4 & & 13878.136 & $197133-33591 \stackrel{\circ}{3}$ & 7156.936 & 300 & 15 & 13968.609 & $13297_{4}-27266_{4}^{\circ}$ \\
\hline 7202.335 & 5 & & 13880.560 & $20566_{4}^{\circ}-34447_{4}$ & 7155.349 & 8 & & 13971.707 & $18809_{4}^{\circ}-32781_{4}$ \\
\hline 7201.803 & 100 & 2 & 13881.586 & $10783_{2}^{\circ}-24664_{3}$ & 7154.952 & 200 & 8 & 13972.482 & $7280_{2}-21252_{2}^{\circ}$ \\
\hline 7201.042 & 50 & 1 & 13883.053 & $159703-29853_{2}^{\circ}$ & 7154.762 & 200 & 20 & 13972.853 & $130888_{3}-27061_{2}^{\circ}$ \\
\hline 7200.815 & 2 & & 13883.490 & $19948_{4}^{\circ}-33831_{4}$ & 7153.587 & 200 & 10 & 13975.149 & $171665-311410^{\circ}$ \\
\hline 7200.171 & 50 & & 13884.732 & $17398_{3}-31283^{\circ}$ & 7151.525 & 20 & 1 & 13979.178 & $20322{ }^{\circ}-34301_{4}$ \\
\hline 7200.041 & 400 & 10 & 13884.983 & $7280_{2}-21165_{3}^{\circ}$ & 7150.285 & 400 & 25 & 13981.602 & $3865_{1}-17847_{2}^{\circ}$ \\
\hline 7198.986 & 5 & & 13887.018 & $21575_{2}-35462_{3}^{\circ}$ & 7149.224 & 5 & & 13983.677 & $22669 \stackrel{\circ}{3}-36653_{2}$ \\
\hline 7198.214 & 4 & & 13888.507 & $19273_{2}-33161_{i}^{\circ}$ & 7148.923 & 8 & 1 & 13984.266 & $18011 \stackrel{\circ}{-31995_{4}}$ \\
\hline 7198.092 & 20 & & 13888.742 & $10783_{2}^{\circ}-24671_{2}$ & 7148.728 & 50 & 1 & 13984.647 & $21165_{3}^{\circ}-35149_{2}$ \\
\hline 7196.791 & 2 & & 13891.253 & $17354^{\circ}-31245_{2}$ & 7148.557 & 400 & 15 & 13984.982 & $10414_{4}^{\circ}-24399_{3}$ \\
\hline 7196.569 & 8 & & 13891.682 & $19516_{2}^{\circ}-33408_{3}$ & 7147.913 & 3 & & 13986.242 & $18809_{4}^{\circ}-327963$ \\
\hline 7195.797 & 4 & & 13893.172 & $14465_{2}^{\circ}-283583$ & 7147.423 & 15 & 1 & 13987.201 & $19227^{\circ}-332145$ \\
\hline 7195.236 & 3 & & 13894.255 & $20566_{4}^{\circ}-344605$ & 7147.167 & 2 & & 13987.702 & $21890_{3}^{\circ}-35877_{2}$ \\
\hline 7193.357 & 1 & & 13897.885 & $22399_{0}^{\circ}-36297_{4}$ & 7146.373 & 8 & 1 & 13989.256 & $15863_{2}-29853_{2}^{\circ}$ \\
\hline 7192.019 & 40 & 2 & 13900.470 & $11802_{2}-25703_{2}^{\circ}$ & 7144.169 & 8 & & 13993.572 & $17959_{4}-31953_{4}^{\circ}$ \\
\hline 7191.585 & 3 & & 13901.309 & $21539_{4}^{\circ}-35440_{3}$ & 7142.328 & 300 & 8 & 13997.179 & $12114_{2}^{\circ}-26111_{1}$ \\
\hline 7190.332 & 2 & & 13903.731 & $22877^{\circ}-36781_{5}$ & 7140.080 & 4 & & 14001.585 & $18011^{\circ}-32012_{4}$ \\
\hline 7190.124 & 5 & & 13904.134 & $19817_{1}^{\circ}-33721_{2}$ & 7138.142 & 20 & & 14005.387 & $19713_{3}-33718_{2}^{\circ}$ \\
\hline 7190.050 & 5 & & 13904.277 & $21890_{3}^{\circ}-35794_{4}$ & 7137.635 & 3 & & 14006.382 & $11802_{2}-25809_{1}^{\circ}$ \\
\hline 7189.407 & 10 & 1 & 13905.520 & $19503_{3}^{\circ}-334083$ & 7137.133 & 5 & & 14007.367 & $18431_{3}-32439_{4}^{\circ}$ \\
\hline 7189.323 & 1 & & 13905.683 & $22141_{3}^{\circ}-36047_{2}$ & 7136.956 & 8 & & 14007.714 & $22877_{i}^{\circ}-36885_{4}$ \\
\hline 7188.530 & 100 & 3 & 13907.217 & $13088_{3}-26995_{3}^{\circ}$ & 7136.841 & 4 & & 14007.940 & $22508_{2}^{\circ}-36515_{3}$ \\
\hline 7185.031 & 3 & & 13913.989 & $15736_{1}^{\circ}-29650_{2}$ & 7134.484 & 8 & & 14012.568 & $11877_{1}^{\circ}-25890_{2}$ \\
\hline 7184.721 & 4 & & 13914.590 & $2869_{3}-16783_{4}^{\circ}$ & 7132.603 & 200 & 4 & 14016.263 & $13175_{4}^{\circ}-271915$ \\
\hline 7184.542 & 3 & & 13914.936 & $19503_{3}^{\circ}-33418_{4}$ & 7130.718 & 200 & 5 & 14019.968 & $132974-27317^{\circ}$ \\
\hline 7184.426 & 4 & & 13915.161 & $16217_{2}^{\circ}-301322$ & 7130.179 & 100 & 4 & 14021.028 & $14206_{4}^{\circ}-28227_{4}$ \\
\hline 7184.111 & 8 & & 13915.771 & $17411_{3}^{\circ}-31326_{4}$ & 7129.871 & 20 & & 14021.634 & $19273_{2}-33294^{\circ}$ \\
\hline 7183.225 & 2 & & 13917.488 & $17224_{2}^{\circ}-31141_{3}$ & 7128.450 & 8 & 1 & 14024.429 & $195033^{\circ}-33527_{4}$ \\
\hline
\end{tabular}


TABle 4. Classified lines of Th $\mathrm{I}$-continued

\begin{tabular}{|c|c|c|c|c|c|c|c|c|c|}
\hline \multirow{2}{*}{$\begin{array}{l}\text { Wavelength } \\
\text { (§) }\end{array}$} & \multicolumn{2}{|c|}{ Intensity } & \multirow{2}{*}{$\begin{array}{l}\text { Wavenumber } \\
\left(\mathrm{cm}^{-1}\right)\end{array}$} & \multirow{2}{*}{ Classification } & \multirow{2}{*}{$\begin{array}{c}\text { Wavelength } \\
\text { (§) }\end{array}$} & \multicolumn{2}{|c|}{ Inten sity } & \multirow{2}{*}{$\begin{array}{l}\text { Wavenumber } \\
\qquad\left(\mathrm{cm}^{-1}\right)\end{array}$} & \multirow{2}{*}{ Classification } \\
\hline & Lamp & Spark & & & & Lamp & Spark & & \\
\hline 7127.640 & 10 & & 14026.022 & $18549_{2}-32575_{2}^{\circ}$ & 7071.096 & 150 & 3 & 14138.181 & $10526^{\circ}-246643$ \\
\hline 7126.814 & 100 & 3 & 14027.648 & $19532_{4}-33560_{4}^{\circ}$ & 7070.984 & 10 & & 14138.405 & $22669_{3}^{\circ}-368083$ \\
\hline 7126.324 & 8 & & 14028.613 & $17166_{5}-31194_{4}^{\circ}$ & 7070.891 & 2 & & 14138.591 & $20322 \circ-344605$ \\
\hline 7126.009 & 5 & & 14029.233 & $14243_{1}^{\circ}-28273_{2}$ & 7070.793 & 1 & & 14138.787 & $215943-35733^{\circ}$ \\
\hline 7125.515 & 150 & 2 & 14030.205 & $12847_{3}-26878_{3}^{\circ}$ & 7070.279 & 2 & & 14139.815 & $210777^{\circ}-352165$ \\
\hline 7125.313 & 10 & 1 & 14030.603 & $8111_{4}-22141_{3}^{\circ}$ & 7068.330 & 2 & & 14143.713 & $18431_{3}-32575_{2}^{\circ}$ \\
\hline 7124.556 & 1000 & 50 & 14032.094 & $0_{2}-14032_{2}^{\circ}$ & 7067.513 & 75 & & 14145.348 & $10526_{3}^{\circ}-24671_{2}$ \\
\hline 7122.887 & 3 & & 14035.382 & $13945_{3}^{\circ}-27980_{3}$ & 7067.247 & 10 & & 14145.881 & $142260-28372^{\circ}$ \\
\hline 7122.098 & 3 & & 14036.937 & $21539_{4}^{\circ}-35576_{3}$ & 7066.996 & 3 & & 14146.383 & $22338_{3}^{\circ}-36485_{2}$ \\
\hline 7121.908 & 8 & & 14037.311 & $75023-21539_{4}^{\circ}$ & 7066.287 & 100 & 2 & 14147.803 & $128473-26995^{\circ}$ \\
\hline 7119.870 & 3 & & 14041.329 & $17224_{2}^{\circ}-31265_{3}$ & 7064.451 & 300 & 10 & 14151.480 & $14206_{4}^{\circ}-283583$ \\
\hline 7119.174 & 2 & & 14042.702 & $21539_{4}^{\circ}-355825$ & 7063.844 & 8 & & 14152.696 & $21252_{2}^{\circ}-35405_{3}$ \\
\hline 7116.992 & 20 & 1 & 14047.007 & $15970_{3}-30017_{3}^{\circ}$ & 7063.585 & 10 & & 14153.214 & $15863_{2}-30017_{3}^{\circ}$ \\
\hline 7116.551 & 8 & & 14047.878 & $19516_{2}^{\circ}-33564_{3}$ & 7063.314 & 8 & & 14153.757 & $19273_{2}-33427_{2}^{\circ}$ \\
\hline 7114.394 & 150 & 3 & 14052.137 & $8111_{4}-22163_{4}^{\circ}$ & 7062.418 & 40 & 1 & 14155.553 & $224011-36556_{1}^{\circ}$ \\
\hline 7113.007 & $5 \mathrm{~h}$ & & 14054.877 & $18699_{2}-32754_{3}^{\circ}$ & 7061.607 & 1 & & 14157.179 & $21890_{3}^{\circ}-36047_{2}$ \\
\hline 7112.917 & 100 & 4 & 14055.055 & $8800_{4}-22855_{3}^{\circ}$ & 7061.392 & 100 & 2 & 14157.610 & $8243_{2}^{\circ}-22401_{1}$ \\
\hline 7111.045 & 75 & 2 & 14058.755 & $195324-33591_{3}^{\circ}$ & 7060.651 & 400 & 10 & 14159.096 & $3687_{2}-17847_{2}^{\circ}$ \\
\hline 7110.278 & 2 & & 14060.271 & $19039_{2}^{\circ}-33099_{3}$ & 7060.039 & 300 & 8 & 14160.323 & $63622-20522_{2}^{\circ}$ \\
\hline 7109.857 & 150 & 4 & 14061.104 & $63622_{2}-20423_{i}^{\circ}$ & 7059.526 & 150 & 3 & 14161.352 & $16346_{4}^{\circ}-305085$ \\
\hline 7109.550 & 40 & 2 & 14061.711 & $19503 \stackrel{\circ}{3}-335643$ & 7058.488 & 150 & 5 & 14163.435 & $11241_{3}^{\circ}-254054$ \\
\hline 7109.320 & 10 & & 14062.166 & $13962_{1}-28024_{i}^{\circ}$ & 7058.221 & 2 & & 14163.970 & $23655_{4}^{\circ}-37819_{4}$ \\
\hline 7109.175 & 50 & & 14062.453 & $15490 \AA-295526$ & 7056.885 & 4 & & 14166.652 & $216454-35812^{\circ}$ \\
\hline 7108.550 & 2 & & 14063.689 & $179594-320230^{\circ}$ & 7056.595 & 10 & & 14167.234 & $20922_{2}^{\circ}-35089_{3}$ \\
\hline 7107.469 & 10 & 1 & 14065.828 & $14465_{2}^{\circ}-28531_{2}$ & 7055.900 & 50 & & 14168.629 & $13175_{4}^{\circ}-273433$ \\
\hline 7105.654 & 10 & & 14069.421 & $16217_{2}^{\circ}-30286_{1}$ & 7055.660 & 1 & & 14169.111 & $18930 \stackrel{\circ}{3}-33099_{3}$ \\
\hline 7104.404 & 41 & & 14071.896 & $18431_{3}-32503_{2}^{\circ}$ & 7054.416 & 150 & 2 & 14171.610 & $13088_{3}-27260_{3}^{\circ}$ \\
\hline 7103.029 & 8 & & 14074.620 & $17354_{i}^{\circ}-314291$ & 7051.787 & 50 & & 14176.893 & $13847_{2}-28024_{1}^{\circ}$ \\
\hline 7102.185 & 10 & & 14076.293 & $18574_{1}-32650_{i}^{\circ}$ & 7051.503 & 8 & & 14177.464 & $13088_{3}-27266_{4}^{\circ}$ \\
\hline 7101.660 & 4 & & 14077.334 & $8800_{4}-22877_{0}^{\circ}$ & 7049.844 & 20 & & 14180.801 & $167833_{4}^{\circ}-309643$ \\
\hline 7100.391 & 2 & & 14079.850 & $22098_{4}-36178_{4}^{\circ}$ & 7047.762 & 2 & & 14184.990 & $20522_{2}^{\circ}-34707_{3}$ \\
\hline 7099.094 & 4 & & 14082.422 & $17847_{2}^{\circ}-31929_{3}$ & 7047.319 & 2 & & 14185.882 & $19503_{3}^{\circ}-33689_{2}$ \\
\hline 7097.645 & 5 & & 14085.297 & $20322^{\circ}-344076$ & 7046.560 & 40 & & 14187.409 & $21902_{4}^{\circ}-36089_{4}$ \\
\hline 7097.032 & 10 & & 14086.513 & $20214_{3}^{\circ}-34301_{4}$ & 7046.124 & 10 & & 14188.287 & $21252_{2}^{\circ}-35440_{3}$ \\
\hline 7096.665 & 5 & & 14087.242 & $22248_{2}^{\circ}-363363$ & 7045.332 & 1 & & 14189.882 & $15970_{3}-30160_{4}^{\circ}$ \\
\hline 7096.478 & 4 & & 14087.613 & $19713_{3}-33800^{\circ}$ & 7044.969 & 50 & 1 & 14190.613 & $18011^{\circ}-322025$ \\
\hline $\begin{array}{l}7096.000 \\
7096\end{array}$ & 4 & & $1408 / .013$ & & 7043.714 & 15 & & 14193.142 & $15493_{4}-29686_{3}^{\circ}$ \\
\hline 7096.000 & 3 & & 14088.562 & $22396_{1}^{\circ}-36485_{2}$ & 7043.228 & 8 & & 14194.121 & $15490 \AA-296845$ \\
\hline 7095.623 & 4 & & 14089.311 & $19516_{2}^{\circ}-33606_{2}$ & 7042.997 & 8 & & 14194.587 & $221411_{3}^{\circ}-363363$ \\
\hline 7094.803 & 3 & & 14090.939 & $18574_{1}-32665_{i}^{\circ}$ & 7042.031 & 3 & & 14196.534 & $230150^{\circ}-372115$ \\
\hline 7093.128 & 3 & & 14094.266 & $21539_{4}^{\circ}-35633_{4}$ & 7040.530 & 2 & & 14199.560 & $21890_{3}^{\circ}-36089_{4}$ \\
\hline 7089.489 & 8 & & 14101.501 & $18549_{2}-32650_{1}^{\circ}$ & 7039.877 & 5 & & 14200.878 & $173983-31599_{2}^{\circ}$ \\
\hline 7089.052 & 8 & & 14102.370 & $11601_{1}-25703_{2}^{\circ}$ & 7038.165 & 40 & & 14204.332 & $19516_{2}^{\circ}-33721_{2}$ \\
\hline 7088.663 & 1 & & 14103.144 & $19503_{3}^{\circ}-33606_{2}$ & 7037.852 & 10 & & 14204.964 & $17224_{2}^{\circ}-31429_{1}$ \\
\hline 7088.330 & 8 & & 14103.807 & $21575_{2}-35678_{1}^{\circ}$ & 7037.781 & 8 & & 14205.107 & $18549_{2}-32754_{3}^{\circ}$ \\
\hline 7087.506 & 3 & & 14105.446 & $22669_{3}^{\circ}-36775_{3}$ & 7037.410 & 4 & & 14205.856 & $16346_{4}^{\circ}-305524$ \\
\hline 7084.165 & 500 & 75 & 14112.099 & $98045-23916_{4}^{\circ}$ & $\begin{array}{l}7036.277 \\
7036.206\end{array}$ & $\begin{array}{c}400 \\
50 \mathrm{~b}\end{array}$ & 8 & $\begin{array}{l}14208.143 \\
14208.287\end{array}$ & \\
\hline 7082.137 & 20 & & 14116.140 & $18549_{2}-32665_{1}^{\circ}$ & $\begin{array}{l}7036.206 \\
7033.646\end{array}$ & $\begin{array}{l}50 \mathrm{~b} \\
50\end{array}$ & 1 & $\begin{array}{l}14208.287 \\
14213.458\end{array}$ & $128473-27061_{2}$ \\
\hline 7077.740 & 4 & & 14124.909 & $20322{ }^{\circ}-344474$ & 7033.352 & 100 & 2 & 14214.052 & $14465_{2}^{\circ}-28679_{2}$ \\
\hline 7077.422 & $50 \mathrm{~b}$ & 3 & 14125.544 & $173983-315233^{\circ}$ & 7032.924 & 100 & 1 & 14214.917 & $8800_{4}-230150$ \\
\hline 7077.380 & $50 \mathrm{~b}$ & 4 & 14125.628 & $17501{ }^{\circ}-316265$ & 7032.677 & 3 & & 14215.416 & $22669_{3}^{\circ}-36885_{4}$ \\
\hline 7077.194 & 4 & & 14125.999 & $17411_{3}^{\circ}-315373$ & 7032.350 & 3 & & 14216.077 & $156183^{\circ}-298353$ \\
\hline 7074.257 & 50 & 1 & 14131.863 & $10783^{\circ}-249153$ & 7032.178 & 15 & 1 & 14216.425 & $20214_{3}^{\circ}-34431_{3}$ \\
\hline 7073.110 & 8 & & 14134.155 & $22163_{4}^{\circ}-36297_{4}$ & 7031.469 & 15 & 1 & 14217.858 & $215943-35812^{\circ}$ \\
\hline 7072.394 & 300 & 8 & 14135.586 & $14206_{4}^{\circ}-283425$ & 7031.310 & 50 & 1 & 14218.180 & $19503_{3}^{\circ}-33721_{2}$ \\
\hline 70071.482 & 100 & & 14137.409 & $15618_{3}^{\circ}-297564$ & 7031.194 & 10 & & 14218.415 & $154934-29711_{0}^{\circ}$ \\
\hline
\end{tabular}


TABLE 4. Classified lines of Th I-continued

\begin{tabular}{|c|c|c|c|c|c|c|c|c|c|}
\hline \multirow{2}{*}{$\begin{array}{l}\text { Wavelength } \\
(\AA)\end{array}$} & \multicolumn{2}{|c|}{ Intensity } & \multirow{2}{*}{$\begin{array}{l}\text { Wavenumber } \\
\left(\mathrm{cm}^{-1}\right)\end{array}$} & \multirow{2}{*}{ Classification } & \multirow{2}{*}{$\begin{array}{c}\text { Wave length } \\
(\AA)\end{array}$} & \multicolumn{2}{|c|}{ Inten sity } & \multirow{2}{*}{$\begin{array}{l}\text { Wavenumber } \\
\left(\mathrm{cm}^{-1}\right)\end{array}$} & \multirow{2}{*}{ Classification } \\
\hline & Lamp & Spark & & & & Lamp & Spark & & \\
\hline 7030.858 & 50 & 1 & 14219.094 & $19948^{\circ}-34167_{3}$ & 6980.543 & 4 & 1 & 14321.583 & $20724 \stackrel{\circ}{-}-35046_{1}$ \\
\hline 7028.017 & 100 & 2 & 14224.842 & $15736_{1}^{\circ}-29961_{1}$ & 6979.944 & 1 & & 14322.812 & $18431_{3}-32754_{3}^{\circ}$ \\
\hline 7026.454 & 100 & 1 & 14228.006 & $8111_{4}-22338^{\circ}$ & 6978.753 & 2 & & 14325.256 & $17959_{4}-32285_{3}^{\circ}$ \\
\hline 7026.041 & 8 & & 14228.842 & $13088_{3}-27317_{3}^{\circ}$ & 6978.241 & 50 & 2 & 14326.307 & $14032_{2}^{\circ}-283583$ \\
\hline 7025.379 & 1 & & 14230.183 & $15618^{\circ}-29849_{4}$ & 6977.875 & 3 & & 14327.059 & $16217_{2}^{\circ}-30544_{2}$ \\
\hline 7025.188 & 4 & & 14230.570 & $22855_{3}^{\circ}-37085_{4}$ & 6977.462 & 25 & 1 & 14327.907 & $13945_{3}^{\circ}-28273_{2}$ \\
\hline 7023.776 & 8 & & 14233.431 & $11802_{2}-26036_{3}^{\circ}$ & 6977.061 & 3 & 1 & 14328.730 & $195033^{\circ}-33831_{4}$ \\
\hline 7023.636 & 25 & 1 & 14233.715 & $11877_{1}^{\circ}-2611_{1}$ & 6976.839 & 2 & & 14329.186 & $22098_{4}-36427_{3}^{\circ}$ \\
\hline 7023.523 & 100 & 3 & 14233.944 & $165546-30788_{0}^{\circ}$ & 6974.446 & 10 & & 14334.103 & $22877_{i}^{\circ}-37211_{5}$ \\
\hline 7023.145 & 100 & 3 & 14234.710 & $19039_{2}^{\circ}-33273_{1}$ & 6974.381 & 1 & & 14334.236 & $17959_{4}-32294_{4}^{\circ}$ \\
\hline 7022.847 & 2 & & 14235.314 & $23655_{4}^{\circ}-37890_{5}$ & 6970.054 & 5 & 1 & 14343.135 & $18069_{3}^{\circ}-32412_{4}$ \\
\hline 7022.619 & 8 & & 14235.776 & $75023-21738_{2}^{\circ}$ & 6969.779 & 2 & & 14343.701 & $18699_{2}-330433^{\circ}$ \\
\hline 7021.899 & 40 & & 14237.236 & $17959_{4}-32197_{3}^{\circ}$ & 6969.301 & 100 & 3 & 14344.685 & $15863_{2}-30208_{2}^{\circ}$ \\
\hline 7021.784 & 2 & & 14237.469 & $21575_{2}-35812_{3}^{\circ}$ & 6966.905 & 3 & & 14349.618 & $22399^{\circ}-36749_{6}$ \\
\hline 7021.668 & 3 & & 14237.704 & $23655_{4}^{\circ}-378924$ & 6966.064 & $3 \mathrm{~h}$ & & 14351.350 & $20522_{2}^{\circ}-348743$ \\
\hline 7021.279 & 150 & 8 & 14238.493 & $15970_{3}-30208_{2}^{\circ}$ & 6965.946 & 5001 & 4 & 14351.593 & $19039_{2}^{\circ}-33390_{1}$ \\
\hline 7020.476 & 100 & 1 & 14240.121 & $154934-297330^{\circ}$ & 6965.240 & 5 & & 14353.048 & $19948{ }_{4}^{\circ}-34301_{4}$ \\
\hline 7019.970 & 4 & & 14241.148 & $14032_{2}^{\circ}-28273_{2}$ & 6964.271 & 50 & 1 & 14355.045 & $2869_{3}-17224_{2}^{\circ}$ \\
\hline 7018.784 & 3 & & 14243.554 & $195880^{\circ}-33831_{4}$ & 6963.930 & 50 & 1 & 14355.748 & $14206_{4}^{\circ}-285624$ \\
\hline 7018.563 & 500 & 75 & 14244.003 & $0_{2}-14243^{\circ}$ & 6962.862 & 75 & 2 & 14357.950 & $16783 \stackrel{\circ}{4}-311413$ \\
\hline 7015.311 & 100 & 3 & 14250.605 & $10414_{4}^{\circ}-24664_{3}$ & 6962.310 & 100 & 4 & 14359.088 & $15490_{0}^{\circ}-29849_{4}$ \\
\hline 7014.965 & 100 & 4 & 14251.308 & $154934-29744^{\circ}$ & 6960.232 & 4 & & 14363.375 & $17411_{3}^{\circ}-317743$ \\
\hline 7014.873 & 50 & 1 & 14251.495 & $15166^{\circ}-29418_{2}$ & 6957.265 & 1 & & 14369.501 & $19039_{2}^{\circ}-33408_{3}$ \\
\hline 7013.617 & 8 & & 14254.047 & $5563_{1}-19817_{i}^{\circ}$ & 6956.113 & 1 & & 14371.880 & $16351_{0}-30723^{\circ}$ \\
\hline 7013.171 & 1 & & 14254.954 & $18699_{2}-32954_{2}^{\circ}$ & 6955.315 & 100 & 3 & 14373.529 & $132974-27670_{3}^{\circ}$ \\
\hline 7007.092 & 100 & 4 & 14267.321 & $195324-33799_{4}^{\circ}$ & 6954.655 & 200 & 5 & 14374.893 & $63622_{2}-20737_{1}^{\circ}$ \\
\hline 7006.655 & 4 & & 14268.210 & $195324-33800_{3}^{\circ}$ & 6954.387 & 2 & & 14375.447 & $20054_{2}-34430_{3}^{\circ}$ \\
\hline 7006.113 & 50 & 2 & 14269.314 & $20054_{2}-34324_{2}^{\circ}$ & 6952.796 & 1 & & 14378.737 & $18930_{3}^{\circ}-33309_{2}$ \\
\hline 7005.825 & 8 & & 14269.901 & $19039_{2}^{\circ}-33309_{2}$ & 6952.189 & 2 & & 14379.992 & $18574_{1}-32954_{2}^{\circ}$ \\
\hline 7002.881 & 200 & 8 & 14275.900 & $14204_{5}-28480_{4}^{\circ}$ & 6951.654 & 20 & 1 & 14381.099 & $3687_{2}-18069_{3}^{\circ}$ \\
\hline 7000.806 & 500 & 75 & 14280.131 & $98045-24084_{6}^{\circ}$ & 6951.259 & 5 & 1 & 14381.916 & $223990^{\circ}-367815$ \\
\hline 6999.624 & 100 & 3 & 14282.543 & $18011^{\circ}-322935$ & 6950.990 & 5 & & 14382.473 & $17398_{3}-31780_{3}^{\circ}$ \\
\hline 6998.247 & 100 & 2 & 14285.353 & $15970_{3}-30255_{3}^{\circ}$ & 6950.990 & 5 & & 14382.473 & $17411_{3}^{\circ}-317934$ \\
\hline 6997.412 & 50 & 1 & 14287.058 & $14243 i-28531_{2}$ & 6949.754 & 10 & & 14385.030 & $139621-28347_{2}^{\circ}$ \\
\hline 6996.812 & 1 & & 14288.283 & $19713_{3}-34001_{4}^{\circ}$ & 6948.391 & 100 & 3 & 14387.852 & $7502_{3}-21890_{3}^{\circ}$ \\
\hline 6996.757 & 501 & & 14288.395 & $8111_{4}-22399_{0}^{\circ}$ & 6948.205 & 300 & 20 & 14388.237 & $154934-29881_{4}^{\circ}$ \\
\hline 6996.202 & 8 & & 14289.528 & $18809_{4}^{\circ}-33099_{3}$ & 6948.090 & 75 & 1 & 14388.475 & $10526_{3}^{\circ}-24915_{3}$ \\
\hline 6995.141 & 75 & 2 & 14291.696 & $21539_{4}^{\circ}-358313$ & 6947.914 & 100 & 3 & 14388.840 & $7280_{2}-21668^{\circ}$ \\
\hline 6994.736 & 100 & 3 & 14292.523 & $17501 \stackrel{\circ}{-}-31793_{4}$ & 6947.783 & 2 & & 14389.111 & $18069_{3}^{\circ}-32458_{4}$ \\
\hline 6993.988 & 100 & 2 & 14294.052 & $11802_{2}-26096_{3}^{\circ}$ & 6946.599 & 50 & 3 & 14391.564 & $15863_{2}-30255^{\circ}$ \\
\hline 6989.657 & 1000 & 150 & 14302.909 & $7795_{4}^{\circ}-22098_{4}$ & 6946.210 & 100 & 3 & 14392.370 & $15618_{3}^{\circ}-30011_{3}$ \\
\hline 6988.871 & 20 & 1 & 14304.517 & $16346_{4}^{\circ}-30651_{3}$ & 6945.488 & 200 & 8 & 14393.866 & $8243_{2}^{\circ}-22637_{3}$ \\
\hline 6987.790 & 20 & 2 & 14306.730 & $20566_{4}^{\circ}-34873_{4}$ & 6944.926 & 2 & & 14395.031 & $21902^{\circ}-36297_{4}$ \\
\hline 6987.444 & 3 & & 14307.439 & $20566_{4}^{\circ}-34874_{3}$ & 6944.773 & 8 & & 14395.348 & $15618{ }^{\circ}-30014_{4}$ \\
\hline 6986.565 & 3 & & 14309.239 & $21738_{2}^{\circ}-360472$ & 6944.611 & 8 & & 14395.684 & $15736_{1}^{\circ}-301322$ \\
\hline 6986.159 & 2 & & 14310.070 & $22508_{2}^{\circ}-36818_{2}$ & 6943.612 & 800 & 200 & 14397.755 & $98045-24202{ }^{\circ}$ \\
\hline 6986.031 & 150 & 4 & 14310.332 & $11802_{2}-26113_{2}^{\circ}$ & 6942.535 & 150 & 8 & 14399.988 & $75023-21902_{4}^{\circ}$ \\
\hline 6985.783 & 10 & 1 & 14310.840 & $21575_{2}-35885_{2}^{\circ}$ & 6940.327 & 50 & 3 & 14404.569 & $18053_{4}^{\circ}-32458_{4}$ \\
\hline 6984.952 & 3 & & 14312.543 & $195324-33844_{0}^{\circ}$ & 6940.037 & 3 & & 14405.171 & $18549_{2}-32954_{2}^{\circ}$ \\
\hline 6984.759 & 5 & & 14312.939 & $17224_{2}^{\circ}-31537_{3}$ & 6937.618 & 10 & & 14410.194 & $139621-28372 i$ \\
\hline 6984.585 & 4 & & 14313.295 & $8800_{4}-23113_{4}^{\circ}$ & 6937.033 & 8 & & 14411.409 & $21165_{3}^{\circ}-35576_{3}$ \\
\hline 6983.965 & 5 & & 14314.566 & $22338_{3}^{\circ}-36653_{2}$ & 6936.651 & 150 & 4 & 14412.203 & $12847_{3}-27260_{3}^{\circ}$ \\
\hline 6982.752 & 3 & & 14317.052 & $20054_{2}-34371_{2}^{\circ}$ & 6936.223 & 10 & & 14413.092 & $13945_{3}^{\circ}-28358_{3}$ \\
\hline 6982.608 & 50 & 2 & 14317.348 & $23015^{\circ}-373326$ & 6934.839 & 4 & 1 & 14415.969 & $22669_{3}^{\circ}-37085_{4}$ \\
\hline 6981.082 & 100 & 3 & 14320.477 & $13175_{4}^{\circ}-27495_{4}$ & 6934.488 & 15 & 1 & 14416.698 & $13175_{4}^{\circ}-275915$ \\
\hline
\end{tabular}


TABLE 4. Classified lines of Th I-continued

\begin{tabular}{|c|c|c|c|c|c|c|c|c|c|}
\hline \multirow{2}{*}{$\begin{array}{l}\text { Wavelength } \\
\text { (A) }\end{array}$} & \multicolumn{2}{|c|}{ Intensity } & \multirow{2}{*}{$\begin{array}{l}\text { Wavenumber } \\
\left(\mathrm{cm}^{-1}\right)\end{array}$} & \multirow{2}{*}{ Classification } & \multirow{2}{*}{$\begin{array}{c}\text { Wavelength } \\
\text { (§) }\end{array}$} & \multicolumn{2}{|c|}{ Inten sity } & \multirow{2}{*}{$\begin{array}{c}\text { Wavenumber } \\
\left(\mathrm{cm}^{-1}\right)\end{array}$} & \multirow{2}{*}{ Classification } \\
\hline & Lamp & Spark & & & & Lamp & Spark & & \\
\hline 6934.274 & $100 \mathrm{~b}$ & 5 & 14417.143 & $15863_{2}-30281_{1}^{\circ}$ & 6882.807 & 200 & 8 & 14524.948 & $13847_{2}-28372_{1}^{\circ}$ \\
\hline 6934.232 & $100 \mathrm{~b}$ & 3 & 14417.231 & $14465_{2}^{\circ}-288822$ & 6881.932 & 2 & & 14526.795 & $203220^{\circ}-348495$ \\
\hline 6933.836 & 2 & & 14418.054 & $12847_{3}-27266_{4}^{\circ}$ & 6881.674 & 5 & & 14527.340 & $19273_{2}-33800_{3}^{\circ}$ \\
\hline 6932.227 & 15 & 1 & 14421.400 & $19986^{\circ}-344076$ & 6881.250 & 5 & & 14528.235 & $20054_{2}-34583_{3}^{\circ}$ \\
\hline 6931.784 & $3 \mathrm{~h}$ & & 14422.322 & $142260-28649^{\circ}$ & 6880.945 & 1 & & 14528.879 & $18930_{3}^{\circ}-33459_{4}$ \\
\hline 6930.094 & 5 & & 14425.839 & $22877_{0}^{\circ}-37303_{4}$ & 6879.376 & 1 & & 14532.193 & $21645_{4}-36178_{4}^{\circ}$ \\
\hline 6928.959 & 3 & & 14428.202 & $216454-36074_{3}^{\circ}$ & 6877.469 & 2 & & 14536.222 & $20054_{2}-34591_{1}^{\circ}$ \\
\hline 6927.721 & 2 & & 14430.780 & $18431_{3}-32862^{\circ}$ & 6875.440 & 2 & & 14540.512 & $16217_{2}^{\circ}-30758_{2}$ \\
\hline 6926.311 & 2 & & 14433.718 & $16217_{2}^{\circ}-30651_{3}$ & 6874.984 & 10 & & 14541.476 & $4961_{4}-19503_{3}^{\circ}$ \\
\hline 6925.566 & 8 & & 14435.271 & $14243 i-28679_{2}$ & 6874.752 & 200 & 10 & 14541.967 & $2869_{3}-17411_{3}^{\circ}$ \\
\hline 6925.471 & 10 & 1 & 14435.469 & $195324-33967_{3}^{\circ}$ & 6874.192 & $50 \mathrm{~b}$ & & 14543.151 & $16783_{4}^{\circ}-31326_{4}$ \\
\hline 6925.040 & 4 & & 14436.367 & $22338{ }^{\circ}-36775_{3}$ & 6872.234 & 8 & & 14547.295 & $15970_{3}-30517_{4}^{\circ}$ \\
\hline 6924.657 & 50 & 1 & 14437.166 & $13175_{4}^{\circ}-276123$ & 6871.271 & 50 & & 14549.334 & $22855_{3}^{\circ}-37404_{3}$ \\
\hline 6920.834 & 1 & & 14445.141 & $19273_{2}-33718_{2}^{\circ}$ & 6870.988 & 150 & 3 & 14549.933 & $15736_{1}^{\circ}-30286_{1}$ \\
\hline 6920.391 & 5 & & 14446.065 & $21890_{3}^{\circ}-363363$ & 6870.911 & 2 & & .14550 .096 & $21539_{4}^{\circ}-36089_{4}$ \\
\hline 6920.037 & 150 & 5 & 14446.804 & $18011 \stackrel{\circ}{-324584}$ & 6870.807 & 2 & & 14550.316 & $17224_{2}^{\circ}-31774_{3}$ \\
\hline 6919.415 & 1 & & 14448.103 & $22855_{3}^{\circ}-373034$ & 6870.727 & 150 & 3 & 14550.486 & $17166_{5}-31716_{0}^{\circ}$ \\
\hline 6916.129 & 200 & 10 & 14454.967 & $98045-24259_{4}^{\circ}$ & 6868.455 & 100 & 3 & 14555.299 & $13297_{4}-27852 \circ$ \\
\hline 6914.713 & 100 & 2 & 14457.928 & $7280_{2}-21738_{2}^{\circ}$ & 6866.756 & $100 \mathrm{~s}$ & 2 & 14558.900 & $8111_{4}-22669_{3}^{\circ}$ \\
\hline 6913.173 & 2 & & 14461.148 & $226693-37131_{3}$ & 6866.368 & 150 & 3 & 14559.723 & $6362_{2}-20922_{2}^{\circ}$ \\
\hline 6913.051 & 4 & & 14461.403 & $19273_{2}-33734_{2}^{\circ}$ & 6866.157 & 100 & 2 & 14560.170 & $11802_{2}-26363_{2}^{\circ}$ \\
\hline 6912.694 & 2 & & 14462.150 & $18699_{2}-33161_{i}^{\circ}$ & 6863.104 & 8 & & 14566.647 & $20522_{2}^{\circ}-35089_{3}$ \\
\hline 6911.222 & $800 \mathrm{r}$ & 200 & 14465.231 & $0_{2}-14465_{2}^{\circ}$ & 6862.873 & 100 & 2 & 14567.138 & $19039_{2}^{\circ}-33606_{2}$ \\
\hline 6909.222 & 1001 & 1 & 14469.418 & $128473-27317^{\circ}$ & 6858.317 & 1 & & 14576.814 & $16351_{0}-30928^{\circ}$ \\
\hline 6908.989 & 100 & 2 & 14469.906 & $98045-24274 \stackrel{\circ}{0}$ & 6857.436 & 2 & & 14578.687 & $21252_{2}^{\circ}-358313$ \\
\hline 6908.630 & 2 & & 14470.658 & $22401_{1}-36871_{2}^{\circ}$ & 6855.689 & 100 & 3 & 14582.402 & $13088_{3}-27670_{3}^{\circ}$ \\
\hline 6908.599 & $2 \mathrm{~h}$ & & 14470.723 & $22401_{1}-36871_{2}^{\circ}$ & 6855.315 & 150 & 4 & 14583.198 & $159703-30553_{2}^{\circ}$ \\
\hline 6906.698 & 20 & 1 & 14474.705 & $19986^{\circ}-344605$ & 6854.107 & 400 & 10 & 14585.768 & $13088_{3}-27674_{2}^{\circ}$ \\
\hline 6905.856 & 40 & 1 & 14476.470 & $14204_{5}-28680_{4}^{\circ}$ & 6853.511 & 100 & 4 & 14587.036 & $165546-311410$ \\
\hline 6904.578 & 50 & & 14479.150 & $17959_{4}-324399$ & 6852.344 & 50 & 2 & 14589.521 & $15166^{\circ}-297564$ \\
\hline 6904.468 & 8 & & 14479.380 & $215943-36074_{3}^{\circ}$ & 6852.099 & 1 & & 14590.042 & $211435-35733^{\circ}$ \\
\hline 6903.317 & 8 & & 14481.795 & $16783^{\circ}-31265_{3}$ & 6850.111 & 8 & & 14594.276 & $21594_{3}-36188^{\circ}$ \\
\hline 6902.957 & 10 & & 14482.550 & $18069_{3}^{\circ}-32551_{3}$ & 6849.623 & 25 & & 14595.316 & $18699_{2}-33294^{\circ}$ \\
\hline 6902.767 & 2 & & 14482.948 & $19948^{\circ}-344313$ & 6848.697 & 3 & & 14597.290 & $18930_{3}^{\circ}-33527_{4}$ \\
\hline 6902.210 & 150 & 3 & 14484.117 & $11802_{2}-26287_{1}^{\circ}$ & 6848.279 & 2 & & 14598.181 & $21738_{2}^{\circ}-363363$ \\
\hline 6901.357 & 10 & & 14485.907 & $22399_{0}^{\circ}-36885_{4}$ & 6847.989 & 3 & & 14598.799 & $18809_{4}^{\circ}-33408_{3}$ \\
\hline 6901.019 & 3 & & 14486.617 & $216454-36132 \circ$ & 6847.517 & 4 & & 14599.805 & $12847_{3}-27447_{2}^{\circ}$ \\
\hline 6900.762 & 100 & 5 & 14487.156 & $16783^{\circ}-312715$ & 6846.603 & 15 & 1 & 14601.754 & $17411_{3}^{\circ}-32012_{4}$ \\
\hline 6900.442 & 10 & & 14487.828 & $18930_{3}^{\circ}-334184$ & 6843.556 & 3 & & 14608.255 & $18809_{4}^{\circ}-33418_{4}$ \\
\hline 6898.073 & 25 & & 14492.804 & $20214_{3}^{\circ}-347073$ & 6842.724 & 50 & & 14610.031 & $7280_{2}-21890_{3}^{\circ}$ \\
\hline 6897.277 & 20 & & 14494.476 & $17501{ }^{\circ}-319954$ & 6841.962 & 8 & & 14611.659 & $18431_{3}-33043_{3}^{\circ}$ \\
\hline 6895.227 & 10 & & 14498.786 & $19948_{4}^{\circ}-344474$ & 6841.690 & 10 & & 14612.239 & $22163_{4}^{\circ}-36775_{3}$ \\
\hline 6895.116 & 8 & & 14499.019 & $21575_{2}-36074_{3}^{\circ}$ & 6840.993 & 2 & & 14613.728 & $21902_{4}^{\circ}-36515_{3}$ \\
\hline 6894.229 & 50 & 4 & 14500.884 & $10414_{4}^{\circ}-249153$ & 6840.899 & 2 & & 14613.929 & $21575_{2}-36188_{2}^{\circ}$ \\
\hline 6893.626 & 10 & 1 & 14502.153 & $20543 \stackrel{\circ}{-350461}$ & 6839.287 & 100 & 2 & 14617.373 & $13945_{3}^{\circ}-285624$ \\
\hline 6892.247 & 200 & 5 & 14505.054 & $171665-31671_{4}^{\circ}$ & 6838.979 & 2 & & 14618.032 & $16346_{4}^{\circ}-30964_{3}$ \\
\hline 6889.052 & 8 & 1 & 14511.781 & 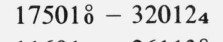 & 6838.902 & 8 & & 14618.196 & $22163_{4}^{\circ}-367815$ \\
\hline 6888.832 & 100 & 3 & 14512.245 & $11601_{1}-26113^{\circ}$ & 6836.881 & 50 & & 14622.518 & $20423 i-350461$ \\
\hline 6888.158 & 50 & 2 & 14513.665 & $15618 \stackrel{\circ}{-301322}$ & 6836.651 & 3 & & 14623.009 & $22508_{2}^{\circ}-37131_{3}$ \\
\hline 6886.404 & 200 & 10 & 14517.362 & $3865_{1}-18382 \AA$ & 6835.613 & 8 & & 14625.230 & $21252_{2}^{\circ}-35877_{2}$ \\
\hline 6885.964 & 2 & & 14518.289 & $17411_{3}^{\circ}-319293$ & 6834.925 & 400 & 50 & 14626.702 & $4961_{4}-195888^{\circ}$ \\
\hline 6885.721 & 1 & & 14518.802 & $209222-354403$ & 6834.780 & $5 b$ & & 14627.012 & $20522_{2}^{\circ}-35149_{2}$ \\
\hline 6883.849 & 75 & & 14522.750 & $20566_{4}^{\circ}-35089_{3}$ & 6834.585 & 1 & & 14627.430 & $19817_{1}^{\circ}-34444_{2}$ \\
\hline 6883.311 & 200 & 10 & 14523.885 & $154934-30017^{\circ}$ & 6833.697 & 3 & & 14629.330 & $216454-36275 \circ$ \\
\hline 6883.135 & 50 & 4 & 14524.256 & $15490 \AA-300144$ & 6833.373 & 10 & & 14630.024 & $17411_{3}^{\circ}-32041_{2}$ \\
\hline
\end{tabular}


TABLE 4. Classified lines of Th $\mathrm{I}$-continued

\begin{tabular}{|c|c|c|c|c|c|c|c|c|c|}
\hline \multirow{2}{*}{$\begin{array}{c}\text { Wavelength } \\
(\AA)\end{array}$} & \multicolumn{2}{|c|}{ Intensity } & \multirow{2}{*}{$\begin{array}{l}\text { Wavenumber } \\
\qquad\left(\mathrm{cm}^{-1}\right)\end{array}$} & \multirow{2}{*}{ Classification } & \multirow{2}{*}{$\begin{array}{c}\text { Wavelength } \\
\text { (Å) }\end{array}$} & \multicolumn{2}{|c|}{ Inten sity } & \multirow{2}{*}{$\begin{array}{l}\text { Wavenumber } \\
\qquad\left(\mathrm{cm}^{-1}\right)\end{array}$} & \multirow{2}{*}{ Classification } \\
\hline & Lamp & Spark & & & & Lamp & Spark & & \\
\hline 6831.719 & 3 & & 14633.566 & $22669_{3}^{\circ}-37303_{4}$ & 6787.913 & 25 & & 14728.004 & $18053_{4}^{\circ}-32781_{4}$ \\
\hline 6831.621 & 2 & & 14633.776 & $22141^{\circ}-367753$ & 6787.734 & 300 & 25 & 14728.392 & $142045-28932^{\circ}$ \\
\hline 6831.249 & 8 & & 14634.573 & $18930_{3}^{\circ}-335643$ & 6784.927 & 10 & & 14734.485 & $19039_{2}^{\circ}-33773_{1}$ \\
\hline 6829.339 & 100 & 3 & 14638.666 & $14206_{4}^{\circ}-28845_{4}$ & 6784.807 & 50 & 2 & 14734.746 & $22669_{3}^{\circ}-37404_{3}$ \\
\hline 6829.212 & 8 & & 14638.938 & $17073_{1}-31712^{\circ}$ & 6782.498 & 1 & & 14739.762 & $220984-36837 \circ$ \\
\hline 6829.037 & 500 & 25 & 14639.313 & $75023-221411^{\circ}$ & 6781.676 & 3 & & 14741.549 & $13847_{2}-28589_{3}^{\circ}$ \\
\hline 6826.296 & 501 & 2 & 14645.191 & $22163_{4}^{\circ}-368083$ & 6781.234 & 3 & & 14742.509 & $18053{ }^{\circ}-327963$ \\
\hline 6824.678 & 400 & 20 & 14648.663 & $11241_{3}^{\circ}-25890_{2}$ & 6780.415 & 400 & 100 & 14744.290 & $8111_{4}-22855_{3}^{\circ}$ \\
\hline 6824.380 & 1 & & 14649.303 & $18809_{4}^{\circ}-33459_{4}$ & 6780.126 & 400 & 1 & 14744.919 & $8243_{2}^{\circ}-22988_{2}$ \\
\hline 6824.097 & 8 & & 14649.911 & $19039_{2}^{\circ}-33689_{2}$ & 6779.323 & 100 & 1 & 14746.665 & $75023-22248_{2}^{\circ}$ \\
\hline 6823.911 & 10 & 1 & 14650.310 & $195324-34182 \circ$ & 6779.085 & 4 & & 14747.183 & $16217_{2}^{\circ}-30964_{3}$ \\
\hline 6823.500 & 150 & 4 & 14651.192 & $13297_{4}-27948_{4}^{\circ}$ & 6778.312 & 400 & 40 & 14748.865 & $38651-18614 i$ \\
\hline 6819.796 & 10 & & 14659.150 & $20214_{3}^{\circ}-348743$ & 6776.230 & 100 & 3 & 14753.396 & $16783^{\circ}-31537_{3}$ \\
\hline 6819.549 & 5 & & 14659.681 & $144816^{\circ}-291415$ & 6775.501 & 2 & & 14754.984 & $18809_{4}^{\circ}-33564_{3}$ \\
\hline 6818.996 & 5 & & 14660.870 & $75023-22163_{4}^{\circ}$ & 6775.101 & 15 & 2 & 14755.855 & $223383-370942$ \\
\hline 6817.248 & 2 & & 14664.629 & $220984-36762^{\circ}$ & 6774.254 & 1 & & 14757.700 & $21539_{4}^{\circ}-362974$ \\
\hline 6816.821 & 25 & & 14665.547 & $13847_{2}-28513_{2}^{\circ}$ & 6774.086 & 8 & & 14758.066 & $224011-37159_{1}^{\circ}$ \\
\hline 6816.510 & 50 & 2 & 14666.216 & $21165^{\circ}-358313$ & 6773.770 & 40 & 1 & 14758.754 & $18930_{3}^{\circ}-33689_{2}$ \\
\hline 6816.510 & 50 & 2 & 14666.216 & $22855^{\circ}-37521_{3}$ & 6772.237 & $50 \mathrm{~b}$ & & 14762.095 & $173983-32160_{2}^{\circ}$ \\
\hline 6816.246 & $50 w$ & & 14666.784 & $154934-30160_{4}^{\circ}$ & 6772.176 & 400 & 15 & 14762.228 & $154934-30255_{3}^{\circ}$ \\
\hline 6815.604 & 100 & 2 & 14668.166 & $15166_{3}^{\circ}-298353$ & 6771.623 & 5 & & 14763.433 & $21890 \AA-36653_{2}$ \\
\hline 6814.184 & 2 & & 14671.222 & $21539_{4}^{\circ}-36210_{5}$ & 6770.175 & 5 & & 14766.591 & $8111_{4}-22877_{0}^{\circ}$ \\
\hline 6813.602 & 10 & & 14672.476 & $16217_{2}^{\circ}-30889_{1}$ & 6768.511 & 3 & & 14770.221 & $18011^{\circ}-32781_{4}$ \\
\hline 6811.958 & 50 & 1 & 14676.017 & $18930_{3}^{\circ}-336062$ & 6765.683 & 200 & 100 & 14776.395 & $18614_{i}^{\circ}-33390_{1}$ \\
\hline 6811.725 & 4 & & 14676.519 & $221411^{\circ}-368182$ & 6764.987 & 1 & & 14777.915 & $21738_{2}^{\circ}-36515_{3}$ \\
\hline 6809.504 & 100 & 3 & 14681.306 & $11241_{3}^{\circ}-259234$ & 6763.532 & 8 & 1 & 14781.094 & $19516_{2}^{\circ}-34298_{1}$ \\
\hline 6809.311 & 150 & 4 & 14681.722 & $12114_{2}^{\circ}-267963$ & 6763.340 & 50 & 2 & 14781.514 & $216454-36427^{\circ}$ \\
\hline 6809.103 & 400 & 10 & 14682.170 & $19039^{\circ}-33721_{2}$ & 6760.667 & 100 & 3 & 14787.358 & $171665-31953^{\circ}$ \\
\hline 6808.530 & 75 & 2 & 14683.406 & $18053^{\circ}-327375$ & 6760.400 & 2 & & 14787.942 & $215943-36382^{\circ}$ \\
\hline 6808.373 & 2 & & 14683.744 & $20737^{\circ}-354211$ & 6758.716 & 2 & & 14791.627 & $159703-307613$ \\
\hline 6807.314 & 100 & 3 & 14686.029 & $11601_{1}-26287^{\circ}$ & 6758.521 & 5 & & 14792.053 & $22338 \stackrel{\circ}{3}-371313$ \\
\hline 6807.108 & 8 & 1 & 14686.473 & $22399_{0}^{\circ}-37085_{4}$ & 6758.202 & 200 & 8 & 14792.752 & $175018-322935$ \\
\hline 6807.035 & 15 & 1 & 14686.631 & $139621-28649 \mathrm{i}$ & 6757.349 & 15 & & 14794.619 & $17959_{4}-32754^{\circ}$ \\
\hline 6805.746 & 100 & 2 & 14689.412 & $15863_{2}-30553_{2}^{\circ}$ & 6757.105 & 100 & 5 & 14795.153 & $16346^{\circ}-31141_{3}$ \\
\hline 6803.292 & 10 & & 14694.711 & $18614_{1}^{\circ}-33309_{2}$ & 6756.454 & $800 \mathrm{~b}$ & 75 & 14796.579 & $25580-17354^{\circ}$ \\
\hline 6802.783 & 100 & 3 & 14695.810 & $130883-27784_{2}^{\circ}$ & 6756.323 & 15 & & 14796.866 & $20054_{2}-34851_{2}^{\circ}$ \\
\hline 6802.410 & 3 & & 14696.616 & $20724^{\circ}-35421_{1}$ & 6756.323 & 15 & & 14796.866 & $20054_{2}-34851_{2}^{\circ}$ \\
\hline 6801.772 & 2 & & 14697.995 & $22396^{\circ}-37094_{2}$ & 6755.663 & 4 & & 14798.311 & $19503 \stackrel{\circ}{3}-343014$ \\
\hline 6800.461 & 100 & 4 & 14700.828 & $17501{ }^{\circ}-322025$ & 6755.475 & 20 & 1 & 14798.723 & $173983-321977_{3}^{\circ}$ \\
\hline 6799.707 & 2 & & 14702.458 & $22098_{4}-36800_{4}^{\circ}$ & 6755.305 & 2 & & 14799.096 & $22508_{2}^{\circ}-37307_{3}$ \\
\hline 6798.743 & 100 & 3 & 14704.543 & $17847_{2}^{\circ}-32551_{3}$ & 6754.265 & 150 & 4 & 14801.374 & $13847_{2}-28649_{1}^{\circ}$ \\
\hline 6798.483 & 150 & 3 & 14705.105 & $11802_{2}-26508_{3}^{\circ}$ & 6753.657 & 150 & 4 & 14802.707 & $63622-21165_{3}^{\circ}$ \\
\hline 6795.797 & 100 & 2 & 14710.917 & $13962_{1}-28673_{2}^{\circ}$ & 6751.426 & 100 & 3 & 14807.598 & $15736_{1}^{\circ}-30544_{2}$ \\
\hline 6795.046 & 75 & 2 & 14712.543 & $18069_{3}^{\circ}-32781_{4}$ & 6749.315 & 50 & 1 & 14812.230 & $19532_{4}-34344_{4}^{\circ}$ \\
\hline 6794.965 & 25 & 1 & 14712.719 & $21165_{3}^{\circ}-35877_{2}$ & 6747.402 & 2 & & 14816.429 & $216681-36485_{2}$ \\
\hline 6794.795 & 8 & 1 & 14713.087 & $19588 \circ-34301_{4}$ & 6747.172 & 5 & & 14816.934 & $17224_{2}^{\circ}-32041_{2}$ \\
\hline 6792.900 & 15 & 1 & 14717.191 & $197133-344303$ & 6746.137 & 75 & 2 & 14819.207 & $19588 \circ-344076$ \\
\hline 6792.674 & 4 & & 14717.681 & $18809_{4}^{\circ}-33527_{4}$ & 6743.199 & 10 & & 14825.664 & $13847_{2}-28673_{2}^{\circ}$ \\
\hline 6791.232 & 400 & 25 & 14720.806 & $88004-23521_{3}^{\circ}$ & 6742.881 & 200 & 8 & 14826.363 & $12847_{3}-27674_{2}^{\circ}$ \\
\hline 6789.000 & 100 & & 14725.645 & $18011^{\circ}-327375$ & 6741.924 & 100 & 2 & 14828.468 & $13847_{2}-28676_{3}^{\circ}$ \\
\hline 6788.837 & 300 & 25 & 14725.999 & $11197^{\circ}-259234$ & 6739.963 & $50 \mathrm{~h}$ & & 14832.782 & $224011-37234_{2}^{\circ}$ \\
\hline 6788.730 & $50 \mathrm{~h}$ & 1 & 14726.231 & $20423^{\circ}-35149_{2}$ & 6738.179 & 200 & 5 & 14836.709 & $75023-223383^{\circ}$ \\
\hline 6788.343 & 20 & & 14727.071 & $18069_{3}^{\circ}-327963$ & 6737.643 & 50 & 8 & 14837.889 & $19273_{2}-34111_{1}^{\circ}$ \\
\hline 6788.180 & 50 & & 14727.424 & $18699_{2}-33427_{2}^{\circ}$ & 6737.281 & 50 & & 14838.687 & $20566_{4}^{\circ}-35405_{3}$ \\
\hline 6787.913 & 25 & & 14728.004 & $14206_{4}^{\circ}-28934_{3}$ & 6735.686 & 2 & & 14842.201 & $7795_{4}^{\circ}-226373$ \\
\hline
\end{tabular}


TABLE 4. Classified lines of $\mathrm{Th} \mathrm{I}$-continued

\begin{tabular}{|c|c|c|c|c|c|c|c|c|c|}
\hline \multirow{2}{*}{$\begin{array}{c}\text { Wavelength } \\
(\AA)\end{array}$} & \multicolumn{2}{|c|}{ Intensity } & \multirow{2}{*}{$\begin{array}{l}\text { Wavenumber } \\
\qquad\left(\mathrm{cm}^{-1}\right)\end{array}$} & \multirow{2}{*}{ Classification } & \multirow{2}{*}{$\begin{array}{c}\text { Wavelength } \\
\text { (§) }\end{array}$} & \multicolumn{2}{|c|}{ Intensity } & \multirow{2}{*}{$\begin{array}{l}\text { Wavenumber } \\
\left(\mathrm{cm}^{-1}\right)\end{array}$} & \multirow{2}{*}{ Classification } \\
\hline & Lamp & Spark & & & & Lamp & Spark & & \\
\hline 6735.369 & 100 & 2 & 14842.899 & $15970_{3}-30812_{2}^{\circ}$ & 6701.373 & 10 & 1 & 14918.196 & $21890 \stackrel{\circ}{3}-368083$ \\
\hline 6735.128 & 100 & 2 & 14843.430 & $13297_{4}-28140_{4}^{\circ}$ & 6701.010 & 2 & & 14919.004 & $16346_{4}^{\circ}-31265_{3}$ \\
\hline 6734.667 & 20 & & 14844.446 & $15166_{3}^{\circ}-30011_{3}$ & 6700.798 & 8 & & 14919.477 & $211435-36062_{4}^{\circ}$ \\
\hline 6734.003 & 4 & & 14845.910 & $22248_{2}^{\circ}-37094_{2}$ & 6699.335 & 2 & & 14922.735 & $22163_{4}^{\circ}-37085_{4}$ \\
\hline 6733.748 & 150 & 5 & 14846.472 & $142045-29050_{0}^{\circ}$ & 6698.610 & 10 & & 14924.350 & $16346_{4}^{\circ}-312715$ \\
\hline 6733.314 & 8 & & 14847.429 & $15166_{3}^{\circ}-30014_{4}$ & 6698.516 & 2 & & 14924.559 & $21165_{3}^{\circ}-36089_{4}$ \\
\hline 6732.650 & 50 & 3 & 14848.893 & $11802_{2}-26651_{2}^{\circ}$ & 6698.029 & 200 & 4 & 14925.644 & $19948_{4}^{\circ}-348743$ \\
\hline 6731.047 & 25 & & 14852.430 & $18574_{1}-33427_{2}^{\circ}$ & 6697.708 & 200 & 5 & 14926.360 & $3687_{2}-18614_{i}^{\circ}$ \\
\hline 6729.932 & 50 & 1 & 14854.890 & $8800_{4}-23655_{4}^{\circ}$ & 6697.139 & 5 & & 14927.628 & $19516_{2}^{\circ}-34444_{2}$ \\
\hline 6728.757 & 300 & 5 & 14857.484 & $171665-320230^{\circ}$ & 6696.996 & 1 & & 14927.947 & $21890_{3}^{\circ}-36818_{2}$ \\
\hline 6728.117 & 150 & 5 & 14858.898 & $13175_{4}^{\circ}-280345$ & 6696.886 & 1 & & 14928.192 & $19503 \stackrel{\circ}{3}-34431_{3}$ \\
\hline 6727.649 & $100 \mathrm{~s}$ & 2 & 14859.931 & $15863_{2}-30723_{i}^{\circ}$ & 6696.140 & 200 & 5 & 14929.855 & $1211_{2}^{\circ}-27044_{3}$ \\
\hline 6727.459 & 500 & 75 & 14860.351 & $5563_{1}-20423_{i}^{\circ}$ & 6695.175 & 8 & 1 & 14932.007 & $20867_{7}^{\circ}-35799_{6}$ \\
\hline 6726.946 & 10 & 2 & 14861.484 & $7280_{2}-22141_{3}^{\circ}$ & 6694.492 & 100 & 2 & 14933.530 & $15618_{3}^{\circ}-30552_{4}$ \\
\hline 6726.310 & 100 & 2 & 14862.889 & $19986_{6}^{\circ}-348495$ & 6694.002 & 100 & 1 & 14934.623 & $14206_{4}^{\circ}-291415$ \\
\hline 6725.482 & 2 & & 14864.719 & $22877^{\circ}-377426$ & 6693.205 & 75 & & 14936.401 & $128473-27784_{2}^{\circ}$ \\
\hline 6725.128 & 1 & & 14865.502 & $18431_{3}-33297_{2}^{\circ}$ & 6690.906 & 50 & & 14941.534 & $22877_{0}^{\circ}-37819_{4}$ \\
\hline 6723.120 & 2 & & 14869.941 & $19713_{3}-34583_{3}^{\circ}$ & 6690.271 & 4 & & 14942.952 & $220984-37041_{4}^{\circ}$ \\
\hline 6722.852 & 1 & & 14870.534 & $22877_{1}^{\circ}-37748_{2}$ & 6689.783 & 5 & & 14944.042 & $19503 \stackrel{\circ}{3}-344474$ \\
\hline 6721.958 & 5 & & 14872.512 & $19588^{\circ}-344605$ & 6687.521 & 150 & 3 & 14949.096 & $15863_{2}-30812_{2}^{\circ}$ \\
\hline 6721.703 & 1 & & 14873.076 & $21902_{4}^{\circ}-367753$ & 6686.214 & 10 & & 14952.019 & $18011{ }^{\circ}-329635$ \\
\hline 6721.089 & 25 & & 14874.435 & $20214_{3}^{\circ}-35089_{3}$ & 6685.701 & 4 & & 14953.166 & $14465_{2}^{\circ}-29418_{2}$ \\
\hline 6720.699 & 1 & & 14875.298 & $23015^{\circ}-378905$ & 6684.687 & 8 & & 14955.434 & $13962_{1}-28917_{2}^{\circ}$ \\
\hline 6719.630 & 20 & & 14877.664 & $185492-33427_{2}^{\circ}$ & 6684.049 & 50 & 2 & 14956.862 & $14465_{2}^{\circ}-29422_{1}$ \\
\hline 6719.307 & 2 & & 14878.380 & $16217_{2}^{\circ}-310951$ & 6683.991 & $8 \mathrm{~b}$ & & 14956.991 & $17501 \stackrel{\circ}{-324584}$ \\
\hline 6719.198 & 200 & 5 & 14878.621 & $10526_{3}^{\circ}-25405_{4}$ & 6683.368 & 200 & 3 & 14958.386 & $8243 \stackrel{\circ}{2}-232013$ \\
\hline 6719.011 & 3 & & 14879.035 & $21902^{\circ}-367815$ & 6682.838 & 5 & & 14959.572 & $5563_{1}-20522_{2}^{\circ}$ \\
\hline 6717.582 & 1 & & 14882.200 & $21165_{3}^{\circ}-36047_{2}$ & 6681.486 & 15 & & 14962.599 & $18699_{2}-33662_{1}^{\circ}$ \\
\hline 6717.384 & 100 & 3 & 14882.639 & $15490 \AA-303726$ & 6680.943 & 2 & & 14963.815 & $22855_{3}^{\circ}-37819_{4}$ \\
\hline 6716.215 & 3 & & 14885.229 & $21890_{3}^{\circ}-36775_{3}$ & 6680.664 & 2 & & 14964.440 & $22338_{3}^{\circ}-37303_{4}$ \\
\hline 6715.515 & 100 & 2 & 14886.781 & $173983-32285^{\circ}$ & 6680.087 & 50 & 1 & 14965.733 & $15166_{3}^{\circ}-30132_{2}$ \\
\hline 6713.967 & 300 & 8 & 14890.213 & $63622-21252_{2}^{\circ}$ & 6678.994 & 1 & & 14968.182 & $22338_{3}^{\circ}-37307_{3}$ \\
\hline 6713.602 & 100 & & 14891.023 & $18382^{\circ}-332731$ & 6678.707 & 200 & 8 & 14968.825 & $7280_{2}-22248 \stackrel{2}{2}$ \\
\hline 6712.838 & 2 & & 14892.717 & $22855_{3}^{\circ}-377482$ & 6677.964 & 15 & & 14970.490 & $14226_{0}-29197^{\circ}$ \\
\hline 6712.194 & 5 & & 14894.146 & $21594_{3}-36488_{2}^{\circ}$ & 6675.341 & 4 & & 14976.373 & $21539_{4}^{\circ}-36515_{3}$ \\
\hline 6711.495 & 50 & & 14895.698 & $173988_{3}-32294_{4}^{\circ}$ & 6675.023 & 8 & & 14977.086 & $23015_{0}^{\circ}-379924$ \\
\hline 6711.249 & 100 & 4 & 14896.243 & $98045-247018$ & 6674.698 & 400 & 8 & 14977.816 & $2869_{3}-17847_{2}^{\circ}$ \\
\hline 6710.865 & 10 & & 14897.096 & $14206_{4}^{\circ}-29104_{4}$ & 6674.339 & 5 & & 14978.621 & $20322{ }^{\circ}-353005$ \\
\hline 6710.545 & 100 & 2 & 14897.806 & $15863_{2}-30761_{3}^{\circ}$ & 6673.590 & 200 & 5 & 14980.302 & $16346_{4}^{\circ}-31326_{4}$ \\
\hline 6710.545 & 100 & 2 & 14897.806 & $195324-34430_{3}^{\circ}$ & 6673.428 & 5 & & 14980.666 & 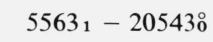 \\
\hline 6710.325 & 3 & & 14898.295 & $20522_{2}^{\circ}-35421_{1}$ & 6672.971 & 2 & & 14981.692 & $21575_{2}-36556^{\circ}$ \\
\hline 6709.443 & 2 & & 14900.253 & $13945_{3}^{\circ}-288454$ & 6672.383 & 1 & & 14983.012 & $21902_{4}^{\circ}-36885_{4}$ \\
\hline 6709.248 & 2 & & 14900.686 & $19948_{4}^{\circ}-34849_{5}$ & 6671.684 & 3 & & 14984.582 & $21668_{1}^{\circ}-36653_{2}$ \\
\hline 6708.839 & 50 & 1 & 14901.595 & $18930_{3}^{\circ}-33831_{4}$ & 6670.741 & 1 & & 14986.700 & $4961_{4}-19948{ }^{\circ}$ \\
\hline 6708.391 & 5 & & 14902.590 & $17959_{4}-32862_{4}^{\circ}$ & 6669.531 & 3 & & 14989.419 & $22141 \stackrel{\circ}{3}-371313$ \\
\hline 6708.283 & 5 & & 14902.830 & $14032_{2}^{\circ}-289343$ & 6669.463 & 8 & & 14989.572 & $13945 \stackrel{\circ}{-289343}$ \\
\hline 6707.733 & $3 \mathrm{~h}$ & & 14904.052 & $223998-373034$ & 6668.826 & 100 & & 14991.004 & $10414_{4}^{\circ}-25405_{4}$ \\
\hline 6706.835 & 15 & 1 & 14906.047 & $21902_{4}^{\circ}-36808_{3}$ & 6668.673 & 8 & & 14991.348 & $19713_{3}-34704_{3}^{\circ}$ \\
\hline 6706.451 & 2 & & 14906.901 & $22877_{1}^{\circ}-37784_{2}$ & 6668.673 & 8 & & 14991.348 & $19713_{3}-34704^{\circ}$ \\
\hline 6705.428 & 8 & & 14909.175 & $20922_{2}^{\circ}-358313$ & 6668.414 & 8 & & 14991.930 & $18614_{1}^{\circ}-33606_{2}$ \\
\hline 6705.151 & 10 & & 14909.791 & $18053 \stackrel{\circ}{4}-329635$ & 6666.987 & 4 & & 14995.139 & $21890_{3}^{\circ}-36885_{4}$ \\
\hline 6703.348 & 5 & & 14913.801 & $21575_{2}-36488_{2}^{\circ}$ & 6663.702 & 50 & 3 & 15002.531 & $8111_{4}-23113^{\circ}$ \\
\hline 6703.091 & 2 & & 14914.373 & $19516_{2}^{\circ}-344313$ & 6662.270 & 800 & 200 & 15005.755 & $7502_{3}-22508_{2}^{\circ}$ \\
\hline 6702.576 & 1 & & 14915.519 & $21738_{2}^{\circ}-36653_{2}$ & 6661.320 & 3 & & 15007.895 & $18382{ }^{\circ}-33390_{1}$ \\
\hline 6701.373 & 10 & 1 & 14918.196 & $20522 \stackrel{\circ}{2}-354403$ & 6659.331 & 3 & & 15012.378 & $19713_{3}-34725_{3}^{\circ}$ \\
\hline
\end{tabular}


TABLE 4. Classified lines of Th I-continued

\begin{tabular}{|c|c|c|c|c|c|c|c|c|c|}
\hline \multirow{2}{*}{$\begin{array}{c}\text { Wavelength } \\
(\AA)\end{array}$} & \multicolumn{2}{|c|}{ Intensity } & \multirow{2}{*}{$\begin{array}{l}\text { Wavenumber } \\
\qquad\left(\mathrm{cm}^{-1}\right)\end{array}$} & \multirow{2}{*}{ Classification } & \multirow{2}{*}{$\begin{array}{c}\text { Wavelength } \\
(\AA)\end{array}$} & \multicolumn{2}{|c|}{ Inten sity } & \multirow{2}{*}{$\begin{array}{l}\text { Wavenumber } \\
\left(\mathrm{cm}^{-1}\right)\end{array}$} & \multirow{2}{*}{ Classification } \\
\hline & Lamp & Spark & & & & Lamp & Spark & & \\
\hline 6659.266 & 2 & & 15012.524 & $21077_{0}^{\circ}-36089_{4}$ & 6615.071 & 50 & 2 & 15112.822 & $18549_{2}-33662_{1}^{\circ}$ \\
\hline 6659.118 & 10 & 2 & 15012.858 & $22877_{0}^{\circ}-37890_{5}$ & 6613.376 & 200 & 8 & 15116.695 & $7280_{2}-22396^{\circ}$ \\
\hline 6658.869 & 2 & & 15013.419 & $22508_{2}^{\circ}-37521_{3}$ & 6613.280 & $4 \mathrm{~h}$ & & 15116.914 & $216454-36762_{3}^{\circ}$ \\
\hline 6658.678 & 400 & 15 & 15013.850 & $11241^{\circ}-26255_{4}$ & 6609.668 & 5 & & 15125.175 & $20922_{2}^{\circ}-36047_{2}$ \\
\hline 6658.071 & 2 & & 15015.219 & $22877_{0}^{\circ}-378924$ & 6609.031 & 5 & 1 & 15126.633 & $15863_{2}-30990_{3}^{\circ}$ \\
\hline 6657.879 & 3 & & 15015.652 & $20566_{4}^{\circ}-355825$ & 6608.438 & 50 & 2 & 15127.991 & $171665-32294_{4}^{\circ}$ \\
\hline 6656.876 & 50 & 1 & 15017.914 & $15490_{0}^{\circ}-305085$ & 6606.790 & 25 & 2 & 15131.764 & $211435-36275 \circ$ \\
\hline 6656.708 & 4 & & 15018.293 & $22669_{3}^{\circ}-376883$ & 6606.597 & 20 & 2 & 15132.206 & $21165_{3}^{\circ}-362974$ \\
\hline 6656.503 & 1 & & 15018.756 & $18699_{2}-33718_{2}^{\circ}$ & 6604.528 & 1 & & 15136.947 & $22855_{3}^{\circ}-37992_{4}$ \\
\hline 6655.773 & 15 & & 15020.403 & $15970_{3}-30990_{3}^{\circ}$ & 6603.788 & 5 & 1 & 15138.643 & $197133-34851_{2}^{\circ}$ \\
\hline 6655.494 & 50 & 2 & 15021.033 & $15736_{1}^{\circ}-30758_{2}$ & 6603.618 & 75 & 5 & 15139.033 & $15618 \stackrel{\circ}{3}-30758_{2}$ \\
\hline 6655.071 & 5 & & 15021.988 & $18809_{4}^{\circ}-33831_{4}$ & 6603.026 & 20 & 2 & 15140.390 & $17411_{3}^{\circ}-32551_{3}$ \\
\hline 6654.236 & 10 & & 15023.872 & $15493_{4}-30517 \%$ & 6602.771 & 100 & 8 & 15140.975 & $19948_{4}^{\circ}-35089_{3}$ \\
\hline 6654.110 & 5 & & 15024.157 & $154934-30517^{\circ}$ & 6601.452 & 3 & 1 & 15144.000 & $22163_{4}^{\circ}-37307_{3}$ \\
\hline 6652.225 & 2 & & 15028.414 & $16217_{2}^{\circ}-31245_{2}$ & 6600.730 & 50 & 2 & 15145.656 & $16783_{4}^{\circ}-31929_{3}$ \\
\hline 6651.371 & 5 & & 15030.344 & $18069_{3}^{\circ}-33099_{3}$ & 6599.183 & 3 & & 15149.207 & $21668_{1}^{\circ}-36818_{2}$ \\
\hline 6650.561 & 50 & 1 & 15032.174 & $15618^{\circ}-306513$ & 6599.183 & 3 & & 15149.207 & $22669_{3}^{\circ}-37819_{4}$ \\
\hline 6649.487 & 1 & & 15034.602 & $211435-36178_{4}^{\circ}$ & 6597.542 & 2 & & 15152.975 & $15736_{1}^{\circ}-30889_{1}$ \\
\hline 6649.278 & 8 & 1 & 15035.075 & $18699_{2}-33734_{2}^{\circ}$ & 6596.750 & 10 & 1 & 15154.794 & $216454-36800_{4}^{\circ}$ \\
\hline 6648.347 & 5 & & 15037.180 & $13847_{2}-28884_{3}^{\circ}$ & 6594.798 & 2 & & 15159.280 & $18614^{\circ}-33773_{1}$ \\
\hline 6646.814 & 8 & & 15040.648 & $17398_{3}-32439_{4}^{\circ}$ & 6594.442 & 8 & 1 & 15160.098 & $18574_{1}-33734_{2}^{\circ}$ \\
\hline 6646.323 & 20 & 1 & 15041.760 & $18549_{2}-33591^{\circ}$ & 6593.940 & 500 & 150 & 15161.252 & $55631-20724{ }^{\circ}$ \\
\hline 6644.839 & 2 & & 15045.119 & $23655_{4}^{\circ}-38700_{4}$ & 6593.464 & 100 & 15 & 15162.347 & $165546-31716_{0}^{\circ}$ \\
\hline 6644.661 & 200 & 150 & 15045.522 & $98045-24850 \%$ & 6591.485 & 400 & 75 & 15166.899 & $0_{2}-15166_{3}^{\circ}$ \\
\hline 6644.550 & 4 & 1 & 15045.773 & $18053_{4}^{\circ}-33099_{3}$ & 6591.278 & 8 & 1 & 15167.375 & $131755_{4}^{\circ}-283425$ \\
\hline 6644.029 & 50 & 2 & 15046.953 & $17411^{\circ}-324584$ & 6591.181 & 100 & 40 & 15167.598 & $75023-22669_{3}^{\circ}$ \\
\hline 6643.501 & 3 & & 15048.149 & $16217_{2}^{\circ}-31265_{3}$ & 6588.540 & $500 \mathrm{r}$ & 200 & 15173.678 & $3865_{1}-19039_{2}^{\circ}$ \\
\hline 6643.120 & 8 & & 15049.012 & $15970_{3}-31019_{4}^{\circ}$ & 6588.340 & 50 & 5 & 15174.139 & $5563_{1}-20737_{1}^{\circ}$ \\
\hline 6642.426 & $40 \mathrm{~b}$ & 1 & 15050.584 & $195324-345833^{\circ}$ & 6588.247 & 1 & & 15174.353 & $14243_{1}^{\circ}-29418_{2}$ \\
\hline 6642.333 & $50 \mathrm{~b}$ & 4 & 15050.795 & $11601_{1}-26651_{2}^{\circ}$ & 6588.063 & 2 & & 15174.777 & $14247^{\circ}-294221$ \\
\hline 6642.333 & $50 \mathrm{~b}$ & 4 & 15050.795 & $19273_{2}-34324_{2}^{\circ}$ & 6586.624 & 4 & & 15178.092 & $142431-294221$ \\
\hline 6641.668 & 4 & & 15052.302 & $13088_{3}-28140_{4}^{\circ}$ & 6585.696 & 100 & 5 & 15180.231 & $19227^{\circ}-344076$ \\
\hline 6641.439 & 4 & & 15052.821 & $13175_{4}^{\circ}-28227_{4}$ & 6584.615 & 100 & 8 & 15182.723 & $13297_{4}-28480_{4}^{\circ}$ \\
\hline 6641.006 & 4 & & 15053.802 & $20522_{2}^{\circ}-355763$ & 6584.374 & 50 & 5 & 15183.279 & $13175_{4}^{\circ}-283583$ \\
\hline 6639.726 & 8 & & 15056.704 & $180110^{\circ}-330686$ & 6583.906 & $400 \mathrm{r}$ & 200 & 15184.358 & $2869_{3}-18053_{4}^{\circ}$ \\
\hline 6638.912 & 150 & 20 & 15058.551 & $11197^{\circ}-26255_{4}$ & 6583.501 & 8 & 2 & 15185.292 & $18549_{2}-33734_{2}^{\circ}$ \\
\hline 6638.774 & 8 & & 15058.864 & $7280_{2}-22338_{3}^{\circ}$ & 6583.177 & 10 & 2 & 15186.039 & $20054_{2}-35240_{3}^{\circ}$ \\
\hline 6637.204 & 15 & 1 & 15062.426 & $15490_{0}^{\circ}-305524$ & 6582.431 & 1 & & 15187.760 & $21575_{2}-36762^{\circ}$ \\
\hline 6636.143 & 2 & & 15064.834 & $15863_{2}-30928^{\circ}$ & 6581.297 & 4 & & 15190.377 & $20214_{3}^{\circ}-35405_{3}$ \\
\hline 6633.784 & 50 & 2 & 15070.191 & $13847_{2}-28917_{2}^{\circ}$ & 6581.135 & 1 & & 15190.751 & $19516_{2}^{\circ}-34707_{3}$ \\
\hline 6633.581 & 8 & & 15070.652 & $14481 \stackrel{\circ}{6}-295526$ & 6580.544 & 3 & & 15192.116 & $216454-36837 \circ$ \\
\hline 6632.110 & 5 & & 15073.995 & $215943-36668_{2}^{\circ}$ & 6580.231 & 75 & 3 & 15192.838 & $11802_{2}-26995_{3}^{\circ}$ \\
\hline 6631.568 & 40 & 1 & 15075.227 & $11802_{2}-26878_{3}^{\circ}$ & 6579.482 & 20 & 2 & 15194.568 & $139621-29157 i$ \\
\hline 6629.447 & 1 & & 15080.050 & $21738_{2}^{\circ}-36818_{2}$ & 6578.980 & 3 & & 15195.727 & $21890_{3}^{\circ}-37085_{4}$ \\
\hline 6626.544 & 2 & & 15086.656 & $17073_{1}-32160_{2}^{\circ}$ & 6577.216 & 100 & 75 & 15199.803 & $2869_{3}-18069_{3}^{\circ}$ \\
\hline 6626.125 & 50 & 1 & 15087.610 & $185741-33662_{1}^{\circ}$ & 6576.123 & 75 & 8 & 15202.329 & $14481 \stackrel{\circ}{6}-296845$ \\
\hline 6623.482 & 2 & & 15093.630 & $21575_{2}-36668_{2}^{\circ}$ & 6575.770 & 2 & & 15203.145 & $18011 \stackrel{\circ}{\circ}-332145$ \\
\hline 6621.329 & 1 & & 15098.538 & $19273_{2}-34371_{2}^{\circ}$ & 6575.148 & 3 & & 15204.583 & $195033_{3}^{\circ}-347073$ \\
\hline 6620.408 & 40 & 1 & 15100.639 & $128477_{3}-27948{ }^{\circ}$ & 6574.542 & 4 & & 15205.985 & $21594_{3}-36800_{4}^{\circ}$ \\
\hline 6620.248 & 2 & & 15101.004 & $18699_{2}-33800_{3}^{\circ}$ & 6572.026 & 10 & 2 & 15211.806 & $16783_{4}^{\circ}-31995_{4}$ \\
\hline 6618.398 & 10 & 1 & 15105.225 & $17398_{3}-32503_{2}^{\circ}$ & 6571.937 & 5 & & 15212.012 & $15863_{2}-31075_{2}^{\circ}$ \\
\hline 6618.163 & 200 & 8 & 15105.761 & $15970_{3}-31075_{2}^{\circ}$ & & & & & \\
\hline 6618.163 & 200 & 8 & 15105.761 & $142045-29310_{4}^{\circ}$ & & & & & \\
\hline 6617.626 & 5 & & 15106.987 & $18614_{1}^{\circ}-33721_{2}$ & & & & & \\
\hline 6617.512 & 75 & 4 & 15107.247 & $10783_{2}^{\circ}-25890_{2}$ & & & & & \\
\hline
\end{tabular}


TABLE 4. Classified lines of Th I-continued

\begin{tabular}{|c|c|c|c|c|c|c|c|c|c|}
\hline \multirow{2}{*}{$\begin{array}{c}\text { Wavelength } \\
\text { (̊̊) }\end{array}$} & \multicolumn{2}{|c|}{ Intensity } & \multirow{2}{*}{$\begin{array}{l}\text { Wavenumber } \\
\left(\mathrm{cm}^{-1}\right)\end{array}$} & \multirow{2}{*}{ Classification } & \multirow{2}{*}{$\begin{array}{c}\text { Wavelength } \\
\text { (§) }\end{array}$} & \multicolumn{2}{|c|}{ Inten sity } & \multirow{2}{*}{$\begin{array}{l}\text { Wavenumber } \\
\qquad\left(\mathrm{cm}^{-1}\right)\end{array}$} & \multirow{2}{*}{ Classification } \\
\hline & Lamp & Spark & & & & Lamp & Spark & & \\
\hline 6568.423 & 3 & 1 & 15220.150 & $21077_{0}^{\circ}-36297_{4}$ & 6515.875 & 5 & & 15342.894 & $22399 \circ-377426$ \\
\hline 6567.213 & 2 & & 15222.954 & $22669_{3}^{\circ}-37892_{4}$ & 6514.699 & 3 & & 15345.663 & $156183-309643$ \\
\hline 6565.068 & 50 & 5 & 15227.928 & $7280_{2}-22508_{2}^{\circ}$ & 6513.200 & 2 & & 15349.195 & $22338_{3}^{\circ}-37688_{3}$ \\
\hline 6564.700 & 2 & & 15228.782 & $21902_{4}^{\circ}-37131_{3}$ & 6513.052 & 20 & 1 & 15349.544 & $13847_{2}-29197^{\circ}$ \\
\hline 6564.443 & 100 & 8 & 15229.378 & $12114_{2}^{\circ}-273433$ & 6512.802 & 4 & & 15350.133 & $13847_{2}-29197_{2}^{\circ}$ \\
\hline 6562.647 & 4 & & 15233.546 & $19227_{6}^{\circ}-344605$ & 6512.366 & 400 & 15 & 15351.161 & $3687_{2}-19039_{2}^{\circ}$ \\
\hline 6562.105 & 50 & & 15234.804 & $13962_{1}-29197^{\circ}$ & 6511.583 & 4 & & 15353.007 & $7502_{3}-22855_{3}^{\circ}$ \\
\hline 6561.854 & 3 & & 15235.387 & $13962_{1}-29197_{2}^{\circ}$ & 6511.050 & 3 & & 15354.263 & $195324-34886^{\circ}$ \\
\hline 6560.849 & 5 & & 15237.720 & $7795_{4}^{\circ}-230324$ & 6510.269 & 5 & & 15356.105 & $173983-32754^{\circ}$ \\
\hline 6559.876 & 5 & 1 & 15239.981 & $18069_{3}^{\circ}-33309_{2}$ & 6509.975 & 1 & & 15356.799 & $21738_{2}^{\circ}-37094_{2}$ \\
\hline 6559.462 & 1 & & 15240.942 & $21890_{3}^{\circ}-37131_{3}$ & 6509.857 & 3 & & 15357.077 & $19516_{2}^{\circ}-348743$ \\
\hline 6559.217 & 50 & 8 & 15241.512 & $22163_{4}^{\circ}-374043$ & 6509.653 & 2 & & 15357.559 & $18809_{4}^{\circ}-34167_{3}$ \\
\hline 6559.120 & $5 \mathrm{~h}$ & & 15241.737 & $21539_{4}^{\circ}-367815$ & 6509.311 & 3 & & 15358.365 & $22163_{4}^{\circ}-375213$ \\
\hline 6558.871 & 100 & 8 & 15242.316 & $3687_{2}-18930_{3}^{\circ}$ & 6509.053 & 150 & 8 & 15358.974 & $5563_{1}-20922_{2}^{\circ}$ \\
\hline 6554.563 & 1 & & 15252.334 & $17847_{2}^{\circ}-33099_{3}$ & 6508.362 & 50 & 1 & 15360.605 & $49614-20322 \circ$ \\
\hline 6554.160 & 150 & 40 & 15253.272 & $4961_{4}-20214_{3}^{\circ}$ & 6508.292 & 2 & & 15360.770 & $16351_{0}-31712^{\circ}$ \\
\hline 6551.917 & 4 & & 15258.493 & $118022-27061_{2}^{\circ}$ & 6507.948 & 4 & 1 & 15361.582 & $20214_{3}^{\circ}-355763$ \\
\hline 6551.703 & 100 & 5 & 15258.992 & $13088_{3}-28347_{2}^{\circ}$ & 6507.409 & 1 & & 15362.854 & $21645_{4}-37008^{\circ}$ \\
\hline 6551.265 & 4 & & 15260.012 & $20322{ }^{\circ}-355825$ & 6506.989 & 100 & 15 & 15363.846 & $10526^{\circ}-25890_{2}$ \\
\hline 6550.335 & 5 & & 15262.178 & $21575_{2}-36837_{1}^{\circ}$ & 6505.221 & 1 & & 15368.022 & $18431_{3}-33799_{4}^{\circ}$ \\
\hline 6549.820 & 4 & & 15263.378 & $21252_{2}^{\circ}-36515_{3}$ & 6504.835 & 2 & & 15368.933 & $18431_{3}-33800_{3}^{\circ}$ \\
\hline 6547.720 & 2 & & 15268.274 & $18699_{2}-33967_{3}^{\circ}$ & 6504.458 & 5 & & 15369.824 & $14465_{2}^{\circ}-29835_{3}$ \\
\hline 6547.618 & 1 & & 15268.512 & $15493_{4}-30761_{3}^{\circ}$ & 6504.226 & 15 & 2 & 15370.372 & $17411_{3}^{\circ}-32781_{4}$ \\
\hline 6547.531 & 3 & & 15268.714 & $21539_{4}^{\circ}-36808_{3}$ & 6501.992 & 100 & 5 & 15375.653 & $6362_{2}-21738_{2}^{\circ}$ \\
\hline 6545.880 & 2 & & 15272.566 & $22248_{2}^{\circ}-37521_{3}$ & 6500.659 & 75 & 3 & 15378.806 & $13297_{4}-28676_{3}^{\circ}$ \\
\hline 6545.719 & 100 & 8 & 15272.941 & $17166_{5}-32439_{4}^{\circ}$ & 6498.769 & 8 & 1 & 15383.279 & $13297_{4}-28680_{4}^{\circ}$ \\
\hline 6545.345 & 1 & & 15273.814 & $20054_{2}-353281$ & 6497.788 & 2 & & 15385.601 & $15166_{3}^{\circ}-305524$ \\
\hline 6543.893 & 1 & & 15277.203 & $215943-36871_{2}^{\circ}$ & 6497.490 & 50 & 3 & 15386.307 & $140322-294182$ \\
\hline 6542.511 & 50 & 3 & 15280.430 & $17501^{\circ}-32781_{4}$ & 6496.965 & 8 & 1 & 15387.550 & $13175_{4}^{\circ}-28562_{4}$ \\
\hline 6542.049 & 50 & 3 & 15281.509 & $116011-26882{ }^{\circ}$ & 6496.028 & 10 & 1 & 15389.770 & $7280_{2}-22669_{3}^{\circ}$ \\
\hline 6540.542 & 15 & 1 & 15285.030 & $118022-27087_{1}^{\circ}$ & 6495.930 & 5 & & 15390.002 & $14032_{2}^{\circ}-29422_{1}$ \\
\hline 6538.301 & 50 & 4 & 15290.269 & $139622_{1}-29252_{2}^{\circ}$ & 6495.595 & 3 & & 15390.796 & $183820^{\circ}-33773_{1}$ \\
\hline 6537.615 & 75 & 5 & 15291.873 & $13297_{4}-28589_{3}^{\circ}$ & 6495.255 & 20 & 2 & 15391.601 & $13088_{3}-28480_{4}^{\circ}$ \\
\hline 6537.178 & 50 & 2 & 15292.896 & $12847_{3}-28140_{4}^{\circ}$ & 6495.004 & 1 & & 15392.196 & $19039_{2}^{\circ}-344313$ \\
\hline 6536.295 & 2 & & 15294.961 & $154934-30788 \circ$ & 6493.702 & 4 & & 15395.282 & $216454-37041^{\circ}$ \\
\hline 6535.486 & 4 & & 15296.855 & $21575_{2}-36871_{2}^{\circ}$ & 6493.198 & 150 & 8 & 15396.477 & $10526^{\circ}-259234$ \\
\hline 6531.342 & $800 \mathrm{r}$ & 200 & 15306.560 & $6362_{2}-21668 i$ & 6491.222 & 2 & & 15401.164 & $21902_{4}^{\circ}-37303_{4}$ \\
\hline 6530.500 & 4 & 1 & 15308.534 & $20522_{2}^{\circ}-35831_{3}$ & 6490.736 & 500 & 50 & 15402.317 & $8800_{4}-24202^{\circ}$ \\
\hline 6530.500 & 4 & 1 & 15308.534 & $22877^{\circ}-38186_{4}$ & 6489.598 & 10 & 1 & 15405.018 & $138472-29252_{2}^{\circ}$ \\
\hline 6530.157 & 20 & 2 & 15309.338 & $13847_{2}-29157_{i}^{\circ}$ & 6489.362 & 2 & & 15405.578 & $18053_{4}^{\circ}-33459_{4}$ \\
\hline 6530.125 & 25 & 3 & 15309.413 & $21902{ }^{\circ}-372115$ & 6488.882 & 50 & 4 & 15406.718 & $7795_{4}^{\circ}-232013$ \\
\hline 6529.994 & 25 & 2 & 15309.720 & $19273_{2}-34583_{3}^{\circ}$ & 6488.882 & 50 & 4 & $15406.71 \delta$ & $18011_{0}^{\circ}-33418_{4}$ \\
\hline 6529.877 & $3 \mathrm{~h}$ & & 15309.994 & $20737_{i}^{\circ}-36047_{2}$ & 6488.785 & 2 & & 15406.948 & $142433^{\circ}-29650_{2}$ \\
\hline 6529.597 & 20 & 2 & 15310.651 & $17959_{4}-33270_{4}^{\circ}$ & 6487.920 & 3 & & 15409.002 & $22338_{3}^{\circ}-37748_{2}$ \\
\hline 6529.193 & 1 & & 15311.598 & $20322{ }^{\circ}-356334$ & 6487.479 & 50 & 4 & 15410.050 & $8111_{4}-23521_{3}^{\circ}$ \\
\hline 6528.592 & 4 & & 15313.008 & $159703-31283_{3}^{\circ}$ & 6486.848 & 4 & & 15411.549 & $18699_{2}-34111^{\circ}$ \\
\hline 6527.858 & 1 & & 15314.730 & $19986^{\circ}-353005$ & 6484.067 & 15 & 3 & 15418.159 & $16783 \stackrel{\circ}{4}-322025$ \\
\hline 6526.584 & 20 & 2 & 15317.719 & $19273_{2}-34591_{i}^{\circ}$ & 6484.067 & 15 & 3 & 15418.159 & $19713_{3}-35131_{4}^{\circ}$ \\
\hline 6525.714 & 2 & & 15319.761 & $16217_{2}^{\circ}-31537_{3}$ & 6483.931 & 2 & & 15418.482 & $18549_{2}-33967_{3}^{\circ}$ \\
\hline 6525.490 & 3 & & 15320.287 & $21165_{3}^{\circ}-36485_{2}$ & 6483.741 & 3 & 1 & 15418.934 & $20214_{3}^{\circ}-35633_{4}$ \\
\hline 6522.499 & 20 & 5 & 15327.312 & $17224_{2}^{\circ}-32551_{3}$ & 6483.618 & 20 & 1 & 15419.226 & $158632-31283^{\circ}$ \\
\hline 6522.044 & 100 & 15 & 15328.381 & $10783_{2}^{\circ}-26111_{1}$ & 6481.299 & 50 & 5 & 15424.743 & $13088_{3}-28513_{2}^{\circ}$ \\
\hline 6520.998 & 4 & & 15330.840 & $22855_{3}^{\circ}-38186_{4}$ & 6480.444 & 5 & 1 & 15426.778 & $17847_{2}^{\circ}-33273_{1}$ \\
\hline 6519.224 & 1 & & 15335.012 & $17959_{4}-33294_{3}^{\circ}$ & 6479.952 & 4 & & 15427.950 & $16346_{4}^{\circ}-317743$ \\
\hline 6517.277 & 3 & & 15339.593 & $18069_{3}^{\circ}-33408_{3}$ & 6478.616 & 8 & 1 & 15431.131 & $192732-34704^{\circ}$ \\
\hline
\end{tabular}


TABLE 4. Classified lines of Th I-continued

\begin{tabular}{|c|c|c|c|c|c|c|c|c|c|}
\hline \multirow{2}{*}{$\begin{array}{l}\text { Wavelength } \\
\text { (Å) }\end{array}$} & \multicolumn{2}{|c|}{ Intensity } & \multirow{2}{*}{$\begin{array}{l}\text { Wavenumber } \\
\quad\left(\mathrm{cm}^{-1}\right)\end{array}$} & \multirow{2}{*}{ Classification } & \multirow{2}{*}{ 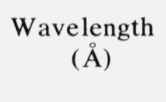 } & \multicolumn{2}{|c|}{ Inten sity } & \multirow{2}{*}{$\begin{array}{c}\text { Wavenumber } \\
\left(\mathrm{cm}^{-1}\right)\end{array}$} & \multirow{2}{*}{ Classification } \\
\hline & Lamp & Spark & & & & Lamp & Spark & & \\
\hline 6474.742 & 50 & 5 & 15440.364 & $15490 \circ-309306$ & 6430.684 & 2 & & 15546.148 & $14465_{2}^{\circ}-30011_{3}$ \\
\hline 6472.193 & 1 & & 15446.445 & $215943-37041_{4}^{\circ}$ & 6430.499 & 5 & 1 & 15546.596 & $22141_{3}^{\circ}-376883$ \\
\hline 6471.936 & 10 & 1 & 15447.058 & $16346^{\circ}-317934$ & 6429.673 & 2 & & 15548.593 & $195324-350818$ \\
\hline 6467.807 & 3 & & 15456.919 & $19948_{4}^{\circ}-35405_{3}$ & 6428.773 & 40 & 2 & 15550.770 & $9804_{5}-25355_{4}^{\circ}$ \\
\hline 6467.671 & 50 & 4 & 15457.244 & $11802_{2}-27260_{3}^{\circ}$ & 6427.507 & 50 & 4 & 15553.832 & $15970_{3}-31523 \stackrel{\circ}{3}$ \\
\hline 6467.145 & 8 & 1 & 15458.502 & $18069_{3}^{\circ}-33527_{4}$ & 6427.507 & 50 & 4 & 15553.832 & $22338 \stackrel{\circ}{3}-37892_{4}$ \\
\hline 6466.712 & 75 & 4 & 15459.537 & $8800_{4}-24259_{4}^{\circ}$ & 6427.264 & 3 & & 15554.421 & $21594_{3}-37149_{2}^{\circ}$ \\
\hline 6466.352 & 50 & 100 & 15460.397 & $11601_{1}-27061_{2}^{\circ}$ & 6426.723 & 1 & & 15555.730 & $21252_{2}^{\circ}-36808_{3}$ \\
\hline 6465.692 & 5 & 1 & 15461.976 & $17847_{2}^{\circ}-33309_{2}$ & 6426.534 & 2 & & 15556.187 & $173983-32954^{\circ}$ \\
\hline 6465.585 & 10 & 2 & 15462.231 & $175018-329635$ & 6426.151 & 5 & & 15557.115 & $16217_{2}^{\circ}-31774_{3}$ \\
\hline 6462.606 & $200 \mathrm{~b}$ & 100 & 15469.359 & $165546-320230^{\circ}$ & 6422.948 & 4 & & 15564.873 & $21594_{3}-37159_{3}^{\circ}$ \\
\hline 6461.454 & 2 & & 15472.117 & $20322 \circ-357944$ & 6422.683 & 2 & & 15565.515 & $21252_{2}^{\circ}-36818_{2}$ \\
\hline 6460.689 & 2 & & 15473.949 & $18053_{4}^{\circ}-33527_{4}$ & 6422.106 & 100 & 8 & 15566.913 & $17501 \stackrel{\circ}{-330686}$ \\
\hline 6460.470 & 50 & 3 & 15474.473 & $8800_{4}-24274{ }^{\circ}$ & 6421.194 & 2 & & 15569.124 & $21738_{2}^{\circ}-37307_{3}$ \\
\hline 6459.295 & 5 & 1 & 15477.288 & $142064-296845$ & 6420.980 & 3 & & 15569.643 & $18431_{3}-34001_{4}^{\circ}$ \\
\hline 6459.116 & 5 & 1 & 15477.717 & $20322 \circ-357996$ & 6420.225 & 2 & & 15571.474 & $13847_{2}-29419_{2}^{\circ}$ \\
\hline 6458.118 & 2 & & 15480.109 & $22338^{\circ}-37819_{4}$ & 6420.080 & 3 & & 15571.826 & $17224_{2}^{\circ}-327963$ \\
\hline 6457.280 & $800 \mathrm{r}$ & 300 & 15482.118 & $7795_{4}^{\circ}-23277_{5}$ & 6419.849 & 5 & 1 & 15572.386 & $19516_{2}^{\circ}-35089_{3}$ \\
\hline 6455.987 & 1 & & 15485.219 & $21645^{\circ}-371313$ & 6419.160 & 2 & & 15574.057 & $21575_{2}-37149_{2}^{\circ}$ \\
\hline 6455.265 & 50 & 4 & 15486.951 & $11601_{1}-27087_{1}^{\circ}$ & 6418.697 & 4 & & 15575.181 & $7280_{2}-22855_{3}^{\circ}$ \\
\hline 6454.629 & 4 & & 15488.477 & $211653_{3}^{\circ}-36653_{2}$ & 6417.908 & 15 & 2 & 15577.096 & $170731-32650_{1}^{\circ}$ \\
\hline 6453.551 & 3 & & 15491.064 & $22399^{\circ}-378905$ & 6416.930 & 3 & & 15579.470 & $20214 \stackrel{\circ}{3}-357944$ \\
\hline 6453.344 & 5 & 1 & 15491.561 & $18809_{4}^{\circ}-34301_{4}$ & 6415.536 & 50 & 5 & 15582.855 & $16346_{4}^{\circ}-31929_{3}$ \\
\hline 6452.951 & 40 & 2 & 15492.504 & $19948_{4}^{\circ}-35440_{3}$ & 6414.703 & 15 & 2 & 15584.878 & $13088_{3}-28673_{2}^{\circ}$ \\
\hline 6452.057 & 25 & 2 & 15494.651 & $11802_{2}-27297_{1}^{\circ}$ & 6414.148 & 75 & 10 & 15586.227 & $19503_{3}^{\circ}-350893$ \\
\hline 6451.582 & 2 & & 15495.792 & $18069_{3}^{\circ}-33564_{3}$ & 6413.615 & 400 & 100 & 15587.522 & $13297_{4}-28884^{\circ}$ \\
\hline 6451.258 & 4 & & 15496.570 & $14465_{2}^{\circ}-29961_{1}$ & 6412.977 & 1 & & 15589.073 & $22508_{2}^{\circ}-38097_{2}$ \\
\hline 6450.956 & 150 & 8 & 15497.295 & $15493_{4}-30990 \stackrel{\circ}{ }$ & 6412.129 & 2 & & 15591.134 & $15166_{3}^{\circ}-30758_{2}$ \\
\hline 6450.697 & 5 & & 15497.917 & $12114_{2}^{\circ}-276123$ & 6411.899 & 500 & 75 & 15591.694 & $75023-23093^{\circ}$ \\
\hline 6450.593 & 5 & & 15498.167 & $8111_{4}-23609 \circ$ & 6411.412 & 2 & & 15592.878 & $22399 \circ-379924$ \\
\hline 6450.007 & 200 & 15 & 15499.575 & $128473-28347_{2}^{\circ}$ & 6410.091 & 1 & & 15596.091 & $19986^{\circ}-355825$ \\
\hline 6449.518 & 5 & & 15500.751 & $130888_{3}-285899_{3}^{\circ}$ & 6410.069 & 2 & & 15596.145 & $199866^{\circ}-355825$ \\
\hline 6449.164 & 2 & & 15501.601 & $17073_{1}-32575_{2}^{\circ}$ & 6409.553 & 3 & & 15597.401 & $7280_{2}-22877_{1}^{\circ}$ \\
\hline 6448.850 & 3 & & 15502.356 & $21902_{4}^{\circ}-374043$ & 6408.425 & 2 & & 15600.146 & $17959_{4}-33560_{4}^{\circ}$ \\
\hline 6446.771 & 100 & 8 & 15507.355 & $142045-29711{ }^{\circ}$ & 6406.446 & 100 & 5 & 15604.965 & $4961_{4}-20566_{4}^{\circ}$ \\
\hline 6446.133 & 25 & 1 & 15508.890 & $10414 \stackrel{\circ}{4}-259234$ & 6403.860 & 4 & & 15611.266 & $75023-23113^{\circ}$ \\
\hline 6445.639 & 75 & 2 & 15510.079 & $16783_{4}^{\circ}-322935$ & 6400.696 & 100 & 8 & 15618.983 & $0_{2}-15618_{3}^{\circ}$ \\
\hline 6444.150 & 1 & & 15513.663 & $21645_{4}-37159^{\circ}$ & 6399.944 & 40 & & 15620.819 & $88004-24421_{3}^{\circ}$ \\
\hline 6444.150 & 1 & & 15513.663 & $21645_{4}-37159_{3}^{\circ}$ & 6399.571 & 20 & & 15621.729 & $19227_{6}^{\circ}-34849_{5}$ \\
\hline 6443.878 & 10 & 1 & 15514.318 & $18930_{3}^{\circ}-34444_{2}$ & 6398.602 & 1 & & 15624.095 & $20054_{2}-35678_{1}^{\circ}$ \\
\hline 6443.819 & 25 & 1 & 15514.460 & $11802_{2}-27317_{3}^{\circ}$ & 6398.451 & 15 & 2 & 15624.463 & $18699_{2}-34324_{2}^{\circ}$ \\
\hline 6443.090 & 4 & & 15516.215 & $18011^{\circ}-33527_{4}$ & 6397.447 & 20 & 2 & 15626.915 & $15618 \stackrel{\circ}{3}-31245_{2}$ \\
\hline 6443.090 & 4 & & 15516.215 & $22669_{3}^{\circ}-381864$ & 6396.951 & 200 & 8 & 15628.127 & $14206_{4}^{\circ}-29835_{3}$ \\
\hline 6440.353 & 5 & 1 & 15522.809 & $15618^{\circ}-31141_{3}$ & 6396.518 & 3 & 1 & 15629.185 & $15970_{3}-31599^{\circ}$ \\
\hline 6440.245 & 1 & & 15523.069 & $20566_{4}^{\circ}-360894$ & 6395.617 & 3 & 1 & 15631.387 & $21890 \AA-37521_{3}$ \\
\hline 6439.418 & 4 & & 15525.063 & $22163_{4}^{\circ}-376883$ & 6395.279 & 3. & & 15632.213 & $128473-28480_{4}^{\circ}$ \\
\hline 6438.918 & 50 & 8 & 15526.268 & $82432-23769_{1}$ & 6395.057 & 75 & 4 & 15632.756 & $19516_{2}^{\circ}-35149_{2}$ \\
\hline 6438.303 & 20 & 2 & 15527.751 & $6362_{2}-21890_{3}^{\circ}$ & 6394.589 & 3 & 1 & 15633.900 & $19948_{4}^{\circ}-355825$ \\
\hline 6437.769 & 200 & 20 & 15529.039 & $142045-297338$ & 6394.045 & 200 & 8 & 15635.230 & $13297_{4}-28932_{4}^{\circ}$ \\
\hline 6437.769 & 200 & 20 & 15529.039 & $19516_{2}^{\circ}-35046_{1}$ & 6393.201 & 5 & 1 & 15637.294 & $18809_{4}^{\circ}-34447_{4}$ \\
\hline 6435.108 & 1 & & 15535.461 & $22248_{2}^{\circ}-37784_{2}$ & 6392.371 & 75 & 8 & 15639.324 & $21594_{3}-37234_{2}^{\circ}$ \\
\hline 6434.800 & 2 & & 15536.204 & $18431_{3}-33967^{\circ}$ & 6391.173 & 50 & 2 & 15642.256 & $14206_{4}^{\circ}-29849_{4}$ \\
\hline 6434.654 & 3 & & 15536.557 & $185741-34111_{1}^{\circ}$ & 6390.388 & 5 & 1 & 15644.177 & $20566_{4}^{\circ}-362105$ \\
\hline 6431.715 & 5 & & 15543.656 & $17847_{2}^{\circ}-33390_{1}$ & 6390.126 & 75 & 4 & 15644.819 & $11802_{2}-27447_{2}^{\circ}$ \\
\hline 6431.518 & 2 & & 15544.132 & $8111_{4}-23655_{4}^{\circ}$ & 6389.391 & $200 \mathrm{~b}$ & 15 & 15646.618 & $156189-31265_{3}$ \\
\hline
\end{tabular}


TABle 4. Classified lines of Th $\mathrm{I}$-continued

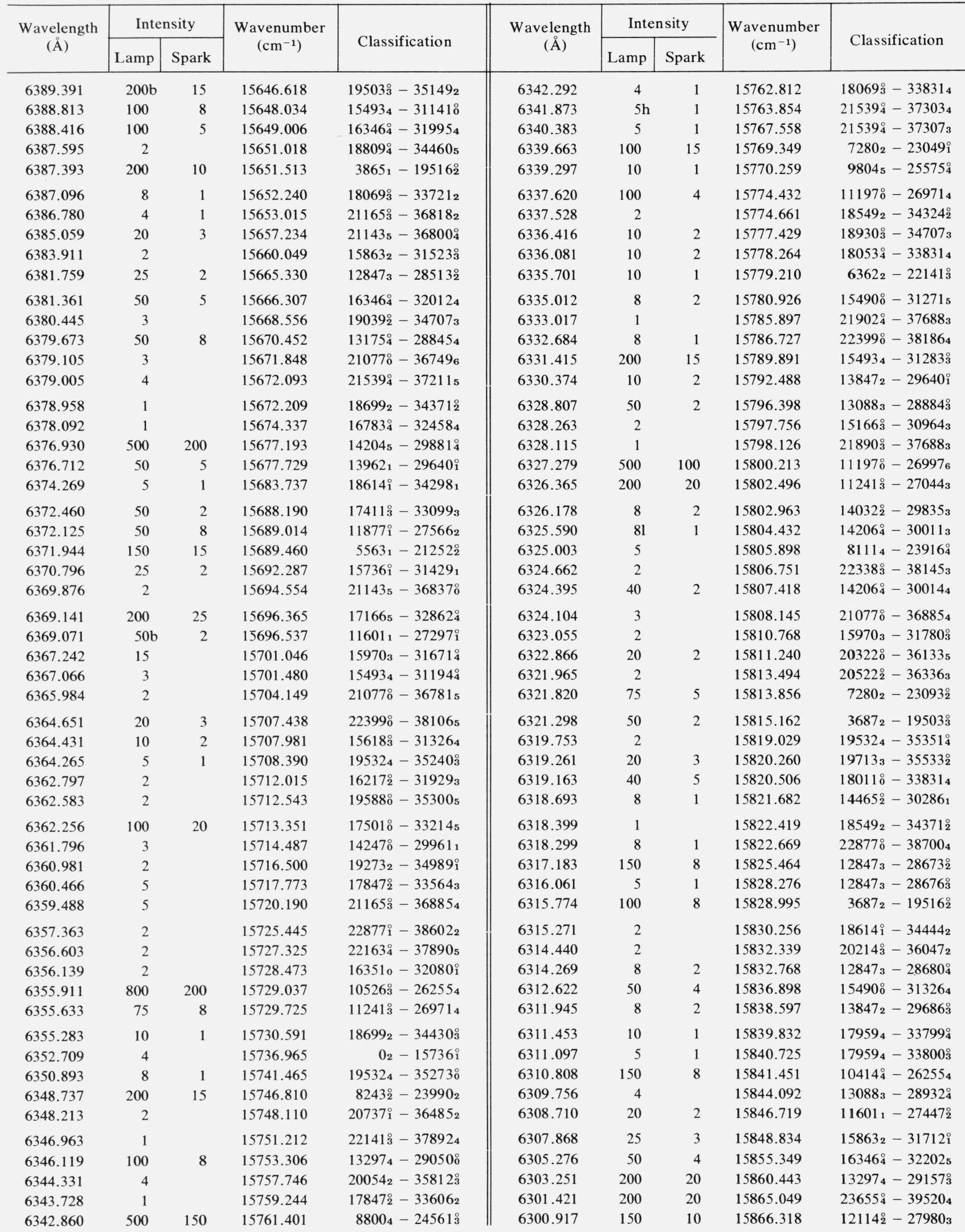


TABLE 4. Classified lines of Th $\mathrm{I}$-continued

\begin{tabular}{|c|c|c|c|c|c|c|c|c|c|}
\hline \multirow{2}{*}{$\begin{array}{l}\text { Wavelength } \\
\text { (§) }\end{array}$} & \multicolumn{2}{|c|}{ Intensity } & \multirow{2}{*}{$\begin{array}{l}\text { Wavenumber } \\
\qquad\left(\mathrm{cm}^{-1}\right)\end{array}$} & \multirow{2}{*}{ Classification } & \multirow{2}{*}{$\begin{array}{c}\text { Wavelength } \\
(\AA)\end{array}$} & \multicolumn{2}{|c|}{ Inten sity } & \multirow{2}{*}{$\begin{array}{l}\text { Wave number } \\
\qquad\left(\mathrm{cm}^{-1}\right)\end{array}$} & \multirow{2}{*}{ Classification } \\
\hline & Lamp & Spark & & & & Lamp & Spark & & \\
\hline 6298.903 & 50 & 5 & 15871.391 & $11802_{2}-27674_{2}^{\circ}$ & 6261.418 & 400 & 150 & 15966.407 & $10414_{4}^{\circ}-26380_{5}$ \\
\hline 6297.777 & 10 & 1 & 15874.229 & $17847_{2}^{\circ}-33721_{2}$ & 6260.218 & 8 & 1 & 15969.467 & $8800_{4}-24769_{3}^{\circ}$ \\
\hline 6297.581 & 1 & & 15874.723 & $20214_{3}^{\circ}-36089_{4}$ & 6258.097 & 2 & & 15974.880 & $15166^{\circ}-31141_{3}$ \\
\hline 6297.426 & 3 & & 15875.113 & $17224_{2}^{\circ}-33099_{3}$ & 6257.424 & 200 & 8 & 15976.598 & $63622-22338_{3}^{\circ}$ \\
\hline 6296.426 & 1 & & 15877.634 & $22401_{1}-38278_{1}^{\circ}$ & 6255.531 & 5 & 1 & 15981.432 & $118022-27784_{2}^{\circ}$ \\
\hline 6295.170 & 10 & 1 & 15880.802 & $17073_{1}-32954_{2}^{\circ}$ & 6255.344 & 4 & 1 & 15981.910 & $21539_{4}^{\circ}-37521_{3}$ \\
\hline 6295.170 & 10 & 1 & 15880.802 & $18549_{2}-34430_{3}^{\circ}$ & 6254.774 & 20 & 2 & 15983.367 & $15970{ }_{3}-31953^{\circ}$ \\
\hline 6294.340 & 2 & & 15882.896 & $19948{ }^{\circ}-358313$ & 6252.887 & 4 & 1 & 15988.190 & $21902_{4}^{\circ}-37890_{5}$ \\
\hline 6294.283 & 2 & & 15883.040 & $22877^{\circ}-38760_{4}$ & 6252.314 & 8 & 1 & 15989.655 & $19227_{6}^{\circ}-352165$ \\
\hline 6293.480 & 5 & & 15885.067 & $179594-33844 \circ$ & 6251.964 & 8 & 2 & 15990.550 & $21902_{4}^{\circ}-37892_{4}$ \\
\hline 6293.241 & 150 & 15 & 15885.670 & $98045-25690_{0}^{\circ}$ & 6250.484 & 50 & 4 & 15994.337 & $11197^{\circ}-271915$ \\
\hline 6293.036 & 3 & & 15886.188 & $20922_{2}^{\circ}-368083$ & 6249.270 & 3 & & 15997.444 & $17411_{3}^{\circ}-33408_{3}$ \\
\hline 6292.891 & 100 & 8 & 15886.554 & $6362_{2}-22248_{2}^{\circ}$ & 6249.146 & 5 & 1 & 15997.761 & $16783_{4}^{\circ}-32781_{4}$ \\
\hline 6292.187 & 4 & & 15888.331 & $19516_{2}^{\circ}-35405_{3}$ & 6247.222 & 3 & & 16002.688 & $21890_{3}^{\circ}-37892_{4}$ \\
\hline 6292.061 & 50 & 3 & 15888.649 & $14243{ }_{1}^{\circ}-301322$ & 6246.664 & 1 & & 16004.117 & $22141_{3}^{\circ}-38145_{3}$ \\
\hline 6291.629 & 50 & 15 & 15889.740 & $13945^{\circ}-29835_{3}$ & 6246.400 & 4 & & 16004.794 & $18699_{2}-34704_{3}^{\circ}$ \\
\hline 6291.192 & 150 & 15 & 15890.844 & $144816^{\circ}-303726$ & 6246.173 & 75 & 8 & 16005.376 & $13847_{2}-29853_{2}^{\circ}$ \\
\hline 6290.576 & 2 & & 15892.400 & $18431_{3}-34324_{2}^{\circ}$ & 6245.848 & 5 & 1 & 16006.208 & $15863_{2}-31870_{2}^{\circ}$ \\
\hline 6289.159 & 2 & & 15895.981 & $20922_{2}^{\circ}-36818_{2}$ & 6245.601 & 50 & 15 & 16006.841 & $19039_{2}^{\circ}-35046_{1}$ \\
\hline 6288.852 & 3 & 1 & 15896.757 & $13847_{2}-29744_{3}^{\circ}$ & 6245.149 & 3 & & 16008.000 & $17959_{4}-33967_{3}^{\circ}$ \\
\hline 6288.424 & 8 & 1 & 15897.839 & $17411_{3}^{\circ}-33309_{2}$ & 6244.875 & 2 & & 16008.702 & $21077_{0}^{\circ}-37085_{4}$ \\
\hline 6288.074 & 2 & & 15898.724 & $17398_{3}-33297_{2}^{\circ}$ & 6244.381 & $8 \mathrm{~h}$ & 2 & 16009.969 & $21738_{2}^{\circ}-37748_{2}$ \\
\hline 6287.570 & 50 & 3 & 15899.998 & $15970_{3}-31870_{2}^{\circ}$ & 6243.953 & 2 & & 16011.066 & $21594_{3}-37605_{4}^{\circ}$ \\
\hline 6287.254 & 75 & 4 & 15900.797 & 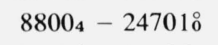 & 6243.358 & 50 & 4 & 16012.592 & $13297_{4}-29310_{4}^{\circ}$ \\
\hline 6286.712 & 3 & & 15902.168 & $19503^{\circ}-35405_{3}$ & 6241.873 & 10 & 3 & 16016.402 & $185741-34591 i$ \\
\hline 6286.043 & 50 & 4 & 15903.860 & $13945^{\circ}-29849_{4}$ & 6240.949 & 150 & 8 & 16018.773 & $75023-23521 \stackrel{\circ}{3}$ \\
\hline 6285.453 & 1 & & 15905.353 & $22855_{3}^{\circ}-38760_{4}$ & 6240.305 & 15 & 2 & 16020.426 & $19713_{3}-35733^{\circ}$ \\
\hline 6282.449 & 2 & & 15912.958 & $18431_{3}-34344_{4}^{\circ}$ & 6239.742 & 2 & & 16021.871 & $22508_{2}^{\circ}-38529_{3}$ \\
\hline 6281.543 & 5 & & 15915.253 & $18382^{\circ}-342981$ & 6239.306 & 5 & 1 & 16022.991 & $22163_{4}^{\circ}-381864$ \\
\hline 6280.875 & $2 b$ & & 15916.946 & $175010^{\circ}-334184$ & 6238.208 & 5 & & 16025.811 & $18699_{2}-34725_{3}^{\circ}$ \\
\hline 6280.359 & 2 & & 15918.254 & $15618^{\circ}-315373$ & 6238.080 & 20 & 2 & 16026.140 & $14481 \stackrel{\circ}{6}-305085$ \\
\hline 6279.982 & 75 & 4 & 15919.209 & $17354_{1}^{\circ}-33273_{1}$ & 6237.982 & 5 & 1 & 16026.392 & $17501^{\circ}-33527_{4}$ \\
\hline 6279.369 & 2 & & 15920.763 & $21165_{3}^{\circ}-37085_{4}$ & 6237.101 & 5 & 1 & 16028.655 & $17398_{3}-33427_{2}^{\circ}$ \\
\hline 6278.125 & 4 & & 15923.918 & $19516_{2}^{\circ}-35440_{3}$ & 6235.176 & 1 & & 16033.604 & $18549_{2}-34583^{\circ}$ \\
\hline 6277.512 & 2 & & 15925.473 & $216454-37571{ }^{\circ}$ & 6234.856 & 400 & 75 & 16034.427 & $6362_{2}-22396 i$ \\
\hline 6276.162 & 100 & 8 & 15928.899 & $13175_{4}^{\circ}-29104_{4}$ & 6234.200 & 150 & 10 & 16036.114 & $17354^{\circ}-33390_{1}$ \\
\hline 6275.849 & 3 & & 15929.693 & $14032_{2}^{\circ}-29961_{1}$ & 6233.858 & 3 & & 16036.994 & $12847_{3}-28884_{3}^{\circ}$ \\
\hline 6275.679 & 2 & & 15930.125 & $22877_{1}^{\circ}-38807_{2}$ & 6233.004 & 8 & & 16039.191 & $23655_{4}^{\circ}-396945$ \\
\hline 6274.523 & 2 & & 15933.059 & $22669_{3}^{\circ}-38602_{2}$ & 6232.845 & 2 & & 16039.600 & $14247^{\circ}-302861$ \\
\hline 6272.676 & 3 & & 15937.751 & $195033^{\circ}-35440_{3}$ & 6232.186 & 8 & 1 & 16041.296 & $21575_{2}-37616_{1}^{\circ}$ \\
\hline 6271.733 & 1 & & 15940.147 & $18431_{3}-34371_{2}^{\circ}$ & 6232.129 & 25 & 1 & 16041.443 & $17959_{4}-34001_{4}^{\circ}$ \\
\hline 6271.544 & 75 & 5 & 15940.628 & $2869_{3}-18809_{4}^{\circ}$ & 6232.068 & 50 & 4 & 16041.600 & $18549_{2}-34591^{\circ}$ \\
\hline 6271.131 & 2 & & 15941.678 & $23015_{0}^{\circ}-389564$ & 6230.933 & 3 & & 16044.522 & $22141_{3}^{\circ}-381864$ \\
\hline 6270.331 & 8 & 2 & 15943.711 & $22163^{\circ}-381065$ & 6230.548 & 1 & & 16045.514 & $19588 \circ-356334$ \\
\hline 6268.923 & 2 & & 15947.292 & $16346_{4}^{\circ}-322935$ & 6230.221 & 1 & & 16046.356 & $21738_{2}^{\circ}-37784_{2}$ \\
\hline 6268.200 & 20 & 2 & 15949.132 & $98045-25753 \stackrel{\circ}{\circ}$ & 6227.179 & 8 & & 16054.194 & $142260-30281^{\circ}$ \\
\hline 6267.800 & 2 & & 15950.150 & $21738_{2}^{\circ}-376883$ & 6226.370 & 100 & 8 & 16056.280 & $25580-18614^{\circ}$ \\
\hline 6267.188 & 2 & & 15951.707 & $3865_{1}-19817_{1}^{\circ}$ & 6225.105 & 5 & 1 & 16059.543 & $19516_{2}^{\circ}-35576_{3}$ \\
\hline 6266.614 & 75 & 5 & 15953.168 & $16783^{\circ}-327375$ & 6224.678 & 8 & 1 & 16060.645 & $19817_{1}^{\circ}-35877_{2}$ \\
\hline 6265.962 & 3 & & 15954.828 & $22098_{4}-38053_{3}^{\circ}$ & 6224.527 & 400 & 40 & 16061.034 & $2869_{3}-18930_{3}^{\circ}$ \\
\hline 6265.604 & 75 & 10 & 15955.740 & $14204_{5}-30160_{4}^{\circ}$ & 6224.043 & 5 & & 16062.283 & $21594_{3}-37656_{3}^{\circ}$ \\
\hline 6264.715 & 100 & 8 & 15958.004 & $17501^{\circ}-33459_{4}$ & 6223.308 & 5 & & 16064.180 & $18809_{4}^{\circ}-348743$ \\
\hline 6262.884 & 3 & & 15962.669 & $20522_{2}^{\circ}-36485_{2}$ & 6222.769 & $4 \mathrm{~h}$ & 1 & 16065.572 & $16346_{4}^{\circ}-32412_{4}$ \\
\hline 6261.584 & $2 \mathrm{~h}$ & & 15965.983 & $21165^{\circ}-371313$ & 6222.588 & 3 & & 16066.039 & $13945_{3}^{\circ}-300113$ \\
\hline 6261.418 & 400 & 150 & 15966.407 & $13175_{4}^{\circ}-291415$ & 6221.426 & 5 & & 16069.040 & $13945_{3}^{\circ}-30014_{4}$ \\
\hline
\end{tabular}


TABle 4. Classified lines of Th $\mathrm{I}$-continued

\begin{tabular}{|c|c|c|c|c|c|c|c|c|c|}
\hline \multirow{2}{*}{$\begin{array}{l}\text { Wavelength } \\
\text { (̊) }\end{array}$} & \multicolumn{2}{|c|}{ Intensity } & \multirow{2}{*}{$\begin{array}{l}\text { Wavenumber } \\
\quad\left(\mathrm{cm}^{-1}\right)\end{array}$} & \multirow{2}{*}{ Classification } & \multirow{2}{*}{$\begin{array}{c}\text { Wavelength } \\
\text { (§) }\end{array}$} & \multicolumn{2}{|c|}{ Inten sity } & \multirow{2}{*}{$\begin{array}{l}\text { Wavenumber } \\
\left(\mathrm{cm}^{-1}\right)\end{array}$} & \multirow{2}{*}{ Classification } \\
\hline & Lamp & Spark & & & & Lamp & Spark & & \\
\hline 6221.320 & 100 & 15 & 16069.313 & $13088_{3}-29157_{3}^{\circ}$ & 6180.285 & 1 & & 16176.007 & $18549_{2}-34725_{3}^{\circ}$ \\
\hline 6221.059 & 75 & 3 & 16069.988 & $12847_{3}-28917_{2}^{\circ}$ & 6179.548 & 4 & & 16177.937 & $154934-31671_{4}^{\circ}$ \\
\hline 6220.011 & 100 & 5 & 16072.695 & $9804_{5}-25877_{4}^{\circ}$ & 6178.930 & 15 & 2 & 16179.555 & $16783{ }_{4}^{\circ}-329635$ \\
\hline 6219.775 & 50 & 3 & 16073.305 & $11601_{1}-27674_{2}^{\circ}$ & 6178.432 & 200 & 20 & 16180.859 & $8800_{4}-24981_{3}^{\circ}$ \\
\hline 6219.672 & 1 & & 16073.571 & $19227^{\circ}-353005$ & 6177.489 & 1 & & 16183.329 & $11601_{1}-27784_{2}^{\circ}$ \\
\hline 6218.862 & 2 & & 16075.665 & $22877^{\circ}-389535$ & 6177.081 & 1 & & 16184.398 & $17224_{2}^{\circ}-33408_{3}$ \\
\hline 6217.572 & 10 & 1 & 16079.000 & $15166_{3}^{\circ}-31245_{2}$ & 6176.490 & 4 & & 16185.946 & $14465_{2}^{\circ}-30651_{3}$ \\
\hline 6217.437 & 1 & & 16079.349 & $14465_{2}^{\circ}-30544_{2}$ & 6175.956 & 10 & 1 & 16187.346 & $13945_{3}^{\circ}-301322$ \\
\hline 6217.437 & 1 & & 16079.349 & $22877_{1}^{\circ}-38956_{4}$ & 6174.797 & 4 & & 16190.384 & $15970_{3}-32160_{2}^{\circ}$ \\
\hline 6216.865 & 15 & 2 & 16080.829 & $20737^{\circ}-36818_{2}$ & 6173.881 & 4 & & 16192.786 & $17398_{3}-33591_{3}^{\circ}$ \\
\hline 6216.441 & 4 & 1 & 16081.925 & $21575_{2}-37656_{3}^{\circ}$ & 6173.004 & 2 & & 16195.087 & $17411_{3}^{\circ}-336062$ \\
\hline 6216.270 & 3 & & 16082.368 & $20214_{3}^{\circ}-36297_{4}$ & 6170.733 & 15 & 2 & 16201.047 & $195324-35733_{4}^{\circ}$ \\
\hline 6215.360 & 1 & & 16084.722 & $17224_{2}^{\circ}-33309_{2}$ & 6170.652 & 15 & 2 & 16201.260 & $7280_{2}-23481_{1}^{\circ}$ \\
\hline 6214.106 & 5 & 1 & 16087.968 & $17073_{1}-33161^{\circ}$ & 6169.822 & 500 & 200 & 16203.439 & $49614-21165_{3}^{\circ}$ \\
\hline 6213.328 & 3 & & 16089.983 & $21902_{4}^{\circ}-37992_{4}$ & 6169.394 & 1 & & 16204.563 & $21902_{4}^{\circ}-381065$ \\
\hline 6212.721 & 100 & 5 & 16091.555 & $8111_{4}-24202_{4}^{\circ}$ & 6168.837 & 4 & 1 & 16206.026 & $19588^{\circ}-35794_{4}$ \\
\hline 6209.949 & 8 & 1 & 16098.738 & $15166_{3}^{\circ}-31265_{3}$ & 6168.482 & 2 & & 16206.959 & $21890_{3}^{\circ}-38097_{2}$ \\
\hline 6209.659 & 2 & & 16099.489 & $19713_{3}-35812_{3}^{\circ}$ & 6166.709 & 4 & 1 & 16211.619 & $19588_{0}^{\circ}-35799_{6}$ \\
\hline 6208.986 & 8 & 1 & 16101.234 & $75023-23603_{2}^{\circ}$ & 6165.538 & 4 & 1 & 16214.697 & $20566_{4}^{\circ}-367815$ \\
\hline 6208.861 & 2 & & 16101.559 & $22855_{3}^{\circ}-389564$ & 6164.842 & 8 & 1 & 16216.528 & $15863_{2}-32080_{1}^{\circ}$ \\
\hline 6208.686 & 100 & 5 & 16102.013 & $11241_{3}^{\circ}-273433$ & 6164.480 & 200 & 20 & 16217.480 & $0_{2}-16217_{2}^{\circ}$ \\
\hline 6207.751 & 150 & 8 & 16104.438 & $17166_{5}-33270_{4}^{\circ}$ & 6163.732 & 100 & 5 & 16219.448 & $18930_{3}^{\circ}-35149_{2}$ \\
\hline 6207.220 & 500 & 200 & 16105.815 & $5563_{1}-21668_{1}^{\circ}$ & 6162.968 & 40 & 3 & 16221.459 & $13088_{3}-29310_{4}^{\circ}$ \\
\hline 6205.861 & 100 & 8 & 16109.342 & $13088_{3}-29197_{2}^{\circ}$ & 6162.854 & 8 & 1 & 16221.759 & $11802_{2}-28024_{1}^{\circ}$ \\
\hline 6205.018 & 50 & 2 & 16111.531 & $16346_{4}^{\circ}-32458_{4}$ & 6162.456 & 4 & & 16222.807 & $17959_{4}-34182 \circ$ \\
\hline 6203.493 & 500 & 100 & 16115.492 & $4961_{4}-21077^{\circ}$ & 6162.261 & 2 & & 16223.320 & $17073_{1}-33297_{2}^{\circ}$ \\
\hline 6203.169 & 3 & 1 & 16116.333 & $17411_{3}^{\circ}-33527_{4}$ & 6161.765 & 50 & 4 & 16224.626 & $19986^{\circ}-36210_{5}$ \\
\hline 6198.223 & 400 & 75 & 16129.193 & $3687_{2}-19817_{1}^{\circ}$ & 6161.646 & 1 & & 16224.939 & $221411_{3}^{\circ}-383664$ \\
\hline 6197.591 & 1 & & 16130.838 & $20522_{2}^{\circ}-366532$ & 6158.829 & 1 & & 16232.361 & $18069_{3}^{\circ}-34301_{4}$ \\
\hline 6196.311 & 5 & & 16134.171 & $20054_{2}-36188_{2}^{\circ}$ & 6157.093 & 100 & 8 & 16236.937 & $7795_{4}^{\circ}-24032_{4}$ \\
\hline 6196.172 & 4 & & 16134.532 & $21077{ }^{\circ}-372115$ & 6156.114 & 8 & 1 & 16239.519 & $21165_{3}^{\circ}-37404_{3}$ \\
\hline 6195.322 & 25 & 3 & 16136.746 & 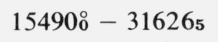 & 6155.587 & 200 & 15 & 16240.910 & $7280_{2}-23521_{3}^{\circ}$ \\
\hline 6194.928 & 1 & & 16137.772 & $22669_{3}^{\circ}-38807_{2}$ & 6155.299 & 8 & 1 & 16241.670 & $20566_{4}^{\circ}-36808_{3}$ \\
\hline 6193.576 & 2 & & 16141.295 & $19948_{4}^{\circ}-36089_{4}$ & 6154.627 & 4 & 1 & 16243.443 & $21902_{4}^{\circ}-38145_{3}$ \\
\hline 6193.290 & 2 & & 16142.040 & $21165_{3}^{\circ}-37307_{3}$ & 6154.515 & 40 & 8 & 16243.738 & $9804_{5}-260484$ \\
\hline 6193.194 & 5 & & 16142.291 & $21594_{3}-37736_{2}^{\circ}$ & 6154.419 & 3 & & 16243.992 & $12114_{2}^{\circ}-283583$ \\
\hline 6191.906 & 400 & 50 & 16145.648 & $6362_{2}-22508_{2}^{\circ}$ & 6154.068 & 100 & 15 & 16244.918 & $144811_{6}^{\circ}-307267$ \\
\hline 6191.261 & 2 & & 16147.330 & $19986^{\circ}-361335$ & 6152.950 & 2 & & 16247.870 & $18053{ }_{4}^{\circ}-343014$ \\
\hline 6190.771 & 10 & 2 & 16148.609 & $21539_{4}^{\circ}-37688_{3}$ & 6151.993 & $150 \mathrm{r}$ & & 16250.398 & $75023-23752_{2}^{\circ}$ \\
\hline 6190.710 & 10 & & 16148.768 & $8111_{4}-24259_{4}^{\circ}$ & 6151.517 & 8 & 2 & 16251.655 & $17354_{1}^{\circ}-33606_{2}$ \\
\hline 6189.145 & 50 & 2 & 16152.851 & $75023-23655_{4}^{\circ}$ & 6151.142 & 1 & & 16252.646 & $20522_{2}^{\circ}-36775_{3}$ \\
\hline 6188.863 & 1 & & 16153.587 & $17411_{3}^{\circ}-335643$ & 6150.683 & 20 & 4 & 16253.859 & $11241_{3}^{\circ}-27495_{4}$ \\
\hline 6188.126 & 200 & 75 & 16155.511 & $8243_{2}^{\circ}-24399_{3}$ & 6150.323 & 4 & 1 & 16254.810 & $14032_{2}^{\circ}-302861$ \\
\hline 6186.763 & 2 & & 16159.070 & $18930_{3}^{\circ}-35089_{3}$ & 6150.120 & 2 & & 16255.347 & $21077^{\circ}-373326$ \\
\hline 6186.380 & 8 & 1 & 16160.070 & $15166_{3}^{\circ}-31326_{4}$ & 6150.031 & 1 & & 16255.582 & $21890_{3}^{\circ}-38145_{3}$ \\
\hline 6185.774 & 25 & 2 & 16161.654 & $17398_{3}-33560_{4}^{\circ}$ & 6148.361 & 3 & 1 & 16259.997 & $19273_{2}-35533_{2}^{\circ}$ \\
\hline 6185.683 & 1 & & 16161.891 & $21575_{2}-37736_{2}^{\circ}$ & 6147.959 & 2 & & 16261.060 & $10783_{2}^{\circ}-27044_{3}$ \\
\hline 6184.985 & 4 & & 16163.715 & $8111_{4}-24274 \circ$ & 6145.128 & 2 & & 16268.551 & $22401_{1}-38669_{2}^{\circ}$ \\
\hline 6184.985 & 4 & & 16163.715 & $8111_{4}-24274 \circ$ & 6145.000 & 2 & & 16268.890 & $21252_{2}^{\circ}-37521_{3}$ \\
\hline 6184.788 & 150 & 15 & 16164.230 & $13088_{3}-29252_{2}^{\circ}$ & 6144.760 & 20 & 3 & 16269.526 & $10526_{3}^{\circ}-267963$ \\
\hline 6183.942 & 5 & & 16166.441 & $17224_{2}^{\circ}-33390_{1}$ & 6144.414 & 1 & & 16270.442 & $20214_{3}^{\circ}-36485_{2}$ \\
\hline 6182.830 & $50 \mathrm{~b}$ & & 16169.349 & $13847_{2}-30017_{3}^{\circ}$ & 6141.928 & 1 & & 16277.027 & $185741-34851_{2}^{\circ}$ \\
\hline 6182.623 & 500 & 1 & 16169.890 & $2869_{3}-19039_{2}^{\circ}$ & 6141.629 & 2 & & 16277.820 & $17411_{3}^{\circ}-33689_{2}$ \\
\hline 6181.537 & 10 & 1 & 16172.731 & $20922_{2}^{\circ}-37094_{2}$ & 6140.994 & 1 & & 16279.503 & $18809_{4}^{\circ}-35089_{3}$ \\
\hline 6180.703 & 200 & 8 & 16174.913 & $5563_{1}-21738_{2}^{\circ}$ & 6140.994 & 1 & & 16279.503 & $21539_{4}^{\circ}-37819_{4}$ \\
\hline
\end{tabular}


TABle 4. Classified lines of Th $\mathrm{I}$-continued

\begin{tabular}{|c|c|c|c|c|c|c|c|c|c|}
\hline \multirow{2}{*}{$\begin{array}{l}\text { Wavelength } \\
\text { (Å) }\end{array}$} & \multicolumn{2}{|c|}{ Intensity } & \multirow{2}{*}{$\begin{array}{c}\text { Wavenumber } \\
\left(\mathrm{cm}^{-1}\right)\end{array}$} & \multirow{2}{*}{ Classification } & \multirow{2}{*}{ 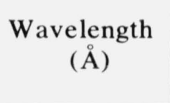 } & \multicolumn{2}{|c|}{ Inten sity } & \multirow{2}{*}{$\begin{array}{l}\text { Wavenumber } \\
\qquad\left(\mathrm{cm}^{-1}\right)\end{array}$} & \multirow{2}{*}{ Classification } \\
\hline & Lamp & Spark & & & & Lamp & Spark & & \\
\hline 6140.884 & 4 & & 16279.795 & $11241_{3}^{\circ}-27521_{4}$ & 6104.572 & 300 & 200 & 16376.631 & $15618_{3}^{\circ}-31995_{4}$ \\
\hline 6140.772 & 25 & 4 & 16280.092 & $19532_{4}-35812_{3}^{\circ}$ & 6104.170 & 4 & 1 & 16377.710 & $180534-344313$ \\
\hline 6140.449 & 1 & & 16280.948 & $22248_{2}^{\circ}-38529_{3}$ & 6104.027 & 5 & 1 & 16378.094 & $18069_{3}^{\circ}-34447_{4}$ \\
\hline 6139.364 & 2 & & 16283.825 & $219024-381864$ & 6103.354 & 2 & & 16379.899 & $21575_{2}-37954^{\circ}$ \\
\hline 6137.025 & 5 & 1 & 16290.031 & $180118-343014$ & 6102.595 & 300 & 50 & 16381.937 & $10414_{4}^{\circ}-267963$ \\
\hline 6135.996 & 5 & 1 & 16292.763 & $14465_{2}^{\circ}-30758_{2}$ & 6101.726 & 100 & 10 & 16384.270 & $8243_{2}^{\circ}-24627_{1}$ \\
\hline 6135.628 & 5 & 1 & 16293.740 & $184313-34725^{\circ}$ & 6101.554 & 3 & 1 & 16384.732 & $179594-34344^{\circ}$ \\
\hline 6135.014 & 2 & & 16295.371 & $20522_{2}^{\circ}-36818_{2}$ & 6100.414 & 3 & 1 & 16387.793 & $19948^{\circ}-363363$ \\
\hline 6134.563 & 8 & 1 & 16296.569 & $158632-32160_{2}^{\circ}$ & 6099.994 & 50 & 3 & 16388.922 & $132974-29686^{\circ}$ \\
\hline 6133.821 & 50 & 2 & 16298.540 & $111978-27495_{4}$ & 6099.458 & 2 & & 16390.362 & $16346_{4}^{\circ}-327375$ \\
\hline 6133.671 & 2 & & 16298.939 & $163510-32650_{1}^{\circ}$ & 6098.126 & 200 & 25 & 16393.942 & $17166_{5}-33560_{4}^{\circ}$ \\
\hline 6133.064 & 75 & 4 & 16300.552 & $14243 i-30544_{2}$ & 6097.821 & 4 & 1 & 16394.762 & $111970^{\circ}-275915$ \\
\hline 6132.874 & 50 & 4 & 16301.057 & $14206_{4}^{\circ}-305085$ & 6097.592 & 5 & & 16395.378 & $11877^{\circ}-28273_{2}$ \\
\hline 6132.874 & 50 & 4 & 16301.057 & $20214_{3}^{\circ}-365153$ & 6097.292 & 4 & & 16396.184 & $18011^{\circ}-344076$ \\
\hline 6132.426 & 4 & 1 & 16302.248 & $18549_{2}-34851_{2}^{\circ}$ & 6095.389 & 1 & & 16401.303 & $17398_{3}-33799^{\circ}$ \\
\hline 6131.753 & 3 & & 16304.037 & $18574_{1}-34878^{\circ}$ & 6095.050 & 20 & 4 & 16402.216 & $17398_{3}-33800_{3}^{\circ}$ \\
\hline 6131.673 & 5 & 1 & 16304.250 & $15736_{1}^{\circ}-32041_{2}$ & 6094.089 & 10 & 1 & 16404.802 & $128473-29252_{2}^{\circ}$ \\
\hline 6130.765 & 25 & 2 & 16306.665 & $20054_{2}-36361_{1}^{\circ}$ & 6093.813 & 5 & 1 & 16405.545 & $19273_{2}-35678_{1}^{\circ}$ \\
\hline 6130.459 & 20 & 1 & 16307.479 & $6362_{2}-22669_{3}^{\circ}$ & 6093.558 & 1 & & 16406.232 & $22877^{\circ}-39283_{4}$ \\
\hline 6129.551 & 75 & 8 & 16309.894 & $128473-29157^{\circ}$ & 6093.240 & 8 & 1 & 16407.088 & $18809_{4}^{\circ}-352165$ \\
\hline 6129.492 & 15 & 2 & 16310.051 & $8111_{4}-24421_{3}^{\circ}$ & 6093.027 & 10 & 1 & 16407.661 & $21738_{2}^{\circ}-381453$ \\
\hline 6129.322 & 2 & & 16310.504 & $156183-31929_{3}$ & 6090.607 & 2 & & 16414.181 & $132974-297110^{\circ}$ \\
\hline 6128.448 & 25 & 2 & 16312.830 & $142045-30517^{\circ}$ & 6090.451 & 15 & 2 & 16414.601 & $7502_{3}-23916^{\circ}$ \\
\hline 6128.344 & 40 & 3 & 16313.107 & $142045-30517^{\circ}$ & 6090.241 & 1 & & 16415.167 & $18574_{1}-34989^{\circ}$ \\
\hline 6128.160 & 10 & 2 & 16313.596 & $163510-32665^{\circ}$ & 6088.828 & 10 & 2 & 16418.976 & $17354^{\circ}-337731$ \\
\hline 6127.899 & 3 & & 16314.291 & $19516_{2}^{\circ}-358313$ & 6088.459 & 4 & 1 & 16419.972 & $18431_{3}-34851_{2}^{\circ}$ \\
\hline 6127.609 & 50 & 2 & 16315.063 & $159700_{3}-32285^{\circ}$ & 6088.203 & 10 & 2 & 16420.662 & $17411^{\circ}-338314$ \\
\hline 6127.425 & 50 & 2 & 16315.553 & $16783{ }^{\circ}-33099_{3}$ & 6088.031 & 400 & 50 & 16421.126 & $8243_{2}^{\circ}-246643$ \\
\hline 6126.509 & 1 & & 16317.993 & $220984-38416_{3}^{\circ}$ & 6086.702 & 8 & 1 & 16424.711 & $14465_{2}^{\circ}-30889_{1}$ \\
\hline 6126.328 & 2 & & 16318.475 & $139621-30281^{\circ}$ & 6085.375 & 100 & 75 & 16428.293 & $8243_{2}^{\circ}-24671_{2}$ \\
\hline 6126.328 & 2 & & 16318.475 & $139621-30281^{\circ}$ & 6084.486 & 2 & & 16430.693 & $167834^{\circ}-332145$ \\
\hline 6125.610 & 4 & & 16320.387 & $17847_{2}^{\circ}-341673$ & 6084.127 & 5 & 1 & 16431.663 & $18614^{\circ}-350461$ \\
\hline 6124.485 & 200 & 15 & 16323.385 & $7280_{2}-23603^{\circ}$ & 6083.533 & 2 & & 16433.267 & $13847_{2}-30281_{1}^{\circ}$ \\
\hline 6124.073 & 50 & 3 & 16324.483 & $111970-27521_{4}$ & 6083.242 & 10 & 2 & 16434.053 & $20054_{2}-36488_{2}^{\circ}$ \\
\hline 6123.838 & 75 & 8 & 16325.110 & $11241_{3}^{\circ}-27566_{2}$ & 6082.910 & $2 \mathrm{~h}$ & & 16434.950 & $16346_{4}^{\circ}-32781_{4}$ \\
\hline 6122.709 & 3 & & 16328.120 & $195033^{\circ}-35831_{3}$ & 6082.669 & 4 & & 16435.601 & $21252_{2}^{\circ}-37688_{3}$ \\
\hline 6121.757 & 2 & & 16330.659 & $130888_{3}-29419_{2}^{\circ}$ & 6082.561 & 10 & 1 & 16435.893 & $132974-297330$ \\
\hline 6120.801 & 50 & 4 & 16333.210 & $15863_{2}-32197^{\circ}$ & 6081.299 & 1 & & 16439.304 & $22401_{1}-38840 \mathrm{i}$ \\
\hline 6120.359 & 8 & 2 & 16334.389 & $17354^{\circ}-33689_{2}$ & 6080.900 & 5 & 1 & 16440.382 & $18549_{2}-34989_{1}^{\circ}$ \\
\hline 6119.649 & 25 & 2 & 16336.285 & $173983-33734_{2}^{\circ}$ & 6079.469 & 50 & 4 & 16444.252 & $14206_{4}^{\circ}-30651_{3}$ \\
\hline 6118.056 & 4 & 1 & 16340.538 & $17224_{2}^{\circ}-33564_{3}$ & 6079.220 & 200 & 25 & 16444.926 & $10526_{3}^{\circ}-26971_{4}$ \\
\hline 6116.172 & 100 & 8 & 16345.572 & $14206_{4}^{\circ}-305524$ & 6078.419 & 200 & 40 & 16447.093 & $132974-29744^{\circ}$ \\
\hline 6114.931 & 8 & 1 & 16348.889 & $19948{ }_{4}^{\circ}-36297_{4}$ & 6077.870 & 150 & 25 & 16448.578 & $14481 \%-309306$ \\
\hline 6114.546 & 150 & 10 & 16349.918 & $128473-29197_{2}^{\circ}$ & 6077.533 & 4 & 1 & 16449.490 & $16346_{4}^{\circ}-327963$ \\
\hline 6113.314 & 3 & & 16353.213 & $170731-33427_{2}^{\circ}$ & 6077.533 & 4 & 1 & 16449.490 & $180110^{\circ}-344605$ \\
\hline 6111.700 & 2 & & 16357.532 & $20737_{1}^{\circ}-37094_{2}$ & 6077.103 & 100 & 15 & 16450.654 & $8111_{4}-24561^{\circ}$ \\
\hline 6110.677 & 3 & & 16360.270 & $215943-37954^{\circ}$ & 6076.984 & 4 & 1 & 16450.977 & $17847_{2}^{\circ}-34298_{1}$ \\
\hline 6110.476 & 2 & & 16360.808 & $138472-30208_{2}^{\circ}$ & 6074.001 & 5 & & 16459.056 & $203220^{\circ}-367815$ \\
\hline 6110.476 & 2 & & 16360.808 & $19516_{2}^{\circ}-35877_{2}$ & 6073.561 & 3 & & 16460.248 & $154934-31953^{\circ}$ \\
\hline 6110.331 & 51 & 1 & 16361.197 & $22399^{\circ}-38760_{4}$ & 6072.894 & 5 & 1 & 16462.056 & $128473-29310^{\circ}$ \\
\hline 6108.293 & 100 & 5 & 16366.655 & $17354_{1}^{\circ}-33721_{2}$ & 6072.799 & 20 & 2 & 16462.313 & $211435-37605^{\circ}$ \\
\hline 6106.919 & 5 & 1 & 16370.338 & $15166_{3}^{\circ}-31537_{3}$ & 6072.061 & 3 & 1 & 16464.314 & $21902{ }^{\circ}-383664$ \\
\hline 6106.847 & 10 & 2 & 16370.531 & $112413^{\circ}-276123$ & 6071.981 & 15 & 2 & 16464.531 & $20867^{\circ}-373326$ \\
\hline 6105.304 & 4 & 1 & 16374.668 & $195033^{\circ}-35877_{2}$ & 6071.904 & 3 & & 16464.740 & $17224_{2}^{\circ}-33689_{2}$ \\
\hline 6104.970 & 2 & & 16375.564 & $18069_{3}^{\circ}-34444_{2}$ & 6071.802 & 1 & & 16465.017 & $197133-36178$ \\
\hline
\end{tabular}


TABle 4. Classified lines of Th I-continued

\begin{tabular}{|c|c|c|c|c|c|c|c|c|c|}
\hline \multirow{2}{*}{$\begin{array}{c}\text { Wavelength } \\
\text { (A) }\end{array}$} & \multicolumn{2}{|c|}{ Intensity } & \multirow{2}{*}{$\begin{array}{l}\text { Wavenumber } \\
\qquad\left(\mathrm{cm}^{-1}\right)\end{array}$} & \multirow{2}{*}{ Classification } & \multirow{2}{*}{$\begin{array}{c}\text { Wavelength } \\
\text { (̊) }\end{array}$} & \multicolumn{2}{|c|}{ Inten sity } & \multirow{2}{*}{$\begin{array}{c}\text { Wavenumber } \\
\left(\mathrm{cm}^{-1}\right)\end{array}$} & \multirow{2}{*}{ Classification } \\
\hline & Lamp & Spark & & & & Lamp & Spark & & \\
\hline 6070.458 & 1 & & 16468.662 & $22338^{\circ}-388072$ & 6024.835 & 2 & & 16593.370 & $20214_{3}^{\circ}-36808_{3}$ \\
\hline 6070.339 & 25 & 2 & 16468.985 & $15970_{3}-32439_{4}^{\circ}$ & 6024.082 & 1 & & 16595.444 & $18809_{4}^{\circ}-35405_{3}$ \\
\hline 6069.844 & 20 & 2 & 16470.328 & $17959_{4}-34430_{3}^{\circ}$ & 6023.332 & $3 b$ & & 16597.510 & $17847_{2}^{\circ}-34444_{2}$ \\
\hline 6069.019 & 100 & 5 & 16472.567 & $7280_{2}-23752_{2}^{\circ}$ & 6023.229 & 100 & 8 & 16597.794 & $13088_{3}-29686_{3}^{\circ}$ \\
\hline 6068.136 & 1 & & 16474.964 & $18930_{3}^{\circ}-35405_{3}$ & 6022.702 & 5 & 1 & 16599.246 & $13945_{3}^{\circ}-305442$ \\
\hline 6064.289 & 1 & & 16485.415 & $22248 \stackrel{\circ}{-}-38734_{3}$ & 6022.387 & 5 & 1 & 16600.114 & $195324-36132 \circ$ \\
\hline 6062.846 & 8 & & 16489.338 & $14481 \stackrel{\circ}{-}-309715$ & 6021.036 & $200 \mathrm{~s}$ & 75 & 16603.839 & $7795_{4}^{\circ}-24399_{3}$ \\
\hline 6062.225 & 1 & & 16491.028 & $18809_{4}^{\circ}-353005$ & 6020.502 & 25 & 2 & 16605.312 & $15970_{3}-32575_{2}^{\circ}$ \\
\hline 6061.535 & 75 & 3 & 16492.905 & $6362_{2}-22855_{3}^{\circ}$ & 6019.821 & 5 & 1 & 16607.190 & $13945_{3}^{\circ}-30552_{4}$ \\
\hline 6059.136 & 10 & 1 & 16499.435 & $14465_{2}^{\circ}-309643$ & 6019.642 & 25 & 3 & 16607.684 & $15166_{3}^{\circ}-317743$ \\
\hline 6058.438 & 4 & 1 & 16501.336 & $19588{ }^{\circ}-36089_{4}$ & 6019.411 & 1 & & 16608.321 & $20522_{2}^{\circ}-371313$ \\
\hline 6056.881 & 5 & & 16505.578 & $15490 \circ-319954$ & 6016.362 & 50 & 4 & 16616.738 & $16346_{4}^{\circ}-329635$ \\
\hline 6055.592 & 50 & 10 & 16509.091 & $13175_{4}^{\circ}-296845$ & 6014.307 & 50 & 2 & 16622.416 & $195888^{\circ}-362105$ \\
\hline 6055.036 & 8 & 1 & 16510.607 & $18930_{3}^{\circ}-35440_{3}$ & 6014.062 & 75 & 5 & 16623.093 & $17959_{4}-345833^{\circ}$ \\
\hline 6054.350 & 1 & & 16512.478 & $14032_{2}^{\circ}-30544_{2}$ & 6013.442 & 8 & 2 & 16624.807 & $16783 \stackrel{\circ}{4}-334083$ \\
\hline 6053.790 & 3 & & 16514.005 & $14243^{\circ}-307582$ & 6012.410 & 3 & 1 & 16627.661 & $21902_{4}^{\circ}-38529_{3}$ \\
\hline 6053.381 & 300 & 15 & 16515.121 & $6362_{2}-22877_{1}^{\circ}$ & 6011.537 & 50 & 5 & 16630.075 & $10414_{4}^{\circ}-270443$ \\
\hline 6052.444 & 8 & 1 & 16517.678 & $10526_{3}^{\circ}-270443$ & 6011.203 & 15 & 3 & 16630.999 & $18809_{4}^{\circ}-354403$ \\
\hline 6050.981 & 100 & 4 & 16521.671 & $8800_{4}-25321_{3}^{\circ}$ & 6010.253 & $50 \mathrm{~b}$ & 8 & 16633.628 & $17166_{5}-33799_{4}^{\circ}$ \\
\hline 6050.545 & 1 & & 16522.862 & $15490^{\circ}-320124$ & 6010.163 & 100 & 10 & 16633.877 & $2869_{3}-19503_{3}^{\circ}$ \\
\hline 6050.457 & 3 & & 16523.102 & $21165_{3}^{\circ}-37688_{3}$ & 6010.028 & 40 & 3 & 16634.251 & $16783_{4}^{\circ}-334184$ \\
\hline 6049.051 & 800 & 100 & 16526.942 & $3687_{2}-20214_{3}^{\circ}$ & 6008.052 & 5 & 1 & 16639.721 & $15863_{2}-32503 \stackrel{2}{2}$ \\
\hline 6047.794 & $10 \mathrm{~s}$ & 1 & 16530.377 & $154934-320230^{\circ}$ & 6007.261 & 10 & 1 & 16641.912 & $21575_{2}-38216_{3}^{\circ}$ \\
\hline 6047.273 & 3 & & 16531.802 & $21252_{2}^{\circ}-37784_{2}$ & 6007.073 & $300 \mathrm{~s}$ & 75 & 16642.433 & $8800_{4}-25442 \stackrel{\circ}{3}$ \\
\hline 6047.159 & 1 & & 16532.113 & $17959_{4}-34492_{4}^{\circ}$ & 6006.294 & 4 & & 16644.592 & $17073_{1}-33718_{2}^{\circ}$ \\
\hline 6046.643 & 10 & 2 & 16533.524 & $159700_{3}-32503_{2}^{\circ}$ & 6005.921 & 1 & & 16645.625 & $19532_{4}-36178_{4}^{\circ}$ \\
\hline 6045.246 & 1 & & 16537.345 & $19039_{2}^{\circ}-35576_{3}$ & 6005.600 & 10 & 1 & 16646.515 & $21539_{4}^{\circ}-381864$ \\
\hline 6044.554 & 8 & & 16539.238 & $19273_{2}-35812^{\circ}$ & 6005.174 & 200 & 15 & 16647.696 & $2869_{3}-19516_{2}^{\circ}$ \\
\hline 6043.676 & $2 \mathrm{~h}$ & & 16541.641 & $195324-36074 \stackrel{\circ}{3}$ & 6003.946 & 5 & & 16651.101 & $139621-306130^{\circ}$ \\
\hline 6042.750 & 2 & & 16544.175 & $19503_{3}^{\circ}-360472$ & 6002.198 & 10 & 1 & 16655.950 & $13088_{3}-29744^{\circ}$ \\
\hline 6042.593 & 75 & 5 & 16544.605 & $11802_{2}-28347_{2}^{\circ}$ & 6001.734 & 10 & 2 & 16657.238 & $3865_{1}-20522_{2}^{\circ}$ \\
\hline 6042.401 & 5 & 1 & 16545.131 & $195888^{\circ}-361335$ & 6001.207 & 300 & 15 & 16658.701 & $8111_{4}-24769_{3}^{\circ}$ \\
\hline 6040.873 & 5 & & 16549.316 & $17224_{2}^{\circ}-33773_{1}$ & 6000.766 & 50 & 8 & 16659.925 & $13175_{4}^{\circ}-29835_{3}$ \\
\hline 6039.228 & 15 & 2 & 16553.824 & $22399{ }^{\circ}-389535$ & 6000.554 & 5 & 1 & 16660.513 & $21645_{4}-38306_{4}^{\circ}$ \\
\hline 6038.681 & 75 & 5 & 16555.323 & $8800_{4}-25355_{4}^{\circ}$ & 6000.418 & 8 & 1 & 16660.891 & $17073_{1}-33734_{2}^{\circ}$ \\
\hline 6037.942 & $3 g$ & & 16557.349 & $10414_{4}^{\circ}-26971_{4}$ & 5999.594 & 5 & 1 & 16663.179 & $18382{ }^{\circ}-35046_{1}$ \\
\hline 6037.697 & 400 & 150 & 16558.021 & $38651-20423 i$ & 5997.788 & 5 & & 16668.197 & $19817_{1}^{\circ}-36485_{2}$ \\
\hline 6036.765 & 50 & 2 & 16560.578 & $10783_{2}^{\circ}-273433$ & 5997.010 & 8 & 1 & 16670.359 & $20214_{3}^{\circ}-36885_{4}$ \\
\hline 6035.365 & 1 & & 16564.419 & $20566^{\circ}-371313$ & 5996.630 & 50 & 4 & 16671.415 & $82432_{2}^{\circ}-249153$ \\
\hline 6035.194 & 75 & 5 & 16564.889 & $12114_{2}^{\circ}-28679_{2}$ & 5995.682 & 10 & & 16674.051 & $13175_{4}^{\circ}-29849_{4}$ \\
\hline 6034.334 & 3 & & 16567.249 & $21539_{4}^{\circ}-381065$ & 5995.221 & 50 & 4 & 16675.333 & $16783_{4}^{\circ}-33459_{4}$ \\
\hline 6034.215 & 5 & & 16567.576 & $19948_{4}^{\circ}-36515_{3}$ & 5994.126 & $500 \mathrm{r}$ & 100 & 16678.380 & 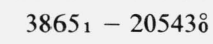 \\
\hline 6033.515 & 15 & 2 & 16569.498 & $173983-33967_{3}^{\circ}$ & 5993.960 & 50 & 8 & 16678.842 & $171665-33844 \circ$ \\
\hline 6033.416 & 50 & 5 & 16569.770 & $11802_{2}-28372_{1}^{\circ}$ & 5993.494 & 40 & 1 & 16680.138 & $75023-241822$ \\
\hline 6032.876 & 75 & 8 & 16571.253 & $128473-29419_{2}^{\circ}$ & 5991.457 & 15 & & 16685.809 & $5563_{1}-22248_{2}^{\circ}$ \\
\hline 6032.555 & 2 & & 16572.135 & $205222-37094_{2}$ & 5991.004 & $400 \mathrm{~s}$ & 50 & 16687.071 & $6362_{2}-23049^{\circ}$ \\
\hline 6032.370 & $50 \mathrm{~b}$ & 8 & 16572.643 & $19227_{6}^{\circ}-35799_{6}$ & 5989.445 & 20 & 2 & 16691.414 & $18549_{2}-35240_{3}^{\circ}$ \\
\hline 6030.448 & $75 \mathrm{~s}$ & 5 & 16577.925 & $4961_{4}-21539_{4}^{\circ}$ & 5986.268 & 50 & 3 & 16700.273 & $75022_{3}-24202^{\circ}$ \\
\hline 6030.189 & 5 & 1 & 16578.637 & $16217_{2}^{\circ}-32796_{3}$ & 5985.679 & 20 & 1 & 16701.916 & $142260-30928^{\circ}$ \\
\hline 6029.651 & 50 & 2 & 16580.116 & $98045-26384_{4}^{\circ}$ & 5984.998 & 15 & 3 & 16703.816 & $21575_{2}-38278^{\circ}$ \\
\hline 6029.228 & 50 & 4 & 16581.279 & $13175_{4}^{\circ}-297564$ & 5984.390 & 31 & & 16705.513 & $13847_{2}-30553_{2}^{\circ}$ \\
\hline 6028.631 & 3 & 1 & 16582.922 & $21165^{\circ}-37748_{2}$ & 5984.278 & 2 & & 16705.826 & $13945_{3}^{\circ}-30651_{3}$ \\
\hline 6026.047 & 50 & 2 & 16590.032 & 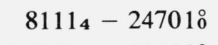 & 5983.163 & 5 & & 16708.939 & $19588^{\circ}-36297_{4}$ \\
\hline 6025.784 & $75 b$ & 5 & 16590.756 & $13962_{1}-30553_{2}^{\circ}$ & 5982.651 & 10 & & 16710.369 & $11802_{2}-285132$ \\
\hline 6025.049 & 1 & & 16592.780 & $22141_{3}^{\circ}-387343$ & 5982.229 & 5 & & 16711.548 & $15863_{2}-32575_{2}^{\circ}$ \\
\hline
\end{tabular}


TABLE 4. Classified lines of Th $\mathrm{I}$-continued

\begin{tabular}{|c|c|c|c|c|c|c|c|c|c|}
\hline \multirow{2}{*}{ 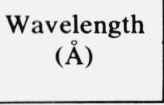 } & \multicolumn{2}{|c|}{ Intensity } & \multirow{2}{*}{$\begin{array}{l}\text { Wavenumber } \\
\qquad\left(\mathrm{cm}^{-1}\right)\end{array}$} & \multirow{2}{*}{ Classification } & \multirow{2}{*}{$\begin{array}{c}\text { Wave length } \\
\text { (§) }\end{array}$} & \multicolumn{2}{|c|}{ Inten sity } & \multirow{2}{*}{$\begin{array}{l}\text { Wavenumber } \\
\qquad\left(\mathrm{cm}^{-1}\right)\end{array}$} & \multirow{2}{*}{ Classification } \\
\hline & Lamp & Spark & & & & Lamp & Spark & & \\
\hline 5982.171 & 5 & & 16711.710 & $215943-38306_{4}^{\circ}$ & 5946.027 & 8 & & 16813.294 & $21077^{\circ}-37890_{5}$ \\
\hline 5982.095 & 50 & 5 & 16711.922 & $15490 \circ-322025$ & 5945.480 & 10 & 1 & 16814.841 & $142045-31019_{4}^{\circ}$ \\
\hline 5981.782 & 1 & & 16712.797 & $218903-38602_{2}$ & 5945.334 & 4 & & 16815.254 & $22141_{3}^{\circ}-389564$ \\
\hline 5981.233 & 2 & & 16714.331 & $197133-36427_{3}^{\circ}$ & 5945.191 & 8 & 1 & 16815.658 & $21077^{\circ}-378924$ \\
\hline 5979.325 & 4 & & 16719.664 & $13297_{4}-30017_{3}^{\circ}$ & 5944.806 & 15 & 1 & 16816.747 & $228770^{\circ}-396945$ \\
\hline 5977.087 & 10 & 2 & 16725.925 & $14032_{2}^{\circ}-30758_{2}$ & 5944.648 & 150 & 40 & 16817.194 & $10526_{3}^{\circ}-27343_{3}$ \\
\hline 5975.066 & 500 & 150 & 16731.582 & $6362_{2}-230932$ & 5943.934 & 1 & & 16819.214 & $19516_{2}^{\circ}-36336_{3}$ \\
\hline 5973.665 & $500 \mathrm{r}$ & 150 & 16735.506 & $3687_{2}-20423 i$ & 5943.496 & 25 & 3 & 16820.454 & $18053_{4}^{\circ}-348743$ \\
\hline 5973.203 & 10 & 1 & 16736.800 & $20566^{\circ}-37303_{4}$ & 5943.470 & 50 & 4 & 16820.528 & $12114_{2}^{\circ}-28934_{3}$ \\
\hline 5972.300 & 3 & & 16739.331 & $216454-383850^{\circ}$ & 5942.259 & 8 & 1 & 16823.956 & $18809_{4}^{\circ}-35633_{4}$ \\
\hline 5971.881 & 3 & & 16740.505 & $20566^{\circ}-37307_{3}$ & 5941.568 & 2 & & 16825.912 & $20922_{2}^{\circ}-37748_{2}$ \\
\hline 5971.369 & 3 & & 16741.941 & $21077 \circ-37819_{4}$ & 5941.188 & 3 & & 16826.988 & $19948_{4}^{\circ}-36775_{3}$ \\
\hline 5971.073 & 4 & & 16742.771 & $195324-36275 \circ$ & 5941.188 & 3 & & 16826.988 & $21539_{4}^{\circ}-38366_{4}$ \\
\hline 5970.456 & 50 & 2 & 16744.501 & $17959_{4}-34704^{\circ}$ & 5941.126 & 5 & 1 & 16827.164 & $21165_{3}^{\circ}-379924$ \\
\hline 5969.742 & 150 & 10 & 16746.504 & $11601_{1}-28347_{2}^{\circ}$ & 5940.567 & 50 & 3 & 16828.747 & $15166^{\circ}-31995_{4}$ \\
\hline 5967.515 & 50 & 2 & 16752.753 & $16346_{4}^{\circ}-33099_{3}$ & 5939.374 & $5 b$ & & 16832.127 & $21902_{4}^{\circ}-387343$ \\
\hline 5966.273 & 5 & & 16756.240 & $17411^{\circ}-34167_{3}$ & 5939.044 & 15 & & 16833.063 & $19503 \stackrel{\circ}{3}-363363$ \\
\hline 5965.832 & 40 & 1 & 16757.479 & $75023-24259_{4}^{\circ}$ & 5938.825 & 400 & 75 & 16833.683 & $5563_{1}-22396_{1}^{\circ}$ \\
\hline 5965.745 & 15 & 1 & 16757.723 & $14206^{\circ}-309643$ & 5938.459 & 150 & 20 & 16834.721 & $3687_{2}-20522_{2}^{\circ}$ \\
\hline 5964.714 & $4 \mathrm{~h}$ & & 16760.620 & $215943-38355_{2}^{\circ}$ & 5938.285 & 15 & 1 & 16835.214 & $171665-34001_{4}^{\circ}$ \\
\hline 5964.481 & 40 & 2 & 16761.275 & $139621-30723 i$ & 5938.041 & 1 & 3 & 16835.906 & $22338^{\circ}-391743$ \\
\hline 5964.012 & 15 & 1 & 16762.593 & $15166_{3}^{\circ}-31929_{3}$ & 5937.925 & 50 & 3 & 16836.235 & $13175_{4}^{\circ}-30011_{3}$ \\
\hline 5963.930 & 15 & 1 & 16762.823 & $19986^{\circ}-367496$ & 5937.665 & 100 & 2 & 16836.972 & $11197 \stackrel{\circ}{-}-280345$ \\
\hline 5963.661 & 10 & 1 & 16763.579 & $20322{ }^{\circ}-37085_{4}$ & 5937.297 & 25 & 2 & 16838.016 & $20566_{4}^{\circ}-37404_{3}$ \\
\hline 5963.308 & 10 & 1 & 16764.572 & $13088_{3}-29853_{2}^{\circ}$ & 5937.166 & 100 & 10 & 16838.387 & $128473-29686_{3}^{\circ}$ \\
\hline 5962.975 & 3 & & 16765.508 & $17959_{4}-34725_{3}^{\circ}$ & 5936.876 & 20 & 2 & 16839.210 & $13175_{4}^{\circ}-30014_{4}$ \\
\hline 5962.773 & 4 & & 16766.076 & $20922_{2}^{\circ}-37688_{3}$ & 5936.876 & 20 & 2 & 16839.210 & $15618^{\circ}-324584$ \\
\hline 5962.579 & 5 & & 16766.621 & $18809_{4}^{\circ}-35576_{3}$ & 5936.387 & 50 & 5 & 16840.597 & $98045-26645 \circ$ \\
\hline 5962.060 & 40 & 3 & 16768.081 & $12114_{2}^{\circ}-288822$ & 5934.458 & $50 \mathrm{~b}$ & 3 & 16846.071 & $15166_{3}^{\circ}-32012_{4}$ \\
\hline 5961.249 & 4 & & 16770.362 & $216454-38416_{3}^{\circ}$ & 5934.412 & $75 b$ & 15 & 16846.201 & $118022_{2}-28649_{i}^{\circ}$ \\
\hline 5960.785 & 50 & & 16771.668 & $11601_{1}-28372{ }_{1}^{\circ}$ & 5934.103 & 1 & & 16847.078 & $22163_{4}^{\circ}-39010_{3}$ \\
\hline 5960.529 & 5 & & 16772.388 & $18809_{4}^{\circ}-35582_{5}$ & 5932.911 & 15 & 1 & 16850.463 & $13962_{1}-30812_{2}^{\circ}$ \\
\hline 5959.681 & 50 & 3 & 16774.774 & $8800_{4}-25575_{4}^{\circ}$ & 5932.426 & 50 & 2 & 16851.841 & $14243^{\circ}-310951$ \\
\hline 5958.811 & 15 & 1 & 16777.223 & $10414_{4}^{\circ}-271915$ & 5932.056 & 4 & & 16852.892 & $22399^{\circ}-392525$ \\
\hline 5957.592 & 50 & 2 & 16780.656 & $14465_{2}^{\circ}-31245_{2}$ & 5930.311 & 10 & 1 & 16857.851 & $14032_{2}^{\circ}-30889_{1}$ \\
\hline 5957.472 & 50 & 2 & 16780.994 & $16783^{\circ}-335643$ & 5929.934 & 100 & 20 & 16858.923 & $3865_{1}-20724 \stackrel{\circ}{0}$ \\
\hline 5956.514 & 5 & & 16783.693 & $10783_{2}^{\circ}-27566_{2}$ & 5929.586 & 10 & 2 & 16859.912 & $19948_{4}^{\circ}-36808_{3}$ \\
\hline 5956.263 & 100 & 8 & 16784.400 & $159703-32754_{3}^{\circ}$ & 5929.328 & 20 & 2 & 16860.646 & $17847_{2}^{\circ}-34707_{3}$ \\
\hline 5955.566 & 100 & 5 & 16786.365 & $11802_{2}-28589_{3}^{\circ}$ & 5928.861 & 20 & 2 & 16861.974 & $18011^{\circ}-34873_{4}$ \\
\hline 5954.588 & 40 & 2 & 16789.122 & $144811^{\circ}-312715$ & 5928.755 & 3 & & 16862.275 & $20922_{2}^{\circ}-37784_{2}$ \\
\hline 5953.587 & 100 & 4 & 16791.945 & $15493_{4}-32285_{3}^{\circ}$ & 5928.654 & 15 & 2 & 16862.563 & $13297_{4}-30160_{4}^{\circ}$ \\
\hline 5953.255 & 50 & 2 & 16792.881 & $130883-29881_{4}^{\circ}$ & 5928.123 & 4 & 1 & 16864.073 & $18930_{3}^{\circ}-35794_{4}$ \\
\hline 5952.807 & 2 & & 16794.145 & $19503 \stackrel{\circ}{3}-36297_{4}$ & 5926.545 & 4 & & 16868.563 & $22141_{3}^{\circ}-39010_{3}$ \\
\hline 5952.456 & 2 & & 16795.135 & $199866-367815$ & 5926.232 & 150 & 40 & 16869.454 & $7795_{4}^{\circ}-24664_{3}$ \\
\hline 5950.641 & 25 & 2 & 16800.258 & $17501^{\circ}-34301_{4}$ & 5926.001 & 50 & 4 & 16870.112 & $8111_{4}-24981_{3}^{\circ}$ \\
\hline 5950.429 & 50 & 2 & 16800.856 & $154934-32294_{4}^{\circ}$ & 5925.719 & 15 & 1 & 16870.914 & $20214_{3}^{\circ}-37085_{4}$ \\
\hline 5950.148 & 15 & 2 & 16801.650 & $15863_{2}-32665_{1}^{\circ}$ & 5925.404 & 100 & 8 & 16871.811 & $3865_{1}-20737_{1}^{\circ}$ \\
\hline 5949.373 & 50 & 5 & 16803.838 & $15490 \AA-322935$ & 5924.519 & 15 & 2 & 16874.332 & $15166_{3}^{\circ}-32041_{2}$ \\
\hline 5949.218 & 15 & 1 & 16804.276 & $18069_{3}^{\circ}-34873_{4}$ & 5924.519 & 15 & 2 & 16874.332 & $20867^{\circ}-377426$ \\
\hline 5948.965 & 5 & & 16804.991 & $18069^{\circ}-348743$ & 5923.919 & 20 & 1 & 16876.041 & $13847_{2}-30723 i$ \\
\hline 5948.803 & 100 & 8 & 16805.448 & $7502_{3}-24307_{2}^{\circ}$ & 5922.860 & 50 & 2 & 16879.058 & $75023-24381_{2}^{\circ}$ \\
\hline 5948.393 & 15 & 1 & 16806.607 & $211435-37950_{4}^{\circ}$ & 5921.102 & 4 & & 16884.069 & $21645_{4}^{\circ}-38529_{3}$ \\
\hline 5947.502 & 4 & & 16809.124 & $18431_{3}-35240 \stackrel{\circ}{\circ}$ & 5920.977 & 15 & 2 & 16884.426 & $22399{ }^{\circ}-39283_{4}$ \\
\hline 5947.243 & 1 & & 16809.857 & $16351 o-33161^{\circ}$ & 5919.224 & 15 & 2 & 16889.426 & $20322{ }^{\circ}-372115$ \\
\hline 5946.239 & 40 & 2 & 16812.695 & $13945_{3}^{\circ}-30758_{2}$ & 5918.946 & 100 & 8 & 16890.220 & $8800_{4}-25690 \circ$ \\
\hline
\end{tabular}


TABLE 4. Classified lines of Th I-continued

\begin{tabular}{|c|c|c|c|c|c|c|c|c|c|}
\hline \multirow{2}{*}{ 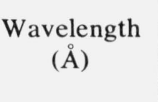 } & \multicolumn{2}{|c|}{ Intensity } & \multirow{2}{*}{$\begin{array}{l}\text { Wavenumber } \\
\left(\mathrm{cm}^{-1}\right)\end{array}$} & \multirow{2}{*}{ Classification } & \multirow{2}{*}{$\begin{array}{c}\text { Wavelength } \\
(\AA)\end{array}$} & \multicolumn{2}{|c|}{ Inten sity } & \multirow{2}{*}{$\begin{array}{l}\text { Wavenumber } \\
\left(\mathrm{cm}^{-1}\right)\end{array}$} & \multirow{2}{*}{ Classification } \\
\hline & Lamp & Spark & & & & Lamp & Spark & & \\
\hline 5918.946 & 100 & 8 & 16890.220 & $17411_{3}^{\circ}-34301_{4}$ & 5874.993 & 150 & 8 & 17016.580 & $17166_{5}-34182{ }^{\circ}$ \\
\hline 5918.189 & 15 & 1 & 16892.380 & $15970_{3}-32862_{4}^{\circ}$ & 5874.040 & 50 & 3 & 17019.341 & $13945_{3}^{\circ}-309643$ \\
\hline 5917.285 & 10 & 1 & 16894.961 & $19532_{4}-36427_{3}^{\circ}$ & 5873.920 & 4 & 1 & 17019.689 & $21645_{4}-386650^{\circ}$ \\
\hline 5916.730 & 100 & 20 & 16896.545 & $12847_{3}-29744_{3}^{\circ}$ & 5873.774 & 8 & 1 & 17020.112 & $17411_{3}^{\circ}-34431_{3}$ \\
\hline 5915.168 & 3 & & 16901.007 & $18930_{3}^{\circ}-35831_{3}$ & 5873.462 & 8 & & 17021.016 & $21165_{3}^{\circ}-381864$ \\
\hline 5914.716 & $50 \mathrm{~b}$ & 7 & 16902.299 & $7280_{2}-24182_{2}^{\circ}$ & 5873.332 & 10 & & 17021.393 & $18809_{4}^{\circ}-35831_{3}$ \\
\hline 5913.362 & 50 & 5 & 16906.169 & $19227^{\circ}-361335$ & 5871.405 & 15 & & 17026.979 & $17847_{2}^{\circ}-348743$ \\
\hline 5913.286 & 8 & 2 & 16906.386 & $17501{ }^{\circ}-344076$ & 5871.186 & 100 & 20 & 17027.614 & $7280_{2}-24307_{2}^{\circ}$ \\
\hline 5911.229 & 50 & 8 & 16912.269 & $11601_{1}-28513_{2}^{\circ}$ & 5870.054 & 20 & 1 & 17030.898 & $11197_{0}^{\circ}-28227_{4}$ \\
\hline 5911.097 & 8 & 1 & 16912.647 & $18549_{2}-35462_{3}^{\circ}$ & 5869.854 & 100 & 5 & 17031.478 & $11241_{3}^{\circ}-28273_{2}$ \\
\hline 5910.640 & 4 & 1 & 16913.954 & $13847_{2}-30761_{3}^{\circ}$ & 5869.200 & $2 b$ & & 17033.376 & $17411_{3}^{\circ}-34444_{2}$ \\
\hline 5910.237 & 15 & 2 & 16915.108 & $21077_{0}^{\circ}-379924$ & 5869.164 & 8 & & 17033.480 & $12847_{3}-29881_{4}^{\circ}$ \\
\hline 5909.887 & 1 & & 16916.109 & $20214_{3}^{\circ}-37131_{3}$ & 5868.382 & 150 & 25 & 17035.750 & $18053_{4}^{\circ}-35089_{3}$ \\
\hline 5908.957 & 200 & & 16918.772 & $75023-24421_{3}^{\circ}$ & 5867.841 & 20 & 1 & 17037.321 & $17073_{1}-34111_{i}^{\circ}$ \\
\hline 5906.550 & 3 & & 16925.666 & $17398_{3}-34324_{2}^{\circ}$ & 5867.542 & 1 & & 17038.189 & $18382^{\circ}-35421_{1}$ \\
\hline 5906.164 & 3 & & 16926.773 & $17959_{4}-34886^{\circ}$ & 5866.814 & 50 & 5 & 17040.303 & $10526_{3}^{\circ}-27566_{2}$ \\
\hline 5905.571 & 150 & 20 & 16928.472 & $4961_{4}-21890_{3}^{\circ}$ & 5864.128 & 4 & & 17048.108 & $11601_{1}-28649_{1}^{\circ}$ \\
\hline 5905.176 & 50 & 2 & 16929.605 & $10414_{4}^{\circ}-273433$ & 5863.720 & 100 & 8 & 17049.294 & $3687_{2}-20737_{1}^{\circ}$ \\
\hline 5903.646 & 2 & & 16933.992 & $21668_{1}^{\circ}-386022$ & 5863.562 & 10 & & 17049.754 & $19713_{3}-36762^{\circ}$ \\
\hline 5903.496 & 20 & 2 & 16934.422 & $21645_{4}-38580_{4}^{\circ}$ & 5863.146 & 3 & 1 & 17050.963 & $21902_{4}^{\circ}-389535$ \\
\hline 5903.337 & 15 & 1 & 16934.879 & $14206_{4}^{\circ}-31141_{3}$ & 5861.894 & 2 & & 17054.605 & $21902_{4}^{\circ}-389564$ \\
\hline 5902.602 & 50 & 2 & 16936.987 & $142045-31141^{\circ}$ & 5861.289 & 8 & & 17056.366 & $16217_{2}^{\circ}-33273_{1}$ \\
\hline 5900.448 & 2 & & 16943.170 & $17224_{2}^{\circ}-34167_{3}$ & 5861.192 & 15 & & 17056.648 & $3865_{1}-20922_{2}^{\circ}$ \\
\hline 5900.355 & 5 & & 16943.437 & $17354^{\circ}-34298_{1}$ & 5860.480 & 15 & 1 & 17058.720 & $14206_{4}^{\circ}-31265_{3}$ \\
\hline 5899.846 & 150 & 5 & 16944.899 & $5563_{1}-22508_{2}^{\circ}$ & 5860.259 & 15 & 1 & 17059.363 & $7502_{3}-24561_{3}^{\circ}$ \\
\hline 5899.521 & 50 & 4 & 16945.832 & $15493_{4}-32439_{4}^{\circ}$ & 5859.348 & 15 & 1 & 17062.016 & $16346_{4}^{\circ}-33408_{3}$ \\
\hline 5899.466 & $8 \mathrm{~b}$ & 1 & 16945.990 & $17501_{0}^{\circ}-34447_{4}$ & 5858.752 & 50 & 3 & 17063.751 & $14032_{2}^{\circ}-31095_{1}$ \\
\hline 5897.246 & 1 & & 16952.370 & $22399^{\circ}-393515$ & 5858.641 & 50 & 2 & 17064.075 & $14206_{4}^{\circ}-31271_{5}$ \\
\hline 5896.786 & 201 & 1 & 16953.692 & $8800_{4}-25753^{\circ}$ & 5857.725 & 2 & & 17066.743 & $21890_{3}^{\circ}-38956_{4}$ \\
\hline 5896.114 & 2 & & 16955.624 & $19713_{3}-36668_{2}^{\circ}$ & 5856.112 & 15 & 1 & 17071.444 & $13088_{3}-30160_{4}^{\circ}$ \\
\hline 5895.283 & 150 & 25 & 16958.014 & $13297_{4}-30255_{3}^{\circ}$ & 5855.914 & 5 & & 17072.021 & $14465_{2}^{\circ}-315373$ \\
\hline 5895.053 & 10 & 1 & 16958.676 & $18574_{1}-35533_{2}^{\circ}$ & 5855.789 & 1 & & 17072.385 & $11601_{1}-28673_{2}^{\circ}$ \\
\hline 5894.699 & 50 & 25 & 16959.694 & $17501_{0}^{\circ}-344605$ & 5854.858 & 3 & & 17075.100 & $21594_{3}-38669_{2}^{\circ}$ \\
\hline 5893.191 & 20 & 2 & 16964.034 & $14465_{2}^{\circ}-31429_{1}$ & 5854.120 & 150 & 8 & 17077.253 & $8800_{4}-25877_{4}^{\circ}$ \\
\hline 5892.780 & 8 & & 16965.217 & $138472-30812 \%$ & 5853.474 & 100 & 5 & 17079.137 & $2869_{3}-19948_{4}^{\circ}$ \\
\hline 5892.434 & 50 & 5 & 16966.213 & $139621-30928_{1}^{\circ}$ & 5852.945 & 10 & & 17080.681 & $18069_{3}^{\circ}-35149_{2}$ \\
\hline 5891.774 & 5 & 1 & 16968.114 & $15490_{0}^{\circ}-324584$ & 5852.844 & 15 & & 17080.976 & $13847_{2}-30928^{\circ}$ \\
\hline 5891.670 & 5 & & 16968.414 & $19516_{2}^{\circ}-36485_{2}$ & 5852.682 & 200 & 25 & 17081.449 & $10414_{4}^{\circ}-27495_{4}$ \\
\hline 5891.451 & 200 & 25 & 16969.044 & $10526^{\circ}-27495_{4}$ & 5852.487 & 100 & 8 & 17082.018 & $11802_{2}-28884_{3}^{\circ}$ \\
\hline 5887.919 & 8 & & 16979.223 & $18699_{2}-356781$ & 5851.214 & 8 & 2 & 17085.734 & $10526_{3}^{\circ}-276123$ \\
\hline 5887.437 & 2 & & 16980.613 & $21165_{3}^{\circ}-381453$ & 5850.566 & 5 & & 17087.627 & $19713_{3}-36800_{4}^{\circ}$ \\
\hline 5886.851 & 5 & & 16982.304 & $22855^{\circ}-39837_{3}$ & 5850.378 & 10 & 1 & 17088.176 & $19273_{2}-36361_{i}^{\circ}$ \\
\hline 5886.456 & 15 & & 16983.443 & $19227_{6}^{\circ}-36210_{5}$ & 5850.270 & 3 & & 17088.491 & $20214_{3}^{\circ}-37303_{4}$ \\
\hline 5886.093 & 15 & 1 & 16984.491 & $15970_{3}-32954_{2}^{\circ}$ & 5849.765 & 8 & 1 & 17089.966 & $17354_{1}^{\circ}-34444_{2}$ \\
\hline 5885.702 & 200 & 50 & 16985.619 & $9804_{5}-26790_{4}^{\circ}$ & 5849.514 & 15 & 1 & 17090.700 & $15863_{2}-32954_{2}^{\circ}$ \\
\hline 5885.502 & 75 & 2 & 16986.196 & $11241_{3}^{\circ}-28227_{4}$ & 5849.217 & 10 & & 17091.567 & $16217_{2}^{\circ}-33309_{2}$ \\
\hline 5884.033 & 50 & 2 & 16990.437 & $142045-31194_{4}^{\circ}$ & 5848.554 & 25 & 2 & 17093.505 & $195324-366250$ \\
\hline 5882.460 & 10 & 1 & 16994.980 & $10526_{3}^{\circ}-27521_{4}$ & 5848.277 & 1 & & 17094.315 & $20054_{2}-37149_{2}^{\circ}$ \\
\hline 5881.988 & 2 & & 16996.344 & $21738_{2}^{\circ}-387343$ & 5848.135 & 4 & & 17094.729 & $21575_{2}-38669_{2}^{\circ}$ \\
\hline 5881.065 & 4 & & 16999.011 & $19516_{2}^{\circ}-36515_{3}$ & 5845.919 & 100 & 5 & 17101.209 & $7280_{2}-24381_{2}^{\circ}$ \\
\hline 5879.127 & 50 & 2 & 17004.615 & $11877_{1}^{\circ}-28882_{2}$ & 5844.873 & 5 & & 17104.270 & $18574_{1}-35678_{1}^{\circ}$ \\
\hline 5878.933 & 100 & 3 & 17005.176 & $128473-298533_{2}^{\circ}$ & 5844.793 & 75 & 3 & 17104.504 & $20054_{2}-37159_{1}^{\circ}$ \\
\hline 5877.186 & 2 & & 17010.231 & $20322^{\circ}-373326$ & 5844.704 & 3 & & 17104.765 & $20054_{2}-37159_{3}^{\circ}$ \\
\hline 5877.013 & 25 & 2 & 17010.732 & $20737_{1}^{\circ}-37748_{2}$ & 5843.805 & 50 & 5 & 17107.396 & $10414_{4}^{\circ}-27521_{4}$ \\
\hline 5876.647 & 3 & & 17011.791 & $22163_{4}^{\circ}-391743$ & 5843.267 & 10 & 1 & 17108.971 & $21077_{0}^{\circ}-38186_{4}$ \\
\hline
\end{tabular}


Table 4. Classified lines of Th $\mathrm{I}$-continued

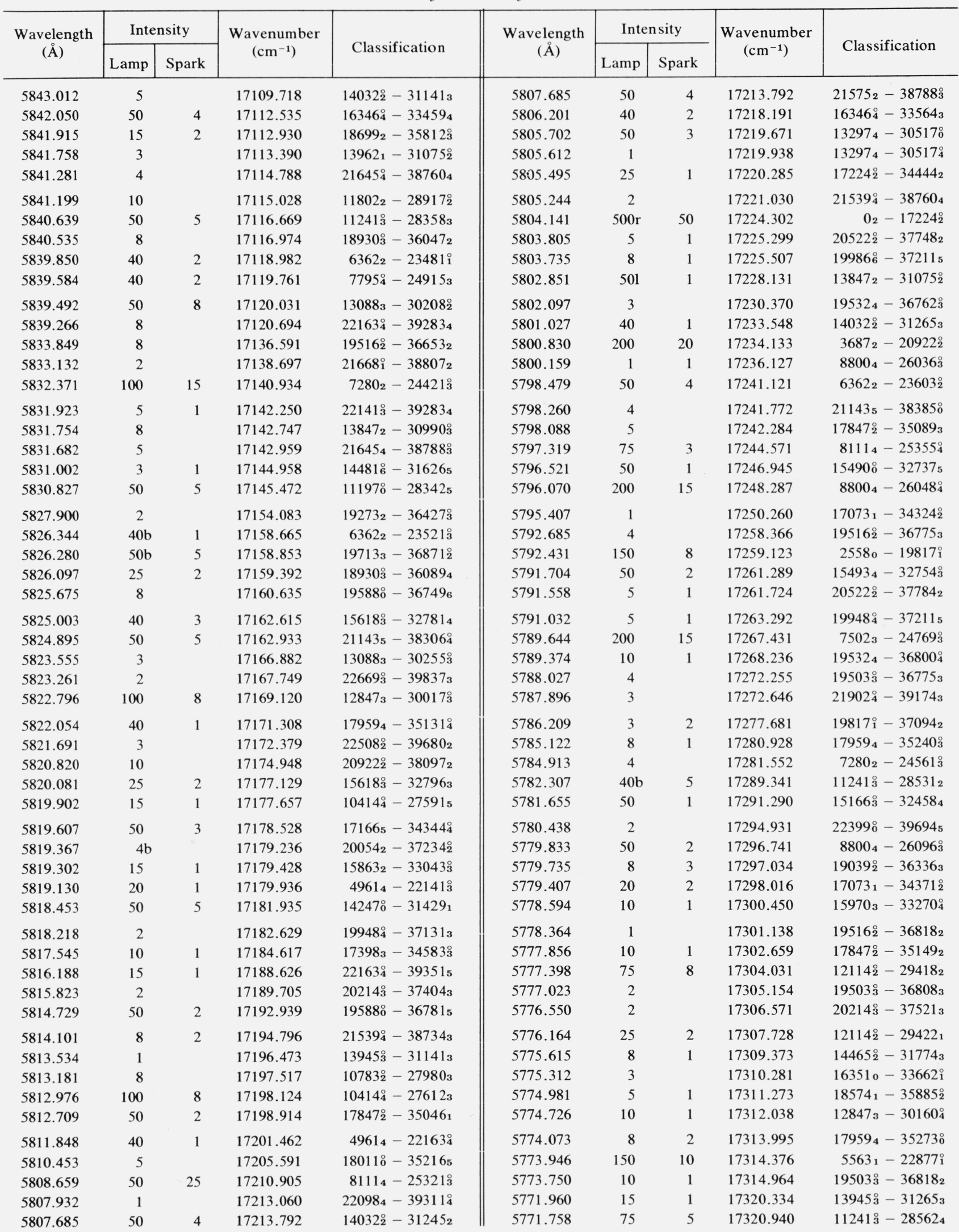


TABLE 4. Classified lines of Th $\mathrm{I}$-continued

\begin{tabular}{|c|c|c|c|c|c|c|c|c|c|}
\hline \multirow{2}{*}{$\begin{array}{l}\text { Wavelength } \\
\text { (§) }\end{array}$} & \multicolumn{2}{|c|}{ Intensity } & \multirow{2}{*}{$\begin{array}{l}\text { Wavenumber } \\
\left(\mathrm{cm}^{-1}\right)\end{array}$} & \multirow{2}{*}{ Classification } & \multirow{2}{*}{$\begin{array}{c}\text { Wavelength } \\
(\AA)\end{array}$} & \multicolumn{2}{|c|}{ Inten sity } & \multirow{2}{*}{$\begin{array}{l}\text { Wavenumber } \\
\left(\mathrm{cm}^{-1}\right)\end{array}$} & \multirow{2}{*}{ Classification } \\
\hline & Lamp & Spark & & & & Lamp & Spark & & \\
\hline 5770.865 & 10 & 2 & 17323.620 & $18809_{4}^{\circ}-361335$ & 5734.579 & 8 & & 17433.236 & $15863_{2}-33297_{2}^{\circ}$ \\
\hline 5770.795 & 5 & 1 & 17323.830 & $20566^{\circ}-378905$ & 5733.887 & 20 & 2 & 17435.340 & $13847_{2}-31283_{3}^{\circ}$ \\
\hline 5770.562 & 4 & 1 & 17324.530 & $20423_{1}^{\circ}-37748_{2}$ & 5733.651 & 4 & 1 & 17436.057 & $19713_{3}-37149_{2}^{\circ}$ \\
\hline 5770.461 & 10 & 1 & 17324.833 & $15970_{3}-33294_{3}^{\circ}$ & 5733.388 & 5 & 1 & 17436.857 & $21738_{2}^{\circ}-391743$ \\
\hline 5770.100 & $15 b$ & & 17325.917 & $17166_{5}-34492_{4}^{\circ}$ & 5733.096 & 20 & 2 & 17437.745 & $4961_{4}-22399^{\circ}$ \\
\hline 5769.730 & 8 & & 17327.028 & $15970_{3}-33297_{2}^{\circ}$ & 5730.307 & 2 & & 17446.232 & $19039_{2}^{\circ}-36485_{2}$ \\
\hline 5769.730 & 8 & & 17327.028 & $173983-34725_{3}^{\circ}$ & 5729.236 & 2 & & 17449.493 & $21902_{4}^{\circ}-39351_{5}$ \\
\hline 5768.633 & 10 & 1 & 17330.323 & $14206_{4}^{\circ}-31537_{3}$ & 5729.119 & 5 & & 17449.850 & $11802_{2}-29252_{2}^{\circ}$ \\
\hline 5768.181 & 200 & 15 & 17331.681 & $8111_{4}-25442_{3}^{\circ}$ & 5727.997 & 8 & & 17453.268 & $173983-34851_{2}^{\circ}$ \\
\hline 5767.779 & 150 & 10 & 17332.889 & $13175_{4}^{\circ}-305085$ & 5727.711 & 40 & 3 & 17454.139 & $10526_{3}^{\circ}-27980_{3}$ \\
\hline 5766.662 & 5 & & 17336.246 & $18069_{3}^{\circ}-35405_{3}$ & 5727.021 & 50 & 3 & 17456.242 & $19948 \stackrel{\circ}{4}-37404_{3}$ \\
\hline 5766.589 & 1 & & 17336.466 & $18549_{2}-35885_{2}^{\circ}$ & 5726.792 & 1 & & 17456.940 & $15970_{3}-33427_{2}^{\circ}$ \\
\hline 5763.529 & 200 & 10 & 17345.670 & $2869_{3}-20214_{3}^{\circ}$ & 5725.390 & 300 & 75 & 17461.215 & $9804_{5}-27266_{4}^{\circ}$ \\
\hline 5763.312 & 5 & 1 & 17346.323 & $199866^{\circ}-373326$ & 5724.863 & 2 & & 17462.822 & $17411_{3}^{\circ}-348743$ \\
\hline 5762.959 & 15 & 1 & 17347.386 & $16217_{2}^{\circ}-33564_{3}$ & 5724.463 & 75 & 4 & 17464.043 & $8111_{4}-25575_{4}^{\circ}$ \\
\hline 5762.796 & 100 & 10 & 17347.876 & $17501{ }^{\circ}-348495$ & 5724.386 & 20 & 2 & 17464.277 & $132974-30761_{3}^{\circ}$ \\
\hline 5762.078 & $3 b$ & & 17350.038 & $21902_{4}^{\circ}-392525$ & 5724.386 & 20 & 2 & 17464.277 & $14465_{2}^{\circ}-31929_{3}$ \\
\hline 5761.527 & 25 & 2 & 17351.697 & $18053^{\circ}-354053$ & 5724.240 & 100 & 10 & 17464.723 & $130883-305532_{2}^{\circ}$ \\
\hline 5760.550 & $600 \mathrm{r}$ & 150 & 17354.640 & $\mathbf{0}_{2}-17354^{\circ}$ & 5723.532 & 10 & 1 & 17466.883 & $142045-31671_{4}^{\circ}$ \\
\hline 5760.447 & $75 b$ & 15 & 17354.950 & $11802_{2}-29157_{3}^{\circ}$ & 5722.012 & 2 & & 17471.523 & $16217_{2}^{\circ}-33689_{2}$ \\
\hline 5759.190 & 5 & 1 & 17358.738 & $19948 \stackrel{\circ}{4}-37307_{3}$ & 5721.425 & 50 & 4 & 17473.316 & $15490 \AA-329635$ \\
\hline 5758.471 & 5 & & 17360.906 & $20423_{1}^{\circ}-37784_{2}$ & 5720.185 & $500 \mathrm{r}$ & 150 & 17477.103 & $3687_{2}-21165_{3}^{\circ}$ \\
\hline 5757.360 & 1 & & 17364.256 & $21645_{4}^{\circ}-39010_{3}$ & 5719.625 & 200 & 50 & 17478.815 & $7502_{3}-24981_{3}^{\circ}$ \\
\hline 5757.336 & 1 & & 17364.328 & $21645_{4}^{\circ}-39010_{3}$ & 5719.098 & 20 & 2 & 17480.425 & $15618_{3}^{\circ}-33099_{3}$ \\
\hline 5756.904 & 4 & 1 & 17365.631 & $11197^{\circ}-285624$ & 5718.657 & 2 & & 17481.773 & $21252_{2}^{\circ}-387343$ \\
\hline 5756.463 & 1 & & 17366.961 & $18930_{3}^{\circ}-36297_{4}$ & 5718.118 & 8 & 1 & 17483.421 & $17224_{2}^{\circ}-34707_{3}$ \\
\hline 5756.445 & 1 & & 17367.016 & $18930_{3}^{\circ}-36297_{4}$ & 5717.526 & 20 & 2 & 17485.231 & $16346_{4}^{\circ}-33831_{4}$ \\
\hline 5754.844 & 8 & 1 & 17371.847 & $18069_{3}^{\circ}-354403$ & 5717.172 & 50 & 15 & 17486.314 & $5563_{1}-23049_{1}^{\circ}$ \\
\hline 5754.734 & 15 & 3 & 17372.179 & $17501{ }^{\circ}-34873_{4}$ & 5716.815 & 10 & 1 & 17487.406 & $18809_{4}^{\circ}-36297_{4}$ \\
\hline 5753.984 & 3 & & 17374.444 & $18699_{2}-36074_{3}^{\circ}$ & 5716.106 & 1 & & 17489.575 & $7280_{2}-24769_{3}^{\circ}$ \\
\hline 5753.026 & 200 & 25 & 17377.337 & $4961_{4}-22338_{3}^{\circ}$ & 5715.948 & 8 & 1 & 17490.058 & $10783_{2}^{\circ}-28273_{2}$ \\
\hline 5752.580 & 15 & 1 & 17378.684 & $63622-23741_{i}^{\circ}$ & 5715.725 & 20 & 2 & 17490.741 & $132974-30788^{\circ}$ \\
\hline 5751.629 & $4 b$ & 1 & 17381.557 & $21902_{4}^{\circ}-392834$ & 5713.732 & 2 & & 17496.842 & $20322 \circ-37819_{4}$ \\
\hline 5751.594 & $5 b$ & 1 & 17381.663 & $13945_{3}^{\circ}-31326_{4}$ & 5711.998 & 50 & 5 & 17502.153 & $17959_{4}-35462_{3}^{\circ}$ \\
\hline 5751.432 & 10 & 2 & 17382.153 & $19503_{3}^{\circ}-36885_{4}$ & 5711.454 & 1 & & 17503.820 & $16217_{2}^{\circ}-33721_{2}$ \\
\hline 5749.782 & $50 \mathrm{~b}$ & 8 & 17387.141 & $3865_{1}-21252_{2}^{\circ}$ & 5711.023 & 1 & & 17505.141 & $14032_{2}^{\circ}-315373$ \\
\hline 5749.733 & $20 \mathrm{~b}$ & 3 & 17387.289 & $18053^{\circ}-354403$ & 5710.270 & 4 & & 17507.449 & $18069_{3}^{\circ}-35576_{3}$ \\
\hline 5749.230 & 15 & 1 & 17388.810 & $16217_{2}^{\circ}-336062$ & 5709.858 & 1 & & 17508.713 & $19532_{4}-37041_{4}^{\circ}$ \\
\hline 5748.741 & 150 & 25 & 17390.289 & $6362_{2}-23752_{2}^{\circ}$ & 5708.679 & 50 & 3 & 17512.329 & $142045-31716^{\circ}$ \\
\hline 5748.323 & 100 & 8 & 17391.554 & $17959_{4}-35351_{4}^{\circ}$ & 5705.866 & 8 & 1 & 17520.962 & $19713_{3}-37234_{2}^{\circ}$ \\
\hline 5747.193 & 50 & 4 & 17394.973 & $118022-29197_{2}^{\circ}$ & 5705.637 & 50 & 4 & 17521.665 & $19227_{6}^{\circ}-36749_{6}$ \\
\hline 5747.058 & 2 & & 17395.382 & $19273_{2}-36668_{2}^{\circ}$ & 5705.483 & 10 & 1 & 17522.138 & $211435-38665^{\circ}$ \\
\hline 5746.468 & 4 & & 17397.168 & $14032_{2}^{\circ}-314291$ & 5705.232 & 4 & 1 & 17522.909 & $18053_{4}^{\circ}-35576_{3}$ \\
\hline 5745.233 & 5 & 1 & 17400.908 & $18809_{4}^{\circ}-36210_{5}$ & 5704.124 & 8 & 1 & 17526.313 & $18809_{4}^{\circ}-36336_{3}$ \\
\hline 5743.583 & 8 & 1 & 17405.906 & $18930_{3}^{\circ}-36336_{3}$ & 5702.650 & 50 & 25 & 17530.843 & $5563_{1}-23093_{2}^{\circ}$ \\
\hline 5743.063 & 15 & 1 & 17407.483 & $128473-30255_{3}^{\circ}$ & 5702.543 & 1 & & 17531.172 & $22163_{4}^{\circ}-396945$ \\
\hline 5741.829 & 50 & 10 & 17411.224 & $0_{2}-17411_{3}^{\circ}$ & 5700.782 & 50 & 8 & 17536.587 & $12114_{2}^{\circ}-29650_{2}$ \\
\hline 5741.715 & 5 & 1 & 17411.569 & $23015 \circ-404264$ & 5698.292 & 50 & 5 & 17544.250 & $11877_{1}^{\circ}-294221$ \\
\hline 5740.196 & 2 & & 17416.177 & $216454-39062{ }^{\circ}$ & 5696.391 & 50 & 8 & 17550.105 & $154934-330433^{\circ}$ \\
\hline 5739.837 & 3 & 1 & 17417.266 & $21539_{4}^{\circ}-389564$ & 5694.434 & 10 & 1 & 17556.136 & $16217_{2}^{\circ}-337731$ \\
\hline 5738.965 & 20 & 2 & 17419.912 & $14206_{4}^{\circ}-316265$ & 5693.759 & 1 & & 17558.218 & $17847_{2}^{\circ}-35405_{3}$ \\
\hline 5737.645 & 2 & & 17423.920 & $21575_{2}-38998_{3}^{\circ}$ & 5693.571 & 8 & & 17558.798 & $7280_{2}-24838_{i}^{\circ}$ \\
\hline 5736.031 & 100 & 8 & 17428.823 & $130883-30517_{4}^{\circ}$ & 5692.159 & 10 & 1 & 17563.153 & $15863_{2}-33427_{2}^{\circ}$ \\
\hline 5735.304 & 8 & 1 & 17431.032 & $15863_{2}-33294_{3}^{\circ}$ & 5691.906 & 3 & & 17563.934 & $19273_{2}-36837_{i}^{\circ}$ \\
\hline 5735.146 & 1 & & 17431.512 & $22248_{2}^{\circ}-39680_{2}$ & 5691.681 & 5 & & 17564.628 & $3687_{2}-21252_{2}^{\circ}$ \\
\hline
\end{tabular}


TABLE 4. Classified lines of $\mathrm{Th} \mathrm{I}-$ continued

\begin{tabular}{|c|c|c|c|c|c|c|c|c|c|}
\hline \multirow{2}{*}{$\begin{array}{l}\text { Wavelength } \\
\text { (Å) }\end{array}$} & \multicolumn{2}{|c|}{ Intensity } & \multirow{2}{*}{$\begin{array}{l}\text { Wavenumber } \\
\qquad\left(\mathrm{cm}^{-1}\right)\end{array}$} & \multirow{2}{*}{ Classification } & \multirow{2}{*}{$\begin{array}{c}\text { Wavelength } \\
\text { (§) }\end{array}$} & \multicolumn{2}{|c|}{ Intensity } & \multirow{2}{*}{$\begin{array}{l}\text { Wavenumber } \\
\qquad\left(\mathrm{cm}^{-1}\right)\end{array}$} & \multirow{2}{*}{ Classification } \\
\hline & Lamp & Spark & & & & Lamp & Spark & & \\
\hline 5691.060 & $10 \mathrm{~b}$ & & 17566.545 & $10414_{4}^{\circ}-27980_{3}$ & 5653.838 & 2 & & 17682.193 & $20054_{2}-37736_{2}^{\circ}$ \\
\hline 5690.693 & 40 & 3 & 17567.678 & $14206_{4}^{\circ}-317743$ & 5653.426 & 2 & & 17683.481 & $21077_{0}^{\circ}-38760_{4}$ \\
\hline 5690.118 & 10 & 3 & 17569.453 & $20214_{3}^{\circ}-37784_{2}$ & 5652.570 & 1 & & 17686.159 & $23015^{\circ}-40701_{4}$ \\
\hline 5689.647 & 10 & 1 & 17570.907 & $18011{ }^{\circ}-355825$ & 5651.326 & 5 & & 17690.052 & $15618_{3}^{\circ}-33309_{2}$ \\
\hline 5689.477 & 1 & & 17571.432 & $22855_{3}^{\circ}-404264$ & 5650.912 & 1 & & 17691.348 & $17354_{i}^{\circ}-350461$ \\
\hline 5688.527 & 1 & & 17574.367 & $20522_{2}^{\circ}-380972$ & 5650.354 & 75 & 15 & 17693.095 & $13297_{4}-30990_{3}^{\circ}$ \\
\hline 5687.997 & $2 \mathrm{c}$ & & 17576.004 & $14465_{2}^{\circ}-32041_{2}$ & 5649.737 & 1 & & 17695.028 & $22401_{1}-40096_{2}^{\circ}$ \\
\hline 5687.350 & 501 & 3 & 17578.004 & $15490 \stackrel{\circ}{-} 330686$ & 5648.990 & 75 & 10 & 17697.368 & $2869_{3}-20566_{4}^{\circ}$ \\
\hline 5686.876 & 10 & 1 & 17579.469 & $8111_{4}-25690_{0}^{\circ}$ & 5647.708 & 20 & 2 & 17701.385 & $10526_{3}^{\circ}-28227_{4}$ \\
\hline 5685.826 & 3 & & 17582.715 & $195033^{\circ}-37085_{4}$ & 5646.455 & 50 & 10 & 17705.313 & $128473-30553_{2}^{\circ}$ \\
\hline 5685.192 & 100 & 8 & 17584.676 & $8800_{4}-26384_{4}^{\circ}$ & 5646.255 & 1 & & 17705.940 & $21645_{4}^{\circ}-393515$ \\
\hline 5684.866 & 4 & & 17585.684 & $18930_{3}^{\circ}-36515_{3}$ & 5646.205 & 1 & & 17706.097 & $18809_{4}^{\circ}-36515_{3}$ \\
\hline 5683.486 & 20 & 2 & 17589.954 & $15970_{3}-33560_{4}^{\circ}$ & 5645.668 & 50 & 20 & 17707.781 & $8800_{4}-26508_{3}^{\circ}$ \\
\hline 5682.846 & 8 & 1 & 17591.935 & $13945_{3}^{\circ}-31537_{3}$ & 5645.529 & 50 & 8 & 17708.217 & $4961_{4}-22669_{3}^{\circ}$ \\
\hline 5682.238 & 4 & & 17593.818 & $17847_{2}^{\circ}-35440_{3}$ & 5643.346 & 8 & 1 & 17715.067 & $19588^{\circ}-37303_{4}$ \\
\hline 5681.441 & 1 & & 17596.286 & $11601_{1}-29197_{1}^{\circ}$ & 5643.110 & 20 & 2 & 17715.808 & $17501_{0}^{\circ}-352165$ \\
\hline 5679.535 & 3 & & 17602.191 & $20054_{2}-37656_{3}^{\circ}$ & 5641.735 & 75 & 8 & 17720.125 & $14481 \stackrel{\circ}{6}-322025$ \\
\hline 5679.004 & 50 & 5 & 17603.836 & $11241_{3}^{\circ}-288454$ & 5641.562 & 50 & 5 & 17720.669 & $12114_{2}^{\circ}-29835_{3}$ \\
\hline 5677.729 & $2 \mathrm{~h}$ & & 17607.790 & $20922_{2}^{\circ}-38529_{3}$ & 5641.242 & 20 & 5 & 17721.674 & $13297_{4}-31019_{4}^{\circ}$ \\
\hline 5677.051 & $200 \mathrm{~b}$ & 8 & 17609.892 & $7795_{4}^{\circ}-25405_{4}$ & 5640.363 & 50 & 2 & 17724.436 & $13088_{3}-30812_{2}^{\circ}$ \\
\hline 5675.701 & 2 & & 17614.081 & $19516^{\circ}-371313$ & 5639.460 & 10 & 1 & 17727.274 & $15863_{2}-3359 !_{3}^{\circ}$ \\
\hline 5675.601 & 2 & & 17614.391 & $19039_{2}^{\circ}-36653_{2}$ & 5638.767 & 1 & & 17729.452 & $17847_{2}^{\circ}-35576_{3}$ \\
\hline 5675.501 & 15 & 2 & 17614.702 & $15166^{\circ}-32781_{4}$ & 5636.625 & 1 & & 17736.190 & $19039_{2}^{\circ}-36775_{3}$ \\
\hline 5674.990 & $200 \mathrm{~b}$ & 25 & 17616.288 & $118022-29419_{2}^{\circ}$ & 5635.889 & 75 & 15 & 17738.506 & $17411_{3}^{\circ}-35149_{2}$ \\
\hline 5674.461 & 15 & 2 & 17617.930 & $21902_{4}^{\circ}-39520_{4}$ & 5635.482 & 2 & & 17739.787 & $19948_{4}^{\circ}-37688_{3}$ \\
\hline 5674.171 & 2 & & 17618.830 & $21645_{4}-39264_{3}^{\circ}$ & 5635.175 & 5 & 1 & 17740.753 & $18053_{4}^{\circ}-35794_{4}$ \\
\hline 5673.955 & 5 & 1 & 17619.501 & $20566_{4}^{\circ}-38186_{4}$ & 5634.619 & 10 & 2 & 17742.504 & $14032_{2}^{\circ}-31774_{3}$ \\
\hline 5673.835 & 50 & 5 & 17619.874 & $10414_{4}^{\circ}-280345$ & 5634.097 & $8 \mathrm{~s}$ & 1 & 17744.148 & $19588^{\circ}-373326$ \\
\hline 5673.009 & 5 & 1 & 17622.439 & $18011 \stackrel{\circ}{-356334}$ & 5633.297 & 75 & 8 & 17746.668 & $10526_{3}^{\circ}-28273_{2}$ \\
\hline 5672.724 & 5 & 1 & 17623.325 & $195888^{\circ}-372115$ & 5632.903 & $2 b$ & & 17747.909 & $10783_{2}^{\circ}-28531_{2}$ \\
\hline 5671.498 & 15 & 2 & 17627.134 & $195324-37159_{3}^{\circ}$ & 5632.776 & 50 & 3 & 17748.309 & $15970_{3}-33718_{2}^{\circ}$ \\
\hline 5671.250 & 10 & 2 & 17627.905 & $195033^{\circ}-371313$ & 5632.494 & 25 & 1 & 17749.198 & $142045-319533^{\circ}$ \\
\hline 5671.072 & 40 & 2 & 17628.458 & $165546-341820^{\circ}$ & 5632.164 & $5 b$ & & 17750.238 & $13962_{1}-31712^{\circ}$ \\
\hline 5670.827 & 3 & & 17629.220 & $15166^{\circ}-327963$ & 5630.297 & 75 & 10 & 17756.123 & $19986^{\circ}-377426$ \\
\hline 5670.199 & 1 & & 17631.172 & $184313-360624$ & 5629.312 & 50 & 3 & 17759.230 & $163510-34111^{\circ}$ \\
\hline 5668.402 & 3 & & 17636.762 & $13962_{1}-31599_{2}^{\circ}$ & 5628.365 & 8 & 1 & 17762.218 & $18069_{3}^{\circ}-35831_{3}$ \\
\hline 5668.016 & 1 & & 17637.963 & $21645_{4}^{\circ}-392834$ & 5627.606 & 3 & & 17764.614 & $15970{ }_{3}-33734_{2}^{\circ}$ \\
\hline 5667.509 & 2 & & 17639.541 & $18549_{2}-36188_{2}^{\circ}$ & 5627.012 & 3 & & 17766.489 & $811_{4}-25877_{4}^{\circ}$ \\
\hline 5667.129 & 150 & 15 & 17640.723 & $11241_{3}^{\circ}-288822$ & 5626.169 & 1 & & 17769.151 & $19039_{2}^{\circ}-368083$ \\
\hline 5666.419 & 10 & 1 & 17642.934 & $8111_{4}-257530$ & 5624.911 & 50 & 3 & 17773.125 & $11877_{1}^{\circ}-29650_{2}$ \\
\hline 5665.181 & 200 & 50 & 17646.789 & $8243_{2}^{\circ}-25890_{2}$ & 5624.775 & 15 & 2 & 17773.555 & $17959_{4}-35733_{4}^{\circ}$ \\
\hline 5664.621 & 75 & 15 & 17648.534 & $11197^{\circ}-28845_{4}$ & 5623.581 & 25 & 2 & 17777.329 & $15493_{4}-33270_{4}^{\circ}$ \\
\hline 5664.228 & 40 & 2 & 17649.758 & $17224_{2}^{\circ}-34874_{3}$ & 5623.474 & 1 & & 17777.667 & $18053_{4}^{\circ}-358313$ \\
\hline 5663.041 & 50 & 3 & 17653.458 & $2869_{3}-20522_{2}^{\circ}$ & 5623.411 & 2 & & 17777.866 & $17073_{1}-34851_{2}^{\circ}$ \\
\hline 5662.949 & 3 & & 17653.745 & $15736_{i}^{\circ}-33390_{1}$ & 5621.788 & 20 & 3 & 17782.998 & $18011 \stackrel{\circ}{-} 357944$ \\
\hline 5660.364 & 1 & & 17661.807 & $18699_{2}-36361_{1}^{\circ}$ & 5621.287 & 8 & 2 & 17784.583 & 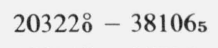 \\
\hline 5659.878 & 2 & & 17663.323 & $16783_{4}^{\circ}-344474$ & 5620.013 & $15 b$ & 5 & 17788.615 & $18011^{\circ}-357996$ \\
\hline 5659.216 & 5 & 1 & 17665.389 & $216454-39311_{4}^{\circ}$ & 5619.976 & $50 \mathrm{~b}$ & 8 & 17788.732 & $14206_{4}^{\circ}-319954$ \\
\hline 5657.926 & 100 & 25 & 17669.417 & $12847_{3}-30517_{4}^{\circ}$ & 5619.719 & $5 b$ & 1 & 17789.546 & $13175_{4}^{\circ}-30964_{3}$ \\
\hline 5656.729 & 40 & 2 & 17673.156 & $13088_{3}-30761_{3}^{\circ}$ & 5619.684 & $4 \mathrm{~b}$ & & 17789.656 & $15618 \stackrel{\circ}{-}-334083$ \\
\hline 5656.302 & 10 & 2 & 17674.490 & $22163_{4}^{\circ}-39837_{3}$ & 5619.526 & 1 & & 17790.156 & $19516_{2}^{\circ}-373073$ \\
\hline 5655.750 & 10 & 3 & 17676.215 & $13847_{2}-31523 \stackrel{\circ}{3}$ & 5619.478 & 1 & & 17790.308 & $21890_{3}^{\circ}-39680_{2}$ \\
\hline 5655.490 & 20 & 2 & 17677.028 & $16783_{4}^{\circ}-344605$ & 5619.017 & 50 & 4 & 17791.768 & $21165_{3}^{\circ}-389564$ \\
\hline 5655.213 & 2 & & 17677.893 & $20214_{3}^{\circ}-378924$ & 5618.927 & 15 & 1 & 17792.053 & $21902_{4}^{\circ}-396945$ \\
\hline 5655.135 & 2 & & 17678.137 & $17411_{3}^{\circ}-35089_{3}$ & 5617.964 & 2 & & 17795.103 & $17354_{1}^{\circ}-35149_{2}$ \\
\hline
\end{tabular}


TABLE 4. Classified lines of Th $\mathrm{I}$-continued

\begin{tabular}{|c|c|c|c|c|c|c|c|c|c|}
\hline \multirow{2}{*}{$\begin{array}{l}\text { Wavelength } \\
(\AA)\end{array}$} & \multicolumn{2}{|c|}{ Intensity } & \multirow{2}{*}{$\begin{array}{l}\text { Wavenumber } \\
\left(\mathrm{cm}^{-1}\right)\end{array}$} & \multirow{2}{*}{ Classification } & \multirow{2}{*}{ 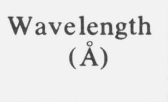 } & \multicolumn{2}{|c|}{ Intensity } & \multirow{2}{*}{$\begin{array}{l}\text { Wave number } \\
\qquad\left(\mathrm{cm}^{-1}\right)\end{array}$} & \multirow{2}{*}{ Classification } \\
\hline & Lamp & Spark & & & & Lamp & Spark & & \\
\hline 5617.291 & 15 & 2 & 17797.235 & $14243 i-32041_{2}$ & 5584.983 & 2 & & 17900.187 & $20054_{2}-37954_{3}^{\circ}$ \\
\hline 5616.945 & 3 & 1 & 17798.331 & $15863_{2}-33662_{1}^{\circ}$ & 5584.430 & 4 & & 17901.960 & $13088_{3}-30990_{3}^{\circ}$ \\
\hline 5616.691 & 20 & 2 & 17799.136 & $15618^{\circ}-33418_{4}$ & 5582.865 & 50 & 2 & 17906.978 & $111970-29104_{4}$ \\
\hline 5616.503 & 10 & 2 & 17799.732 & $17501{ }^{\circ}-353005$ & 5582.678 & 50 & 3 & 17907.578 & $139621-31870_{2}^{\circ}$ \\
\hline 5616.438 & 2 & & 17799.938 & $20566_{4}^{\circ}-38366_{4}$ & 5582.367 & 75 & 4 & 17908.575 & $15618_{3}^{\circ}-33527_{4}$ \\
\hline 5616.328 & 8 & 2 & 17800.286 & $19503^{\circ}-373034$ & 5580.756 & 100 & 10 & 17913.745 & $128473-30761_{3}^{\circ}$ \\
\hline 5615.879 & 10 & 3 & 17801.709 & $154934-33294^{\circ}$ & 5580.395 & 50 & 10 & 17914.904 & 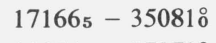 \\
\hline 5615.320 & $400 \mathrm{r}$ & 15 & 17803.482 & $3865_{1}-21668^{\circ}$ & 5580.221 & 3 & & 17915.462 & $200542-37970_{2}^{\circ}$ \\
\hline 5614.515 & 5 & 1 & 17806.034 & $14206_{4}^{\circ}-32012_{4}$ & 5580.076 & 50 & 2 & 17915.928 & $4961_{4}-228778$ \\
\hline 5613.659 & 5 & & 17808.749 & $18069_{3}^{\circ}-35877_{2}$ & 5579.990 & 4 & & 17916.204 & $21645_{4}-39562 \stackrel{\circ}{4}$ \\
\hline 5612.619 & 75 & 3 & 17812.049 & $14481{ }^{\circ}-322935$ & 5579.359 & 300 & 40 & 17918.230 & $55633_{1}-23481 i$ \\
\hline 5612.619 & 75 & 3 & 17812.049 & $18549_{2}-36361_{i}^{\circ}$ & 5578.091 & 2 & & 17922.303 & $21252_{2}^{\circ}-391743$ \\
\hline 5612.068 & 150 & 20 & 17813.798 & $10414_{4}^{\circ}-28227_{4}$ & 5577.602 & 10 & & 17923.875 & $16783_{4}^{\circ}-34707_{3}$ \\
\hline 5610.685 & 200 & 20 & 17818.189 & $11601_{1}-29419_{2}^{\circ}$ & 5577.115 & 5 & 1 & 17925.440 & $17224_{2}^{\circ}-35149_{2}$ \\
\hline 5610.329 & 50 & 3 & 17819.320 & $142045-320230^{\circ}$ & 5576.300 & 40 & 3 & 17928.060 & 154900 - 334184 \\
\hline 5610.235 & 75 & 5 & 17819.618 & $7502{ }_{3}-25321_{3}^{\circ}$ & 5576.205 & 100 & 10 & 17928.365 & $10414_{4}^{\circ}-283425$ \\
\hline 5610.111 & 75 & 4 & 17820.012 & $63622-24182_{2}^{\circ}$ & 5575.524 & 3 & & 17930.555 & $13088_{3}-31019_{4}^{\circ}$ \\
\hline 5609.857 & 15 & 1 & 17820.819 & $16346_{4}^{\circ}-34167_{3}$ & 5575.446 & 4 & & 17930.806 & $20214_{3}^{\circ}-38145_{3}$ \\
\hline 5609.585 & 150 & 10 & 17821.683 & $17224_{2}^{\circ}-35046_{1}$ & 5574.028 & 2 & & 17935.367 & $21902_{4}^{\circ}-39837_{3}$ \\
\hline 5607.197 & 15 & 1 & 17829.273 & $13945^{\circ}-317743$ & 5573.599 & 2 & & 17936.748 & $158632-338003$ \\
\hline 5607.087 & 10 & 1 & 17829.623 & $15970_{3}-33799_{4}^{\circ}$ & 5573.355 & 400 & 20 & 17937.533 & $8111_{4}-26048{ }^{\circ}$ \\
\hline 5606.390 & 100 & 10 & 17831.839 & $10526_{3}^{\circ}-28358_{3}$ & 5572.766 & $10 \mathrm{~s}$ & 1 & 17939.429 & $18549_{2}-36488_{2}^{\circ}$ \\
\hline 5604.814 & 2 & & 17836.853 & $21575_{2}-39411^{\circ}$ & 5572.466 & 200 & 8 & 17940.394 & $75023-25442 \stackrel{\circ}{3}$ \\
\hline 5603.764 & 20 & 1 & 17840.195 & $15618_{3}^{\circ}-33459_{4}$ & 5572.095 & 20 & 1 & 17941.589 & $11802_{2}-29744^{\circ}$ \\
\hline 5603.060 & $8 \mathrm{~s}$ & & 17842.437 & $17398_{3}-35240_{3}^{\circ}$ & 5571.253 & $75 b$ & 10 & 17944.301 & $10414_{4}^{\circ}-283583$ \\
\hline 5602.627 & 15 & 1 & 17843.816 & $132974-31141{ }^{\circ}$ & 5570.928 & 50 & 2 & 17945.347 & $6362_{2}-24307_{2}^{\circ}$ \\
\hline 5602.216 & $2 b$ & & 17845.125 & $8800_{4}-26645 \circ$ & 5570.762 & 8 & 1 & 17945.882 & $15618_{3}^{\circ}-33564_{3}$ \\
\hline 5601.934 & 2 & & 17846.023 & $22855_{3}^{\circ}-40701_{4}$ & 5570.265 & 1 & & 17947.483 & $21890_{3}^{\circ}-39837_{3}$ \\
\hline 5601.604 & 200 & 8 & 17847.074 & $0_{2}-17847_{2}^{\circ}$ & 5569.486 & 2 & & 17949.993 & $16217_{2}^{\circ}-34167_{3}$ \\
\hline 5601.604 & 200 & 8 & 17847.074 & $55631-23410 \circ$ & 5568.840 & 15 & & 17952.076 & $15736 i-33689_{2}$ \\
\hline 5601.506 & $8 \mathrm{~b}$ & & 17847.387 & $12114_{2}^{\circ}-29961_{1}$ & 5568.542 & 50 & 3 & 17953.036 & $173983-35351_{4}^{\circ}$ \\
\hline 5601.191 & 4 & & 17848.390 & $13945_{3}^{\circ}-31793_{4}$ & 5568.000 & $300 \mathrm{~b}$ & 150 & 17954.784 & $16346_{4}^{\circ}-34301_{4}$ \\
\hline 5599.865 & 3 & & 17852.617 & $17959_{4}-35812_{3}^{\circ}$ & 5567.942 & $25 b$ & 10 & 17954.971 & $18930_{3}^{\circ}-368854$ \\
\hline 5599.655 & 50 & 2 & 17853.286 & $75023-25355_{4}^{\circ}$ & 5564.806 & 25 & 2 & 17965.089 & $171665-35131^{\circ}$ \\
\hline 5599.573 & 2 & & 17853.548 & $142260-32080^{\circ}$ & 5564.691 & 2 & & 17965.461 & $18809_{4}^{\circ}-36775_{3}$ \\
\hline 5599.274 & $10 \mathrm{~b}$ & & 17854.501 & $15863_{2}-33718_{2}^{\circ}$ & 5563.582 & 4 & & 17969.042 & $18699_{2}-36668_{2}^{\circ}$ \\
\hline 5598.445 & 4 & & 17857.145 & $18699_{2}-36556_{1}^{\circ}$ & 5562.847 & 5 & & 17971.416 & $18809_{4}^{\circ}-36781_{5}$ \\
\hline 5596.836 & 5 & & 17862.278 & $11241_{3}^{\circ}-291044$ & 5560.000 & $3 \mathrm{~h}$ & & 17980.618 & $21539_{4}^{\circ}-39520_{4}$ \\
\hline 5595.962 & 75 & 8 & 17865.068 & $17224_{2}^{\circ}-35089_{3}$ & 5559.894 & 150 & 8 & 17980.961 & $3687_{2}-21668^{\circ}$ \\
\hline 5595.847 & 50 & 3 & 17865.435 & $25580-20423^{\circ}$ & 5559.526 & 3 & & 17982.151 & $18574_{1}-36556^{\circ}$ \\
\hline 5595.778 & 5 & & 17865.656 & $20737_{1}^{\circ}-38602_{2}$ & 5558.882 & $8 b$ & 3 & 17984.234 & $17847_{2}^{\circ}-35831_{3}$ \\
\hline 5595.064 & 300 & 25 & 17867.935 & $8243_{2}^{\circ}-26111_{1}$ & 5558.848 & $75 b$ & 3 & 17984.344 & $15736_{1}^{\circ}-33721_{2}$ \\
\hline 5594.161 & 15 & 1 & 17870.820 & $15863_{2}-33734_{2}^{\circ}$ & 5558.848 & $75 b$ & 3 & 17984.344 & $192277_{6}^{\circ}-372115$ \\
\hline 5592.597 & 2 & & 17875.817 & $19273_{2}-37149_{2}^{\circ}$ & 5558.433 & $75 b$ & $25 \mathrm{~b}$ & 17985.687 & $13297_{4}-31283^{\circ}$ \\
\hline 5591.907 & $15 b$ & 3 & 17878.023 & $18549_{2}-36427_{3}^{\circ}$ & 5558.342 & $400 \mathrm{~b}$ & 50 & 17985.981 & $8111_{4}-26096_{3}^{\circ}$ \\
\hline 5591.907 & $15 b$ & 3 & 17878.023 & $18930_{3}^{\circ}-36808_{3}$ & 5557.924 & 25 & 5 & 17987.334 & $13088_{3}-31075_{2}^{\circ}$ \\
\hline 5590.215 & 20 & & 17883.434 & $11802_{2}-29686_{3}^{\circ}$ & 5557.045 & 300 & 15 & 17990.179 & $8800_{4}-26790_{4}^{\circ}$ \\
\hline 5589.560 & 1 & & 17885.530 & $20922_{2}^{\circ}-388072$ & 5555.839 & 10 & 1 & 17994.084 & $17411_{3}^{\circ}-35405_{3}$ \\
\hline 5589.412 & 2 & & 17886.003 & $19273_{2}-37159^{\circ}$ & 5555.534 & 75 & 4 & 17995.072 & $14206_{4}^{\circ}-322025$ \\
\hline 5589.328 & 5 & 1 & 17886.272 & $19273_{2}-37159_{3}^{\circ}$ & 5554.695 & 5 & & 17997.790 & $159703-33967_{3}^{\circ}$ \\
\hline 5587.027 & $500 \mathrm{r}$ & 50 & 17893.638 & $4961_{4}-22855_{3}^{\circ}$ & 5554.546 & $10 \mathrm{~b}$ & 2 & 17998.273 & $20054_{2}-38053 \stackrel{\circ}{3}$ \\
\hline 5586.255 & 2 & & 17896.111 & $10783_{2}^{\circ}-28679_{2}$ & 5554.502 & 20 & 4 & 17998.415 & $18809_{4}^{\circ}-36808_{3}$ \\
\hline 5585.981 & 4 & & 17896.989 & $12114_{2}^{\circ}-30011_{3}$ & 5552.624 & 75 & 4 & 18004.503 & $10526 \stackrel{8}{3}-28531_{2}$ \\
\hline 5585.884 & $5 b$ & & 17897.300 & $132974-31194_{4}^{\circ}$ & 5551.744 & 25 & 5 & 18007.357 & $18549_{2}-36556^{\circ}$ \\
\hline 5585.848 & 151 & 1 & 17897.415 & $14032_{2}^{\circ}-31929_{3}$ & 5551.193 & 40 & 2 & 18009.144 & $140322_{2}^{\circ}-32041_{2}$ \\
\hline
\end{tabular}


TABLE 4. Classified lines of $\mathrm{Th} \mathrm{I}$-continued

\begin{tabular}{|c|c|c|c|c|c|c|c|c|c|}
\hline \multirow{2}{*}{$\begin{array}{l}\text { Wavelength } \\
(\AA)\end{array}$} & \multicolumn{2}{|c|}{ Intensity } & \multirow{2}{*}{$\begin{array}{l}\text { Wavenumber } \\
\left(\mathrm{cm}^{-1}\right)\end{array}$} & \multirow{2}{*}{ Classification } & \multirow{2}{*}{$\begin{array}{c}\text { Wavelength } \\
(\AA)\end{array}$} & \multicolumn{2}{|c|}{ Intensity } & \multirow{2}{*}{$\begin{array}{l}\text { Wavenumber } \\
\left(\mathrm{cm}^{-1}\right)\end{array}$} & \multirow{2}{*}{ Classification } \\
\hline & Lamp & Spark & & & & Lamp & Spark & & \\
\hline 5550.988 & 2 & & 18009.809 & $21165^{\circ}-391743$ & 5523.539 & 8 & 2 & 18099.307 & $10783_{2}^{\circ}-28882_{2}$ \\
\hline 5550.476 & 2 & & 18011.470 & $21668_{1}^{\circ}-39680_{2}$ & 5523.451 & 5 & & 18099.596 & $21738_{2}^{\circ}-39837_{3}$ \\
\hline 5550.456 & 1 & & 18011.535 & $21668^{\circ}-39680_{2}$ & 5523.164 & 25 & 1 & 18100.536 & $16346 \stackrel{\circ}{4}-34447_{4}$ \\
\hline 5548.377 & 15 & 5 & 18018.284 & $12114_{2}^{\circ}-301322$ & 5522.616 & 8 & & 18102.332 & $15618 \stackrel{\circ}{3}-33721_{2}$ \\
\hline 5548.176 & $400 \mathrm{r}$ & $40 b$ & 18018.937 & $6362_{2}-24381_{2}^{\circ}$ & 5522.416 & 3 & & 18102.988 & $17959_{4}-36062_{4}^{\circ}$ \\
\hline 5547.654 & 15 & 1 & 18020.632 & $18069_{3}^{\circ}-36089_{4}$ & 5522.104 & 8 & & 18104.010 & $15863_{2}-33967_{3}^{\circ}$ \\
\hline 5547.135 & 75 & 3 & 18022.318 & $13847_{2}-31870_{2}^{\circ}$ & 5521.751 & 200 & 8 & 18105.168 & $19227^{\circ}-373326$ \\
\hline 5546.638 & 4 & & 18023.933 & $19713_{3}-37736_{2}^{\circ}$ & 5520.954 & 5 & & 18107.781 & $171665-352738$ \\
\hline 5545.806 & 15 & & 18026.637 & $7280_{2}-25306_{2}^{\circ}$ & 5518.988 & $200 b$ & 50 & 18114.232 & $16346_{4}^{\circ}-344605$ \\
\hline 5545.586 & 2 & & 18027.352 & $22399_{0}^{\circ}-404264$ & 5517.874 & 3 & & 18117.889 & $139621-320801$ \\
\hline 5544.879 & 1 & & 18029.651 & $17411_{3}^{\circ}-354403$ & 5517.616 & 4 & & 18118.736 & $21165_{3}^{\circ}-39283_{4}$ \\
\hline 5544.543 & 2 & & 18030.743 & $17847_{2}^{\circ}-35877_{2}$ & 5517.455 & 4 & & 18119.265 & $18549_{2}-36668_{2}^{\circ}$ \\
\hline 5544.385 & 3 & & 18031.257 & $159703-340011_{4}^{\circ}$ & 5515.845 & 4 & & 18124.554 & $19532_{4}-37656_{3}^{\circ}$ \\
\hline 5544.385 & 3 & & 18031.257 & $159703-34001_{4}^{\circ}$ & 5514.873 & $200 \mathrm{~b}$ & 10 & 18127.748 & $77955_{4}^{\circ}-259234$ \\
\hline 5544.252 & 4 & 1 & 18031.690 & $22855^{\circ}-40886_{2}$ & 5513.370 & 2 & & 18132.690 & $17501 \stackrel{\circ}{\circ}-356334$ \\
\hline 5543.480 & 10 & 1 & 18034.201 & $20054_{2}-38088_{2}^{\circ}$ & 5513.084 & 2 & & 18133.630 & $20566_{4}^{\circ}-38700_{4}$ \\
\hline 5542.890 & 300 & 10 & 18036.121 & $10526^{\circ}-28562_{4}$ & 5510.495 & 20 & 1 & 18142.150 & $15166_{3}^{\circ}-33309_{2}$ \\
\hline 5542.027 & 3 & & 18038.929 & $195324-37571_{0}^{\circ}$ & 5510.372 & 15 & 1 & 18142.555 & $12847_{3}-30990_{3}^{\circ}$ \\
\hline 5541.936 & 50 & 2 & 18039.225 & $11601_{1}-29640_{1}^{\circ}$ & 5509.993 & 400 & 20 & 18143.803 & $98045-27948^{\circ}$ \\
\hline 5541.936 & 50 & 2 & 18039.225 & $18614^{\circ}-366532$ & 5508.557 & 75 & 5 & 18148.533 & $10414_{4}^{\circ}-285624$ \\
\hline 5541.581 & 50 & 2 & 18040.381 & $5563_{1}-23603_{2}^{\circ}$ & 5508.050 & 8 & 1 & 18150.203 & $21902 \stackrel{\circ}{4}-400524$ \\
\hline 5541.146 & 50 & 2 & 18041.797 & $7280_{2}-25321_{3}^{\circ}$ & 5507.542 & 1001 & 2 & 18151.877 & $4961_{4}-23113_{4}^{\circ}$ \\
\hline 5540.521 & 2 & & 18043.832 & $19948{ }^{\circ}-37992_{4}$ & 5507.542 & 1001 & 2 & 18151.877 & $13175_{4}^{\circ}-313264$ \\
\hline 5540.380 & 1 & & 18044.292 & $20322{ }^{\circ}-38366_{4}$ & 5507.295 & 1 & & 18152.691 & $10526_{3}^{\circ}-28679_{2}$ \\
\hline 5539.262 & $500 \mathrm{r}$ & 50 & 18047.933 & $98045-27852 \circ$ & 5506.919 & 50 & 5 & 18153.931 & $19588 \circ-377426$ \\
\hline 5538.608 & 75 & 4 & 18050.065 & $3687_{2}-21738_{2}^{\circ}$ & 5506.676 & 1 & & 18154.732 & $21539_{4}^{\circ}-396945$ \\
\hline 5537.749 & 1 & & 18052.864 & $2869_{3}-20922_{2}^{\circ}$ & 5506.423 & 3 & & 18155.566 & $18930_{3}^{\circ}-37085_{4}$ \\
\hline 5537.555 & 50 & 2 & 18053.497 & $4961_{4}-230158$ & 5506.078 & 5 & & 18156.703 & $21165_{3}^{\circ}-39321_{3}$ \\
\hline 5536.436 & 4 & & 18057.146 & $18431_{3}-36488_{2}^{\circ}$ & 5505.934 & 5 & & 18157.178 & $18053 \stackrel{\circ}{4}-362105$ \\
\hline 5535.970 & 50 & 1 & 18058.666 & $63622-24421_{3}^{\circ}$ & 5505.547 & 2 & & 18158.455 & $19948_{4}^{\circ}-381065$ \\
\hline 5534.586 & 5 & & 18063.181 & $18699_{2}-36762_{3}^{\circ}$ & 5504.302 & 200 & 20 & 18162.562 & $7280_{2}-25442{ }_{3}^{\circ}$ \\
\hline 5534.439 & 5 & & 18063.661 & $17398_{3}-35462_{3}^{\circ}$ & 5503.698 & 2 & & 18164.555 & $18930_{3}^{\circ}-37094_{2}$ \\
\hline 5533.963 & 4 & & 18065.215 & $16783_{4}^{\circ}-34849_{5}$ & 5503.473 & 3 & & 18165.298 & $17411_{3}^{\circ}-355763$ \\
\hline 5533.608 & 5 & & 18066.374 & $17354_{i}^{\circ}-35421_{1}$ & 5502.718 & 5 & & 18167.790 & $20566_{4}^{\circ}-38734_{3}$ \\
\hline 5533.467 & 5 & 1 & 18066.834 & $15493_{4}-33560_{4}^{\circ}$ & 5501.701 & 8 & 2 & 18171.148 & $12847_{3}-31019^{\circ}$ \\
\hline 5533.219 & $10 \mathrm{~b}$ & & 18067.644 & $13945^{\circ}-320124$ & 5501.467 & 5 & & 18171.921 & $220984-40270_{4}^{\circ}$ \\
\hline 5532.780 & 5 & & 18069.077 & $0_{2}-18069_{3}^{\circ}$ & 5501.281 & 100 & 10 & 18172.536 & $12114_{2}^{\circ}-30286_{1}$ \\
\hline 5532.481 & 1 & & 18070.054 & $15618_{3}^{\circ}-33689_{2}$ & 5500.032 & 8 & & 18176.662 & $11241_{3}^{\circ}-29418_{2}$ \\
\hline 5531.483 & 1 & & 18073.314 & $195324-37605_{4}^{\circ}$ & 5499.647 & 75 & 5 & 18177.935 & $55633_{1}-23741^{\circ}$ \\
\hline 5530.843 & 2 & & 18075.405 & $18809_{4}^{\circ}-36885_{4}$ & 5499.255 & 300 & 40 & 18179.230 & $25580-20737^{\circ}$ \\
\hline 5530.075 & 50 & 2 & 18077.916 & $88004-26878_{3}^{\circ}$ & 5498.716 & 8 & 1 & 18181.012 & $17224_{2}^{\circ}-35405_{3}$ \\
\hline 5529.472 & 8 & & 18079.887 & $18053_{4}^{\circ}-361335$ & 5497.401 & $2 b$ & & 18185.361 & $171665-35351^{\circ}$ \\
\hline 5529.096 & 75 & 10 & 18081.117 & $17501 \stackrel{\circ}{-355825}$ & 5496.138 & 200 & 10 & 18189.540 & $5563_{1}-23752_{2}^{\circ}$ \\
\hline 5528.226 & $50 \mathrm{~b}$ & 5 & 18083.962 & $11877_{1}^{\circ}-29961_{1}$ & 5494.797 & 2 & & 18193.979 & $20566_{4}^{\circ}-38760_{4}$ \\
\hline 5527.480 & 10 & 1 & 18086.403 & $14465_{2}^{\circ}-325513$ & 5494.619 & 5 & & 18194.569 & $13088_{3}-31283_{3}^{\circ}$ \\
\hline 5527.295 & 200 & 8 & 18087.008 & $14206_{4}^{\circ}-322935$ & 5494.330 & 75 & 10 & 18195.526 & $8800_{4}-26995 \stackrel{\circ}{\circ}$ \\
\hline 5527.074 & 2 & & 18087.731 & $22338^{\circ}-404264$ & 5493.973 & 5 & & 18196.708 & $17224_{2}^{\circ}-35421_{1}$ \\
\hline 5526.983 & 1 & & 18088.029 & $20922_{2}^{\circ}-390103$ & 5493.594 & 10 & 1 & 18197.963 & $13962_{1}-32160_{2}^{\circ}$ \\
\hline 5526.528 & 50 & 8 & 18089.518 & $16783_{4}^{\circ}-34873_{4}$ & 5493.196 & 100 & 8 & 18199.282 & $6362_{2}-24561^{\circ}$ \\
\hline 5526.434 & 8 & 1 & 18089.826 & $14204_{5}-32294_{4}^{\circ}$ & 5492.912 & 15 & 3 & 18200.223 & $17847_{2}^{\circ}-36047_{2}$ \\
\hline 5526.313 & 8 & & 18090.222 & $16783^{\circ}-348743$ & 5492.642 & 100 & 10 & 18201.117 & $75023-25703_{2}^{\circ}$ \\
\hline 5525.799 & 2 & & 18091.905 & $19039_{2}^{\circ}-37131_{3}$ & 5492.332 & 40 & 20 & 18202.145 & $3687_{2}-21890_{3}^{\circ}$ \\
\hline 5525.155 & 20 & 2 & 18094.013 & $18574_{1}-36668_{2}^{\circ}$ & 5489.086 & 75 & 5 & 18212.909 & $15618{ }_{3}^{\circ}-33831_{4}$ \\
\hline 5524.583 & $150 \mathrm{~b}$ & $10 \mathrm{~b}$ & 18095.887 & $13175^{\circ}-312715$ & 5488.795 & 3 & & 18213.874 & $16217_{2}^{\circ}-34431_{3}$ \\
\hline 5523.951 & 10 & & 18097.957 & $154934-33591{ }^{\circ}$ & 5488.207 & 4 & & 18215.826 & $23015 \circ-41230_{4}$ \\
\hline
\end{tabular}


TABLE 4. Classified lines of Th $\mathrm{I}$-continued

\begin{tabular}{|c|c|c|c|c|c|c|c|c|c|}
\hline \multirow{2}{*}{$\begin{array}{l}\text { Wavelength } \\
\text { (Å) }\end{array}$} & \multicolumn{2}{|c|}{ Intensity } & \multirow{2}{*}{$\begin{array}{l}\text { Wavenumber } \\
\left(\mathrm{cm}^{-1}\right)\end{array}$} & \multirow{2}{*}{ Classification } & \multirow{2}{*}{$\begin{array}{c}\text { Wavelength } \\
\text { (A) }\end{array}$} & \multicolumn{2}{|c|}{ Inten sity } & \multirow{2}{*}{$\begin{array}{l}\text { Wavenumber } \\
\qquad\left(\mathrm{cm}^{-1}\right)\end{array}$} & \multirow{2}{*}{ Classification } \\
\hline & Lamp & Spark & & & & Lamp & Spark & & \\
\hline 5487.971 & 15 & 1 & 18216.609 & $17224_{2}^{\circ}-35440_{3}$ & 5449.058 & 1 & & 18346.697 & $12847_{3}-31194_{4}^{\circ}$ \\
\hline 5487.837 & 15 & 2 & 18217.054 & $22669_{3}^{\circ}-40886_{2}$ & 5448.272 & 50 & 3 & 18349.344 & $13847_{2}-32197_{3}^{\circ}$ \\
\hline 5487.505 & 15 & 2 & 18218.156 & $17959_{4}-36178^{\circ}$ & 5447.574 & 2 & & 18351.695 & 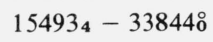 \\
\hline 5485.714 & 4 & & 18224.104 & $20054_{2}-38278^{\circ}$ & 5447.417 & 2 & & 18352.223 & $17224_{2}^{\circ}-35576_{3}$ \\
\hline 5484.988 & 50 & 3 & 18226.516 & $13297_{4}-31523_{3}^{\circ}$ & 5447.071 & 1 & & 18353.389 & $228770^{\circ}-41230_{4}$ \\
\hline 5484.556 & 20 & 1 & 18227.951 & $12847_{3}-31075_{2}^{\circ}$ & 5446.894 & 10 & & 18353.986 & $15970_{3}-34324_{2}^{\circ}$ \\
\hline 5484.469 & 8 & 1 & 18228.241 & $18069_{3}^{\circ}-36297_{4}$ & 5446.321 & 8 & & 18355.917 & $10526^{\circ}-28882_{2}$ \\
\hline 5483.709 & 2 & & 18230.767 & $19588_{0}^{\circ}-37819_{4}$ & 5444.668 & 2 & & 18361.489 & $20054_{2}-38416{ }^{\circ}$ \\
\hline 5483.623 & 3 & & 18231.053 & $19516_{2}^{\circ}-37748_{2}$ & 5444.477 & 5 & 1 & 18362.133 & $13175_{4}^{\circ}-31537_{3}$ \\
\hline 5483.143 & 50 & 2 & 18232.649 & $13847_{2}-32080_{1}^{\circ}$ & 5444.421 & 1 & & 18362.322 & $22338_{3}^{\circ}-40701_{4}$ \\
\hline 5482.498 & 75 & 8 & 18234.794 & $14204_{5}-32439_{4}^{\circ}$ & 5443.481 & 1 & & 18365.493 & $19039_{2}^{\circ}-374043$ \\
\hline 5481.828 & 8 & 1 & 18237.023 & $19713_{3}-37950_{4}^{\circ}$ & 5441.213 & 75 & 8 & 18373.148 & $18930_{3}^{\circ}-37303_{4}$ \\
\hline 5481.612 & 2 & & 18237.741 & $19948{ }^{\circ}-381864$ & 5441.042 & 25 & 1 & 18373.726 & $13297_{4}-31671_{4}^{\circ}$ \\
\hline 5481.211 & 5 & & 18239.075 & $16351_{0}-34591_{1}^{\circ}$ & 5440.796 & 3 & & 18374.556 & $15970_{3}-34344_{4}^{\circ}$ \\
\hline 5480.402 & 4 & 1 & 18241.768 & $15166_{3}^{\circ}-33408_{3}$ & 5440.600 & 50 & 2 & 18375.218 & $75023-25877_{4}^{\circ}$ \\
\hline 5479.462 & 8 & & 18244.897 & $19503_{3}^{\circ}-37748_{2}$ & 5440.385 & 4 & & 18375.944 & $19713_{3}-38088_{2}^{\circ}$ \\
\hline 5479.072 & 75 & 75 & 18246.196 & $7280_{2}-25526_{1}^{\circ}$ & 5440.116 & 4 & & 18376.853 & $18930_{3}^{\circ}-37307_{3}$ \\
\hline 5478.742 & 50 & 3 & 18247.295 & $15863_{2}-34111_{i}^{\circ}$ & 5439.495 & 2 & & 18378.951 & $22508_{2}^{\circ}-40886_{2}$ \\
\hline 5477.550 & 40 & 2 & 18251.266 & $14206_{4}^{\circ}-324584$ & 5438.250 & 10 & 1 & 18383.159 & $17411_{3}^{\circ}-35794_{4}$ \\
\hline 5476.482 & 20 & 2 & 18254.825 & $11877_{1}^{\circ}-301322$ & 5437.958 & 3 & & 18384.146 & $20423 i-388072$ \\
\hline 5476.482 & 20 & 2 & 18254.825 & $17073_{1}-35328_{1}^{\circ}$ & 5437.232 & 10 & 1 & 18386.600 & $20566_{4}^{\circ}-389535$ \\
\hline 5475.767 & $4 h$ & & 18257.208 & $19713_{3}-37970_{2}^{\circ}$ & 5436.814 & 2 & & 18388.014 & $20214_{3}^{\circ}-38602_{2}$ \\
\hline 5474.145 & 2 & & 18262.618 & $18574_{1}-36837_{1}^{\circ}$ & 5436.326 & 2 & & 18389.665 & $19503_{3}^{\circ}-37892_{4}$ \\
\hline 5473.849 & 10 & 1 & 18263.606 & $22163_{4}^{\circ}-404264$ & 5436.146 & 1 & & 18390.273 & $20566_{4}^{\circ}-389564$ \\
\hline 5472.701 & 8 & & 18267.437 & $19516_{2}^{\circ}-37784_{2}$ & 5434.152 & 100 & 10 & 18397.022 & $811_{4}-26508_{3}^{\circ}$ \\
\hline 5472.535 & 25 & 1 & 18267.991 & $19039_{2}^{\circ}-37307_{3}$ & 5433.876 & 15 & 2 & 18397.956 & $15166_{3}^{\circ}-33564_{3}$ \\
\hline 5470.759 & 150 & 10 & 18273.921 & $8111_{4}-26384_{4}^{\circ}$ & 5432.127 & 2 & & 18403.879 & $19588^{\circ}-379924$ \\
\hline 5470.145 & 50 & 8 & 18275.972 & $18809_{4}^{\circ}-37085_{4}$ & 5431.605 & 15 & 2 & 18405.648 & $11802_{2}-30208_{2}^{\circ}$ \\
\hline 5468.569 & 1 & & 18281.239 & $19503_{3}^{\circ}-37784_{2}$ & 5431.112 & 150 & 40 & 18407.319 & $6362_{2}-24769_{3}^{\circ}$ \\
\hline 5468.166 & 2 & & 18282.586 & $18053_{4}^{\circ}-363363$ & 5430.807 & 4 & & 18408.353 & $10526_{3}^{\circ}-28934_{3}$ \\
\hline 5467.403 & 5 & 1 & 18285.138 & $22141_{3}^{\circ}-404264$ & 5430.593 & 10 & & 18409.078 & $11877_{i}^{\circ}-30286_{1}$ \\
\hline 5467.167 & 75 & 4 & 18285.927 & $18011^{\circ}-36297_{4}$ & 5430.121 & 3 & & 18410.678 & $21575_{2}-39985_{3}^{\circ}$ \\
\hline 5466.602 & 5 & 1 & 18287.817 & $18549_{2}-36837_{1}^{\circ}$ & 5429.105 & 100 & 5 & 18414.124 & $17398_{3}-35812^{\circ}$ \\
\hline 5465.268 & 5 & & 18292.281 & $15166_{3}^{\circ}-33459_{4}$ & 5428.069 & 10 & & 18417.638 & $19532_{4}-37950_{4}^{\circ}$ \\
\hline 5464.205 & $200 w$ & 8 & 18295.839 & $2869_{3}-21165_{3}^{\circ}$ & 5427.767 & 15 & 1 & 18418.663 & $211435-39562{ }^{\circ}$ \\
\hline 5463.771 & 50 & 4 & 18297.293 & $18574_{1}-36871_{2}^{\circ}$ & 5427.618 & 20 & 2 & 18419.168 & $132974-31716^{\circ}$ \\
\hline 5463.320 & 40 & $10 \mathrm{~h}$ & 18298.803 & $17501^{\circ}-357996$ & 5427.352 & $2 \mathrm{~h}$ & & 18420.071 & $17411_{3}^{\circ}-35831_{3}$ \\
\hline 5462.809 & $3 b$ & & 18300.515 & $20054_{2}-38355_{2}^{\circ}$ & 5426.621 & $15 b$ & 1 & 18422.552 & $195324-37954^{\circ}$ \\
\hline 5462.336 & 50 & 15 & 18302.099 & $19588^{\circ}-37890_{5}$ & 5426.574 & $10 \mathrm{~b}$ & & 18422.712 & $17959_{4}-36382_{4}^{\circ}$ \\
\hline 5461.636 & 5 & $2 b$ & 18304.445 & $19588_{0}^{\circ}-37892_{4}$ & 5426.407 & 50 & 3 & 18423.279 & $7280_{2}-257032$ \\
\hline 5461.314 & 3 & & 18305.524 & $16783_{4}^{\circ}-35089_{3}$ & 5426.170 & 50 & 2 & 18424.084 & $142260-32650_{i}^{\circ}$ \\
\hline 5461.019 & 5 & & 18306.513 & $15493_{4}-33799_{4}^{\circ}$ & 5424.368 & 5 & & 18430.204 & $12114_{2}^{\circ}-30544_{2}$ \\
\hline 5459.171 & 15 & 3 & 18312.710 & $13847_{2}-32160_{2}^{\circ}$ & 5424.008 & 300 & 40 & 18431.427 & $10414_{4}^{\circ}-28845_{4}$ \\
\hline 5458.492 & 1 & & 18314.988 & $20214_{3}^{\circ}-38529_{3}$ & 5423.501 & $20 \mathrm{~b}$ & 3 & 18433.150 & $16783_{4}^{\circ}-35216_{5}$ \\
\hline 5457.289 & 50 & 3 & 18319.025 & $10526_{3}^{\circ}-28845_{4}$ & 5422.914 & 15 & 1 & 18435.146 & $128473-31283_{3}^{\circ}$ \\
\hline 5456.654 & 8 & 1 & 18321.157 & $18809_{4}^{\circ}-37131_{3}$ & 5422.847 & $8 \mathrm{~b}$ & & 18435.373 & $13088_{3}-315233^{\circ}$ \\
\hline 5456.255 & 40 & 2 & 18322.497 & $18549_{2}-36871_{2}^{\circ}$ & 5422.250 & 2 & & 18437.403 & $13847_{2}-32285_{3}^{\circ}$ \\
\hline 5454.919 & 10 & 1 & 18326.984 & $22098_{4}-40425_{4}^{\circ}$ & 5421.966 & 5 & 1 & 18438.369 & $20322{ }^{\circ}-38760_{4}$ \\
\hline 5453.757 & 1 & & 18330.889 & $14465_{2}^{\circ}-32796_{3}$ & 5421.664 & 100 & 5 & 18439.396 & $15166_{3}^{\circ}-33606_{2}$ \\
\hline 5453.686 & 4 & 1 & 18331.128 & $18431_{3}-36762_{3}^{\circ}$ & 5420.451 & 4 & & 18443.522 & $20566_{4}^{\circ}-39010_{3}$ \\
\hline 5453.297 & 5 & & 18332.435 & $165546-34886^{\circ}$ & 5419.463 & 1 & & 18446.884 & $18069_{3}^{\circ}-36515_{3}$ \\
\hline 5452.219 & 300 & 40 & 18336.060 & $9804_{5}-28140_{4}^{\circ}$ & 5418.702 & 3 & & 18449.475 & $18699_{2}-37149_{2}^{\circ}$ \\
\hline 5451.091 & 15 & 2 & 18339.854 & $21645_{4}-39985_{3}^{\circ}$ & 5417.808 & 75 & 1 & 18452.520 & $11802_{2}-30255_{3}^{\circ}$ \\
\hline 5450.511 & 25 & & 18341.806 & $15490_{0}^{\circ}-33831_{4}$ & 5417.486 & $400 \mathrm{r}$ & 40 & 18453.616 & $3687_{2}-22141_{3}^{\circ}$ \\
\hline 5450.146 & 5 & & 18343.034 & $19273_{2}-37616_{1}^{\circ}$ & 5415.775 & 4 & & 18459.446 & $17073_{1}-35533_{2}^{\circ}$ \\
\hline
\end{tabular}


TABLE 4. Classified lines of Th $\mathrm{I}$-continued

\begin{tabular}{|c|c|c|c|c|c|c|c|c|c|}
\hline \multirow{2}{*}{$\begin{array}{l}\text { Wavelength } \\
\text { (§) }\end{array}$} & \multicolumn{2}{|c|}{ Intensity } & \multirow{2}{*}{$\begin{array}{l}\text { Wavenumber } \\
\left(\mathrm{cm}^{-1}\right)\end{array}$} & \multirow{2}{*}{ Classification } & \multirow{2}{*}{$\begin{array}{c}\text { Wavelength } \\
\text { (§) }\end{array}$} & \multicolumn{2}{|c|}{ Inten sity } & \multirow{2}{*}{$\begin{array}{l}\text { Wavenumber } \\
\qquad\left(\mathrm{cm}^{-1}\right)\end{array}$} & \multirow{2}{*}{ Classification } \\
\hline & Lamp & Spark & & & & Lamp & Spark & & \\
\hline 5415.711 & 8 & & 18459.664 & $18699_{2}-37159_{1}^{\circ}$ & 5379.112 & 300 & 20 & 18585.261 & $7795_{4}^{\circ}-263805$ \\
\hline 5415.558 & $75 b$ & & 18460.186 & $15863_{2}-34324_{2}^{\circ}$ & 5378.837 & 400 & 40 & 18586.211 & $14481 \stackrel{\circ}{6}-330686$ \\
\hline 5415.528 & $200 \mathrm{~b}$ & 2 & 18460.288 & $7795_{4}^{\circ}-26255_{4}$ & 5378.169 & 50 & 4 & 18588.520 & $17501^{\circ}-36089_{4}$ \\
\hline 5414.920 & 5 & 1 & 18462.361 & $18053^{\circ}-365153$ & 5377.970 & 10 & 1 & 18589.207 & $14206_{4}^{\circ}-327963$ \\
\hline 5414.920 & 5 & 1 & 18462.361 & $21738^{\circ}-402003$ & 5376.778 & 200 & 50 & 18593.328 & $11241_{3}^{\circ}-29835_{3}$ \\
\hline 5413.917 & 10 & 1 & 18465.781 & $8800_{4}-27266_{4}^{\circ}$ & 5376.600 & 4 & & 18593.944 & $19503_{3}^{\circ}-38097_{2}$ \\
\hline 5411.406 & 3 & & 18474.350 & $18930_{3}^{\circ}-374043$ & 5376.366 & $2 b$ & & 18594.753 & $18809_{4}^{\circ}-374043$ \\
\hline 5411.311 & 5 & & 18474.674 & $154934-33967_{3}^{\circ}$ & 5375.497 & 20 & & 18597.759 & $19588^{\circ}-38186_{4}$ \\
\hline 5410.769 & 400 & 15 & 18476.525 & $6362_{2}-24838^{\circ}$ & 5374.998 & 50 & $8 \mathrm{~h}$ & 18599.486 & $13175_{4}^{\circ}-317743$ \\
\hline 5410.313 & $15 b$ & & 18478.082 & $11802_{2}-30281_{1}^{\circ}$ & 5373.394 & 40 & 2 & 18605.038 & $17073_{1}-35678_{i}$ \\
\hline 5409.603 & 2 & & 18480.507 & $18614^{\circ}-370942$ & 5372.703 & 200 & 25 & 18607.431 & $11241_{3}^{\circ}-29849_{4}$ \\
\hline 5409.304 & 4 & & 18481.528 & $14481^{\circ}-329635$ & 5372.230 & 1 & & 18609.069 & $216454-40254^{\circ}$ \\
\hline 5408.749 & 20 & $2 \mathrm{~h}$ & 18483.425 & $13297_{4}-31780_{3}^{\circ}$ & 5372.117 & 3 & & 18609.460 & $18431_{3}-37041_{4}^{\circ}$ \\
\hline 5408.192 & 2 & & 18485.328 & $20214_{3}^{\circ}-38700_{4}$ & 5371.999 & 8 & & 18609.869 & $18549_{2}-37159_{1}^{\circ}$ \\
\hline 5407.652 & $400 \mathrm{~b}$ & $40 \mathrm{~b}$ & 18487.175 & $111977^{\circ}-296845$ & 5371.678 & 3 & & 18610.981 & $75023-26113_{2}^{\circ}$ \\
\hline 5407.567 & $75 b$ & $10 \mathrm{~b}$ & 18487.465 & $20522_{2}^{\circ}-39010_{3}$ & 5371.124 & 50 & 1 & 18612.901 & $13962_{1}-32575_{2}^{\circ}$ \\
\hline 5406.755 & 50 & 4 & 18490.241 & $16217_{2}^{\circ}-34707_{3}$ & 5371.124 & 50 & 1 & 18612.901 & $15970_{3}-34583^{\circ}$ \\
\hline 5405.787 & 20 & 1 & 18493.552 & $18809_{4}^{\circ}-37303_{4}$ & 5370.709 & 150 & 2 & 18614.339 & $0_{2}-18614^{\circ}$ \\
\hline 5404.708 & 15 & & 18497.244 & $18809_{4}^{\circ}-37307_{3}$ & 5370.522 & 5 & & 18614.987 & $20054_{2}-38669_{2}^{\circ}$ \\
\hline 5403.200 & 200 & 15 & 18502.407 & $16346_{4}^{\circ}-348495$ & 5369.890 & 10 & 1 & 18617.178 & $21077 \circ-396945$ \\
\hline 5401.582 & $8 b$ & $1 \mathrm{u}$ & 18507.949 & $15863_{2}-34371_{2}^{\circ}$ & 5369.447 & 200 & 5 & 18618.714 & $6362_{2}-24981_{3}^{\circ}$ \\
\hline 5401.536 & $10 \mathrm{~b}$ & 1 & 18508.107 & $15493_{4}-34001_{4}^{\circ}$ & 5369.284 & 200 & 8 & 18619.279 & $5563_{1}-24182_{2}^{\circ}$ \\
\hline 5400.775 & 8 & & 18510.714 & $13088_{3}-31599_{2}^{\circ}$ & 5368.902 & 5 & & 18620.604 & $20054_{2}-38675_{3}^{\circ}$ \\
\hline 5400.145 & 150 & 5 & 18512.874 & $13945_{3}^{\circ}-324584$ & 5368.654 & 10 & 1 & 18621.464 & $16783^{\circ}-35405_{3}$ \\
\hline 5399.620 & 50 & 100 & 18514.674 & $112411_{3}^{\circ}-297564$ & 5367.849 & 3 & & 18624.257 & $21645_{4}-40270_{4}^{\circ}$ \\
\hline 5399.427 & $8 \mathrm{~h}$ & & 18515.336 & $21165_{3}^{\circ}-39680_{2}$ & 5367.234 & 51 & & 18626.391 & $220984-40724{ }^{\circ}$ \\
\hline 5398.920 & 400 & 40 & 18517.075 & $16783_{4}^{\circ}-353005$ & 5366.557 & 2 & & 18628.740 & $19516_{2}^{\circ}-38145_{3}$ \\
\hline 5398.704 & 150 & 4 & 18517.815 & $6362_{2}-24880_{1}^{\circ}$ & 5365.913 & 2 & & 18630.976 & $20322{ }^{\circ}-389535$ \\
\hline 5398.510 & 8 & 1 & 18518.481 & $195880^{\circ}-381065$ & 5365.520 & $50 \mathrm{~b}$ & $3 b$ & 18632.341 & $17501 \stackrel{\circ}{-}-361335$ \\
\hline 5398.205 & 150 & 4 & 18519.527 & $14032_{2}^{\circ}-32551_{3}$ & 5364.985 & 4 & 1 & 18634.199 & $14465_{2}^{\circ}-33099_{3}$ \\
\hline 5397.890 & 20 & 1 & 18520.608 & $195322_{4}-38053_{3}^{\circ}$ & 5364.682 & $8 b$ & & 18635.251 & $10783_{2}^{\circ}-29418_{2}$ \\
\hline 5397.731 & 1 & & 18521.153 & $21575_{2}-40096_{2}^{\circ}$ & 5364.447 & 15 & 5 & 18636.067 & $17411_{3}^{\circ}-36047_{2}$ \\
\hline 5397.499 & 2 & & 18521.949 & $15970_{3}-34492_{4}^{\circ}$ & 5363.931 & 5 & & 18637.860 & $16351_{0}-34989^{\circ}$ \\
\hline 5397.445 & 5 & & 18522.135 & $15166^{\circ}-33689_{2}$ & 5363.880 & 5 & & 18638.037 & $22248_{2}^{\circ}-40886_{2}$ \\
\hline 5397.139 & 201 & & 18523.185 & $17354_{1}^{\circ}-35877_{2}$ & 5362.576 & 200 & 5 & 18642.570 & $3865_{1}-22508_{2}^{\circ}$ \\
\hline 5396.109 & 50 & 4 & 18526.720 & $16346_{4}^{\circ}-34873_{4}$ & 5361.156 & 75 & 3 & 18647.507 & $4961_{4}-236098$ \\
\hline 5396.109 & 50 & 4 & 18526.720 & $16351_{0}-34878^{\circ}$ & 5360.697 & 8 & & 18649.104 & $19039_{2}^{\circ}-37688_{3}$ \\
\hline 5394.761 & 300 & 20 & 18531.350 & $38651-22396 i$ & 5360.150 & 300 & 5 & 18651.007 & $3687_{2}-22338^{\circ}$ \\
\hline 5393.972 & 150 & 8 & 18534.060 & $75023-26036_{3}^{\circ}$ & 5359.827 & 100 & 4 & 18652.131 & 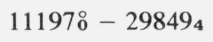 \\
\hline 5393.873 & $50 \mathrm{~b}$ & 3 & 18534.401 & $8111_{4}-26645^{\circ}$ & 5359.432 & 5 & & 18653.506 & $17224_{2}^{\circ}-35877_{2}$ \\
\hline 5393.873 & $50 \mathrm{~b}$ & 3 & 18534.401 & $18699_{2}-37234_{2}^{\circ}$ & 5358.713 & 300 & 15 & 18656.009 & $132974-31953_{4}^{\circ}$ \\
\hline 5392.768 & 5 & & 18538.198 & $22163_{4}^{\circ}-407014$ & 5358.558 & 2 & & 18656.548 & $16217_{2}^{\circ}-348743$ \\
\hline 5390.575 & 2 & & 18545.740 & $20214_{3}^{\circ}-38760_{4}$ & 5358.415 & 3 & & 18657.046 & $16783_{4}^{\circ}-35440_{3}$ \\
\hline 5390.429 & 300 & 75 & 18546.242 & $7502_{3}-26048_{4}^{\circ}$ & 5358.081 & 5 & & 18658.209 & $142045-32862_{4}^{\circ}$ \\
\hline 5388.057 & 300 & 20 & 18554.407 & $15166_{3}^{\circ}-33721_{2}$ & 5357.743 & 8 & 1 & 18659.386 & $18574_{1}-37234_{2}^{\circ}$ \\
\hline 5386.611 & 500 & 20 & 18559.388 & $4961_{4}-23521_{3}^{\circ}$ & 5357.338 & 2 & & 18660.797 & $21539_{4}^{\circ}-40200_{3}$ \\
\hline 5386.611 & 500 & 20 & 18559.388 & $11197^{\circ}-29756_{4}$ & 5356.672 & 10 & & 18663.117 & $19227^{\circ}-378905$ \\
\hline 5386.149 & $2 b$ & & 18560.980 & $3687_{2}-22248_{2}^{\circ}$ & 5356.278 & 1 & & 18664.490 & $17398_{3}-36062_{4}^{\circ}$ \\
\hline 5386.111 & 201 & & 18561.110 & $15736^{\circ}-34298_{1}$ & 5356.137 & 10 & & 18664.981 & $15166_{3}^{\circ}-33831_{4}$ \\
\hline 5384.301 & 100 & 15 & 18567.350 & $171665-35733^{\circ}$ & 5355.638 & 100 & 3 & 18666.720 & $11877 i-30544_{2}$ \\
\hline 5382.175 & $15 b$ & $1 \mathrm{u}$ & 18574.684 & $14206_{4}^{\circ}-32781_{4}$ & 5354.602 & 40 & 1 & 18670.332 & $2869_{3}-21539_{4}^{\circ}$ \\
\hline 5381.368 & $25 b$ & 1 & 18577.470 & $10526^{\circ}-291044$ & 5353.977 & $5 \mathrm{~h}$ & & 18672.511 & $21165_{3}^{\circ}-39837_{3}$ \\
\hline 5379.884 & 8 & 1 & 18582.594 & $13088_{3}-31671_{4}^{\circ}$ & 5353.154 & 2 & & 18675.382 & $21594_{3}-40270_{4}^{\circ}$ \\
\hline 5379.325 & $8 b$ & & 18584.525 & $18069_{3}^{\circ}-36653_{2}$ & 5353.154 & 2 & & 18675.382 & $9804_{5}-28480_{4}^{\circ}$ \\
\hline 5379.285 & $10 \mathrm{~b}$ & 1 & 18584.663 & $18574_{1}-37159_{1}^{\circ}$ & 5353.073 & 50 & 4 & 18675.664 & $17398_{3}-36074_{3}^{\circ}$ \\
\hline
\end{tabular}


TABLE 4. Classified lines of Th $\mathrm{I}$-continued

\begin{tabular}{|c|c|c|c|c|c|c|c|c|c|}
\hline \multirow{2}{*}{$\begin{array}{l}\text { Wavelength } \\
\text { (A) }\end{array}$} & \multicolumn{2}{|c|}{ Intensity } & \multirow{2}{*}{$\begin{array}{l}\text { Wavenumber } \\
\left(\mathrm{cm}^{-1}\right)\end{array}$} & \multirow{2}{*}{ Classification } & \multirow{2}{*}{$\begin{array}{c}\text { Wavelength } \\
\text { (̊) }\end{array}$} & \multicolumn{2}{|c|}{ Inten sity } & \multirow{2}{*}{$\begin{array}{l}\text { Wavenumber } \\
\left(\mathrm{cm}^{-1}\right)\end{array}$} & \multirow{2}{*}{ Classification } \\
\hline & Lamp & Spark & & & & Lamp & Spark & & \\
\hline 5352.992 & $50 \mathrm{~b}$ & 15 & 18675.947 & $12847_{3}-31523 \stackrel{\circ}{3}$ & 5319.023 & 2 & & 18795.216 & $20214_{3}^{\circ}-39010_{3}$ \\
\hline 5352.304 & $3 \mathrm{~b}$ & & 18678.347 & $22098_{4}-40776_{4}^{\circ}$ & 5318.110 & 75 & 3 & 18798.443 & $16783_{4}^{\circ}-355825$ \\
\hline 5352.272 & $5 b$ & & 18678.459 & $17411_{3}^{\circ}-36089_{4}$ & 5317.912 & 8 & & 18799.143 & $22401_{1}-41200_{2}^{\circ}$ \\
\hline 5351.996 & 10 & & 18679.423 & $8111_{4}-26790_{4}^{\circ}$ & 5317.494 & 200 & 4 & 18800.620 & $8243^{\circ}-270443$ \\
\hline 5351.839 & 150 & 8 & 18679.970 & $11601_{1}-30281_{1}^{\circ}$ & 5316.783 & 15 & & 18803.135 & $13847_{2}-32650_{1}^{\circ}$ \\
\hline 5351.126 & 200 & 25 & 18682.459 & $15618_{3}^{\circ}-34301_{4}$ & 5315.834 & 5 & & 18806.491 & $17847_{2}^{\circ}-36653_{2}$ \\
\hline 5350.538 & 50 & 4 & 18684.512 & $195324-38216^{\circ}$ & 5315.230 & 150 & & 18808.629 & $14465_{2}^{\circ}-33273_{1}$ \\
\hline 5350.206 & 50 & 1 & 18685.672 & $20566_{4}^{\circ}-392525$ & 5314.251 & $8 b$ & 1 & 18812.093 & $17073_{1}-35885_{2}^{\circ}$ \\
\hline 5349.425 & 3 & & 18688.400 & $13962_{1}-32650_{1}^{\circ}$ & 5314.205 & $10 \mathrm{~b}$ & 2 & 18812.256 & $19948_{4}^{\circ}-38760_{4}$ \\
\hline 5349.003 & 75 & 3 & 18689.874 & $10414_{4}^{\circ}-291044$ & 5314.178 & $10 \mathrm{~b}$ & & 18812.352 & $15618_{3}^{\circ}-344313$ \\
\hline 5348.532 & 4 & 1 & 18691.520 & $22669_{3}^{\circ}-413613$ & 5313.233 & $5 \mathrm{~h}$ & & 18815.698 & $19273_{2}-38088_{2}^{\circ}$ \\
\hline 5348.310 & 50 & 1 & 18692.296 & $13088_{3}-31780_{3}^{\circ}$ & 5312.904 & 200 & 15 & 18816.863 & $7280_{2}-26096_{3}^{\circ}$ \\
\hline 5347.973 & 150 & 5 & 18693.474 & $4961_{4}-23655_{4}^{\circ}$ & 5312.781 & 50 & 3 & 18817.298 & $111970^{\circ}-30014_{4}$ \\
\hline 5346.766 & 50 & 2 & 18697.694 & $23015^{\circ}-417125$ & 5312.643 & 150 & 15 & 18817.787 & $13847_{2}-32665^{\circ}$ \\
\hline 5345.241 & 5 & & 18703.028 & $13962_{1}-32665_{1}^{\circ}$ & 5312.532 & 400 & 40 & 18818.180 & $55631-24381_{1}^{\circ}$ \\
\hline 5344.308 & 3 & & 18706.293 & $18069_{3}^{\circ}-36775_{3}$ & 5312.001 & $400 \mathrm{r}$ & 25 & 18820.062 & $3687_{2}-22508_{2}^{\circ}$ \\
\hline 5343.581 & $500 \mathrm{r}$ & 40 & 18708.838 & $3687_{2}-22396_{1}^{\circ}$ & 5311.870 & $75 b$ & 2 & 18820.526 & $13175_{4}^{\circ}-31995_{4}$ \\
\hline 5343.361 & $50 \mathrm{~b}$ & & 18709.608 & $17501 \stackrel{\circ}{\circ}-362105$ & 5311.174 & 20 & 1 & 18822.992 & $17224_{2}^{\circ}-36047_{2}$ \\
\hline 5341.206 & 75 & 3 & 18717.157 & $20566_{4}^{\circ}-392834$ & 5310.432 & 5 & & 18825.622 & $15618^{\circ}-344442$ \\
\hline 5340.650 & 201 & & 18719.106 & $15863_{2}-34583 \stackrel{\circ}{3}$ & 5309.616 & 150 & 5 & 18828.515 & $16217_{2}^{\circ}-350461$ \\
\hline 5340.501 & 200 & 10 & 18719.628 & $165546-352730^{\circ}$ & 5309.052 & $2 \mathrm{~h}$ & & 18830.515 & $215943-40425^{\circ}$ \\
\hline 5339.896 & 50 & 5 & 18721.749 & $18053_{4}^{\circ}-36775_{3}$ & 5308.808 & 10 & 1 & 18831.381 & $22855_{3}^{\circ}-41686_{3}$ \\
\hline 5338.641 & 40 & 1 & 18726.150 & $132974-32023{ }^{\circ}$ & 5308.726 & 10 & 1 & 18831.672 & $18053 \stackrel{\circ}{4}-36885_{4}$ \\
\hline 5338.366 & 40 & 15 & 18727.114 & $15863_{2}-34591_{i}$ & 5308.310 & 100 & 10 & 18833.147 & $7280_{2}-26113_{2}^{\circ}$ \\
\hline 5338.285 & $25 b$ & 1 & 18727.399 & $10414_{4}^{\circ}-291415$ & 5306.987 & 75 & 4 & 18837.842 & $13175_{4}^{\circ}-320124$ \\
\hline 5338.215 & $50 \mathrm{~b}$ & 1 & 18727.644 & $13847_{2}-32575_{2}^{\circ}$ & 5306.166 & 5 & & 18840.757 & $17959_{4}-36800_{4}^{\circ}$ \\
\hline 5337.502 & 2 & & 18730.146 & $211435-398730^{\circ}$ & 5305.304 & 51 & & 18843.818 & $14465_{2}^{\circ}-33309_{2}$ \\
\hline 5336.785 & 50 & 1 & 18732.662 & $14481{ }^{\circ}-33214_{5}$ & 5303.557 & 20 & 1 & 18850.025 & $16783_{4}^{\circ}-35633_{4}$ \\
\hline 5336.315 & 40 & 1 & 18734.312 & $159703-34704_{3}^{\circ}$ & 5303.483 & 75 & 3 & 18850.288 & $12114_{2}^{\circ}-30964_{3}$ \\
\hline 5335.373 & 10 & 3 & 18737.620 & $18011^{\circ}-367496$ & 5303.334 & 50 & 2 & 18850.818 & $13945_{3}^{\circ}-32796_{3}$ \\
\hline 5334.145 & 751 & 1 & 18741.933 & $202143_{3}^{\circ}-389564$ & 5303.162 & 40 & 1 & 18851.429 & $154934-34344_{4}^{\circ}$ \\
\hline 5333.386 & $75 b$ & 1 & 18744.601 & $5563_{1}-24307_{2}^{\circ}$ & 5302.779 & 1 & & 18852.791 & $195324-38385^{\circ}$ \\
\hline 5333.200 & $8 \mathrm{~b}$ & & 18745.254 & $19039_{2}^{\circ}-37784_{2}$ & 5302.407 & 10 & & 18854.114 & $18930_{3}^{\circ}-37784_{2}$ \\
\hline 5332.120 & 2 & & 18749.051 & $18069_{3}^{\circ}-36818_{2}$ & 5300.524 & 300 & 5 & 18860.811 & $75023-26363_{2}^{\circ}$ \\
\hline 5331.749 & 50 & 2 & 18750.356 & $11802_{2}-305532$ & 5300.321 & 4 & & 18861.534 & $15863_{2}-34725_{3}^{\circ}$ \\
\hline 5331.481 & 8 & & 18751.298 & $12847_{3}-31599_{2}^{\circ}$ & 5299.787 & 2 & & 18863.434 & $195033_{3}^{\circ}-383664$ \\
\hline 5330.517 & 8 & & 18754.689 & $18053_{4}^{\circ}-368083$ & 5299.376 & 2 & & 18864.897 & $13088_{3}-31953_{4}^{\circ}$ \\
\hline 5330.340 & 51 & & 18755.312 & $15970_{3}-34725_{3}^{\circ}$ & 5298.714 & 8 & 2 & 18867.254 & $19713_{3}-38580_{4}^{\circ}$ \\
\hline 5330.082 & $200 \mathrm{~b}$ & 5 & 18756.220 & $7280_{2}-26036_{3}^{\circ}$ & 5298.559 & 75 & 2 & 18867.806 & $10783_{2}^{\circ}-29650_{2}$ \\
\hline 5330.016 & $50 \mathrm{~b}$ & & 18756.452 & $14206^{\circ}-329635$ & 5298.284 & 200 & 4 & 18868.785 & $2869_{3}-21738_{2}^{\circ}$ \\
\hline 5329.605 & 2 & & 18757.899 & $18930_{3}^{\circ}-37688_{3}$ & 5297.844 & $100 \mathrm{~b}$ & 5 & 18870.352 & $16346_{4}^{\circ}-352165$ \\
\hline 5328.009 & 8 & & 18763.517 & $211435-39906_{4}^{\circ}$ & 5297.746 & $300 \mathrm{~b}$ & 15 & 18870.701 & $8800_{4}-27670_{3}^{\circ}$ \\
\hline 5327.862 & 10 & & 18764.035 & $14032_{2}^{\circ}-327963$ & 5296.845 & 50 & 2 & 18873.911 & $18011^{\circ}-36885_{4}$ \\
\hline 5326.976 & $500 \mathrm{r}$ & 40 & 18767.156 & $8111_{4}-26878 \stackrel{\circ}{3}$ & 5296.279 & 300 & 5 & 18875.928 & $9804_{5}-28680_{4}^{\circ}$ \\
\hline 5326.278 & $100 \mathrm{~b}$ & 8 & 18769.615 & $11241_{3}^{\circ}-300113$ & 5295.674 & $50 \mathrm{~b}$ & $4 \mathrm{~b}$ & 18878.085 & $179594-36837^{\circ}$ \\
\hline 5326.192 & 50 & 2 & 18769.918 & $18011{ }^{\circ}-367815$ & 5295.089 & 50 & 1 & 18880.170 & $11877_{1}^{\circ}-30758_{2}$ \\
\hline 5325.434 & 100 & 2 & 18772.590 & $11241_{3}^{\circ}-30014_{4}$ & 5294.697 & $25 b$ & 1 & 18881.568 & $15970{ }_{3}-34851_{2}^{\circ}$ \\
\hline 5324.591 & 10 & & 18775.562 & $12114_{2}^{\circ}-30889_{1}$ & 5294.397 & 200 & 3 & 18882.638 & $7502_{3}-26384_{4}^{\circ}$ \\
\hline 5323.851 & 151 & 3 & 18778.172 & $19588 \circ-383664$ & 5294.070 & 50 & 1 & 18883.804 & $19532_{4}-38416_{3}^{\circ}$ \\
\hline 5323.527 & 8 & 1 & 18779.315 & $21645_{4}-40425_{4}^{\circ}$ & 5293.435 & 3 & 1 & 18886.070 & $17411_{3}^{\circ}-36297_{4}$ \\
\hline 5323.406 & 150 & 3 & 18779.741 & $19273_{2}-38053_{3}^{\circ}$ & 5293.137 & 50 & 1 & 18887.133 & $21539_{4}^{\circ}-40426_{4}$ \\
\hline 5322.901 & 200 & 5 & 18781.523 & $13088_{3}-31870_{2}^{\circ}$ & 5293.077 & 3 & & 18887.347 & $21165_{3}^{\circ}-400524$ \\
\hline 5321.873 & 2 & & 18785.151 & $20566_{4}^{\circ}-393515$ & 5292.666 & 3 & & 18888.814 & $18930_{3}^{\circ}-37819_{4}$ \\
\hline 5321.691 & 3 & & 18785.793 & $19817_{1}^{\circ}-38602_{2}$ & 5292.077 & 10 & & 18890.916 & $11241_{3}^{\circ}-301322$ \\
\hline 5319.742 & 8 & & 18792.676 & $16783{ }^{\circ}-355763$ & 5291.818 & 200 & 5 & 18891.840 & $10526^{\circ}-29418_{2}$ \\
\hline
\end{tabular}


TABLE 4. Classified lines of Th I-continued

\begin{tabular}{|c|c|c|c|c|c|c|c|c|c|}
\hline \multirow{2}{*}{$\begin{array}{c}\text { Wavelength } \\
\text { (Å) }\end{array}$} & \multicolumn{2}{|c|}{ Intensity } & \multirow{2}{*}{$\begin{array}{l}\text { Wavenumber } \\
\left(\mathrm{cm}^{-1}\right)\end{array}$} & \multirow{2}{*}{ Classification } & \multirow{2}{*}{$\begin{array}{c}\text { Wavelength } \\
\text { (§) }\end{array}$} & \multicolumn{2}{|c|}{ Inten sity } & \multirow{2}{*}{$\begin{array}{l}\text { Wavenumber } \\
\left(\mathrm{cm}^{-1}\right)\end{array}$} & \multirow{2}{*}{ Classification } \\
\hline & Lamp & Spark & & & & Lamp & Spark & & \\
\hline 5291.638 & 3 & & 18892.483 & $14206_{4}^{\circ}-33099_{3}$ & 5253.534 & 8 & & 19029.509 & $20322{ }^{\circ}-393515$ \\
\hline 5289.624 & 75 & 2 & 18899.676 & $13297_{4}-32197_{3}^{\circ}$ & 5252.568 & 15 & & 19033.009 & $2869_{3}-21902_{4}^{\circ}$ \\
\hline 5287.648 & 3 & & 18906.739 & $13847_{2}-32754_{3}^{\circ}$ & 5251.373 & 501 & 2 & 19037.340 & $18699_{2}-37736_{2}^{\circ}$ \\
\hline 5285.183 & $3 \mathrm{~h}$ & & 18915.557 & $20922_{2}^{\circ}-39837_{3}$ & 5250.874 & 50 & 1 & 19039.149 & $0_{2}-19039_{2}^{\circ}$ \\
\hline 5283.697 & 50 & 2 & 18920.877 & $11802_{2}-30723 i$ & 5250.171 & 10 & & 19041.698 & $18574_{1}-37616^{\circ}$ \\
\hline 5282.552 & 15 & & 18924.978 & $17411_{3}^{\circ}-36336_{3}$ & 5248.587 & 10 & & 19047.445 & $16783^{\circ}-358313$ \\
\hline 5282.402 & 200 & 4 & 18925.515 & $14465_{2}^{\circ}-33390_{1}$ & 5248.199 & 20 & & 19048.853 & $17959_{4}-37008 \circ$ \\
\hline 5281.070 & 300 & 2 & 18930.288 & $0_{2}-18930_{3}^{\circ}$ & 5247.361 & 40 & & 19051.895 & $10783 \stackrel{2}{2}-298353$ \\
\hline 5280.520 & 50 & & 18932.260 & $16217_{2}^{\circ}-35149_{2}$ & 5247.200 & $50 \mathrm{~b}$ & 2 & 19052.480 & $8800_{4}-278520^{\circ}$ \\
\hline 5280.346 & 50 & 2 & 18932.884 & $12847_{3}-31780_{3}^{\circ}$ & 5245.698 & 10 & & 19057.935 & $19039_{2}^{\circ}-38097_{2}$ \\
\hline 5279.766 & 2 & & 18934.964 & $142260-33161_{1}^{\circ}$ & 5244.647 & $8 \mathrm{~b}$ & 1 & 19061.754 & $19948_{4}^{\circ}-39010_{3}$ \\
\hline 5279.200 & 50 & 1 & 18936.994 & $154934-34430_{3}^{\circ}$ & 5244.594 & $10 \mathrm{~b}$ & 1 & 19061.947 & $18069_{3}^{\circ}-37131_{3}$ \\
\hline 5277.404 & 10 & & 18943.438 & $14465_{2}^{\circ}-33408_{3}$ & 5244.594 & $10 \mathrm{~b}$ & 1 & 19061.947 & $18930_{3}^{\circ}-37992_{4}^{4}$ \\
\hline 5277.341 & 3 & & 18943.665 & $19273_{2}-38216_{3}^{\circ}$ & 5243.746 & 50 & 1 & 19065.029 & $14243 i-33309_{2}$ \\
\hline 5277.147 & 75 & 1 & 18944.361 & $63622-25306_{2}^{\circ}$ & 5243.406 & 8 & & 19066.265 & $142045-33270_{4}^{\circ}$ \\
\hline 5276.198 & 2 & & 18947.768 & $21252_{2}^{\circ}-40200_{3}$ & 5243.237 & 3 & & 19066.880 & $18549_{2}-37616_{1}^{\circ}$ \\
\hline 5274.948 & 8 & 1 & 18952.258 & $11601_{1}-30553_{2}^{\circ}$ & 5243.120 & 3 & & 19067.305 & $14032_{2}^{\circ}-33099_{3}$ \\
\hline 5274.581 & 5 & & 18953.577 & $20566_{4}^{\circ}-39520_{4}$ & 5242.687 & 20 & & 19068.880 & $20214^{\circ}-392834$ \\
\hline 5274.391 & 75 & 3 & 18954.260 & $16346 \stackrel{\circ}{-}-35300_{5}$ & 5241.858 & 50 & 20 & 19071.896 & $13088_{3}-32160_{2}^{\circ}$ \\
\hline 5274.121 & 200 & 5 & 18955.230 & $4961_{4}-23916_{4}^{\circ}$ & 5241.223 & 8 & 1 & 19074.206 & $17411_{3}^{\circ}-36485_{2}$ \\
\hline 5273.595 & 2 & & 18957.121 & $15490 \AA-34447_{4}$ & 5241.153 & 8 & 1 & 19074.461 & $18011^{\circ}-370854$ \\
\hline 5273.531 & 40 & & 18957.351 & $18699_{2}-37656_{3}^{\circ}$ & 5240.789 & 3 & & 19075.786 & $19713_{3}-38788_{3}^{\circ}$ \\
\hline 5273.134 & $100 \mathrm{~b}$ & 10 & 18958.778 & $11802_{2}-30761_{3}^{\circ}$ & 5240.789 & 3 & & 19075.786 & $19713_{3}-38788^{\circ}$ \\
\hline 5272.929 & 100 & 20 & 18959.515 & $63622-253213$ & 5240.341 & 2 & & 19077.417 & $18053{ }^{\circ}-371313$ \\
\hline 5272.092 & 5 & & 18962.525 & $18930_{3}^{\circ}-37892_{4}$ & 5239.984 & 2 & & 19078.717 & $216454-407240^{\circ}$ \\
\hline 5271.023 & 20 & 3 & 18966.371 & $171665-36132{ }^{\circ}$ & 5239.550 & 50 & 3 & 19080.297 & $63622_{2}-25442{ }_{3}^{\circ}$ \\
\hline 5270.832 & 50 & 2 & 18967.058 & $199866-389535$ & 5239.079 & 2 & & 19082.012 & $19273_{2}-38355_{2}^{\circ}$ \\
\hline 5269.794 & 75 & 4 & 18970.794 & $15490 \circ-344605$ & 5238.812 & $75 \mathrm{~h}$ & 10 & 19082.985 & $7280_{2}-26363_{2}^{\circ}$ \\
\hline 5268.539 & 2 & & 18975.313 & $21077 \circ-400524$ & 5237.989 & 3 & & 19085.983 & $19516_{2}^{\circ}-38602_{2}$ \\
\hline 5268.158 & 2 & & 18976.685 & $16351_{0}-35328^{\circ}$ & 5237.229 & 5 & 1 & 19088.753 & $15618_{3}^{\circ}-347073$ \\
\hline 5266.829 & 2 & & 18981.474 & $1214_{2}^{\circ}-310951$ & 5236.766 & 10 & 1 & 19090.440 & $17398_{3}-36488_{2}^{\circ}$ \\
\hline 5266.710 & 300 & 15 & 18981.902 & $3687_{2}-22669_{3}^{\circ}$ & 5235.720 & 4 & 1 & 19094.254 & $16346_{4}^{\circ}-35440_{3}$ \\
\hline 5266.073 & 1 & & 18984.199 & $173983-36382_{4}^{\circ}$ & 5234.243 & 75 & & 19099.642 & $14465_{2}^{\circ}-335643$ \\
\hline 5265.081 & 10 & & 18987.775 & $158632-34851_{2}^{\circ}$ & 5234.107 & 100 & 1 & 19100.138 & $8243_{2}^{\circ}-273433$ \\
\hline 5264.335 & 20 & & 18990.466 & $19817_{1}^{\circ}-38807_{2}$ & 5232.841 & 8 & & 19104.759 & $17411_{3}^{\circ}-365153$ \\
\hline 5263.892 & $15 b$ & 1 & 18992.064 & $13962_{1}-32954_{2}^{\circ}$ & 5232.341 & $40 \mathrm{~b}$ & 2 & 19106.585 & $19039_{2}^{\circ}-38145_{3}$ \\
\hline 5262.621 & $50 \mathrm{~b}$ & 4 & 18996.651 & $13297_{4}-32294_{4}^{\circ}$ & 5232.278 & $25 b$ & 1 & 19106.815 & $13847_{2}-32954_{2}^{\circ}$ \\
\hline 5262.024 & 5 & 1 & 18998.806 & $154934-34492_{4}^{\circ}$ & 5231.800 & 40 & 1 & 19108.561 & $13088_{3}-32197_{3}^{\circ}$ \\
\hline 5261.530 & $50 \mathrm{~b}$ & 1 & 19000.590 & $15166_{3}^{\circ}-341673$ & 5231.659 & 3 & & 19109.076 & $171665-362758$ \\
\hline 5261.474 & $50 \mathrm{~b}$ & 3 & 19000.792 & $7795_{4}^{\circ}-26796_{3}$ & 5231.160 & $1000 \mathrm{r}$ & 40 & 19110.899 & $25580-21668^{\circ}$ \\
\hline 5260.106 & 200 & 8 & 19005.734 & $7502_{3}-26508_{3}^{\circ}$ & 5230.012 & 15 & & 19115.093 & $17073_{1}-36188_{2}^{\circ}$ \\
\hline 5259.779 & 75 & 2 & 19006.915 & $7280_{2}-26287_{i}^{\circ}$ & 5228.996 & 75 & 1 & 19118.807 & $13175_{4}^{\circ}-322935$ \\
\hline 5259.587 & 20 & 1 & 19007.609 & $14206^{\circ}-33214_{5}$ & 5227.904 & 8 & & 19122.801 & $11601_{1}-30723{ }^{\circ}$ \\
\hline 5259.353 & 3 & & 19008.455 & $19948{ }^{\circ}-389564$ & 5227.461 & 8 & & 19124.421 & $10526_{3}^{\circ}-29650_{2}$ \\
\hline 5258.908 & 8 & & 19010.063 & $11802_{2}-30812_{2}^{\circ}$ & 5227.078 & 40 & & 19125.823 & $11802{ }_{2}-30928^{\circ}$ \\
\hline 5258.779 & 5 & & 19010.530 & $16783_{4}^{\circ}-35794_{4}$ & 5226.527 & 50 & & 19127.839 & $98045-28932 \stackrel{\circ}{9}$ \\
\hline 5258.361 & $400 \mathrm{r}$ & 25 & 19012.041 & $38651-22877 i$ & 5225.755 & 5 & 1 & 19130.665 & $21645_{4}-40776_{4}^{\circ}$ \\
\hline 5258.212 & $150 \mathrm{~b}$ & 3 & 19012.580 & $116011-30613{ }^{\circ}$ & 5225.518 & 4 & & 19131.532 & $12114_{2}^{\circ}-31245_{2}$ \\
\hline 5257.607 & 4 & & 19014.767 & $15863_{2}-34878_{1}^{\circ}$ & 5224.928 & 3 & & 19133.693 & $18614_{1}^{\circ}-37748_{2}$ \\
\hline 5257.051 & 10 & 1 & 19016.778 & $18069_{3}^{\circ}-37085_{4}$ & 5224.693 & 5 & & 19134.553 & $15166^{\circ}-343014$ \\
\hline 5255.921 & $10 \mathrm{~b}$ & & 19020.867 & $2869_{3}-21890_{3}^{\circ}$ & 5222.909 & 2 & & 19141.089 & $14465_{2}^{\circ}-33606_{2}$ \\
\hline 5255.576 & 100 & 3 & 19022.115 & $12847_{3}-31870_{2}^{\circ}$ & 5222.765 & 40 & 1 & 19141.617 & $13297_{4}-32439_{4}^{\circ}$ \\
\hline 5254.262 & 1501 & 8 & 19026.872 & $13175_{4}^{\circ}-322025$ & 5222.268 & 50 & 1 & 19143.438 & $142470^{\circ}-33390_{1}$ \\
\hline 5254.111 & 40 & 4 & 19027.419 & $12114_{2}^{\circ}-31141_{3}$ & 5220.928 & 100 & 1 & 19148.352 & $8800_{4}-27948_{4}^{\circ}$ \\
\hline 5253.682 & 50 & 2 & 19028.973 & $17398_{3}-36427^{\circ}$ & 5220.707 & 100 & 1 & 19149.162 & $8111_{4}-27260_{3}^{\circ}$ \\
\hline
\end{tabular}


TABle 4. Classified lines of Th $\mathrm{I}$-continued

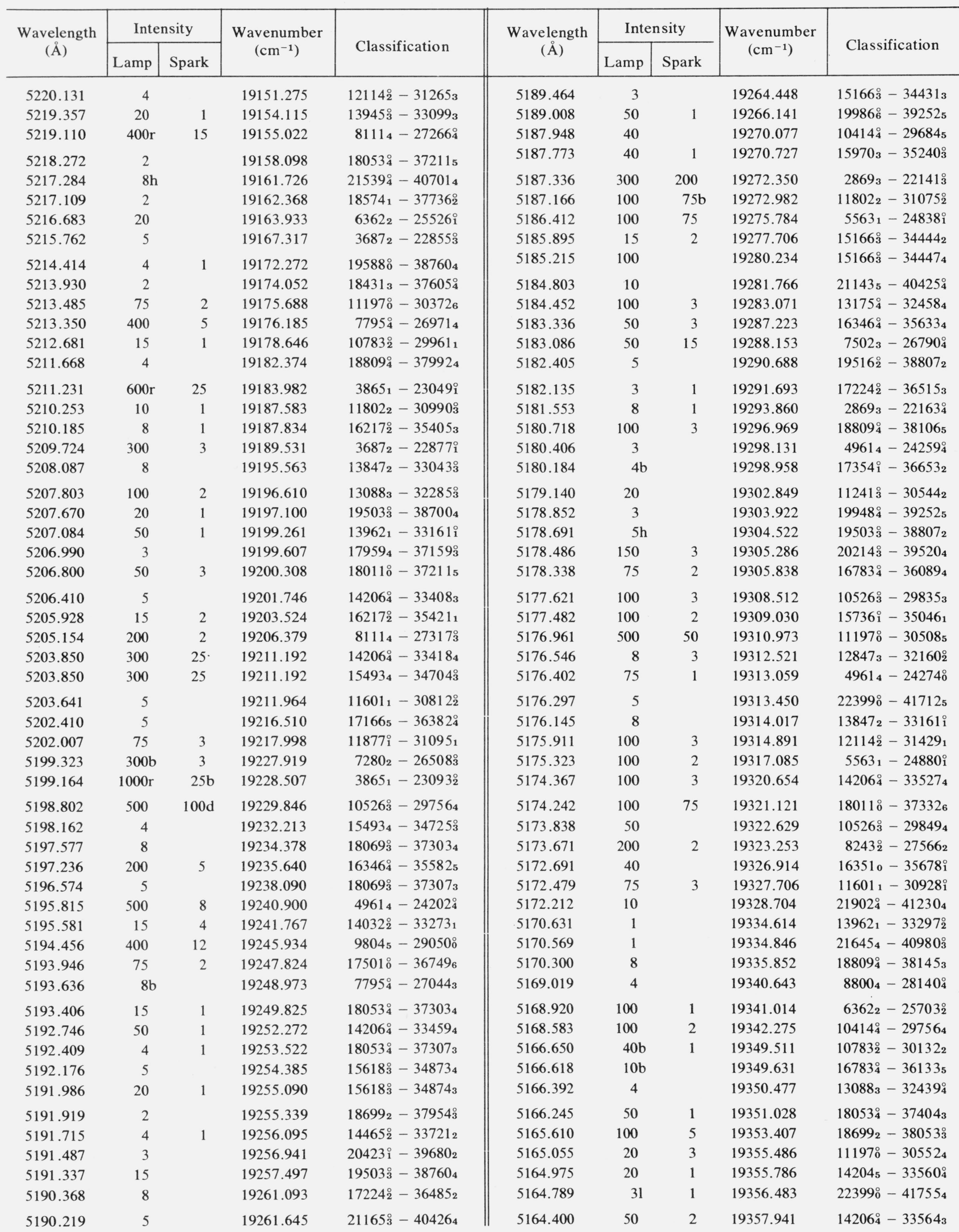


TABLE 4. Classified lines of Th $\mathrm{I}$-continued

\begin{tabular}{|c|c|c|c|c|c|c|c|c|c|}
\hline \multirow{2}{*}{$\begin{array}{c}\text { Wavelength } \\
(\AA)\end{array}$} & \multicolumn{2}{|c|}{ Intensity } & \multirow{2}{*}{$\begin{array}{l}\text { Wavenumber } \\
\left(\mathrm{cm}^{-1}\right)\end{array}$} & \multirow{2}{*}{ Classification } & \multirow{2}{*}{$\begin{array}{c}\text { Wavelength } \\
(\AA)\end{array}$} & \multicolumn{2}{|c|}{ Intensity } & \multirow{2}{*}{$\begin{array}{l}\text { Wavenumber } \\
\left(\mathrm{cm}^{-1}\right)\end{array}$} & \multirow{2}{*}{ Classification } \\
\hline & Lamp & Spark & & & & Lamp & Spark & & \\
\hline 5164.206 & 40 & 1 & 19358.669 & $14032_{2}^{\circ}-33390_{1}$ & 5133.389 & 10 & & 19474.882 & $11601_{1}-31075_{2}^{\circ}$ \\
\hline 5164.108 & 40 & 1 & 19359.036 & $16217_{2}^{\circ}-355763$ & 5132.748 & 50 & 1 & 19477.314 & $14243 i-33721_{2}$ \\
\hline 5163.459 & 400 & 250 & 19361.469 & $3687_{2}-23049_{1}^{\circ}$ & 5131.992 & 150 & 1 & 19480.183 & $11802_{2}-31283^{\circ}$ \\
\hline 5163.236 & 25 & 1 & 19362.305 & $14243 i-33606_{2}$ & 5131.257 & 5 & & 19482.973 & $17073_{1}-36556 i$ \\
\hline 5162.852 & 8 & & 19363.745 & $13945_{3}^{\circ}-33309_{2}$ & 5130.814 & $20 \mathrm{~b}$ & & 19484.656 & $16346_{4}^{\circ}-35831_{3}$ \\
\hline 5162.740 & 4 & & 19364.166 & $17411^{\circ}-367753$ & 5130.777 & $20 \mathrm{~b}$ & & 19484.796 & $10526_{3}^{\circ}-30011_{3}$ \\
\hline 5162.673 & 15 & 1 & 19364.417 & $173983-36762_{3}^{\circ}$ & 5130.235 & 150 & 2 & 19486.855 & $13088_{3}-32575_{2}^{\circ}$ \\
\hline 5162.553 & 50 & 8 & 19364.867 & $19588 \circ-389535$ & 5129.988 & 8 & & 19487.793 & $10526^{\circ}-30014_{4}$ \\
\hline 5162.356 & 75 & 5 & 19365.606 & $19986^{\circ}-393515$ & 5129.656 & 20 & & 19489.054 & $216454-41134{ }^{\circ}$ \\
\hline 5161.540 & 400 & 8 & 19368.667 & $8243_{2}^{\circ}-276123$ & 5129.206 & 50 & 1 & 19490.764 & $19039_{2}^{\circ}-38529_{3}$ \\
\hline 5160.718 & 300 & 50 & 19371.752 & $7280_{2}-26651_{2}^{\circ}$ & 5128.891 & 2 & & 19491.961 & $159700_{3}-35462_{3}^{\circ}$ \\
\hline 5160.280 & 5 & & 19373.396 & $19948{ }^{\circ}-393213$ & 5128.574 & 20 & & 19493.166 & $19516_{2}^{\circ}-39010_{3}$ \\
\hline 5159.621 & 100 & 2 & 19375.871 & $75023-26878_{3}^{\circ}$ & 5128.489 & 300 & 5 & 19493.489 & $7502_{3}-26995_{3}^{\circ}$ \\
\hline 5159.451 & 20 & 2 & 19376.509 & $13175_{4}^{\circ}-32551_{3}$ & 5125.950 & 200 & 3 & 19503.144 & $0_{2}-19503 \stackrel{\circ}{3}$ \\
\hline 5159.339 & 15 & & 19376.930 & $15863_{2}-35240_{3}^{\circ}$ & 5125.827 & $50 \mathrm{~b}$ & 1 & 19503.612 & $18549_{2}-38053 \stackrel{\circ}{3}$ \\
\hline 5158.605 & $800 \mathrm{r}$ & 50 & 19379.687 & $2869_{3}-22248_{2}^{\circ}$ & 5125.797 & $50 \mathrm{~b}$ & 2 & 19503.727 & $10783_{2}^{\circ}-30286_{1}$ \\
\hline 5157.426 & 100 & 2 & 19384.117 & $175018-368854$ & 5125.307 & $5 b$ & & 19505.591 & $21077_{0}^{\circ}-405825$ \\
\hline 5154.243 & 500 & 40 & 19396.088 & $7795_{4}^{\circ}-271915$ & 5124.925 & 3 & & 19507.045 & $19503_{3}^{\circ}-39010_{3}$ \\
\hline 5153.975 & $4 \mathrm{~b}$ & & 19397.096 & $17411_{3}^{\circ}-368083$ & 5123.239 & 50 & 3 & 19513.464 & $16783_{4}^{\circ}-36297_{4}$ \\
\hline 5152.305 & 4 & & 19403.383 & $19948 \stackrel{\circ}{4}-393515$ & 5123.131 & 50 & & 19513.876 & $13945_{3}^{\circ}-33459_{4}$ \\
\hline 5151.612 & 500 & 15 & 19405.993 & $3687_{2}-23093_{2}^{\circ}$ & 5123.006 & 8 & & 19514.352 & $18574_{1}-38088_{2}^{\circ}$ \\
\hline 5151.382 & 50 & 1 & 19406.860 & $17411^{\circ}-36818_{2}$ & 5122.226 & 10 & & 19517.323 & $18699_{2}-38216_{3}^{\circ}$ \\
\hline 5150.701 & 50 & 2 & 19409.426 & $11241_{3}^{\circ}-306513$ & 5121.953 & 4 & & 19518.364 & $18431_{3}-37950_{4}^{\circ}$ \\
\hline 5149.816 & 100 & 10 & 19412.761 & $15736_{1}^{\circ}-35149_{2}$ & 5121.699 & 2 & & 19519.332 & $22508_{2}^{\circ}-42027_{2}$ \\
\hline 5149.210 & 300 & 3 & 19415.046 & $130883-32503_{2}^{\circ}$ & 5120.593 & 4 & & 19523.548 & $22163{ }^{\circ}-416863$ \\
\hline 5147.135 & 40 & 1 & 19422.873 & $12114_{2}^{\circ}-31537_{3}$ & 5119.867 & 20 & & 19526.316 & $14247{ }^{\circ}-33773_{1}$ \\
\hline 5146.059 & 200 & 10 & 19426.934 & $16783^{\circ}-362105$ & 5119.000 & 1 & & 19529.623 & $14243 i-33773_{1}$ \\
\hline 5143.916 & $300 \mathrm{~b}$ & 15 & 19435.027 & $10414_{4}^{\circ}-29849_{4}$ & 5118.176 & 15 & & 19532.767 & $14032_{2}^{\circ}-335643$ \\
\hline 5143.823 & $150 \mathrm{~b}$ & 4 & 19435.379 & $142260-33662_{1}^{\circ}$ & 5116.661 & $2 \mathrm{~h}$ & & 19538.551 & $18431_{3}-37970_{2}^{\circ}$ \\
\hline 5141.264 & 50 & 1 & 19445.052 & $14243_{i}^{\circ}-33689_{2}$ & 5115.045 & $500 \mathrm{r}$ & 40 & 19544.724 & $3865_{1}-234108$ \\
\hline 5140.983 & 3 & 1 & 19446.115 & $128473-32294_{4}^{\circ}$ & 5114.065 & 8 & 1 & 19548.469 & $7795_{4}^{\circ}-27343_{3}$ \\
\hline 5140.771 & $200 \mathrm{~b}$ & 5 & 19446.917 & $6362_{2}-25809_{1}^{\circ}$ & 5113.733 & 2 & & 19549.738 & $22163_{4}^{\circ}-417125$ \\
\hline 5140.716 & $100 \mathrm{~b}$ & $20 \mathrm{~b}$ & 19447.125 & $13847_{2}-33294_{3}^{\circ}$ & 5113.050 & 10 & 3 & 19552.349 & $16783_{4}^{\circ}-363363$ \\
\hline 5140.543 & 50 & 1 & 19447.779 & $16346_{4}^{\circ}-35794_{4}$ & 5111.911 & 4 & & 19556.706 & $18809_{4}^{\circ}-38366_{4}$ \\
\hline 5140.126 & 40 & 50 & 19449.357 & $13847_{2}-33297_{2}^{\circ}$ & 5111.685 & 25 & 2 & 19557.570 & $17847_{2}^{\circ}-374043$ \\
\hline 5139.314 & 3 & & 19452.430 & $18069_{3}^{\circ}-37521_{3}$ & 5111.280 & 150 & 4 & 19559.120 & $7502_{3}-27061_{2}^{\circ}$ \\
\hline 5138.961 & 15 & & 19453.766 & $19503 \stackrel{\circ}{-}-389564$ & 5111.063 & 200 & 5 & 19559.951 & $8111_{4}-27670_{3}^{\circ}$ \\
\hline 5138.934 & 15 & & 19453.868 & $21902^{\circ}-413565$ & 5110.555 & 150 & 2 & 19561.895 & $13175_{4}^{\circ}-32737_{5}$ \\
\hline 5138.086 & 8 & 1 & 19457.079 & $13297_{4}-32754_{3}^{\circ}$ & 5110.216 & 25 & & 19563.192 & $15970_{3}-35533_{2}^{\circ}$ \\
\hline 5137.531 & $10 \mathrm{~b}$ & & 19459.181 & $21902_{4}^{\circ}-41361_{3}$ & 5110.056 & 50 & 2 & 19563.805 & $19039_{2}^{\circ}-38602_{2}$ \\
\hline 5137.475 & $150 \mathrm{~b}$ & 1 & 19459.393 & $4961_{4}-24421_{3}^{\circ}$ & 5109.735 & 200 & 3 & 19565.034 & $13297_{4}-32862_{4}^{\circ}$ \\
\hline 5137.375 & 2 & & 19459.772 & $17166_{5}-36625^{\circ}$ & 5107.970 & 3 & & 19571.794 & $19948_{4}^{\circ}-39520_{4}$ \\
\hline 5137.357 & 1 & & 19459.840 & $171665-36625^{\circ}$ & 5107.338 & 5 & & 19574.216 & $14032_{2}^{\circ}-33606_{2}$ \\
\hline 5137.300 & 3 & & 19460.056 & $17847_{2}^{\circ}-37307_{3}$ & 5106.023 & 5 & & 19579.257 & $13847_{2}-33427_{2}^{\circ}$ \\
\hline 5136.396 & 15 & & 19463.481 & $17354_{1}^{\circ}-36818_{2}$ & 5105.242 & 5 & & 19582.252 & $13945^{\circ}-33527_{4}$ \\
\hline 5136.121 & 100 & 1 & 19464.523 & $13962_{1}-33427_{2}^{\circ}$ & 5102.509 & 3 & & 19592.741 & $22163_{4}^{\circ}-41755_{4}$ \\
\hline 5136.067 & $10 \mathrm{~b}$ & & 19464.728 & $15863_{2}-35328_{1}^{\circ}$ & 5102.228 & 2 & & 19593.820 & $17224_{2}^{\circ}-36818_{2}$ \\
\hline 5135.856 & 5 & 1 & 19465.527 & $20214_{3}^{\circ}-39680_{2}$ & 5101.799 & 40 & 1 & 19595.468 & $142045-33799^{\circ}$ \\
\hline 5135.585 & 75 & 1 & 19466.554 & $19532_{4}-38998_{3}^{\circ}$ & 5101.344 & 40 & 1 & 19597.215 & $10414_{4}^{\circ}-300113$ \\
\hline 5134.746 & 300 & 3 & 19469.735 & $2869_{3}-22338^{\circ}$ & 5101.130 & 300 & 4 & 19598.037 & $7280_{2}-26878_{3}^{\circ}$ \\
\hline 5134.572 & 50 & 2 & 19470.395 & $15618_{3}^{\circ}-35089_{3}$ & 5100.621 & $300 \mathrm{~b}$ & & 19599.993 & $4961_{4}-24561_{3}^{\circ}$ \\
\hline 5134.337 & 15 & 1 & 19471.286 & $21890_{3}^{\circ}-41361_{3}$ & 5100.573 & $150 \mathrm{~b}$ & $2 b$ & 19600.178 & $10414_{4}^{\circ}-30014_{4}$ \\
\hline 5133.922 & 3 & & 19472.860 & $13945_{3}^{\circ}-33418_{4}$ & 5099.030 & 50 & 1 & 19606.109 & $10526^{\circ}-30132_{2}$ \\
\hline 5133.751 & 50 & 1 & 19473.509 & $173983-36871_{2}^{\circ}$ & 5098.933 & 150 & 2 & 19606.482 & $13175_{4}^{\circ}-32781_{4}$ \\
\hline 5133.602 & 5 & & 19474.074 & $17411_{3}^{\circ}-36885_{4}$ & 5097.644 & 8 & 1 & 19611.439 & 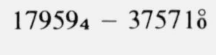 \\
\hline
\end{tabular}


TABle 4. Classified lines of Th $\mathrm{I}-$ continued

\begin{tabular}{|c|c|c|c|c|c|c|c|c|c|}
\hline \multirow{2}{*}{$\begin{array}{l}\text { Wavelength } \\
\text { (Å) }\end{array}$} & \multicolumn{2}{|c|}{ Intensity } & \multirow{2}{*}{$\begin{array}{l}\text { Wavenumber } \\
\left(\mathrm{cm}^{-1}\right)\end{array}$} & \multirow{2}{*}{ Classification } & \multirow{2}{*}{$\begin{array}{c}\text { Wavelength } \\
\text { (§) }\end{array}$} & \multicolumn{2}{|c|}{ Inten sity } & \multirow{2}{*}{$\begin{array}{l}\text { Wavenumber } \\
\left(\mathrm{cm}^{-1}\right)\end{array}$} & \multirow{2}{*}{ Classification } \\
\hline & Lamp & Spark & & & & Lamp & Spark & & \\
\hline 5097.039 & 2 & & 19613.767 & $16217_{2}^{\circ}-35831_{3}$ & 5062.494 & 50 & 2 & 19747.604 & $15493_{4}-35240_{3}^{\circ}$ \\
\hline 5096.484 & 500 & 5 & 19615.903 & $38651-23481_{1}^{\circ}$ & 5061.654 & 200 & 5 & 19750.881 & $6362_{2}-26113^{\circ}$ \\
\hline 5095.644 & 5 & 1 & 19619.137 & $18069_{3}^{\circ}-37688_{3}$ & 5060.366 & 3 & & 19755.908 & $13962_{1}-33718_{2}^{\circ}$ \\
\hline 5095.539 & 3 & & 19619.541 & $13945_{3}^{\circ}-335643$ & 5059.861 & $400 \mathrm{~b}$ & 75 & 19757.880 & $75023-27260_{3}^{\circ}$ \\
\hline 5095.149 & $20 \mathrm{~h}$ & & 19621.043 & $13175_{4}^{\circ}-327963$ & 5059.021 & 8 & 1 & 19761.161 & $17398_{3}-37159_{3}^{\circ}$ \\
\hline 5095.074 & 300 & 50 & 19621.331 & $18431_{3}-38053_{3}^{\circ}$ & 5058.446 & 100 & 3 & 19763.407 & $17073_{1}-36837_{1}^{\circ}$ \\
\hline 5094.546 & 20 & 3 & 19623.365 & $21738_{2}^{\circ}-41361_{3}$ & 5058.446 & 100 & 3 & 19763.407 & $19588_{0}^{\circ}-393515$ \\
\hline 5094.334 & 2 & & 19624.182 & $21077_{0}^{\circ}-40701_{4}$ & 5058.359 & 200 & 3 & 19763.747 & $7502_{3}-27266_{4}^{\circ}$ \\
\hline 5094.127 & 200 & 50 & 19624.979 & $14206_{4}^{\circ}-33831_{4}$ & 5057.137 & 5 & & 19768.522 & $19039_{2}^{\circ}-38807_{2}$ \\
\hline 5092.023 & 1 & & 19633.088 & $211435-40776_{4}^{\circ}$ & 5056.766 & 10 & & 19769.973 & $18930_{3}^{\circ}-38700_{4}$ \\
\hline 5091.641 & $2 \mathrm{~h}$ & & 19634.561 & $171665-36800_{4}^{\circ}$ & 5056.194 & 50 & 2 & 19772.209 & $13962_{1}-33734_{2}^{\circ}$ \\
\hline 5090.548 & 300 & 2 & 19638.777 & $2869_{3}-22508_{2}^{\circ}$ & 5055.751 & $10 \mathrm{~b}$ & & 19773.942 & $13088_{3}-32862_{4}^{\circ}$ \\
\hline 5090.052 & 400 & 5 & 19640.690 & $142045-33844^{\circ}$ & 5055.225 & 300 & 4 & 19775.999 & $13945_{3}^{\circ}-33721_{2}$ \\
\hline 5086.180 & 150 & 3 & 19655.642 & $128473-325032$ & 5054.493 & 3 & & 19778.863 & $195324-39311^{\circ}$ \\
\hline 5085.839 & 15 & 1 & 19656.960 & $14032_{2}^{\circ}-33689_{2}$ & 5053.067 & 3 & & 19784.445 & $21902_{4}^{\circ}-41686_{3}$ \\
\hline 5085.761 & 4 & & 19657.261 & $18431_{3}-38088_{2}^{\circ}$ & 5052.854 & 8 & 1 & 19785.279 & $18431_{3}-38216_{3}^{\circ}$ \\
\hline 5084.997 & 200 & 8 & 19660.215 & $12114_{2}^{\circ}-31774_{3}$ & 5052.448 & 15 & & 19786.869 & $16346_{4}^{\circ}-361335$ \\
\hline 5084.800 & 150 & & 19660.976 & $13945_{3}^{\circ}-336062$ & 5051.885 & 500 & 5 & 19789.074 & $8800_{4}-28589_{3}^{\circ}$ \\
\hline 5084.038 & 15 & 1 & 19663.923 & $19588{ }^{\circ}-392525$ & 5050.780 & 300 & 5 & 19793.403 & $3687_{2}-23481_{1}^{\circ}$ \\
\hline 5083.516 & 1 & & 19665.942 & $130883_{3}-32754_{3}^{\circ}$ & 5050.029 & $5 \mathrm{~b}$ & & 19796.347 & $11802_{2}-31599_{2}^{\circ}$ \\
\hline 5082.625 & 100 & 8. & 19669.390 & $15863_{2}-35533_{2}^{\circ}$ & 5049.976 & $10 \mathrm{~b}$ & & 19796.554 & $7795_{4}^{\circ}-275915$ \\
\hline 5082.005 & 20 & 8 & 19671.789 & $195033^{\circ}-391743$ & 5049.976 & $10 \mathrm{~b}$ & & 19796.554 & $21890_{3}^{\circ}-416863$ \\
\hline 5081.778 & 15 & 2 & 19672.668 & $18930_{3}^{\circ}-38602_{2}$ & 5049.841 & $50 \mathrm{~b}$ & 1 & 19797.083 & $142045-34001^{\circ}$ \\
\hline 5081.449 & 200 & 4 & 19673.942 & $6362_{2}-26036_{3}^{\circ}$ & 5049.580 & 40 & 8 & 19798.107 & $170731-36871_{2}^{\circ}$ \\
\hline 5081.326 & 2 & & 19674.418 & $17847_{2}^{\circ}-37521_{3}$ & 5048.937 & 20 & 1 & 19800.628 & $2869_{3}-22669_{3}^{\circ}$ \\
\hline 5080.157 & 1 & & 19678.945 & $18069_{3}^{\circ}-37748_{2}$ & 5047.600 & 2 & & 19805.873 & $18549_{2}-38355_{2}^{\circ}$ \\
\hline 5079.911 & 15 & 2 & 19679.898 & $8800_{4}-28480_{4}^{\circ}$ & 5047.122 & $5 \mathrm{~h}$ & 1 & 19807.748 & $18011^{\circ}-37819_{4}$ \\
\hline 5078.848 & 40 & 1 & 19684.017 & $15736_{1}^{\circ}-35421_{1}$ & 5047.047 & $150 \mathrm{~b}$ & 5 & 19808.043 & $4961_{4}-24769^{\circ}$ \\
\hline 5077.510 & 5 & 1 & 19689.204 & $14032_{2}^{\circ}-33721_{2}$ & 5045.417 & 3 & & 19814.442 & $13847_{2}-33662_{1}^{\circ}$ \\
\hline 5075.955 & 50 & 1 & 19695.236 & $19039_{2}^{\circ}-38734_{3}$ & 5045.252 & 100 & 5 & 19815.090 & $7502_{3}-27317^{\circ}$ \\
\hline 5074.807 & 10 & & 19699.691 & $13962_{1}-33662_{1}^{\circ}$ & 5044.715 & $400 \mathrm{r}$ & 8 & 19817.199 & $0_{2}-19817 i$ \\
\hline 5074.649 & 25 & 1 & 19700.304 & $7795_{4}^{\circ}-27495_{4}$ & 5044.349 & 5 & 1 & 19818.637 & $19503_{3}^{\circ}-39321_{3}$ \\
\hline 5072.885 & 150 & 1 & 19707.155 & $15166_{3}^{\circ}-348743$ & 5043.520 & 75 & 151 & 19821.895 & $15618_{3}^{\circ}-35440_{3}$ \\
\hline 5070.781 & 75 & 2 & 19715.332 & $18069_{3}^{\circ}-37784_{2}$ & 5041.514 & 2 & & 19829.782 & $16217_{2}^{\circ}-36047_{2}$ \\
\hline 5070.456 & 50 & 1 & 19716.595 & $18699_{2}-38416_{3}^{\circ}$ & 5041.377 & 3 & & 19830.320 & $18930_{3}^{\circ}-38760_{4}$ \\
\hline 5069.626 & 1 & & 19719.823 & $17411_{3}^{\circ}-371313$ & 5041.126 & 150 & 5 & 19831.308 & $175018-373326$ \\
\hline 5069.337 & 200 & 4 & 19720.948 & $165546-36275^{\circ}$ & 5040.683 & 251 & 1 & 19833.051 & $3687_{2}-23521_{3}^{\circ}$ \\
\hline 5069.093 & 20 & 2 & 19721.897 & $21165_{3}^{\circ}-40886_{2}$ & 5040.032 & 2 & & 19835.612 & $173983-37234_{2}^{\circ}$ \\
\hline 5068.829 & 25 & 2 & 19722.924 & $11241_{3}^{\circ}-309643$ & 5039.717 & 1 & & 19836.852 & $18053_{4}^{\circ}-378905$ \\
\hline 5067.974 & $1000 \mathrm{r}$ & 75 & 19726.251 & $7795_{4}^{\circ}-27521_{4}$ & 5039.529 & 50 & 1 & 19837.592 & $8111_{4}-27948$ \\
\hline 5067.807 & 20 & & 19726.901 & $15490 \AA-352165$ & 5039.231 & $300 \mathrm{r}$ & 81 & 19838.765 & $25580-22396 i$ \\
\hline 5067.667 & 5 & 5 & 19727.446 & $128477_{3}-32575_{2}^{\circ}$ & 5038.618 & 1 & & 19841.179 & $17847_{2}^{\circ}-37688_{3}$ \\
\hline 5067.138 & 50 & 2 & 19729.506 & $18549_{2}-38278_{1}^{\circ}$ & 5038.306 & 75 & 3 & 19842.407 & $15970_{3}-35812^{\circ}$ \\
\hline 5066.959 & 80 & 20 & 19730.203 & $20322{ }^{\circ}-400524$ & 5036.623 & 3 & & 19849.038 & $19713_{3}-39562^{\circ}$ \\
\hline 5066.777 & 200 & 40 & 19730.911 & $18011{ }^{\circ}-377426$ & 5035.466 & 5 & & 19853.598 & $21902 \stackrel{\circ}{4}-41755_{4}$ \\
\hline 5066.461 & 4 & & 19732.142 & $16783_{4}^{\circ}-36515_{3}$ & 5034.297 & 100 & 2 & 19858.209 & $154934-35351_{4}^{\circ}$ \\
\hline 5066.134 & 300 & 10 & 19733.416 & $111970^{\circ}-30930_{6}$ & 5033.010 & 2 & & 19863.286 & $19817_{1}^{\circ}-39680_{2}$ \\
\hline 5065.190 & 300 & 5 & 19737.093 & $8243_{2}^{\circ}-27980_{3}$ & 5032.786 & 1 & & 19864.171 & $16346_{4}^{\circ}-36210_{5}$ \\
\hline 5064.945 & $300 \mathrm{r}$ & 8 & 19738.048 & $3865_{1}-23603_{2}^{\circ}$ & 5032.119 & 4 & & 19866.803 & $18549_{2}-38416^{\circ}$ \\
\hline 5064.601 & 300 & 40 & 19739.389 & $49614-24701{ }^{\circ}$ & 5031.815 & 10 & & 19868.004 & $107832-306513$ \\
\hline 5064.004 & $100 \mathrm{~b}$ & $2 \mathrm{~d}$ & 19741.716 & $8111_{4}-27852^{\circ}$ & 5031.148 & 20 & & 19870.638 & $13847_{2}-33718_{2}^{\circ}$ \\
\hline 5063.658 & 8 & 1 & 19743.065 & $16346 \stackrel{\circ}{4}-36089_{4}$ & 5030.116 & 2 & & 19874.714 & $18431_{3}-38306_{4}^{\circ}$ \\
\hline 5063.565 & $20 \mathrm{~h}$ & & 19743.427 & $13847_{2}-33591_{3}^{\circ}$ & 5030.031 & 3 & & 19875.050 & $17166_{5}-37041^{\circ}$ \\
\hline 5063.514 & 2001 & 501 & 19743.626 & $5563_{1}-25306_{2}^{\circ}$ & 5029.893 & 200 & 3 & 19875.595 & $38651-23741$ \\
\hline 5062.932 & 300 & 5 & 19745.896 & $132974-33043_{3}^{\circ}$ & 5029.013 & 75 & 2 & 19879.073 & $18011 \stackrel{\circ}{-378905}$ \\
\hline
\end{tabular}


TABLE 4. Classified lines of Th $\mathrm{I}$-continued

\begin{tabular}{|c|c|c|c|c|c|c|c|c|c|}
\hline \multirow{2}{*}{$\begin{array}{c}\text { Wavelength } \\
\text { (§) }\end{array}$} & \multicolumn{2}{|c|}{ Intensity } & \multirow{2}{*}{$\begin{array}{l}\text { Wavenumber } \\
\left(\mathrm{cm}^{-1}\right)\end{array}$} & \multirow{2}{*}{ Classification } & \multirow{2}{*}{$\begin{array}{c}\text { Wavelength } \\
(\AA)\end{array}$} & \multicolumn{2}{|c|}{ Inten sity } & \multirow{2}{*}{$\begin{array}{c}\text { Wavenumber } \\
\left(\mathrm{cm}^{-1}\right)\end{array}$} & \multirow{2}{*}{ Classification } \\
\hline & Lamp & Spark & & & & Lamp & Spark & & \\
\hline 5028.656 & $300 \mathrm{~b}$ & 15 & 19880.485 & $8800_{4}-28680_{4}^{\circ}$ & 4997.566 & 3 & & 20004.160 & $11241_{3}^{\circ}-312452$ \\
\hline 5027.679 & 5 & & 19884.348 & $142260-34111^{\circ}$ & 4996.229 & 5 & 1 & 20009.513 & $163510-36361^{\circ}$ \\
\hline 5027.318 & 2 & & 19885.776 & $22141_{3}^{\circ}-42027_{2}$ & 4994.982 & 10 & & 20014.509 & $128473-32862{ }^{\circ}$ \\
\hline 5027.122 & 4 & & 19886.551 & $13945^{\circ}-33831_{4}$ & 4994.891 & 5 & & 20014.873 & $15618_{3}^{\circ}-35633_{4}$ \\
\hline 5027.024 & 5 & & 19886.939 & $13847_{2}-33734_{2}^{\circ}$ & 4994.576 & 10 & 3 & 20016.135 & $20566_{4}^{\circ}-405825$ \\
\hline 5026.956 & 5 & & 19887.208 & $38651-237522_{2}^{\circ}$ & 4994.105 & 50 & 2 & 20018.023 & $10526_{3}^{\circ}-30544_{2}$ \\
\hline 5026.159 & 5 & & 19890.361 & $18809_{4}^{\circ}-38700_{4}$ & 4993.749 & 50 & 4 & 20019.450 & $4961_{4}-24981_{3}^{\circ}$ \\
\hline 5024.110 & 8 & & 19898.473 & $19713_{3}-39611^{\circ}$ & 4993.111 & 1 & & 20022.008 & $15863_{2}-35885_{2}^{\circ}$ \\
\hline 5023.709 & 50 & 1 & 19900.061 & $11241 \stackrel{\circ}{3}-311413$ & 4992.638 & 20 & 5 & 20023.905 & $11241_{3}^{\circ}-31265_{3}$ \\
\hline 5023.486 & 50 & 1 & 19900.945 & $17847_{2}^{\circ}-37748_{2}$ & 4992.375 & 8 & 2 & 20024.960 & $19227_{6}^{\circ}-392525$ \\
\hline 5022.070 & 5 & & 19906.556 & $128473-327543$ & 4992.125 & 15 & 2 & 20025.963 & $10526_{3}^{\circ}-30552_{4}$ \\
\hline 5022.005 & 75 & 2 & 19906.813 & 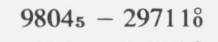 & 4991.603 & 1 & & 20028.057 & $18069_{3}^{\circ}-38097_{2}$ \\
\hline 5021.254 & 75 & 2 & 19909.791 & $11802_{2}-31712_{1}^{\circ}$ & 4989.309 & 150 & 10 & 20037.265 & $7280_{2}-27317_{3}^{\circ}$ \\
\hline 5019.806 & 200 & 25 & 19915.534 & $3687_{2}-23603_{2}^{\circ}$ & 4988.772 & 4 & & 20039.422 & $13175_{4}^{\circ}-332145$ \\
\hline 5019.752 & $10 \mathrm{~b}$ & & 19915.748 & $15970_{3}-35885_{2}^{\circ}$ & 4988.262 & 1 & & 20041.471 & $20054_{2}-40096_{2}^{\circ}$ \\
\hline 5018.059 & 150 & 5 & 19922.467 & $15166_{3}^{\circ}-35089_{3}$ & 4985.948 & 50 & 3 & 20050.772 & $14247_{0}^{\circ}-342981$ \\
\hline 5017.877 & 10 & 2 & 19923.190 & $18069_{3}^{\circ}-37992_{4}$ & 4985.373 & 300 & 10 & 20053.085 & $3687_{2}-23741^{\circ}$ \\
\hline 5017.769 & 10 & 2 & 19923.619 & $18431_{3}-38355_{2}^{\circ}$ & 4985.125 & 8 & & 20054.082 & $14243^{\circ}-342981$ \\
\hline 5017.589 & 5 & & 19924.333 & $13175_{4}^{\circ}-33099_{3}$ & 4982.488 & 150 & $25 \mathrm{~h}$ & 20064.696 & $3687_{2}-237522_{2}^{\circ}$ \\
\hline 5017.510 & 100 & 15 & 19924.647 & $6362_{2}-26287_{i}^{\circ}$ & 4981.877 & 41 & & 20067.157 & $11802_{2}-31870_{2}^{\circ}$ \\
\hline 5016.951 & 8 & & 19926.867 & $12114_{2}^{\circ}-32041_{2}$ & 4980.757 & 20 & 3 & 20071.669 & $165546-366258$ \\
\hline 5016.535 & 40 & 2 & 19928.520 & $98045-29733{ }^{\circ}$ & 4980.186 & 150 & 10 & 20073.970 & $11197{ }^{\circ}-312715$ \\
\hline 5012.274 & 5 & & 19945.461 & $75023-27447_{2}^{\circ}$ & 4979.521 & $4 b$ & & 20076.651 & $98045-29881_{4}^{\circ}$ \\
\hline 5011.478 & 75 & 4 & 19948.629 & $15863_{2}-35812 \stackrel{\circ}{3}$ & 4979.521 & $4 b$ & & 20076.651 & $18069_{3}^{\circ}-38145_{3}$ \\
\hline 5011.478 & 75 & 4 & 19948.629 & $21738_{2}^{\circ}-416863$ & 4978.727 & 2 & & 20079.853 & $18930_{3}^{\circ}-39010_{3}$ \\
\hline 5010.951 & 100 & 2 & 19950.727 & $18809_{4}^{\circ}-38760_{4}$ & 4977.522 & 5 & & 20084.714 & $8800_{4}-28884_{3}^{\circ}$ \\
\hline 5010.417 & 50 & 4 & 19952.853 & $13847_{2}-33800 \stackrel{\circ}{\circ}$ & 4977.389 & 8 & 1 & 20085.251 & $11241 \stackrel{\circ}{3}-313264$ \\
\hline 5009.938 & $50 \mathrm{~b}$ & & 19954.761 & $13088_{3}-33043_{3}^{\circ}$ & 4976.471 & 1 & & 20088.956 & $20522_{2}^{\circ}-40611_{2}$ \\
\hline 5009.240 & 10 & 1 & 19957.541 & $15618_{3}^{\circ}-355763$ & 4976.406 & 1 & & 20089.218 & $18699_{2}-38788_{3}^{\circ}$ \\
\hline 5008.481 & 2 & & 19960.565 & $14206_{4}^{\circ}-341673$ & 4975.524 & 5 & & 20092.779 & $15970_{3}-36062_{4}^{\circ}$ \\
\hline 5007.087 & 5 & & 19966.123 & $14465^{\circ}-344313$ & 4975.442 & 3 & & 20093.110 & $17959_{4}-38053_{3}^{\circ}$ \\
\hline 5006.405 & 5 & & 19968.842 & $154934-354623$ & 4975.254 & 2 & & 20093.869 & $10414_{4}^{\circ}-305085$ \\
\hline 5006.073 & 1 & & 19970.167 & $18699_{2}-38669_{2}^{\circ}$ & 4975.088 & 10 & 1 & 20094.540 & $14206_{4}^{\circ}-343014$ \\
\hline 5005.970 & 20 & 5 & 19970.578 & $20522_{2}^{\circ}-404933$ & 4972.754 & 20 & 2 & 20103.971 & $159700_{3}-36074{ }^{\circ}$ \\
\hline 5005.334 & 15 & 1 & 19973.115 & $13297_{4}-33270_{4}^{\circ}$ & 4972.258 & 15 & 1 & 20105.977 & $195880^{\circ}-396945$ \\
\hline 5004.900 & 2 & & 19974.847 & $10783_{2}^{\circ}-307582$ & 4972.057 & 40 & 1 & 20106.789 & $10783_{2}^{\circ}-30889_{1}$ \\
\hline 5004.685 & 1 & & 19975.705 & $18699_{2}-38675_{3}^{\circ}$ & 4971.553 & 2 & & 20108.828 & $21252_{2}^{\circ}-41361_{3}$ \\
\hline 5004.128 & 150 & 10 & 19977.929 & $11802_{2}-31780_{3}^{\circ}$ & 4970.074 & $150 \mathrm{~b}$ & 2 & 20114.812 & $8243_{2}^{\circ}-283583$ \\
\hline 5004.001 & 50 & 3 & 19978.436 & $142045-34182{ }^{\circ}$ & 4969.518 & 2 & & 20117.062 & $18069_{3}^{\circ}-38186_{4}$ \\
\hline 5003.857 & 20 & 2 & 19979.011 & $14481 \stackrel{\circ}{-}-344605$ & 4968.753 & 200 & 20 & 20120.159 & $13847_{2}-33967_{3}^{\circ}$ \\
\hline 5003.767 & 3 & & 19979.370 & $14465_{2}^{\circ}-34444_{2}$ & & & & & \\
\hline 5003.597 & 75 & 4 & 19980.049 & $7280_{2}-27260_{3}^{\circ}$ & 4967.649 & 5 & & 20124.631 & $10526_{3}^{\circ}-306513$ \\
\hline 5003.385 & $10 \mathrm{~s}$ & 2 & 19980.895 & $18011^{\circ}-379924$ & 4967.334 & 5 & 1 & 20125.907 & $18549_{2}-38675_{3}^{\circ}$ \\
\hline 5002.898 & 15 & 3 & 19982.840 & $15166_{3}^{\circ}-35149_{2}$ & 4967.334 & 5 & 1 & 20125.907 & $21594_{3}-41720_{2}^{\circ}$ \\
\hline 5002.472 & 3 & & 19984.542 & $18431_{3}-38416_{3}^{\circ}$ & 4965.731 & 200 & 10 & 20132.404 & $8800_{4}-28932_{4}^{\circ}$ \\
\hline 5002.225 & 3 & & 19985.529 & $20214_{3}^{\circ}-40200_{3}$ & 4964.538 & 2 & & 20137.242 & $21890_{3}^{\circ}-42027_{2}$ \\
\hline 5002.097 & $500 \mathrm{r}$ & 40 & 19986.040 & $2869_{3}-22855_{3}^{\circ}$ & 4964.255 & 10 & 2 & 20138.390 & $10414_{4}^{\circ}-30552_{4}$ \\
\hline 5001.450 & 8 & 1 & 19988.626 & $18614_{1}^{\circ}-38602_{2}$ & 4963.763 & 75 & 50 & 20140.386 & $142045-34344_{4}^{\circ}$ \\
\hline 5001.068 & 2 & & 19990.152 & $17959_{4}-37950_{4}^{\circ}$ & 4963.031 & 8 & & 20143.356 & $18809_{4}^{\circ}-389535$ \\
\hline 5001.068 & 2 & & 19990.152 & $17959_{4}-37950_{4}^{\circ}$ & 4962.466 & 5 & & 20145.650 & $6362_{2}-26508_{3}^{\circ}$ \\
\hline 5000.730 & 15 & 1 & 19991.504 & $16783_{4}^{\circ}-36775_{3}$ & 4961.724 & 200 & 10 & 20148.662 & $139621-34111_{1}^{\circ}$ \\
\hline 5000.252 & 100 & $25 \mathrm{~d}$ & 19993.414 & $17411_{3}^{\circ}-374043$ & 4958.878 & 2 & & 20160.226 & $17073_{1}-37234_{2}^{\circ}$ \\
\hline 4999.841 & 2 & & 19995.058 & $17959_{4}-37954_{3}^{\circ}$ & 4958.093 & 50 & 2 & 20163.418 & $11877_{1}^{\circ}-32041_{2}$ \\
\hline 4999.239 & 10 & 2 & 19997.466 & $16783^{\circ}-367815$ & 4957.057 & 15 & & 20167.632 & $7280_{2}-27447_{2}^{\circ}$ \\
\hline 4998.419 & 1 & & 20000.746 & $6362_{2}-263632$ & 4956.798 & $20 \mathrm{~b}$ & & 20168.685 & $7502_{3}-27670_{3}^{\circ}$ \\
\hline
\end{tabular}


TABLE 4. Classified lines of Th I-continued

\begin{tabular}{|c|c|c|c|c|c|c|c|c|c|}
\hline \multirow{2}{*}{$\begin{array}{c}\text { Wavelength } \\
(\AA)\end{array}$} & \multicolumn{2}{|c|}{ Intensity } & \multirow{2}{*}{$\begin{array}{l}\text { Wavenumber } \\
\left(\mathrm{cm}^{-1}\right)\end{array}$} & \multirow{2}{*}{ Classification } & \multirow{2}{*}{$\begin{array}{c}\text { Wavelength } \\
\text { (§) }\end{array}$} & \multicolumn{2}{|c|}{ Intensity } & \multirow{2}{*}{$\begin{array}{l}\text { Wavenumber } \\
\qquad\left(\mathrm{cm}^{-1}\right)\end{array}$} & \multirow{2}{*}{ Classification } \\
\hline & Lamp & Spark & & & & Lamp & Spark & & \\
\hline 4956.637 & 50 & 2 & 20169.340 & $16346_{4}^{\circ}-36515_{3}$ & 4924.246 & $75 b$ & 2 & 20302.010 & $16783_{4}^{\circ}-37085_{4}$ \\
\hline 4955.303 & 3 & & 20174.770 & $18011^{\circ}-381864$ & 4923.690 & 5 & & 20304.302 & $15490 \AA-35794_{4}$ \\
\hline 4953.936 & 25 & 3 & 20180.337 & $17224_{2}^{\circ}-37404_{3}$ & 4922.338 & 2 & & 20309.879 & $15490 \AA-35799_{6}$ \\
\hline 4953.646 & 2 & & 20181.519 & $10783_{2}^{\circ}-309643$ & 4922.231 & 5 & & 20310.321 & $15736_{1}^{\circ}-36047_{2}$ \\
\hline 4953.529 & 50 & 10 & 20181.995 & $13088_{3}-33270_{4}^{\circ}$ & 4921.653 & $40 \mathrm{~b}$ & & 20312.706 & $10783_{2}^{\circ}-31095_{1}$ \\
\hline 4952.688 & 40 & 3 & 20185.422 & $7795_{4}^{\circ}-27980_{3}$ & 4920.624 & 50 & 2 & 20316.954 & $3865_{1}-24182_{2}^{\circ}$ \\
\hline 4952.009 & 2 & & 20188.190 & $20423^{\circ}-40611_{2}$ & 4920.053 & $75 b$ & $25 \mathrm{~s}$ & 20319.312 & $15493_{4}-35812^{\circ}$ \\
\hline 4950.755 & 8 & & 20193.303 & $18614_{1}^{\circ}-38807_{2}$ & 4920.018 & $75 b$ & 2 & 20319.456 & $25580-22877^{\circ}$ \\
\hline 4950.250 & 150 & 10 & 20195.363 & $128473-33043 \stackrel{\circ}{3}$ & 4918.659 & 3 & & 20325.070 & $15863_{2}-36188_{2}^{\circ}$ \\
\hline 4949.065 & 2 & & 20200.199 & $20054_{2}-40254_{3}^{\circ}$ & 4916.383 & $2 \mathrm{~h}$ & & 20334.479 & $19503_{3}^{\circ}-39837_{3}$ \\
\hline 4948.964 & 8 & & 20200.611 & $14243 i-344442$ & 4915.823 & 10 & 1 & 20336.796 & $17411_{3}^{\circ}-37748_{2}$ \\
\hline 4948.565 & 1 & & 20202.240 & $23655_{4}^{\circ}-43857_{5}$ & 4915.416 & 50 & 1 & 20338.480 & $13088_{3}-33427_{2}^{\circ}$ \\
\hline 4947.015 & 5 & & 20208.570 & $13088_{3}-33297_{2}^{\circ}$ & 4913.480 & 5 & 1 & 20346.493 & $17959_{4}-38306_{4}^{\circ}$ \\
\hline 4946.220 & 2 & & 20211.818 & $20214_{3}^{\circ}-404264$ & 4912.043 & 10 & 1 & 20352.445 & $13175_{4}^{\circ}-33527_{4}$ \\
\hline 4945.458 & $200 \mathrm{r}$ & 10 & 20214.932 & $0_{2}-20214_{3}^{\circ}$ & 4911.784 & 2 & & 20353.519 & $18930_{3}^{\circ}-39283_{4}$ \\
\hline 4943.130 & $50 \mathrm{~b}$ & 2 & 20224.452 & $14206_{4}^{\circ}-344313$ & 4911.380 & 200 & 10 & 20355.193 & $9804_{5}-30160_{4}^{\circ}$ \\
\hline 4943.064 & $200 \mathrm{~b}$ & 10 & 20224.722 & $2869_{3}-23093_{2}^{\circ}$ & 4911.380 & 200 & 10 & 20355.193 & $18011{ }^{\circ}-383664$ \\
\hline 4941.415 & 15 & 1 & 20231.471 & $10526_{3}^{\circ}-30758_{2}$ & 4910.807 & $100 \mathrm{~b}$ & 2 & 20357.568 & $11802_{2}-32160_{2}^{\circ}$ \\
\hline 4940.907 & 20 & 2 & 20233.551 & $13175_{4}^{\circ}-334083$ & 4910.792 & $100 \mathrm{~b}$ & 2 & 20357.630 & $8800_{4}-29157^{\circ}$ \\
\hline 4940.057 & 5 & & 20237.033 & $10414_{4}^{\circ}-306513$ & 4910.549 & 15 & 1 & 20358.637 & $10783_{2}^{\circ}-31141_{3}$ \\
\hline 4939.642 & $300 \mathrm{r}$ & 15 & 20238.733 & $7795_{4}^{\circ}-280345$ & 4910.158 & 100 & 4 & 20360.259 & $49614-25321_{3}^{\circ}$ \\
\hline 4939.270 & 75 & 2 & 20240.257 & $14206^{\circ}-34447_{4}$ & 4909.844 & 50 & 3 & 20361.561 & $139621-34324_{2}^{\circ}$ \\
\hline 4939.059 & 10 & 1 & 20241.122 & $17501{ }^{\circ}-377426$ & 4909.213 & 15 & 1 & 20364.178 & $142260-34591^{\circ}$ \\
\hline 4938.720 & 3 & & 20242.511 & $14465_{2}^{\circ}-34707_{3}$ & 4909.010 & 8 & 1 & 20365.020 & $18809_{4}^{\circ}-391743$ \\
\hline 4938.597 & 15 & 1 & 20243.015 & $13175_{4}^{\circ}-33418_{4}$ & 4908.478 & 100 & 1 & 20367.227 & $14481 \stackrel{\circ}{-348495}$ \\
\hline 4938.439 & 10 & 1 & 20243.663 & $18431_{3}-38675_{3}^{\circ}$ & 4908.012 & 5 & & 20369.161 & $8111_{4}-28480_{4}^{\circ}$ \\
\hline 4938.283 & 15 & & 20244.302 & $2869_{3}-23113^{\circ}$ & 4907.044 & 50 & 2 & 20373.179 & $17411_{3}^{\circ}-377842$ \\
\hline 4938.206 & 20 & 2 & 20244.618 & $18930_{3}^{\circ}-391743$ & 4906.731 & 2 & & 20374.479 & $195324-39906^{\circ}$ \\
\hline 4937.829 & 300 & 8 & 20246.164 & $5563_{1}-25809_{1}^{\circ}$ & 4905.631 & 2 & & 20379.047 & $203220^{\circ}-407014$ \\
\hline 4936.889 & 25 & 1 & 20250.019 & $17847_{2}^{\circ}-38097_{2}$ & 4904.407 & 1 & & 20384.133 & $21902^{\circ}-422863$ \\
\hline 4936.775 & 150 & 100 & 20250.486 & 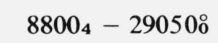 & 4903.057 & 8 & 1 & 20389.746 & $13175_{4}^{\circ}-335643$ \\
\hline 4935.929 & 20 & 1 & 20253.957 & $14206^{\circ}-344605$ & 4902.793 & 75 & 5 & 20390.843 & $7280_{2}-27670_{3}^{\circ}$ \\
\hline 4935.177 & 5 & & 20257.043 & $17959_{4}-38216_{3}^{\circ}$ & 4902.600 & 50 & 5 & 20391.646 & $175010^{\circ}-378924$ \\
\hline 4934.807 & 3 & & 20258.562 & $17398_{3}-37656_{3}^{\circ}$ & 4902.177 & 2 & & 20393.406 & $17354_{1}^{\circ}-37748_{2}$ \\
\hline 4934.748 & 1 & & 20258.804 & $15618_{3}^{\circ}-35877_{2}$ & 4902.051 & 75 & 5 & 20393.930 & $49614-25355_{4}^{\circ}$ \\
\hline 4934.337 & 15 & 1 & 20260.492 & $20322^{\circ}-405825$ & 4901.987 & 50 & 2 & 20394.196 & $7280_{2}-27674_{2}^{\circ}$ \\
\hline 4933.825 & $15 b$ & & 20262.594 & $13297_{4}-33560_{4}^{\circ}$ & 4901.987 & 50 & 2 & 20394.196 & $11802_{2}-32197^{\circ}$ \\
\hline 4933.283 & 3 & & 20264.820 & $18549_{2}-38814_{2}^{\circ}$ & 4901.479 & 4 & & 20396.310 & $21890_{3}^{\circ}-42286_{3}$ \\
\hline 4933.003 & 20 & 1 & 20265.970 & $14032_{2}^{\circ}-342981$ & 4901.371 & 3 & & 20396.759 & $20214^{\circ}-406112$ \\
\hline 4932.250 & 5 & & 20269.064 & $11601_{1}-31870_{2}^{\circ}$ & 4899.336 & 50 & 3 & 20405.231 & $171665-37571{ }^{\circ}$ \\
\hline 4931.373 & $10 \mathrm{~b}$ & & 20272.669 & $19713_{3}-39985_{3}^{\circ}$ & 4898.697 & 10 & 1 & 20407.893 & $17411_{3}^{\circ}-37819_{4}$ \\
\hline 4931.050 & 10 & & 20273.997 & $15166_{3}^{\circ}-35440_{3}$ & 4898.358 & $25 b$ & 1 & 20409.305 & $139621-34371_{2}^{\circ}$ \\
\hline 4930.322 & 3 & & 20276.990 & $17411_{3}^{\circ}-37688_{3}$ & 4897.586 & 8 & 1 & 20412.522 & $14032_{2}^{\circ}-34444_{2}$ \\
\hline 4929.832 & 5 & & 20279.006 & $21077^{\circ}-413565$ & 4897.586 & 8 & 1 & 20412.522 & $15970_{3}-36382^{\circ}$ \\
\hline 4929.086 & 100 & 3 & 20282.075 & $7502_{3}-27784_{2}^{\circ}$ & 4895.168 & 3 & & 20422.605 & $128473-33270_{4}^{\circ}$ \\
\hline 4928.949 & 5 & & 20282.639 & $19039_{2}^{\circ}-393213$ & 4894.955 & $400 \mathrm{r}$ & 10 & 20423.494 & $0_{2}-20423^{\circ}$ \\
\hline 4928.683 & 50 & 2 & 20283.733 & $165546-36837 \circ$ & 4894.520 & 4 & & 20425.309 & $17959_{4}-38385^{\circ}$ \\
\hline 4928.604 & 8 & & 20284.058 & $13175_{4}^{\circ}-33459_{4}$ & 4893.910 & 4 & & 20427.855 & $16783^{\circ}-372115$ \\
\hline 4927.781 & 200 & 5 & 20287.446 & $82432^{\circ}-285312$ & 4893.704 & 10 & 1 & 20428.715 & $16346^{\circ}-36775_{3}$ \\
\hline 4927.299 & 50 & 1 & 20289.431 & $63622-26651_{2}^{\circ}$ & 4893.444 & 50 & 2 & 20429.800 & $11197 \stackrel{\circ}{-3}-316265$ \\
\hline 4926.254 & 40 & 2 & 20293.734 & $132974-335911_{3}^{\circ}$ & 4892.758 & 50 & 2 & 20432.664 & $7795_{4}^{\circ}-28227_{4}$ \\
\hline 4925.822 & $8 b$ & $1 b$ & 20295.514 & $11241_{3}^{\circ}-31537_{3}$ & 4892.415 & 1 & & 20434.097 & $21252_{2}^{\circ}-41686_{3}$ \\
\hline 4925.339 & 5 & 2 & 20297.505 & $18069 \stackrel{\circ}{-}-383664$ & 4892.276 & 5 & 1 & 20434.677 & $16346_{4}^{\circ}-367815$ \\
\hline 4925.097 & $3 b$ & & 20298.502 & $16217_{2}^{\circ}-36515_{3}$ & 4892.038 & 5 & & 20435.671 & $8243_{2}^{\circ}-28679_{2}$ \\
\hline 4924.896 & 8 & 1 & 20299.330 & $18699_{2}-38998_{3}^{\circ}$ & 4891.658 & 15 & 1 & 20437.259 & $12114_{2}^{\circ}-32551_{3}$ \\
\hline
\end{tabular}


TABLE 4. Classified lines of Th $\mathrm{I}$-continued

\begin{tabular}{|c|c|c|c|c|c|c|c|c|c|}
\hline \multirow{2}{*}{$\begin{array}{l}\text { Wavelength } \\
(\AA)\end{array}$} & \multicolumn{2}{|c|}{ Intensity } & \multirow{2}{*}{$\begin{array}{l}\text { Wavenumber } \\
\left(\mathrm{cm}^{-1}\right)\end{array}$} & \multirow{2}{*}{ Classification } & \multirow{2}{*}{$\begin{array}{c}\text { Wavelength } \\
\text { (Å) }\end{array}$} & \multicolumn{2}{|c|}{ Inten sity } & \multirow{2}{*}{$\begin{array}{l}\text { Wavenumber } \\
\qquad\left(\mathrm{cm}^{-1}\right)\end{array}$} & \multirow{2}{*}{ Classification } \\
\hline & Lamp & Spark & & & & Lamp & Spark & & \\
\hline 4891.453 & 4 & & 20438.115 & $10526_{3}^{\circ}-30964_{3}$ & 4862.597 & 1 & & 20559.399 & $11601_{1}-32160_{2}^{\circ}$ \\
\hline 4890.455 & 50 & 8 & 20442.286 & $3865_{1}-24307_{2}^{\circ}$ & 4861.720 & $75 b$ & $20 \mathrm{~h}$ & 20563.108 & $7795_{4}^{\circ}-283583$ \\
\hline 4889.489 & 50 & 2 & 20446.325 & $7502_{3}-27948_{4}^{\circ}$ & 4861.635 & 50 & 2 & 20563.467 & $15863_{2}-36427_{3}^{\circ}$ \\
\hline 4888.811 & 4 & & 20449.161 & $12847_{3}-33297_{2}^{\circ}$ & 4861.223 & $300 \mathrm{r}$ & 50 & 20565.210 & $8111_{4}-286763$ \\
\hline 4888.714 & 4 & & 20449.566 & $18549_{2}-38998^{\circ}$ & 4860.738 & 3 & 1 & 20567.262 & $18431_{3}-38998 \stackrel{\circ}{3}$ \\
\hline 4887.822 & 1 & & 20453.298 & $19532_{4}-39985_{3}^{\circ}$ & 4860.173 & 5 & 2 & 20569.653 & $15493_{4}-36062_{4}^{\circ}$ \\
\hline 4887.538 & 1 & & 20454.486 & $165546-37008 \circ$ & 4857.949 & 5 & 1 & 20579.070 & $12847_{3}-33427_{2}^{\circ}$ \\
\hline 4887.096 & 50 & 2 & 20456.336 & $17959_{4}-38416_{3}^{\circ}$ & 4857.536 & 100 & 15 & 20580.819 & $14465_{2}^{\circ}-35046_{1}$ \\
\hline 4886.869 & 50 & 2 & 20457.287 & $15970_{3}-36427_{3}^{\circ}$ & 4857.536 & 100 & 15 & 20580.819 & $15493_{4}-360743^{\circ}$ \\
\hline 4886.014 & $3 b$ & & 20460.867 & $18069_{3}^{\circ}-38529_{3}$ & 4852.868 & 200 & 50 & 20600.616 & $16217_{2}^{\circ}-36818_{2}$ \\
\hline 4885.825 & 41 & & 20461.658 & $16346_{4}^{\circ}-36808_{3}$ & 4851.676 & 5 & 2 & 20605.677 & $17501 \stackrel{\circ}{\circ}-381065$ \\
\hline 4885.565 & $5 b$ & 1 & 20462.747 & $10783_{2}^{\circ}-31245_{2}$ & 4849.861 & 50 & 15 & 20613.389 & $4961_{4}-25575_{4}^{\circ}$ \\
\hline 4885.290 & 2 & & 20463.899 & $17224_{2}^{\circ}-376883$ & 4849.417 & 2 & & 20615.276 & $10526_{3}^{\circ}-31141_{3}$ \\
\hline 4885.241 & 3 & & 20464.104 & $19588^{\circ}-400524$ & 4848.363 & $400 \mathrm{r}$ & 150 & 20619.758 & $3687_{2}-24307_{2}^{\circ}$ \\
\hline 4884.549 & 50 & 2 & 20467.003 & $19227_{6}^{\circ}-39694_{5}$ & 4848.216 & 20 & 8 & 20620.383 & $17959_{4}-38580_{4}^{\circ}$ \\
\hline 4883.663 & 2 & & 20470.716 & $15618_{3}^{\circ}-36089_{4}$ & 4847.330 & 150 & 15 & 20624.152 & $14465_{2}^{\circ}-35089_{3}$ \\
\hline 4883.490 & 3 & 1 & 20471.441 & $13088_{3}-33560_{4}^{\circ}$ & 4847.144 & 20 & 2 & 20624.943 & $15863_{2}-36488_{2}^{\circ}$ \\
\hline 4882.894 & 5 & & 20473.940 & $18809_{4}^{\circ}-39283_{4}$ & 4845.990 & 1 & & 20629.854 & $13088_{3}-33718_{2}^{\circ}$ \\
\hline 4882.328 & 3 & & 20476.313 & $13847_{2}-34324_{2}^{\circ}$ & 4845.161 & 100 & 10 & 20633.384 & $6362_{2}-26995_{3}^{\circ}$ \\
\hline 4882.328 & 3 & & 20476.313 & $18053_{4}^{\circ}-38529_{3}$ & 4844.929 & 75 & 5 & 20634.372 & $19948{ }_{4}^{\circ}-405825$ \\
\hline 4881.853 & 75 & 2 & 20478.306 & $8111_{4}-28589_{3}^{\circ}$ & 4844.612 & $5 b$ & & 20635.722 & $21077_{0}^{\circ}-417125$ \\
\hline 4881.597 & 50 & 2 & 20479.380 & $11601_{1}-32080_{1}^{\circ}$ & 4843.938 & $100 \mathrm{~b}$ & $50 \mathrm{~b}$ & 20638.594 & $7502_{3}-28140_{4}^{\circ}$ \\
\hline 4881.204 & 75 & 2 & 20481.029 & $4961_{4}-25442{ }^{\circ}$ & 4843.876 & $75 b$ & $10 \mathrm{~b}$ & 20638.858 & $8243_{2}^{\circ}-288822$ \\
\hline 4881.070 & 3 & & 20481.591 & $17411_{3}^{\circ}-378924$ & 4843.103 & 3 & 1 & 20642.152 & $14206_{4}^{\circ}-34849_{5}$ \\
\hline 4880.912 & $50 \mathrm{~b}$ & 2 & 20482.254 & $11802_{2}-32285_{3}^{\circ}$ & 4840.843 & $400 \mathrm{r}$ & 75 & 20651.789 & $2869_{3}-23521_{3}^{\circ}$ \\
\hline 4880.859 & $25 b$ & 2 & 20482.476 & $10783_{2}^{\circ}-31265_{3}$ & 4839.780 & 2 & & 20656.324 & $20922_{2}^{\circ}-415782$ \\
\hline 4880.190 & $15 b$ & 1 & 20485.284 & $16351_{0}-36837^{\circ}$ & 4839.674 & 5 & $2 b$ & 20656.777 & $13175_{4}^{\circ}-33831_{4}$ \\
\hline 4880.010 & 5 & 1 & 20486.040 & $13945^{\circ}-344313$ & 4837.903 & 1 & & 20664.339 & $20566_{4}^{\circ}-41230_{4}$ \\
\hline 4878.733 & $300 \mathrm{~b}$ & 8 & 20491.402 & $25580-23049_{1}^{\circ}$ & 4837.409 & 40 & 4 & 20666.449 & $14206_{4}^{\circ}-348734$ \\
\hline 4878.009 & 100 & 3 & 20494.443 & $3687_{2}-24182_{2}^{\circ}$ & 4837.245 & 20 & 3 & 20667.150 & $14206_{4}^{\circ}-348743$ \\
\hline 4877.265 & 20 & 1 & 20497.569 & $15863_{2}-36361_{1}^{\circ}$ & 4836.091 & 3 & & 20672.081 & $20214_{3}^{\circ}-40886_{2}$ \\
\hline 4876.856 & 4 & & 20499.288 & $13945_{3}^{\circ}-34444_{2}$ & 4834.632 & 2 & & 20678.319 & $15618^{\circ}-36297_{4}$ \\
\hline 4876.495 & 100 & 5 & 20500.806 & $14206_{4}^{\circ}-347073$ & 4834.537 & 3 & 1 & 20678.726 & $21077^{\circ}-41755_{4}$ \\
\hline 4876.244 & 50 & 2 & 20501.861 & $13945_{3}^{\circ}-34447_{4}$ & 4833.674 & $20 \mathrm{~s}$ & $4 \mathrm{~s}$ & 20682.418 & $14204_{5}-34886^{\circ}$ \\
\hline 4876.142 & 3 & & 20502.290 & $13297_{4}-33799_{4}^{\circ}$ & 4833.179 & $200 b$ & 50 & 20684.536 & $14465_{2}^{\circ}-35149_{2}$ \\
\hline 4876.067 & 4 & & 20502.605 & $13088_{3}-33591_{3}^{\circ}$ & 4833.112 & $50 \mathrm{~b}$ & 8 & 20684.823 & $15493_{4}-36178_{4}^{\circ}$ \\
\hline 4875.677 & 4 & & 20504.245 & $7280_{2}-27784_{2}^{\circ}$ & 4832.423 & 51 & 21 & 20687.772 & $11241_{3}^{\circ}-31929_{3}$ \\
\hline 4874.365 & 150 & 10 & 20509.764 & $8800_{4}-29310_{4}^{\circ}$ & 4831.767 & 1 & & 20690.580 & $17398_{3}-38088_{2}^{\circ}$ \\
\hline 4873.855 & 2 & & 20511.910 & $18809_{4}^{\circ}-393213$ & 4831.597 & $300 \mathrm{r}$ & 150 & 20691.309 & $8243_{2}^{\circ}-289343$ \\
\hline 4872.922 & $300 \mathrm{r}$ & $25 b$ & 20515.838 & $3865_{1}-24381_{2}^{\circ}$ & 4831.121 & $500 \mathrm{r}$ & 200 & 20693.347 & $3687_{2}-24381_{2}^{\circ}$ \\
\hline 4872.229 & $10 \mathrm{~b}$ & 1 & 20518.756 & $15970_{3}-36488_{2}^{\circ}$ & 4830.206 & 1 & & 20697.267 & $19503 \stackrel{\circ}{3}-402003$ \\
\hline 4872.030 & 100 & 4 & 20519.594 & $16783^{\circ}-373034$ & 4829.796 & 50 & 8 & 20699.024 & $6362_{2}-27061_{2}^{\circ}$ \\
\hline 4871.558 & 3 & & 20521.582 & $21165_{3}^{\circ}-41686_{3}$ & 4829.407 & 5 & 2 & 20700.691 & $11802_{2}-32503_{2}^{\circ}$ \\
\hline 4871.286 & 50 & 2 & 20522.728 & $0_{2}-20522_{2}^{\circ}$ & 4828.658 & 20 & 4 & 20703.902 & $132974-34001_{4}^{\circ}$ \\
\hline 4868.882 & 150 & 10 & 20532.860 & $11241_{3}^{\circ}-317743$ & 4828.246 & 1 & & 20705.669 & $17959_{4}-386655^{\circ}$ \\
\hline 4868.636 & $8 \mathrm{~h}$ & 1 & 20533.898 & $18069_{3}^{\circ}-38602_{2}$ & 4826.700 & $200 \mathrm{~b}$ & $50 \mathrm{~b}$ & 20712.301 & $30517{ }^{\circ}-98045$ \\
\hline 4867.502 & $5 b$ & $1 b$ & 20538.682 & $16346_{4}^{\circ}-36885_{4}$ & 4826.637 & $50 \mathrm{~b}$ & $15 b$ & 20712.571 & $9804_{5}-30517_{4}^{\circ}$ \\
\hline 4866.740 & 2 & & 20541.898 & $18809_{4}^{\circ}-39351_{5}$ & 4826.008 & 50 & 4 & 20715.271 & $18549_{2}-39264_{3}^{\circ}$ \\
\hline 4865.477 & $300 \mathrm{r}$ & $150 \mathrm{~b}$ & 20547.230 & $7795_{4}^{\circ}-283425$ & 4825.553 & 5 & 1 & 20717.224 & $15618_{3}^{\circ}-36336_{3}$ \\
\hline 4865.415 & $75 b$ & $15 \mathrm{~b}$ & 20547.492 & $13297_{4}-33844^{\circ}$ & 4824.736 & 1 & & 20720.732 & $15490{ }^{\circ}-36210_{5}$ \\
\hline 4864.985 & 2 & & 20549.308 & $19503_{3}^{\circ}-400524$ & 4823.997 & $200 \mathrm{r}$ & $100 \mathrm{~h}$ & 20723.906 & $5563_{1}-26287_{1}^{\circ}$ \\
\hline 4864.789 & 2 & & 20550.136 & $5563_{1}-26113_{2}^{\circ}$ & 4823.604 & $300 \mathrm{r}$ & 75 & 20725.595 & $63622_{2}-27087^{\circ}$ \\
\hline 4864.433 & 8 & 2 & 20551.640 & $17398_{3}-37950_{4}^{\circ}$ & 4823.122 & $5 \mathrm{~b}$ & 1 & 20727.666 & $10414_{4}^{\circ}-31141_{3}$ \\
\hline 4864.357 & 3 & 1 & 20551.961 & $11241_{3}^{\circ}-31793_{4}$ & 4822.855 & $300 \mathrm{r}$ & 75 & 20728.814 & $49614-25690_{0}^{\circ}$ \\
\hline 4862.962 & 4 & & 20557.856 & $16217_{2}^{\circ}-36775_{3}$ & 4821.862 & 15 & 2 & 20733.082 & $3687_{2}-24421_{3}^{\circ}$ \\
\hline
\end{tabular}


TABle 4. Classified lines of Th $\mathrm{I}$-continued

\begin{tabular}{|c|c|c|c|c|c|c|c|c|c|}
\hline \multirow{2}{*}{$\begin{array}{c}\text { Wavelength } \\
(\AA)\end{array}$} & \multicolumn{2}{|c|}{ Intensity } & \multirow{2}{*}{$\begin{array}{l}\text { Wavenumber } \\
\left(\mathrm{cm}^{-1}\right)\end{array}$} & \multirow{2}{*}{ Classification } & \multirow{2}{*}{$\begin{array}{c}\text { Wavelength } \\
\text { (§) }\end{array}$} & \multicolumn{2}{|c|}{ Inten sity } & \multirow{2}{*}{$\begin{array}{l}\text { Wavenumber } \\
\left(\mathrm{cm}^{-1}\right)\end{array}$} & \multirow{2}{*}{ Classification } \\
\hline & Lamp & Spark & & & & Lamp & Spark & & \\
\hline 4821.587 & 150 & 25 & 20734.265 & $2869_{3}-23603_{2}^{\circ}$ & 4785.007 & 1 & & 20892.770 & $195324-40425_{4}^{\circ}$ \\
\hline 4821.387 & 50 & 15 & 20735.125 & $144816^{\circ}-35216_{5}$ & 4784.163 & $2 \mathrm{~h}$ & & 20896.456 & $17073_{1}-37970_{2}^{\circ}$ \\
\hline 4820.884 & 100 & 200 & 20737.288 & $0_{2}-20737_{1}^{\circ}$ & 4784.038 & 150 & 20 & 20897.002 & $15618_{3}^{\circ}-36515_{3}$ \\
\hline 4820.788 & $2 \mathrm{~h}$ & 1 & 20737.701 & $16783^{\circ}-37521_{3}$ & 4783.864 & 1501 & $10 \mathrm{~b}$ & 20897.762 & $63622-27260 \AA$ \\
\hline 4820.463 & $150 \mathrm{~b}$ & $40 \mathrm{~h}$ & 20739.099 & $10526^{\circ}-31265_{3}$ & 4783.605 & $2 b$ & & 20898.893 & $15863_{2}-36762_{3}^{\circ}$ \\
\hline 4819.508 & 10 & 3 & 20743.209 & $12847_{3}-33591_{3}^{\circ}$ & 4782.936 & 5 & 2 & 20901.816 & $159703-36871_{2}^{\circ}$ \\
\hline 4819.191 & 150 & 15 & 20744.573 & $7280_{2}-28024_{i}$ & 4781.671 & 8 & 2 & 20907.346 & $18930_{3}^{\circ}-39837_{3}$ \\
\hline 4818.294 & 5 & 2 & 20748.435 & $15736^{\circ}-36485_{2}$ & 4781.527 & 100 & 5 & 20907.976 & $17398_{3}-38306_{4}^{\circ}$ \\
\hline 4818.102 & 4 & 2 & 20749.262 & $18011^{\circ}-38760_{4}$ & 4781.363 & 2 & & 20908.693 & $203220^{\circ}-41230_{4}$ \\
\hline 4817.017 & 75 & 8 & 20753.935 & $11241_{3}^{\circ}-31995_{4}$ & 4780.751 & 75 & 8 & 20911.369 & $8800_{4}-29711^{\circ}$ \\
\hline 4816.566 & 1 & & 20755.879 & $17847_{2}^{\circ}-38602_{2}$ & 4780.633 & 3 & & 20911.885 & $18699_{2}-39611_{3}^{\circ}$ \\
\hline 4815.046 & 5 & 2 & 20762.431 & $13945_{3}^{\circ}-34707_{3}$ & 4780.427 & 150 & 25 & 20912.786 & $13088_{3}-34001_{4}^{\circ}$ \\
\hline 4814.919 & 15 & 3 & 20762.978 & $14226 o-34989^{\circ}$ & 4779.728 & 50 & 5 & 20915.845 & $4961_{4}-25877_{4}^{\circ}$ \\
\hline 4814.553 & 2 & & 20764.557 & $20922_{2}^{\circ}-41686_{3}$ & 4779.661 & 4 & 1 & 20916.138 & $13962_{1}-34878_{1}^{\circ}$ \\
\hline 4813.893 & 200 & 100 & 20767.404 & $7795_{4}^{\circ}-285624$ & 4779.566 & $2 b$ & & 20916.554 & $15736^{\circ}-36653_{2}$ \\
\hline 4813.499 & 1 & & 20769.103 & $18699_{2}-39468_{3}^{\circ}$ & 4778.453 & 5 & 2 & 20921.425 & $17224_{2}^{\circ}-38145_{3}$ \\
\hline 4813.004 & 75 & 15 & 20771.239 & $112413-320124$ & 4778.294 & $400 \mathrm{r}$ & 50 & 20922.122 & $0_{2}-20922_{2}^{\circ}$ \\
\hline 4812.712 & 1 & & 20772.500 & $11802_{2}-32575_{2}^{\circ}$ & 4778.021 & 2 & & 20923.317 & $25580-23481^{\circ}$ \\
\hline 4812.373 & 150 & 25 & 20773.963 & $8111_{4}-28884^{\circ}$ & 4777.421 & 1 & & 20925.945 & $20054_{2}-40980_{3}^{\circ}$ \\
\hline & & & (2) & 201104200070 & 4777.192 & 300 & 75 & 20926.948 & $142045-35131{ }^{\circ}$ \\
\hline 4810.519 & 8 & 4 & 20781.969 & $154934-36275^{\circ}$ & 4775.793 & 100 & 15 & 20933.078 & $8800_{4}-297330^{\circ}$ \\
\hline 4809.615 & 200 & 50 & 20785.875 & $2869_{3}-23655_{4}^{\circ}$ & 4775.313 & 1001 & $5 b$ & 20935.182 & $6362_{2}-27297^{\circ}$ \\
\hline 4808.764 & 5 & 2 & 20789.554 & $20566^{\circ}-413565$ & 4774.275 & $40 \mathrm{~b}$ & & 20939.734 & $8111_{4}-29050_{0}^{\circ}$ \\
\hline 4808.134 & $500 \mathrm{r}$ & 150 & 20792.278 & $49614-257538$ & 4773.241 & 100 & 8 & 20944.270 & $8800_{4}-29744_{3}^{\circ}$ \\
\hline 4807.550 & 4 & 2 & 20794.803 & $20566_{4}^{\circ}-41361_{3}$ & 4772.962 & 3 & 0 & 20945.494 & 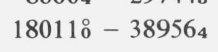 \\
\hline 4806.459 & 5 & 2 & 20799.524 & $11241_{3}^{\circ}-32041_{2}$ & 4771.533 & 4 & 1 & 20951.767 & $128477_{3}-33799_{4}^{\circ}$ \\
\hline 4806.355 & 5 & 1 & 20799.974 & $5563_{1}-26363_{2}^{\circ}$ & 4771.330 & 8 & 2 & 20952.658 & $128473-33800_{3}^{\circ}$ \\
\hline 4806.248 & 3 & 1 & 20800.437 & $10526^{\circ}-313264$ & 4770.796 & 15 & 1 & 20955.003 & $63622-27317_{3}^{\circ}$ \\
\hline 4805.244 & 15 & 3 & 20804.782 & $15863_{2}-366682$ & 4770.440 & $10 \mathrm{~b}$ & 3 & 20956.567 & $18053_{4}^{\circ}-39010_{3}$ \\
\hline 4802.670 & 10 & 3 & 20815.933 & 111970 - 320124 & 4770.390 & $15 b$ & 3 & 20956.787 & $16346^{\circ}-37303_{4}$ \\
\hline 4801.951 & 2 & & 20819.050 & $14481^{\circ}-353005$ & 4769.543 & 50 & 3 & 20960.508 & $16346_{4}^{\circ}-37307_{3}$ \\
\hline 4801.349 & 4 & 1 & 20821.660 & $8111_{4}-28932_{4}^{\circ}$ & 4766.600 & $300 \mathrm{r}$ & 100 & 20973.450 & $3865_{1}-24838_{1}^{\circ}$ \\
\hline 4801.054 & 25 & 4 & 20822.939 & $19273_{2}-40096_{2}^{\circ}$ & 4766.382 & 2 & & 20974.409 & $11601_{1}-32575_{2}^{\circ}$ \\
\hline 4799.670 & 3 & 1 & 20828.943 & $17959_{4}-38788_{3}^{\circ}$ & 4766.098 & 1 & & 20975.659 & $14465_{2}^{\circ}-35440_{3}$ \\
\hline 4798.734 & 4 & 2 & 20833.006 & $18431_{3}-39264_{3}^{\circ}$ & 4765.593 & $100 \mathrm{~b}$ & 100 & 20977.881 & $75023-28480{ }_{4}^{\circ}$ \\
\hline 4797.497 & 75 & 50 & 20838.378 & $19588^{\circ}-404264$ & 4763.963 & 4 & 1 & 20985.059 & $12114_{2}^{\circ}-33099_{3}$ \\
\hline 4796.667 & 3 & 1 & 20841.983 & $14032_{2}^{\circ}-348743$ & 4762.805 & 2 & & 20990.161 & $19503 \stackrel{\circ}{3}-404933$ \\
\hline 4795.913 & 150 & 75 & 20845.260 & $75023-28347_{2}^{\circ}$ & 4762.302 & 8 & 2 & 20992.378 & $13175_{4}^{\circ}-34167_{3}$ \\
\hline 4795.289 & 3 & 1 & 20847.973 & $11802_{2}-32650_{1}^{\circ}$ & 4759.687 & 75 & 10 & 21003.911 & $138472-34851_{2}^{\circ}$ \\
\hline 4794.474 & 2 & & 20851.516 & $10414_{4}^{\circ}-312653$ & 4759.441 & 2 & & 21004.997 & $111977^{\circ}-322025$ \\
\hline 4793.285 & 8 & & 20856.689 & $13847_{2}-34704_{3}^{\circ}$ & 4758.286 & 50 & 15 & 21010.095 & $14206_{4}^{\circ}-35216_{5}$ \\
\hline 4793.246 & 1001 & 25 & 20856.858 & $10414^{\circ}-312715$ & 4758.150 & 100 & 75 & 21010.696 & $10526_{3}^{\circ}-31537_{3}$ \\
\hline 4792.007 & 3 & & 20862.251 & $21165_{3}^{\circ}-42027_{2}$ & 4758.075 & 50 & 15 & 21011.027 & $75023-285132$ \\
\hline 4791.921 & 5 & 1 & 20862.625 & $11802_{2}-32665^{\circ}$ & 4757.415 & 15 & 2 & 21013.942 & $14032_{2}^{\circ}-350461$ \\
\hline 4791.362 & 8 & 3 & 20865.059 & $16346_{4}^{\circ}-372115$ & 4757.133 & 50 & 5 & 21015.187 & $17073_{1}-38088_{2}^{\circ}$ \\
\hline 4789.387 & $400 \mathrm{r}$ & 75 & 20873.663 & $3687_{2}-24561_{3}^{\circ}$ & 4756.699 & 100 & 25 & 21017.105 & $165546-375718$ \\
\hline 4788.678 & 25 & 5 & 20876.754 & $142045-35081{ }^{\circ}$ & 4756.532 & 75 & & 21017.843 & $17398_{3}-38416_{3}^{\circ}$ \\
\hline 4788.542 & 3 & 1 & 20877.347 & $16217_{2}^{\circ}-37094_{2}$ & 4753.581 & 21 & & 21030.890 & $13847_{2}-34878^{\circ}$ \\
\hline 4788.472 & 2 & & 20877.652 & $13847_{2}-34725^{\circ}$ & 4752.900 & 10 & 3 & 21033.904 & $20322{ }^{\circ}-41356_{5}$ \\
\hline 4788.079 & 1 & & 20879.366 & $13088_{3}-33967_{3}^{\circ}$ & 4752.588 & $50 \mathrm{~b}$ & $10 \mathrm{~b}$ & 21035.284 & $16783_{4}^{\circ}-37819_{4}$ \\
\hline 4787.148 & 150 & 25 & 20883.426 & $2869_{3}-23752_{2}^{\circ}$ & 4752.199 & 2 & & 21037.006 & $18431_{3}-39468_{3}^{\circ}$ \\
\hline 4786.914 & 5 & 1 & 20884.447 & $18809_{4}^{\circ}-396945$ & 4749.968 & $300 \mathrm{~b}$ & 50 & 21046.887 & $8111_{4}-29157^{\circ}$ \\
\hline 4786.724 & 75 & 5 & 20885.276 & $13297_{4}-34182^{\circ}$ & 4749.892 & 20 & 4 & 21047.224 & $132974-34344_{4}^{\circ}$ \\
\hline 4786.532 & 200 & 50 & 20886.114 & $8800_{4}-29686_{3}^{\circ}$ & 4749.795 & 25 & 3 & 21047.654 & $2869_{3}-23916_{4}^{\circ}$ \\
\hline 4785.780 & 5 & 2 & 20889.395 & $15493_{4}-36382_{4}^{\circ}$ & 4749.466 & $3 b$ & 1 & 21049.111 & $21575_{2}-42624_{1}^{\circ}$ \\
\hline
\end{tabular}


TABLE 4. Classified lines of Th $\mathrm{I}$-continued

\begin{tabular}{|c|c|c|c|c|c|c|c|c|c|}
\hline \multirow{2}{*}{$\begin{array}{l}\text { Wavelength } \\
\text { (A) }\end{array}$} & \multicolumn{2}{|c|}{ Intensity } & \multirow{2}{*}{$\begin{array}{l}\text { Wavenumber } \\
\left(\mathrm{cm}^{-1}\right)\end{array}$} & \multirow{2}{*}{ Classification } & \multirow{2}{*}{$\begin{array}{c}\text { Wavelength } \\
\text { (A) }\end{array}$} & \multicolumn{2}{|c|}{ Intensity } & \multirow{2}{*}{$\begin{array}{l}\text { Wavenumber } \\
\left(\mathrm{cm}^{-1}\right)\end{array}$} & \multirow{2}{*}{ Classification } \\
\hline & Lamp & Spark & & & & Lamp & Spark & & \\
\hline 4749.287 & 5 & & 21049.905 & $11601_{1}-32650_{1}^{\circ}$ & 4718.051 & 100 & 5 & 21189.264 & $20566_{4}^{\circ}-41755_{4}$ \\
\hline 4749.200 & $400 \mathrm{r}$ & 150 & 21050.290 & $7795_{4}^{\circ}-28845_{4}$ & 4717.487 & 3 & 1 & 21191.798 & $17411_{3}^{\circ}-38602_{2}$ \\
\hline 4747.617 & 100 & 4 & 21057.309 & $14032_{2}^{\circ}-35089_{3}$ & 4717.390 & 20 & 2 & 21192.233 & $3687_{2}-24880_{1}^{\circ}$ \\
\hline 4745.629 & 8 & 2 & 21066.130 & $18614^{\circ}-39680_{2}$ & 4716.862 & $5 b$ & & 21194.605 & $13297_{4}-34492^{\circ}$ \\
\hline 4745.331 & $200 \mathrm{~b}$ & $25 b$ & 21067.453 & $7280_{2}-28347_{2}^{\circ}$ & 4716.849 & $8 \mathrm{~b}$ & 1 & 21194.664 & $12114_{2}^{\circ}-33309_{2}$ \\
\hline 4744.838 & 5 & 1 & 21069.642 & $142045-352730^{\circ}$ & 4715.955 & $5 b$ & 1 & 21198.682 & $18053_{4}^{\circ}-392525$ \\
\hline 4744.797 & 5 & 2 & 21069.824 & $19817_{1}^{\circ}-40886_{2}$ & 4715.856 & 8 & 1 & 21199.127 & $15618^{\circ}-36818_{2}$ \\
\hline 4744.706 & 3 & & 21070.228 & $13945^{\circ}-35015_{2}$ & 4714.673 & 150 & 8 & 21204.446 & $13945_{3}^{\circ}-35149_{2}$ \\
\hline 4744.516 & 2 & & 21071.072 & $15970_{3}-37041_{4}^{\circ}$ & 4713.789 & 25 & $4 b$ & 21208.422 & $16783 \stackrel{\circ}{4}-37992_{4}$ \\
\hline 4742.114 & 200 & 8 & 21081.745 & $3687_{2}-24769_{3}^{\circ}$ & 4712.839 & 100 & 8 & 21212.698 & $10414 \stackrel{\circ}{4}-316265$ \\
\hline 4741.301 & 150 & 40 & 21085.360 & $63622-27447_{2}^{\circ}$ & 4712.481 & $300 \mathrm{~b}$ & 100 & 21214.309 & $98045-31019_{4}^{\circ}$ \\
\hline 4740.951 & $200 \mathrm{~b}$ & 15 & 21086.916 & $4961_{4}-26048_{4}^{\circ}$ & 4712.004 & 50 & 4 & 21216.456 & $11241_{3}^{\circ}-32458_{4}$ \\
\hline 4740.929 & $100 \mathrm{~b}$ & 8 & 21087.014 & $75023-28589 \stackrel{\circ}{3}$ & 4711.917 & 5 & 1 & 21216.848 & $8800_{4}-30017^{\circ}$ \\
\hline 4740.332 & 15 & 3 & 21089.670 & $16217_{2}^{\circ}-37307_{3}$ & 4711.417 & 75 & 5 & 21219.100 & $171665-38385 \circ$ \\
\hline 4739.674 & 300 & 40 & 21092.598 & $7280_{2}-28372_{1}^{\circ}$ & 4708.948 & 3 & 1 & 21230.225 & $18053_{4}^{\circ}-39283_{4}$ \\
\hline 4739.353 & 5 & 2 & 21094.026 & $14206_{4}^{\circ}-35300_{5}$ & 4708.294 & 200 & 25 & 21233.174 & $7280_{2}-28513_{2}^{\circ}$ \\
\hline 4738.839 & 8 & 2 & 21096.314 & $21539_{4}^{\circ}-42635_{5}$ & 4707.772 & $25 b$ & 3 & 21235.529 & $13088_{3}-34324_{2}^{\circ}$ \\
\hline 4737.915 & 150 & 15 & 21100.428 & $14481 \stackrel{\circ}{-}-355825$ & 4706.691 & 10 & 2 & 21240.406 & $118022-33043 \stackrel{\circ}{3}$ \\
\hline 4737.604 & 101 & 2 & 21101.813 & $142260-35328^{\circ}$ & 4706.575 & 100 & 5 & 21240.929 & 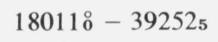 \\
\hline 4737.529 & 20 & 3 & 21102.148 & $17959_{4}-39062^{\circ}$ & 4705.873 & 8 & 1 & 21244.098 & $195324-40776_{4}^{\circ}$ \\
\hline 4736.523 & 10 & 2 & 21106.629 & $16783_{4}^{\circ}-37890_{5}$ & 4704.997 & 5 & 1 & 21248.053 & $10526_{3}^{\circ}-317743$ \\
\hline 4735.995 & 200 & 20 & 21108.982 & $16783^{\circ}-37892_{4}$ & 4704.935 & 8 & 2 & 21248.333 & $17354_{1}^{\circ}-38602_{2}$ \\
\hline 4735.474 & 4 & 1 & 21111.305 & $14465_{2}^{\circ}-35576_{3}$ & 4703.990 & $500 \mathrm{r}$ & 200 & 21252.602 & $0_{2}-21252_{2}^{\circ}$ \\
\hline 4734.046 & 200 & 10 & 21117.673 & $14032_{2}^{\circ}-35149_{2}$ & 4703.213 & 50 & 2 & 21256.112 & $13088_{3}-34344_{4}^{\circ}$ \\
\hline 4733.536 & 2 & & 21119.948 & $12847_{3}-33967_{3}^{\circ}$ & 4703.190 & 50 & 5 & 21256.216 & $13175_{4}^{\circ}-344313$ \\
\hline 4733.504 & 1 & & 21120.091 & $20566_{4}^{\circ}-41686_{3}$ & 4702.778 & 15 & 2 & 21258.079 & $10783_{2}^{\circ}-32041_{2}$ \\
\hline 4733.224 & 1 & & 21121.340 & $180534^{\circ}-391743$ & 4702.591 & 25 & 3 & 21258.924 & $15490 \circ-367496$ \\
\hline 4732.826 & 4 & 1 & 21123.116 & $10414_{4}^{\circ}-31537_{3}$ & 4702.475 & 25 & 3 & 21259.449 & $175018-38760_{4}$ \\
\hline 4732.103 & 40 & 4 & 21126.344 & $13175^{\circ}-343014$ & 4701.388 & 20 & 4 & 21264.364 & $163510-37616^{\circ}$ \\
\hline 4731.192 & 20 & 3 & 21130.411 & $15166_{3}^{\circ}-36297_{4}$ & 4700.959 & 50 & 4 & 21266.304 & $15618^{\circ}-36885_{4}$ \\
\hline 4730.657 & 150 & 8 & 21132.801 & $132974-34430_{3}^{\circ}$ & 4700.772 & 200 & 8 & 21267.150 & $10526_{3}^{\circ}-317934$ \\
\hline 4730.089 & 40 & 5 & 21135.339 & $4961_{4}-26096_{3}^{\circ}$ & 4700.655 & 2 & & 21267.679 & $19713_{3}-40980_{3}^{\circ}$ \\
\hline 4729.128 & $400 \mathrm{~b}$ & $100 \mathrm{~b}$ & 21139.634 & $7795_{4}^{\circ}-28934_{3}$ & 4700.234 & 8 & 1 & 21269.584 & $15493_{4}-36762^{\circ}$ \\
\hline 4728.985 & 50 & 8 & 21140.273 & $17166_{5}-38306_{4}^{\circ}$ & 4699.602 & 10 & 2 & 21272.445 & $18011^{\circ}-39283_{4}$ \\
\hline 4728.591 & 40 & 3 & 21142.034 & $13847_{2}-34989_{1}^{\circ}$ & 4698.732 & $25 b$ & 3 & 21276.383 & $1214_{2}^{\circ}-33390_{1}$ \\
\hline 4728.133 & 300 & $25 \mathrm{~d}$ & 21144.082 & $13945_{3}^{\circ}-35089_{3}$ & 4697.600 & 10 & 2 & 21281.510 & $17073_{1}-38355_{2}^{\circ}$ \\
\hline 4727.619 & $3 \mathrm{~h}$ & & 21146.381 & $10783_{2}^{\circ}-31929_{3}$ & 4697.359 & 2 & & 21282.602 & $19948_{4}^{\circ}-41230_{4}$ \\
\hline 4727.440 & 75 & 4 & 21147.182 & $142045-35351_{4}^{\circ}$ & 4697.212 & 10 & 3 & 21283.268 & $13088_{3}-34371_{2}^{\circ}$ \\
\hline 4726.598 & 5 & 2 & 21150.949 & $3687_{2}-24838_{1}^{\circ}$ & 4696.697 & $15 b$ & $4 b$ & 21285.602 & $13297_{4}-34583^{\circ}$ \\
\hline 4726.441 & 8 & & 21151.651 & $11802_{2}-32954_{2}^{\circ}$ & 4696.667 & $25 b$ & $8 b$ & 21285.738 & $13175_{4}^{\circ}-34460_{5}$ \\
\hline 4726.054 & 4 & & 21153.383 & $128473-34001_{4}^{\circ}$ & 4696.588 & 2 & & 21286.096 & $18699_{2}-39985_{3}^{\circ}$ \\
\hline 4725.384 & 8 & 2 & 21156.383 & $15618^{\circ}-36775_{3}$ & 4695.705 & 1 & & 21290.099 & $20737_{1}^{\circ}-42027_{2}$ \\
\hline 4724.691 & 2 & & 21159.486 & $1214_{2}^{\circ}-33273_{1}$ & 4695.456 & 200 & 50 & 21291.228 & $15490 \circ-36781_{5}$ \\
\hline 4724.292 & 2 & & 21161.273 & $19039_{2}^{\circ}-40200_{3}$ & 4695.039 & $300 \mathrm{r}$ & 100 & 21293.119 & $3687_{2}-24981_{3}^{\circ}$ \\
\hline 4723.438 & $800 \mathrm{r}$ & 200 & 21165.099 & $0_{2}-21165_{3}^{\circ}$ & 4694.537 & 5 & 1 & 21295.396 & $15863_{2}-37159^{\circ}$ \\
\hline 4722.499 & 75 & 5 & 21169.307 & $15166_{3}^{\circ}-36336_{3}$ & 4694.478 & 15 & $3 b$ & 21295.663 & $15863_{2}-37159_{3}^{\circ}$ \\
\hline 4722.089 & 300 & 50 & 21171.145 & $75023-28673_{2}^{\circ}$ & 4692.465 & 3 & 1 & 21304.798 & $17959_{4}-39264_{3}^{\circ}$ \\
\hline 4721.456 & 3 & & 21173.983 & $75023-28676_{3}^{\circ}$ & 4691.883 & 100 & 8 & 21307.441 & $15493_{4}-36800_{4}^{\circ}$ \\
\hline 4721.276 & 200 & 15 & 21174.791 & $8243^{\circ}-29418_{2}$ & 4691.631 & $300 \mathrm{~b}$ & $50 \mathrm{~b}$ & 21308.586 & $6362_{2}-27670_{3}^{\circ}$ \\
\hline 4720.780 & 150 & 15 & 21177.015 & $14243^{\circ}-35421_{1}$ & 4691.603 & $50 \mathrm{~b}$ & $8 \mathrm{~b}$ & 21308.713 & $7795_{4}^{\circ}-29104_{4}$ \\
\hline 4720.457 & 75 & 8 & 21178.465 & $7502_{3}-28680_{4}^{\circ}$ & 4691.500 & 100 & 15 & 21309.181 & $7280_{2}-28589_{3}^{\circ}$ \\
\hline 4720.338 & 3 & 1 & 21178.998 & $15970_{3}-37149_{2}^{\circ}$ & 4691.346 & 100 & 15 & 21309.880 & $11241_{3}^{\circ}-32551_{3}$ \\
\hline 4720.160 & 50 & 3 & 21179.797 & $18431_{3}-39611_{3}^{\circ}$ & 4690.894 & 15 & 2 & 21311.934 & $63622-27674_{2}^{\circ}$ \\
\hline 4719.692 & 150 & 5 & 21181.897 & $173983-38580_{4}^{\circ}$ & 4690.623 & 300 & $40 \mathrm{~b}$ & 21313.165 & $2869_{3}-24182_{2}^{\circ}$ \\
\hline 4719.441 & 20 & 2 & 21183.024 & $25580-23741_{i}^{\circ}$ & 4689.532 & 4 & & 21318.123 & $14481 \stackrel{\circ}{6}-357996$ \\
\hline
\end{tabular}


TABLE 4. Classified lines of Th $\mathrm{I}$-continued

\begin{tabular}{|c|c|c|c|c|c|c|c|c|c|}
\hline \multirow{2}{*}{$\begin{array}{c}\text { Wavelength } \\
(\AA)\end{array}$} & \multicolumn{2}{|c|}{ Intensity } & \multirow{2}{*}{$\begin{array}{l}\text { Wavenumber } \\
\left(\mathrm{cm}^{-1}\right)\end{array}$} & \multirow{2}{*}{ Classification } & \multirow{2}{*}{$\begin{array}{c}\text { Wavelength } \\
\text { (̊) }\end{array}$} & \multicolumn{2}{|c|}{ Inten sity } & \multirow{2}{*}{$\begin{array}{c}\text { Wavenumber } \\
\left(\mathrm{cm}^{-1}\right)\end{array}$} & \multirow{2}{*}{ Classification } \\
\hline & Lamp & Spark & & & & Lamp & Spark & & \\
\hline 4689.451 & 50 & 5 & 21318.491 & $15166^{\circ}-36485_{2}$ & 4659.568 & 200 & 40 & 21455.210 & $8800_{4}-30255^{\circ}$ \\
\hline 4689.252 & 2001 & $50 \mathrm{~b}$ & 21319.396 & 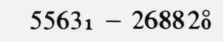 & 4659.458 & 5 & & 21455.717 & $17501{ }^{\circ}-389564$ \\
\hline 4688.458 & $5 \mathrm{~h}$ & 2 & 21323.006 & $16783^{\circ}-381065$ & 4657.033 & 1 & & 21466.889 & $15618 \stackrel{\circ}{3}-37085_{4}$ \\
\hline 4686.195 & $500 \mathrm{r}$ & 50 & 21333.303 & $2869_{3}-24202^{\circ}$ & 4656.548 & 50 & 4 & 21469.125 & $10526 \stackrel{8}{3}-31995_{4}$ \\
\hline 4684.634 & 40 & 3 & 21340.412 & $18011{ }^{\circ}-393515$ & 4655.974 & 3 & & 21471.771 & $20214_{3}^{\circ}-41686_{3}$ \\
\hline 4684.362 & $50 \mathrm{~b}$ & 3 & 21341.651 & $13088_{3}-34430_{3}^{\circ}$ & 4655.818 & 4 & 1 & 21472.491 & $16346_{4}^{\circ}-37819_{4}$ \\
\hline 4683.349 & 200 & 20 & 21346.267 & $77955^{\circ}-291415$ & 4655.328 & 1 & & 21474.751 & $17847_{2}^{\circ}-39321_{3}$ \\
\hline 4682.728 & 75 & 5 & 21349.098 & $15166_{3}^{\circ}-36515_{3}$ & 4655.210 & 100 & 20 & 21475.295 & $18431_{3}-39906^{\circ}$ \\
\hline 4682.662 & $3 b$ & & 21349.399 & $17411_{3}^{\circ}-38760_{4}$ & 4655.030 & 75 & 10 & 21476.126 & $12847_{3}-34324_{2}^{\circ}$ \\
\hline 4682.229 & 150 & 15 & 21351.373 & $179594-39311_{4}^{\circ}$ & 4654.002 & 50 & 3 & 21480.869 & $13847_{2}-35328^{\circ}$ \\
\hline 4681.743 & 1 & & 21353.590 & $11601_{1}-32954_{2}^{\circ}$ & 4652.743 & 20 & 2 & 21486.682 & $15166_{3}^{\circ}-366532$ \\
\hline 4680.588 & $50 \mathrm{~b}$ & $10 \mathrm{~b}$ & 21358.859 & $11802_{2}-33161^{\circ}$ & 4651.584 & $75 b$ & & 21492.036 & $11802_{2}-33294_{3}^{\circ}$ \\
\hline 4680.387 & 100 & 8 & 21359.776 & $8800_{4}-30160_{4}^{\circ}$ & 4651.112 & 75 & 5 & 21494.216 & $11802_{2}-33297_{2}^{\circ}$ \\
\hline 4680.235 & 200 & 15 & 21360.470 & $10414_{4}^{\circ}-317743$ & 4651.062 & $75 b$ & 4 & 21494.447 & $13088_{3}-34583^{\circ}$ \\
\hline 4679.919 & 3 & & 21361.912 & $16783_{4}^{\circ}-38145_{3}$ & 4650.628 & 50 & 4 & 21496.453 & $18930_{3}^{\circ}-404264$ \\
\hline 4679.575 & 4 & 2 & 21363.482 & $20214^{\circ}-41578_{2}$ & 4650.233 & 100 & 5 & 21498.279 & $55631-27061_{2}^{\circ}$ \\
\hline 4679.390 & 3 & & 21364.327 & $20922_{2}^{\circ}-422863$ & 4649.975 & 75 & 8 & 21499.472 & $171665-386650$ \\
\hline 4679.001 & 20 & 1 & 21366.103 & $13962_{1}-35328^{\circ}$ & 4647.953 & 100 & 5 & 21508.825 & $18011^{\circ}-39520_{4}$ \\
\hline 4678.358 & 5 & 2 & 21369.040 & $7280_{2}-28649_{i}^{\circ}$ & 4647.249 & 150 & 20 & 21512.083 & $28699_{3}-24381_{2}^{\circ}$ \\
\hline 4678.233 & 150 & $20 \mathrm{~b}$ & 21369.611 & $142064-355763$ & 4647.249 & 150 & 20 & 21512.083 & $15618 \stackrel{\circ}{3}-37131_{3}$ \\
\hline 4678.145 & 8 & 2 & 21370.012 & $19516_{2}^{\circ}-40886_{2}$ & 4647.068 & 50 & 2 & 21512.921 & $11877_{1}^{\circ}-333901$ \\
\hline 4676.971 & 150 & $25 b$ & 21375.377 & $14206^{\circ}-355825$ & 4646.685 & 150 & 15 & 21514.694 & $10526_{3}^{\circ}-32041_{2}$ \\
\hline 4676.248 & 2 & & 21378.681 & $17224_{2}^{\circ}-386022$ & 4646.538 & $75 b$ & 4 & 21515.375 & $10414_{4}^{\circ}-31929_{3}$ \\
\hline 4676.248 & 2 & & 21378.681 & $17224_{2}^{\circ}-38602_{2}$ & 4646.505 & $8 \mathrm{~b}$ & 1 & 21515.528 & $154934-370080$ \\
\hline 4676.056 & $500 \mathrm{r}$ & 150 & 21379.559 & $10414_{4}^{\circ}-317934$ & 4645.186 & 3 & & 21521.637 & $18574_{1}-40096_{2}^{\circ}$ \\
\hline 4675.375 & 200 & 15 & 21382.673 & $75023-28884_{3}^{\circ}$ & 4644.707 & 150 & 50 & 21523.856 & $12847_{3}-34371_{2}^{\circ}$ \\
\hline 4675.115 & 3 & 2 & 21383.862 & $19503_{3}^{\circ}-408862$ & 4644.495 & 4 & & 21524.839 & $5563_{1}-27087_{1}^{\circ}$ \\
\hline 4674.006 & 3 & & 21388.936 & $14032_{2}^{\circ}-35421_{1}$ & 4643.557 & 100 & 10 & 21529.187 & $142045-35733{ }^{\circ}$ \\
\hline 4673.805 & 2 & & 21389.856 & $98045-31194_{4}^{\circ}$ & 4643.266 & 1 & & 21530.536 & $16217_{2}^{\circ}-37748_{2}$ \\
\hline 4673.661 & $800 r$ & 150 & 21390.515 & $2869_{3}-24259_{4}^{\circ}$ & 4642.816 & 3 & & 21532.623 & $13175_{4}^{\circ}-34707_{3}$ \\
\hline 4673.042 & 200 & 25 & 21393.348 & $7280_{2}-28673 \stackrel{\circ}{2}$ & 4641.687 & 5 & 1 & 21537.860 & $223999^{\circ}-439376$ \\
\hline 4672.632 & 8 & 1 & 21395.225 & $15490 \AA-36885_{4}$ & 4641.244 & $300 \mathrm{~b}$ & $150 \mathrm{~b}$ & 21539.916 & $11241_{3}^{\circ}-32781_{4}$ \\
\hline 4672.436 & 50 & 20 & 21396.123 & $7280_{2}-28676_{3}^{\circ}$ & 4641.024 & 2 & & 21540.937 & $20214_{3}^{\circ}-41755_{4}$ \\
\hline 4672.370 & 2 & & 21396.425 & $17411_{3}^{\circ}-388072$ & 4640.396 & 1 & & 21543.852 & $16346_{4}^{\circ}-37890_{5}$ \\
\hline 4672.332 & 5 & 1 & 21396.599 & $18699_{2}-40096_{2}^{\circ}$ & 4639.896 & 75 & 25 & 21546.174 & $16346_{4}^{\circ}-37892_{4}$ \\
\hline 4671.091 & 2 & & 21402.284 & $16783^{\circ}-381864$ & 4639.857 & $40 \mathrm{~b}$ & 5 & 21546.355 & $4961_{4}-26508 \stackrel{8}{3}$ \\
\hline 4670.942 & 50 & 3 & 21402.966 & $10526_{3}^{\circ}-31929_{3}$ & 4639.755 & 5 & & 21546.828 & $18549_{2}-40096_{2}^{\circ}$ \\
\hline 4670.832 & 10 & 1 & 21403.471 & $13088_{3}-34492_{4}^{\circ}$ & 4639.520 & 200 & 50 & 21547.920 & $154934-37041_{4}^{\circ}$ \\
\hline 4670.059 & $15 b$ & 1 & 21407.013 & $13297_{4}-34704_{3}^{\circ}$ & 4638.686 & 150 & 10 & 21551.794 & $2869_{3}-24421_{3}^{\circ}$ \\
\hline 4669.984 & $500 \mathrm{r}$ & 100 & 21407.357 & $8243 \stackrel{\circ}{-}-29650_{2}$ & 4638.127 & 150 & 10 & 21554.391 & $11241_{3}^{\circ}-327963$ \\
\hline 4669.665 & 75 & 4 & 21408.819 & $14032_{2}^{\circ}-35440_{3}$ & 4637.191 & 50 & 2 & 21558.742 & $21077_{0}^{\circ}-42635_{5}$ \\
\hline 4668.492 & 3 & & 21414.198 & $171665-38580_{4}^{\circ}$ & 4636.758 & 4 & 1 & 21560.755 & $11601_{1}-33161_{1}^{\circ}$ \\
\hline 4668.172 & $800 \mathrm{r}$ & 150 & 21415.666 & $75023-28917_{2}^{\circ}$ & 4634.605 & 8 & 2 & 21570.771 & $13962_{1}-35533^{\circ}$ \\
\hline 4666.797 & $500 \mathrm{r}$ & 150 & 21421.976 & $6362_{2}-27784_{2}^{\circ}$ & 4634.227 & 3 & & 21572.530 & $19039_{2}^{\circ}-40611_{2}$ \\
\hline 4666.515 & 200 & 8 & 21423.270 & $4961_{4}-26384_{4}^{\circ}$ & 4633.765 & $300 \mathrm{~b}$ & $75 b$ & 21574.681 & $12114_{2}^{\circ}-33689_{2}$ \\
\hline 4665.713 & 40 & 3 & 21426.953 & $14206_{4}^{\circ}-356334$ & 4633.620 & 150 & 8 & 21575.356 & $8111_{4}-29686^{\circ}$ \\
\hline 4665.483 & 5 & 1 & 21428.009 & $13297_{4}-34725_{3}^{\circ}$ & 4632.294 & 3 & & 21581.532 & $10414_{4}^{\circ}-319954$ \\
\hline 4664.968 & 100 & 4 & 21430.375 & $75023-28932^{\circ}$ & 4632.173 & $5 b$ & 1 & 21582.096 & $14465_{2}^{\circ}-36047_{2}$ \\
\hline 4664.784 & 25 & 2 & 21431.220 & $11877_{i}^{\circ}-33309_{2}$ & 4632.140 & $8 \mathrm{~b}$ & 2 & 21582.250 & $12847_{3}-34430_{3}^{\circ}$ \\
\hline 4663.670 & 5 & 2 & 21436.339 & $18549_{2}-39985_{3}^{\circ}$ & 4632.038 & 5 & 1 & 21582.725 & $16783 \stackrel{\circ}{4}-38366_{4}$ \\
\hline 4663.202 & $300 \mathrm{r}$ & 10 & 21438.490 & $2869_{3}-24307_{2}^{\circ}$ & 4631.901 & 2 & & 21583.363 & $17224_{2}^{\circ}-38807_{2}$ \\
\hline 4662.590 & 20 & 2 & 21441.304 & $3865_{1}-25306_{2}^{\circ}$ & 4631.644 & 100 & 50 & 21584.561 & $11197{ }^{\circ}-32781_{4}$ \\
\hline 4660.254 & 20 & 2 & 21452.052 & $142260-35678^{\circ}$ & 4631.021 & 50 & 3 & 21587.464 & $14206_{4}^{\circ}-35794_{4}$ \\
\hline 4660.254 & 20 & 2 & 21452.052 & $17501{ }^{\circ}-389535$ & 4630.640 & 8 & 1 & 21589.241 & $13297_{4}-34886^{\circ}$ \\
\hline 4660.039 & 4 & 1 & 21453.042 & $17354_{1}^{\circ}-388072$ & 4630.165 & 5 & & 21591.455 & $8243 \stackrel{2}{2}-298353$ \\
\hline
\end{tabular}


TABLE 4. Classified lines of $\mathrm{Th} \mathrm{I}$-continued

\begin{tabular}{|c|c|c|c|c|c|c|c|c|c|}
\hline \multirow{2}{*}{$\begin{array}{l}\text { Wavelength } \\
(\AA)\end{array}$} & \multicolumn{2}{|c|}{ Intensity } & \multirow{2}{*}{$\begin{array}{l}\text { Wavenumber } \\
\left(\mathrm{cm}^{-1}\right)\end{array}$} & \multirow{2}{*}{ Classification } & \multirow{2}{*}{$\begin{array}{c}\text { Wavelength } \\
\text { (§) }\end{array}$} & \multicolumn{2}{|c|}{ Inten sity } & \multirow{2}{*}{$\begin{array}{c}\text { Wavenumber } \\
\left(\mathrm{cm}^{-1}\right)\end{array}$} & \multirow{2}{*}{ Classification } \\
\hline & Lamp & Spark & & & & Lamp & Spark & & \\
\hline 4629.199 & 15 & & 21595.961 & $17073_{1}-38669_{2}^{\circ}$ & 4601.104 & 4 & 1 & 21727.827 & $19503_{3}^{\circ}-41230_{4}$ \\
\hline 4628.579 & $15 b$ & & 21598.854 & $10414_{4}^{\circ}-320124$ & 4600.969 & 3 & & 21728.464 & $11877^{\circ}-33606_{2}$ \\
\hline 4628.558 & $75 b$ & 40 & 21598.952 & $17411_{3}^{\circ}-39010_{3}$ & 4600.872 & 100 & 8 & 21728.922 & $14481 \stackrel{\circ}{6}-362105$ \\
\hline 4628.202 & 200 & 4 & 21600.613 & $8111_{4}-29711^{\circ}$ & 4599.704 & 100 & 10 & 21734.440 & $5563_{1}-27297^{\circ}$ \\
\hline 4627.503 & 3 & & 21603.876 & $20423^{\circ}-42027_{2}$ & 4598.939 & 40 & 2 & 21738.055 & $0_{2}-21738_{2}^{\circ}$ \\
\hline 4627.298 & 200 & 20 & 21604.833 & $7280_{2}-28884_{3}^{\circ}$ & 4598.888 & 4 & 1 & 21738.296 & $19948 \stackrel{\circ}{4}-416863$ \\
\hline 4626.521 & 5 & 1 & 21608.461 & $15166^{\circ}-36775_{3}$ & 4598.434 & 50 & 4 & 21740.442 & $17073_{1}-38814_{2}^{\circ}$ \\
\hline 4625.893 & 2 & & 21611.395 & $18069_{3}^{\circ}-39680_{2}$ & 4597.243 & 4 & & 21746.075 & $16783_{4}^{\circ}-38529_{3}$ \\
\hline 4625.274 & 100 & 5 & 21614.287 & $13847_{2}-35462_{3}^{\circ}$ & 4596.308 & 200 & 50 & 21750.498 & $75023-29252_{2}^{\circ}$ \\
\hline 4624.939 & 5 & & 21615.852 & $130883-34704_{3}^{\circ}$ & 4596.176 & 100 & 8 & 21751.123 & $17501 \stackrel{\circ}{-392525}$ \\
\hline 4624.314 & 75 & 4 & 21618.774 & $3687_{2}-25306_{2}^{\circ}$ & 4595.902 & 3 & & 21752.420 & $15863_{2}-37616_{1}^{\circ}$ \\
\hline 4623.553 & $8 \mathrm{~b}$ & 1 & 21622.332 & $8111_{4}-29733^{\circ}$ & 4595.420 & $800 \mathrm{r}$ & 200 & 21754.701 & $3687_{2}-25442^{\circ}$ \\
\hline 4623.169 & $10 \mathrm{~b}$ & 1 & 21624.128 & $11802_{2}-33427_{2}^{\circ}$ & 4594.260 & 2 & & 21760.194 & $16346^{\circ}-381065$ \\
\hline 4623.118 & $50 \mathrm{~b}$ & 5 & 21624.367 & $14206_{4}^{\circ}-35831_{3}$ & 4594.046 & 2 & & 21761.208 & $19817_{1}^{\circ}-41578_{2}$ \\
\hline 4621.657 & 50 & 2 & 21631.203 & $13945_{3}^{\circ}-35576_{3}$ & 4593.645 & 300 & 150 & 21763.107 & $130888_{3}-34851_{2}^{\circ}$ \\
\hline 4621.165 & 150 & 40 & 21633.506 & $8111_{4}-29744{ }_{3}^{\circ}$ & 4593.524 & 4 & 2 & 21763.681 & $17411^{\circ}-391743$ \\
\hline 4621.076 & 50 & 2 & 21633.922 & $3687_{2}-25321_{3}^{\circ}$ & 4593.362 & 8 & 1 & 21764.448 & $19948{ }^{\circ}-417125$ \\
\hline 4620.705 & $3 b$ & & 21635.659 & $15970_{3}-37605_{4}^{\circ}$ & 4592.958 & 100 & 5 & 21766.362 & $111978-329635$ \\
\hline 4620.448 & 150 & 10 & 21636.862 & $13088_{3}-34725_{3}^{\circ}$ & 4592.877 & 3 & & 21766.746 & $17073_{1}-38840_{1}^{\circ}$ \\
\hline 4620.241 & 200 & 15 & 21637.832 & $7280_{2}-28917_{2}^{\circ}$ & 4592.851 & 1 & & 21766.869 & $15970_{3}-37736_{2}^{\circ}$ \\
\hline 4619.624 & 50 & 4 & 21640.722 & $18053_{4}^{\circ}-396945$ & 4592.668 & $500 \mathrm{r}$ & 150 & 21767.737 & $8243_{2}^{\circ}-300113$ \\
\hline 4619.223 & 2 & & 21642.600 & $19588^{\circ}-41230_{4}$ & 4592.519 & 15 & 2 & 21768.443 & $10783_{2}^{\circ}-325513$ \\
\hline 4617.384 & 3 & & 21651.220 & $15166^{\circ}-36818_{2}$ & 4592.097 & 8 & & 21770.444 & $8111_{4}-29881^{\circ}$ \\
\hline 4617.295 & 25 & 2 & 21651.637 & $14481^{\circ}-361335$ & 4591.972 & 1 & & 21771.036 & $18930_{3}^{\circ}-40701_{4}$ \\
\hline 4617.295 & 25 & 2 & 21651.637 & $17959_{4}-39611_{3}^{\circ}$ & 4591.581 & 8 & 1 & 21772.890 & $18809_{4}^{\circ}-405825$ \\
\hline 4616.451 & 75 & 100 & 21655.596 & $75023-29157_{3}^{\circ}$ & 4589.526 & 3 & & 21782.639 & $17501 \stackrel{\circ}{-39283_{4}}$ \\
\hline 4615.669 & 50 & 4 & 21659.265 & $12114_{2}^{\circ}-33773_{1}$ & 4589.328 & 5 & & 21783.579 & $132974-35081{ }^{\circ}$ \\
\hline 4615.334 & 150 & 15 & 21660.837 & $38651-25526 i$ & 4588.846 & 8 & 1 & 21785.867 & $17224_{2}^{\circ}-39010_{3}$ \\
\hline 4615.025 & 200 & 40 & 21662.287 & $63622_{2}-28024_{i}^{\circ}$ & 4588.426 & $500 \mathrm{r}$ & 200 & 21787.861 & $10414{ }_{4}^{\circ}-322025$ \\
\hline 4614.538 & 2 & & 21664.573 & $184313-40096_{2}^{\circ}$ & 4588.350 & $100 \mathrm{~b}$ & 25 & 21788.222 & $11802_{2}-33591_{3}^{\circ}$ \\
\hline 4614.278 & 8 & 2 & 21665.794 & $20054_{2}-41720_{2}^{\circ}$ & 4587.332 & 3 & & 21793.057 & $15863_{2}-37656_{3}^{\circ}$ \\
\hline 4614.162 & 50 & 4 & 21666.339 & $15493_{4}-37159_{3}^{\circ}$ & 4586.047 & $50 \mathrm{~b}$ & 2 & 21799.163 & $14032_{2}^{\circ}-35831_{3}$ \\
\hline 4613.605 & 150 & 15 & 21668.954 & $0_{2}-21668_{1}^{\circ}$ & 4585.175 & 2 & & 21803.309 & $14243^{\circ}-36047_{2}$ \\
\hline 4610.628 & $300 \mathrm{~b}$ & 50 & 21682.945 & $18011^{\circ}-396945$ & 4584.300 & 5 & 2 & 21807.470 & $19948^{\circ}-41755_{4}$ \\
\hline 4610.532 & 10 & 1 & 21683.397 & $18809_{4}^{\circ}-404933$ & 4584.247 & 10 & 2 & 21807.722 & $75023-29310_{4}^{\circ}$ \\
\hline 4610.456 & 2 & & 21683.754 & $4961_{4}-26645_{0}^{\circ}$ & 4583.514 & 1 & & 21811.210 & $11877_{1}^{\circ}-33689_{2}$ \\
\hline 4610.307 & 5 & 1 & 21684.455 & $15618 \stackrel{\circ}{-3} 37303_{4}$ & 4583.063 & 5 & & 21813.356 & $15490 \AA-37303_{4}$ \\
\hline 4610.078 & 100 & 4 & 21685.532 & $13847_{2}-355333_{2}^{\circ}$ & 4580.981 & 3 & & 21823.270 & $18431_{3}-40254^{\circ}$ \\
\hline 4609.796 & 2 & & 21686.859 & $15970_{3}-37656_{3}^{\circ}$ & 4580.405 & 15 & 2 & 21826.014 & $11601_{1}-33427_{2}^{\circ}$ \\
\hline 4609.516 & 2 & & 21688.176 & $15618_{3}^{\circ}-3730 \dot{7}_{3}$ & 4579.829 & 200 & 15 & 21828.759 & $4961_{4}-26790_{4}^{\circ}$ \\
\hline 4609.439 & 2 & & 21688.538 & $13945_{3}^{\circ}-35633_{4}$ & 4579.367 & 75 & 3 & 21830.961 & $165546-38385 \circ$ \\
\hline 4608.620 & 150 & 8 & 21692.392 & $2869_{3}-24561_{3}^{\circ}$ & 4578.778 & $5 \mathrm{~b}$ & & 21833.769 & $132974-35131_{4}^{\circ}$ \\
\hline 4607.935 & 300 & 20 & 21695.617 & $75023-29197_{2}^{\circ}$ & 4577.905 & 100 & 3 & 21837.933 & $3865_{1}-25703_{2}^{\circ}$ \\
\hline 4607.834 & 50 & 5 & 21696.093 & $11601_{1}-33297_{2}^{\circ}$ & 4577.823 & $100 \mathrm{~s}$ & 4 & 21838.324 & $3687_{2}-25526_{1}^{\circ}$ \\
\hline 4607.377 & 150 & 50 & 21698.245 & $13175_{4}^{\circ}-34873_{4}$ & 4577.582 & 150 & 5 & 21839.474 & $16346 \stackrel{\circ}{4}-381864$ \\
\hline 4607.224 & 3 & & 21698.965 & $13175^{\circ}-348743$ & 4576.964 & 4 & & 21842.423 & $15490 \AA-373326$ \\
\hline 4605.825 & 40 & 2 & 21705.556 & $18549_{2}-40254_{3}^{\circ}$ & 4576.742 & 100 & 2 & 21843.482 & $11877_{1}^{\circ}-33721_{2}$ \\
\hline 4605.425 & 2 & & 21707.441 & $19273_{2}-40980_{3}^{\circ}$ & 4576.542 & 20 & 2 & 21844.437 & $19516_{2}^{\circ}-41361_{3}$ \\
\hline 4603.537 & 2 & & 21716.344 & $13962_{1}-35678_{1}^{\circ}$ & 4576.272 & 8 & 2 & 21845.726 & $14032_{2}^{\circ}-35877_{2}$ \\
\hline 4603.434 & 1 & & 21716.830 & $8800_{4}-30517^{\circ}$ & 4575.821 & 1 & & 21847.879 & $19039_{2}^{\circ}-40886_{2}$ \\
\hline 4603.143 & $300 \mathrm{~b}$ & $100 \mathrm{~b}$ & 21718.202 & $824320-299611$ & 4575.247 & 200 & 50 & 21850.620 & $17501 \stackrel{\circ}{-393515}$ \\
\hline 4603.113 & $50 \mathrm{~b}$ & $8 b$ & 21718.344 & $15166_{3}^{\circ}-36885_{4}$ & 4574.028 & 200 & 20 & 21856.443 & $128477_{3}-34704^{\circ}$ \\
\hline 4602.795 & 2 & & 21719.845 & $20566_{4}^{\circ}-422863$ & 4573.574 & 75 & 3 & 21858.613 & $142045-36062^{\circ}$ \\
\hline 4602.420 & 20 & 2 & 21721.614 & $15490_{0}^{\circ}-372115$ & 4573.433 & 3 & & 21859.286 & $118022_{2}-33662_{1}^{\circ}$ \\
\hline 4601.347 & 40 & 4 & 21726.680 & $19986^{\circ}-417125$ & 4571.959 & 100 & 20 & 21866.334 & $98045-31671_{4}^{\circ}$ \\
\hline
\end{tabular}


TABle 4. Classified lines of Th $\mathrm{I}$-continued

\begin{tabular}{|c|c|c|c|c|c|c|c|c|c|}
\hline \multirow{2}{*}{$\begin{array}{l}\text { Wavelength } \\
\text { (Å) }\end{array}$} & \multicolumn{2}{|c|}{ Intensity } & \multirow{2}{*}{$\begin{array}{l}\text { Wavenumber } \\
\left(\mathrm{cm}^{-1}\right)\end{array}$} & \multirow{2}{*}{ Classification } & \multirow{2}{*}{$\begin{array}{c}\text { Wavelength } \\
\text { (§) }\end{array}$} & \multicolumn{2}{|c|}{ Inten sity } & \multirow{2}{*}{$\begin{array}{l}\text { Wavenumber } \\
\left(\mathrm{cm}^{-1}\right)\end{array}$} & \multirow{2}{*}{ Classification } \\
\hline & Lamp & Spark & & & & Lamp & Spark & & \\
\hline 4570.972 & $500 \mathrm{r}$ & 200 & 21871.055 & $11197 \circ-330686$ & 4541.501 & 10 & 2 & 22012.980 & $10783_{2}^{\circ}-327963$ \\
\hline 4570.655 & $20 \mathrm{~h}$ & 3 & 21872.572 & $17411_{3}^{\circ}-392834$ & 4541.039 & $8 b$ & & 22015.219 & $14032_{2}^{\circ}-360472$ \\
\hline 4570.551 & 2 & & 21873.070 & $15863_{2}-37736_{2}^{\circ}$ & 4540.999 & $300 \mathrm{r}$ & 75 & 22015.413 & $3687_{2}-25703_{2}^{\circ}$ \\
\hline 4569.736 & 100 & 5 & 21876.971 & $7280_{2}-29157_{1}^{\circ}$ & 4540.566 & 75 & 5 & 22017.513 & $11197^{\circ}-332145$ \\
\hline 4569.636 & 20 & 2 & 21877.449 & $12847_{3}-34725_{3}^{\circ}$ & 4540.255 & $50 \mathrm{~b}$ & 15 & 22019.021 & $17501_{0}^{\circ}-39520_{4}$ \\
\hline 4569.574 & 5 & 2 & 21877.746 & $7280_{2}-29157_{3}^{\circ}$ & 4540.070 & 4 & & 22019.918 & $16346_{4}^{\circ}-38366_{4}$ \\
\hline 4569.148 & 150 & 20 & 21879.786 & $10414^{\circ}-322935$ & 4537.143 & 100 & 3 & 22034.123 & $4961_{4}-26995_{3}^{\circ}$ \\
\hline 4568.523 & 100 & 10 & 21882.779 & $14206_{4}^{\circ}-360894$ & 4536.322 & 2 & & 22038.111 & $13847_{2}-35885_{2}^{\circ}$ \\
\hline 4568.143 & 200 & 40 & 21884.599 & $5563_{1}-27447_{2}^{\circ}$ & 4535.979 & 100 & 8 & 22039.778 & $7795_{4}^{\circ}-29835_{3}$ \\
\hline 4567.856 & 4 & 1 & 21885.974 & $13945_{3}^{\circ}-35831_{3}$ & 4535.546 & 100 & 8 & 22041.882 & $13175_{4}^{\circ}-352165$ \\
\hline 4567.240 & 300 & 100 & 21888.926 & $7795_{4}^{\circ}-296845$ & 4535.388 & 10 & 3 & 22042.650 & $13088_{3}-35131_{4}^{\circ}$ \\
\hline 4566.988 & 10 & & 21890.134 & $\mathbf{0}_{2}-21890_{3}^{\circ}$ & 4535.255 & $300 \mathrm{r}$ & 150 & 22043.296 & $8243_{2}^{\circ}-30286_{1}$ \\
\hline 4566.713 & 15 & 4 & 21891.452 & $18809_{4}^{\circ}-40701_{4}$ & 4535.103 & 2 & & 22044.035 & $10414_{4}^{\circ}-324584$ \\
\hline 4565.805 & $40 \mathrm{~b}$ & 5 & 21895.806 & $11877_{1}^{\circ}-33773_{1}$ & 4534.402 & 50 & 4 & 22047.443 & $15736_{1}^{\circ}-37784_{2}$ \\
\hline 4565.783 & $75 b$ & 10 & 21895.911 & $171665-39062 \circ$ & 4533.717 & 4 & & 22050.774 & $14465_{2}^{\circ}-36515_{3}$ \\
\hline 4564.835 & 150 & 10 & 21900.458 & $2869_{3}-24769_{3}^{\circ}$ & 4533.071 & 300 & 75 & 22053.916 & $7795_{4}^{\circ}-29849_{4}$ \\
\hline 4563.658 & $400 \mathrm{r}$ & $200 b$ & 21906.106 & $8111_{4}-30017_{3}^{\circ}$ & 4531.574 & 3 & & 22061.202 & $11601_{1}-33662^{\circ}$ \\
\hline 4562.727 & 8 & 1 & 21910.576 & $17411_{3}^{\circ}-39321_{3}$ & 4531.531 & 1 & & 22061.411 & $19516_{2}^{\circ}-415782$ \\
\hline 4562.476 & 75 & 5 & 21911.782 & $98045-31716^{\circ}$ & 4530.319 & 200 & 100 & 22067.313 & $11241_{3}^{\circ}-33309_{2}$ \\
\hline 4562.081 & 200 & 50 & 21913.679 & 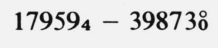 & 4529.922 & 40 & 5 & 22069.247 & $15618_{3}^{\circ}-376883$ \\
\hline 4561.704 & 5 & 2 & 21915.490 & $11802_{2}-33718_{2}^{\circ}$ & 4527.115 & 21 & & 22082.930 & $159703-38053_{3}^{\circ}$ \\
\hline 4561.495 & 200 & 25 & 21916.494 & $4961_{4}-26878^{\circ}$ & 4525.447 & 40 & 3 & 22091.069 & $15863_{2}-37954_{3}^{\circ}$ \\
\hline 4561.397 & $100 \mathrm{~b}$ & 10 & 21916.965 & $75023-29419_{2}^{\circ}$ & 4524.129 & 100 & 10 & 22097.505 & $17224_{2}^{\circ}-39321_{3}$ \\
\hline 4561.348 & $500 \mathrm{r}$ & 200 & 21917.200 & $7280_{2}-29197_{1}^{\circ}$ & 4523.211 & 2 & & 22101.990 & $13945_{3}^{\circ}-36047_{2}$ \\
\hline 4561.228 & 150 & 10 & 21917.777 & $7280_{2}-29197_{2}^{\circ}$ & 4522.315 & 50 & 4 & 22106.369 & $15863_{2}-37970_{2}^{\circ}$ \\
\hline 4560.981 & 2 & & 21918.964 & $15166_{3}^{\circ}-37085_{4}$ & 4521.332 & $15 b$ & 4 & 22111.175 & $5563_{1}-27674_{2}^{\circ}$ \\
\hline 4560.067 & 3 & & 21923.357 & $13962_{1}-35885_{2}^{\circ}$ & 4521.305 & 1501 & 20 & 22111.307 & $165546-38665^{\circ}$ \\
\hline 4559.396 & 2 & & 21926.583 & $14206^{\circ}-361335$ & 4521.196 & $500 \mathrm{r}$ & 75 & 22111.840 & $2869_{3}-24981_{3}^{\circ}$ \\
\hline 4559.312 & 50 & 5 & 21926.987 & $16351_{0}-38278^{\circ}$ & 4521.059 & 50 & 5 & 22112.510 & $15493_{4}-37605_{4}^{\circ}$ \\
\hline 4559.108 & 1 & & 21927.969 & $15166_{3}^{\circ}-370942$ & 4519.259 & 300 & 75 & 22121.317 & $3687_{2}-25809_{1}^{\circ}$ \\
\hline 4559.058 & 100 & 3 & 21928.209 & $16217_{2}^{\circ}-38145_{3}$ & 4518.609 & $5 b$ & & 22124.499 & $19588^{\circ}-417125$ \\
\hline 4558.346 & 300 & 40 & 21931.634 & $10526^{\circ}-324584$ & 4518.342 & 50 & & 22125.807 & $13175_{4}^{\circ}-35300_{5}$ \\
\hline 4555.903 & 300 & 150 & 21943.394 & $13297_{4}-35240_{3}^{\circ}$ & 4517.725 & $4 b$ & 2 & 22128.829 & $19227 \%-413565$ \\
\hline 4555.809 & $500 \mathrm{r}$ & 100 & 21943.847 & $3865_{1}-25809^{\circ}$ & 4517.680 & $4 \mathrm{~b}$ & & 22129.049 & $15618_{3}^{\circ}-37748_{2}$ \\
\hline 4555.143 & 100 & 5 & 21947.055 & $17959_{4}-39906_{4}^{\circ}$ & 4517.629 & $8 \mathrm{~b}$ & 3 & 22129.299 & $14206_{4}^{\circ}-363363$ \\
\hline 4554.415 & 100 & 3 & 21950.563 & $16783 \stackrel{9}{4}-387343$ & 4516.736 & 3 & & 22133.674 & $116011-33734_{2}^{\circ}$ \\
\hline 4553.474 & 3 & & 21955.099 & $21902_{4}^{\circ}-43857_{5}$ & 4516.540 & 40 & 4 & 22134.634 & $142260-36361_{1}^{\circ}$ \\
\hline 4552.222 & $100 \mathrm{~b}$ & 8 & 21961.138 & $7795_{4}^{\circ}-29756_{4}$ & 4515.629 & 10 & 2 & 22139.100 & $7280_{2}-29419_{2}^{\circ}$ \\
\hline 4552.154 & $300 \mathrm{r}$ & 150 & 21961.466 & $8800_{4}-30761_{3}^{\circ}$ & 4515.401 & 10 & 2 & 22140.218 & $15166_{3}^{\circ}-37307_{3}$ \\
\hline 4551.597 & 25 & 5 & 21964.153 & $15166_{3}^{\circ}-371313$ & 4515.118 & $300 \mathrm{r}$ & 50 & 22141.605 & $0_{2}-22141_{3}^{\circ}$ \\
\hline 4551.473 & 200 & 75 & 21964.751 & $13847_{2}-35812_{3}^{\circ}$ & 4514.548 & 10 & 2 & 22144.401 & $139455_{3}^{\circ}-36089_{4}$ \\
\hline 4549.833 & 40 & 2 & 21972.669 & $7280_{2}-29252_{2}^{\circ}$ & 4514.398 & 3 & 1 & 22145.137 & $171665-39311^{\circ}$ \\
\hline 4549.606 & $5 b$ & & 21973.765 & $142045-36178_{4}^{\circ}$ & 4514.059 & 75 & 5 & 22146.800 & $18053_{4}^{\circ}-40200_{3}$ \\
\hline 4549.052 & 2 & & 21976.441 & 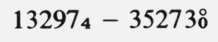 & 4513.681 & $400 \mathrm{r}$ & 150 & 22148.654 & $98045-31953^{\circ}$ \\
\hline 4548.979 & 20 & 4 & 21976.794 & $16783_{4}^{\circ}-38760_{4}$ & 4513.224 & 200 & 50 & 22150.897 & $63622-28513_{2}^{\circ}$ \\
\hline 4548.326 & $100 \mathrm{~b}$ & 10 & 21979.949 & $15970_{3}-37950_{4}^{\circ}$ & 4512.950 & 2 & & 22152.242 & $13088_{3}-35240_{3}^{\circ}$ \\
\hline 4547.611 & 5 & 1 & 21983.404 & $18069_{3}^{\circ}-400524$ & 4510.611 & 75 & & 22163.729 & $15493_{4}-37656_{3}^{\circ}$ \\
\hline 4547.248 & 200 & 25 & 21985.160 & $6362_{2}-28347_{2}^{\circ}$ & 4510.611 & 75 & & 22163.729 & $17398_{3}-39562_{4}^{\circ}$ \\
\hline 4546.675 & 75 & & 21987.930 & $8800_{4}-30788 \circ$ & 4510.433 & 4 & & 22164.604 & $13297_{4}-35462_{3}^{\circ}$ \\
\hline 4544.729 & $3 b$ & & 21997.345 & $18614_{i}^{\circ}-406112$ & 4509.960 & 100 & 8 & 22166.928 & $112413-334083$ \\
\hline 4544.657 & 20 & 4 & 21997.694 & $11802_{2}-33800_{3}^{\circ}$ & 4509.844 & 20 & 4 & 22167.498 & $19588^{\circ}-41755_{4}$ \\
\hline 4544.422 & 2 & & 21998.831 & $18053_{4}^{\circ}-40052_{4}$ & 4509.461 & 2 & & 22169.381 & $16783_{4}^{\circ}-38953_{5}$ \\
\hline 4543.412 & $100 \mathrm{~b}$ & $8 \mathrm{~b}$ & 22003.721 & $12847_{3}-34851_{2}^{\circ}$ & 4509.390 & 2 & & 22169.730 & $19516_{2}^{\circ}-41686_{3}$ \\
\hline 4543.384 & $20 \mathrm{~b}$ & 5 & 22003.857 & $14206^{\circ}-362105$ & 4508.715 & 2 & & 22173.049 & $16783_{4}^{\circ}-38956_{4}$ \\
\hline 4542.049 & 8 & 1 & 22010.324 & $6362_{2}-28372_{i}^{\circ}$ & 4508.035 & 100 & 20 & 22176.394 & $11241 \stackrel{\circ}{3}-334184$ \\
\hline
\end{tabular}


TABle 4. Classified lines of Th $\mathrm{I}$-continued

\begin{tabular}{|c|c|c|c|c|c|c|c|c|c|}
\hline \multirow{2}{*}{$\begin{array}{l}\text { Wavelength } \\
\text { (Å) }\end{array}$} & \multicolumn{2}{|c|}{ Intensity } & \multirow{2}{*}{$\begin{array}{l}\text { Wavenumber } \\
\left(\mathrm{cm}^{-1}\right)\end{array}$} & \multirow{2}{*}{ Classification } & \multirow{2}{*}{$\begin{array}{c}\text { Wavelength } \\
\text { (̊̊) }\end{array}$} & \multicolumn{2}{|c|}{ Inten sity } & \multirow{2}{*}{$\begin{array}{l}\text { Wavenumber } \\
\left(\mathrm{cm}^{-1}\right)\end{array}$} & \multirow{2}{*}{ Classification } \\
\hline & Lamp & Spark & & & & Lamp & Spark & & \\
\hline 4507.639 & 2 & & 22178.342 & $142045-36382^{\circ}$ & 4477.865 & 3 & & 22325.806 & $17354 i-39680_{2}$ \\
\hline 4506.549 & 150 & 40 & 22183.706 & $12114_{2}^{\circ}-342981$ & 4477.216 & 50 & 8 & 22329.043 & $15490 \AA-37819_{4}$ \\
\hline 4506.474 & 200 & 75 & 22184.075 & $75023-29686_{3}^{\circ}$ & 4477.037 & 8 & & 22329.935 & $142260-36556^{\circ}$ \\
\hline 4505.607 & 8 & 3 & 22188.344 & $14465_{2}^{\circ}-366532$ & 4476.977 & 5 & 2 & 22330.235 & $12114_{2}^{\circ}-34444_{2}$ \\
\hline 4505.448 & 2 & & 22189.127 & $15863_{2}-38053_{3}^{\circ}$ & 4475.765 & 75 & 8 & 22336.281 & $159703-38306_{4}^{\circ}$ \\
\hline 4505.216 & $500 \mathrm{r}$ & 100 & 22190.269 & $8800_{4}-30990_{3}^{\circ}$ & 4475.410 & 2 & & 22338.053 & $170731-39411_{1}^{\circ}$ \\
\hline 4504.630 & 100 & 20 & 22193.156 & $17501 \stackrel{\circ}{-} 396945$ & 4475.410 & 2 & & 22338.053 & $19948 \stackrel{\circ}{4}-422863$ \\
\hline 4503.214 & 15 & 3 & 22200.135 & $15618^{\circ}-37819_{4}$ & 4475.221 & 200 & 40 & 22338.996 & $\mathbf{0}_{2}-22338^{\circ}$ \\
\hline 4501.177 & 4 & 2 & 22210.181 & $19817_{1}^{\circ}-42027_{2}$ & 4474.819 & 50 & 8 & 22341.003 & 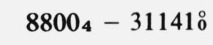 \\
\hline 4500.581 & 20 & 3 & 22213.122 & $173983-39611_{3}^{\circ}$ & 4474.789 & 10 & & 22341.153 & $13847_{2}-36188_{2}^{\circ}$ \\
\hline 4499.984 & 500 & 200 & 22216.069 & $7795^{\circ}-300113$ & 4474.403 & 5 & & 22343.080 & $14465_{2}^{\circ}-36808_{3}$ \\
\hline 4499.705 & 50 & 5 & 22217.446 & $11241^{\circ}-334594$ & 4473.337 & 2 & & 22348.405 & $3687_{2}-26036_{3}^{\circ}$ \\
\hline 4499.431 & 15 & 3 & 22218.799 & $98045-32023{ }^{\circ}$ & 4472.844 & 200 & 50 & 22350.868 & $75023-29853^{\circ}$ \\
\hline 4499.383 & 20 & 8 & 22219.037 & $7795^{\circ}-30014_{4}$ & 4472.620 & 20 & 5 & 22351.987 & $13945_{3}^{\circ}-36297_{4}$ \\
\hline 4498.947 & $800 r$ & 200 & 22221.190 & $5563_{1}-27784_{2}^{\circ}$ & 4472.348 & 3 & & 22353.347 & $17847_{2}^{\circ}-40200_{3}$ \\
\hline 4498.156 & 40 & 3 & 22225.097 & $15863_{2}-38088_{2}^{\circ}$ & 4471.871 & 25 & 2 & 22355.731 & $4961_{4}-27317^{\circ}$ \\
\hline 4497.908 & $150 \mathrm{~b}$ & 50 & 22226.323 & $16783^{\circ}-39010_{3}$ & 4471.483 & 3 & & 22357.671 & $180699_{3}^{\circ}-404264$ \\
\hline 4497.908 & $150 \mathrm{~b}$ & 50 & 22226.323 & $13847_{2}-36074_{3}^{\circ}$ & 4470.990 & 200 & 25 & 22360.136 & $7280_{2}-29640_{1}^{\circ}$ \\
\hline 4497.888 & $50 \mathrm{~b}$ & & 22226.421 & $13962_{1}-36188_{2}^{\circ}$ & 4470.990 & 200 & 25 & 22360.136 & $15736_{1}^{\circ}-38097_{2}$ \\
\hline 4497.780 & 3 & 1 & 22226.955 & $6362_{2}-28589_{3}^{\circ}$ & 4470.105 & 5 & 2 & 22364.563 & $11241_{3}^{\circ}-336062$ \\
\hline 4497.125 & 4 & 2 & 22230.192 & $13175_{4}^{\circ}-35405_{3}$ & 4469.526 & $400 \mathrm{r}$ & 150 & 22367.460 & $10414_{4}^{\circ}-327814$ \\
\hline 4495.598 & 15 & 4 & 22237.743 & $15166^{\circ}-374043$ & 4468.320 & 200 & 50 & 22373.497 & $13088_{3}-35462^{\circ}$ \\
\hline 4494.863 & 2 & & 22241.380 & $14243 i-36485_{2}$ & 4467.188 & 2 & & 22379.166 & $75023-29881_{4}^{\circ}$ \\
\hline 4494.692 & 100 & 8 & 22242.225 & $75023-29744_{3}^{\circ}$ & 4465.975 & 150 & 2 & 22385.245 & $159703-38355_{2}^{\circ}$ \\
\hline 4493.763 & 2 & & 22246.824 & $15970_{3}-38216_{3}^{\circ}$ & 4465.471 & 3 & & 22387.771 & $16346_{4}^{\circ}-38734_{3}$ \\
\hline 4493.568 & 100 & 20 & 22247.789 & $38651-26113_{2}^{\circ}$ & 4464.848 & 50 & 20 & 22390.895 & $139453_{3}^{\circ}-36336_{3}$ \\
\hline 4493.334 & $1000 \mathrm{r}$ & 300 & 22248.948 & $0_{2}-22248_{2}^{\circ}$ & 4464.140 & 10 & 3 & 22394.446 & $8800_{4}-31194_{4}^{\circ}$ \\
\hline 4492.677 & 20 & 5 & 22252.201 & $15490^{\circ}-377426$ & 4463.834 & 200 & 100 & 22395.981 & $17166_{5}-39562^{\circ}$ \\
\hline 4492.573 & 8 & 3 & 22252.716 & $19503_{3}^{\circ}-41755_{4}$ & 4463.668 & 200 & 75 & 22396.814 & $\mathbf{0}_{2}-22396^{\circ}$ \\
\hline 4492.102 & 200 & 20 & 22255.050 & $10526^{\circ}-32781_{4}$ & 4463.245 & 4 & 2 & 22398.937 & $139621-36361 i$ \\
\hline 4490.673 & 200 & 4 & 22262.131 & 111970 - 334594 & 4462.757 & 3 & & 22401.386 & $13175_{4}^{\circ}-35576_{3}$ \\
\hline 4490.524 & 2 & & 22262.870 & $130883-35351_{4}^{\circ}$ & 4461.815 & $15 b$ & & 22406.115 & $8111_{4}-30517^{\circ}$ \\
\hline 4489.664 & 200 & 100 & 22267.134 & $14481 \stackrel{\circ}{-}-367496$ & 4461.789 & $200 \mathrm{~b}$ & 100 & 22406.246 & $7280_{2}-29686_{3}^{\circ}$ \\
\hline 4489.245 & 2 & & 22269.213 & $17411^{\circ}-39680_{2}$ & 4461.602 & 15 & & 22407.185 & $13175_{4}^{\circ}-355825$ \\
\hline 4488.554 & 5 & 2 & 22272.641 & $18614 i-408862$ & 4461.241 & 200 & 100 & 22408.998 & $3687_{2}-26096_{3}^{\circ}$ \\
\hline 4488.312 & 200 & 100 & 22273.842 & $15618{ }^{\circ}-378924$ & 4459.976 & 15 & 4 & 22415.354 & $18011{ }^{\circ}-404264$ \\
\hline 4486.898 & 300 & 100 & 22280.861 & $25580-24838^{\circ}$ & 4458.736 & 150 & 75 & 22421.588 & $3865_{1}-26287_{i}^{\circ}$ \\
\hline 4486.419 & 8 & 2 & 22283.240 & $128473-35131_{4}^{\circ}$ & 4458.002 & $600 \mathrm{r}$ & 200 & 22425.279 & $36872-26113^{\circ}$ \\
\hline 4485.898 & 5 & 2 & 22285.828 & $11241^{\circ}-33527_{4}$ & 4457.782 & 2 & & 22426.386 & $17411_{3}^{\circ}-39837_{3}$ \\
\hline 4485.713 & 150 & 40 & 22286.747 & $6362_{2}-28649_{1}^{\circ}$ & 4456.809 & 2 & & 22431.282 & $185492-40980^{\circ}$ \\
\hline 4484.038 & 200 & & 22295.072 & $17959_{4}-40254_{3}^{\circ}$ & 4455.867 & 150 & 25 & 22436.024 & $13297_{4}-35733^{\circ}$ \\
\hline 4483.346 & 300 & 1 & 22298.513 & $4961_{4}-27260_{3}^{\circ}$ & 4455.148 & 2 & & 22439.645 & $18053 \stackrel{\circ}{4}-404933$ \\
\hline 4482.911 & 5 & 2 & 22300.677 & $18930_{3}^{\circ}-41230_{4}$ & 4454.139 & 75 & 10 & 22444.728 & $130883-35533^{\circ}$ \\
\hline 4482.855 & 3 & 1 & 22300.955 & $82432-305442$ & 4453.860 & 8 & 3 & 22446.134 & $15970_{3}-38416_{3}^{\circ}$ \\
\hline 4482.169 & $400 \mathrm{r}$ & 100 & 22304.368 & $4961_{4}-27266_{4}^{\circ}$ & 4452.565 & 200 & 100 & 22452.662 & $2869_{3}-25321_{3}^{\circ}$ \\
\hline 4481.391 & 20 & 3 & 22308.241 & $11802_{2}-34111_{1}^{\circ}$ & 4452.437 & 50 & 4 & 22453.308 & $14032_{2}^{\circ}-36485_{2}$ \\
\hline 4481.226 & 10 & 8 & 22309.062 & $14206^{\circ}-36515_{3}$ & 4451.871 & 1 & & 22456.162 & $17224_{2}^{\circ}-39680_{2}$ \\
\hline 4481.010 & 8 & 3 & 22310.137 & $14465_{2}^{\circ}-36775_{3}$ & 4451.740 & 20 & 4 & 22456.823 & $154934-37950_{4}^{\circ}$ \\
\hline 4480.547 & 251 & 10 & 22312.443 & $16217_{2}^{\circ}-38529_{3}$ & 4451.359 & 40 & 15 & 22458.745 & $13175_{4}^{\circ}-35633_{4}$ \\
\hline 4480.308 & 1 & & 22313.633 & $20322 \stackrel{\circ}{-}-426355$ & 4450.802 & 100 & 40 & 22461.556 & $55631-28024^{\circ}$ \\
\hline 4480.267 & 25 & 3 & 22313.837 & $6362_{2}-28676_{3}^{\circ}$ & 4450.238 & 5 & & 22464.402 & $7280_{2}-29744_{3}^{\circ}$ \\
\hline 4479.781 & 15 & 4 & 22316.258 & $10783_{2}^{\circ}-33099_{3}$ & 4450.066 & 2 & & 22465.271 & $17959_{4}-40425^{\circ}$ \\
\hline 4479.637 & 200 & 75 & 22316.975 & $12114_{2}^{\circ}-344313$ & 4449.437 & 5 & 1 & 22468.446 & $16783 \stackrel{\circ}{4}-392525$ \\
\hline 4478.594 & 150 & 40 & 22322.172 & $25580-24880^{\circ}$ & 4447.230 & 150 & 25 & 22479.597 & $112411_{3}^{\circ}-33721_{2}$ \\
\hline 4478.402 & 150 & 40 & 22323.130 & $11241^{\circ}-335643$ & 4446.584 & 100 & 15 & 22482.862 & $8800_{4}-31283_{3}^{\circ}$ \\
\hline
\end{tabular}


TABLE 4. Classified lines of Th $\mathrm{I}$-continued

\begin{tabular}{|c|c|c|c|c|c|c|c|c|c|}
\hline \multirow{2}{*}{ 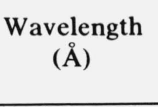 } & \multicolumn{2}{|c|}{ Intensity } & \multirow{2}{*}{$\begin{array}{l}\text { Wavenumber } \\
\left(\mathrm{cm}^{-1}\right)\end{array}$} & \multirow{2}{*}{ Classification } & \multirow{2}{*}{$\begin{array}{c}\text { Wavelength } \\
\text { (A) }\end{array}$} & \multicolumn{2}{|c|}{ Intensity } & \multirow{2}{*}{$\begin{array}{c}\text { Wavenumber } \\
\left(\mathrm{cm}^{-1}\right)\end{array}$} & \multirow{2}{*}{ Classification } \\
\hline & Lamp & Spark & & & & Lamp & Spark & & \\
\hline 4445.901 & 200 & 150 & 22486.316 & $2869_{3}-25355_{4}^{\circ}$ & 4414.486 & $500 \mathrm{~d}$ & 150 & 22646.334 & $8243_{2}^{\circ}-30889_{1}$ \\
\hline 4445.447 & 3 & 1 & 22488.612 & $163510-38840^{\circ}$ & 4414.216 & 75 & 5 & 22647.719 & $18053_{4}^{\circ}-40701_{4}$ \\
\hline 4445.309 & $200 \mathrm{~b}$ & $40 b$ & 22489.310 & $98045-32294 \stackrel{\circ}{9}$ & 4414.142 & 15 & 3 & 22648.098 & $18930_{3}^{\circ}-41578_{2}$ \\
\hline 4445.032 & 200 & 75 & 22490.712 & $10783_{2}^{\circ}-332731$ & 4413.632 & 200 & 25 & 22650.715 & $8111_{4}-30761_{3}^{\circ}$ \\
\hline 4444.901 & 1 & & 22491.375 & $15863_{2}-38355_{2}^{\circ}$ & 4413.589 & 1 & & 22650.936 & $185492-41200_{2}^{\circ}$ \\
\hline 4443.665 & 150 & 100 & 22497.631 & $3865_{1}-26363_{2}^{\circ}$ & 4413.343 & $10 b$ & 3 & 22652.199 & $15166_{3}^{\circ}-37819_{4}$ \\
\hline 4443.203 & 4 & 2 & 22499.970 & $16783_{4}^{\circ}-39283_{4}$ & 4412.565 & $300 \mathrm{~b}$ & $50 \mathrm{~b}$ & 22656.193 & $13175_{4}^{\circ}-35831_{3}$ \\
\hline 4442.513 & 20 & 5 & 22503.465 & $12847_{3}-35351_{4}^{\circ}$ & 4412.270 & $25 \mathrm{~s}$ & 2 & 22657.707 & $7502_{3}-30160_{4}^{\circ}$ \\
\hline 4441.659 & 50 & 4 & 22507.791 & $165546-39062{ }^{\circ}$ & 4411.139 & 50 & 5 & 22663.517 & $16346_{4}^{\circ}-39010_{3}$ \\
\hline 4441.609 & 100 & 5 & 22508.045 & $\mathbf{0}_{2}-22508_{2}^{\circ}$ & 4409.899 & 100 & 5 & 22669.889 & $0_{2}-22669_{3}^{\circ}$ \\
\hline 4441.512 & 25 & 3 & 22508.536 & $17398_{3}-39906_{4}^{\circ}$ & 4408.883 & $800 \mathrm{r}$ & 200 & 22675.113 & $3687_{2}-26363_{2}^{\circ}$ \\
\hline 4441.198 & 40 & 5 & 22510.128 & $11601_{1}-34111^{\circ}$ & 4408.481 & 300 & 150 & 22677.181 & $8111_{4}-30788 \circ$ \\
\hline 4441.149 & 15 & 3 & 22510.376 & $19516^{\circ}-42027_{2}$ & 4408.251 & 20 & 2 & 22678.364 & $14206_{4}^{\circ}-368854$ \\
\hline 4440.353 & 300 & 75 & 22514.411 & $8243_{2}^{\circ}-307582$ & 4406.472 & 5 & 2 & 22687.520 & $19948 \stackrel{\circ}{4}-426355$ \\
\hline 4440.276 & 300 & 75 & 22514.802 & $75023-30017_{3}^{\circ}$ & 4405.999 & 15 & 3 & 22689.955 & $18011{ }^{\circ}-40701_{4}$ \\
\hline 4440.230 & 2 & & 22515.035 & $132974-35812^{\circ}$ & 4404.820 & 15 & 3 & 22696.028 & $15490 \AA-381864$ \\
\hline 4439.858 & 100 & 4 & 22516.921 & $16217^{\circ}-387343$ & 4404.474 & 1 & & 22697.811 & $17398_{3}-40096_{2}^{\circ}$ \\
\hline 4439.000 & 8 & 2 & 22521.273 & $15166^{\circ}-376883$ & 4404.113 & 300 & 40 & 22699.672 & $15970_{3}-38669_{2}^{\circ}$ \\
\hline 4438.746 & 400 & 200 & 22522.562 & $6362_{2}-28884_{3}^{\circ}$ & 4403.425 & 50 & 3 & 22703.218 & $18431_{3}-41134_{4}^{\circ}$ \\
\hline 4438.092 & 150 & 8 & 22525.881 & $107832-333092$ & 4402.927 & 500 & 150 & 22705.786 & $2869_{3}-25575_{4}^{\circ}$ \\
\hline 4437.446 & 300 & 20 & 22529.160 & $18053_{4}^{\circ}-405825$ & 4402.601 & 75 & 10 & 22707.467 & $171665-39873{ }^{\circ}$ \\
\hline 4435.705 & 4 & 1 & 22538.003 & $16783^{\circ}-393213$ & 4402.448 & 50 & 5 & 22708.256 & $13945_{3}^{\circ}-36653_{2}$ \\
\hline 4435.296 & 100 & 10 & 22540.081 & $13945_{3}^{\circ}-36485_{2}$ & 4402.246 & 200 & 50 & 22709.299 & $4961_{4}-27670 \stackrel{8}{\circ}$ \\
\hline 4434.075 & 150 & 40 & 22546.288 & $18809_{4}^{\circ}-41356_{5}$ & 4401.581 & 500 & 200 & 22712.729 & $7795_{4}^{\circ}-305085$ \\
\hline 4433.490 & 150 & 50 & 22549.263 & $10414{ }^{\circ}-329635$ & 4399.968 & 150 & 50 & 22721.055 & $8243_{2}^{\circ}-309643$ \\
\hline 4432.252 & 400 & 150 & 22555.561 & $6362_{2}-28917_{2}^{\circ}$ & 4399.583 & 8 & 2 & 22723.044 & $11601_{1}-34324^{\circ}$ \\
\hline 4431.416 & 100 & 8 & 22559.816 & $15493_{4}-38053_{3}^{\circ}$ & 4399.453 & 15 & 3 & 22723.715 & $15493_{4}-38216_{3}^{\circ}$ \\
\hline 4429.979 & 100 & 8 & 22567.134 & $15618^{\circ}-381864$ & 4399.027 & 50 & 15 & 22725.916 & $15166_{3}^{\circ}-37892_{4}$ \\
\hline 4429.822 & 200 & 20 & 22567.934 & $16783{ }^{\circ}-393515$ & 4398.272 & 15 & 3 & 22729.817 & $14481 \stackrel{\circ}{-372115}$ \\
\hline 4429.722 & 8 & 2 & 22568.443 & $14206_{4}^{\circ}-36775_{3}$ & 4397.009 & $200 w$ & 40 & 22736.346 & $16783^{\circ}-39520_{4}$ \\
\hline 4429.633 & 200 & $20 \mathrm{~h}$ & 22568.897 & $11802_{2}-34371_{2}^{\circ}$ & 4396.140 & 100 & 10 & 22740.840 & $17166_{5}-39906_{4}^{\circ}$ \\
\hline 4428.828 & 5 & 2 & 22572.999 & $7280_{2}-29853 \stackrel{\circ}{2}$ & 4395.674 & 10 & 2 & 22743.251 & $14032_{2}^{\circ}-36775_{3}$ \\
\hline 4428.744 & 100 & 5 & 22573.427 & $2869_{3}-25442 \stackrel{\circ}{3}$ & 4394.838 & 50 & 15 & 22747.577 & $15618_{3}^{\circ}-38366_{4}$ \\
\hline 4428.553 & 200 & 40 & 22574.400 & $14206_{4}^{\circ}-367815$ & 4393.759 & 400 & 150 & 22753.163 & $7502 \mathrm{~s}-30255_{3}^{\circ}$ \\
\hline 4427.530 & $100 \mathrm{~b}$ & 8 & 22579.616 & $13847_{2}-36427_{3}^{\circ}$ & 4393.131 & 4 & 2 & 22756.416 & $18930_{3}^{\circ}-416863$ \\
\hline 4427.232 & 100 & 15 & 22581.136 & $15166_{3}^{\circ}-37748_{2}$ & 4392.974 & 400 & 100 & 22757.229 & $7795_{4}^{\circ}-305524$ \\
\hline 4426.019 & 1 & & 22587.325 & $17398_{3}-39985_{3}^{\circ}$ & 4392.495 & $100 \mathrm{~b}$ & 10 & 22759.710 & $12114_{2}^{\circ}-34874_{3}$ \\
\hline 4425.460 & 200 & 20 & 22590.177 & $16217_{2}^{\circ}-38807_{2}$ & 4391.531 & 100 & 25 & 22764.706 & $179594-40724{ }^{\circ}$ \\
\hline 4424.836 & $200 \mathrm{~b}$ & 50 & 22593.363 & $12114^{\circ}-34707_{3}$ & 4391.390 & 150 & 50 & 22765.437 & $132974-36062_{4}^{\circ}$ \\
\hline 4424.661 & 4 & 2 & 22594.257 & $13962_{1}-36556_{1}^{\circ}$ & 4390.613 & 15 & 3 & 22769.466 & $19516_{2}^{\circ}-42286_{3}$ \\
\hline 4424.243 & 75 & 5 & 22596.392 & $142045-36800_{4}^{\circ}$ & 4389.308 & 3 & 1 & 22776.236 & $140322_{2}^{\circ}-368083$ \\
\hline 4423.719 & 400 & 150 & 22599.068 & $3687_{2}-26287_{i}^{\circ}$ & 4389.234 & 8 & 2 & 22776.620 & $132974-360743^{\circ}$ \\
\hline 4423.263 & 100 & 10 & 22601.398 & $14206_{4}^{\circ}-36808_{3}$ & 4388.569 & 10 & 3 & 22780.071 & $11802_{2}-34583_{3}^{\circ}$ \\
\hline 4422.048 & 600 & 200 & 22607.608 & $10783^{\circ}-33390_{1}$ & 4388.541 & 1 & & 22780.216 & $21077^{\circ}-43857_{5}$ \\
\hline 4420.925 & 100 & 8 & 22613.350 & $17224_{2}^{\circ}-39837_{3}$ & 4388.101 & 50 & 5 & 22782.500 & $10526^{\circ}-33309_{2}$ \\
\hline 4420.782 & $300 \mathrm{~b}$ & $100 \mathrm{~b}$ & 22614.082 & $12847_{3}-35462_{3}^{\circ}$ & 4387.734 & 150 & 40 & 22784.406 & $55631-28347_{2}^{\circ}$ \\
\hline 4420.256 & 200 & 20 & 22616.773 & $15490 \circ-381065$ & 4387.028 & $100 \mathrm{~b}$ & 40 & 22788.072 & $118022-345911$ \\
\hline 4420.112 & 100 & 10 & 22617.509 & $15166_{3}^{\circ}-377842$ & 4386.138 & 3 & & 22792.696 & $16217_{2}^{\circ}-39010_{3}$ \\
\hline 4419.768 & 100 & 20 & 22619.270 & $13175_{4}^{\circ}-357944$ & 4385.753 & 25 & 5 & 22794.697 & $6362_{2}-29157_{1}^{\circ}$ \\
\hline 4418.554 & 75 & 3 & 22625.484 & $10783_{2}^{\circ}-334083$ & 4384.656 & $150 \mathrm{~b}$ & $50 \mathrm{~b}$ & 22800.400 & $10414 \stackrel{\circ}{4}-332145$ \\
\hline 4418.201 & 15 & 2 & 22627.292 & $11802_{2}-34430_{3}^{\circ}$ & 4383.872 & 100 & 40 & 22804.478 & $142045-37008{ }^{\circ}$ \\
\hline 4417.229 & 10 & 3 & 22632.271 & $18069_{3}^{\circ}-40701_{4}$ & 4383.602 & 15 & 3 & 22805.882 & $15863_{2}-38669_{2}^{\circ}$ \\
\hline 4416.946 & 25 & 3 & 22633.721 & $142045-36837^{\circ}$ & 4382.893 & 100 & 15 & 22809.571 & $5563_{1}-28372{ }^{\circ}$ \\
\hline 4416.844 & 500 & 100 & 22634.244 & $98045-32439 \stackrel{\circ}{9}$ & 4382.526 & 3 & & 22811.481 & $15863_{2}-38675_{3}^{\circ}$ \\
\hline 4414.767 & 75 & 10 & 22644.892 & $13088_{3}-35733^{\circ}$ & 4382.205 & 20 & 5 & 22813.152 & $154934-383064$ \\
\hline
\end{tabular}


TABLE 4. Classified lines of Th $\mathrm{I}$-continued

\begin{tabular}{|c|c|c|c|c|c|c|c|c|c|}
\hline \multirow{2}{*}{ 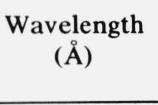 } & \multicolumn{2}{|c|}{ Intensity } & \multirow{2}{*}{$\begin{array}{l}\text { Wavenumber } \\
\quad\left(\mathrm{cm}^{-1}\right)\end{array}$} & \multirow{2}{*}{ Classification } & \multirow{2}{*}{$\begin{array}{c}\text { Wavelength } \\
\text { (§) }\end{array}$} & \multicolumn{2}{|c|}{ Inten sity } & \multirow{2}{*}{$\begin{array}{l}\text { Wavenumber } \\
\left(\mathrm{cm}^{-1}\right)\end{array}$} & \multirow{2}{*}{ Classification } \\
\hline & Lamp & Spark & & & & Lamp & Spark & & \\
\hline 4381.130 & 2 & & 22818.750 & $15970_{3}-38788_{3}^{\circ}$ & 4351.466 & 100 & 50 & 22974.303 & $13088_{3}-36062_{4}^{\circ}$ \\
\hline 4380.879 & 4 & 1 & 22820.057 & $3687_{2}-26508^{\circ}$ & 4351.274 & 200 & 50 & 22975.317 & $7280_{2}-30255_{3}^{\circ}$ \\
\hline 4380.716 & 15 & & 22820.906 & $13847_{2}-36668_{2}^{\circ}$ & 4351.120 & 8 & 3 & 22976.130 & $17224_{2}^{\circ}-40200_{3}$ \\
\hline 4380.289 & 200 & 25 & 22823.131 & $10783^{\circ}-33606_{2}$ & 4351.017 & 50 & 10 & 22976.673 & $15863_{2}-38840_{1}^{\circ}$ \\
\hline 4379.860 & 20 & 4 & 22825.366 & $15166^{\circ}-379924$ & 4350.272 & 100 & 10 & 22980.609 & $8800_{4}-31780_{3}^{\circ}$ \\
\hline 4378.177 & $500 \mathrm{r}$ & 100 & 22834.140 & $2869_{3}-25703_{2}^{\circ}$ & 4349.630 & 40 & 5 & 22984.000 & $15618 \stackrel{\circ}{3}-386022$ \\
\hline 4378.026 & 100 & 3 & 22834.928 & $6362_{2}-29197_{1}^{\circ}$ & 4349.346 & 20 & 4 & 22985.501 & $13088_{3}-36074 \stackrel{\circ}{3}$ \\
\hline 4377.650 & 8 & & 22836.889 & $142045-37041^{\circ}$ & 4349.072 & 400 & 40 & 22986.949 & $4961_{4}-27948{ }^{\circ}$ \\
\hline 4376.682 & 8 & 2 & 22841.940 & $14465_{2}^{\circ}-37307_{3}$ & 4348.828 & 3 & 1 & 22988.239 & $19039_{2}^{\circ}-42027_{2}$ \\
\hline 4376.257 & 25 & 5 & 22844.158 & $15970_{3}-38814_{2}^{\circ}$ & 4348.599 & 400 & 75 & 22989.449 & $13847_{2}-36837^{\circ}$ \\
\hline 4374.710 & 8 & 3 & 22852.236 & $8243_{2}^{\circ}-310951$ & 4348.498 & 4 & 1 & 22989.983 & $116011-34591^{\circ}$ \\
\hline 4374.124 & 600 & 150 & 22855.298 & $\mathbf{0}_{2}-22855_{3}^{\circ}$ & 4348.403 & 3 & 1 & 22990.485 & $10783_{2}^{\circ}-33773_{1}$ \\
\hline 4374.013 & 150 & 40 & 22855.878 & $7795_{4}^{\circ}-306513$ & 4347.638 & 100 & 15 & 22994.531 & $10414_{4}^{\circ}-334083$ \\
\hline 4373.206 & 40 & 8 & 22860.095 & $210778-439376$ & 4346.432 & $500 \mathrm{r}$ & 200 & 23000.911 & $7280_{2}-30281_{i}^{\circ}$ \\
\hline 4372.067 & 1 & & 22866.051 & $157361-386022$ & 4346.410 & $15 b$ & & 23001.028 & $10526^{\circ}-33527_{4}$ \\
\hline 4371.140 & 75 & 8 & 22870.900 & $8800_{4}-31671_{4}^{\circ}$ & 4345.851 & 150 & 50 & 23003.986 & $10414_{4}^{\circ}-334184$ \\
\hline 4370.980 & 10 & 3 & 22871.737 & $173983-40270_{4}^{\circ}$ & 4345.702 & 25 & 10 & 23004.775 & $14206_{4}^{\circ}-372115$ \\
\hline 4370.068 & 5 & 2 & 22876.510 & $15490 \circ-38366_{4}$ & 4343.744 & 5 & & 23015.144 & $7502{ }_{3}-30517^{\circ}$ \\
\hline 4369.876 & 200 & 50 & 22877.515 & $0_{2}-22877_{1}^{\circ}$ & 4343.381 & 100 & 25 & 23017.068 & $38651-26882 \circ$ \\
\hline 4369.602 & 50 & 25 & 22878.950 & $14206_{4}^{\circ}-370854$ & 4342.973 & 15 & 4 & 23019.230 & $15166_{3}^{\circ}-381864$ \\
\hline 4369.496 & 100 & 25 & 22879.505 & $8111_{4}-30990_{3}^{\circ}$ & 4342.674 & 100 & 20 & 23020.815 & $17959_{4}-40980_{3}^{\circ}$ \\
\hline 4369.288 & $50 \mathrm{~b}$ & 25 & 22880.594 & $132974-36178_{4}^{\circ}$ & 4342.445 & $500 \mathrm{r}$ & 100 & 23022.029 & $8243_{2}^{\circ}-31265_{3}$ \\
\hline 4368.995 & 20 & 4 & 22882.128 & $10526^{\circ}-33408_{3}$ & 4342.049 & 50 & 4 & 23024.128 & $13847_{2}-36871_{2}^{\circ}$ \\
\hline 4368.352 & 5 & 2 & 22885.497 & $128473-35733^{\circ}$ & 4340.896 & 200 & 40 & 23030.244 & $8111_{4}-311418$ \\
\hline 4367.418 & 100 & 15 & 22890.391 & $6362_{2}-29252_{2}^{\circ}$ & 4339.924 & 10 & 2 & 23035.402 & $12114_{2}^{\circ}-35149_{2}$ \\
\hline 4367.287 & 50 & 15 & 22891.077 & $4961_{4}-27852^{\circ}$ & 4339.449 & 8 & 2 & 23037.923 & $12847_{3}-35885_{2}^{\circ}$ \\
\hline 4367.191 & 5 & 2 & 22891.581 & $10526^{\circ}-33418_{4}$ & 4339.374 & 15 & 3 & 23038.321 & $10526_{3}^{\circ}-33564_{3}$ \\
\hline 4367.113 & 3 & 1 & 22891.989 & $154934-383850$ & 4339.069 & 3 & 1 & 23039.941 & $17847_{2}^{\circ}-40886_{2}$ \\
\hline 4365.930 & 600 & 150 & 22898.192 & $8243_{2}^{\circ}-31141_{3}$ & 4338.108 & 400 & 100 & 23045.045 & $10414_{4}^{\circ}-33459_{4}$ \\
\hline 4365.302 & 4 & 1 & 22901.486 & $11802_{2}-34704_{3}^{\circ}$ & 4337.639 & 15 & 4 & 23047.536 & $19588^{\circ}-426355$ \\
\hline 4365.020 & 25 & 8 & 22902.966 & $18809_{4}^{\circ}-417125$ & 4337.277 & $800 \mathrm{r}$ & 100 & 23049.460 & $0_{2}-23049 i$ \\
\hline 4364.506 & 8 & 3 & 22905.663 & $16346_{4}^{\circ}-392525$ & 4336.986 & 50 & 10 & 23051.006 & $7502_{3}-30553_{2}^{\circ}$ \\
\hline 4364.461 & 5 & 2 & 22905.899 & $10783_{2}^{\circ}-33689_{2}$ & 4335.992 & 5 & 2 & 23056.291 & $14465_{2}^{\circ}-375213$ \\
\hline 4364.040 & 25 & 4 & 22908.109 & $8111_{4}-31019_{4}^{\circ}$ & 4335.731 & $200 \mathrm{~b}$ & $50 \mathrm{~b}$ & 23057.678 & $98045-32862_{4}^{\circ}$ \\
\hline 4363.798 & 2 & & 22909.379 & $13962_{1}-36871_{2}^{\circ}$ & 4335.347 & $25 b$ & 8 & 23059.721 & $11241 \stackrel{\circ}{3}-343014$ \\
\hline 4363.589 & 1 & & 22910.476 & $16783^{\circ}-396945$ & 4333.552 & 10 & 3 & 23069.272 & $20867_{7}^{\circ}-439376$ \\
\hline 4363.500 & 8 & 2 & 22910.944 & $\begin{array}{l}15618_{3}^{\circ}-38529_{3} \\
13847_{2}-36762_{3}^{\circ}\end{array}$ & 4333.284 & 75 & 4 & 23070.699 & $15736^{\circ}-38807_{2}$ \\
\hline $\begin{array}{l}4362.720 \\
4362.472\end{array}$ & 15 & $\begin{array}{l}3 \\
8\end{array}$ & $\begin{array}{l}22915.040 \\
22916.343\end{array}$ & $\begin{array}{r}13847_{2}-36762_{3}^{\circ} \\
8800_{4}-31716^{\circ}\end{array}$ & 4332.339 & 150 & 40 & 23075.731 & $11802_{2}-34878_{i}^{\circ}$ \\
\hline & 50 & 8 & 22916.343 & $\begin{array}{r}88004-317160^{\circ} \\
15493_{4}-38416^{\circ}\end{array}$ & 4331.582 & 5 & 1 & 23079.764 & $10526_{3}^{\circ}-33606_{2}$ \\
\hline 4361.201 & 4 & 1 & 22923.021 & $154934-384163$ & 4331.291 & $4 b$ & $2 b$ & 23081.314 & $15618_{3}^{\circ}-38700_{4}$ \\
\hline 4360.991 & 5 & 1 & 22924.125 & $14206_{4}^{\circ}-371313$ & & & & & \\
\hline 4360.825 & 3 & 1 & 22924.997 & $15863_{2}-38788_{3}^{\circ}$ & 4330.844 & 300 & 25 & 23083.697 & $8111_{4}-31194_{4}^{\circ}$ \\
\hline 4360.167 & 100 & 15 & 22928.457 & $7280_{2}-30208_{2}^{\circ}$ & 4330.565 & $8 b$ & 3 & 23085.184 & $132974-36382_{4}^{\circ}$ \\
\hline 4359.836 & 10 & 2 & 22930.198 & $15166_{3}^{\circ}-38097_{2}$ & 4330.412 & 50 & 20 & 23086.000 & $5563_{1}-28649^{\circ}$ \\
\hline 4359.558 & 15 & 4 & 22931.660 & $1214_{2}^{\circ}-35046_{1}$ & 4328.915 & $300 \mathrm{r}$ & 25 & 23093.983 & $0_{2}-23093_{2}^{\circ}$ \\
\hline 4359.372 & $600 \mathrm{r}$ & 100 & 22932.638 & $10526_{3}^{\circ}-33459_{4}$ & 4328.436 & 3 & 1 & 23096.539 & $14206_{4}^{\circ}-37303_{4}$ \\
\hline 4358.321 & 500 & 150 & 22938.168 & $10783_{2}^{\circ}-33721_{2}$ & 4327.981 & 15 & 3 & 23098.966 & $14032_{2}^{\circ}-37131_{3}$ \\
\hline 4358.082 & 15 & 5 & 22939.426 & $14465_{2}^{\circ}-37404_{3}$ & 4327.715 & 150 & 25 & 23100.386 & $13088_{3}-36188^{\circ}$ \\
\hline 4357.974 & 8 & 2 & 22939.995 & $13945_{3}^{\circ}-36885_{4}$ & 4327.036 & 100 & 25 & 23104.011 & $171665-40270_{4}^{\circ}$ \\
\hline 4356.835 & 3 & 1 & 22945.992 & $18809_{4}^{\circ}-41755_{4}$ & 4325.860 & 150 & 8 & 23110.292 & $5563_{1}-28673_{2}^{\circ}$ \\
\hline 4356.045 & 75 & 20 & 22950.153 & $5563_{1}-28513_{2}^{\circ}$ & 4325.274 & 200 & 50 & 23113.423 & $10414_{4}^{\circ}-33527_{4}$ \\
\hline 4354.660 & 5 & 2 & 22957.452 & $16217_{2}^{\circ}-39174_{3}$ & 4324.901 & 20 & 4 & 23115.416 & $15618 \stackrel{\circ}{3}-387343$ \\
\hline 4354.483 & 400 & 100 & 22958.386 & $13175_{4}^{\circ}-361335$ & 4323.634 & 20 & 5 & 23122.190 & $13175_{4}^{\circ}-362974$ \\
\hline 4353.449 & $300 \mathrm{~b}$ & $100 \mathrm{~b}$ & 22963.838 & $3687_{2}-26651_{2}^{\circ}$ & 4322.181 & 3 & & 23129.963 & $13297_{4}-36427_{3}^{\circ}$ \\
\hline 4352.613 & 150 & $25 \mathrm{~b}$ & 22968.249 & $25580-25526^{\circ}$ & 4321.224 & 5 & 2 & 23135.085 & $15863_{2}-38998^{\circ}$ \\
\hline
\end{tabular}


TABLE 4. Classified lines of Th $\mathrm{I}$-continued

\begin{tabular}{|c|c|c|c|c|c|c|c|c|c|}
\hline \multirow{2}{*}{$\begin{array}{l}\text { Wavelength } \\
\text { (Å) }\end{array}$} & \multicolumn{2}{|c|}{ Intensity } & \multirow{2}{*}{$\begin{array}{l}\text { Wavenumber } \\
\left(\mathrm{cm}^{-1}\right)\end{array}$} & \multirow{2}{*}{ Classification } & \multirow{2}{*}{$\begin{array}{c}\text { Wavelength } \\
\text { (§) }\end{array}$} & \multicolumn{2}{|c|}{ Inten sity } & \multirow{2}{*}{$\begin{array}{l}\text { Wavenumber } \\
\left(\mathrm{cm}^{-1}\right)\end{array}$} & \multirow{2}{*}{ Classification } \\
\hline & Lamp & Spark & & & & Lamp & Spark & & \\
\hline 4318.523 & 20 & 8 & 23149.555 & $13945 \stackrel{8}{3}-370942$ & 4288.471 & $200 \mathrm{~b}$ & $50 \mathrm{~b}$ & 23311.775 & $13847_{2}-37159_{3}^{\circ}$ \\
\hline 4317.838 & 100 & 5 & 23153.227 & $8800_{4}-31953_{4}^{\circ}$ & 4287.082 & 200 & 40 & 23319.328 & $165546-39873 \circ$ \\
\hline 4316.371 & 25 & 5 & 23161.096 & $13175_{4}^{\circ}-363363$ & 4286.229 & $200 b$ & $75 b$ & 23323.969 & $6362_{2}-29686 \stackrel{8}{\circ}$ \\
\hline 4316.218 & 10 & 3 & 23161.917 & $18069_{3}^{\circ}-41230_{4}$ & 4285.757 & 3 & 1 & 23326.537 & $12114 \stackrel{2}{2}-354403$ \\
\hline 4316.109 & 200 & 50 & 23162.502 & $10526_{3}^{\circ}-336892$ & 4285.398 & 10 & 3 & 23328.491 & $13297_{4}-366258$ \\
\hline 4315.254 & $500 \mathrm{r}$ & 150 & 23167.091 & $2869_{3}-26036_{3}^{\circ}$ & 4283.103 & $200 \mathrm{~b}$ & $50 \mathrm{~b}$ & 23340.991 & $12847_{3}-36188_{2}^{\circ}$ \\
\hline 4315.052 & 100 & 15 & 23168.176 & $11877^{\circ}-350461$ & 4283.073 & $50 \mathrm{~b}$ & $20 \mathrm{~b}$ & 23341.155 & $15970_{3}-39311_{4}^{\circ}$ \\
\hline 4314.491 & 8 & 3 & 23171.188 & $18549_{2}-41720_{2}^{\circ}$ & 4282.084 & $150 \mathrm{~b}$ & $50 \mathrm{~b}$ & 23346.546 & $7795_{4}^{\circ}-311413$ \\
\hline 4314.319 & 300 & 50 & 23172.112 & $8111_{4}-312833$ & 4280.568 & $300 \mathrm{r}$ & 100 & 23354.814 & $5563_{1}-28917_{2}^{\circ}$ \\
\hline 4314.279 & 2 & & 23172.327 & $154934-386658$ & 4280.322 & 8 & 3 & 23356.156 & $18930_{3}^{\circ}-42286_{3}$ \\
\hline 4314.053 & 20 & 8 & 23173.541 & $16346_{4}^{\circ}-39520_{4}$ & 4279.281 & 25 & 3 & 23361.837 & $13945_{3}^{\circ}-37307_{3}$ \\
\hline 4313.778 & 25 & 8 & 23175.018 & $179594-41134_{4}^{\circ}$ & 4279.064 & 300 & 40 & 23363.022 & $15166_{3}^{\circ}-38529_{3}$ \\
\hline 4313.335 & 2 & & 23177.398 & $18053_{4}^{\circ}-41230_{4}$ & 4278.324 & $500 \mathrm{r}$ & 200 & 23367.063 & $142045-37571 \%$ \\
\hline 4312.992 & $800 \mathrm{r}$ & 200 & 23179.241 & $2869_{3}-26048{ }_{4}^{\circ}$ & 4275.567 & 50 & 4 & 23382.130 & $6362_{2}-29744_{3}^{\circ}$ \\
\hline 4312.992 & $800 \mathrm{r}$ & 200 & 23179.241 & $4961_{4}-28140 \stackrel{\circ}{\circ}$ & 4275.165 & 15 & 2 & 23384.329 & $107833_{2}^{\circ}-341673$ \\
\hline 4311.798 & 300 & 100 & 23185.660 & $82432^{\circ}-314291$ & 4274.817 & 200 & 25 & 23386.233 & $13847_{2}-37234_{2}^{\circ}$ \\
\hline 4311.625 & $150 \mathrm{~b}$ & 25 & 23186.590 & $139621-37149_{2}^{\circ}$ & 4273.911 & 15 & 3 & 23391.190 & $15618_{3}^{\circ}-39010_{3}$ \\
\hline 4311.576 & $100 \mathrm{~b}$ & 25 & 23186.854 & $11802_{2}-34989_{1}^{\circ}$ & 4272.875 & $400 \mathrm{r}$ & 50 & 23396.862 & $8800_{4}-32197_{3}^{\circ}$ \\
\hline 4311.062 & 150 & 15 & 23189.618 & $112413-344313$ & 4272.303 & $300 \mathrm{r}$ & 100 & 23399.994 & $3687_{2}-27087_{1}^{\circ}$ \\
\hline 4310.957 & 75 & 5 & 23190.183 & $3687_{2}-26878_{3}^{\circ}$ & 4270.734 & 100 & 15 & 23408.591 & $144811^{\circ}-378905$ \\
\hline 4310.101 & 15 & 5 & 23194.788 & $10526_{3}^{\circ}-33721_{2}$ & 4269.943 & 300 & 75 & 23412.927 & $8111_{4}-315233^{\circ}$ \\
\hline 4309.886 & 150 & 50 & 23195.945 & $38651-27061_{2}^{\circ}$ & 4269.272 & 3 & 1 & 23416.607 & $16783_{4}^{\circ}-40200_{3}$ \\
\hline 4309.557 & 25 & 4 & 23197.716 & $14206 \stackrel{8}{-}-374043$ & 4269.063 & 100 & 5 & 23417.753 & $10414_{4}^{\circ}-33831_{4}$ \\
\hline 4308.599 & $100 \mathrm{~b}$ & 20 & 23202.874 & $11241_{3}^{\circ}-344442$ & 4266.447 & 15 & 3 & 23432.111 & $3865_{1}-27297_{i}^{\circ}$ \\
\hline 4308.121 & 300 & 100 & 23205.449 & $11241_{3}^{\circ}-34447_{4}$ & 4264.338 & 2001 & 20 & 23443.700 & $7280_{2}-30723^{\circ}$ \\
\hline 4307.176 & $600 \mathrm{r}$ & 200 & 23210.540 & $11197^{\circ}-344076$ & 4261.492 & 150 & 4 & 23459.356 & $13945_{3}^{\circ}-37404_{3}$ \\
\hline 4305.495 & 5 & 2 & 23219.602 & $18011{ }^{\circ}-41230_{4}$ & 4260.984 & 100 & 2 & 23462.153 & $12114_{2}^{\circ}-35576_{3}$ \\
\hline 4304.955 & 150 & 10 & 23222.514 & $3865_{1}-27087_{1}^{\circ}$ & 4260.802 & 150 & 1 & 23463.155 & $15490 \AA-389535$ \\
\hline 4304.868 & 8 & 3 & 23222.984 & $14465_{2}^{\circ}-37688_{3}$ & 4260.395 & 15 & 1 & 23465.397 & $13297_{4}-36762_{3}^{\circ}$ \\
\hline 4304.801 & 8 & 3 & 23223.345 & $8800_{4}-320230^{\circ}$ & 4260.332 & $800 \mathrm{r}$ & 5 & 23465.744 & $98045-33270_{4}^{\circ}$ \\
\hline 4304.290 & 8 & 3 & 23226.102 & $128473-360743^{\circ}$ & 4260.288 & $75 b$ & 1 & 23465.986 & $11241_{3}^{\circ}-347073$ \\
\hline 4303.988 & 75 & 5 & 23227.732 & $2869_{3}-26096_{3}^{\circ}$ & 4260.138 & 20 & & 23466.812 & $154908-389564$ \\
\hline 4300.370 & 3 & 1 & 23247.273 & $19039_{2}^{\circ}-422863$ & 4259.494 & 200 & 1 & 23470.360 & $7795_{4}^{\circ}-31265_{3}$ \\
\hline 4299.839 & $500 \mathrm{r}$ & 100 & 23250.144 & $11197^{\circ}-34447_{4}$ & 4258.521 & 400 & 2 & 23475.723 & $7795^{\circ}-312715$ \\
\hline 4299.633 & 200 & 50 & 23251.258 & $25580-25809^{\circ}$ & 4257.496 & $600 \mathrm{r}$ & 4 & 23481.375 & $0_{2}-23481^{\circ}$ \\
\hline 4298.121 & 75 & 5 & 23259.437 & $75023-307611^{\circ}$ & 4257.459 & $10 \mathrm{~h}$ & & 23481.579 & $7280_{2}-30761_{3}^{\circ}$ \\
\hline 4297.938 & 100 & 20 & 23260.428 & $144816-377426$ & 4256.254 & $500 \mathrm{r}$ & 8 & 23488.226 & $7502_{3}-30990_{3}^{\circ}$ \\
\hline 4297.307 & $300 \mathrm{~b}$ & 75 & 23263.843 & $11197 \stackrel{\circ}{-}-344605$ & 4256.039 & 10 & & 23489.413 & $14032_{2}^{\circ}-375213$ \\
\hline 4295.902 & 2 & 1 & 23271.451 & $13962_{1}-37234_{2}^{\circ}$ & 4255.795 & $150 \mathrm{~b}$ & 4 & 23490.760 & $6362_{2}-29853_{2}^{\circ}$ \\
\hline 4295.816 & 100 & 40 & 23271.917 & $11877^{\circ}-35149_{2}$ & 4255.759 & $50 \mathrm{~b}$ & 3 & 23490.958 & $16346_{4}^{\circ}-39837_{3}$ \\
\hline 4295.584 & 200 & 25 & 23273.174 & $7280_{2}-30553_{2}^{\circ}$ & 4255.237 & 200 & 3 & 23493.840 & $2869_{3}-26363_{2}^{\circ}$ \\
\hline 4295.236 & 25 & 5 & 23275.060 & $14032_{2}^{\circ}-373073$ & 4254.380 & 100 & 1 & 23498.572 & $159700_{3}-39468_{3}^{\circ}$ \\
\hline 4294.756 & $75 b$ & $25 b$ & 23277.661 & $11601_{1}-34878 i$ & 4253.539 & $600 \mathrm{r}$ & 20 & 23503.218 & $13297_{4}-36800_{4}^{\circ}$ \\
\hline 4294.720 & $100 \mathrm{~b}$ & 25 & 23277.856 & $6362_{2}-29640_{1}^{\circ}$ & 4253.388 & $10 \mathrm{~b}$ & & 23504.053 & $14243 i-37748_{2}$ \\
\hline 4293.800 & $8 b$ & 3 & 23282.844 & $14465_{2}^{\circ}-377482$ & 4251.421 & 5 & & 23514.927 & $10783_{2}^{\circ}-34298_{1}$ \\
\hline 4292.681 & 4 & 1 & 23288.913 & $184313-41720_{2}^{\circ}$ & 4251.286 & 50 & & 23515.674 & $2869_{3}-26384_{4}^{\circ}$ \\
\hline 4292.305 & 50 & 10 & 23290.953 & $12114_{2}^{\circ}-35405_{3}$ & 4251.077 & 4 & & 23516.830 & $7502_{3}-31019_{4}^{\circ}$ \\
\hline 4291.810 & 400 & 100 & 23293.639 & $82432^{\circ}-315373$ & 4250.775 & 100 & 2 & 23518.501 & $4961_{4}-28480_{4}^{\circ}$ \\
\hline 4291.635 & 50 & 10 & 23294.589 & $159703-39264_{3}^{\circ}$ & 4250.315 & $800 \mathrm{r}$ & 75 & 23521.046 & $\mathbf{0}_{2}-23521_{3}^{\circ}$ \\
\hline 4290.394 & 200 & 50 & 23301.327 & $13847_{2}-37149_{2}^{\circ}$ & 4249.476 & 150 & 1 & 23525.690 & $118022-353281$ \\
\hline 4289.655 & 100 & 40 & 23305.341 & $105263-338314$ & 4248.518 & 50 & 1 & 23530.995 & $8243_{2}^{\circ}-31774_{3}$ \\
\hline 4289.414 & 8 & 3 & 23306.650 & $12114_{2}^{\circ}-35421_{1}$ & 4248.391 & 200 & 3 & 23531.698 & $7795_{4}^{\circ}-313264$ \\
\hline 4289.204 & 8 & 2 & 23307.791 & $3687_{2}-26995_{3}^{\circ}$ & 4248.274 & 4 & & 23532.346 & $17354_{1}^{\circ}-40886_{2}$ \\
\hline 4289.204 & 8 & 2 & 23307.791 & $18053^{\circ}-41361_{3}$ & 4248.181 & 5 & & 23532.861 & $7280_{2}-30812_{2}^{\circ}$ \\
\hline 4288.669 & 200 & 75 & 23310.699 & $75023-308122$ & 4247.861 & 150 & 2 & 23534.634 & $128473-36382{ }^{\circ}$ \\
\hline
\end{tabular}


TABLE 4. Classified lines of Th I-continued

\begin{tabular}{|c|c|c|c|c|c|c|c|c|c|}
\hline \multirow{2}{*}{$\begin{array}{l}\text { Wavelength } \\
\text { (Å) }\end{array}$} & \multicolumn{2}{|c|}{ Intensity } & \multirow{2}{*}{$\begin{array}{l}\text { Wavenumber } \\
\left(\mathrm{cm}^{-1}\right)\end{array}$} & \multirow{2}{*}{ Classification } & \multirow{2}{*}{$\begin{array}{c}\text { Wavelength } \\
\text { (§) }\end{array}$} & \multicolumn{2}{|c|}{ Intensity } & \multirow{2}{*}{$\begin{array}{l}\text { Wavenumber } \\
\qquad\left(\mathrm{cm}^{-1}\right)\end{array}$} & \multirow{2}{*}{ Classification } \\
\hline & Lamp & Spark & & & & Lamp & Spark & & \\
\hline 4247.777 & 10 & & 23535.099 & $20322{ }^{\circ}-438575$ & 4215.210 & 4 & & 23716.930 & $12114_{2}^{\circ}-35831_{3}$ \\
\hline 4246.796 & $10 \mathrm{~b}$ & & 23540.536 & $13297_{4}-36837^{\circ}$ & 4214.828 & 200 & 4 & 23719.079 & $4961_{4}-28680_{4}^{\circ}$ \\
\hline 4246.324 & 20 & 1 & 23543.153 & $11877^{\circ}-35421_{1}$ & 4213.315 & 15 & 1 & 23727.596 & $11601_{1}-35328^{\circ}$ \\
\hline 4245.451 & 100 & 3 & 23547.994 & $15863_{2}-39411_{1}^{\circ}$ & 4213.067 & 400 & 5 & 23728.993 & $25580-26287^{\circ}$ \\
\hline 4244.022 & 25 & 1 & 23555.922 & $15618^{\circ}-39174_{3}$ & 4212.923 & 50 & 1 & 23729.804 & $17501 \stackrel{\circ}{\circ}-41230_{4}$ \\
\hline 4243.261 & 100 & 2 & 23560.147 & $8111_{4}-31671_{4}^{\circ}$ & 4212.653 & 4 & & 23731.325 & $17847_{2}^{\circ}-41578_{2}$ \\
\hline 4241.697 & 4 & & 23568.834 & $154934-39062 \circ$ & 4210.923 & $900 \mathrm{r}$ & 10 & 23741.075 & $0_{2}-23741$ i \\
\hline 4241.096 & 100 & 2 & 23572.173 & $3687_{2}-27260_{3}^{\circ}$ & 4210.765 & 400 & 10 & 23741.965 & $7795_{4}^{\circ}-31537_{3}$ \\
\hline 4239.791 & 15 & & 23579.429 & $12847_{3}-36427_{3}^{\circ}$ & 4210.600 & 2 & & 23742.896 & $13945_{3}^{\circ}-37688_{3}$ \\
\hline 4239.276 & 4 & & 23582.293 & $3865_{1}-27447_{2}^{\circ}$ & 4210.455 & 75 & 2 & 23743.713 & $13297_{4}-37041_{4}^{\circ}$ \\
\hline 4239.276 & 4 & & 23582.293 & $17398_{3}-40980_{3}^{\circ}$ & 4210.089 & 75 & 3 & 23745.778 & $142045-37950_{4}^{\circ}$ \\
\hline 4237.535 & 10 & & 23591.982 & $15970_{3}-39562_{4}^{\circ}$ & 4208.921 & $100 \mathrm{~b}$ & $10 \mathrm{~b}$ & 23752.367 & $14032_{2}^{\circ}-37784_{2}$ \\
\hline 4237.182 & 20 & & 23593.947 & $5563_{1}-29157_{1}^{\circ}$ & 4208.867 & $200 b$ & & 23752.672 & $0_{2}-23752_{2}^{\circ}$ \\
\hline 4236.049 & 50 & 1 & 23600.258 & $13175_{4}^{\circ}-36775_{3}$ & 4208.750 & $100 \mathrm{~b}$ & 5 & 23753.332 & $10414_{4}^{\circ}-34167_{3}$ \\
\hline 4235.463 & $600 \mathrm{r}$ & 5 & 23603.523 & $0_{2}-23603_{2}^{\circ}$ & 4208.412 & 150 & 4 & 23755.240 & $98045-33560_{4}^{\circ}$ \\
\hline 4235.238 & 20 & 1 & 23604.777 & $15863_{2}-39468_{3}^{\circ}$ & 4207.612 & 25 & 1 & 23759.756 & $3687_{2}-27447_{2}^{\circ}$ \\
\hline 4235.092 & 50 & 1 & 23605.591 & $8111_{4}-31716^{\circ}$ & 4207.176 & 20 & 1 & 23762.218 & $15490 \AA-392525$ \\
\hline 4234.231 & 50 & 1 & 23610.390 & $171665-40776_{4}^{\circ}$ & 4206.058 & 10 & 1 & 23768.535 & $13847_{2}-37616_{1}^{\circ}$ \\
\hline 4233.907 & 50 & 1 & 23612.197 & $14206_{4}^{\circ}-37819_{4}$ & 4205.540 & 10 & & 23771.462 & $154934-392643$ \\
\hline 4232.478 & 1 & & 23620.169 & $16217_{2}^{\circ}-39837_{3}$ & 4205.012 & 4 & & 23774.447 & $139621-37736_{2}^{\circ}$ \\
\hline 4231.617 & 10 & & 23624.975 & $144816^{\circ}-381065$ & 4204.930 & 3 & & 23774.911 & $10526_{3}^{\circ}-343014$ \\
\hline 4231.136 & 4 & & 23627.661 & $4961_{4}-28589_{3}^{\circ}$ & 4203.884 & 75 & 2 & 23780.826 & $75023-312833^{\circ}$ \\
\hline 4230.823 & 150 & 1 & 23629.409 & $3687_{2}-27317_{3}^{\circ}$ & 4203.575 & 15 & 1 & 23782.574 & $2869_{3}-26651_{2}^{\circ}$ \\
\hline 4230.427 & 500 & 5 & 23631.621 & $11241_{3}^{\circ}-34873_{4}$ & 4203.437 & 4 & 1 & 23783.355 & $13088_{3}-36871_{2}^{\circ}$ \\
\hline 4230.299 & 15 & & 23632.336 & $11241_{3}^{\circ}-348743$ & 4203.085 & 15 & 1 & 23785.346 & $14206_{4}^{\circ}-379924$ \\
\hline 4229.961 & 10 & & 23634.224 & $5563_{1}-29197_{i}^{\circ}$ & 4202.266 & 50 & 2 & 23789.982 & $15166_{3}^{\circ}-389564$ \\
\hline 4229.864 & 20 & & 23634.766 & $5563_{1}-29197_{2}^{\circ}$ & 4200.686 & 2 & & 23798.930 & $16783_{4}^{\circ}-405825$ \\
\hline 4229.148 & 300 & 4 & 23638.767 & $2869_{3}-26508_{3}^{\circ}$ & 4200.149 & 2 & & 23801.973 & $173983-41200_{2}^{\circ}$ \\
\hline 4228.761 & $150 \mathrm{~b}$ & 3 & 23640.931 & $10526_{3}^{\circ}-34167_{3}$ & 4200.017 & 2 & & 23802.721 & $13945_{3}^{\circ}-37748_{2}$ \\
\hline 4228.677 & $25 b$ & 2 & 23641.400 & $15970_{3}-39611_{3}^{\circ}$ & 4198.936 & 20 & 1 & 23808.849 & $38651-27674_{2}^{\circ}$ \\
\hline 4228.418 & 100 & 3 & 23642.848 & $16783_{4}^{\circ}-404264$ & 4198.876 & 15 & 1 & 23809.189 & $13847_{2}-37656_{3}^{\circ}$ \\
\hline 4227.387 & 400 & 8 & 23648.614 & $7280_{2}-30928^{\circ}$ & 4197.317 & 2 & & 23818.032 & $154934-39311_{4}^{\circ}$ \\
\hline 4226.775 & 100 & 2 & 23652.038 & $11197^{\circ}-348,495$ & 4197.016 & 2 & & 23819.740 & $17411_{3}^{\circ}-41230_{4}$ \\
\hline 4226.298 & 200 & & 23654.708 & $6362_{2}-30017_{3}^{\circ}$ & 4196.846 & $10 \mathrm{~s}$ & & 23820.705 & $12847_{3}-36668_{2}^{\circ}$ \\
\hline 4226.047 & 10 & & 23656.113 & $14032_{2}^{\circ}-37688_{3}$ & 4194.936 & 200 & 5 & 23831.551 & $7795_{4}^{\circ}-316265$ \\
\hline 4225.489 & 501 & 2 & 23659.236 & $18053_{4}^{\circ}-41712_{5}$ & 4193.601 & 1 & & 23839.137 & $139453_{3}^{\circ}-37784_{2}$ \\
\hline 4225.095 & 3 & & 23661.443 & $10783_{2}^{\circ}-34444_{2}$ & 4193.518 & 15 & 2 & 23839.609 & $17847_{2}^{\circ}-416863$ \\
\hline 4224.871 & 3 & & 23662.697 & $17224_{2}^{\circ}-40886_{2}$ & 4193.017 & 600 & 15 & 23842.457 & $8111_{4}-31953^{\circ}$ \\
\hline 4224.488 & 3 & & 23664.843 & $15618_{3}^{\circ}-39283_{4}$ & 4192.877 & 2 & & 23843.253 & $15166^{\circ}-39010_{3}$ \\
\hline 4223.589 & $150 \mathrm{~b}$ & 3 & 23669.880 & $8111_{4}-31780_{3}^{\circ}$ & 4192.362 & 150 & 3 & 23846.182 & $6362_{2}-30208_{2}^{\circ}$ \\
\hline 4222.439 & 20 & & 23676.326 & $11197{ }^{\circ}-34873_{4}$ & 4191.146 & 15 & 1 & 23853.101 & $14243 i-38097_{2}$ \\
\hline 4221.691 & 75 & 2 & 23680.521 & $14465_{2}^{\circ}-38145_{3}$ & 4191.028 & 3 & & 23853.772 & $163469-40200_{3}$ \\
\hline 4221.151 & 100 & 2 & 23683.550 & $14206_{4}^{\circ}-378905$ & 4190.813 & 5 & 1 & 23854.996 & $17501{ }^{\circ}-413565$ \\
\hline 4220.730 & 150 & 2 & 23685.913 & $8243^{\circ}-319293$ & 4190.620 & 15 & 1 & 23856.095 & $5563_{1}-29419_{2}^{\circ}$ \\
\hline 4220.730 & 150 & 2 & 23685.913 & $14206^{\circ}-378924$ & 4189.633 & 5 & 1 & 23861.715 & $15490 \AA-393515$ \\
\hline 4220.570 & 3 & & 23686.810 & $18069_{3}^{\circ}-41755_{4}$ & 4189.563 & 100 & 3 & 23862.113 & $13297_{4}-37159_{3}^{\circ}$ \\
\hline 4220.064 & 200 & 15 & 23689.650 & $5563_{1}-29252_{2}^{\circ}$ & 4187.512 & 1 & & 23873.800 & $13945_{3}^{\circ}-37819_{4}$ \\
\hline 4219.569 & 20 & 1 & 23692.430 & $75023-31194_{4}^{\circ}$ & 4187.142 & 50 & 3 & 23875.910 & $11802_{2}-35678^{\circ}$ \\
\hline 4217.185 & $100 \mathrm{~b}$ & $2 b$ & 23705.823 & $16346_{4}^{\circ}-400524$ & 4185.143 & 50 & 2 & 23887.314 & $10414 \stackrel{\circ}{4}-34301_{4}$ \\
\hline 4216.543 & 3 & & 23709.432 & $16783_{4}^{\circ}-404933$ & 4184.138 & 200 & 4 & 23893.052 & $63622-30255_{3}^{\circ}$ \\
\hline 4216.371 & 2001 & 3 & 23710.399 & $7280_{2}-30990 \stackrel{8}{3}$ & 4182.933 & 20 & 2 & 23899.934 & $14206{ }_{4}^{\circ}-381065$ \\
\hline 4216.205 & 75 & 1 & 23711.333 & $13297_{4}-37008_{0}^{\circ}$ & 4182.707 & 4 & & 23901.226 & $15618^{\circ}-39520_{4}$ \\
\hline 4216.068 & $300 \mathrm{~s}$ & 5 & 23712.103 & $13088_{3}-36800_{4}^{\circ}$ & 4182.080 & 50 & 2 & 23904.809 & $10526^{\circ}-34431_{3}$ \\
\hline 4215.625 & 150 & 1 & 23714.595 & $4961_{4}-28676_{3}^{\circ}$ & 4181.518 & 2 & & 23908.022 & $11241_{3}^{\circ}-35149_{2}$ \\
\hline 4215.383 & 3 & & 23715.956 & $14032_{2}^{\circ}-37748_{2}$ & 4180.720 & 10 & 1 & 23912.585 & $81114-320230^{\circ}$ \\
\hline
\end{tabular}


TABLE 4. Classified lines of Th $\mathrm{I}$-continued

\begin{tabular}{|c|c|c|c|c|c|c|c|c|c|}
\hline \multirow{2}{*}{$\begin{array}{c}\text { Wavelength } \\
(\AA)\end{array}$} & \multicolumn{2}{|c|}{ Intensity } & \multirow{2}{*}{$\begin{array}{l}\text { Wavenumber } \\
\left(\mathrm{cm}^{-1}\right)\end{array}$} & \multirow{2}{*}{ Classification } & \multirow{2}{*}{$\begin{array}{c}\text { Wavelength } \\
\text { (Å) }\end{array}$} & \multicolumn{2}{|c|}{ Intensity } & \multirow{2}{*}{$\begin{array}{c}\text { Wavenumber } \\
\left(\mathrm{cm}^{-1}\right)\end{array}$} & \multirow{2}{*}{ Classification } \\
\hline & Lamp & Spark & & & & Lamp & Spark & & \\
\hline 4180.326 & 20 & 2 & 23914.839 & $12847_{3}-36762_{3}^{\circ}$ & 4143.648 & 100 & 2 & 24126.520 & $2869_{3}-26995_{3}^{\circ}$ \\
\hline 4179.861 & 1 & & 23917.499 & $16783^{\circ}-40701_{4}$ & 4143.337 & 5 & 1 & 24128.331 & $13175_{4}^{\circ}-37303_{4}$ \\
\hline 4179.760 & 10 & 2 & 23918.077 & $10526^{\circ}-34444_{2}$ & 4142.325 & 100 & 3 & 24134.225 & $7795_{4}^{\circ}-31929_{3}$ \\
\hline 4179.620 & $200 \mathrm{~b}$ & 5 & 23918.878 & $3865_{1}-27784_{2}^{\circ}$ & 4141.719 & 100 & 3 & 24137.757 & $14465_{2}^{\circ}-38602_{2}$ \\
\hline 4179.314 & 100 & 8 & 23920.630 & $10526^{\circ}-34447_{4}$ & 4138.040 & 200 & 3 & 24159.216 & $3865_{1}-28024_{1}^{\circ}$ \\
\hline 4179.220 & 150 & 3 & 23921.168 & $2869_{3}-26790_{4}^{\circ}$ & 4136.286 & 300 & 8 & 24169.461 & $11877_{1}^{\circ}-36047_{2}$ \\
\hline 4178.848 & 200 & 3 & 23923.297 & $4961_{4}-28884_{3}^{\circ}$ & 4136.132 & 8 & 1 & 24170.361 & $165546-40724 \circ$ \\
\hline 4178.624 & 2 & & 23924.579 & $10783_{2}^{\circ}-34707_{3}$ & 4135.480 & 300 & 3 & 24174.171 & $8111_{4}-32285_{3}^{\circ}$ \\
\hline 4177.281 & 4 & 1 & 23932.271 & $11601_{1}-35533_{2}^{\circ}$ & 4134.323 & 50 & 3 & 24180.936 & $142045-383855^{\circ}$ \\
\hline 4177.165 & 100 & 4 & 23932.936 & $12114_{2}^{\circ}-36047_{2}$ & 4134.062 & $200 \mathrm{~b}$ & $10 \mathrm{~b}$ & 24182.463 & $0_{2}-24182_{2}^{\circ}$ \\
\hline 4176.480 & 10 & 1 & 23936.861 & $15970_{3}-39906_{4}^{\circ}$ & 4133.958 & 200 & 10 & 24183.071 & $8111_{4}-32294_{4}^{\circ}$ \\
\hline 4175.322 & 4 & & 23943.499 & $15736^{\circ}-39680_{2}$ & 4132.620 & 150 & 3 & 24190.901 & $6362_{2}-30553_{2}^{\circ}$ \\
\hline 4174.620 & 2 & & 23947.526 & $13945_{3}^{\circ}-37892_{4}$ & 4131.710 & 150 & 3 & 24196.229 & $4961_{4}-29157^{\circ}$ \\
\hline 4173.720 & 25 & 1 & 23952.690 & $12847_{3}-36800_{4}^{\circ}$ & 4131.662 & $200 \mathrm{~b}$ & 3 & 24196.510 & $98045-34001_{4}^{\circ}$ \\
\hline 4173.448 & 50 & 4 & 23954.251 & $8800_{4}-32754_{3}^{\circ}$ & 4131.207 & 25 & 2 & 24199.175 & $11241 \stackrel{\circ}{3}-354403$ \\
\hline 4170.533 & $500 \mathrm{~b}$ & 25 & 23970.993 & $4961_{4}-28932_{4}^{\circ}$ & 4131.002 & 800 & 15 & 24200.375 & $7795_{4}^{\circ}-31995_{4}$ \\
\hline 4169.762 & 4 & & 23975.425 & $154934-39468^{\circ}$ & 4130.168 & 10 & 1 & 24205.262 & $13847_{2}-380533_{3}^{\circ}$ \\
\hline 4169.085 & 15 & 1 & 23979.319 & $7795_{4}^{\circ}-317743$ & 4129.073 & 3 & & 24211.681 & $17501 \stackrel{\circ}{\circ}-417125$ \\
\hline 4168.455 & 50 & 4 & 23982.943 & $16217_{2}^{\circ}-40200_{3}$ & 4128.050 & 100 & 2 & 24217.681 & $7795_{4}^{\circ}-32012_{4}$ \\
\hline 4167.864 & 20 & 1 & 23986.343 & $3687_{2}-27674_{2}^{\circ}$ & 4127.345 & $150 \mathrm{~b}$ & 10 & 24221.818 & $12114_{2}^{\circ}-363363$ \\
\hline 4165.766 & $800 \mathrm{r}$ & 15 & 23998.423 & $7795_{4}^{\circ}-317934$ & 4126.032 & 10 & 1 & 24229.526 & $13175_{4}^{\circ}-37404_{3}$ \\
\hline 4165.496 & 3 & & 23999.979 & $11877_{1}^{\circ}-35877_{2}$ & 4124.105 & $5 b$ & & 24240.847 & $13945_{3}^{\circ}-381864$ \\
\hline 4164.972 & 50 & 3 & 24002.998 & $7280_{2}-31283 \stackrel{\circ}{3}$ & 4124.043 & 150 & 5 & 24241.211 & $13847_{2}-38088_{2}^{\circ}$ \\
\hline 4163.947 & 100 & 2 & 24008.907 & $2869_{3}-26878_{3}^{\circ}$ & 4123.726 & 100 & 3 & 24243.074 & $8800_{4}-33043 \stackrel{\circ}{3}$ \\
\hline 4163.827 & 10 & 1 & 24009.599 & $11802_{2}-35812_{3}^{\circ}$ & 4123.601 & 150 & 3 & 24243.809 & $7280_{2}-315233^{\circ}$ \\
\hline 4162.782 & 75 & 5 & 24015.626 & $15970_{3}-39985_{3}^{\circ}$ & 4121.744 & 3 & & 24254.732 & $17501^{\circ}-417554$ \\
\hline 4162.509 & 400 & 15 & 24017.201 & $10414_{4}^{\circ}-34431_{3}$ & 4120.364 & 15 & 1 & 24262.855 & $10783_{2}^{\circ}-350461$ \\
\hline 4162.029 & 3 & & 24019.971 & $11197^{\circ}-352165$ & 4118.490 & 200 & 4 & 24273.895 & $132974-37571^{\circ}$ \\
\hline 4161.738 & 150 & 5 & 24021.650 & $75023-31523 \stackrel{\circ}{3}$ & 4118.225 & 5 & 1 & 24275.457 & $17411_{3}^{\circ}-41686_{3}$ \\
\hline 4161.344 & 2 & & 24023.925 & $128473-36871_{2}^{\circ}$ & 4117.698 & $4 b$ & 1 & 24278.564 & $75023-31780_{3}^{\circ}$ \\
\hline 4160.272 & $2 b$ & & 24030.115 & $15490 \AA-39520_{4}$ & 4116.635 & $25 b$ & $4 b$ & 24284.833 & $159700_{3}-40254_{3}^{\circ}$ \\
\hline 4159.764 & 20 & 1 & 24033.049 & $10414_{4}^{\circ}-34447_{4}$ & 4116.635 & $25 b$ & $4 b$ & 24284.833 & $11601_{1}-35885_{2}^{\circ}$ \\
\hline 4159.152 & 75 & 2 & 24036.586 & $13175^{\circ}-372115$ & 4115.759 & $500 \mathrm{r}$ & 50 & 24290.002 & $5563_{1}-29853_{2}^{\circ}$ \\
\hline 4158.535 & 800 & 20 & 24040.152 & $98045-33844{ }^{\circ}$ & 4113.873 & 20 & 1 & 24301.137 & $12847_{3}-37149_{2}^{\circ}$ \\
\hline 4157.391 & $150 \mathrm{~b}$ & 3 & 24046.767 & $10414_{4}^{\circ}-344605$ & 4112.753 & $600 \mathrm{~b}$ & 15 & 24307.755 & $0_{2}-24307_{2}^{\circ}$ \\
\hline 4157.363 & $25 b$ & $2 \mathrm{~b}$ & 24046.929 & $13945_{3}^{\circ}-37992_{4}$ & 4112.713 & $300 \mathrm{~b}$ & & 24307.991 & $8243_{2}^{\circ}-32551_{3}$ \\
\hline 4156.481 & $50 \mathrm{~b}$ & $10 \mathrm{~b}$ & 24052.031 & $142260-38278^{\circ}$ & 4112.665 & $40 \mathrm{~b}$ & 2 & 24308.275 & $13297_{4}-37605_{4}^{\circ}$ \\
\hline 4155.010 & 5 & & 24060.546 & $13088_{3}-37149_{2}^{\circ}$ & 4112.104 & 10 & 1 & 24311.591 & $12847_{3}-37159_{3}^{\circ}$ \\
\hline 4154.719 & 200 & 5 & 24062.232 & $8800_{4}-32862_{4}^{\circ}$ & 4110.829 & $200 \mathrm{~b}$ & $10 \mathrm{~b}$ & 24319.131 & $7280_{2}-31599_{2}^{\circ}$ \\
\hline 4153.573 & 8 & 1 & 24068.870 & $154934-39562_{4}^{\circ}$ & 4110.317 & 2 & & 24322.160 & $173983-41720_{2}^{\circ}$ \\
\hline 4153.204 & 5 & & 24071.009 & $13088{ }_{3}-37159_{3}^{\circ}$ & 4110.174 & 4 & & 24323.007 & $14206_{4}^{\circ}-38529_{3}$ \\
\hline 4152.561 & 2 & & 24074.736 & $18549_{2}-42624_{i}^{\circ}$ & 4109.323 & $400 \mathrm{r}$ & 5 & 24328.044 & $8111_{4}-32439_{4}^{\circ}$ \\
\hline 4152.147 & 15 & & 24077.137 & $5563_{1}-29640_{1}^{\circ}$ & 4108.183 & 4 & & 24334.794 & $11241_{3}^{\circ}-355763$ \\
\hline 4152.032 & 3 & & 24077.803 & $11601_{1}-35678 i$ & 4107.861 & 100 & 3 & 24336.702 & $3687_{2}-28024^{\circ}$ \\
\hline 4151.144 & $50 \mathrm{~b}$ & 2 & 24082.954 & $11802_{2}-35885_{2}^{\circ}$ & 4106.522 & 4 & & 24344.637 & $17411_{3}^{\circ}-41755_{4}$ \\
\hline 4150.598 & 5 & & 24086.122 & $8111_{4}-32197_{3}^{\circ}$ & 4106.154 & $15 b$ & $2 b$ & 24346.819 & $10526^{\circ}-348734$ \\
\hline 4150.087 & $200 \mathrm{~b}$ & 5 & 24089.088 & $4961_{4}-29050_{0}^{\circ}$ & 4106.034 & 10 & 1 & 24347.530 & $10526_{3}^{\circ}-348743$ \\
\hline 4148.829 & 50 & 3 & 24096.392 & $3687_{2}-27784_{2}^{\circ}$ & 4105.894 & $25 b$ & & 24348.361 & $4961_{4}-29310_{4}^{\circ}$ \\
\hline 4148.724 & 100 & 3 & 24097.001 & $75023-31599_{2}^{\circ}$ & 4105.062 & 25 & 2 & 24353.295 & $15166_{3}^{\circ}-39520_{4}$ \\
\hline 4147.538 & 100 & 3 & 24103.892 & $11197 \circ-353005$ & 4103.692 & $20 \mathrm{~b}$ & 2 & 24361.425 & $63622-30723 i$ \\
\hline 4146.968 & 15 & 1 & 24107.205 & $13847_{2}-37954_{3}^{\circ}$ & 4102.820 & 20 & & 24366.603 & $10783_{2}^{\circ}-35149_{2}$ \\
\hline 4145.858 & $5 b$ & & 24113.659 & $14032_{2}^{\circ}-38145_{3}$ & 4102.617 & $400 \mathrm{r}$ & 3 & 24367.809 & $7502_{3}-31870_{2}^{\circ}$ \\
\hline 4145.296 & 20 & 2 & 24116.928 & $15166^{\circ}-39283_{4}$ & 4101.234 & 5 & & 24376.026 & $142045-38580^{\circ}$ \\
\hline 4144.342 & 40 & 3 & 24122.480 & $13847_{2}-37970_{2}^{\circ}$ & 4100.919 & 40 & 2 & 24377.898 & $98045-34182 \circ$ \\
\hline 4143.711 & 5 & & 24126.153 & $159703-40096_{2}^{\circ}$ & 4100.341 & $1000 \mathrm{r}$ & 20 & 24381.334 & $0_{2}-24381_{2}^{\circ}$ \\
\hline
\end{tabular}


TABLE 4. Classified lines of $\mathrm{Th} \mathrm{I}-$ continued

\begin{tabular}{|c|c|c|c|c|c|c|c|c|c|}
\hline \multirow{2}{*}{$\begin{array}{l}\text { Wavelength } \\
\text { (Å) }\end{array}$} & \multicolumn{2}{|c|}{ Intensity } & \multirow{2}{*}{$\begin{array}{l}\text { Wavenumber } \\
\qquad\left(\mathrm{cm}^{-1}\right)\end{array}$} & \multirow{2}{*}{ Classification } & \multirow{2}{*}{$\begin{array}{c}\text { Wavelength } \\
\text { (A) }\end{array}$} & \multicolumn{2}{|c|}{ Intensity } & \multirow{2}{*}{$\begin{array}{l}\text { Wavenumber } \\
\qquad\left(\mathrm{cm}^{-1}\right)\end{array}$} & \multirow{2}{*}{ Classification } \\
\hline & Lamp & Spark & & & & Lamp & Spark & & \\
\hline 4099.553 & 100 & 4 & 24386.021 & $11802_{2}-36188_{2}^{\circ}$ & 4060.062 & 200 & 1 & 24623.211 & $10526_{3}^{\circ}-35149_{2}$ \\
\hline 4099.553 & 100 & 4 & 24386.021 & $12847_{3}-37234_{2}^{\circ}$ & 4059.847 & 200 & 10 & 24624.515 & $11802_{2}-36427_{3}^{\circ}$ \\
\hline 4098.730 & $100 \mathrm{~s}$ & 2 & 24390.917 & $2869_{3}-27260_{3}^{\circ}$ & 4059.253 & $1500 \mathrm{r}$ & 20 & 24628.119 & $63622_{2}-30990_{3}^{\circ}$ \\
\hline 4097.747 & $500 \mathrm{~b}$ & 8 & 24396.768 & $2869_{3}-27266_{4}^{\circ}$ & 4058.936 & 75 & 8 & 24630.042 & $19227_{6}^{\circ}-438575$ \\
\hline 4097.321 & 200 & 8 & 24399.305 & $63622_{2}-307611^{\circ}$ & 4057.940 & 200 & 2 & 24636.087 & $11241_{3}^{\circ}-35877_{2}$ \\
\hline 4096.077 & 150 & 4 & 24406.714 & $7795_{4}^{\circ}-322025$ & 4056.718 & 10 & & 24643.508 & $8111_{4}-32754 \stackrel{3}{3}$ \\
\hline 4094.901 & 50 & 3 & 24413.724 & $15493_{4}-39906_{4}^{\circ}$ & 4056.636 & 200 & 2 & 24644.006 & $13175_{4}^{\circ}-37819_{4}$ \\
\hline 4093.672 & 40 & 2 & 24421.053 & $0_{2}-24421_{3}^{\circ}$ & 4056.399 & 100 & 2 & 24645.446 & $5563_{1}-30208_{2}^{\circ}$ \\
\hline 4091.988 & 8 & 1 & 24431.103 & $13847_{2}-38278_{1}^{\circ}$ & 4056.007 & 150 & 1 & 24647.828 & $3865_{1}-28513_{2}^{\circ}$ \\
\hline 4091.737 & 40 & 2 & 24432.602 & $7280_{2}-31712_{i}^{\circ}$ & 4055.908 & 200 & 1 & 24648.430 & $130883-37736_{2}^{\circ}$ \\
\hline 4091.590 & 10 & 1 & 24433.479 & $15618_{3}^{\circ}-400524$ & 4054.387 & 200 & 8 & 24657.676 & $13945_{3}^{\circ}-38602_{2}$ \\
\hline 4089.138 & 400 & 8 & 24448.130 & $2869_{3}-27317_{3}^{\circ}$ & 4054.302 & 300 & 8 & 24658.193 & $7502_{3}-32160_{2}^{\circ}$ \\
\hline 4088.728 & 500 & 8 & 24450.582 & $63622_{2}-30812_{2}^{\circ}$ & 4054.080 & 20 & & 24659.543 & $3687_{2}-28347_{2}^{\circ}$ \\
\hline 4088.629 & 100 & 3 & 24451.174 & $75023-31953^{\circ}$ & 4053.841 & 40 & & 24660.997 & $12114_{2}^{\circ}-36775_{3}$ \\
\hline 4087.285 & 200 & 5 & 24459.214 & $10414_{4}^{\circ}-348734$ & 4053.527 & $1000 \mathrm{r}$ & 75 & 24662.907 & $7795_{4}^{\circ}-324584$ \\
\hline 4087.166 & 501 & 2 & 24459.926 & $10414_{4}^{\circ}-348743$ & 4052.240 & $100 \mathrm{~b}$ & & 24670.740 & $15166_{3}^{\circ}-398373$ \\
\hline 4086.936 & 15 & 1 & 24461.303 & $142045-386658$ & 4051.913 & 40 & & 24672.731 & $17354_{1}^{\circ}-42027_{2}$ \\
\hline 4085.434 & 500 & 10 & 24470.296 & $8800_{4}-33270_{4}^{\circ}$ & 4051.500 & 500 & 15 & 24675.246 & $10414_{4}^{\circ}-35089_{3}$ \\
\hline 4085.257 & 200 & 8 & 24471.356 & $14481 \stackrel{\circ}{-}-389535$ & 4049.944 & 500 & 40 & 24684.726 & $3687_{2}-28372_{i}^{\circ}$ \\
\hline 4083.469 & 500 & 8 & 24482.071 & $3865_{1}-28347_{2}^{\circ}$ & 4049.535 & 200 & 25 & 24687.219 & $98045-34492_{4}^{\circ}$ \\
\hline 4081.592 & 50 & 2 & 24493.329 & $14206_{4}^{\circ}-38700_{4}$ & 4048.432 & 800 & 50 & 24693.945 & $12114_{2}^{\circ}-36808_{3}$ \\
\hline 4081.368 & 800 & 8 & 24494.673 & $8800_{4}-33294^{\circ}$ & 4048.288 & 800 & 40 & 24694.824 & $75023-32197_{3}^{\circ}$ \\
\hline 4080.707 & 400 & 20 & 24498.641 & $7795^{\circ}-322935$ & 4047.058 & 50 & 1 & 24702.329 & $14032_{2}^{\circ}-387343$ \\
\hline 4080.359 & 150 & 4 & 24500.730 & $7280_{2}-31780_{3}^{\circ}$ & 4046.824 & 200 & 15 & 24703.757 & $12114_{2}^{\circ}-36818_{2}$ \\
\hline 4079.273 & $25 b$ & 1 & 24507.253 & $3865_{1}-28372_{1}^{\circ}$ & 4046.252 & 200 & 8 & 24707.249 & $13962_{1}-38669_{2}^{\circ}$ \\
\hline 4078.302 & 100 & 2 & 24513.087 & $13175_{4}^{\circ}-37688_{3}$ & 4045.848 & $40 \mathrm{~b}$ & 2 & 24709.717 & $14465_{2}^{\circ}-391743$ \\
\hline 4077.623 & 15 & 1 & 24517.169 & $13088_{3}-37605_{4}^{\circ}$ & 4045.818 & $50 \mathrm{~b}$ & 3 & 24709.900 & $19227^{\circ}-439376$ \\
\hline 4075.907 & 150 & 5 & 24527.491 & $14206^{\circ}-38734_{3}$ & 4045.226 & 200 & 2 & 24713.516 & $63622-31075_{2}^{\circ}$ \\
\hline 4075.503 & $400 \mathrm{r}$ & 10 & 24529.922 & $25580-27087_{i}^{\circ}$ & 4044.926 & 300 & 8 & 24715.349 & $13175_{4}^{\circ}-37890_{5}$ \\
\hline 4073.960 & 25 & 1 & 24539.213 & $12114_{2}^{\circ}-366532$ & 4044.515 & $200 b$ & 2 & 24717.860 & $55631-30281_{1}^{\circ}$ \\
\hline 4073.856 & 200 & 3 & 24539.839 & $98045-34344_{4}^{\circ}$ & 4043.394 & $1000 \mathrm{r}$ & 50 & 24724.713 & $4961_{4}-29686_{3}^{\circ}$ \\
\hline 4073.007 & 20 & 1 & 24544.954 & $14465_{2}^{\circ}-39010_{3}$ & 4040.971 & 100 & & 24739.538 & $25580-27297^{\circ}$ \\
\hline 4071.750 & $200 \mathrm{~b}$ & 15 & 24552.532 & $8243^{\circ}-327963$ & 4039.865 & 800 & 20 & 24746.311 & $14206_{4}^{\circ}-389535$ \\
\hline 4071.731 & $100 \mathrm{~b}$ & & 24552.646 & $11241_{3}^{\circ}-35794_{4}$ & 4039.268 & 100 & 2 & 24749.968 & $4961_{4}-29711^{\circ}$ \\
\hline 4071.553 & 100 & 1 & 24553.719 & $14206_{4}^{\circ}-38760_{4}$ & 4039.268 & 100 & 2 & 24749.968 & $14206_{4}^{\circ}-38956_{4}$ \\
\hline 4070.756 & 100 & 2 & 24558.527 & $11802_{2}-36361_{i}^{\circ}$ & 4039.022 & 150 & 1 & 24751.475 & $8111_{4}-32862_{4}^{\circ}$ \\
\hline 4070.238 & 150 & 3 & 24561.652 & $0_{2}-24561_{3}^{\circ}$ & 4038.635 & 100 & & 24753.847 & $11802_{2}-36556_{1}^{\circ}$ \\
\hline 4070.115 & 25 & 1 & 24562.394 & $15490_{0}^{\circ}-400524$ & 4038.456 & 300 & 2 & 24754.944 & $13945_{3}^{\circ}-38700_{4}$ \\
\hline 4070.041 & 75 & 1 & 24562.841 & $10526_{3}^{\circ}-35089_{3}$ & 4038.228 & 300 & 10 & 24756.342 & $7795_{4}^{\circ}-32551_{3}$ \\
\hline 4069.901 & 5 & & 24563.686 & $14243^{\circ}-38807_{2}$ & 4037.993 & 15 & & 24757.783 & $12847_{3}-37605_{4}^{\circ}$ \\
\hline 4069.461 & $500 \mathrm{r}$ & 15 & 24566.342 & $6362_{2}-30928_{1}^{\circ}$ & 4037.663 & 100 & 2 & 24759.806 & $8800_{4}-33560_{4}^{\circ}$ \\
\hline 4069.112 & 50 & 2 & 24568.449 & $13847_{2}-38416_{3}^{\circ}$ & 4037.562 & 500 & 15 & 24760.425 & $11601_{1}-36361_{1}^{\circ}$ \\
\hline 4068.705 & 75 & & 24570.906 & $14032_{2}^{\circ}-38602_{2}$ & 4036.047 & $2000 \mathrm{r}$ & 50 & 24769.719 & $\mathbf{0}_{2}-24769_{3}^{\circ}$ \\
\hline 4068.470 & 50 & 2 & 24572.325 & $16783^{\circ}-413565$ & 4035.931 & 200 & 8 & 24770.431 & $14481 \stackrel{\circ}{6}-392525$ \\
\hline 4067.451 & $400 \mathrm{r}$ & 5 & 24578.481 & $2869_{3}-27447_{2}^{\circ}$ & 4035.730 & 300 & 1 & 24771.665 & $49614-297330$ \\
\hline 4066.961 & 50 & 1 & 24581.442 & $15618_{3}^{\circ}-40200_{3}$ & 4035.089 & 100 & & 24775.600 & $14032_{2}^{\circ}-38807_{2}$ \\
\hline 4066.822 & 100 & 2 & 24582.283 & $18053^{\circ}-426355$ & 4034.878 & 200 & 8 & 24776.896 & $15493_{4}-40270 \stackrel{\circ}{\circ}$ \\
\hline 4066.435 & 25 & & 24584.622 & $13945^{\circ}-38529_{3}$ & 4033.907 & $600 \mathrm{r}$ & 8 & 24782.860 & $4961_{4}-29744 \stackrel{\circ}{3}$ \\
\hline 4065.889 & 100 & 3 & 24587.923 & $11601_{1}-36188_{2}^{\circ}$ & 4033.776 & 500 & 8 & 24783.664 & $3865_{1}-28649^{\circ}$ \\
\hline 4065.618 & 100 & 4 & 24589.562 & $11241_{3}^{\circ}-35831_{3}$ & 4032.596 & $600 \mathrm{r}$ & 200 & 24790.916 & $8800_{4}-33591_{3}^{\circ}$ \\
\hline 4065.551 & 50 & 1 & 24589.968 & $7280_{2}-31870_{2}^{\circ}$ & 4032.199 & 20 & & 24793.357 & $10783_{2}^{\circ}-355763$ \\
\hline 4064.332 & 400 & 10 & 24597.343 & $111977^{\circ}-35794_{4}$ & 4030.842 & $2000 \mathrm{r}$ & 50 & 24801.704 & $2869_{3}-27670_{3}^{\circ}$ \\
\hline 4063.407 & $1500 \mathrm{r}$ & 75 & 24602.942 & $11197^{\circ}-35799_{6}$ & 4030.590 & 20 & & 24803.254 & $14206_{4}^{\circ}-39010_{3}$ \\
\hline 4061.625 & 300 & 3 & 24613.736 & $142260-38840^{\circ}$ & 4030.292 & 300 & 40 & 24805.088 & $2869_{3}-27674_{2}^{\circ}$ \\
\hline 4060.241 & 5 & & 24622.126 & $10783^{\circ}-35405_{3}$ & 4030.214 & 100 & 1 & 24805.568 & $11241_{3}^{\circ}-36047_{2}$ \\
\hline
\end{tabular}


TABLE 4. Classified lines of $\mathrm{Th} \mathrm{I}$-continued

\begin{tabular}{|c|c|c|c|c|c|c|c|c|c|}
\hline \multirow{2}{*}{$\begin{array}{c}\text { Wavelength } \\
\text { (§) }\end{array}$} & \multicolumn{2}{|c|}{ Intensity } & \multirow{2}{*}{$\begin{array}{l}\text { Wavenumber } \\
\left(\mathrm{cm}^{-1}\right)\end{array}$} & \multirow{2}{*}{ Classification } & \multirow{2}{*}{$\begin{array}{c}\text { Wavelength } \\
\text { (§) }\end{array}$} & \multicolumn{2}{|c|}{ Inten sity } & \multirow{2}{*}{$\begin{array}{l}\text { Wavenumber } \\
\left(\mathrm{cm}^{-1}\right)\end{array}$} & \multirow{2}{*}{ Classification } \\
\hline & Lamp & Spark & & & & Lamp & Spark & & \\
\hline 4030.078 & 25 & & 24806.405 & $15970_{3}-40776_{4}^{\circ}$ & 4000.445 & 200 & 3 & 24990.153 & $55631-30553_{2}^{\circ}$ \\
\hline 4029.827 & $400 \mathrm{r}$ & 15 & 24807.951 & $3865_{1}-28673_{2}^{\circ}$ & 4000.280 & 800 & 20 & 24991.184 & $10414_{4}^{\circ}-35405_{3}$ \\
\hline 4029.658 & 400 & 15 & 24808.991 & $12847_{3}-37656_{3}^{\circ}$ & 4000.041 & $200 \mathrm{~b}$ & & 24992.677 & $15618_{3}^{\circ}-40611_{2}$ \\
\hline 4028.334 & 200 & 10 & 24817.145 & $13175_{4}^{\circ}-37992_{4}$ & 4000.024 & $100 \mathrm{~b}$ & & 24992.783 & $13847_{2}-38840^{\circ}$ \\
\hline 4027.546 & 1501 & 20 & 24822.000 & $13847_{2}-38669_{2}^{\circ}$ & 3998.953 & 600 & 20 & 24999.477 & $8800_{4}-33799_{4}^{\circ}$ \\
\hline 4027.007 & $500 \mathrm{r}$ & 10 & 24825.322 & $3687_{2}-285132$ & 3998.800 & $100 \mathrm{~b}$ & 5 & 25000.433 & $13088_{3}-38088_{2}^{\circ}$ \\
\hline 4026.644 & 4 & & 24827.560 & $13847_{2}-38675_{3}^{\circ}$ & 3998.733 & $400 \mathrm{~b}$ & 5 & 25000.852 & $7795_{4}^{\circ}-327963$ \\
\hline 4024.801 & $300 \mathrm{r}$ & 8 & 24838.929 & $0_{2}-24838$ i & 3998.657 & $300 \mathrm{~s}$ & 2 & 25001.327 & $75023-32503_{2}^{\circ}$ \\
\hline 4023.338 & 400 & 10 & 24847.961 & $112413-36089_{4}$ & 3998.061 & 300 & 5 & 25005.054 & $7280_{2}-322853$ \\
\hline 4022.728 & 75 & 1 & 24851.729 & $13962_{1}-38814_{2}^{\circ}$ & 3997.443 & $15 b$ & & 25008.920 & $13297_{4}-38306_{4}^{\circ}$ \\
\hline 4022.067 & $500 \mathrm{~b}$ & & 24855.813 & $8243 \stackrel{\circ}{-}-33099_{3}$ & 3997.347 & 200 & 4 & 25009.521 & $16346 \stackrel{\circ}{-4}-413565$ \\
\hline 4021.940 & $200 \mathrm{~b}$ & 4 & 24856.598 & $14465_{2}^{\circ}-39321_{3}$ & 3997.172 & 50 & 1 & 25010.615 & $15970_{3}-40980_{3}^{\circ}$ \\
\hline 4021.750 & 200 & 8 & 24857.772 & $142045-390620^{\circ}$ & 3997.109 & 300 & 5 & 25011.009 & $13175_{4}^{\circ}-38186_{4}$ \\
\hline 4021.149 & 300 & 10 & 24861.487 & $13088_{3}-37950_{4}^{\circ}$ & 3997.019 & 300 & 8 & 25011.573 & $13945_{3}^{\circ}-38956_{4}$ \\
\hline 4021.007 & 75 & 2 & 24862.365 & $13945_{3}^{\circ}-38807_{2}$ & 3996.670 & 500 & 15 & 25013.757 & $111970^{\circ}-36210_{5}$ \\
\hline 4020.462 & 100 & & 24865.735 & $11802_{2}-36668_{2}^{\circ}$ & 3996.508 & 100 & 2 & 25014.771 & $16346 \stackrel{4}{4}-41361_{3}$ \\
\hline 4020.354 & 500 & 20 & 24866.403 & $13088_{3}-37954_{3}^{\circ}$ & 3996.203 & 300 & 25 & 25016.680 & $12114_{2}^{\circ}-37131_{3}$ \\
\hline 4019.787 & 200 & 5 & 24869.911 & $144811^{\circ}-393515$ & 3994.038 & 5 & & 25030.240 & $82432-332731$ \\
\hline 4018.932 & 25 & 2 & 24875.201 & $17411_{3}^{\circ}-422863$ & 3993.515 & 200 & 15 & 25033.518 & $15166_{3}^{\circ}-40200_{3}$ \\
\hline 4018.354 & 200 & 15 & 24878.779 & $10526^{\circ}-35405_{3}$ & 3993.397 & 100 & & 25034.258 & $11802_{2}-36837_{1}^{\circ}$ \\
\hline 4018.118 & $50 \mathrm{~b}$ & & 24880.241 & $0_{2}-24880_{1}^{\circ}$ & 3991.731 & $800 r$ & 40 & 25044.706 & 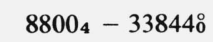 \\
\hline 4018.099 & $400 \mathrm{~b}$ & 15 & 24880.358 & $7280_{2}-32160_{2}^{\circ}$ & 3991.624 & $300 \mathrm{~b}$ & 40 & 25045.377 & $14206^{\circ}-39252_{5}$ \\
\hline 4017.461 & 8 & & 24884.309 & $163469-41230_{4}^{\circ}$ & 3991.289 & 15 & & 25047.479 & $18809_{4}^{\circ}-43857_{5}$ \\
\hline 4017.257 & 100 & 2 & 24885.573 & $15166_{3}^{\circ}-40052_{4}$ & 3990.892 & 300 & 15 & 25049.971 & $10526_{3}^{\circ}-35576_{3}$ \\
\hline 4017.062 & 400 & 5 & 24886.781 & $10414_{4}^{\circ}-353005$ & 3990.809 & 300 & 25 & 25050.492 & $55631-30613 \circ$ \\
\hline 4016.896 & 10 & & 24887.809 & $11601_{1}-36488_{2}^{\circ}$ & 3990.492 & $800 r$ & 10 & 25052.482 & $3865_{1}-28917_{2}^{\circ}$ \\
\hline 4016.112 & 100 & 2 & 24892.668 & $111970-36089_{4}$ & 3990.020 & $150 \mathrm{~b}$ & 40 & 25055.445 & $4961_{4}-30017_{3}^{\circ}$ \\
\hline 4014.716 & 400 & 10 & 24901.323 & $3687_{2}-28589_{3}^{\circ}$ & 3990.002 & $20 b$ & 3 & 25055.558 & $11241_{3}^{\circ}-36297_{4}$ \\
\hline 4014.472 & 40 & 2 & 24902.837 & $16783_{4}^{\circ}-416863$ & 3988.953 & 25 & & 25062.147 & $17224_{2}^{\circ}-42286_{3}$ \\
\hline 4012.610 & 25 & 2 & 24914.392 & $10526_{3}^{\circ}-35440_{3}$ & 3988.519 & 25 & 4 & 25064.874 & $13945_{3}^{\circ}-39010_{3}$ \\
\hline 4012.495 & $2000 r$ & 150 & 24915.106 & $2869_{3}-27784_{2}^{\circ}$ & 3988.429 & 75 & & 25065.440 & $8243_{2}^{\circ}-33309_{2}$ \\
\hline 4012.190 & 150 & 2 & 24917.000 & $7280_{2}-32197^{\circ}$ & 3988.078 & $10 \mathrm{~b}$ & $8 b$ & 25067.646 & $11601_{1}-36668_{2}^{\circ}$ \\
\hline 4011.739 & $800 \mathrm{r}$ & $5 b$ & 24919.801 & $4961_{4}-29881_{4}^{\circ}$ & 3987.867 & 150 & 2 & 25068.972 & $11802_{2}-36871_{2}^{\circ}$ \\
\hline 4011.591 & $500 \mathrm{r}$ & 40 & 24920.721 & $63622-312833_{3}^{\circ}$ & 3987.206 & $200 \mathrm{~b}$ & & 25073.128 & $7502_{3}-32575_{2}^{\circ}$ \\
\hline 4009.816 & $400 \mathrm{~b}$ & 10 & 24931.752 & $13175_{4}^{\circ}-381065$ & 3986.604 & 200 & 25 & 25076.914 & $14206_{4}^{\circ}-39283_{4}$ \\
\hline 4009.783 & $200 \mathrm{~b}$ & 2 & 24931.957 & $154934-40425^{\circ}$ & 3986.215 & 25 & & 25079.361 & $2869_{3}-27948_{4}^{\circ}$ \\
\hline 4009.724 & 400 & 10 & 24932.324 & $8111_{4}-33043^{\circ}$ & 3985.815 & 8 & & 25081.878 & $98045-34886 \circ$ \\
\hline 4009.057 & $1500 \mathrm{r}$ & 100 & 24936.472 & $111977^{\circ}-361335$ & 3985.737 & 4 & & 25082.369 & $15618 \stackrel{\circ}{3}-40701_{4}$ \\
\hline 4009.012 & $300 \mathrm{r}$ & 50 & 24936.752 & $75023-32439_{4}^{\circ}$ & 3984.879 & 300 & 40 & 25087.769 & $13297_{4}-383850$ \\
\hline 4008.443 & 10 & & 24940.292 & $11877_{1}^{\circ}-368182$ & 3984.095 & 200 & & 25092.706 & $15490 \%-405825$ \\
\hline 4008.312 & 100 & & 24941.107 & $13847_{2}-38788_{3}^{\circ}$ & 3983.815 & 2 & & 25094.470 & $11241_{3}^{\circ}-36336_{3}$ \\
\hline 4008.210 & 1500 & 100 & 24941.741 & $7795_{4}^{\circ}-327375$ & 3983.783 & 10 & & 25094.671 & $10783_{2}^{\circ}-35877_{2}$ \\
\hline 4005.960 & 300 & 8 & 24955.750 & $11601_{1}-36556_{1}^{\circ}$ & 3982.894 & 200 & 8 & 25100.272 & $11197^{\circ}-362974$ \\
\hline 4005.297 & 75 & 1 & 24959.881 & $11802_{2}-36762_{3}^{\circ}$ & 3982.607 & 100 & 2 & 25102.081 & $12847_{3}-37950_{4}^{\circ}$ \\
\hline 4005.091 & $500 \mathrm{r}$ & 10 & 24961.165 & $3687_{2}-28649^{\circ}$ & 3981.827 & 300 & 100 & 25106.998 & $12847_{3}-37954^{\circ}$ \\
\hline 4004.560 & 20 & & 24964.474 & $130888_{3}-38053_{3}^{\circ}$ & 3980.575 & 15 & & 25114.895 & $14206^{\circ}-393213$ \\
\hline 4004.238 & 8 & 1 & 24966.482 & $13847_{2}-38814_{2}^{\circ}$ & 3979.956 & 200 & 8 & 25118.801 & $13297_{4}-38416_{3}^{\circ}$ \\
\hline 4003.993 & 4 & & 24968.009 & $142069-391743$ & 3979.402 & 75 & 2 & 25122.298 & $12847_{3}-37970_{2}^{\circ}$ \\
\hline 4003.574 & 400 & 25 & 24970.622 & $13175_{4}^{\circ}-38145_{3}$ & 3978.438 & 50 & 1 & 25128.385 & $13088_{3}-38216_{3}^{\circ}$ \\
\hline 4002.376 & 75 & 3 & 24978.096 & $14032_{2}^{\circ}-39010_{3}$ & 3977.434 & 15 & & 25134.728 & $17501 \stackrel{\circ}{\circ}-426355$ \\
\hline 4001.992 & 50 & & 24980.493 & $12114_{2}^{\circ}-37094_{2}$ & 3976.152 & $50 \mathrm{~b}$ & 1 & 25142.831 & $14032_{2}^{\circ}-391743$ \\
\hline 4001.894 & 300 & 2 & 24981.105 & $0_{2}-24981_{3}^{\circ}$ & 3975.976 & 20 & & 25143.944 & $16217_{2}^{\circ}-41361_{3}$ \\
\hline 4001.198 & $100 \mathrm{~h}$ & & 24985.450 & $3687_{2}-28673_{2}^{\circ}$ & 3975.831 & 150 & 5 & 25144.862 & $14206_{4}^{\circ}-393515$ \\
\hline 4001.058 & $800 r$ & 40 & 24986.324 & $7795_{4}^{\circ}-32781_{4}$ & 3975.468 & 200 & 20 & 25147.157 & $8243 \stackrel{\circ}{2}-33390_{1}$ \\
\hline 4000.748 & 200 & & 24988.261 & $3687_{2}-28676_{3}^{\circ}$ & 3975.008 & $5 \mathrm{~s}$ & & 25150.067 & $15736_{1}^{\circ}-40886_{2}$ \\
\hline
\end{tabular}


TABLE 4. Classified lines of $\mathrm{Th} \mathrm{I}$-continued

\begin{tabular}{|c|c|c|c|c|c|c|c|c|c|}
\hline \multirow{2}{*}{$\begin{array}{l}\text { Wavelength } \\
(\AA)\end{array}$} & \multicolumn{2}{|c|}{ Intensity } & \multirow{2}{*}{$\begin{array}{l}\text { Wavenumber } \\
\qquad\left(\mathrm{cm}^{-1}\right)\end{array}$} & \multirow{2}{*}{ Classification } & \multirow{2}{*}{$\begin{array}{c}\text { Wavelength } \\
\text { (̊̊) }\end{array}$} & \multicolumn{2}{|c|}{ Inten sity } & \multirow{2}{*}{$\begin{array}{l}\text { Wavenumber } \\
\qquad\left(\mathrm{cm}^{-1}\right)\end{array}$} & \multirow{2}{*}{ Classification } \\
\hline & Lamp & Spark & & & & Lamp & Spark & & \\
\hline 3974.829 & 200 & 4 & 25151.200 & $13847_{2}-38998_{3}^{\circ}$ & 3941.682 & 10 & & 25362.701 & $8243 \stackrel{2}{2}-336062$ \\
\hline 3973.507 & 25 & 2 & 25159.568 & $8111_{4}-33270_{4}^{\circ}$ & 3941.232 & 100 & 2 & 25365.597 & $5563_{1}-30928_{1}^{\circ}$ \\
\hline 3973.333 & 500 & 10 & 25160.669 & $55631-30723 i$ & 3941.137 & 100 & 3 & 25366.208 & $16346_{4}^{\circ}-417125$ \\
\hline 3973.196 & $1000 \mathrm{r}$ & 50 & 25161.537 & $63622-31523_{3}^{\circ}$ & 3940.837 & 200 & 5 & 25368.139 & $13297_{4}-38665^{\circ}$ \\
\hline 3973.063 & $40 \mathrm{~b}$ & 1 & 25162.379 & $10414^{\circ}-35576_{3}$ & 3940.705 & 200 & 4 & 25368.989 & $128473-38216_{3}^{\circ}$ \\
\hline 3972.640 & 500 & 75 & 25165.058 & $8243^{\circ}-334083$ & 3940.422 & 5 & & 25370.811 & $7280_{2}-32650_{1}^{\circ}$ \\
\hline 3972.230 & 75 & 2 & 25167.656 & $8800_{4}-33967_{3}^{\circ}$ & 3939.538 & 75 & 2 & 25376.503 & $13945_{3}^{\circ}-393213$ \\
\hline 3972.153 & $1500 \mathrm{r}$ & 300 & 25168.144 & $7795_{4}^{\circ}-329635$ & 3939.318 & 150 & 8 & 25377.921 & $13297_{4}-38675^{\circ}$ \\
\hline 3972.153 & $1500 \mathrm{r}$ & 300 & 25168.144 & $10414_{4}^{\circ}-355825$ & 3938.956 & 75 & 2 & 25380.253 & $10414_{4}^{\circ}-357944$ \\
\hline 3969.665 & 300 & 15 & 25183.918 & $8111_{4}-33294_{3}^{\circ}$ & 3938.614 & $400 \mathrm{~b}$ & 150 & 25382.457 & $8800_{4}-34182{ }^{\circ}$ \\
\hline 3967.610 & 200 & 2 & 25196.961 & $3687_{2}-28884_{3}^{\circ}$ & 3938.152 & 200 & 8 & 25385.434 & $7280_{2}-32665_{1}^{\circ}$ \\
\hline 3967.392 & $2000 \mathrm{r}$ & 300 & 25198.346 & $4961_{4}-30160_{4}^{\circ}$ & 3937.861 & 100 & $2 b$ & 25387.310 & $3865_{1}-29252_{2}^{\circ}$ \\
\hline 3966.334 & 200 & 4 & 25205.067 & $128473-38053 \stackrel{\circ}{3}$ & 3934.465 & 15 & 1 & 25409.223 & $16346_{4}^{\circ}-41755_{4}$ \\
\hline 3965.483 & 100 & 3 & 25210.476 & $19227^{\circ}-444376$ & 3934.060 & 150 & 3 & 25411.838 & $11241_{3}^{\circ}-36653_{2}$ \\
\hline 3964.455 & 75 & 2 & 25217.013 & $11877^{\circ}-37094_{2}$ & 3933.238 & 400 & 40 & 25417.149 & $10414 \stackrel{\circ}{4}-358313$ \\
\hline 3964.329 & 200 & 3 & 25217.814 & $13088_{3}-38306_{4}^{\circ}$ & 3933.034 & 200 & 5 & 25418.467 & $6362_{2}-31780_{3}^{\circ}$ \\
\hline 3964.030 & 300 & 15 & 25219.717 & $10414_{4}^{\circ}-35633_{4}$ & 3932.911 & $1500 \mathrm{r}$ & 200 & 25419.262 & $7795_{4}^{\circ}-332145$ \\
\hline 3962.475 & 100 & 3 & 25229.613 & $13945^{\circ}-391743$ & 3931.085 & 150 & 3 & 25431.069 & $118022-37234_{2}^{\circ}$ \\
\hline 3962.420 & $400 \mathrm{r}$ & 15 & 25229.964 & $3687_{2}-28917_{2}^{\circ}$ & 3930.252 & 150 & 4 & 25436.459 & $14243 i-39680_{2}$ \\
\hline 3962.371 & 75 & $5 \mathrm{~b}$ & 25230.276 & $159703-41200_{2}^{\circ}$ & 3929.291 & 300 & 8 & 25442.680 & $0_{2}-25442 \stackrel{\circ}{3}$ \\
\hline 3962.197 & 200 & 8 & 25231.384 & $154934-40724^{\circ}$ & 3928.967 & $200 \mathrm{~b}$ & $40 \mathrm{~b}$ & 25444.778 & $151663-40611_{2}$ \\
\hline 3960.685 & 10 & & 25241.015 & $12847_{3}-38088^{\circ}$ & 3928.865 & 300 & 50 & 25445.439 & $82432-33689_{2}$ \\
\hline 3960.270 & 500 & 50 & 25243.661 & $11241 \stackrel{\circ}{3}-364852$ & 3928.308 & 100 & 10 & 25449.046 & $8111_{4}-33560_{4}^{\circ}$ \\
\hline 3959.300 & $1000 \mathrm{r}$ & 100 & 25249.845 & $5563_{1}-30812_{2}^{\circ}$ & 3927.806 & 400 & 10 & 25452.299 & $75023-32954_{2}^{\circ}$ \\
\hline 3958.928 & 200 & 3 & 25252.217 & $75023-327543$ & 3926.864 & 200 & 8 & 25458.404 & $128473-38306_{4}^{\circ}$ \\
\hline 3957.736 & 40 & & 25259.823 & $15166^{\circ}-404264$ & 3925.596 & 300 & 8 & 25466.628 & $25580-28024_{i}^{\circ}$ \\
\hline 3957.059 & 100 & 20 & 25264.144 & $10783_{2}^{\circ}-36047_{2}$ & 3925.219 & 400 & 50 & 25469.074 & $98045-352730^{\circ}$ \\
\hline 3956.481 & 150 & 20 & 25267.835 & $10526^{\circ}-35794_{4}$ & 3925.093 & $1000 \mathrm{r}$ & $200 \mathrm{~b}$ & 25469.891 & $3687_{2}-29157^{\circ}$ \\
\hline 3956.005 & 400 & 10 & 25270.875 & $11601_{1}-36871_{2}^{\circ}$ & 3924.404 & 5001 & 10 & 25474.363 & $7280_{2}-32754^{\circ}$ \\
\hline 3955.891 & 400 & $50 \mathrm{~b}$ & 25271.604 & $2869_{3}-281400_{4}^{\circ}$ & 3923.799 & $500 \mathrm{r}$ & 20 & 25478.290 & $2869_{3}-28347_{2}^{\circ}$ \\
\hline 3955.477 & 15 & & 25274.249 & $11241 \stackrel{\circ}{3}-36515_{3}$ & 3923.510 & 100 & 2 & 25480.167 & $8111_{4}-33591{ }_{3}^{\circ}$ \\
\hline 3955.170 & 800 & 40 & 25276.210 & $98045-35081 \stackrel{\circ}{\circ}$ & 3922.394 & 100 & 8 & 25487.416 & $14206_{4}^{\circ}-396945$ \\
\hline 3954.130 & 100 & 20 & 25282.858 & $13297_{4}-38580_{4}^{\circ}$ & 3921.782 & 25 & 4 & 25491.394 & $13297_{4}-38788_{3}^{\circ}$ \\
\hline 3954.068 & 100 & 10 & 25283.255 & $15493_{4}-40776_{4}^{\circ}$ & 3921.731 & 15 & & 25491.725 & $13088_{3}-38580_{4}^{\circ}$ \\
\hline 3953.056 & 4 & & 25289.727 & $14032_{2}^{\circ}-393213$ & 3920.058 & 300 & 10 & 25502.604 & $16783 \stackrel{\circ}{4}-422863$ \\
\hline 3952.967 & 75 & 1 & 25290.296 & $12114_{2}^{\circ}-37404_{3}$ & 3919.275 & 200 & 5 & 25507.699 & $6362_{2}-31870_{2}^{\circ}$ \\
\hline 3952.761 & $500 \mathrm{r}$ & 10 & 25291.614 & $3865_{1}-29157_{i}^{\circ}$ & 3919.023 & $1000 \mathrm{r}$ & 100 & 25509.339 & $3687_{2}-29197^{\circ}$ \\
\hline 3952.421 & 25 & & 25293.790 & $4961_{4}-30255_{3}^{\circ}$ & 3918.934 & 300 & 8 & 25509.919 & $3687_{2}-29197_{2}^{\circ}$ \\
\hline 3950.804 & 300 & 8 & 25304.142 & $7795_{4}^{\circ}-33099_{3}$ & 3917.270 & 500 & 50 & 25520.755 & $10526_{3}^{\circ}-36047_{2}$ \\
\hline 3950.707 & $100 \mathrm{~s}$ & 3 & 25304.763 & $10526_{3}^{\circ}-358313$ & 3916.597 & 200 & 8 & 25525.140 & $13175_{4}^{\circ}-38700_{4}$ \\
\hline 3950.393 & $1000 \mathrm{r}$ & 200 & 25306.775 & $\mathbf{0}_{2}-25306_{2}^{\circ}$ & 3916.417 & $500 \mathrm{r}$ & 25 & 25526.313 & $0_{2}-25526^{\circ}$ \\
\hline 3948.133 & 500 & 40 & 25321.261 & $82432-335643$ & 3915.848 & 200 & 15 & 25530.022 & $8243_{2}^{\circ}-33773_{1}$ \\
\hline 3948.029 & $600 \mathrm{r}$ & 15 & 25321.928 & $\mathbf{0}_{2}-25321_{3}^{\circ}$ & 3915.295 & 300 & 8 & 25533.628 & $11241_{3}^{\circ}-36775_{3}$ \\
\hline 3947.329 & $1000 \mathrm{r}$ & 100 & 25326.418 & $98045-351314$ & 3915.172 & 5 & & 25534.430 & $15166_{3}^{\circ}-40701_{4}$ \\
\hline 3947.136 & 500 & 50 & 25327.656 & $13088_{3}-38416_{3}^{\circ}$ & 3914.163 & $200 \mathrm{~s}$ & 8 & 25541.012 & $75023-330433^{\circ}$ \\
\hline 3946.481 & 400 & 10 & 25331.860 & $38651-29197_{i}^{\circ}$ & 3913.645 & 2001 & 5 & 25544.393 & $8800_{4}-34344_{4}^{\circ}$ \\
\hline 3946.391 & 300 & 25 & 25332.438 & $38651-29197_{2}^{\circ}$ & 3913.302 & 75 & 2 & 25546.632 & $98045-35351_{4}^{\circ}$ \\
\hline 3945.206 & 200 & 8 & 25340.046 & $16346_{4}^{\circ}-416863$ & 3913.082 & 300 & 25 & 25548.068 & $11601_{1}-37149_{2}^{\circ}$ \\
\hline 3944.253 & 500 & 20 & 25346.169 & $11802_{2}-37149_{2}^{\circ}$ & 3912.738 & 75 & 3 & 25550.314 & $17073_{1}-42624^{\circ}$ \\
\hline 3943.605 & 300 & 25 & 25350.333 & $63622-31712^{\circ}$ & 3912.485 & 751 & 1 & 25551.966 & $111970^{\circ}-367496$ \\
\hline 3943.459 & 300 & 40 & 25351.272 & $10526_{3}^{\circ}-35877_{2}$ & 3912.211 & 75 & 2 & 25553.756 & $3865_{1}-29419_{2}^{\circ}$ \\
\hline 3942.907 & 300 & 20 & 25354.821 & $13175_{4}^{\circ}-38529_{3}$ & 3911.950 & $300 \mathrm{~b}$ & 25 & 25555.460 & $4961_{4}-30517^{\circ}$ \\
\hline 3942.628 & 100 & & 25356.615 & $11802_{2}-37159_{3}^{\circ}$ & 3911.910 & $500 \mathrm{r}$ & 40 & 25555.722 & $4961_{4}-30517_{4}^{\circ}$ \\
\hline 3942.072 & $500 \mathrm{~b}$ & 50 & 25360.192 & $75023-32862_{4}^{\circ}$ & 3911.523 & 200 & 3 & 25558.250 & $11601_{1}-37159^{\circ}$ \\
\hline 3941.962 & 10 & 1 & 25360.899 & $16217_{2}^{\circ}-41578_{2}$ & 3911.364 & 15 & & 25559.289 & $13175^{\circ}-387343$ \\
\hline
\end{tabular}


TABLE 4. Classified lines of Th $\mathrm{I}$-continued

\begin{tabular}{|c|c|c|c|c|c|c|c|c|c|}
\hline \multirow{2}{*}{$\begin{array}{l}\text { Wavelength } \\
(\AA)\end{array}$} & \multicolumn{2}{|c|}{ Intensity } & \multirow{2}{*}{$\begin{array}{l}\text { Wavenumber } \\
\qquad\left(\mathrm{cm}^{-1}\right)\end{array}$} & \multirow{2}{*}{ Classification } & \multirow{2}{*}{$\begin{array}{c}\text { Wavelength } \\
\text { (§) }\end{array}$} & \multicolumn{2}{|c|}{ Inten sity } & \multirow{2}{*}{$\begin{array}{l}\text { Wavenumber } \\
\qquad\left(\mathrm{cm}^{-1}\right)\end{array}$} & \multirow{2}{*}{ Classification } \\
\hline & Lamp & Spark & & & & Lamp & Spark & & \\
\hline 3910.773 & 300 & 8 & 25563.152 & $10526^{\circ}-36089_{4}$ & 3877.610 & $500 \mathrm{~b}$ & 15 & 25781.774 & $13175_{4}^{\circ}-38956_{4}$ \\
\hline 3910.622 & 50 & 2 & 25564.139 & $13847_{2}-394111$ & 3877.462 & 500 & 20 & 25782.758 & $8800_{4}-34583_{3}^{\circ}$ \\
\hline 3910.521 & 100 & 2 & 25564.799 & $3687_{2}-29252_{2}^{\circ}$ & 3875.646 & $500 \mathrm{r}$ & 25 & 25794.838 & $7502_{3}-33297_{2}^{\circ}$ \\
\hline 3910.251 & 50 & 2 & 25566.564 & $11241_{3}^{\circ}-36808_{3}$ & 3875.374 & $1500 \mathrm{r}$ & 200 & 25796.649 & $10414 \stackrel{\circ}{4}-362105$ \\
\hline 3909.991 & 100 & 8 & 25568.264 & $128473-38416_{3}^{\circ}$ & 3875.157 & 300 & 2 & 25798.093 & $6362_{2}-32160_{2}^{\circ}$ \\
\hline 3909.139 & 300 & 20 & 25573.837 & $12114_{2}^{\circ}-37688_{3}$ & 3874.862 & $1500 \mathrm{r}$ & 200 & 25800.057 & $4961_{4}-30761_{3}^{\circ}$ \\
\hline 3908.979 & 300 & 25 & 25574.883 & $13945_{3}^{\circ}-39520_{4}$ & 3874.244 & $1000 \mathrm{r}$ & 50 & 25804.173 & $2869_{3}-28673_{2}^{\circ}$ \\
\hline 3908.749 & 500 & 15 & 25576.388 & $11241_{3}^{\circ}-368182$ & 3873.821 & $2000 \mathrm{r}$ & 500 & 25806.990 & $2869_{3}-28676_{3}^{\circ}$ \\
\hline 3908.014 & 75 & 1 & 25581.198 & $130883-38669_{2}^{\circ}$ & 3873.473 & $500 \mathrm{r}$ & 50 & 25809.309 & $0_{2}-25809_{1}^{\circ}$ \\
\hline 3907.543 & 50 & 5 & 25584.282 & $11197 \stackrel{\circ}{-}-367815$ & 3873.421 & $400 \mathrm{~b}$ & 20 & 25809.655 & $10526_{3}^{\circ}-36336_{3}$ \\
\hline 3907.352 & 2 & & 25585.532 & $13175_{4}^{\circ}-38760_{4}$ & 3873.147 & 400 & 10 & 25811.481 & $2869_{3}-28680_{4}^{\circ}$ \\
\hline 3907.160 & 200 & 10 & 25586.790 & $13088_{3}-38675_{3}^{\circ}$ & 3872.861 & 200 & 4 & 25813.387 & $11802_{2}-37616_{i}^{\circ}$ \\
\hline 3903.481 & 300 & 3 & 25610.905 & $2869_{3}-28480_{4}^{\circ}$ & 3872.672 & $200 \mathrm{~b}$ & 5 & 25814.647 & $25580-28372_{1}^{\circ}$ \\
\hline 3903.315 & 200 & 5 & 25611.994 & $15618^{\circ}-41230_{4}$ & 3870.891 & 75 & & 25826.524 & $4961_{4}-30788_{0}^{\circ}$ \\
\hline 3903.103 & $1000 \mathrm{r}$ & 150 & 25613.385 & $7795_{4}^{\circ}-334083$ & 3870.763 & 75 & 2 & 25827.378 & $12847_{3}-38675_{3}^{\circ}$ \\
\hline 3901.957 & 20 & 2 & 25620.907 & $13847_{2}-39468_{3}^{\circ}$ & 3869.663 & $800 \mathrm{r}$ & 50 & 25834.720 & $63622-32197_{3}^{\circ}$ \\
\hline 3901.662 & 300 & 15 & 25622.844 & $7795_{4}^{\circ}-334184$ & 3868.254 & 400 & 10 & 25844.130 & $11241_{3}^{\circ}-37085_{4}$ \\
\hline 3900.577 & $600 \mathrm{r}$ & 50 & 25629.971 & $8800_{4}-34430_{3}^{\circ}$ & 3868.040 & 100 & 3 & 25845.559 & $14206^{\circ}-40052_{4}$ \\
\hline 3900.464 & 40 & 1 & 25630.714 & $14206^{\circ}-39837_{3}$ & 3867.974 & 100 & 200 & 25846.001 & $18011^{\circ}-438575$ \\
\hline 3898.795 & 300 & 100 & 25641.686 & $154934-411344_{4}^{\circ}$ & 3867.069 & 40 & 10 & 25852.049 & $167833_{4}^{\circ}-426355$ \\
\hline 3898.509 & $300 \mathrm{~b}$ & 100 & 25643.567 & $11241_{3}^{\circ}-36885_{4}$ & 3866.907 & $500 \mathrm{~b}$ & 50 & 25853.132 & $11241_{3}^{\circ}-37094_{2}$ \\
\hline 3898.438 & $500 \mathrm{r}$ & 25 & 25644.034 & $2869_{3}-285132$ & 3866.771 & 200 & 2 & 25854.041 & $11802_{2}-37656_{3}^{\circ}$ \\
\hline 3897.778 & 200 & 5 & 25648.376 & $14032_{2}^{\circ}-39680_{2}$ & 3866.375 & 100 & 2 & 25856.689 & $15863_{2}-41720_{2}^{\circ}$ \\
\hline 3895.418 & $2000 \mathrm{r}$ & 200 & 25663.914 & $7795_{4}^{\circ}-33459_{4}$ & 3866.338 & 40 & 1 & 25856.937 & $8111_{4}-33967_{3}^{\circ}$ \\
\hline 3894.601 & 8 & & 25669.298 & $142045-39873{ }^{\circ}$ & 3864.970 & $200 \mathrm{~b}$ & $40 \mathrm{~b}$ & 25866.089 & $15490 \AA-413565$ \\
\hline 3893.817 & 200 & 8 & 25674.466 & $7280_{2}-32954_{2}^{\circ}$ & 3862.654 & $200 \mathrm{~b}$ & 200 & 25881.597 & $7280_{2}-33161_{i}^{\circ}$ \\
\hline 3893.651 & 300 & 40 & 25675.561 & $10414_{4}^{\circ}-36089_{4}$ & 3862.419 & $500 \mathrm{~b}$ & $250 \mathrm{~b}$ & 25883.172 & $10414_{4}^{\circ}-36297_{4}$ \\
\hline 3891.725 & 400 & 10 & 25688.267 & $11197{ }^{\circ}-36885_{4}$ & 3861.574 & $300 \mathrm{~b}$ & 40 & 25888.835 & $11197^{\circ}-37085_{4}$ \\
\hline 3891.657 & 75 & 1 & 25688.716 & $8111_{4}-33799_{4}^{\circ}$ & 3861.502 & $300 \mathrm{~b}$ & 40 & 25889.318 & $11241_{3}^{\circ}-37131_{3}$ \\
\hline 3891.520 & 300 & 3 & 25689.621 & $8111_{4}-33800_{3}^{\circ}$ & 3861.351 & 300 & 10 & 25890.331 & $8111_{4}-34001_{4}^{\circ}$ \\
\hline 3889.906 & 300 & 4 & 25700.279 & $13088_{3}-38788_{3}^{\circ}$ & 3861.051 & 200 & 2 & 25892.342 & $13945_{3}^{\circ}-39837_{3}$ \\
\hline 3889.715 & 100 & 2 & 25701.541 & $13297_{4}-38998^{\circ}$ & 3859.289 & 20 & & 25904.163 & $8800_{4}-34704_{3}^{\circ}$ \\
\hline 3889.609 & 150 & 3 & 25702.242 & $10783_{2}^{\circ}-36485_{2}$ & 3858.927 & $100 \mathrm{~b}$ & 10 & 25906.593 & $11877^{\circ}-37784_{2}$ \\
\hline 3889.542 & 200 & 8 & 25702.685 & $142045-39906^{\circ}$ & 3858.358 & 200 & 150 & 25910.414 & $13088_{3}-38998_{3}^{\circ}$ \\
\hline 3889.432 & 15 & & 25703.411 & $02-257032$ & 3856.622 & 300 & 15 & 25922.077 & $10414_{4}^{\circ}-363363$ \\
\hline 3887.220 & 40 & & 25718.037 & $6362_{2}-32080_{1}^{\circ}$ & 3856.516 & 300 & 50 & 25922.789 & $6362_{2}-32285_{3}^{\circ}$ \\
\hline 3887.019 & $800 \mathrm{r}$ & 100 & 25719.367 & $104144-361335$ & 3856.354 & $400 \mathrm{r}$ & 40 & 25923.878 & $8243 \stackrel{\circ}{-}-341673$ \\
\hline 3886.917 & $800 \mathrm{r}$ & 500 & 25720.042 & $2869_{3}-28589_{3}^{\circ}$ & 3856.222 & 100 & $8 \mathrm{~b}$ & 25924.766 & $7502_{3}-33427_{2}^{\circ}$ \\
\hline 3886.064 & 75 & 25 & 25725.688 & $13088_{3}-38814_{2}^{\circ}$ & 3853.985 & 100 & 8 & 25939.813 & $16346_{4}^{\circ}-42286_{3}$ \\
\hline 3885.224 & 200 & 5 & 25731.250 & $3687_{2}-29419_{2}^{\circ}$ & 3853.825 & $200 \mathrm{~b}$ & 15 & 25940.890 & $12847_{3}-38788_{3}^{\circ}$ \\
\hline 3885.066 & 200 & 10 & 25732.296 & $7795_{4}^{\circ}-33527_{4}$ & 3852.135 & $800 \mathrm{r}$ & 50 & 25952.270 & $3687_{2}-29640^{\circ}$ \\
\hline 3884.985 & 25 & & 25732.833 & $10783^{\circ}-36515_{3}$ & 3851.155 & 300 & 15 & 25958.874 & $10526_{3}^{\circ}-36485_{2}$ \\
\hline 3884.629 & 200 & 8 & 25735.191 & $13945_{3}^{\circ}-39680_{2}$ & 3851.072 & 300 & 20 & 25959.434 & $15618_{3}^{\circ}-41578_{2}$ \\
\hline 3884.629 & 200 & 8 & 25735.191 & $14465_{2}^{\circ}-40200_{3}$ & 3850.058 & 20 & & 25966.270 & $12847_{3}-38814_{2}^{\circ}$ \\
\hline 3883.767 & 200 & 15 & 25740.902 & $15490 \AA-412304$ & 3849.910 & 400 & 15 & 25967.269 & $13297_{4}-39264_{3}^{\circ}$ \\
\hline 3883.536 & 200 & 5 & 25742.433 & $15618_{3}^{\circ}-41361_{3}$ & 3847.617 & 500 & 10 & 25982.743 & $12114_{2}^{\circ}-38097_{2}$ \\
\hline 3882.324 & 200 & 8 & 25750.470 & $159703-41720_{2}^{\circ}$ & 3846.886 & $1000 \mathrm{r}$ & 100 & 25987.681 & $3865_{1}-29853_{2}^{\circ}$ \\
\hline 3880.404 & 50 & 2 & 25763.211 & $7280_{2}-330433^{\circ}$ & 3846.625 & 400 & 50 & 25989.444 & $10526_{3}^{\circ}-36515_{3}$ \\
\hline 3880.323 & 100 & 3 & 25763.748 & $13847_{2}-39611_{3}^{\circ}$ & 3846.024 & 15 & & 25993.505 & $14206_{4}^{\circ}-40200_{3}$ \\
\hline 3880.194 & 300 & 15 & 25764.605 & $132974-39062{ }^{\circ}$ & 3845.302 & 100 & 2 & 25998.386 & $3687_{2}-29686_{3}^{\circ}$ \\
\hline 3879.644 & $1000 \mathrm{r}$ & 200 & 25768.257 & $7502_{3}-33270_{4}^{\circ}$ & 3845.092 & 400 & 10 & 25999.805 & $13175_{4}^{\circ}-39174_{3}$ \\
\hline 3879.445 & $400 \mathrm{~b}$ & 10 & 25769.579 & $7795_{4}^{\circ}-335643$ & 3843.019 & 300 & 40 & 26013.830 & $132974-39311_{4}^{\circ}$ \\
\hline 3879.269 & 400 & 25 & 25770.748 & $10526^{\circ}-36297_{4}$ & 3842.898 & $400 \mathrm{r}$ & $75 b$ & 26014.649 & $11197 \stackrel{\circ}{-}-372115$ \\
\hline 3878.662 & 400 & 150 & 25774.781 & $38651-29640_{1}^{\circ}$ & 3842.803 & 200 & 5 & 26015.292 & $11601_{1}-37616_{1}^{\circ}$ \\
\hline 3878.160 & 100 & 3 & 25778.117 & $13175_{4}^{\circ}-389535$ & 3842.742 & 200 & 4 & 26015.705 & $2869_{3}-28884_{3}^{\circ}$ \\
\hline
\end{tabular}


TABLE 4. Classified lines of Th $\mathrm{I}$-continued

\begin{tabular}{|c|c|c|c|c|c|c|c|c|c|}
\hline \multirow{2}{*}{$\begin{array}{l}\text { Wavelength } \\
(\AA)\end{array}$} & \multicolumn{2}{|c|}{ Intensity } & \multirow{2}{*}{$\begin{array}{l}\text { Wavenumber } \\
\left(\mathrm{cm}^{-1}\right)\end{array}$} & \multirow{2}{*}{ Classification } & \multirow{2}{*}{$\begin{array}{c}\text { Wave length } \\
\text { (§) }\end{array}$} & \multicolumn{2}{|c|}{ Inten sity } & \multirow{2}{*}{$\begin{array}{l}\text { Wavenumber } \\
\qquad\left(\mathrm{cm}^{-1}\right)\end{array}$} & \multirow{2}{*}{ Classification } \\
\hline & Lamp & Spark & & & & Lamp & Spark & & \\
\hline 3842.549 & 400 & 40 & 26017.012 & $7280_{2}-33297_{2}^{\circ}$ & 3807.700 & 200 & $5 b$ & 26255.120 & $13945_{3}^{\circ}-40200_{3}$ \\
\hline 3840.918 & 25 & 10 & 26028.059 & $14465_{2}^{\circ}-404933$ & 3807.273 & 400 & 50 & 26258.065 & $98045-36062^{\circ}$ \\
\hline 3840.799 & $600 \mathrm{r}$ & 40 & 26028.866 & $4961_{4}-30990_{3}^{\circ}$ & 3806.317 & 100 & 1 & 26264.660 & $132974-39562^{\circ}$ \\
\hline 3840.430 & 300 & 250 & 26031.367 & $12114_{2}^{\circ}-38145_{3}$ & 3806.153 & 200 & 8 & 26265.791 & $15490 \AA-41755_{4}$ \\
\hline 3839.898 & 50 & 10 & 26034.973 & $10783_{2}^{\circ}-36818_{2}$ & 3805.227 & 200 & 10 & 26272.183 & $16351_{0}-42624^{\circ}$ \\
\hline 3839.695 & $800 \mathrm{r}$ & 200 & 26036.349 & $0_{2}-26036 \stackrel{\circ}{\circ}$ & 3804.127 & 400 & 20 & 26279.779 & $11241{ }_{3}^{\circ}-375213$ \\
\hline 3837.875 & $1500 \mathrm{r}$ & 200 & 26048.696 & $2869_{3}-28917_{2}^{\circ}$ & 3803.985 & $600 \mathrm{r}$ & 150 & 26280.761 & 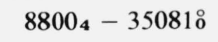 \\
\hline 3837.024 & 200 & 3 & 26054.473 & $8243_{2}^{\circ}-34298_{1}$ & 3803.839 & 400 & 25 & 26281.769 & $10526_{3}^{\circ}-368083$ \\
\hline 3836.721 & 300 & 20 & 26056.531 & $3687_{2}-29744^{\circ}$ & 3803.173 & $5 b$ & 3 & 26286.371 & $14206_{4}^{\circ}-404933$ \\
\hline 3836.584 & $800 \mathrm{r}$ & 100 & 26057.461 & $4961_{4}-31019 \stackrel{\circ}{\circ}$ & 3803.075 & $3000 \mathrm{r}$ & 200 & 26287.049 & $0_{2}-26287^{\circ}$ \\
\hline 3836.542 & $1000 \mathrm{r}$ & $150 \mathrm{~b}$ & 26057.747 & $75023-33560_{4}^{\circ}$ & 3802.864 & 500 & 10 & 26288.507 & $6362_{2}-32650_{1}^{\circ}$ \\
\hline 3835.960 & 75 & 8 & 26061.700 & $11241_{3}^{\circ}-37303_{4}$ & 3802.589 & 3 & & 26290.408 & $15736_{1}^{\circ}-42027_{2}$ \\
\hline 3835.711 & 200 & 5 & 26063.392 & $2869_{3}-28932{ }_{4}^{\circ}$ & 3802.420 & 400 & 25 & 26291.577 & $10526_{3}^{\circ}-36818_{2}$ \\
\hline 3835.414 & 200 & 10 & 26065.410 & $11241_{3}^{\circ}-37307_{3}$ & 3801.571 & $400 \mathrm{r}$ & 100 & 26297.448 & $75023-33799_{4}^{\circ}$ \\
\hline 3835.349 & 200 & 10 & 26065.852 & $14204_{5}-40270_{4}^{\circ}$ & 3801.443 & $800 \mathrm{r}$ & 100 & 26298.334 & $75023-338003$ \\
\hline 3835.077 & 100 & 2 & 26067.700 & $15618^{\circ}-416863$ & 3800.745 & 2 & & 26303.163 & $6362_{2}-32665^{\circ}$ \\
\hline 3834.890 & 100 & 5 & 26068.971 & $16217_{2}^{\circ}-422863$ & 3800.197 & 400 & 15 & 26306.956 & $5563_{1}-31870_{2}^{\circ}$ \\
\hline 3834.488 & 100 & 75 & 26071.704 & $8111_{4}-34182^{\circ}$ & 3799.166 & 75 & 1 & 26314.095 & $132974-39611^{\circ}$ \\
\hline 3832.323 & 75 & $3 b$ & 26086.433 & $8800_{4}-34886_{0}^{\circ}$ & 3798.432 & 200 & 75 & 26319.180 & $8111_{4}-344303$ \\
\hline 3831.962 & 100 & 2 & 26088.890 & $75023-33591_{3}^{\circ}$ & 3798.103 & $500 \mathrm{r}$ & 150 & 26321.460 & $49614-312833$ \\
\hline 3831.640 & 300 & 50 & 26091.083 & $25580-28649^{\circ}$ & 3797.205 & 200 & 10 & 26327.684 & $98045-36132 \circ$ \\
\hline 3830.774 & $800 \mathrm{r}$ & 150 & 26096.981 & $0_{2}-26096_{3}^{\circ}$ & 3796.997 & 200 & 10 & 26329.126 & $3687_{2}-30017^{\circ}$ \\
\hline 3830.198 & 50 & 2 & 26100.905 & $14481 \stackrel{\circ}{6}-405825$ & 3796.731 & $500 \mathrm{~b}$ & 20 & 26330.971 & $8800_{4}-35131_{4}^{\circ}$ \\
\hline 3830.061 & $500 \mathrm{r}$ & 50 & 26101.839 & $10414_{4}^{\circ}-36515_{3}$ & 3794.981 & 200 & 3 & 26343.113 & $3865_{1}-30208_{2}^{\circ}$ \\
\hline 3829.393 & $50 \mathrm{~b}$ & & 26106.392 & 111970 - 373034 & 3794.697 & $1500 \mathrm{r}$ & 100 & 26345.084 & $13175_{4}^{\circ}-39520_{4}$ \\
\hline 3829.283 & $150 \mathrm{~b}$ & $4 b$ & 26107.142 & $139453-400524$ & 3793.097 & 300 & 10 & 26356.197 & $17501 \stackrel{\circ}{\circ}-438575$ \\
\hline 3828.385 & $3000 \mathrm{r}$ & 300 & 26113.265 & $0_{2}-26113_{2}^{\circ}$ & 3792.730 & 4001 & 75 & 26358.747 & $10526^{\circ}-36885_{4}$ \\
\hline 3826.368 & $400 \mathrm{r}$ & 75 & 26127.030 & $10526_{3}^{\circ}-36653_{2}$ & 3792.371 & $1000 \mathrm{r}$ & 100 & 26361.242 & $10414_{4}^{\circ}-36775_{3}$ \\
\hline 3825.132 & $500 \mathrm{r}$ & 200 & 26135.472 & $11197 \stackrel{\circ}{-}-373326$ & 3792.104 & 150 & 3 & 26363.098 & $0_{2}-26363_{2}^{\circ}$ \\
\hline 3824.927 & 20 & 2 & 26136.873 & $15618{ }^{\circ}-41755_{4}$ & 3791.517 & 300 & 10 & 26367.180 & $10414_{4}^{\circ}-367815$ \\
\hline 3823.527 & 10 & & 26146.443 & $14465_{2}^{\circ}-40611_{2}$ & 3791.221 & 150 & 8 & 26369.238 & $11601_{1}-37970_{2}^{\circ}$ \\
\hline 3823.458 & 300 & 25 & 26146.915 & $7280_{2}-33427_{2}^{\circ}$ & 3790.794 & $1500 \mathrm{r}$ & 200 & 26372.209 & $7795_{4}^{\circ}-34167_{3}$ \\
\hline 3823.067 & $500 \mathrm{r}$ & 100 & 26149.589 & $5563_{1}-31712_{1}^{\circ}$ & 3790.646 & 100 & 5 & 26373.238 & $98045-36178{ }^{\circ}$ \\
\hline 3822.863 & 300 & 40 & 26150.984 & $12847_{3}-38998_{3}^{\circ}$ & 3790.269 & 300 & 10 & 26375.861 & $14206_{4}^{\circ}-405825$ \\
\hline 3822.709 & 300 & 25 & 26152.038 & $11802_{2}-379543_{3}^{\circ}$ & 3789.659 & 50 & 1 & 26380.107 & $13088_{3}-39468^{\circ}$ \\
\hline 3821.119 & 15 & & 26162.920 & $11241_{3}^{\circ}-374043$ & 3789.529 & 75 & 2 & 26381.012 & $8111_{4}-34492^{\circ}$ \\
\hline 3820.792 & $600 \mathrm{r}$ & 75 & 26165.159 & $3687_{2}-29853_{2}^{\circ}$ & 3789.372 & 300 & 10 & 26382.105 & $7280_{2}-33662_{i}^{\circ}$ \\
\hline 3820.474 & $300 \mathrm{~b}$ & 10 & 26167.337 & $11802_{2}-37970_{2}^{\circ}$ & 3789.167 & $500 \mathrm{r}$ & 100 & 26383.532 & $2869_{3}-29252_{2}^{\circ}$ \\
\hline 3820.327 & 200 & 2 & 26168.343 & $14032_{2}^{\circ}-40200_{3}$ & 3787.934 & 300 & 3 & 26392.120 & $63622-32754 \stackrel{\circ}{\circ}$ \\
\hline 3819.904 & 500 & $100 \mathrm{~b}$ & 26171.241 & $132974-39468{ }^{\circ}$ & 3787.638 & 300 & 40 & 26394.182 & $10414_{4}^{\circ}-368083$ \\
\hline 3819.189 & 200 & 10 & 26176.141 & $13088_{3}-39264_{3}^{\circ}$ & 3785.771 & 100 & 2 & 26407.199 & $13847_{2}-40254^{\circ}$ \\
\hline 3819.112 & 300 & 10 & 26176.668 & $13175_{4}^{\circ}-393515$ & 3785.154 & 200 & 2 & 26411.503 & $15166_{3}^{\circ}-41578_{2}$ \\
\hline 3818.685 & $400 \mathrm{r}$ & 15 & 26179.595 & $4961_{4}-311418$ & 3784.793 & 300 & 15 & 26414.022 & $11802_{2}-38216^{\circ}$ \\
\hline 3817.495 & 100 & & 26187.756 & $8243_{2}^{\circ}-344313$ & 3784.574 & 400 & 20 & 26415.551 & $38651-30281_{1}^{\circ}$ \\
\hline 3816.510 & 15 & 1 & 26194.514 & $15166_{3}^{\circ}-41361_{3}$ & 3784.574 & 400 & 20 & 26415.551 & $12114_{2}^{\circ}-38529_{3}$ \\
\hline 3815.566 & 500 & 50 & 26200.995 & $8243_{2}^{\circ}-344442$ & 3784.406 & 100 & 3 & 26416.723 & $128477_{3}-39264_{3}^{\circ}$ \\
\hline 3813.815 & $500 \mathrm{r}$ & 50 & 26213.024 & $6362_{2}-32575_{2}^{\circ}$ & 3783.680 & $50 \mathrm{~b}$ & & 26421.792 & $14465_{2}^{\circ}-40886_{2}$ \\
\hline 3812.905 & 75 & 2 & 26219.280 & $11877_{1}^{\circ}-38097_{2}$ & 3781.638 & 10 & & 26436.059 & 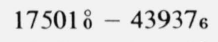 \\
\hline 3812.827 & 15 & & 26219.816 & $14206_{4}^{\circ}-404264$ & 3781.318 & 300 & 75 & 26438.296 & $7280_{2}-33718_{2}^{\circ}$ \\
\hline 3812.667 & 75 & 5 & 26220.917 & $142045-40425^{\circ}$ & 3780.966 & $300 \mathrm{r}$ & $20 b$ & 26440.757 & $2869_{3}-29310_{4}^{\circ}$ \\
\hline 3812.398 & 400 & 40 & 26222.767 & $15490 \AA-417125$ & 3780.147 & 200 & 8 & 26446.486 & $11241 \stackrel{\circ}{3}-37688_{3}$ \\
\hline 3810.995 & $600 \mathrm{r}$ & 150 & 26232.420 & $75023-33734_{2}^{\circ}$ & 3778.987 & 200 & 3 & 26454.603 & $7280_{2}-33734_{2}^{\circ}$ \\
\hline 3810.906 & 75 & 1 & 26233.033 & $4961_{4}-31194_{4}^{\circ}$ & 3778.045 & 300 & 15 & 26461.199 & $14032_{2}^{\circ}-404933$ \\
\hline 3808.614 & 500 & 20 & 26248.819 & $10526^{\circ}-367753$ & 3777.745 & 200 & 40 & 26463.301 & $128477_{3}-39311_{4}^{\circ}$ \\
\hline 3808.430 & 15 & & 26250.088 & $11802_{2}-380533^{\circ}$ & 3777.626 & $50 \mathrm{~b}$ & & 26464.134 & $8243_{2}^{\circ}-34707_{3}$ \\
\hline
\end{tabular}


TABle 4. Classified lines of Th $\mathrm{I}$-continued

\begin{tabular}{|c|c|c|c|c|c|c|c|c|c|}
\hline \multirow{2}{*}{$\begin{array}{l}\text { Wavelength } \\
(\AA)\end{array}$} & \multicolumn{2}{|c|}{ Intensity } & \multirow{2}{*}{$\begin{array}{l}\text { Wavenumber } \\
\left(\mathrm{cm}^{-1}\right)\end{array}$} & \multirow{2}{*}{ Classification } & \multirow{2}{*}{$\begin{array}{c}\text { Wavelength } \\
\text { (Å) }\end{array}$} & \multicolumn{2}{|c|}{ Intensity } & \multirow{2}{*}{$\begin{array}{l}\text { Wavenumber } \\
\qquad\left(\mathrm{cm}^{-1}\right)\end{array}$} & \multirow{2}{*}{ Classification } \\
\hline & Lamp & Spark & & & & Lamp & Spark & & \\
\hline 3777.415 & 300 & 40 & 26465.612 & $7502_{3}-33967_{3}^{\circ}$ & 3748.820 & 100 & 8 & 26667.480 & $15618^{\circ}-42286_{3}$ \\
\hline 3776.732 & 10 & & 26470.399 & $98045-36275^{\circ}$ & 3748.225 & $20 \mathrm{~b}$ & 5 & 26671.713 & $10414_{4}^{\circ}-37085_{4}$ \\
\hline 3776.622 & 300 & $40 \mathrm{~b}$ & 26471.169 & $10414_{4}^{\circ}-36885_{4}$ & 3746.929 & 4 & & 26680.938 & $6362_{2}-33043_{3}^{\circ}$ \\
\hline 3776.502 & 300 & 8 & 26472.010 & $8111_{4}-34583_{3}^{\circ}$ & 3745.176 & 4001 & 20 & 26693.427 & $11197^{\circ}-378905$ \\
\hline 3776.271 & $600 \mathrm{r}$ & 150 & 26473.630 & 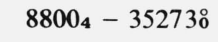 & 3744.847 & 100 & 5 & 26695.772 & $11197^{\circ}-378924$ \\
\hline 3775.937 & 15 & & 26475.971 & $118022-38278^{\circ}$ & 3742.923 & $1000 \mathrm{r}$ & 100 & 26709.494 & $4961_{4}-31671_{4}^{\circ}$ \\
\hline 3775.157 & 150 & 15 & 26481.442 & $13945_{3}^{\circ}-404264$ & 3742.275 & 200 & 5 & 26714.119 & $12847_{3}-39562^{\circ}$ \\
\hline 3774.223 & $50 \mathrm{~b}$ & & 26487.995 & $11601_{1}-38088_{2}^{\circ}$ & 3741.884 & 200 & 8 & 26716.910 & $10414_{4}^{\circ}-37131_{3}$ \\
\hline 3774.133 & 100 & 8 & 26488.627 & $12114_{2}^{\circ}-38602_{2}$ & 3740.731 & 40 & 2 & 26725.145 & $11877_{1}^{\circ}-38602_{2}$ \\
\hline 3773.306 & 200 & 8 & 26494.432 & $14206^{\circ}-40701_{4}$ & 3737.514 & $600 \mathrm{r}$ & 75 & 26748.147 & $38651-30613 \AA$ \\
\hline 3772.649 & 400 & 10 & 26499.046 & $7502_{3}-34001_{4}^{\circ}$ & 3736.655 & 100 & 5 & 26754.296 & $11601_{1}-38355_{2}^{\circ}$ \\
\hline 3771.629 & $100 \mathrm{~b}$ & 5 & 26506.212 & $7795_{4}^{\circ}-34301_{4}$ & 3736.566 & 50 & 1 & 26754.933 & $4961_{4}-31716^{\circ}$ \\
\hline 3771.616 & $300 \mathrm{~b}$ & 10 & 26506.303 & $11241_{3}^{\circ}-377482$ & 3736.411 & 5 & & 26756.043 & $13945_{3}^{\circ}-40701_{4}$ \\
\hline 3771.370 & $1500 \mathrm{r}$ & 100 & 26508.032 & $0_{2}-265083$ & 3735.827 & $50 \mathrm{~b}$ & 2 & 26760.226 & $15863_{2}-42624^{\circ}$ \\
\hline 3770.056 & $1500 \mathrm{r}$ & 200 & 26517.271 & $5563_{1}-32080^{\circ}$ & 3735.363 & 100 & 3 & 26763.550 & $128473-39611_{3}^{\circ}$ \\
\hline 3769.778 & 50 & 1 & 26519.226 & $13175_{4}^{\circ}-396945$ & 3733.672 & $300 \mathrm{r}$ & 25 & 26775.671 & $8111_{4}-348860$ \\
\hline 3769.697 & 75 & 10 & 26519.796 & $15166^{\circ}-416863$ & 3733.501 & 10 & 1 & 26776.897 & $10526^{\circ}-373034$ \\
\hline 3769.615 & $200 \mathrm{~b}$ & 40 & 26520.373 & $142045-40724 \stackrel{\circ}{ }$ & 3732.985 & $400 \mathrm{r}$ & 40 & 26780.599 & $10526_{3}^{\circ}-37307_{3}$ \\
\hline 3769.592 & $400 \mathrm{~b}$ & 50 & 26520.535 & $7280_{2}-33800_{3}^{\circ}$ & 3730.946 & 50 & 3 & 26795.234 & $11197^{\circ}-379924$ \\
\hline 3769.101 & 75 & 1 & 26523.990 & $10783_{2}^{\circ}-373073$ & 3730.623 & 75 & 4 & 26797.554 & $10414_{4}^{\circ}-372115$ \\
\hline 3766.447 & 300 & 10 & 26542.679 & $11241_{3}^{\circ}-377842$ & 3730.368 & $800 \mathrm{r}$ & 2001 & 26799.386 & $63622-33161_{i}^{\circ}$ \\
\hline 3766.083 & $300 \mathrm{~b}$ & 50 & 26545.244 & $11197{ }^{\circ}-377426$ & 3729.945 & 400 & 40 & 26802.425 & $8243_{2}^{\circ}-350461$ \\
\hline 3765.696 & 200 & 4 & 26547.972 & $13945_{3}^{\circ}-40493_{3}$ & 3727.903 & $1000 \mathrm{r}$ & 150 & 26817.106 & $2869_{3}-29686_{3}^{\circ}$ \\
\hline 3765.412 & $400 \mathrm{r}$ & 50 & 26549.975 & $2869_{3}-29419_{2}^{\circ}$ & 3727.725 & 75 & 2 & 26818.386 & $13088_{3}-39906_{4}^{\circ}$ \\
\hline 3765.241 & $800 \mathrm{r}$ & 200 & 26551.181 & $8800_{4}-35351^{\circ}$ & 3727.612 & $400 \mathrm{r}$ & 40 & 26819.199 & $4961_{4}-31780_{3}^{\circ}$ \\
\hline 3765.067 & 150 & 2 & 26552.408 & $118022-38355_{2}^{\circ}$ & 3727.346 & 300 & 40 & 26821.113 & $98045-36625 \circ$ \\
\hline 3764.089 & 4 & & 26559.306 & $10526_{3}^{\circ}-370854$ & 3727.251 & 300 & 40 & 26821.797 & $75023-34324^{\circ}$ \\
\hline 3763.668 & 300 & 10 & 26562.277 & $4961_{4}-315233^{\circ}$ & 3725.966 & 75 & 2 & 26831.047 & $7280_{2}-34111^{\circ}$ \\
\hline 3762.934 & $500 \mathrm{r}$ & 100 & 26567.458 & $3687_{2}-30255_{3}^{\circ}$ & 3724.397 & 100 & 2 & 26842.350 & $75023-34344_{4}^{\circ}$ \\
\hline 3762.815 & $300 \mathrm{~b}$ & 25 & 26568.298 & $10526^{\circ}-37094_{2}$ & 3723.921 & $300 \mathrm{~s}$ & 8 & 26845.781 & $8243_{2}^{\circ}-35089_{3}$ \\
\hline 3762.257 & 200 & 8 & 26572.239 & $142045-40776_{4}^{\circ}$ & 3722.655 & 75 & 2 & 26854.910 & $14032_{2}^{\circ}-40886_{2}$ \\
\hline 3761.704 & 200 & 20 & 26576.145 & $132974-39873 \circ$ & 3722.591 & $100 \mathrm{~b}$ & 5 & 26855.372 & $11241_{3}^{\circ}-38097_{2}$ \\
\hline 3761.527 & 200 & 20 & 26577.396 & $11241_{3}^{\circ}-378194$ & 3722.179 & $300 \mathrm{~b}$ & $25 b$ & 26858.344 & $38651-30723 i$ \\
\hline 3761.470 & 200 & 25 & 26577.798 & $98045-36382_{4}^{\circ}$ & 3721.881 & $10 \mathrm{~b}$ & & 26860.495 & $15166_{3}^{\circ}-42027_{2}$ \\
\hline 3761.217 & 200 & 8 & 26579.586 & $14032_{2}^{\circ}-40611_{2}$ & 3721.219 & $500 \mathrm{r}$ & 50 & 26865.273 & $3687_{2}-305532$ \\
\hline 3759.886 & 50 & & 26588.995 & $15166_{3}^{\circ}-41755_{4}$ & 3721.003 & 100 & 10 & 26866.833 & $11802_{2}-38669_{2}^{\circ}$ \\
\hline 3759.433 & $500 \mathrm{r}$ & 75 & 26592.199 & $6362_{2}-32954_{2}^{\circ}$ & 3720.628 & 20 & 15 & 26869.540 & $75023-34371_{2}^{\circ}$ \\
\hline 3759.314 & $200 \mathrm{r}$ & 100 & 26593.041 & $3687_{2}-30281_{1}^{\circ}$ & 3719.837 & $400 \mathrm{r}$ & 40 & 26875.254 & $2869_{3}-29744_{3}^{\circ}$ \\
\hline 3759.262 & $300 \mathrm{r}$ & 100 & 26593.408 & $8111_{4}-34704_{3}^{\circ}$ & 3719.546 & 300 & 15 & 26877.356 & $13175_{4}^{\circ}-400524$ \\
\hline 3758.706 & 400 & 20 & 26597.342 & $5563_{1}-32160_{2}^{\circ}$ & 3719.435 & 3000 & 200 & 26878.158 & $0_{2}-26878_{3}^{\circ}$ \\
\hline 3758.467 & $400 \mathrm{r}$ & 25 & 26599.033 & $25580-29157^{\circ}$ & 3717.895 & 50 & 2 & 26889.291 & $10414_{4}^{\circ}-37303_{4}$ \\
\hline 3757.694 & $1000 \mathrm{r}$ & 200 & 26604.505 & $10526^{\circ}-37131_{3}$ & 3717.384 & 100 & 3 & 26892.988 & $10414_{4}^{\circ}-37307_{3}$ \\
\hline 3756.451 & 200 & 8 & 26613.308 & $11802_{2}-38416_{3}^{\circ}$ & 3716.992 & $100 \mathrm{~b}$ & 4 & 26895.824 & $12114_{2}^{\circ}-39010_{3}$ \\
\hline 3756.293 & 400 & 25 & 26614.428 & $8111_{4}-34725_{3}^{\circ}$ & 3716.942 & 100 & 2 & 26896.186 & $14465_{2}^{\circ}-41361_{3}$ \\
\hline 3755.498 & $25 \mathrm{~s}$ & 3 & 26620.061 & $12114_{2}^{\circ}-38734_{3}$ & 3716.808 & 100 & 2 & 26897.155 & $13088_{3}-39985_{3}^{\circ}$ \\
\hline 3755.211 & $800 \mathrm{r}$ & 250 & 26622.096 & $11197^{\circ}-37819_{4}$ & 3715.862 & 300 & 20 & 26904.003 & $11241_{3}^{\circ}-38145_{3}$ \\
\hline 3754.030 & $500 \mathrm{r}$ & 50 & 26630.471 & $8243_{2}^{\circ}-348743$ & 3715.561 & 400 & 50 & 26906.182 & $8243_{2}^{\circ}-35149_{2}$ \\
\hline 3753.241 & 300 & 40 & 26636.069 & $7795_{4}^{\circ}-344313$ & 3715.060 & 100 & 2 & 26909.810 & $11197^{\circ}-381065$ \\
\hline 3752.789 & 300 & 8 & 26639.277 & $25580-29197^{\circ}$ & 3714.696 & 100 & 3 & 26912.447 & $7795_{4}^{\circ}-34707_{3}$ \\
\hline 3752.265 & 10 & 1 & 26642.997 & $14243 i-408862$ & 3712.299 & 40 & 2 & 26929.824 & $11877_{1}^{\circ}-38807_{2}$ \\
\hline 3751.121 & 300 & 25 & 26651.122 & $11241_{3}^{\circ}-37892_{4}$ & 3712.187 & 25 & 8 & 26930.636 & $14204_{5}-41134_{4}^{\circ}$ \\
\hline 3751.021 & $300 \mathrm{r}$ & 10 & 26651.833 & $0_{2}-26651 \stackrel{\circ}{2}$ & 3711.926 & 200 & 5 & 26932.530 & $63622-33294_{3}^{\circ}$ \\
\hline 3749.617 & 300 & 20 & 26661.812 & $8800_{4}-35462_{3}^{\circ}$ & 3711.833 & 200 & 15 & 26933.205 & $8800_{4}-35733^{\circ}$ \\
\hline 3749.514 & 300 & 10 & 26662.544 & $13175_{4}^{\circ}-39837_{3}$ & 3711.623 & $600 \mathrm{r}$ & 50 & 26934.729 & $63622-33297_{2}^{\circ}$ \\
\hline 3749.083 & 200 & 25 & 26665.609 & $7795^{\circ}-344605$ & 3711.357 & 75 & $4 b$ & 26936.659 & $17501 \stackrel{\circ}{-4} 444376$ \\
\hline
\end{tabular}


TABLE 4. Classified lines of Th $\mathrm{I}$-continued

\begin{tabular}{|c|c|c|c|c|c|c|c|c|c|}
\hline \multirow{2}{*}{$\begin{array}{l}\text { Wavelength } \\
\text { (A) }\end{array}$} & \multicolumn{2}{|c|}{ Intensity } & \multirow{2}{*}{$\begin{array}{l}\text { Wavenumber } \\
\qquad\left(\mathrm{cm}^{-1}\right)\end{array}$} & \multirow{2}{*}{ Classification } & \multirow{2}{*}{$\begin{array}{c}\text { Wavelength } \\
\text { (̊) }\end{array}$} & \multicolumn{2}{|c|}{ Inten sity } & \multirow{2}{*}{$\begin{array}{l}\text { Wavenumber } \\
\qquad\left(\mathrm{cm}^{-1}\right)\end{array}$} & \multirow{2}{*}{ Classification } \\
\hline & Lamp & Spark & & & & Lamp & Spark & & \\
\hline 3710.830 & 100 & 1 & 26940.484 & $5563_{1}-32503_{2}^{\circ}$ & 3679.970 & 75 & 2 & 27166.400 & $13088_{3}-40254_{3}^{\circ}$ \\
\hline 3710.665 & 1001 & 2 & 26941.682 & $13945_{3}^{\circ}-40886_{2}$ & 3679.544 & $400 \mathrm{r}$ & 150 & 27169.545 & $11197^{\circ}-38366_{4}$ \\
\hline 3710.292 & 300 & 8 & 26944.391 & $112413^{\circ}-381864$ & 3678.478 & $400 \mathrm{r}$ & $50 \mathrm{~b}$ & 27177.418 & $8243_{2}^{\circ}-35421_{1}$ \\
\hline 3709.861 & 300 & 25 & 26947.521 & $3865_{1}-30812_{2}^{\circ}$ & 3675.960 & 400 & 50 & 27196.034 & $11802_{2}-38998^{\circ}$ \\
\hline 3708.483 & 75 & 2 & 26957.534 & $13297_{4}-40254 \stackrel{9}{3}$ & 3675.790 & $400 \mathrm{r}$ & 50 & 27197.292 & $8243_{2}^{\circ}-354403$ \\
\hline 3707.465 & 200 & 8 & 26964.936 & $10783_{2}^{\circ}-37748_{2}$ & 3675.137 & $500 \mathrm{r}$ & 50 & 27202.124 & $75023-34704_{3}^{\circ}$ \\
\hline 3706.767 & $2000 \mathrm{r}$ & 300 & 26970.013 & $8111_{4}-350818$ & 3674.891 & $300 \mathrm{r}$ & 50 & 27203.945 & $98045-37008_{0}^{\circ}$ \\
\hline 3706.402 & 200 & 75 & 26972.669 & $13297_{4}-40270_{4}^{\circ}$ & 3674.420 & 50 & 5 & 27207.432 & $12114_{2}^{\circ}-39321_{3}$ \\
\hline 3704.862 & $500 \mathrm{r}$ & 50 & 26983.880 & $2869_{3}-29853_{2}^{\circ}$ & 3674.013 & $300 \mathrm{r}$ & $50 \mathrm{~b}$ & 27210.446 & $3865_{1}-31075_{2}^{\circ}$ \\
\hline 3704.584 & 100 & 5 & 26985.905 & $11802_{2}-38788_{3}^{\circ}$ & 3673.639 & 50 & 1 & 27213.216 & $11601_{1}-38814_{2}^{\circ}$ \\
\hline 3704.147 & 200 & 50 & 26989.089 & $111970-381864$ & 3672.522 & 500 & 25 & 27221.493 & $10526^{\circ}-37748_{2}$ \\
\hline 3703.951 & $75 b$ & & 26990.517 & $10414_{4}^{\circ}-374043$ & 3672.522 & 500 & 25 & 27221.493 & $14465_{2}^{\circ}-41686_{3}$ \\
\hline 3703.775 & $500 \mathrm{r}$ & 50 & 26991.800 & $4961_{4}-319534$ & 3672.300 & $500 \mathrm{r}$ & 50 & 27223.138 & $75023-34725_{3}^{\circ}$ \\
\hline 3703.342 & 200 & 2 & 26994.955 & $10526^{\circ}-375213$ & 3671.540 & $600 \mathrm{r}$ & 150 & 27228.773 & $63622_{2}-33591 \stackrel{\circ}{3}$ \\
\hline 3703.230 & $400 \mathrm{r}$ & 25 & 26995.772 & $0_{2}-26995_{3}^{\circ}$ & 3671.242 & 75 & 2 & 27230.983 & $14481 \%-417125$ \\
\hline 3702.479 & 100 & 2 & 27001.247 & $10783_{2}^{\circ}-37784_{2}$ & 3670.520 & 200 & 4 & 27236.340 & $98045-37041_{4}^{\circ}$ \\
\hline 3701.594 & $150 \mathrm{~b}$ & 3 & 27007.703 & $13088_{3}-40096_{2}^{\circ}$ & 3669.968 & $2000 \mathrm{r}$ & 100 & 27240.436 & $8111_{4}-35351_{4}^{\circ}$ \\
\hline 3701.099 & 200 & 2 & 27011.315 & $11802_{2}-38814_{2}^{\circ}$ & 3668.914 & 100 & 4 & 27248.261 & $12847_{3}-40096_{2}^{\circ}$ \\
\hline 3700.978 & $500 \mathrm{r}$ & 100 & 27012.198 & $2869_{3}-29881_{4}^{\circ}$ & 3667.621 & 500 & 40 & 27257.867 & $10526^{\circ}-37784_{2}$ \\
\hline 3699.881 & $500 \mathrm{r}$ & 50 & 27020.207 & $8111_{4}-35131_{4}^{\circ}$ & 3667.311 & 5 & 1 & 27260.171 & $0_{2}-27260_{3}^{\circ}$ \\
\hline 3699.355 & 300 & 5 & 27024.048 & $14206_{4}^{\circ}-41230_{4}$ & 3666.981 & $800 \mathrm{r}$ & 75 & 27262.625 & $8800_{4}-36062_{4}^{\circ}$ \\
\hline 3699.182 & 500 & 75 & 27025.312 & $13175_{4}^{\circ}-40200_{3}$ & 3665.476 & $100 \mathrm{~b}$ & $10 \mathrm{~b}$ & 27273.818 & $8800_{4}-36074_{3}^{\circ}$ \\
\hline 3698.106 & $1000 \mathrm{r}$ & 150 & 27033.176 & $98045-36837 \circ$ & 3665.443 & $150 \mathrm{~b}$ & 500 & 27274.064 & $10414_{4}^{\circ}-376883$ \\
\hline 3697.743 & 300 & 10 & 27035.829 & $3687_{2}-30723 i$ & 3663.543 & 300 & 15 & 27288.208 & $11241^{\circ}-38529_{3}$ \\
\hline 3697.497 & 300 & 3 & 27037.628 & $11802_{2}-38840^{\circ}$ & 3663.201 & $1500 \mathrm{r}$ & 100 & 27290.756 & $2869_{3}-30160_{4}^{\circ}$ \\
\hline 3695.288 & $500 \mathrm{r}$ & 75 & 27053.790 & $7795_{4}^{\circ}-348495$ & 3662.955 & 150 & 15 & 27292.589 & $10526_{3}^{\circ}-37819_{4}$ \\
\hline 3694.579 & 50 & 2 & 27058.982 & $12847_{3}-39906_{4}^{\circ}$ & 3662.750 & $300 \mathrm{r}$ & 20 & 27294.116 & $7795_{4}^{\circ}-35089_{3}$ \\
\hline 3694.365 & $300 \mathrm{r}$ & 50 & 27060.549 & $12114_{2}^{\circ}-391743$ & 3662.283 & 100 & 3 & 27297.596 & $0_{2}-27297^{\circ}$ \\
\hline 3694.246 & $300 \mathrm{r}$ & 10 & 27061.421 & $0_{2}-27061_{2}^{\circ}$ & 3661.982 & 150 & 15 & 27299.840 & $6362_{2}-33662_{1}^{\circ}$ \\
\hline 3694.177 & $400 \mathrm{r}$ & 75 & 27061.926 & $49614-320238$ & 3661.622 & $500 \mathrm{r}$ & 75 & 27302.524 & $3687_{2}-30990_{3}^{\circ}$ \\
\hline 3693.993 & $400 \mathrm{r}$ & 200 & 27063.274 & $3865_{1}-30928^{\circ}$ & 3661.575 & $500 \mathrm{r}$ & 200 & 27302.874 & $7280_{2}-34583^{\circ}$ \\
\hline 3693.804 & $200 \mathrm{~b}$ & 25 & 27064.659 & $6362_{2}-33427_{2}^{\circ}$ & 3660.500 & 400 & 25 & 27310.892 & $7280_{2}-34591_{i}^{\circ}$ \\
\hline 3693.247 & 200 & 10 & 27068.741 & $11601_{1}-38669_{2}^{\circ}$ & 3660.091 & $200 \mathrm{~b}$ & 4 & 27313.944 & $10783_{2}^{\circ}-38097_{2}$ \\
\hline 3692.566 & $1000 \mathrm{r}$ & 100 & 27073.733 & $3687_{2}-30761_{3}^{\circ}$ & 3659.629 & $600 \mathrm{r}$ & 150 & 27317.392 & $0_{2}-27317^{\circ}$ \\
\hline 3691.972 & $300 \mathrm{~b}$ & 5 & 27078.088 & $7795_{4}^{\circ}-348734$ & 3658.808 & $400 \mathrm{r}$ & 25 & 27323.522 & $4961_{4}-32285_{3}^{\circ}$ \\
\hline 3691.876 & $400 \mathrm{r}$ & 100 & 27078.792 & $7795^{\circ}-348743$ & 3657.641 & $200 \mathrm{~s}$ & 10 & 27332.240 & $8800_{4}-36132 \circ$ \\
\hline 3691.613 & $500 \mathrm{r}$ & 200 & 27080.722 & $75023-34583 \stackrel{\circ}{3}$ & 3657.054 & 300 & 20 & 27336.627 & $13088_{3}-40425_{4}^{\circ}$ \\
\hline 3691.411 & $500 \mathrm{r}$ & 50 & 27082.203 & $25580-29640 \mathrm{i}$ & 3656.693 & $500 \mathrm{r}$ & 50 & 27339.325 & $2869_{3}-30208_{2}^{\circ}$ \\
\hline 3690.649 & $100 \mathrm{~b}$ & $8 b$ & 27087.795 & $5563_{1}-32650_{1}^{\circ}$ & 3655.125 & 300 & 50 & 27351.053 & $8111_{4}-35462_{3}^{\circ}$ \\
\hline 3690.624 & $600 \mathrm{r}$ & 4 & 27087.978 & $0_{2}-27087^{\circ}$ & 3654.915 & 3 & & 27352.624 & $13847_{2}-41200_{2}^{\circ}$ \\
\hline 3690.115 & 200 & 300 & 27091.715 & $7280_{2}-343712$ & 3654.461 & $500 \mathrm{r}$ & 50 & 27356.023 & $6362_{2}-33718_{2}^{\circ}$ \\
\hline 3688.658 & 300 & 20 & 27102.416 & $5563_{1}-32665_{1}^{\circ}$ & 3653.586 & 40 & & 27362.574 & $10783_{2}^{\circ}-38145_{3}$ \\
\hline 3687.983 & $400 \mathrm{r}$ & 75 & 27107.376 & $10414^{\circ}-375213$ & 3653.090 & 100 & 2 & 27366.289 & $10526_{3}^{\circ}-37892_{4}$ \\
\hline 3687.193 & 4001 & 20 & 27113.184 & $14465_{2}^{\circ}-415782$ & 365.9284 & 20 & 1 & 27372.328 & $6362_{2}-33734_{2}^{\circ}$ \\
\hline 3686.326 & 2 & & 27119.560 & $15166^{\circ}-422863$ & 3650.436 & 200 & 50 & 27386.185 & $2869_{3}-30255_{3}^{\circ}$ \\
\hline 3685.586 & $300 \mathrm{~b}$ & 101 & 27125.005 & $3687_{2}-30812_{2}^{\circ}$ & 3650.204 & 3001 & 2 & 27387.925 & $3687_{2}-31075_{2}^{\circ}$ \\
\hline 3685.214 & 200 & 4 & 27127.743 & $13297_{4}-40425_{4}^{\circ}$ & 3649.735 & $1000 \mathrm{r}$ & 100 & 27391.445 & $5563_{1}-32954_{2}^{\circ}$ \\
\hline 3684.932 & 300 & 20 & 27129.819 & $8111_{4}-35240_{3}^{\circ}$ & 3647.932 & 300 & 50 & 27404.982 & $10414_{4}^{\circ}-37819_{4}$ \\
\hline 3683.856 & 20 & & 27137.743 & $12847_{3}-39985_{3}^{\circ}$ & 3647.662 & 25 & & 27407.011 & $12847_{3}-40254_{3}^{\circ}$ \\
\hline 3682.759 & $10 b$ & & 27145.827 & $15490 \circ-426355$ & 3647.575 & 300 & 15 & 27407.665 & $13175_{4}^{\circ}-405825$ \\
\hline 3682.486 & $800 \mathrm{r}$ & 50 & 27147.839 & $2869_{3}-30017_{3}^{\circ}$ & 3646.452 & 15 & & 27416.105 & $139453^{\circ}-41361_{3}$ \\
\hline 3682.295 & 300 & 20 & 27149.247 & $14206_{4}^{\circ}-413565$ & 3645.706 & 300 & 15 & 27421.715 & $7795_{4}^{\circ}-352165$ \\
\hline 3682.179 & 300 & 50 & 27150.103 & $7280_{2}-344303$ & 3645.648 & 200 & 8 & 27422.151 & $12847_{3}-40270_{4}^{\circ}$ \\
\hline 3680.605 & $400 \mathrm{r}$ & 40 & 27161.713 & $8243_{2}^{\circ}-35405_{3}$ & 3645.365 & $200 \mathrm{~b}$ & $25 b$ & 27424.280 & $7280_{2}-34704_{3}^{\circ}$ \\
\hline 3680.448 & $500 \mathrm{r}$ & 50 & 27162.871 & $8111_{4}-352738$ & 3644.981 & 200 & 3 & 27427.169 & $132974-407240^{\circ}$ \\
\hline
\end{tabular}


TABLE 4. Classified lines of Th I-continued

\begin{tabular}{|c|c|c|c|c|c|c|c|c|c|}
\hline \multirow{2}{*}{$\begin{array}{c}\text { Wavelength } \\
\text { (Å) }\end{array}$} & \multicolumn{2}{|c|}{ Intensity } & \multirow{2}{*}{$\begin{array}{l}\text { Wavenumber } \\
\left(\mathrm{cm}^{-1}\right)\end{array}$} & \multirow{2}{*}{ Classification } & \multirow{2}{*}{$\begin{array}{c}\text { Wavelength } \\
\text { (§) }\end{array}$} & \multicolumn{2}{|c|}{ Inten sity } & \multirow{2}{*}{$\begin{array}{l}\text { Wavenumber } \\
\left(\mathrm{cm}^{-1}\right)\end{array}$} & \multirow{2}{*}{ Classification } \\
\hline & Lamp & Spark & & & & Lamp & Spark & & \\
\hline 3643.512 & $600 \mathrm{r}$ & 50 & 27438.227 & $6362_{2}-33800_{3}^{\circ}$ & 3604.965 & 20 & & 27731.609 & $10414_{4}^{\circ}-38145_{3}$ \\
\hline 3642.573 & $500 \mathrm{r}$ & 50 & 27445.300 & $7280_{2}-34725_{3}^{\circ}$ & 3604.680 & $600 \mathrm{r}$ & 40 & 27733.802 & $3865_{1}-31599_{2}^{\circ}$ \\
\hline 3642.249 & $2000 \mathrm{r}$ & 100 & 27447.741 & $0_{2}-27447_{2}^{\circ}$ & 3604.657 & 100 & & 27733.979 & $5563_{1}-33297_{2}^{\circ}$ \\
\hline 3640.820 & 10 & & 27458.514 & $11241_{3}^{\circ}-38700_{4}$ & 3604.064 & $200 b$ & & 27738.542 & $7502_{3}-35240_{3}^{\circ}$ \\
\hline 3640.390 & 100 & 3 & 27461.757 & $11802_{2}-39264_{3}^{\circ}$ & 3603.694 & 150 & 4 & 27741.390 & $13945_{3}^{\circ}-41686_{3}$ \\
\hline 3639.867 & 4001 & 40 & 27465.703 & $10526_{3}^{\circ}-37992_{4}$ & 3601.770 & 200 & 10 & 27756.208 & $11197_{0}^{\circ}-389535$ \\
\hline 3638.644 & $500 \mathrm{r}$ & 50 & 27474.935 & $8800_{4}-36275_{0}^{\circ}$ & 3601.295 & 300 & 10 & 27759.869 & $11197^{\circ}-38956_{4}$ \\
\hline 3638.460 & 40 & 2 & 27476.324 & $10414_{4}^{\circ}-378905$ & 3600.431 & 300 & 50 & 27766.531 & $98045-37571{ }^{\circ}$ \\
\hline 3638.319 & $400 \mathrm{r}$ & 25 & 27477.389 & $4961_{4}-32439_{4}^{\circ}$ & 3600.182 & 100 & 25 & 27768.451 & $11241_{3}^{\circ}-39010_{3}$ \\
\hline 3638.146 & 200 & 4 & 27478.695 & $10414_{4}^{\circ}-378924$ & 3599.723 & 300 & 20 & 27771.992 & $10414_{4}^{\circ}-381864$ \\
\hline 3638.097 & 150 & 4 & 27479.066 & $132974-40776_{4}^{\circ}$ & 3598.523 & 150 & 10 & 27781.252 & $7795_{4}^{\circ}-35576_{3}$ \\
\hline 3638.004 & 75 & 4 & 27479.768 & $14206_{4}^{\circ}-41686_{3}$ & 3598.245 & 75 & 10 & 27783.399 & $14243 i-420272$ \\
\hline 3636.295 & 150 & 3 & 27492.682 & $11241_{3}^{\circ}-387343$ & 3598.120 & $2000 \mathrm{r}$ & 50 & 27784.364 & $0_{2}-27784_{2}^{\circ}$ \\
\hline 3634.902 & 200 & 4 & 27503.218 & $111977^{\circ}-38700_{4}$ & 3597.776 & 10 & & 27787.020 & $7795_{4}^{\circ}-355825$ \\
\hline 3634.582 & $600 \mathrm{r}$ & $100 \mathrm{~b}$ & 27505.640 & $7795_{4}^{\circ}-353005$ & 3597.021 & 75 & 2 & 27792.853 & $4961_{4}-32754_{3}^{\circ}$ \\
\hline 3634.546 & $500 \mathrm{~b}$ & $50 \mathrm{~b}$ & 27505.912 & $14206_{4}^{\circ}-417125$ & 3595.975 & 150 & 5 & 27800.937 & $9804_{5}-37605_{4}^{\circ}$ \\
\hline 3633.910 & 100 & 3 & 27510.726 & $16346_{4}^{\circ}-43857_{5}$ & 3595.756 & 100 & 10 & 27802.630 & $11877_{i}^{\circ}-39680_{2}$ \\
\hline 3632.830 & $1000 \mathrm{r}$ & 100 & 27518.905 & $11241_{3}^{\circ}-38760_{4}$ & 3595.617 & $500 \mathrm{r}$ & 100 & 27803.705 & $8243_{2}^{\circ}-36047_{2}$ \\
\hline 3631.863 & 150 & 8 & 27526.231 & $13175_{4}^{\circ}-40701_{4}$ & 3594.985 & 300 & 20 & 27808.593 & $11802_{2}-39611_{3}^{\circ}$ \\
\hline 3629.214 & 15 & & 27546.323 & $14032^{\circ}-415782$ & 3593.535 & 15 & & 27819.813 & $10783_{2}^{\circ}-386022$ \\
\hline 3628.867 & 100 & 4 & 27548.956 & $14206_{4}^{\circ}-41755_{4}$ & 3593.352 & 5 & & 27821.230 & $14465_{2}^{\circ}-42286_{3}$ \\
\hline 3626.939 & 300 & 50 & 27563.600 & $111977^{\circ}-38760_{4}$ & 3592.777 & $800 \mathrm{r}$ & 100 & 27825.682 & $8800_{4}-366250^{\circ}$ \\
\hline 3626.631 & $200 \mathrm{~b}$ & 10 & 27565.941 & $11241_{3}^{\circ}-38807_{2}$ & 3591.452 & $800 \mathrm{r}$ & 50 & 27835.948 & $3687_{2}-315233^{\circ}$ \\
\hline 3626.613 & $50 \mathrm{~b}$ & & 27566.078 & $12114_{2}^{\circ}-39680_{2}$ & 3591.253 & 300 & 50 & 27837.490 & $132974-41134_{4}^{\circ}$ \\
\hline 3626.025 & 50 & 3 & 27570.548 & $10526_{3}^{\circ}-38097_{2}$ & 3590.924 & 400 & & 27840.041 & $10526_{3}^{\circ}-38366_{4}$ \\
\hline $\begin{array}{l}3620.025 \\
3625.895\end{array}$ & $\begin{array}{c}50 \\
500 \mathrm{r}\end{array}$ & $400 \mathrm{~b}$ & 27571.537 & $\begin{array}{r}105203-3809 / 2 \\
7280_{2}-34851_{2}^{\circ}\end{array}$ & 3589.992 & 300 & 20 & 27847.268 & $38651-31712_{1}^{\circ}$ \\
\hline 3625.150 & 300 & 75 & 27577.203 & $12847_{3}-40425_{4}^{\circ}$ & 3589.748 & $1000 \mathrm{r}$ & 75 & 27849.161 & $7502_{3}-35351_{4}^{\circ}$ \\
\hline 3625.026 & $300 \mathrm{r}$ & 100 & 27578.146 & $104144^{\circ}-379924$ & 3586.703 & 100 & & 27872.803 & $138472-41720_{2}^{\circ}$ \\
\hline 3624.472 & 300 & 50 & 27582.361 & $8800_{4}-36382_{4}^{\circ}$ & 3584.175 & $800 \mathrm{r}$ & 50 & 27892.462 & $2869_{3}-30761_{3}^{\circ}$ \\
\hline & & 50 & 27302.501 & $88004-503824$ & 3583.100 & $600 \mathrm{r}$ & 25 & 27900.830 & $4961_{4}-32862^{\circ}$ \\
\hline 3623.773 & 300 & 25 & 27587.681 & $8243^{\circ}-35831_{3}$ & 3581.754 & 200 & 10 & 27911.315 & $3687_{2}-31599_{2}^{\circ}$ \\
\hline 3622.795 & $600 \mathrm{r}$ & 50 & 27595.129 & $3687_{2}-31283_{3}^{\circ}$ & 3579.543 & 300 & 50 & 27928.554 & $128473-40776_{4}^{\circ}$ \\
\hline 3622.338 & $500 \mathrm{r}$ & $20 \mathrm{~b}$ & 27598.610 & $55631-33161^{\circ}$ & 3578.947 & 400 & 10 & 27933.205 & $11241_{3}^{\circ}-391743$ \\
\hline 3621.435 & 50 & & 27605.492 & $6362_{2}-33967^{\circ}$ & 3577.596 & 200 & 10 & 27943.753 & $2869_{3}-30812_{2}^{\circ}$ \\
\hline 3620.977 & 5 & & 27608.983 & $118022-39411^{\circ}$ & 3576.633 & 300 & 40 & 27951.277 & $10783_{2}^{\circ}-387343$ \\
\hline 3620.839 & $300 \mathrm{r}$ & 50 & 27610.035 & $7795_{4}^{\circ}-35405_{3}$ & 3576.557 & $400 \mathrm{r}$ & 150 & 27951.871 & $8111_{4}-36062_{4}^{\circ}$ \\
\hline 3619.633 & 100 & 20 & 27619.234 & $10526^{\circ}-38145_{3}$ & 3576.481 & 300 & & 27952.465 & $10414_{4}^{\circ}-383664$ \\
\hline 3619.211 & 200 & 40 & 27622.455 & $8111_{4}-35733_{4}^{\circ}$ & 3575.546 & 50 & & 27959.774 & $75023-35462 \stackrel{\circ}{3}$ \\
\hline 3618.597 & 200 & 10 & 27627.142 & $8800_{4}-36427_{3}^{\circ}$ & 3575.427 & 100 & 10 & 27960.705 & $7280_{2}-35240_{3}^{\circ}$ \\
\hline 3618.363 & $800 \mathrm{r}$ & 50 & 27628.928 & $75023-35131 \stackrel{\circ}{\circ}$ & 3575.301 & $50 \mathrm{~b}$ & & 27961.690 & $6362_{2}-34324_{2}^{\circ}$ \\
\hline 3617.818 & 150 & 10 & 27633.090 & $13945_{3}^{\circ}-41578_{2}$ & 3575.121 & $500 \mathrm{r}$ & 50 & 27963.098 & $8111_{4}-360743$ \\
\hline 3617.671 & 300 & 8 & 27634.213 & $8243_{2}^{\circ}-35877_{2}$ & 3571.008 & 300 & 10 & 27995.304 & $14032_{2}^{\circ}-420272$ \\
\hline 3616.177 & 200 & 8 & 27645.629 & $7795_{4}^{\circ}-35440_{3}$ & 3570.523 & 300 & 10 & 27999.107 & $7795_{4}^{\circ}-35794_{4}$ \\
\hline 3615.849 & 200 & 4 & 27648.137 & $2869_{3}-30517_{4}^{\circ}$ & 3570.357 & 500 & 10 & 28000.408 & $8800_{4}-36800_{4}^{\circ}$ \\
\hline 3615.004 & 100 & 5 & 27654.600 & $14032_{2}^{\circ}-416863$ & 3569.977 & 100 & 5 & 28003.389 & $105263_{3}^{\circ}-38529_{3}$ \\
\hline 3614.352 & $500 \mathrm{r}$ & 75 & 27659.588 & $105263^{\circ}-381864$ & 3569.820 & $1000 \mathrm{r}$ & 25 & 28004.620 & $3865_{1}-31870_{2}^{\circ}$ \\
\hline 3613.547 & 200 & 5 & 27665.750 & $11802_{2}-394688^{\circ}$ & 3569.207 & 75 & & 28009.430 & $6362_{2}-34371_{2}^{\circ}$ \\
\hline 3612.864 & $600 \mathrm{r}$ & 5 & 27670.980 & $0_{2}-27670_{3}^{\circ}$ & 3567.672 & 200 & 20 & 28021.481 & $8111_{4}-36132{ }^{\circ}$ \\
\hline 3612.427 & $1500 \mathrm{r}$ & 75 & 27674.327 & $0_{2}-27674_{2}^{\circ}$ & 3567.264 & $1000 \mathrm{r}$ & 50 & 28024.686 & $0_{2}-28024^{\circ}$ \\
\hline 3611.257 & 25 & 5 & 27683.293 & $13297_{4}-40980_{3}^{\circ}$ & 3566.459 & 20 & 1 & 28031.011 & $75023-35533^{\circ}$ \\
\hline 3611.158 & $200 \mathrm{~s}$ & 10 & 27684.052 & $2869_{3}-30553_{2}^{\circ}$ & 3565.822 & 300 & 15 & 28036.018 & $7795_{4}^{\circ}-35831_{3}$ \\
\hline 3610.650 & 300 & 20 & 27687.947 & $13088_{3}-40776_{4}^{\circ}$ & 3565.604 & 200 & 10 & 28037.732 & $8800_{4}-36837_{0}^{\circ}$ \\
\hline 3608.880 & 300 & 4 & 27701.526 & $8111_{4}-35812^{\circ}$ & 3565.049 & 40 & & 28042.097 & $11241_{3}^{\circ}-39283_{4}$ \\
\hline 3607.819 & 75 & & 27709.673 & $7280_{2}-34989_{1}^{\circ}$ & 3564.508 & 100 & 10 & 28046.353 & $13088_{3}-41134_{4}^{\circ}$ \\
\hline 3606.090 & 200 & 3 & 27722.958 & $25580-30281^{\circ}$ & 3564.235 & 200 & 8 & 28048.501 & $7280_{2}-35328_{1}^{\circ}$ \\
\hline
\end{tabular}


TABLE 4. Classified lines of Th I-continued

\begin{tabular}{|c|c|c|c|c|c|c|c|c|c|}
\hline \multirow{2}{*}{$\begin{array}{c}\text { Wavelength } \\
\text { (§) }\end{array}$} & \multicolumn{2}{|c|}{ Intensity } & \multirow{2}{*}{$\begin{array}{l}\text { Wavenumber } \\
\left(\mathrm{cm}^{-1}\right)\end{array}$} & \multirow{2}{*}{ Classification } & \multirow{2}{*}{$\begin{array}{c}\text { Wavelength } \\
\text { (A) }\end{array}$} & \multicolumn{2}{|c|}{ Inten sity } & \multirow{2}{*}{$\begin{array}{c}\text { Wavenumber } \\
\left(\mathrm{cm}^{-1}\right)\end{array}$} & \multirow{2}{*}{ Classification } \\
\hline & Lamp & Spark & & & & Lamp & Spark & & \\
\hline 3563.375 & $600 \mathrm{r}$ & $75 b$ & 28055.270 & $11197 \AA-392525$ & 3526.764 & 300 & & 28346.501 & $10414 \stackrel{\circ}{4}-38760_{4}$ \\
\hline 3563.299 & 300 & 25 & 28055.869 & $13175_{4}^{\circ}-41230_{4}$ & 3526.634 & $500 \mathrm{r}$ & 10 & 28347.546 & $0_{2}-28347_{2}^{\circ}$ \\
\hline 3561.879 & 40 & & 28067.053 & $8111_{4}-36178^{\circ}$ & 3526.029 & 151 & 1 & 28352.410 & $128477_{3}-41200_{2}^{\circ}$ \\
\hline 3561.780 & $500 \mathrm{r}$ & 75 & 28067.833 & $6362_{2}-34430_{3}^{\circ}$ & 3525.171 & 8 & & 28359.310 & $8800_{4}-37159_{3}^{\circ}$ \\
\hline 3560.688 & 200 & 5 & 28076.441 & $19526^{\circ}-386022$ & 3524.708 & 300 & 8 & 28363.035 & $6362_{2}-34725_{3}^{\circ}$ \\
\hline 3560.295 & 20 & & 28079.540 & $14206_{4}^{\circ}-42286_{3}$ & 3524.178 & 300 & 25 & 28367.301 & $154900^{\circ}-438575$ \\
\hline 3560.226 & 40 & 5 & 28080.084 & $11241_{3}^{\circ}-39321_{3}$ & 3523.758 & 300 & 8 & 28370.682 & $25580-30928^{\circ}$ \\
\hline 3560.021 & $300 \mathrm{r}$ & 50 & 28081.701 & $4961_{4}-33043_{3}^{\circ}$ & 3523.505 & 300 & & 28372.719 & $0_{2}-28372^{\circ}$ \\
\hline 3559.378 & $200 \mathrm{~b}$ & & 28086.774 & $11197 \circ-39283_{4}^{\circ}$ & 3522.154 & 100 & 2 & 28383.602 & $7502_{3}-35885_{2}^{\circ}$ \\
\hline 3558.638 & 100 & & 28092.614 & $82432_{2}^{\circ}-363363$ & 3521.141 & 100 & 2 & 28391.767 & $10783_{2}^{\circ}-391743$ \\
\hline 3558.599 & 8 & & 28092.922 & $3687_{2}-31780_{3}^{\circ}$ & 3521.059 & $500 \mathrm{r}$ & 25 & 28392.428 & $3687_{2}-32080_{i}^{\circ}$ \\
\hline 3557.818 & 150 & 5 & 28099.089 & $5563_{1}-33662_{1}^{\circ}$ & 3520.453 & 5 & & 28397.315 & $142260-42624_{i}^{\circ}$ \\
\hline 3556.206 & 8 & & 28111.826 & $130883-41200_{2}^{\circ}$ & 3520.277 & 15 & & 28398.735 & $7280_{2}-35678_{1}^{\circ}$ \\
\hline 3555.705 & $500 \mathrm{r}$ & 50 & 28115.787 & $10414_{4}^{\circ}-38529_{3}$ & 3518.885 & 4001 & 50 & 28409.969 & $8243_{2}^{\circ}-36653_{2}$ \\
\hline 3555.013 & $1000 \mathrm{r}$ & 50 & 28121.259 & $28699_{3}-30990_{3}^{\circ}$ & 3518.404 & $1000 \mathrm{r}$ & 40 & 28413.853 & $2869_{3}-31283^{\circ}$ \\
\hline 3551.984 & 20 & & 28145.239 & $9804_{5}-37950_{4}^{\circ}$ & 3518.197 & 100 & 5 & 28415.524 & $7795_{4}^{\circ}-36210_{5}$ \\
\hline 3551.401 & $1000 \mathrm{r}$ & 150 & 28149.860 & $2869_{3}-31019_{4}^{\circ}$ & 3516.530 & 3 & & 28428.994 & $14206_{4}^{\circ}-426355$ \\
\hline 3550.878 & 300 & 40 & 28154.006 & $14481 \stackrel{\circ}{-}-426355$ & 3515.327 & 100 & 40 & 28438.723 & $11241_{3}^{\circ}-39680_{2}$ \\
\hline 3550.783 & 300 & 40 & 28154.759 & $11197 \stackrel{\circ}{-}-393515$ & 3514.285 & 200 & 10 & 28447.155 & $15490 \AA-439376$ \\
\hline 3550.718 & $100 \mathrm{~s}$ & & 28155.274 & $5563_{1}-33718_{2}^{\circ}$ & 3513.682 & $75 \mathrm{~s}$ & 4 & 28452.037 & $11802_{2}-40254_{3}^{\circ}$ \\
\hline 3549.596 & $1000 \mathrm{r}$ & 75 & 28164.174 & $8111_{4}-36275^{\circ}$ & 3511.157 & $800 \mathrm{r}$ & 25 & 28472.497 & $3687_{2}-32160_{2}^{\circ}$ \\
\hline 3549.396 & 50 & 25 & 28165.761 & $25580-30723^{\circ}$ & 3509.784 & 10 & & 28483.635 & $10526_{3}^{\circ}-39010_{3}$ \\
\hline 3548.664 & 300 & 8 & 28171.570 & $55631-33734_{2}^{\circ}$ & 3509.089 & 500 & 25 & 28489.276 & $63622-34851_{2}^{\circ}$ \\
\hline 3547.469 & 1501 & & 28181.060 & $13175_{4}^{\circ}-413565$ & 3508.360 & 300 & 3 & 28495.196 & $11601_{1}-40096_{2}^{\circ}$ \\
\hline 3547.364 & $300 \mathrm{~b}$ & & 28181.894 & $7280_{2}-35462_{3}^{\circ}$ & 3508.100 & $300 \mathrm{~b}$ & & 28497.307 & $11197^{\circ}-396945$ \\
\hline 3547.338 & $200 \mathrm{~b}$ & & 28182.100 & $3687_{2}-31870_{2}^{\circ}$ & 3507.520 & 100 & & 28502.020 & $7795_{4}^{\circ}-36297_{4}$ \\
\hline 3547.252 & 100 & 5 & 28182.784 & $11802_{2}-39985_{3}^{\circ}$ & 3506.645 & 300 & 5 & 28509.131 & $3687_{2}-32197_{3}^{\circ}$ \\
\hline 3546.809 & 75 & & 28186.304 & $13175^{\circ}-413613$ & 3506.345 & 150 & 10 & 28511.571 & $13175_{4}^{\circ}-416863$ \\
\hline 3544.250 & 150 & 3 & 28206.654 & $2869_{3}-31075_{2}^{\circ}$ & 3506.132 & 300 & 3 & 28513.303 & 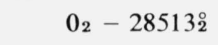 \\
\hline 3544.097 & 200 & & 28207.872 & $10526^{\circ}-387343$ & 3505.934 & 10 & & 28514.913 & $8111_{4}-36625^{\circ}$ \\
\hline 3544.018 & $800 \mathrm{r}$ & 50 & 28208.500 & $8800_{4}-37008 \circ$ & 3505.767 & 150 & 5 & 28516.271 & $6362_{2}-34878_{1}^{\circ}$ \\
\hline 3543.208 & 150 & 3 & 28214.949 & $38651-32080_{1}^{\circ}$ & 3503.863 & 75 & & 28531.766 & $8243_{2}^{\circ}-36775_{3}$ \\
\hline 3542.498 & $500 \mathrm{r}$ & 50 & 28220.604 & $63622-34583_{3}^{\circ}$ & 3503.786 & $500 \mathrm{r}$ & 40 & 28532.393 & $7280_{2}-35812_{3}^{\circ}$ \\
\hline 3541.692 & 40 & & 28227.026 & $107832-390103$ & 3503.130 & 200 & 15 & 28537.736 & $13175_{4}^{\circ}-41712_{5}$ \\
\hline 3541.493 & 150 & & 28228.612 & $63622-345911$ & 3503.017 & 100 & 5 & 28538.657 & $10783_{2}^{\circ}-393213$ \\
\hline 3540.807 & 2 & & 28234.081 & $10526_{3}^{\circ}-38760_{4}$ & 3502.963 & 200 & 10 & 28539.097 & $10414 \stackrel{\circ}{4}-389535$ \\
\hline 3539.951 & $8 b$ & & 28240.908 & $8800_{4}-37041_{4}^{\circ}$ & 3502.739 & $200 \mathrm{~b}$ & & 28540.922 & $7795_{4}^{\circ}-363363$ \\
\hline 3539.843 & 300 & 25 & 28241.770 & $8243_{2}^{\circ}-36485_{2}$ & 3502.514 & 100 & 2 & 28542.755 & $10414_{4}^{\circ}-389564$ \\
\hline 3538.415 & 3 & & 28253.167 & $7280_{2}-35533_{2}^{\circ}$ & 3501.866 & $150 \mathrm{~b}$ & 25 & 28548.037 & $5563_{1}-34111_{1}^{\circ}$ \\
\hline 3538.264 & 15 & 2 & 28254.372 & $14032_{2}^{\circ}-422863$ & 3500.326 & 50 & 10 & 28560.596 & $7502_{3}-36062_{4}^{\circ}$ \\
\hline 3536.009 & $500 \mathrm{r}$ & 20 & 28272.390 & $82432^{\circ}-365153$ & 3499.821 & 200 & 5 & 28564.717 & $8243_{2}^{\circ}-368083$ \\
\hline 3535.250 & $150 \mathrm{~b}$ & & 28278.460 & $11241_{3}^{\circ}-39520_{4}$ & 3498.956 & $200 \mathrm{~b}$ & $20 b$ & 28571.779 & $75023-36074_{3}^{\circ}$ \\
\hline 3534.916 & 15 & & 28281.132 & $105263-38807_{2}$ & 3498.621 & $800 \mathrm{r}$ & 75 & 28574.514 & $8243_{2}^{\circ}-36818_{2}$ \\
\hline 3533.396 & 40 & 1 & 28293.298 & $11802_{2}-40096_{2}^{\circ}$ & 3497.901 & 50 & 10 & 28580.396 & $98045-38385^{\circ}$ \\
\hline 3533.181 & $200 \mathrm{~b}$ & 10 & 28295.019 & $3865_{1}-32160_{2}^{\circ}$ & 3497.856 & 50 & 3 & 28580.764 & $13175_{4}^{\circ}-41755_{4}$ \\
\hline 3531.450 & $1000 \mathrm{r}$ & 50 & 28308.888 & $4961_{4}-33270_{4}^{\circ}$ & 3496.811 & $500 \mathrm{r}$ & 15 & 28589.305 & $0_{2}-28589_{3}^{\circ}$ \\
\hline 3531.282 & 200 & 8 & 28310.235 & $75023-35812^{\circ}$ & 3496.003 & 200 & 10 & 28595.912 & $11241_{3}^{\circ}-39837_{3}$ \\
\hline 3530.514 & $800 \mathrm{r}$ & 50 & 28316.393 & $8111_{4}-36427_{3}^{\circ}$ & 3495.847 & 15 & & 28597.188 & $3687_{2}-32285_{3}^{\circ}$ \\
\hline 3530.030 & 50 & 3 & 28320.276 & $10414_{4}^{\circ}-387343$ & 3495.700 & $800 \mathrm{r}$ & 25 & 28598.390 & $4961_{4}-33560_{4}^{\circ}$ \\
\hline 3529.670 & $200 \mathrm{~b}$ & 15 & 28323.164 & $11197^{\circ}-39520_{4}$ & 3494.801 & 100 & & 28605.747 & $7280_{2}-35885_{2}^{\circ}$ \\
\hline 3529.385 & 3001 & 40 & 28325.451 & $2869_{3}-31194_{4}^{\circ}$ & 3492.158 & 25 & & 28627.396 & $6362_{2}-34989_{1}^{\circ}$ \\
\hline 3528.411 & 300 & 50 & 28333.270 & $4961_{4}-33294^{\circ}$ & 3491.900 & 500 & 2 & 28629.511 & $4961_{4}-33591_{3}^{\circ}$ \\
\hline 3527.793 & 200 & 10 & 28338.233 & $7795_{4}^{\circ}-361335$ & 3490.848 & 20 & 1 & 28638.139 & $3865_{1}-32503_{2}^{\circ}$ \\
\hline 3527.434 & $4 \mathrm{~s}$ & & 28341.117 & $13945_{3}^{\circ}-422863$ & 3489.602 & 1001 & 1 & 28648.364 & $10526^{\circ}-391743$ \\
\hline 3527.322 & 200 & 50 & 28342.017 & $63622-34704_{3}^{\circ}$ & 3489.509 & 400 & 2 & 28649.128 & $0_{2}-28649^{\circ}$ \\
\hline
\end{tabular}


TABLE 4. Classified lines of Th $\mathrm{I}$-continued

\begin{tabular}{|c|c|c|c|c|c|c|c|c|c|}
\hline \multirow{2}{*}{$\begin{array}{c}\text { Wavelength } \\
\text { (Å) }\end{array}$} & \multicolumn{2}{|c|}{ Intensity } & \multirow{2}{*}{$\begin{array}{l}\text { Wavenumber } \\
\left(\mathrm{cm}^{-1}\right)\end{array}$} & \multirow{2}{*}{ Classification } & \multirow{2}{*}{$\begin{array}{c}\text { Wavelength } \\
\text { (§) }\end{array}$} & \multicolumn{2}{|c|}{ Inten sity } & \multirow{2}{*}{$\begin{array}{l}\text { Wavenumber } \\
\left(\mathrm{cm}^{-1}\right)\end{array}$} & \multirow{2}{*}{ Classification } \\
\hline & Lamp & Spark & & & & Lamp & Spark & & \\
\hline 3489.185 & 500 & 4 & 28651.788 & $8111_{4}-36762_{3}^{\circ}$ & 3442.578 & $800 \mathrm{r}$ & 20 & 29039.676 & $4961_{4}-34001_{4}^{\circ}$ \\
\hline 3488.834 & 500 & 3 & 28654.670 & $2869_{3}-31523 \stackrel{\circ}{3}$ & 3441.527 & 200 & 5 & 29048.544 & $8111_{4}-37159_{3}^{\circ}$ \\
\hline 3487.991 & 1501 & 4 & 28661.596 & $13962_{1}-42624_{i}^{\circ}$ & 3440.827 & 8 & & 29054.453 & $10783_{2}^{\circ}-39837_{3}$ \\
\hline 3486.551 & $1000 \mathrm{r}$ & $10 \mathrm{~b}$ & 28673.433 & $0_{2}-28673 \stackrel{2}{2}$ & 3439.748 & $100 \mathrm{~b}$ & & 29063.567 & $82432^{\circ}-37307_{3}$ \\
\hline 3486.269 & 50 & 1 & 28675.752 & $75023-36178_{4}^{\circ}$ & 3439.399 & $400 \mathrm{~b}$ & 8 & 29066.516 & $3687_{2}-32754_{3}^{\circ}$ \\
\hline 3486.210 & 50 & 1 & 28676.238 & $0_{2}-28676_{3}^{\circ}$ & 3437.645 & $50 \mathrm{~b}$ & $5 b$ & 29081.346 & $7280_{2}-36361_{i}^{\circ}$ \\
\hline 3484.943 & 100 & 1 & 28686.663 & $75023-36188_{2}^{\circ}$ & 3437.307 & $2000 \mathrm{r}$ & 75 & 29084.206 & $2869_{3}-31953_{4}^{\circ}$ \\
\hline 3484.580 & 8 & & 28689.651 & $8111_{4}-36800_{4}^{\circ}$ & 3436.726 & $1500 \mathrm{r}$ & 50 & 29089.122 & $3865_{1}-32954_{2}^{\circ}$ \\
\hline 3480.810 & 100 & & 28720.723 & $7795^{\circ}-36515_{3}$ & 3436.621 & 200 & 4 & 29090.011 & $7795_{4}^{\circ}-36885_{4}$ \\
\hline 3480.052 & 500 & 3 & 28726.979 & $8111_{4}-36837^{\circ}$ & 3435.487 & 251 & & 29099.613 & $6362_{2}-35462_{3}^{\circ}$ \\
\hline 3479.684 & 300 & 2 & 28730.017 & $2869_{3}-31599_{2}^{\circ}$ & 3434.727 & $200 \mathrm{~b}$ & 2 & 29106.052 & $10414_{4}^{\circ}-39520_{4}$ \\
\hline 3479.221 & $300 \mathrm{~b}$ & 2 & 28733.840 & $11877_{1}^{\circ}-40611_{2}$ & 3434.103 & $50 \mathrm{~b}$ & & 29111.340 & $13175_{4}^{\circ}-42286_{3}$ \\
\hline 3476.385 & 200 & 3 & 28757.280 & $10526^{\circ}-39283_{4}$ & 3429.871 & $100 \mathrm{~b}$ & & 29147.259 & $7280_{2}-36427_{3}^{\circ}$ \\
\hline 3475.961 & $100 \mathrm{~b}$ & & 28760.788 & $10414_{4}^{\circ}-391743$ & 3429.574 & 200 & 10 & 29149.783 & $8800_{4}-37950_{4}^{\circ}$ \\
\hline 3475.943 & $100 \mathrm{~b}$ & & 28760.937 & $5563_{1}-34324_{2}^{\circ}$ & 3429.086 & 50 & & 29153.931 & $10526_{3}^{\circ}-39680_{2}$ \\
\hline 3474.717 & 200 & 10 & 28771.084 & $8800_{4}-37571^{\circ}$ & 3428.998 & $1500 \mathrm{r}$ & 50 & 29154.679 & $25580-31712_{1}^{\circ}$ \\
\hline 3474.530 & 200 & 3 & 28772.633 & $12114_{2}^{\circ}-40886_{2}$ & 3428.714 & $300 \mathrm{r}$ & 8 & 29157.094 & $0_{2}-29157^{\circ}$ \\
\hline 3474.188 & 3 & & 28775.465 & $9804_{5}-38580_{4}^{\circ}$ & 3428.621 & $300 \mathrm{r}$ & 4 & 29157.885 & $0_{2}-29157_{3}^{\circ}$ \\
\hline 3472.989 & $15 b$ & & 28785.399 & $3865_{1}-32650_{1}^{\circ}$ & 3428.248 & 50 & 3 & 29161.057 & $8243_{2}^{\circ}-37404_{3}$ \\
\hline 3471.959 & $500 \mathrm{r}$ & 501 & 28793.938 & $7280_{2}-36074_{3}^{\circ}$ & 3427.619 & 5 & & 29166.408 & $7502_{3}-36668^{\circ}$ \\
\hline 3471.800 & $50 \mathrm{~b}$ & & 28795.257 & $10526^{\circ}-39321_{3}$ & 3427.089 & $200 \mathrm{~s}$ & & 29170.919 & $63622-35533_{2}^{\circ}$ \\
\hline 3471.216 & $1000 \mathrm{r}$ & $50 \mathrm{~b}$ & 28800.102 & $3865_{1}-32665_{i}^{\circ}$ & 3426.285 & 2 & & 29177.764 & $11802_{2}-40980 \stackrel{\circ}{3}$ \\
\hline 3471.001 & 300 & 15 & 28801.885 & $2869_{3}-31671_{4}^{\circ}$ & 3425.435 & 150 & 15 & 29185.004 & $11241_{3}^{\circ}-40426_{4}$ \\
\hline 3470.568 & 300 & 20 & 28805.479 & $8800_{4}-37605_{4}^{\circ}$ & 3423.990 & $1500 \mathrm{r}$ & 50 & 29197.320 & $\mathbf{0}_{\mathbf{2}}-29197^{\circ}$ \\
\hline 3470.181 & 5 & 2 & 28808.691 & $5563_{1}-34371_{2}^{\circ}$ & 3423.923 & $200 \mathrm{~b}$ & & 29197.891 & $0_{2}-29197_{2}^{\circ}$ \\
\hline 3469.345 & $1000 \mathrm{r}$ & 150 & 28815.633 & $3687_{2}-32503_{2}^{\circ}$ & 3422.655 & $1000 \mathrm{r}$ & 40 & 29208.708 & $7280_{2}-36488_{2}^{\circ}$ \\
\hline 3466.644 & $600 \mathrm{r}$ & 100 & 28838.083 & $4961_{4}-33799^{\circ}$ & 3421.210 & $2000 \mathrm{r}$ & & 29221.044 & $4961_{4}-34182 \circ$ \\
\hline 3466.538 & $600 \mathrm{r}$ & 40 & 28838.965 & $4961_{4}-33800_{3}^{\circ}$ & 3420.196 & 100 & 3 & 29229.707 & $111970^{\circ}-404264$ \\
\hline 3465.061 & $200 \mathrm{~b}$ & 25 & 28851.258 & $8243^{\circ}-370942$ & 3418.170 & 15 & & 29247.032 & $12114_{2}^{\circ}-41361_{3}$ \\
\hline 3464.560 & 25 & 2 & 28855.430 & $111977^{\circ}-40052_{4}$ & 3417.642 & 100 & & 29251.550 & $11241 \stackrel{\circ}{3}-404933$ \\
\hline 3463.921 & 50 & 2 & 28860.753 & $98045-386655^{\circ}$ & 3417.498 & $400 \mathrm{r}$ & 10 & 29252.783 & $0_{2}-29252_{2}^{\circ}$ \\
\hline 3461.801 & 8 & & 28878.426 & $6362_{2}-35240_{3}^{\circ}$ & 3416.595 & 150 & 3 & 29260.514 & $7502_{3}-36762_{3}^{\circ}$ \\
\hline 3461.574 & 200 & 3 & 28880.320 & $7502_{3}-36382^{\circ}$ & 3415.885 & $500 \mathrm{r}$ & 75 & 29266.595 & $3687_{2}-32954_{2}^{\circ}$ \\
\hline 3461.215 & $2000 \mathrm{r}$ & 75 & 28883.315 & $4961_{4}-33844^{\circ}$ & 3414.712 & 200 & 2 & 29276.649 & $7280_{2}-36556^{\circ}$ \\
\hline 3461.018 & $1500 \mathrm{r}$ & 50 & 28884.959 & $0_{2}-28884_{3}^{\circ}$ & 3414.565 & $100 \mathrm{~b}$ & 1 & 29277.909 & $8243 \stackrel{\circ}{2}-37521_{3}$ \\
\hline 3460.724 & 75 & 2 & 28887.413 & $3687_{2}-32575_{2}^{\circ}$ & 3414.299 & 75 & & 29280.190 & $10414_{4}^{\circ}-396945$ \\
\hline 3460.724 & 75 & 2 & 28887.413 & $82432^{\circ}-37131_{3}$ & 3413.327 & 100 & 3 & 29288.528 & $5563_{1}-34851_{2}^{\circ}$ \\
\hline 3459.536 & $50 \mathrm{~b}$ & & 28897.333 & $10783_{2}^{\circ}-39680_{2}$ & 3413.086 & $300 \mathrm{~b}$ & 25 & 29290.596 & $7795_{4}^{\circ}-37085_{4}$ \\
\hline 3459.488 & 75 & 8 & 28897.734 & $8111_{4}-370080$ & 3413.013 & $1000 \mathrm{r}$ & 25 & 29291.222 & $2869_{3}-32160_{2}^{\circ}$ \\
\hline 3458.299 & 20 & & 28907.669 & $10414_{4}^{\circ}-393213$ & 3412.422 & 15 & & 29296.295 & $38651-33161^{\circ}$ \\
\hline 3457.829 & 75 & & 28911.598 & $2869_{3}-31780_{3}^{\circ}$ & 3412.181 & 200 & & 29298.364 & $7502_{3}-36800^{\circ}$ \\
\hline 3457.069 & $400 \mathrm{r}$ & 25 & 28917.954 & $0_{2}-28917_{2}^{\circ}$ & 3410.699 & 25 & & 29311.094 & $10526_{3}^{\circ}-39837_{3}$ \\
\hline 3456.217 & 2 & & 28925.082 & $7502_{3}-36427^{\circ}$ & 3410.184 & 150 & 3 & 29315.521 & $5563_{1}-34878_{i}$ \\
\hline 3455.612 & $200 \mathrm{~b}$ & 10 & 28930.146 & $8111_{4}-37041_{4}^{\circ}$ & 3410.076 & 300 & 50 & 29316.449 & $6362_{2}-35678 i$ \\
\hline 3453.513 & $300 \mathrm{~b}$ & 10 & 28947.729 & $15490 \AA-444376$ & 3408.750 & $1000 \mathrm{r}$ & 25 & 29327.853 & $2869_{3}-32197_{3}^{\circ}$ \\
\hline 3452.207 & 75 & 1 & 28958.680 & $11241_{3}^{\circ}-40200_{3}$ & 3405.558 & $1500 \mathrm{r}$ & 50 & 29355.341 & $3687_{2}-33043_{3}^{\circ}$ \\
\hline 3451.702 & $800 \mathrm{r}$ & 100 & 28962.916 & $3687_{2}-32650_{1}^{\circ}$ & 3403.903 & 300 & 8 & 29369.613 & $7502_{3}-36871_{2}^{\circ}$ \\
\hline 3451.308 & 100 & & 28966.223 & $6362_{2}-35328_{1}^{\circ}$ & 3403.222 & 15 & & 29375.490 & $14481 \stackrel{\circ}{6}-438575$ \\
\hline 3449.949 & 200 & 5 & 28977.623 & $3687_{2}-32665_{1}$ & 3402.354 & 15 & & 29382.984 & $49614-34344_{4}^{\circ}$ \\
\hline 3448.949 & 300 & 10 & 28986.034 & $7795^{\circ}-367815$ & 3401.711 & $500 \mathrm{r}$ & 25 & 29388.538 & $7280_{2}-36668_{2}^{\circ}$ \\
\hline 3448.891 & $15 \mathrm{~h}$ & & 28986.522 & $7502_{3}-36488^{\circ}$ & 3400.680 & 25 & & 29397.447 & $11802_{2}-41200_{2}^{\circ}$ \\
\hline 3447.189 & 40 & & 29000.833 & $2869_{3}-31870_{2}^{\circ}$ & 3398.545 & $1500 \mathrm{r}$ & 50 & 29415.914 & $2869_{3}-32285_{3}^{\circ}$ \\
\hline 3446.546 & 300 & 8 & 29006.243 & $4961_{4}-33967_{3}^{\circ}$ & 3398.490 & $150 \mathrm{~b}$ & 4 & 29416.390 & $7795^{\circ}-372115$ \\
\hline 3446.200 & 15 & & 29009.155 & $11877_{1}^{\circ}-40886_{2}$ & 3398.457 & $100 \mathrm{~b}$ & 4 & 29416.676 & $8800_{4}-38216_{3}^{\circ}$ \\
\hline 3443.980 & 100 & 8 & 29027.854 & $5563_{1}-34591_{1}^{\circ}$ & 3398.162 & 25 & & 29419.230 & $0_{2}-29419_{2}^{\circ}$ \\
\hline
\end{tabular}


TABLE 4. Classified lines of Th $\mathrm{I}$-continued

\begin{tabular}{|c|c|c|c|c|c|c|c|c|c|}
\hline \multirow{2}{*}{$\begin{array}{c}\text { Wavelength } \\
\text { (Å) }\end{array}$} & \multicolumn{2}{|c|}{ Intensity } & \multirow{2}{*}{$\begin{array}{l}\text { Wavenumber } \\
\left(\mathrm{cm}^{-1}\right)\end{array}$} & \multirow{2}{*}{ Classification } & \multirow{2}{*}{$\begin{array}{c}\text { Wavelength } \\
\text { (A) }\end{array}$} & \multicolumn{2}{|c|}{ Intensity } & \multirow{2}{*}{$\begin{array}{l}\text { Wavenumber } \\
\left(\mathrm{cm}^{-1}\right)\end{array}$} & \multirow{2}{*}{ Classification } \\
\hline & Lamp & Spark & & & & Lamp & Spark & & \\
\hline 3397.516 & $1500 \mathrm{r}$ & $50 \mathrm{~b}$ & 29424.823 & $2869_{3}-32294_{4}^{\circ}$ & 3346.992 & 100 & 3 & 29868.987 & $7280_{2}-37149_{2}^{\circ}$ \\
\hline 3397.306 & 50 & 1 & 29426.642 & $5563_{1}-34989_{1}^{\circ}$ & 3346.966 & 300 & 3 & 29869.219 & $3865_{1}-33734_{2}^{\circ}$ \\
\hline 3396.727 & $800 \mathrm{r}$ & 50 & 29431.658 & $3865_{1}-33297_{2}^{\circ}$ & 3345.851 & $200 \mathrm{r}$ & $8 \mathrm{~b}$ & 29879.172 & $7280_{2}-37159^{\circ}$ \\
\hline 3395.234 & 20 & 1 & 29444.600 & $8243_{2}^{\circ}-376883$ & 3345.822 & $200 \mathrm{r}$ & 8 & 29879.431 & $7280_{2}-37159_{3}^{\circ}$ \\
\hline 3393.993 & 200 & 2 & 29455.365 & $14481{ }^{\circ}-439376$ & 3345.170 & 150 & 3 & 29885.255 & $2869_{3}-32754^{\circ}$ \\
\hline 3393.421 & 150 & 10 & 29460.331 & $8111_{4}-375718$ & 3344.310 & 20 & 2 & 29892.940 & $7795_{4}^{\circ}-37688_{3}$ \\
\hline 3393.373 & $4 b$ & & 29460.747 & $13175_{4}^{\circ}-426355$ & 3343.499 & 150 & 5 & 29900.190 & $10526_{3}^{\circ}-404264$ \\
\hline 3392.993 & 25 & & 29464.046 & $12114_{2}^{\circ}-41578_{2}$ & 3343.281 & $100 \mathrm{~b}$ & & 29902.140 & $8243_{2}^{\circ}-38145_{3}$ \\
\hline 3392.473 & 100 & 2 & 29468.563 & $4961_{4}-34430_{3}^{\circ}$ & 3343.164 & 300 & 5 & 29903.186 & $3687_{2}-33591_{3}^{\circ}$ \\
\hline 3391.872 & $400 \mathrm{r}$ & 40 & 29473.784 & $3687_{2}-33161^{\circ}$ & 3342.069 & 200 & 10 & 29912.984 & $12114_{2}^{\circ}-42027_{2}$ \\
\hline 3390.849 & 200 & 8 & 29482.676 & $7280_{2}-36762_{3}^{\circ}$ & 3341.550 & 10 & 2 & 29917.630 & $11802_{2}-41720_{2}^{\circ}$ \\
\hline 3389.463 & $800 \mathrm{r}$ & 40 & 29494.731 & $8111_{4}-37605^{\circ}$ & 3340.724 & $300 \mathrm{r}$ & 5 & 29925.026 & $4961_{4}-34886_{0}^{\circ}$ \\
\hline 3388.348 & 50 & & 29504.437 & $8243_{2}^{\circ}-37748_{2}$ & 3337.505 & 10 & & 29953.888 & $7280_{2}-37234_{2}^{\circ}$ \\
\hline 3388.154 & $150 \mathrm{~b}$ & 5 & 29506.126 & $8800_{4}-38306_{4}^{\circ}$ & 3337.275 & 200 & 5 & 29955.952 & $144811^{\circ}-444376$ \\
\hline 3388.117 & $40 \mathrm{~b}$ & 2 & 29506.448 & $98045-39311^{\circ}$ & 3336.074 & 150 & & 29966.736 & $10526^{\circ}-404933$ \\
\hline 3387.920 & $600 \mathrm{r}$ & 15 & 29508.164 & $7795^{\circ}-37303_{4}$ & 3335.694 & 150 & 4 & 29970.150 & $5563_{1}-35533_{2}^{\circ}$ \\
\hline 3387.493 & $50 \mathrm{~b}$ & & 29511.883 & $7795_{4}^{\circ}-37307_{3}$ & 3335.238 & 300 & 5 & 29974.247 & $3687_{2}-33662_{1}^{\circ}$ \\
\hline 3386.291 & 5 & & 29522.359 & $25580-32080_{1}^{\circ}$ & 3333.571 & 10 & 1 & 29989.236 & $11241_{3}^{\circ}-41230_{4}$ \\
\hline 3386.159 & 15 & 5 & 29523.509 & $6362_{2}-35885_{2}^{\circ}$ & 3333.128 & $1000 \mathrm{r}$ & $20 \mathrm{~b}$ & 29993.222 & $2869_{3}-32862_{4}^{\circ}$ \\
\hline 3385.373 & 4 & & 29530.364 & $4961_{4}-34492_{4}^{\circ}$ & 3332.479 & $100 \mathrm{~b}$ & 1 & 29999.062 & $6362_{2}-36361^{\circ}$ \\
\hline 3384.403 & 200 & 4 & 29538.827 & $75023-37041_{4}^{\circ}$ & 3330.976 & 150 & 4 & 30012.598 & $10414_{4}^{\circ}-404264$ \\
\hline 3384.177 & 15 & & 29540.800 & $8243^{\circ}-37784_{2}$ & 3330.477 & $1500 \mathrm{r}$ & 40 & 30017.095 & $0_{2}-30017^{\circ}$ \\
\hline 3383.586 & 200 & 8 & 29545.959 & $8111_{4}-37656_{3}^{\circ}$ & 3329.728 & $400 \mathrm{r}$ & 75 & 30023.847 & $7795_{4}^{\circ}-37819_{4}$ \\
\hline 3382.310 & 4 & & 29557.106 & $7280_{2}-36837_{1}^{\circ}$ & 3328.997 & $10 \mathrm{~s}$ & & 30030.439 & $3687_{2}-33718_{2}^{\circ}$ \\
\hline 3381.800 & 2 & & 29561.563 & $3865_{1}-33427_{2}^{\circ}$ & 3328.608 & 4 & & 30033.949 & $11197^{\circ}-41230_{4}$ \\
\hline 3380.859 & $600 \mathrm{r}$ & 20 & 29569.790 & $2869_{3}-32439_{4}^{\circ}$ & 3327.193 & $800 \mathrm{r}$ & 25 & 30046.721 & $3687_{2}-33734_{2}^{\circ}$ \\
\hline 3380.571 & 3 & & 29572.309 & $12114_{2}^{\circ}-41686_{3}$ & 3322.945 & $200 \mathrm{~b}$ & & 30085.131 & $105263^{\circ}-40611_{2}$ \\
\hline 3379.124 & $10 \mathrm{~b}$ & & 29584.973 & $8800_{4}-38385^{\circ}$ & 3322.924 & 10 & & 30085.321 & $2869_{3}-32954_{2}^{\circ}$ \\
\hline 3378.348 & 2 & & 29591.768 & $7280_{2}-36871_{2}^{\circ}$ & 3322.093 & $400 \mathrm{r}$ & 10 & 30092.847 & $25580-32650^{\circ}$ \\
\hline 3377.483 & 100 & 15 & 29599.346 & $11601_{1}-41200_{2}^{\circ}$ & 3321.835 & 5 & 1 & 30095.184 & $7795_{4}^{\circ}-378905$ \\
\hline 3376.617 & 25 & & 29606.937 & $3687_{2}-33294_{3}^{\circ}$ & 3321.574 & $300 \mathrm{r}$ & 40 & 30097.549 & $7795_{4}^{\circ}-37892_{4}$ \\
\hline 3376.367 & 300 & 1 & 29609.130 & $3687_{2}-33297_{2}^{\circ}$ & 3321.067 & 3 & & 30102.143 & $98045-39906_{4}^{\circ}$ \\
\hline 3375.587 & $200 \mathrm{~b}$ & & 29615.971 & $8800_{4}-38416_{3}^{\circ}$ & 3320.879 & 20 & & 30103.847 & $10783_{2}^{\circ}-40886_{2}$ \\
\hline 3374.975 & $1500 \mathrm{r}$ & 75 & 29621.341 & $4961_{4}-34583_{3}^{\circ}$ & 3320.476 & $400 \mathrm{r}$ & 25 & 30107.501 & $25580-32665^{\circ}$ \\
\hline 3373.493 & $800 \mathrm{r}$ & 75 & 29634.354 & $2869_{3}-32503_{2}^{\circ}$ & 3319.910 & $400 \mathrm{r}$ & $75 b$ & 30112.634 & $3687_{2}-33800_{3}^{\circ}$ \\
\hline 3372.822 & $400 \mathrm{r}$ & 100 & 29640.249 & $0_{2}-29640 i$ & 3319.572 & 50 & & 30115.700 & $5563_{1}-35678^{\circ}$ \\
\hline 3372.253 & 10 & & 29645.250 & $11241_{3}^{\circ}-40886_{2}$ & 3319.149 & $25 b$ & & 30119.538 & $11601_{1}-41720_{2}^{\circ}$ \\
\hline 3372.077 & 8 & & 29646.798 & $75023-37149_{2}^{\circ}$ & 3319.133 & $300 \mathrm{~b}$ & 40 & 30119.683 & $11241_{3}^{\circ}-41361_{3}$ \\
\hline 3371.664 & 10 & & 29650.429 & $14206^{\circ}-438575$ & 3318.390 & 200 & 5 & 30126.426 & $6362_{2}-36488^{\circ}$ \\
\hline 3370.887 & 200 & 5 & 29657.263 & $75023-37159_{3}^{\circ}$ & 3315.848 & 200 & 2 & 30149.521 & $11877_{1}^{\circ}-42027_{2}$ \\
\hline 3369.000 & 200 & 4 & 29673.874 & $105263-40200_{3}$ & 3314.793 & $400 \mathrm{r}$ & & 30159.117 & $11197 \stackrel{\circ}{-}-413565$ \\
\hline 3367.582 & $400 \mathrm{r}$ & 10 & 29686.368 & $\mathbf{0}_{2}-29686_{3}^{\circ}$ & 3313.744 & $20 \mathrm{~b}$ & & 30168.663 & $10414 \stackrel{\circ}{4}-405825$ \\
\hline 3365.337 & $400 \mathrm{r}$ & 50 & 29706.171 & $2869_{3}-32575_{2}^{\circ}$ & 3313.647 & $500 \mathrm{r}$ & & 30169.546 & $4961_{4}-35131^{\circ}$ \\
\hline 3364.888 & 25 & & 29710.135 & $10783_{2}^{\circ}-404933$ & 3313.367 & $25 b$ & & 30172.096 & $12114_{2}^{\circ}-422863$ \\
\hline 3362.443 & $10 \mathrm{~b}$ & & 29731.738 & $75023-37234_{2}^{\circ}$ & 3313.151 & 5 & 1 & 30174.063 & $2869_{3}-330433^{\circ}$ \\
\hline 3361.196 & $300 \mathrm{r}$ & 25 & 29742.768 & $49614-34704_{3}^{\circ}$ & 3313.073 & 3001 & 40 & 30174.773 & $10526^{\circ}-40701_{4}$ \\
\hline 3360.997 & $400 \mathrm{r}$ & 150 & 29744.529 & $0_{2}-29744_{3}^{\circ}$ & 3310.922 & 8 & 2 & 30194.376 & $63622-36556_{1}^{\circ}$ \\
\hline 3356.986 & 8 & & 29780.068 & $8800_{4}-38580_{4}^{\circ}$ & 3310.814 & 25 & 4 & 30195.361 & $8111_{4}-383064$ \\
\hline 3355.106 & $500 \mathrm{r}$ & 10 & 29796.754 & $38651-33662_{1}^{\circ}$ & 3310.637 & $150 \mathrm{~d}$ & $15 \mathrm{~b}$ & 30196.975 & $7795_{4}^{\circ}-379924$ \\
\hline 3351.753 & 3 & & 29826.561 & $6362_{2}-36188^{\circ}$ & 3310.446 & 200 & $10 \mathrm{~b}$ & 30198.717 & $8800_{4}-38998_{3}^{\circ}$ \\
\hline 3350.352 & 200 & 5 & 29839.033 & $8111_{4}-37950_{4}^{\circ}$ & 3309.365 & $1000 \mathrm{r}$ & 40 & 30208.582 & $0_{2}-30208^{\circ}$ \\
\hline 3349.800 & $40 \mathrm{~b}$ & & 29843.950 & $8111_{4}-37954^{\circ}$ & 3306.506 & $2 b$ & & 30234.701 & $75023-37736_{2}^{\circ}$ \\
\hline 3348.790 & $400 \mathrm{~b}$ & & 29852.950 & $38651-33718^{\circ}$ & 3305.303 & $400 \mathrm{r}$ & 40 & 30245.705 & $38651-34111 i$ \\
\hline 3348.770 & $1500 \mathrm{r}$ & 20 & 29853.129 & $0_{2}-29853_{2}^{\circ}$ & 3304.238 & $3000 \mathrm{r}$ & 200 & 30255.453 & $\mathbf{0}_{2}-30255^{\circ}$ \\
\hline 3347.403 & 150 & & 29865.319 & $8800_{4}-38665_{0}^{\circ}$ & 3303.546 & 20 & & 30261.791 & $8800_{4}-39062 \circ$ \\
\hline
\end{tabular}


TABLE 4. Classified lines of $\mathrm{Th} \mathrm{I}$-continued

\begin{tabular}{|c|c|c|c|c|c|c|c|c|c|}
\hline \multirow{2}{*}{$\begin{array}{l}\text { Wavelength } \\
(\AA)\end{array}$} & \multicolumn{2}{|c|}{ Intensity } & \multirow{2}{*}{$\begin{array}{l}\text { Wavenumber } \\
\left(\mathrm{cm}^{-1}\right)\end{array}$} & \multirow{2}{*}{ Classification } & \multirow{2}{*}{$\begin{array}{c}\text { Wavelength } \\
\text { ( })\end{array}$} & \multicolumn{2}{|c|}{ Inten sity } & \multirow{2}{*}{$\begin{array}{l}\text { Wavenumber } \\
\left(\mathrm{cm}^{-1}\right)\end{array}$} & \multirow{2}{*}{ Classification } \\
\hline & Lamp & Spark & & & & Lamp & Spark & & \\
\hline 3302.192 & $400 \mathrm{r}$ & 5 & 30274.198 & $8111_{4}-383850$ & 3257.366 & $1000 \mathrm{r}$ & 50 & 30690.800 & $2869_{3}-33560_{4}^{\circ}$ \\
\hline 3301.651 & $1500 \mathrm{r}$ & 150 & 30279.159 & $4961_{4}-35240 \AA$ & 3255.920 & 200 & 8 & 30704.430 & $10526_{3}^{\circ}-41230_{4}$ \\
\hline 3301.570 & 200 & 8 & 30279.902 & $3687_{2}-33967_{3}^{\circ}$ & 3254.834 & $8 b$ & & 30714.674 & $75023-38216_{3}^{\circ}$ \\
\hline 3301.449 & 150 & 3 & 30281.011 & $02-30281 i$ & 3254.067 & $300 r$ & 20 & 30721.914 & $2869_{3}-33591_{3}^{\circ}$ \\
\hline 3300.870 & 20 & 4 & 30286.323 & $8243_{2}^{\circ}-385293$ & 3253.866 & $300 \mathrm{r}$ & 50 & 30723.811 & $0_{2}-30723 i$ \\
\hline 3300.775 & 150 & 4 & 30287.194 & $10414_{4}^{\circ}-40701_{4}$ & 3253.684 & $300 r$ & 40 & 30725.530 & $38651-34591 \stackrel{\circ}{1}$ \\
\hline 3298.811 & 300 & 8 & 30305.226 & $8111_{4}-38416_{3}^{\circ}$ & 3251.916 & $2000 \mathrm{r}$ & 200 & 30742.234 & $3687_{2}-34430_{3}^{\circ}$ \\
\hline 3298.697 & 75 & 4 & 30306.273 & $6362_{2}-36668 \stackrel{\circ}{2}$ & 3249.855 & $800 r$ & 25 & 30761.730 & $0_{2}-30761_{3}^{\circ}$ \\
\hline 3298.119 & 75 & 3 & 30311.584 & $7795_{4}^{\circ}-381065$ & 3249.344 & 50 & 3 & 30766.567 & $8243_{2}^{\circ}-39010_{3}$ \\
\hline 3298.050 & $500 \mathrm{r}$ & 40 & 30312.218 & $4961_{4}-352738$ & 3248.790 & 3 & & 30771.813 & $4961_{4}-35733^{\circ}$ \\
\hline 3296.901 & $30 \mathrm{~h}$ & 2 & 30322.782 & $5563_{1}-35885_{2}^{\circ}$ & 3247.331 & 15 & & 30785.639 & $11241_{3}^{\circ}-42027_{2}$ \\
\hline 3295.444 & 25 & & 30336.188 & $7280_{2}-37616 i$ & 3247.218 & 75 & 4 & 30786.710 & $6362_{2}-37149_{2}^{\circ}$ \\
\hline 3292.926 & 300 & 4 & 30359.384 & $8243{ }_{2}^{\circ}-38602_{2}$ & 3246.319 & 5 & 1 & 30795.235 & $10783_{2}^{\circ}-41578_{2}$ \\
\hline 3292.810 & $50 \mathrm{~b}$ & 4 & 30360.454 & $10526_{3}^{\circ}-40886_{2}$ & 3246.144 & 75 & 4 & 30796.895 & $6362_{2}-37159_{1}^{\circ}$ \\
\hline 3291.037 & 401 & 1 & 30376.810 & $7280_{2}-37656_{3}^{\circ}$ & 3246.116 & 20 & 2 & 30797.161 & $6362_{2}-37159_{3}^{\circ}$ \\
\hline 3289.631 & 1501 & 10 & 30389.792 & $4961_{4}-35351_{4}^{\circ}$ & 3245.994 & 200 & 8 & 30798.319 & $5561_{1}-36361_{1}^{\circ}$ \\
\hline 3289.517 & 150 & 10 & 30390.845 & $7795_{4}^{\circ}-38186_{4}$ & 3245.386 & 3 & 1 & 30804.088 & $7502_{3}-38306_{4}^{\circ}$ \\
\hline 3288.478 & 2 & & 30400.447 & $6362_{2}-36762_{3}^{\circ}$ & 3244.883 & 100 & 5 & 30808.863 & $7280_{2}-38088^{\circ}$ \\
\hline 3288.387 & $300 \mathrm{r}$ & 8 & 30401.288 & $2869_{3}-33270_{4}^{\circ}$ & 3244.628 & 4 & & 30811.284 & $8800_{4}-39611_{3}^{\circ}$ \\
\hline 3286.019 & 20 & 3 & 30423.196 & $3687_{2}-34111^{\circ}$ & 3244.448 & $1000 \mathrm{r}$ & 50 & 30812.994 & $0_{2}-30812_{2}^{\circ}$ \\
\hline 3285.752 & $800 \mathrm{r}$ & 20 & 30425.668 & $2869_{3}-33294_{3}^{\circ}$ & 3244.043 & 150 & 10 & 30816.840 & $10414_{4}^{\circ}-41230_{4}$ \\
\hline 3285.514 & $200 \mathrm{r}$ & 5 & 30427.872 & $2869_{3}-33297_{2}^{\circ}$ & 3243.585 & 40 & 2 & 30821.192 & $118022-42624^{\circ}$ \\
\hline 3283.671 & 100 & 4 & 30444.949 & $11241^{\circ}-416863$ & 3242.145 & 50 & $15 b$ & 30834.880 & $10526_{3}^{\circ}-41361_{3}$ \\
\hline 3283.369 & 8 & 2 & 30447.749 & $7502_{3}-37950_{4}^{\circ}$ & 3240.644 & $400 \mathrm{r}$ & $40 \mathrm{~b}$ & 30849.162 & $2869_{3}-33718_{2}^{\circ}$ \\
\hline 3282.837 & 40 & 2 & 30452.683 & $75023-37954_{3}^{\circ}$ & 3240.236 & 40 & 3 & 30853.046 & $7502_{3}-38355_{2}^{\circ}$ \\
\hline 3282.385 & 50 & 3 & 30456.877 & $7280_{2}-37736_{2}^{\circ}$ & 3238.934 & $400 \mathrm{r}$ & 20 & 30865.448 & $2869_{3}-33734_{2}^{\circ}$ \\
\hline 3282.198 & 8 & 1 & 30458.612 & $38651-34324^{\circ}$ & 3238.287 & 25 & 3 & 30871.615 & $6362_{2}-37234_{2}^{\circ}$ \\
\hline 3281.189 & 300 & 5 & 30467.978 & $75023-37970_{2}^{\circ}$ & 3236.573 & 100 & $8 b$ & 30887.963 & $8111_{4}-38998_{3}^{\circ}$ \\
\hline 3281.049 & $400 \mathrm{r}$ & 25 & 30469.278 & $8111_{4}-38580_{4}^{c}$ & 3234.996 & $400 \mathrm{r}$ & 20 & 30903.020 & $3687_{2}-34591_{1}^{\circ}$ \\
\hline 3280.451 & 20 & & 30474.832 & $63622-36837_{1}^{\circ}$ & 3234.791 & 10 & 2 & 30904.978 & $7795_{4}^{\circ}-38700_{4}$ \\
\hline 3278.733 & 200 & 25 & 30490.800 & $82432_{2}^{\circ}-387343$ & 3233.853 & 5 & 2 & 30913.942 & $7502_{3}-38416_{3}^{\circ}$ \\
\hline 3277.702 & 50 & 3 & 30500.390 & $4961_{4}-35462^{\circ}$ & 3233.240 & 15 & 2 & 30919.803 & $98045-40724{ }^{\circ}$ \\
\hline 3277.062 & 200 & 4 & 30506.347 & $38651-34371_{2}^{\circ}$ & 3232.305 & $500 \mathrm{r}$ & 25 & 30928.747 & $02-30928^{\circ}$ \\
\hline 3276.721 & 300 & 5 & 30509.521 & $6362_{2}-36871_{2}^{\circ}$ & 3232.124 & $300 \mathrm{r}$ & & 30930.479 & $2869_{3}-33799_{4}^{\circ}$ \\
\hline 3276.562 & 25 & 3 & 30511.002 & $8800_{4}-39311^{\circ}$ & 3232.031 & $200 \mathrm{r}$ & & 30931.368 & $2869_{3}-33800_{3}^{\circ}$ \\
\hline 3276.225 & 200 & 8 & 30514.140 & $11241_{3}^{\circ}-41755_{4}$ & 3231.461 & 75 & & 30936.824 & $7280_{2}-38216_{3}^{\circ}$ \\
\hline 3276.045 & 50 & 5 & 30515.817 & $11197{ }^{\circ}-417125$ & 3231.221 & 5 & & 30939.122 & $7795_{4}^{\circ}-38734_{3}$ \\
\hline 3272.301 & 5 & & 30550.730 & $75023-380533_{3}^{\circ}$ & 3230.368 & 25 & 2 & 30947.291 & $10414_{4}^{\circ}-41361_{3}$ \\
\hline 3272.027 & $800 r$ & 75 & 30553.288 & $\mathbf{0}_{2}-30553_{2}^{\circ}$ & 3228.485 & 25 & 3 & 30965.341 & $7795_{4}^{\circ}-38760_{4}$ \\
\hline 3271.892 & $300 \mathrm{r}$ & 100 & 30554.549 & $8111_{4}-38665^{\circ}$ & 3227.820 & 10 & & 30971.720 & $98045-40776_{4}^{\circ}$ \\
\hline 3271.546 & 50 & 3 & 30557.780 & $2869_{3}-33427_{2}^{\circ}$ & 3225.863 & 50 & 8 & 30990.509 & $0_{2}-30990_{3}^{\circ}$ \\
\hline 3271.433 & 2 & & 30558.836 & $111978-41755_{4}$ & 3225.539 & 100 & 8 & 30993.621 & $5563_{1}-36556 i$ \\
\hline 3270.874 & 150 & 10 & 30564.058 & $8243 \stackrel{\circ}{-}-38807_{2}$ & 3225.007 & 100 & 5 & 30998.734 & $7280_{2}-38278 i$ \\
\hline 3270.100 & 100 & 5 & 30571.292 & $7795_{4}^{\circ}-38366_{4}$ & 3223.504 & 8 & 2 & 31013.187 & $3865_{1}-34878 i$ \\
\hline 3269.352 & 3 & & 30578.286 & $10783_{2}^{\circ}-41361_{3}$ & 3223.168 & $200 \mathrm{r}$ & 5 & 31016.420 & $3687_{2}-34704_{3}^{\circ}$ \\
\hline 3268.453 & 300 & 75 & 30586.697 & $7502_{3}-38088_{2}^{\circ}$ & 3222.475 & 101 & & 31023.090 & $11601_{1}-42624^{\circ}$ \\
\hline 3266.635 & $500 \mathrm{r}$ & 50 & 30603.719 & $25580-33161^{\circ}$ & 3220.986 & $75 b$ & & 31037.431 & $3687_{2}-347253$ \\
\hline 3264.859 & 40 & 3 & 30620.366 & $98045-40425_{4}^{\circ}$ & 3219.490 & $20 \mathrm{~h}$ & 4 & 31051.852 & $10526_{3}^{\circ}-41578_{2}$ \\
\hline 3263.183 & $400 \mathrm{r}$ & 20 & 30636.092 & $3687_{2}-34324^{\circ}$ & 3217.265 & 40 & 2 & 31073.326 & $8800_{4}-39873 \circ$ \\
\hline 3259.744 & 25 & 2 & 30668.412 & $8800_{4}-39468_{3}^{\circ}$ & 3216.998 & 75 & 3 & 31075.905 & $0_{2}-31075_{2}^{\circ}$ \\
\hline 3259.061 & $600 \mathrm{r}$ & 50 & 30674.839 & $7280_{2}-37954_{3}^{\circ}$ & 3216.761 & 40 & & 31078.195 & $8243_{2}^{\circ}-393213$ \\
\hline 3258.744 & 100 & 3 & 30677.823 & $8111_{4}-38788^{\circ}$ & 3214.648 & 150 & 5 & 31098.622 & $2869_{3}-33967_{3}^{\circ}$ \\
\hline 3258.274 & 15 & 2 & 30682.248 & $13175_{4}^{\circ}-43857_{5}$ & 3214.381 & $400 \mathrm{r}$ & 25 & 31101.205 & $4961_{4}-36062_{4}^{\circ}$ \\
\hline 3258.103 & $400 \mathrm{r}$ & & 30683.858 & $3687_{2}-34371_{2}^{\circ}$ & 3214.076 & $400 \mathrm{r}$ & 50 & 31104.156 & $25580-33662^{\circ}$ \\
\hline 3257.435 & 200 & 75 & 30690.150 & $7280_{2}-37970_{2}^{\circ}$ & 3213.937 & 8 & 1 & 31105.501 & $5563_{1}-36668_{2}^{\circ}$ \\
\hline
\end{tabular}


TABLE 4. Classified lines of Th I-continued

\begin{tabular}{|c|c|c|c|c|c|c|c|c|c|}
\hline \multirow{2}{*}{$\begin{array}{l}\text { Wavelength } \\
\text { (§) }\end{array}$} & \multicolumn{2}{|c|}{ Intensity } & \multirow{2}{*}{$\begin{array}{l}\text { Wavenumber } \\
\left(\mathrm{cm}^{-1}\right)\end{array}$} & \multirow{2}{*}{ Classification } & \multirow{2}{*}{$\begin{array}{c}\text { Wavelength } \\
\text { (Å) }\end{array}$} & \multicolumn{2}{|c|}{ Intensity } & \multirow{2}{*}{$\begin{array}{l}\text { Wavenumber } \\
\left(\mathrm{cm}^{-1}\right)\end{array}$} & \multirow{2}{*}{ Classification } \\
\hline & Lamp & Spark & & & & Lamp & Spark & & \\
\hline 3213.818 & 3 & & 31106.653 & $8800_{4}-39906_{4}^{\circ}$ & 3152.295 & 100 & 3 & 31713.736 & $2869_{3}-34583_{3}^{\circ}$ \\
\hline 3211.997 & $200 \mathrm{r}$ & 5 & 31124.288 & $3865_{1}-34989^{\circ}$ & 3151.184 & 300 & 5 & 31724.917 & $7795_{4}^{\circ}-39520_{4}$ \\
\hline 3211.195 & $400 \mathrm{r}$ & 25 & 31132.061 & $28699_{3}-34001{ }_{4}^{\circ}$ & 3151.017 & 200 & & 31726.598 & $6362_{2}-38088_{2}^{\circ}$ \\
\hline 3210.779 & 200 & 5 & 31136.095 & $7280_{2}-38416_{3}^{\circ}$ & 3147.449 & 200 & 25 & 31762.562 & $81114-398730^{\circ}$ \\
\hline 3208.971 & 15 & & 31153.636 & $8111_{4}-39264_{3}^{\circ}$ & 3146.310 & $200 \mathrm{r}$ & 8 & 31774.060 & $3687_{2}-35462^{\circ}$ \\
\hline 3208.527 & 100 & 3 & 31157.947 & $7795_{4}^{\circ}-389535$ & 3145.637 & $400 \mathrm{r}$ & 25 & 31780.858 & $0_{2}-31780_{3}^{\circ}$ \\
\hline 3208.152 & 25 & & 31161.589 & $7795_{4}^{\circ}-389564$ & 3144.145 & 100 & 3 & 31795.939 & $8111_{4}-39906^{\circ}$ \\
\hline 3207.938 & 100 & 5 & 31163.668 & $3687_{2}-348511^{\circ}$ & 3143.632 & 1 & & 31801.127 & $4961_{4}-36762^{\circ}$ \\
\hline 3207.548 & 4 & & 31167.457 & $75023-38669_{2}^{\circ}$ & 3142.422 & 100 & 5 & 31813.372 & $3865_{1}-35678^{\circ}$ \\
\hline 3205.696 & 50 & 3 & 31185.462 & $8800_{4}-39985_{3}^{\circ}$ & 3140.272 & $600 \mathrm{r}$ & 50 & 31835.152 & $2869_{3}-34704^{\circ}$ \\
\hline 3205.162 & 3 & & 31190.658 & $3687_{2}-34878_{1}^{\circ}$ & 3139.892 & 150 & 100 & 31839.005 & $4961_{4}-36800_{4}^{\circ}$ \\
\hline 3202.521 & $200 \mathrm{r}$ & 8 & 31216.379 & $4961_{4}-36178_{4}^{\circ}$ & 3139.273 & 150 & & 31845.282 & $3687_{2}-35533^{\circ}$ \\
\hline 3199.669 & 1 & & 31244.202 & $10783_{2}^{\circ}-42027_{2}$ & 3138.359 & 40 & 2 & 31854.556 & $6362_{2}-38216^{\circ}$ \\
\hline 3196.611 & 20 & 2 & 31274.091 & $5563_{1}-36837_{i}^{\circ}$ & 3138.200 & 100 & 10 & 31856.170 & $2869_{3}-34725^{\circ}$ \\
\hline 3195.688 & $400 \mathrm{r}$ & 50 & 31283.123 & $\mathbf{0}_{2}-31283^{\circ}$ & 3136.829 & $400 \mathrm{r}$ & 8 & 31870.093 & $\mathbf{0}_{2}-31870_{2}^{\circ}$ \\
\hline 3194.518 & 5 & & 31294.580 & $6362_{2}-37656_{3}^{\circ}$ & 3136.612 & 10 & & 31872.298 & $10414_{4}^{\circ}-422863$ \\
\hline 3194.096 & 200 & 5 & 31298.715 & $104144^{\circ}-417125$ & 3136.216 & $800 \mathrm{r}$ & 100 & 31876.322 & $4961_{4}-36837_{0}^{\circ}$ \\
\hline 3193.781 & $200 \mathrm{r}$ & 5 & 31301.802 & $3687_{2}-34989_{1}^{\circ}$ & 3133.980 & 200 & 5 & 31899.064 & $7795_{4}^{\circ}-39694_{5}$ \\
\hline 3192.586 & $400 \mathrm{r}$ & 20 & 31313.518 & $49614-36275_{0}^{\circ}$ & 3131.496 & 20 & 2 & 31924.366 & $8800_{4}-407240^{\circ}$ \\
\hline 3190.895 & $150 \mathrm{~b}$ & 2 & 31330.112 & $9804_{5}-41134_{4}^{\circ}$ & 3128.314 & 100 & 3 & 31956.838 & $8243_{2}^{\circ}-402003$ \\
\hline 3189.711 & 200 & 8 & 31341.741 & $10414_{4}^{\circ}-41755_{4}$ & 3126.414 & 200 & 2 & 31976.258 & $8800_{4}-40776_{4}^{\circ}$ \\
\hline 3188.092 & $8 \mathrm{~b}$ & & 31357.656 & $8111_{4}-39468 \stackrel{\circ}{\circ}$ & 3125.812 & 150 & 2 & 31982.416 & $2869_{3}-34851_{2}^{\circ}$ \\
\hline 3186.371 & 75 & 2 & 31374.592 & $6362_{2}-37736_{2}^{\circ}$ & 3125.601 & $500 \mathrm{r}$ & 20 & 31984.575 & $7280_{2}-39264^{\circ}$ \\
\hline 3185.857 & 1 & & 31379.654 & $7795_{4}^{\circ}-391743$ & 3124.986 & 75 & 2 & 31990.869 & $3687_{2}-35678_{1}^{\circ}$ \\
\hline 3184.843 & 300 & 8 & 31389.644 & $7280_{2}-38669_{2}^{\circ}$ & 3122.102 & $15 b$ & & 32020.419 & $3865_{1}-35885_{2}^{\circ}$ \\
\hline 3184.277 & 300 & $15 b$ & 31395.223 & $7280_{2}-38675_{3}^{\circ}$ & 3120.881 & $400 \mathrm{r}$ & 25 & 32032.946 & $25580-34591^{\circ}$ \\
\hline 3181.670 & $500 \mathrm{r}$ & 20 & 31420.947 & $4961_{4}-36382_{4}^{\circ}$ & 3119.963 & 100 & 20 & 32042.371 & $7795_{4}^{\circ}-39837_{3}$ \\
\hline 3180.062 & 100 & 5 & 31436.835 & $8243_{2}^{\circ}-39680_{2}$ & 3119.504 & $200 \mathrm{r}$ & & 32047.085 & $4961_{4}-37008^{\circ}$ \\
\hline 3179.858 & 25 & 2 & 31438.851 & $11197^{\circ}-426355$ & 3118.848 & 15 & 1 & 32053.826 & $6362_{2}-38416^{\circ}$ \\
\hline 3178.622 & 200 & 4 & 31451.076 & $8111_{4}-39562 \stackrel{\circ}{9}$ & 3118.266 & 4 & & 32059.808 & $75023-39562_{4}^{\circ}$ \\
\hline 3178.244 & $600 \mathrm{r}$ & 100 & 31454.816 & $2869_{3}-34324_{2}^{\circ}$ & 3116.352 & $300 \mathrm{r}$ & 10 & 32079.498 & $4961_{4}-37041_{4}^{\circ}$ \\
\hline 3178.018 & 3 & & 31457.053 & $7795_{4}^{\circ}-392525$ & 3116.263 & $500 \mathrm{r}$ & & 32080.414 & $0_{2}-32080_{1}^{\circ}$ \\
\hline 3177.402 & 100 & 2 & 31463.151 & $3865_{1}-35328^{\circ}$ & 3111.982 & 50 & 2 & 32124.544 & $3687_{2}-35812^{\circ}$ \\
\hline 3176.723 & 4 & & 31469.876 & $8800_{4}-40270_{4}^{\circ}$ & 3111.280 & 300 & 8 & 32131.792 & $7280_{2}-39411_{1}^{\circ}$ \\
\hline 3176.167 & $200 \mathrm{r}$ & 5 & 31475.385 & $2869_{3}-34344_{4}^{\circ}$ & 3110.102 & 200 & & 32143.962 & $8111_{4}-40254_{3}^{\circ}$ \\
\hline 3174.840 & 200 & 4 & 31488.540 & $7795_{4}^{\circ}-39283_{4}$ & 3108.636 & 200 & 3 & 32159.120 & $8111_{4}-40270_{4}^{\circ}$ \\
\hline 3174.020 & 300 & 8 & 31496.675 & $7502_{3}-38998_{3}^{\circ}$ & 3108.504 & $300 \mathrm{r}$ & 10 & 32160.485 & $0_{2}-32160_{2}^{\circ}$ \\
\hline 3173.634 & 2 & & 31500.506 & $8111_{4}-39611_{3}^{\circ}$ & 3107.214 & 3 & & 32173.837 & $5563_{1}-37736_{2}^{\circ}$ \\
\hline 3173.427 & $500 \mathrm{r}$ & 50 & 31502.560 & $2869_{3}-34371_{2}^{\circ}$ & 3105.794 & $200 \mathrm{~b}$ & & 32188.546 & $7280_{2}-39468_{3}^{\circ}$ \\
\hline 3172.808 & 8 & & 31508.706 & $7280_{2}-38788^{\circ}$ & 3104.966 & $200 \mathrm{r}$ & 3 & 32197.130 & $0_{2}-32197^{\circ}$ \\
\hline 3171.276 & $500 \mathrm{r}$ & 75 & 31523.927 & $0_{2}-31523^{\circ}$ & 3104.892 & $200 \mathrm{r}$ & 25 & 32197.897 & $4961_{4}-37159_{3}^{\circ}$ \\
\hline 3171.015 & 10 & 3 & 31526.521 & $7795_{4}^{\circ}-39321_{3}$ & 3102.595 & 20 & & 32221.734 & $10414_{4}^{\circ}-426355$ \\
\hline 3170.250 & 5 & 1 & 31534.129 & $7280_{2}-38814_{2}^{\circ}$ & 3099.902 & 201 & & 32249.725 & $8243_{2}^{\circ}-40493_{3}$ \\
\hline 3168.342 & 100 & 2 & 31553.118 & $25580-34111^{\circ}$ & 3099.184 & 100 & 2 & 32257.196 & $7795_{4}^{\circ}-40052_{4}$ \\
\hline 3165.048 & 150 & 3 & 31585.956 & $55631-37149_{2}^{\circ}$ & 3098.726 & $300 \mathrm{r}$ & 5 & 32261.963 & $2869_{3}-35131_{4}^{\circ}$ \\
\hline 3164.386 & 25 & 3 & 31592.564 & $6362_{2}-37954_{3}^{\circ}$ & 3096.498 & 25 & & 32285.176 & $0_{2}-32285_{3}^{\circ}$ \\
\hline 3164.239 & 200 & 3 & 31594.031 & $8243_{2}^{\circ}-398373$ & 3094.373 & 2 & & 32307.346 & $6362_{2}-38669_{2}^{\circ}$ \\
\hline 3163.715 & 200 & 3 & 31599.264 & $0_{2}-31599_{2}^{\circ}$ & 3093.832 & 4 & & 32312.995 & $6362_{2}-38675_{3}^{\circ}$ \\
\hline 3162.856 & $100 \mathrm{~b}$ & & 31607.846 & $6362_{2}-37970_{2}^{\circ}$ & 3093.719 & 200 & 3 & 32314.176 & $8111_{4}-40425_{4}^{\circ}$ \\
\hline 3161.364 & 200 & 5 & 31622.762 & $2869_{3}-34492_{4}^{\circ}$ & 3093.102 & 15 & & 32320.621 & $25580-34878^{\circ}$ \\
\hline 3161.148 & 100 & & 31624.923 & $8800_{4}-40425_{4}^{\circ}$ & 3092.828 & $300 \mathrm{r}$ & 8 & 32323.484 & $3865_{1}-36188^{\circ}$ \\
\hline 3159.577 & 10 & & 31640.647 & $3687_{2}-35328_{1}^{\circ}$ & 3088.567 & $200 \mathrm{r}$ & 10 & 32368.076 & $8243_{2}^{\circ}-40611_{2}$ \\
\hline 3157.221 & $300 \mathrm{r}$ & 8 & 31664.257 & $4961_{4}-36625 \circ$ & 3088.235 & 15 & 1 & 32371.556 & $2869_{3}-35240_{3}^{\circ}$ \\
\hline 3156.866 & 300 & & 31667.818 & $38651-35533_{2}^{\circ}$ & 3086.852 & 50 & 1 & 32386.059 & $3687_{2}-36074_{3}^{\circ}$ \\
\hline 3152.395 & 100 & 3 & 31712.730 & $0_{2}-31712 i$ & 3085.084 & $3 b$ & & 32404.618 & $7502_{3}-39906^{\circ}$ \\
\hline
\end{tabular}


TABLE 4. Classified lines of $\mathrm{Th} \mathrm{I}$-continued

\begin{tabular}{|c|c|c|c|c|c|c|c|c|c|}
\hline \multirow{2}{*}{$\begin{array}{l}\text { Wavelength } \\
\text { (§) }\end{array}$} & \multicolumn{2}{|c|}{ Intensity } & \multirow{2}{*}{$\begin{array}{l}\text { Wavenumber } \\
\quad\left(\mathrm{cm}^{-1}\right)\end{array}$} & \multirow{2}{*}{ Classification } & \multirow{2}{*}{$\begin{array}{c}\text { Wavelength } \\
\text { (§̊) }\end{array}$} & \multicolumn{2}{|c|}{ Inten sity } & \multirow{2}{*}{$\begin{array}{c}\text { Wavenumber } \\
\left(\mathrm{cm}^{-1}\right)\end{array}$} & \multirow{2}{*}{ Classification } \\
\hline & Lamp & Spark & & & & Lamp & Spark & & \\
\hline 3085.038 & 4 & & 32405.101 & $7795_{4}^{\circ}-402003$ & 3025.448 & $200 \mathrm{r}$ & 2 & 33043.334 & $0_{2}-33043_{3}^{\circ}$ \\
\hline 3083.003 & $75 b$ & & 32426.490 & $6362_{2}-38788^{\circ}$ & 3024.883 & 150 & & 33049.506 & $6362_{2}-39411^{\circ}$ \\
\hline 3082.507 & $150 \mathrm{r}$ & 3 & 32431.707 & $25580-34989^{\circ}$ & 3022.567 & 3 & 1 & 33074.829 & $3687_{2}-36762_{3}^{\circ}$ \\
\hline 3080.593 & 3 & & 32451.857 & $63622-38814_{2}^{\circ}$ & 3021.056 & 300 & 1 & 33091.370 & $4961_{4}-38053_{3}^{\circ}$ \\
\hline 3078.100 & 3 & & 32478.139 & $63622-38840 i$ & 3018.644 & 100 & & 33117.810 & $8243_{2}^{\circ}-41361_{3}$ \\
\hline 3077.718 & $300 \mathrm{r}$ & 5 & 32482.170 & $28693-35351_{4}^{\circ}$ & 3015.785 & 40 & 3 & 33149.205 & $3687_{2}-36837_{i}^{\circ}$ \\
\hline 3077.600 & 25 & 1 & 32483.415 & $75023-39985_{3}^{\circ}$ & 3014.641 & $150 \mathrm{r}$ & 1 & 33161.784 & $0_{2}-33161^{\circ}$ \\
\hline 3076.410 & 150 & 30 & 32495.980 & $38651-36361_{1}^{\circ}$ & 3012.630 & 150 & & 33183.919 & $3687_{2}-36871_{2}^{\circ}$ \\
\hline 3075.938 & 5 & & 32500.966 & $3687_{2}-36188_{2}^{\circ}$ & 3010.736 & $200 \mathrm{r}$ & 2 & 33204.794 & $2869_{3}-36074_{3}^{\circ}$ \\
\hline 3075.687 & 100 & 3 & 32503.618 & $\mathbf{0}_{2}-32503_{2}^{\circ}$ & 3007.476 & 100 & 1 & 33240.785 & $111977^{\circ}-444376$ \\
\hline 3073.589 & 2 & & 32525.804 & $5563_{1}-38088_{2}^{\circ}$ & 3006.720 & 3 & & 33249.143 & $63622-39611 \stackrel{\circ}{3}$ \\
\hline 3068.908 & $300 \mathrm{r}$ & 5 & 32575.414 & $\mathbf{0}_{2}-32575_{2}^{\circ}$ & 3006.164 & $300 \mathrm{r}$ & 2 & 33255.292 & $4961_{4}-38216^{\circ}$ \\
\hline 3067.270 & 25 & & 32592.809 & $2869_{3}-35462_{3}^{\circ}$ & 3004.163 & $3 b$ & & 33277.442 & $5563_{1}-38840^{\circ}$ \\
\hline 3067.163 & $100 \mathrm{~b}$ & & 32593.946 & $75023-40096_{2}^{\circ}$ & 3003.605 & 200 & 1 & 33283.624 & $3865_{1}-37149_{2}^{\circ}$ \\
\hline 3065.683 & $200 \mathrm{r}$ & 2 & 32609.680 & $4961_{4}-37571^{\circ}$ & 3002.687 & $200 \mathrm{r}$ & 2 & 33293.799 & $38651-37159^{\circ}$ \\
\hline 3065.314 & $15 b$ & 4 & 32613.606 & $81114-40724{ }^{\circ}$ & 3002.390 & $100 \mathrm{~b}$ & 10 & 33297.092 & $0_{2}-33297_{2}^{\circ}$ \\
\hline 3064.396 & 2 & & 32623.375 & $38651-36488_{2}^{\circ}$ & 3001.336 & $50 \mathrm{r}$ & & 33308.785 & $2869_{3}-36178_{4}^{\circ}$ \\
\hline 3063.637 & $8 \mathrm{~b}$ & & 32631.457 & $7795_{4}^{\circ}-404264$ & 3000.353 & $100 \mathrm{~b}$ & & 33319.697 & $2869_{3}-36188_{2}^{\circ}$ \\
\hline 3063.157 & $75 b$ & & 32636.570 & $63622-389983$ & 2998.103 & $150 \mathrm{~b}$ & & 33344.702 & $4961_{4}-38306^{\circ}$ \\
\hline 3062.517 & 100 & 10 & 32643.391 & $8243_{2}^{\circ}-40886_{2}$ & 2995.962 & 100 & 1 & 33368.530 & $3865_{1}-37234_{2}^{\circ}$ \\
\hline 3062.454 & $200 \mathrm{r}$ & 2 & 32644.062 & $4961_{4}-37605_{4}^{\circ}$ & 2991.032 & $75 b$ & & 33423.528 & $4961_{4}-38385 \circ$ \\
\hline 3061.812 & $400 \mathrm{r}$ & 3 & 32650.907 & $0_{2}-32650_{i}^{\circ}$ & 2990.717 & 75 & & 33427.048 & $\mathbf{0}_{2}-33427_{2}^{\circ}$ \\
\hline 3060.928 & 100 & & 32660.336 & $111977^{\circ}-43857_{5}$ & 2989.272 & 401 & & 33443.206 & $10414_{4}^{\circ}-438575$ \\
\hline 3060.582 & 75 & & 32664.028 & $2869_{3}-35533_{2}^{\circ}$ & 2988.255 & $300 \mathrm{r}$ & & 33454.587 & $4961_{4}-38416_{3}^{\circ}$ \\
\hline 3060.440 & $500 \mathrm{r}$ & 10 & 32665.543 & $0_{2}-32665^{\circ}$ & 2987.672 & $300 \mathrm{r}$ & 2 & 33461.115 & $3687_{2}-37149_{2}^{\circ}$ \\
\hline 3059.698 & 200 & 1 & 32673.465 & $3687_{2}-36361_{i}^{\circ}$ & 2986.740 & $100 \mathrm{r}$ & 2 & 33471.556 & $3687_{2}-37159^{\circ}$ \\
\hline 3058.029 & $200 \mathrm{r}$ & 2 & 32691.297 & $3865_{1}-36556_{i}^{\circ}$ & 2986.127 & 2 & & 33478.427 & $7502_{3}-40980_{3}^{\circ}$ \\
\hline 3057.651 & 100 & & 32695.338 & $4961_{4}-37656_{3}^{\circ}$ & 2980.112 & $200 \mathrm{r}$ & 2 & 33545.996 & $3687_{2}-37234_{2}^{\circ}$ \\
\hline 3056.693 & 150 & 1 & 32705.585 & $7280_{2}-39985_{3}^{\circ}$ & 2979.035 & 100 & & 33558.123 & $2869_{3}-36427_{3}^{\circ}$ \\
\hline 3053.536 & 200 & 1 & 32739.397 & $3687_{2}-36427_{3}^{\circ}$ & 2978.791 & 75 & 2 & 33560.872 & $7795_{4}^{\circ}-413565$ \\
\hline 3053.462 & 150 & 1 & 32740.190 & $11197 \circ-439376$ & 2978.325 & 100 & 1 & 33566.122 & $7795_{4}^{\circ}-41361_{3}$ \\
\hline 3052.296 & 3 & & 32752.697 & $75023-40254_{3}^{\circ}$ & 2976.103 & $200 \mathrm{r}$ & 3 & 33591.182 & $\mathbf{0}_{2}-33591_{3}^{\circ}$ \\
\hline 3052.128 & 100 & 1 & 32754.500 & $\mathbf{0}_{2}-32754_{3}^{\circ}$ & 2973.675 & 100 & 1 & 33618.608 & $4961_{4}-38580_{4}^{\circ}$ \\
\hline 3050.892 & $4 b$ & & 32767.769 & $75023-40270_{4}^{\circ}$ & 2973.590 & 15 & & 33619.569 & $2869_{3}-36488_{2}^{\circ}$ \\
\hline 3050.631 & $200 \mathrm{r}$ & 2 & 32770.572 & $25580-35328^{\circ}$ & 2972.438 & 10 & & 33632.598 & $75023-41134_{4}^{\circ}$ \\
\hline 3049.054 & $100 \mathrm{~b}$ & & 32787.521 & $7795_{4}^{\circ}-405825$ & 2969.822 & $200 \mathrm{r}$ & 4 & 33662.223 & $0_{2}-33662 i$ \\
\hline 3048.621 & 10 & & 32792.177 & $55631-38355_{2}^{\circ}$ & 2966.662 & 5 & & 33698.077 & $7502_{3}-41200_{2}^{\circ}$ \\
\hline 3047.816 & $500 \mathrm{r}$ & 2 & 32800.838 & $3687_{2}-36488_{2}^{\circ}$ & 2966.441 & 8 & & 33700.587 & $7280_{2}-409803$ \\
\hline 3046.401 & 4 & & 32816.073 & $7280_{2}-40096_{2}^{\circ}$ & 2966.150 & 1001 & 5 & 33703.894 & $4961_{4}-38665 \circ$ \\
\hline 3041.941 & 100 & & 32864.185 & $2869_{3}-35733_{4}^{\circ}$ & 2965.290 & 15 & & 33713.668 & $4961_{4}-38675^{\circ}$ \\
\hline 3041.515 & $300 \mathrm{r}$ & 100 & 32868.788 & $3687_{2}-36556_{1}^{\circ}$ & 2963.521 & 2 & & 33733.792 & $6362_{2}-40096_{2}^{\circ}$ \\
\hline 3041.433 & $40 \mathrm{~b}$ & & 32869.674 & $8111_{4}-40980_{3}^{\circ}$ & 2963.442 & 75 & & 33734.691 & $0_{2}-33734_{2}^{\circ}$ \\
\hline 3038.417 & 1001 & & 32902.300 & $6362_{2}-39264_{3}^{\circ}$ & 2959.137 & 200 & 2 & 33783.767 & $8243 \stackrel{\circ}{2}-420272$ \\
\hline 3038.070 & 100 & & 32906.057 & $7795_{4}^{\circ}-40701_{4}$ & 2957.767 & 75 & 3 & 33799.414 & $2869_{3}-36668_{2}^{\circ}$ \\
\hline 3036.518 & $10 \mathrm{~b}$ & 2 & 32922.875 & $75023-40425_{4}^{\circ}$ & 2957.660 & $100 \mathrm{r}$ & 5 & 33800.637 & $0_{2}-33800_{3}^{\circ}$ \\
\hline 3034.639 & $400 \mathrm{r}$ & 2 & 32943.260 & $2869_{3}-35812_{3}^{\circ}$ & 2957.421 & $10 \mathrm{~b}$ & & 33803.368 & $25580-36361{ }^{\circ}$ \\
\hline 3033.595 & 15 & & 32954.597 & $02-32954_{2}^{\circ}$ & 2955.339 & 10 & & 33827.181 & $4961_{4}-38788{ }^{\circ}$ \\
\hline 3032.016 & $100 \mathrm{r}$ & 2 & 32971.758 & $3865_{1}-36837_{i}^{\circ}$ & 2953.454 & 25 & 1 & 33848.770 & $5563_{1}-39411^{\circ}$ \\
\hline 3031.733 & $75 b$ & & 32974.836 & $7280_{2}-40254_{3}^{\circ}$ & 2951.471 & 50 & 1 & 33871.511 & $38651-377362$ \\
\hline 3031.195 & $100 \mathrm{r}$ & 2 & 32980.688 & $3687_{2}-36668_{2}^{\circ}$ & 2949.736 & 20 & 2 & 33891.433 & $7795_{4}^{\circ}-41686_{3}$ \\
\hline 3030.487 & $400 \mathrm{r}$ & 8 & 32988.393 & $4961_{4}-37950_{4}^{\circ}$ & 2949.639 & 50 & 2 & 33892.547 & $6362_{2}-40254 \stackrel{\circ}{\circ}$ \\
\hline 3030.035 & 75 & & 32993.314 & $4961_{4}-37954_{3}^{\circ}$ & 2949.551 & $100 \mathrm{r}$ & $15 b$ & 33893.559 & $2869_{3}-367623$ \\
\hline 3028.830 & 200 & 2 & 33006.439 & $38651-36871_{2}^{\circ}$ & 2947.462 & 100 & 3 & 33917.579 & $7795_{4}^{\circ}-417125$ \\
\hline 3027.896 & 150 & 3 & 33016.620 & $2869_{3}-35885_{2}^{\circ}$ & 2947.229 & 200 & 25 & 33920.261 & $7280_{2}-412002$ \\
\hline 3027.228 & $200 \mathrm{~b}$ & 15 & 33023.906 & $8111_{4}-41134_{4}^{\circ}$ & 2946.528 & 100 & 20 & 33928.330 & $3687_{2}-37616_{i}^{\circ}$ \\
\hline
\end{tabular}


TABLE 4. Classified lines of Th I-continued

\begin{tabular}{|c|c|c|c|c|c|c|c|c|c|}
\hline \multirow{2}{*}{$\begin{array}{l}\text { Wavelength } \\
\text { (Å) }\end{array}$} & \multicolumn{2}{|c|}{ Intensity } & \multirow{2}{*}{$\begin{array}{l}\text { Wavenumber } \\
\left(\mathrm{cm}^{-1}\right)\end{array}$} & \multirow{2}{*}{ Classification } & \multirow{2}{*}{$\begin{array}{c}\text { Wavelength } \\
\text { (§) }\end{array}$} & \multicolumn{2}{|c|}{ Intensity } & \multirow{2}{*}{$\begin{array}{l}\text { Wavenumber } \\
\left(\mathrm{cm}^{-1}\right)\end{array}$} & \multirow{2}{*}{ Classification } \\
\hline & Lamp & Spark & & & & Lamp & Spark & & \\
\hline 2946.258 & $200 \mathrm{r}$ & 50 & 33931.439 & $2869_{3}-36800_{4}^{\circ}$ & 2838.483 & $300 \mathrm{r}$ & 2 & 35219.729 & $2869_{3}-38088_{2}^{\circ}$ \\
\hline 2943.729 & 400 & 3 & 33960.589 & $7795_{4}^{\circ}-41755_{4}$ & 2836.783 & 3 & & 35240.834 & $0_{2}-35240_{3}^{\circ}$ \\
\hline 2943.095 & $300 \mathrm{r}$ & 2 & 33967.904 & $\mathbf{0}_{2}-33967_{3}^{\circ}$ & 2832.568 & 100 & & 35293.271 & $4961_{4}-40254_{3}^{\circ}$ \\
\hline 2943.000 & $300 \mathrm{r}$ & 20 & 33969.001 & $3687_{2}-37656_{3}^{\circ}$ & 2831.146 & 20 & 4 & 35310.997 & $3687_{2}-38998_{3}^{\circ}$ \\
\hline 2940.432 & $40 \mathrm{~h}$ & & 33998.666 & $25580-36556^{\circ}$ & 2829.732 & $150 \mathrm{r}$ & 2 & 35328.641 & $0_{2}-35328{ }^{\circ}$ \\
\hline 2940.088 & $800 \mathrm{r}$ & 50 & 34002.644 & $2869_{3}-36871_{2}^{\circ}$ & 2828.502 & 2001 & 4 & 35344.003 & $7280_{2}-42624^{\circ}$ \\
\hline 2937.095 & 5 & & 34037.292 & $4961_{4}-38998_{3}^{\circ}$ & 2828.206 & $150 \mathrm{r}$ & 4 & 35347.702 & $2869_{3}-38216_{3}^{\circ}$ \\
\hline 2936.617 & $150 \mathrm{~h}$ & 3 & 34042.832 & $8243_{2}^{\circ}-422863$ & 2821.072 & $100 \mathrm{~s}$ & 2 & 35437.086 & $2869_{3}-38306_{4}^{\circ}$ \\
\hline 2936.089 & 500 & 5 & 34048.954 & $3687_{2}-37736_{2}^{\circ}$ & 2818.971 & 15 & & 35463.496 & $4961_{4}-40425^{\circ}$ \\
\hline 2931.663 & $150 \mathrm{~b}$ & 25 & 34100.356 & $4961_{4}-39062 \circ$ & 2817.180 & 5 & & 35486.041 & $2869_{3}-38355_{2}^{\circ}$ \\
\hline 2931.281 & $300 \mathrm{r}$ & 2 & 34104.800 & $3865_{1}-37970_{2}^{\circ}$ & 2813.434 & 100 & & 35533.287 & $0_{2}-35533_{2}^{\circ}$ \\
\hline 2930.735 & 4 & & 34111.153 & $\mathbf{0}_{2}-34111$ i & 2812.395 & $8 b$ & & 35546.414 & $38651-39411^{\circ}$ \\
\hline 2925.526 & $500 \mathrm{r}$ & 5 & 34171.887 & $28699_{3}-37041_{4}^{\circ}$ & 2810.002 & 100 & & 35576.683 & $3687_{2}-39264_{3}^{\circ}$ \\
\hline 2921.560 & $15 b$ & & 34218.273 & $7502_{3}-41720_{2}^{\circ}$ & 2805.228 & 4 & 1 & 35637.226 & $5563_{1}-41200_{2}^{\circ}$ \\
\hline 2916.369 & $500 \mathrm{r}$ & 10 & 34279.177 & $25580-36837^{\circ}$ & 2801.957 & 25 & & 35678.826 & $0_{2}-35678^{\circ}$ \\
\hline 2916.313 & $75 b$ & 2 & 34279.835 & $2869_{3}-37149_{2}^{\circ}$ & 2799.432 & 100 & 1 & 35711.006 & $2869_{3}-38580_{4}^{\circ}$ \\
\hline 2916.105 & 100 & 2 & 34282.280 & $3687_{2}-37970_{2}^{\circ}$ & 2798.421 & 75 & & 35723.907 & $3687_{2}-39411^{\circ}$ \\
\hline 2915.424 & 100 & 2 & 34290.287 & $2869_{3}-37159_{3}^{\circ}$ & 2795.368 & 100 & & 35762.921 & $4961_{4}-407248$ \\
\hline 2912.553 & 100 & 3 & 34324.087 & $0_{2}-34324_{2}^{\circ}$ & 2792.433 & 15 & 1 & 35800.508 & $2869_{3}-38669_{2}^{\circ}$ \\
\hline 2910.390 & 150 & 2 & 34349.595 & $4961_{4}-39311_{4}^{\circ}$ & 2791.999 & 50 & 1 & 35806.073 & $2869_{3}-38675_{3}^{\circ}$ \\
\hline 2909.103 & $300 \mathrm{~d}$ & $8 d$ & 34364.791 & $2869_{3}-37234_{2}^{\circ}$ & 2791.499 & $100 \mathrm{~b}$ & & 35812.486 & $0_{2}-35812^{\circ}$ \\
\hline 2909.080 & $50 \mathrm{~b}$ & $5 b$ & 34365.063 & $3687_{2}-38053 \stackrel{0}{3}$ & 2782.871 & 100 & & 35923.513 & $3687_{2}-39611^{\circ}$ \\
\hline 2908.507 & $200 \mathrm{r}$ & 20 & 34371.833 & $0_{2}-34371_{2}^{\circ}$ & 2781.209 & 50 & & 35944.979 & $2869_{3}-38814_{2}^{\circ}$ \\
\hline 2902.714 & 300 & 2 & 34440.426 & $7280_{2}-41720_{2}^{\circ}$ & 2775.488 & 100 & & 36019.067 & $4961_{4}-40980_{3}^{\circ}$ \\
\hline 2898.556 & 100 & 2 & 34489.829 & $3865_{1}-38355_{2}^{\circ}$ & 2772.176 & 4 & & 36062.098 & $7795_{4}^{\circ}-43857_{5}$ \\
\hline 2898.443 & $8 \mathrm{~b}$ & & 34491.174 & $7795_{4}^{\circ}-422863$ & 2771.257 & 75 & & 36074.056 & $0_{2}-36074_{3}^{\circ}$ \\
\hline 2897.112 & $40 \mathrm{~b}$ & $20 \mathrm{~b}$ & 34507.019 & $4961_{4}-39468_{3}^{\circ}$ & 2766.988 & 50 & & 36129.709 & $2869_{3}-38998^{\circ}$ \\
\hline 2895.271 & 150 & 3 & 34528.960 & $3687_{2}-38216_{3}^{\circ}$ & 2763.655 & $40 \mathrm{~b}$ & & 36173.280 & $49614-41134^{\circ}$ \\
\hline 2890.746 & 40 & & 34583.007 & $0_{2}-34583_{3}^{\circ}$ & 2762.458 & $100 \mathrm{r}$ & & 36188.953 & $0_{2}-36188_{2}^{\circ}$ \\
\hline 2890.078 & $150 \mathrm{r}$ & 5 & 34591.000 & $02-34591 i$ & 2759.272 & 8 & & 36230.737 & $3865_{1}-40096_{2}^{\circ}$ \\
\hline 2889.290 & $150 \mathrm{r}$ & 15 & 34600.433 & $4961_{4}-39562^{\circ}$ & 2756.912 & 3 & & 36261.750 & $6362_{2}-42624^{\circ}$ \\
\hline 2885.169 & 75 & & 34649.852 & $4961_{4}-39611_{3}^{\circ}$ & 2755.336 & 75 & & 36282.490 & $2558 \mathrm{o}-38840^{\circ}$ \\
\hline 2883.713 & $200 w$ & 2 & 34667.346 & $3687_{2}-38355_{2}^{\circ}$ & 2754.179 & 8 & & 36297.731 & $3687_{2}-39985^{\circ}$ \\
\hline 2880.632 & $200 \mathrm{r}$ & 3 & 34704.423 & $0_{2}-34704_{3}^{\circ}$ & 2749.351 & 50 & 2 & 36361.468 & $02-36361 i$ \\
\hline 2878.657 & $150 \mathrm{~b}$ & & 34728.232 & $3687_{2}-38416_{3}^{\circ}$ & 2746.786 & 100 & & 36395.421 & $2869_{3}-39264_{3}^{\circ}$ \\
\hline 2877.974 & 5 & & 34736.473 & $2869_{3}-37605_{4}^{\circ}$ & 2744.376 & 20 & & 36427.381 & $0_{2}-36427_{3}^{\circ}$ \\
\hline 2873.734 & $75 b$ & & 34787.722 & $2869_{3}-37656_{3}^{\circ}$ & 2743.274 & 3 & & 36442.013 & $2869_{3}-39311_{4}^{\circ}$ \\
\hline 2869.589 & 2 & & 34837.969 & $6362_{2}-41200_{2}^{\circ}$ & 2739.753 & 50 & & 36488.844 & $0_{2}-36488_{2}^{\circ}$ \\
\hline 2869.374 & 5 & & 34840.580 & $7795_{4}^{\circ}-42635_{5}$ & 2734.661 & 40 & 2 & 36556.783 & $0_{2}-36556^{\circ}$ \\
\hline 2868.460 & $300 \mathrm{r}$ & 10 & 34851.681 & $0_{2}-34851_{2}^{\circ}$ & 2733.900 & 50 & & 36566.959 & $3687_{2}-40254_{3}^{\circ}$ \\
\hline 2867.141 & $40 \mathrm{~b}$ & & 34867.713 & $2869_{3}-37736_{2}^{\circ}$ & 2726.317 & 201 & & 36668.661 & $0_{2}-36668_{2}^{\circ}$ \\
\hline 2866.241 & 40 & & 34878.661 & $0_{2}-34878_{i}^{\circ}$ & 2724.522 & 25 & & 36692.818 & $2869_{3}-39562_{4}^{\circ}$ \\
\hline 2863.510 & 10 & & 34911.924 & $4961_{4}-398730^{\circ}$ & 2720.857 & 50 & & 36742.241 & $2869_{3}-39611_{3}^{\circ}$ \\
\hline 2860.776 & 100 & 2 & 34945.287 & $4961_{4}-39906^{\circ}$ & 2719.333 & $.15 b$ & & 36762.831 & $0_{2}-36762^{\circ}$ \\
\hline 2860.491 & 150 & 3 & 34948.769 & $38651-38814_{2}^{\circ}$ & 2713.843 & 75 & & 36837.197 & $0_{2}-36837^{\circ}$ \\
\hline 2857.336 & 10 & & 34987.356 & $3687_{2}-38675_{3}^{\circ}$ & 2712.620 & 2 & & 36853.804 & $25580-39411^{\circ}$ \\
\hline 2857.138 & 100 & 2 & 34989.781 & $0_{2}-34989_{1}^{\circ}$ & 2711.288 & 5 & & 36871.909 & $0_{2}-36871_{2}^{\circ}$ \\
\hline 2854.342 & 300 & 3 & 35024.054 & $4961_{4}-39985_{3}^{\circ}$ & 2699.153 & 2 & & 37037.670 & $2869_{3}-39906_{4}^{\circ}$ \\
\hline 2851.558 & $50 \mathrm{r}$ & 4 & 35058.246 & $25580-37616_{i}^{\circ}$ & 2693.425 & $50 \mathrm{~h}$ & & 37116.433 & $2869_{3}-39985_{3}^{\circ}$ \\
\hline 2849.725 & 10 & 1 & 35080.796 & $2869_{3}-37950_{4}^{\circ}$ & 2691.057 & $200 \mathrm{r}$ & & 37149.091 & $0_{2}-37149_{2}^{\circ}$ \\
\hline 2849.326 & 100 & 25 & 35085.708 & $2869_{3}-37954_{3}^{\circ}$ & 2690.319 & 10 & & 37159.281 & $0_{2}-37159^{\circ}$ \\
\hline 2848.084 & $300 \mathrm{r}$ & 2 & 35101.007 & $2869_{3}-37970_{2}^{\circ}$ & 2685.426 & 3 & & 37226.984 & $2869_{3}-40096_{2}^{\circ}$ \\
\hline 2846.036 & 150 & 2 & 35126.265 & $3687_{2}-38814_{2}^{\circ}$ & 2680.688 & $100 \mathrm{r}$ & & 37292.777 & $3687_{2}-409803$ \\
\hline 2843.906 & $300 \mathrm{r}$ & 2 & 35152.572 & $3687_{2}-38840_{1}^{\circ}$ & 2677.666 & 10 & & 37334.863 & $3865_{1}-41200_{2}^{\circ}$ \\
\hline 2841.383 & $150 \mathrm{~h}$ & 2 & 35183.784 & $2869_{3}-38053 \stackrel{\circ}{3}$ & 2674.029 & 3 & & 37385.640 & $2869_{3}-402543$ \\
\hline
\end{tabular}


TABLE 4. Classified lines of $\mathrm{Th} \mathrm{I}$-continued

\begin{tabular}{|c|c|c|c|c|c|c|c|c|c|}
\hline \multirow{2}{*}{$\begin{array}{l}\text { Wavelength } \\
\text { (§) }\end{array}$} & \multicolumn{2}{|c|}{ Intensity } & \multirow{2}{*}{$\begin{array}{l}\text { Wavenumber } \\
\left(\mathrm{cm}^{-1}\right)\end{array}$} & \multirow{2}{*}{ Classification } & \multirow{2}{*}{$\begin{array}{c}\text { Wavelength } \\
\text { (§) }\end{array}$} & \multicolumn{2}{|c|}{ Inten sity } & \multirow{2}{*}{$\begin{array}{l}\text { Wavenumber } \\
\quad\left(\mathrm{cm}^{-1}\right)\end{array}$} & \multirow{2}{*}{ Classification } \\
\hline & Lamp & Spark & & & & Lamp & Spark & & \\
\hline 2664.996 & 8 & & 37512.351 & $3687_{2}-41200_{2}^{\circ}$ & 2577.288 & $75 r$ & 3 & 38788.861 & $0_{2}-38788^{\circ}$ \\
\hline 2657.633 & 3 & & 37616.274 & $0_{2}-37616_{i}^{\circ}$ & 2575.599 & 3 & & 38814.295 & $0_{2}-38814_{2}^{\circ}$ \\
\hline 2654.762 & 4 & & 37656.952 & $0_{2}-37656^{\circ}$ & 2573.854 & 3 & & 38840.609 & $0_{2}-38840$ i \\
\hline 2640.865 & 10 & & 37855.102 & $3865_{1}-41720_{2}^{\circ}$ & 2563.402 & 2 & & 38998.967 & $0_{2}-38998_{3}^{\circ}$ \\
\hline 2633.914 & 2 & & 37954.997 & $0_{2}-37954_{3}^{\circ}$ & 2546.052 & 100 & & 39264.707 & $02-39264_{3}^{\circ}$ \\
\hline 2632.854 & 20 & 1 & 37970.277 & $0_{2}-37970_{2}^{\circ}$ & 2536.540 & 25 & & 39411.940 & $0_{2}-39411$ i \\
\hline 2627.128 & 5 & & 38053.031 & $0_{2}-380533^{\circ}$ & 2523.760 & 25 & & 39611.503 & $02-39611_{3}^{\circ}$ \\
\hline 2624.647 & $100 \mathrm{r}$ & & 38088.999 & $0_{2}-38088_{2}^{\circ}$ & 2500.142 & 10 & & 39985.674 & $0_{2}-39985_{3}^{\circ}$ \\
\hline 2623.100 & $100 \mathrm{r}$ & & 38111.461 & $2869_{3}-40980_{3}^{\circ}$ & 2495.126 & 10 & & 40066.053 & $25580-42624 i$ \\
\hline 2615.858 & 5 & & 38216.966 & $0_{2}-38216^{\circ}$ & 2493.250 & 100 & & 40096.198 & $0_{2}-40096_{2}^{\circ}$ \\
\hline 2611.626 & 15 & 2 & 38278.891 & $02-38278_{1}^{\circ}$ & 2483.418 & 50 & & 40254.930 & $0_{2}-40254_{3}^{\circ}$ \\
\hline 2606.425 & 20 & & 38355.270 & $02-38355_{2}^{\circ}$ & 2439.433 & $150 \mathrm{r}$ & & 40980.707 & $0_{2}-40980_{3}^{\circ}$ \\
\hline 2602.290 & 4 & & 38416.212 & $02-38416_{3}^{\circ}$ & 2345.372 & 50 & & 42624.104 & $0_{2}-42624_{i}^{\circ}$ \\
\hline 2585.224 & 3 & & 38669.796 & $02-38669_{2}^{\circ}$ & & & & & \\
\hline 2584.851 & 2 & & 38675.375 & $0_{2}-38675_{3}^{\circ}$ & & & & & \\
\hline
\end{tabular}

The late Mrs. Ruth L. Peterson carried out the data processing work. It is a pleasure to record recognition of her work. The author is grateful to S. Tomkins and M. Fred for making it possible to observe the Zeeman effect at Argonne National Laboratory; to S. P. Davis for furnishing spectrograms; and to Lucy Hagan, Charles H. Corliss, and William C. Martin for their suggestions and reviewing of the mansucript. The manuscript was typed by Miss Marsha C. Ahalt; her assistance is appreciated.

\section{References}

Avni, R., and Klein, F. S., Spectrochim. Acta 28B, 331 (1973).

Brewer, L., J. Opt. Soc. Am. 61, 1101 (1971).

Charles, G. W., A Compilation of Data on Some Spectra of Thorium, Oak Ridge National Laboratory, ORNL-2319 (1958).

Giacchetti, A., Argonne National Laboratory, Report, ANL-7209 (1966).

Giacchetti, A., and Blaise, J., European Group for Atomic Spectroscopy, Paper 57 (1970).

Giacchetti, A., Blaise, J., Corliss, C. H., and Zalubas, R., J. Res.

Nat. Bur. Stand. (U.S.), 78A (Phys. and Chem.), No. 2, 247 -249 (Mar.-Apr. 1947).
Giacchetti, A., Stanley, R. W., and Zalubas, R., J. Opt. Soc. Am. 69, 474 (1970).

Ionov, N. I., and Mittsev, M. A., Soviet Phys.-JETP 13, 513 (1961). Klinkenberg, P. F. A., Physica 16, 618 (1950).

Lier, J. N., Disertation University of Amsterdam, Holland (1939).

Martin, W. C., J. Opt. Soc. Am. 53, 1047 (1963).

Meggers, W. F., Corliss, C. H., and Scribner, B. F., Nat. Bur. Stand. (U.S.) Monogr. 32, Part I, 473 pp. (1961).

Moore, B. E., Astrophys. J. 30, 143 (1909).

Rauh, E. G., and Ackermann, J. Chem. Phys. 60, 1396 (1973).

Schuurmans, Ph. D. Thesis, Univ. Amsterdam (1946).

Smith, D. H., and Hertel, G. R., J. Chem. Phys. 51, 3105 (1969).

Steers, E. B. M., Spectrochim. Acta 23B, 135 (1967).

Stukenbroeker, G. L., and McNally, J. R., Jr., J. Opt. Soc. Am. 43 36 (1953).

Sugar, J., private communication (1970).

Sugar, J., J. Chem. Phys. 59, 788 (1973).

Sugar, J., J. Chem. Phys. 60, 4103 (1974).

Tech, J. L., private communication (1967).

Trees, R. E., Physica 26, 353 (1960).

Zalubas, R., J. Res. Nat. Bur. Stand. (U.S.) 63A (Phys. and Chem.), No. 3, 275-278 (Nov.-Dec. 1959).

Zalubas, R., Nat. Bur. Stand. (U.S.), Monogr. 17, 130 (1960).

Zalubas, R., J. Opt. Soc. Am. 58, 1195 (1968).

Zalubas, R., and Corliss, C. H., J. Res. Nat. Bur. Stand. (U.S.), 78A (Phys. and Chem.), No. 2, 163-249 (Mar.-Apr. 1974).

(Paper 80A2-889) 